Adamczak-Krysztofowicz/Szczepaniak-Kozak/Rybszleger (Hrsg.)

\title{
Angewandte Linguistik - Neue Herausforderungen und Konzepte
}

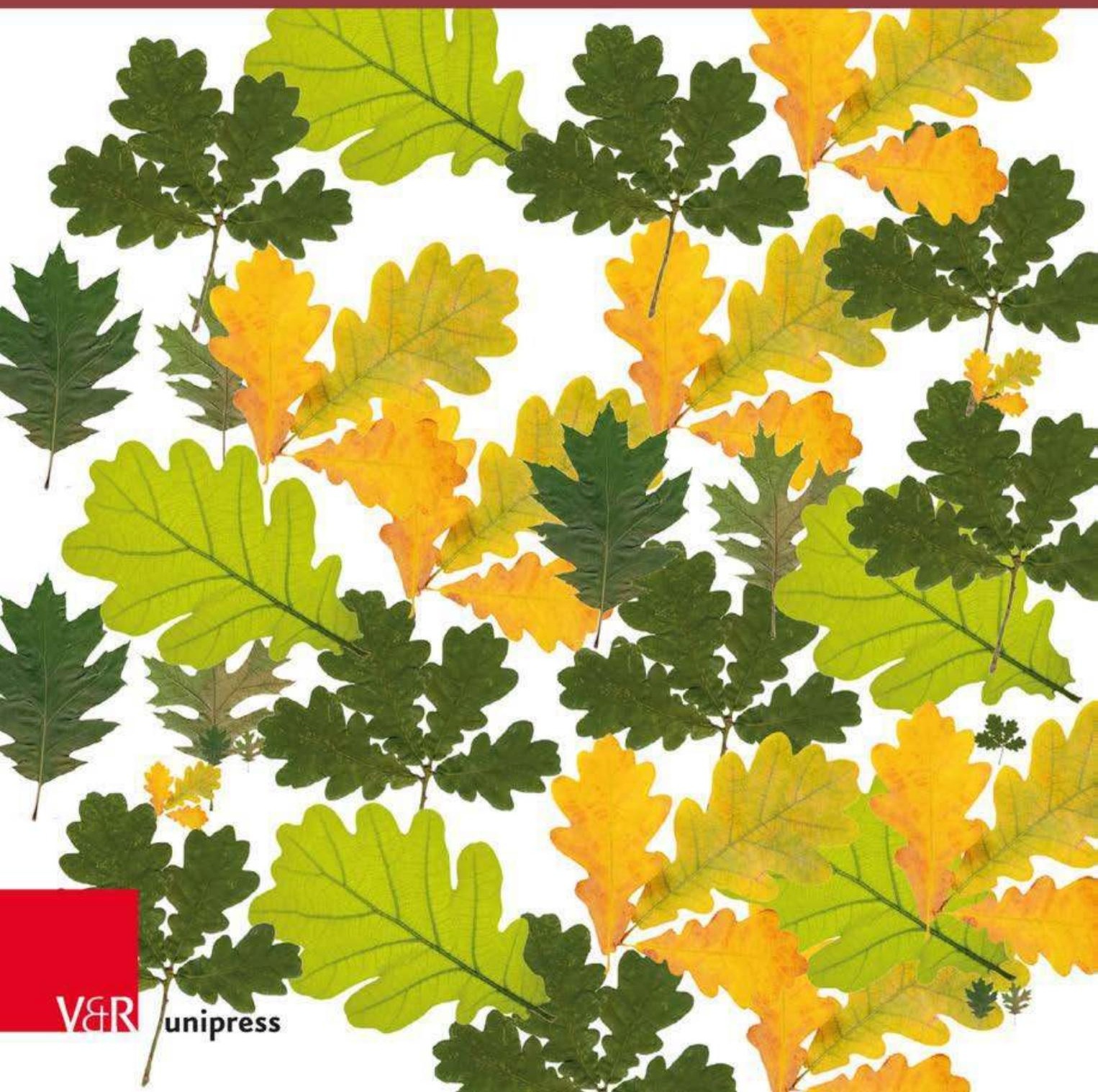




\section{V\&R unipress}

Open-Access-Publikation im Sinne der CC-Lizenz BY-NC-ND 4.0

() 2020, Vandenhoeck \& Ruprecht GmbH \& Co. KG, Göttingen

ISBN Print: 9783847111863 - ISBN E-Lib: 9783737011860 
Open-Access-Publikation im Sinne der CC-Lizenz BY-NC-ND 4.0

(C) 2020, Vandenhoeck \& Ruprecht GmbH \& Co. KG, Göttingen ISBN Print: 9783847111863 - ISBN E-Lib: 9783737011860 
Sylwia Adamczak-Krysztofowicz I

Anna Szczepaniak-Kozak /

Paweł Rybszleger (Hrsg.)

\section{Angewandte Linguistik - \\ Neue Herausforderungen und Konzepte}

Festschrift für Izabela Prokop zum 70. Geburtstag

Mit drei Abbildungen

V\&R unipress 
Bibliografische Information der Deutschen Nationalbibliothek

Die Deutsche Nationalbibliothek verzeichnet diese Publikation in der Deutschen

Nationalbibliografie; detaillierte bibliografische Daten sind im Internet über

https://dnb.de abrufbar.

Diese Publikation wurde von der Adam-Mickiewicz-Universität Poznań finanziell unterstützt.

Diese Publikation ist peer-reviewed (Begutachter: Prof. Roman Lewicki, Państwowa Wyższa Szkoła Zawodowa Konin).

(C) 2020, Vandenhoeck \& Ruprecht GmbH \& Co. KG, Theaterstraße 13, D-37073 Göttingen Dieses Werk ist als Open-Access-Publikation im Sinne der Creative-Commons-Lizenz BY-NC-ND International 4.0 („Namensnennung - Nicht kommerziell - Keine Bearbeitungen“) unter dem DOI 10.14220/9783737011860 abzurufen. Um eine Kopie dieser Lizenz zu sehen, besuchen Sie https://creativecommons.org/licenses/by-nc-nd/4.0/.

Jede Verwertung in anderen als den durch diese Lizenz zugelassenen Fällen bedarf der vorherigen schriftlichen Einwilligung des Verlages.

Umschlagabbildung: ㄷ Paweł Rybszleger

Vandenhoeck \& Ruprecht Verlage | www.vandenhoeck-ruprecht-verlage.com

ISBN 978-3-7370-1186-0 


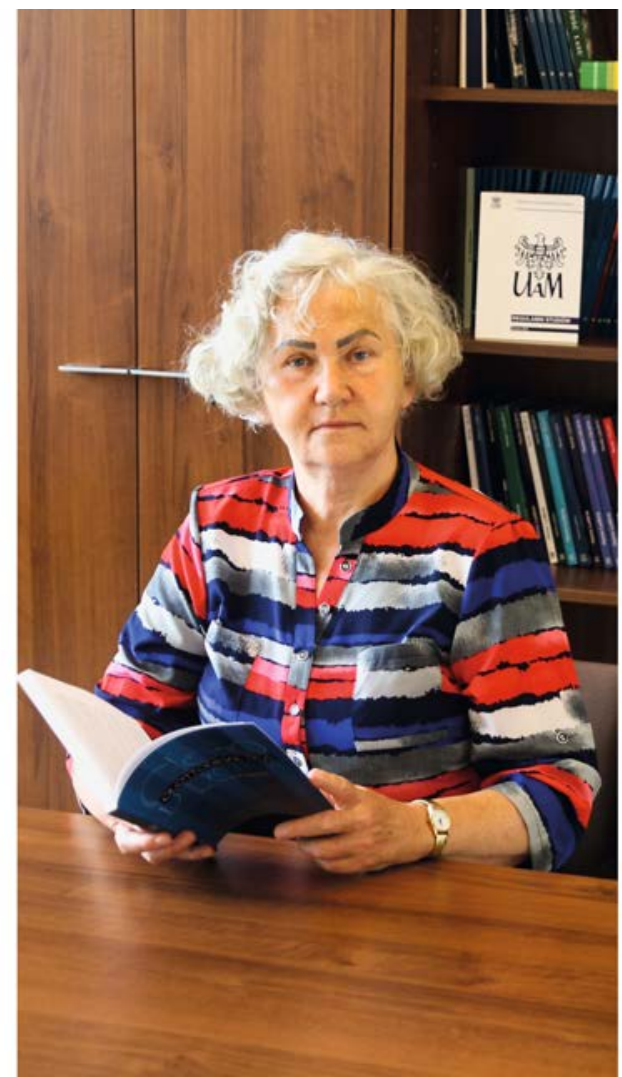

Prof. Dr. habil. Izabela Prokop

Professor Izabela Prokop zu ihrem 70. Geburtstag gewidmet

HerausgeberInnen und AutorInnen 
Open-Access-Publikation im Sinne der CC-Lizenz BY-NC-ND 4.0

(C) 2020, Vandenhoeck \& Ruprecht GmbH \& Co. KG, Göttingen ISBN Print: 9783847111863 - ISBN E-Lib: 9783737011860 


\section{Inhalt}

Tabula Gratulatoria . . . . . . . . . . . . . . 11

Wissenschaftlicher Werdegang von Frau Professor Izabela Prokop . . . 15

Liste der Veröffentlichungen von Frau Professor Izabela Prokop ～. . . . 19

Sylwia Adamczak-Krysztofowicz / Anna Szczepaniak-Kozak /

Paweł Rybszleger (Uniwersytet im. Adama Mickiewicza w Poznaniu)

Neue Herausforderungen und aktuelle Konzepte der Angewandten

Linguistik - einleitende Bemerkungen . . . . . . . . . . . . . . 29

\section{Teil 1: Pragma- und Soziolinguistikstudien}

Barbara Kryk-Kastovsky (Universität Wien)

Impoliteness as a result of power asymmetry in selected social contexts $\quad$. 41

Anna Szczepaniak-Kozak

(Uniwersytet im. Adama Mickiewicza w Poznaniu)

Pragmatic accent: Sociolinguistic and pragmalinguistic examples . . . . 57

Anna Pieczyńska-Sulik (Uniwersytet im. Adama Mickiewicza w Poznaniu)

Ikonische Dimensionen der Sprachpragmatik am Beispiel

von Repräsentativa . . . . . . . . . . . . . . . 7

Paweł Rybszleger (Uniwersytet im. Adama Mickiewicza w Poznaniu)

Pragmatik von Hashtags am Beispiel von Twitter als multimodales

Diskurssystem ........................ 87 


\section{Teil 2: Kontrastive Studien}

Norbert Nübler (Christian-Albrechts-Universität zu Kiel)

Der polnische Verbalaspekt im Fremdsprachenunterricht . . . . . . 105

Magdalena Jurewicz / Martyna Gabrych / Justyna Sobańska

(Uniwersytet im. Adama Mickiewicza w Poznaniu)

Sprachliches Weltbild in ausgewählten gegenwärtigen polnischen und deutschen parallelen Gebrauchshypertexten . . . . . . . . . . . 121

Elżbieta Dziurewicz (Uniwersytet im. Adama Mickiewicza w Poznaniu) Idiome im deutsch-polnischen Kontrast als Herausforderung für Studierende der Angewandten Linguistik. Ergebnisse einer Umfrage und Postulate für den Einsatz im universitären Unterricht $\ldots$. . . . . . . 137

\section{Teil 3: Interkulturalität}

Sylwia Adamczak-Krysztofowicz (Uniwersytet im. Adama Mickiewicza w Poznaniu) / Krystyna Mihułka (Uniwersytet Rzeszowski) Interkulturelle Kommunikation und Angewandte Linguistik: Zur Positionierung der beiden Disziplinen und ihrer Studienschwerpunkte unter Berücksichtigung der neuen Reform im polnischen Hochschulwesen . . . . . . . . . . . . . . . . . . . . . . . 159

Stephan Wolting (Uniwersytet im. Adama Mickiewicza w Poznaniu) Fremdes und Eigenes als „Fremdes verstehen“ Neuere anwendungsbezogene fremdkulturwissenschaftliche Konzepte in Zeichen und Zeiten der Krise . . . . . . . . . . . . . . . 181

\section{Teil 4: Spracherwebs- und Sprachlehrforschung}

Aldona Sopata / Aleksandra Putowska

(Uniwersytet im. Adama Mickiewicza w Poznaniu)

Polnisch als Herkunftssprache in Deutschland - Einfluss der

Familiensprachenpolitik auf die Sprachentwicklung der Kinder . . . . . 203

Małgorzata Bielicka (Uniwersytet im. Adama Mickiewicza w Poznaniu) Immersion als eine innovative Methode der Fremdsprachenvermittlung auf der Elementar- und Primarstufe in Polen . . . . . . . . . . . . 217

Teresa Siek-Piskozub / Aleksandra Wach (Uniwersytet im. Adama Mickiewicza w Poznaniu)

EFL learner agency from the perspective of learners' autobiographies . . . 229 
Katarzyna Bieniecka-Drzymała

(Uniwersytet im. Adama Mickiewicza w Poznaniu)

Fragen, die mehr können? Zum Potential von Coaching-Gesprächen für die Entwicklung der Sprachlernbewusstheit und Lernerautonomie bei Fremdsprachenstudierenden ................ 24

Magdalena Aleksandrzak

(Uniwersytet im. Adama Mickiewicza w Poznaniu)

Developing speaking skills at advanced level - the perspective of language department students and the implications for language teaching . . . . . 257

\section{Teil 5: Fachsprachen}

Klaus-Dieter Baumann (Universität Leipzig)

Thought patterns in natural scientific and technical scientific

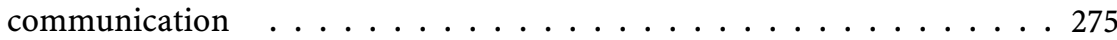

Paweł Kubiak (Uniwersytet im. Adama Mickiewicza w Poznaniu) Politische Begriffe als Herausforderung für die Didaktik der Angewandten Linguistik . . . . . . . . . . . . . . . . . . 295

Joanna Kic-Drgas / Joanna Woźniak

(Uniwersytet im. Adama Mickiewicza w Poznaniu)

Fachsprachendidaktik an europäischen Hochschulen - Stand und

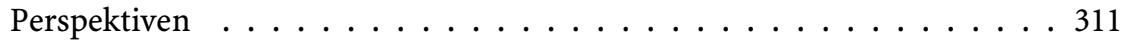

\section{Teil 6: Übersetzen und Dolmetschen}

Joanna Kubaszczyk (Uniwersytet im. Adama Mickiewicza w Poznaniu) Zur Textur des Originalwerkes und der Übersetzung - über die lautlichen und graphematischen Herausforderungen der Übersetzung . . . . . . . . 329

Hanka Błaszkowska (Uniwersytet im. Adama Mickiewicza w Poznaniu) Translation und Transkreation: Zu Differenzen der Begriffssystematik zwischen Theorie und Praxis . . . . . . . . . . . . . . . 343

Alicja Sakaguchi (Uniwersytet im. Adama Mickiewicza w Poznaniu) Die Kunst des Verstehens und Übersetzens sakraler Texte. Ein kritischer Blick auf die Wiedergabe einiger Schlüsselinhalte auf der Grundlage der deutschen, polnischen und Esperanto-Bibelübersetzungen . . . . . . . 355 
Lucyna Krenz-Brzozowska

(Uniwersytet im. Adama Mickiewicza w Poznaniu)

Postulate hinsichtlich der Entwicklung der Fähigkeit zur Selbstbewertung eigener Dolmetschleistungen - Vorschlag einer Feldübung für

Studierende der Angewandten Linguistik mit dem Schwerpunkt

Übersetzen/Dolmetschen . . . . . . . . . . . . . . . . . . . . . . 385

Augustyn Surdyk (Uniwersytet im. Adama Mickiewicza w Poznaniu)

Culture in translation: A comparative analysis of English originals and

Polish dubbed versions of computer-animated film productions . . . . 399

\section{Teil 7: Linguistic landscapes}

Grzegorz Lisek (Universität Greifswald)

Neue Herausforderungen für Angewandte Linguistik -

Sprachlandschaften? Visuelle Mehrsprachigkeit in den Linguistic

Landscapes der deutsch-polnischen Grenzregion . . . . . . . . . . . 417

Danuta Wiśniewska (Uniwersytet im. Adama Mickiewicza w Poznaniu)

Linguistic landscape, murals and language learning . . . . . . . . . 429

Autorenverzeichnis . . . . . . . . . . . . . . . . . 447 


\section{Tabula Gratulatoria}

Sylwia Adamczak-Krysztofowicz, Poznań Ruth Albert, Marburg

Magdalena Aleksandrzak, Poznań

Bolesław Andrzejewski, Poznań

Jarosław Aptacy, Poznań

Jolanta Bachan, Poznań

Catharina Badstübner-Kizik, Poznań

Pascale Bali, Poznań

Sandra Ballweg, Bielefeld

Klaus-Dieter Baumann, Leipzig

Jerzy Bańczerowski, Poznań

Iwona Bartoszewicz, Wrocław

Paweł Bąk, Rzeszów

Marta Bąkiewicz, Poznań

Marwa Belal, Kairo

Sadia Belkhir, Tizi Ouzou

Anna Berezowska, Poznań

Małgorzata Bielicka, Poznań

Katarzyna Bieniecka-Drzymała, Poznań

Zofia Bilut-Homplewicz, Rzeszów

Hanka Błaszkowska, Poznań

Agnieszka Błażek, Poznań

Ines Bose, Halle

Bernhard Brehmer, Greifswald

Halina Bułczyńska-Zgółka, Poznań

Kamila Chmielewska, Poznań

Luiza Ciepielewska-Kaczmarek, Poznań

Lesław Cirko, Wrocław

Małgorzata Czekańska, Poznań

Joanna Czerwińska, Poznań/Bydgoszcz

Maria Dakowska, Warszawa

Andrzej Danielak, Poznań

Ulrich Dausendschön-Gay, Bielefeld

Igor Deiana, Perugia
Kamil Długosz, Poznań

Dmitrij Dobrovolskij, Moskwa/Wiedeń

Ewa Donder, Poznań

Piotr Dranikowski, Poznań

Maria Drażyńska-Deja, Poznań

Martina Drescher, Bayreuth

Justyna Duch-Adamczyk, Poznań

Michael Düring, Kiel

Katarzyna Dziubalska-Kołaczyk, Poznań

Elżbieta Dziurewicz, Poznań

Yury Fedorushkov, Poznań

Reinhard Fiehler, Mannheim/Heidelberg

Maria Carmen Fonseca Mora, Huelva

Izabella Gajewska-Głodek, Poznań

Marian Glinka, Poznań

Gabriela Gorąca-Sawczyk, Poznań

Karolina Gortych-Michalak, Poznań

Albert Gouaffo, Dschang

Joanna Górecka, Poznań

Johann Görzen, Poznań

Piotr Grablunas, Poznań

Barbara Grucza, Warszawa

Franciszek Grucza, Warszawa

Sambor Grucza, Warszawa

Milena Hadryan, Poznań

Aleksandra Hans, Poznań

Anna Hanus, Rzeszów

Gisela Hartung, Halle

Heiko Hausendorf, Zürich

Jana Holšánová, Lund

Paweł Hostyński, Poznań

Britta Hufeisen, Darmstadt

Catalina Jiménez Hurtado, Granada

Jan Iluk, Katowice 
Danuta Jabłońska, Poznań

Marta Janachowska-Budych, Poznań

Barbara Jańczak, Poznań

Ewa Jarmołowicz-Nowikow, Poznań

Magdalena Jaszczyk-Grzyb, Poznań

Sabine Jentges, Nijmegen

Limin Jin, Beijing

Alina Jurasz, Wrocław

Magdalena Jurewicz, Poznań

Karolina Kaczmarek, Poznań

Dorota Kalecińska, Poznań

Victoria Kamasa, Poznań

Bogumiła Kaniewska, Poznań

Czesław Karolak, Poznań

Katarzyna Karpińska-Szaj, Poznań

Maciej Karpiński, Poznań

Mary Kastner, Bielefeld

Andrzej Kątny, Gdańsk

Inken Keim, Mannheim

Maria Kempa, Poznań

Joanna Kic-Drgas, Poznań

Ursula Kiermeier, Kraków

Ferit Kilickaya, Burdur

Gabriella B. Klein, Perugia

Katarzyna Klessa, Poznań

Graham Knox-Crawford, Poznań

Kurt Kohn, Tübingen

Martyna Kokotkiewicz, Poznań

Hanna Komorowska, Warszawa

Małgorzata Komorowska, Poznań

Hanka Konieczna-Zięta, Poznań

Paweł Kornatowski, Poznań

Jan Korzeniewski, Poznań

Tetyana Kosmeda, Poznań

Olga Kostrova, Samara

Monika Kowalonek-Janczarek, Poznań

Katarzyna Kozińska, Poznań

Lucyna Krenz-Brzozowska, Poznań

Marcin Krygier, Poznań

Barbara Kryk-Kastovsky, Wien

Dorota Krystosiak, Poznań

Artur Kubacki, Kraków

Katarzyna Kubaszczyk, Poznań

Paweł Kubiak, Poznań

Grażyna Kunigiel-Trąpczyńska, Poznań

Anna Kurzaj, Poznań
Agnieszka Kuśnierkiewicz, Poznań

Ksenia Kuzminykh, Göttingen

Kwiryna Kuźmar, Poznań

Hadrian Lankiewicz, Gdańsk

Marcin Lewandowski, Poznań

Grażyna Lewicka, Konin

Roman Lewicki, Konin

Gero Lietz, Frankfurt/Oder

Ryszard Lipczuk, Szczecin

Wojciech Lipoński, Poznań

Tomasz Lis, Kiel

Grzegorz Lisek, Greifswald

Mirosław Loba, Poznań

Mateusz Ławniczak, Poznań

Daniel Łubiński, Poznań

Marcin Maciejewski, Poznań

Jacek Makowski, Łódź

Łukasz Małecki, Poznań

Anna Małgorzewicz, Wrocław

Anna Marko, Poznań

Antoni Markunas, Poznań

Andrzej Marniok, Poznań

Elżbieta Marszałek, Poznań

Aleksandra Matulewska, Poznań

Marta Mazurek, Poznań

Paweł Mecner, Szczecin

Krystyna Mihułka, Rzeszów

Marcin Michalski, Poznań

Beata Mikołajczyk, Poznań

Tomasz Mizerkiewicz, Poznań

Michał Młodecki, Poznań

Aleksandra Muga-Bartkowiak, Poznań

Bernd Müller-Jacquier, Bayreuth

Dorothea Müller-Ott, Wien

Andrzej Narloch, Poznań

Marek Nawrocki, Poznań

Magdalena Nawrotek, Poznań

Jiří Nekvapil, Praha

Jowita Niewulis-Grablunas, Poznań

Piotr Nowak, Poznań

Joanna Nowak-Michalska, Poznań

Agnieszka Nowicka, Poznań

Norbert Nübler, Kiel

Magdalena Olpińska-Szkiełko, Warszawa

Slavo Ondrejovič, Bratislava

Izabela Orchowska, Poznań 
Hubert Orłowski, Poznań

Agnieszka Otwinowska-Kasztelanic, Warszawa

Dorota Owczarek, Poznań

Gina Oxbrow, Las Palmas de Gran Canaria

Kiriakos Papoulidis, Poznań

Margita Pätzold, Kassel

Ingwer Paul, Bielefeld

Joanna Pawelczyk, Poznań

Miroslaw Pawlak, Poznań

Waldemar Pfeiffer, Poznań

Elżbieta Piasecka, Poznań

Eliza Pieciul-Karmińska, Poznań

Anna Pieczyńska-Sulik, Poznań

Sławomir Piontek, Poznań

Kinga Piotrowiak-Junkiert, Poznań

Wawrzyniec Popiel-Machnicki, Poznań

Agnieszka Poźlewicz, Poznań

Ewa Półtorak, Katowice

Ludmila Pöppel, Stokholm

Aleksandra Przysiecka, Poznań

Stanisław Puppel, Poznań

Joanna Puppel-Wobalis, Poznań

Aleksandra Putowska, Poznań

Wen Qiufang, Beijing

Eugeniusz Rajnik, Poznań

Olimpia Rasom, Bozen

Claudia Riemer, Bielefeld

Bogusława Rolek, Rzeszów

Paweł Rybszleger, Poznań

Danuta Rytel-Schwarz, Leipzig

Roman Sadziński, Łódź

Alicja Sakaguchi, Poznań

Irena Sarnowska-Giefing, Poznań

Paul Sars, Nijmegen

Maria Sawicka, Poznań

Tabea Laura Schleinitz, Poznań

Angela Schmidt-Bernhardt, Marburg

Reinhold Schmitt, Mannheim

Joanna Schulz-Andrzejewska, Poznań

Teresa Siek-Piskozub, Poznań

Liliana Sikorska, Poznań

David S. Singleton, Dublin

Wanda Sitarz, Poznań

Grzegorz Skommer, Poznań

Barbara Skowronek, Poznań
Dominika Skrzypek, Poznań

Maciej Smuk, Warszawa

Hassan Soleimani, Tehran

Aldona Sopata, Poznań

Stefania Spina, Perugia

Joachim Stephan, Poznań

Victoria Storozenko, Marburg

Britta Stöckmann, München

Krzysztof Stroński, Poznań

Augustyn Surdyk, Poznań

Anna Szczepaniak-Kozak, Poznań

Marian Szczodrowski, Gdańsk

Sławomira Szubartowicz, Poznań

Janusz Taborek, Poznań

Eva Teshajev-Sunderland, Kaliningrad

Magdalena Thien-Pieklarz, Białystok

Teresa Tomaszkiewicz, Poznań

Kamil Trąba, Poznań

Paula Trzaskawka, Poznań

Freiderikos Valetopoulos, Poitiers

Grażyna Vetulani, Poznań

Aleksandra Wach, Poznań

Barbara Walkiewicz, Poznań

Tadeusz Wallas, Poznań

Elżbieta Magdalena Wąsik, Poznań

Emilia Wąsikiewicz-Firlej, Poznań

Anna Weigl, Kiel

Zenon Weigt, Łódź

Dorota Werbińska, Stupsk

Iwar Werlen, Bern

Tomasz Wicherkiewicz, Poznań

Elwira Wilczyńska, Poznań

Weronika Wilczyńska, Poznań

Danuta Wiśniewska, Poznań

Jacek Witkoś, Poznań

Bernadeta Wojciechowska, Poznań

Emilia Wojtasik-Dziekan, Poznań

Agata Wolarska-Sobocińska, Poznań

Ricarda Wolf, Berlin

Stephan Wolting, Poznań

Erika Worbs, Mainz

Justyna Woszczyk, Poznań

Joanna Woźniak, Poznań

Magdalena Wrembel, Poznań

Władysław Zabrocki, Poznań

Esther Zarraua Isasi-Isasmenti, Poznań 
Marta Zawacka-Najgeburska, Poznań

Joanna Zawodniak, Zielona Góra

Tadeusz Zgółka, Poznań

Joanna Ziemska, Wiedeń
Nadja Zuzok, Poznań

Jerzy Żmudzki, Lubin

Sylwiusz Żychliński, Poznań 


\section{Wissenschaftlicher Werdegang von Frau Professor Izabela Prokop}

Frau Professor Dr. habil. Izabela Prokop schloss 1974 ihr Studium der Germanistik an der Adam-Mickiewicz-Universität Poznań ab. Gleich nach dem Studienabschluss war sie in den Jahren 1974-1981 als Lektorin für Deutsch als Fremdsprache an der Akademie für Landwirtschaft in Poznań tätig. In dieser Zeit wurde ihre wissenschaftliche Arbeit von Professor Andrzej Z. Bzdęga an der Adam-Mickiewicz-Universität Poznań betreut. Ihre Dissertation unter dem Titel „Deutsch-polnische Übersetzungsäquivalenz im Bereich der Dialogstrukturen“ wurde im Jahre 1982 verteidigt. Die Erlangung des Doktortitels ebnete Professor Izabela Prokop den Weg zur Oberassistenz und Assistenzprofessur am Institut für Germanistik, Lehrstuhl Westgermanische Sprachen (Zakład Języków Zachodniogermańskich), der Adam-Mickiewicz-Universität Poznań, wo sie in den Jahren 1981-1992 arbeitete. 1992 wechselte sie zum Lehrstuhl für Glottodidaktik der Adam-Mickiewicz-Universität Poznań. Mit der Schrift „Erotetische Sprechakte im Deutschen und im Polnischen anhand natürlicher Gespräche“ habilitierte sie im Jahr 1995. Zwei Jahre später wurde sie außerordentliche Professorin an der Adam-Mickiewicz-Universität Poznań, wo sie in den Jahren 1997-2005 den Lehrstuhl für Glottodidaktik und Translatorik (Katedra Glottodydaktyki i Translatoryki) und ab 1999 auch den Lehrstuhl für Linguistische Übersetzungsforschung (Zakład Lingwistycznych Studiów nad Przekładem) leitete.

Frau Professor Izabela Prokop entwarf das Curriculum des Studiengangs der Angewandten Linguistik und führte das modulare Punktesystem ein. Sie war ebenfalls verantwortlich für die Einführung der ECTS-Bewertung (1996), erstellte das Programm des Postgraduiertenstudiums der Glottodidaktik (Förderung aus Mitteln des polnischen Bildungsministeriums MEN 1999) und und arbeitete als Mitglied einer Fakultätsarbeitsgruppe am Curriculum für das Zwei-Fach-Studium für (angehende) Fremdsprachenlehrer (Förderung aus Mitteln des polnischen Bildungs- und Sportministeriums MENIS 2004).

Im Rahmen ihrer Lehrtätigkeit beschäftigt sich Frau Professor Izabela Prokop mit folgenden Themenbereichen: Einführung in die Linguistik, Beschreibende Grammatik der deutschen Sprache aus traditioneller und strukturalistischer 
Sicht, Deutsch-polnische kontrastive Grammatik, Erforschung nationaler Stereotype, Einführung in die Translationswissenschaft, Linguistische Grundlagen der Übersetzung, Sprachkultur und lehrt Master- und Bachelor-Seminare im Bereich Linguistik und Translationswissenschaft mit den Schwerpunkten Pragmalinguistik und Sprechakttheorie, Textlinguistik und Alternative Lehrmethoden im Fremdsprachenunterricht.

$\mathrm{Zu}$ den Schwerpunkten ihrer Forschung gehören: Linguistische Übersetzungsforschung, Empirische Linguistik, Deutsch-polnische kontrastive Grammatik, Pragmalinguistik und Sprechakttheorie, Textlinguistik, Erforschung gesprochener Sprache, Methoden der Diskursanalyse, Dolmetschen und alternative Methoden des Fremdsprachenunterrichts, darunter Suggestionspädagogik, Offene Didaktik und Förderung der Lernerautonomie bei Studierenden.

Ihr vielfältiges wissenschaftliches Interesse war Grund für zahlreiche Einladungen zu wissenschaftlichen Vorträgen in Polen und im Ausland. Professor Izabela Prokop hat Gastvorlesungen an der Europa-Universität Viadrina Frankfurt (Oder) und am Deutschen Poleninstitut in Darmstadt gehalten. Im Sommersemester 2003 war sie Gastprofessorin an der Johannes GutenbergUniversität Mainz im Fachbereich Translations-, Sprach- und Kulturwissenschaft in Germersheim. Zuvor war sie 1989-1990 Stipendiatin der ChristianAlbrechts-Universität zu Kiel und hatte von Februar bis Juli 1993 ein Forschungsstipendium am Zentrum für interdisziplinäre Forschung der Universität Bielefeld.

Im Rahmen der internationalen Zusammenarbeit forschte Professor Izabela Prokop in den Jahren 1982-1989 in Halle zur gesprochenen Sprache und 1988 in Zusammenarbeit mit dem Institut für Deutsche Sprache in Mannheim zur deutsch-polnischen kontrastiven Grammatik. In den Jahren 1992-1995 arbeitete sie am Zentrum für interdisziplinäre Forschung der Universität Bielefeld mit Wissenschaftlern von verschiedenen europäischen Universitäten zu nationalen Auto- und Heterostereotypen in Konversationen zusammen. Derzeit arbeitet Professor Izabela Prokop zusammen mit Professor Olga Kostrova von der Universität Samara in Russland an einem deutschen Grammatiklehrbuch für polnische und russische Studierende. Gleichzeitig forscht sie über das Thema „Der Idiolekt eines Imkers".

Professor Izabela Prokop veröffentlichte drei Monographien, über vierzig Artikel, Berichte sowie Rezensionen und Übersetzungen in polnischen und internationalen Publikationen. In den Jahren 1995-2005 war Professor Izabela Prokop Chefredakteurin der Zeitschrift Glottodidactica. An International Journal of Applied Linguistics und initiierte die Herausgabe der Reihe Wissenschaftliche Hefte des Lehrstuhls für Glottodidaktik und Translatorik (Zeszyty Naukowe Katedry Glottodydaktyki i Translatoryki). Seit 2015 ist sie gemeinsam mit Aldona Sopata Mitherausgeberin der Reihe Sprache-Kultur-Kommunikation. 
Seit 2001 hat Professor Izabela Prokop fünfzehn Doktorandinnen und Doktoranden betreut: Eliza Pieciul (2001), Magdalena Jurewicz (2001), Marcin Maciejewski (2001), Agnieszka Nowicka (2002), Michał Młodecki (2003), Augustyn Surdyk (2003), Magdalena Aleksandrzak (2003), Joanna Kubaszczyk (2003), Joanna Andrzejewska-Kwiatkowska (2003), Paweł Rybszleger (2005), Paweł Kubiak (2006), Małgorzata Bielicka (2006), Katarzyna Malesa (2007) und Magdalena Witkowska (2014) und Tomasz Janiak (2020).

Die wissenschaftliche Tätigkeit von Professor Izabela Prokop wurde im Jahre 2000 mit dem Goldenen Verdienstkreuz der Republik Polen sowie mit dem Preis des Ministers für Hochschulwesen (Preis für die Forschergruppe Kontrastive Deutsch-Polnische Grammatik) geehrt. Im Jahre 2005 wurde sie mit der Medaille der Nationalen Bildungskommission ausgezeichnet. Im November 2013 wurde sie vom Staatspräsidenten der Republik Polen Bronisław Komorowski zur ordentlichen Professorin ernannt.

Seit 2010 leitet Professor Izabela Prokop mit großem Engagement und Erfolg das Institut für Angewandte Linguistik und den Lehrstuhl für Linguistische Übersetzungsforschung. Ihre hervorragende Leitungsfähigkeit, ihr tatkräftiges Handeln, ihre Unterstützung aller Mitarbeiterinnen und Mitarbeiter sowie ihr freundliches und zuvorkommendes Wesen ermöglichen dem Institut eine dynamische Entwicklung. 
Open-Access-Publikation im Sinne der CC-Lizenz BY-NC-ND 4.0

(C) 2020, Vandenhoeck \& Ruprecht GmbH \& Co. KG, Göttingen ISBN Print: 9783847111863 - ISBN E-Lib: 9783737011860 


\section{Liste der Veröffentlichungen von Frau Professor Izabela Prokop}

Insgesamt über 60 Publikationen, darunter 3 Bücher, 3 Lehrwerke, 3 Übersetzungen, 7 Rezensionen sowie über 50 veröffentlichte Artikel.

\section{Bücher}

\section{5}

Erotetische Sprechakte im Deutschen und im Polnischen anhand natürlicher Gespräche. Poznań: Wydawnictwo Naukowe Uniwersytetu im. Adama Mickiewicza.

\section{0}

Aspekty analizy pragmalingwistycznej (Aspekte der pragmalinguistischen Analyse). Poznań: Wydawnictwo Naukowe Uniwersytetu im. Adama Mickiewicza.

\section{6}

Pragmalingwistyka antropocentryczna (Anthropozentrische Pragmalinguistik). Poznań: Wydawnictwo Naukowe Uniwersytetu im. Adama Mickiewicza.

\section{Lehrbücher}

(für $\mathrm{DaF}$ an polnischen Hochschulen)

\section{8}

Wybór tekstów z języka niemieckiego dla studentów technologii drewna (Auswahl von Texten in deutscher Sprache für Studierende der Holztechnologie). Poznań: Wydawnictwo Naukowe Akademii Rolniczej. 
1981

Język niemiecki dla studentów technologii żywności (Deutsch für Studierende der Lebensmitteltechnologie). Poznań: Wydawnictwo Naukowe Akademii Rolniczej.

1991

Altdeutsche Chrestomathie. T. 1, Gotisch, Althochdeutsch, Altniederdeutsch. [H. 1], Lesestücke; [H. 2], Glossar, mit A. Z. Bzdęga. Poznań: Wydawnictwo Naukowe Uniwersytetu im. Adama Mickiewicza.

\section{Artikel}

\section{3}

Sprachliche Merkmale des Dialogs. In: H. Ziebart (Hrsg.), Germanistisches Jahrbuch DDR$V R P, 1983$ (S. 36-40). Warszawa: Deutschlektorat beim Kultur- und Informationszentrum der Deutschen Demokratischen Republik in Warszawa.

\section{4}

Ellipse in den deutschen und polnischen Dialogen. Studia Germanica Posnaniensia, XIII, 103-108.

\section{6}

Exozentrische Konstruktionen im Deutschen und Polnischen. Studia Germanica Posnaniensia, XV, 93-116.

Antworten auf Ergänzungsfragen im Deutschen und Polnischen. Studia Germanica Posnaniensia, XV, 117-127.

\section{8}

Stand, Aufgaben und Probleme der Erforschung der gesprochenen Sprache in der VR Polen - ein Bericht. Wissenschaftliche Zeitschrift der Martin-Luther-Universität HalleWittenberg, 25/1, 61-65.

Zum Problem der Textsegmentation. In: H. Stetter (Hrsg.), Proben. Konferenzbeiträge (S. 70-80). Warszawa: Deutschlektorat beim Kultur- und Informationszentrum der DDR in Warszawa. 


\section{9}

Kommunikation im Büro - erste Analyseergebnisse. In: M. Pätzold/P. Lindemann (Hrsg.), Kommunikationstagung 1989. Internationale Arbeitstagung in Wulkow 18.-20. April 1989 (S. 116-123). Berlin: Akademie der Wissenschaften der DDR, Zentralinstitut für Sprachwissenschaft.

Zur Konzeption eines zweisprachigen deutsch-polnischen Textkorpus. In: A. Kątny (Hrsg.), Theorie und Praxis der deutsch-polnischen Konfrontation und Translation. Festschrift für Prof. Dr. habil. Andrzej Zdzisław Bzdega zum 60. Geburtstag (S. 221-230). Rzeszów: Wydawnictwo Wyższej Szkoły Pedagogicznej.

Sprachhandlung Einladung/Vorschlag/Verabredung im Deutschen und Polnischen. Studia Germanica Posnaniensia, XVI, 195-207.

Komposita mit -weise im Deutschen und ihre Entsprechungen im Polnischen. In: H. Stetter (Hrsg.), Proben. Konferenzbeiträge (S. 64-77). Warszawa: Deutschlektorat beim Kulturund Informationszentrum der DDR.

\section{0}

Versuch einer Typologie mündlicher Texte. In: I. Prokop (Hrsg.), Gesprochene Sprache 1. Materialien des I. wissenschaftlichen Symposiums im Rahmen des Forschungsprojekts „Linguistische Studien zur gesprochenen Sprache“, Poznań, 23-29.06. 1988 (S. 37-45). Poznań: Wydawnictwo Naukowe Uniwersytetu im. Adama Mickiewicza.

\section{1}

Zur Struktur der Ratschläge im Deutschen und Polnischen. Studia Germanica Posnaniensia, XVII und XVIII, 81-88.

\section{2}

Zum Begriff „Höflichkeit“. In: J. Papiór (Hrsg.), Untersuchungen zur polnisch-deutschen Kulturkontrastivik (S. 189-196). Poznań: Wydawnictwo Naukowe Uniwersytetu im. Adama Mickiewicza.

\section{3}

Idiolekt und Erstellung von Textkorpora. In: G. Richter (Hrsg.), Methodische Grundfragen der Erforschung gesprochener Sprache (S. 22-27). Frankfurt a.M. u. a.: Peter Lang.

Polenstereotyp Anno Domini 1991. In: J. Darski/Z. Vetulani (Hrsg.), Sprache - Kommunikation - Informatik. Akten des 26. Linguistischen Kolloquiums, Poznań 1991, Bd. 1 (S. 407-410). Tübingen: Niemeyer.

Sprachliche Realisierung des Heterostereotyps in schriftlichen und mündlichen Äußerungen. Lingua ac Communitas, 1, 129-136. 


\section{4}

Gesprächsanalyse in Polen. In: K. Ehlich (Hrsg.), Diskursanalyse in Europa (S. 133-139). Frankfurt a.M. u. a.: Peter Lang.

\section{5}

Interaktionsschemata als Gliederungsprinzip von Korpora gesprochener Sprache. Studia Germanica Posnaniensia, XXI, 97-108.

Kontrastive Pragmatik (Arbeitspapier). Bielefeld: Zentrum für interdisziplinäre Forschung der Universität Bielefeld.

Bausteine des Diskurses über Ethnien (Arbeitspapier). Bielefeld: Zentrum für interdisziplinäre Forschung der Universität Bielefeld.

Die Funktionen von Stereotypen im Text (Arbeitspapier). Bielefeld: Zentrum für interdisziplinäre Forschung der Universität Bielefeld.

Fehlschläge in der interkulturellen Kommunikation zwischen Deutschen und Polen. In: W. Pfeiffer (Hrsg.), Wissenschaftseinrichtungen und Strukturentwicklung in der Grenzregion. Modellfall Europa-Universität Viadrina Frankfurt (Oder) (S. 165-172). Poznań: Wydawnictwo Naukowe Uniwersytetu im. Adama Mickiewicza.

Fremdbilder, Stereotype, Vorurteile. In: M. Czyżewski/E. Gülich/H. Hausendorf/M. Kastner (Hrsg.), Nationale Selbst- und Fremdbilder im Gespräch. Kommunikative Prozesse nach der Wiedervereinigung Deutschlands und dem Systemwandel in Ostmitteleuropa (S. 180-202). Opladen: Westdeutscher Verlag.

Wie sind die Deutschen? Einige Überlegungen zur Stereotypenproblematik. Neofilolog, 11, $42-47$.

Wie sind die Ausländer? Einige Überlegungen zur Stereotypenproblematik. FIPLV WORLD NEWS, Dez. 1995, 15-20 (verkürzter Abdruck aus: Neofilolog, 11, 42-47).

\section{6}

Erotetyczne akty mowy na lekcji języka obcego (Erotetische Sprechakte im Fremdsprachenunterricht). Neofilolog, 13, 76-81.

1997

Zur Tiefenstruktur des Gesprächs. Studia Germanica Posnaniensia, XXIII, 145-168.

\section{8}

Zagadnienie propozycji w erotetycznych aktach mowy (Die Frage der Proposition in erotetischen Sprechakten). In: S. Puppel und T. Tomaszkiewicz (Hrsg.), Scripta Manent. Publikacja wydziałowa z okazji 25-lecia istnienia Wydziału Neofilologii UAM (19881998) (S. 175-181). Poznań: Wydział Neofilologii Uniwersytetu im. Adama Mickiewicza w Poznaniu. 
Editorial. Glottodidactica, XXVI, 5.

\section{9}

Das Tertium comparationis in der Diskursanalyse. In: J. Bańczerowski/T. Zgółka (Hrsg.), Linguam amicabilem facere - Ludovico Zabrocki in memoriam (S. 391-396). Poznań: Wydawnictwo Naukowe Uniwersytetu im. Adama Mickiewicza.

Zum Aufbau des Gesprächs über Ethnien. In: Z. Bilut-Homplewicz (Hrsg.), Zur Mehrdimensionalität des Textes. Repräsentationsformen, Kommunikationsbereiche, Handlungsfunktionen (S. 159-176). Rzeszów: Wydawnictwo Wyższej Szkoły Pedagogicznej w Rzeszowie.

Relewancja w dialogu (Relevanz im Dialog). Scripta Neophilologica Posnaniensia. Rocznik poświęcony językoznawstwu, literaturoznawstwu i kulturoznawstwu, 1, 31-38.

Kulturbedingtheit des Translationsprozesses. In: A. Kątny/Ch. Schatte (Hrsg.), Das Deutsche von innen und von außen. Ulrich Engel zum 70. Geburtstag (S. 235-242). Poznań: Wydawnictwo Naukowe Uniwersytetu im. Adama Mickiewicza.

Präpositionen. In: U. Engel/D. Rytel-Kuc/L. Cirko/A. Dębski/A. Gaca/A. Jurasz/A. Kątny/P. Mecner/I. Prokop/R. Sadziński/Ch. Schatte/Cz. Schatte/E. Tomiczek/D. Weiss, Deutschpolnische kontrastive Grammatik. Bd. 2 (S. 1001-1026). Heidelberg: Julius Groos (polnische Ausgabe: Warszawa: PWN 2000).

Apposition. In: U. Engel/D. Rytel-Kuc/L. Cirko/A. Dębski/A. Gaca/A. Jurasz/A. Kątny/P. Mecner/I. Prokop/R. Sadziński/Ch. Schatte/Cz. Schatte/E. Tomiczek/D. Weiss, Deutschpolnische kontrastive Grammatik. Bd. 2 (S. 1231-1239). Heidelberg: Julius Groos (polnische Ausgabe: Warszawa: PWN 2000).

\section{0}

W poszukiwaniu lingwistyki (do)stosowanej (Auf der Suche nach einer (angemessenen) angewandten Linguistik). Scripta Neophilologica Posnaniensia. Rocznik poświęcony językoznawstwu, literaturoznawstwu i kulturoznawstwu, 2, 259-274.

Kształcenie tłumaczy i nauczycieli w systemie modułowo-punktowym (Ausbildung von Übersetzern und Lehrern in einem modul- und punktebasierten System). In: G. Szpila (Hrsg.), Język a komunikacja 1. Zbiór referatów z konferencji „Język trzeciego tysiąclecia“, Kraków, 2.-4. marca 2000 (S. 405-424). Kraków: Krakowskie Towarzystwo Wiedzy o Komunikacji Językowej Tertium.

2001

Translatorik und Hochschuldidaktik. In: F. Grucza (Hrsg.), Tausend Jahre polnisch-deutsche Beziehungen. Sprache - Literatur - Kultur - Politik. Materialien des MillenniumKongresses 5.-8. April 2000 (S. 803-810). Warszawa: Graf-Punkt.

Sugestopedia (Suggestopädie). In: I. Prokop (Hrsg.), Materiały I Meetingu Glottodydaktycznego, zorganizowanego w ramach Podyplomowego Studium Glottodydaktyki 1999/ 
2000 w Katedrze Glottodydaktyki i Translatoryki w dniach 24-25 czerwca 2000 (S. 25-

33). Poznań: Wydawnictwo Naukowe Uniwersytetu im. Adama Mickiewicza.

Typy interakcji w dydaktyce (Typen von Interaktion in der Didaktik). Neofilolog, 20, 13-22.

2002

Typologia interakcji dydaktycznych (Typologie didaktischer Interaktionen). In: W. Wilczyńska (Hrsg.), Autonomizacja w dydaktyce języków obcych. Doskonalenie się w komunikacji ustnej (S. 159-174). Poznań: Wydawnictwo Naukowe Uniwersytetu im. Adama Mickiewicza.

Metodologia i narzędzia badawcze zastosowane w projekcie (Die im Projekt angewandte Methodologie und die Forschungsinstrumente), mit D. Wiśniewska. In: W. Wilczyńska (Hrsg.), Autonomizacja $w$ dydaktyce języków obcych. Doskonalenie się w komunikacji ustnej (S. 109-128). Poznań: Wydawnictwo Naukowe Uniwersytetu im. Adama Mickiewicza.

Kierunki i sposoby usprawniania dydaktyki obcojęzycznej na poziomie zaawansowanym (Richtungen und Methoden der Verbesserung des Fremdsprachenunterrichts für Fortgeschrittene), mit M. Glinka und J. Puppel. In: W. Wilczyńska (Hrsg.), Autonomizacja $w$ dydaktyce języków obcych. Doskonalenie się w komunikacji ustnej (S. 129147). Poznań: Wydawnictwo Naukowe Uniwersytetu im. Adama Mickiewicza.

2003

Skuteczna szkoła - mrzonka to czy nadzieja? (Eine erfolgreiche Schule - Wunschtraum oder Hoffnung?). In: P. Hostyński (Hrsg.), Szukamy nowych dróg. Materiały II meetingu glottodydaktycznego zorganizowanego w ramach Podyplomowego Studium Glottodydaktyki 2000/2001 w Katedrze Glottodydaktyki i Translatoryki w dniach 26-27.06. 2001 (S. 23-30). Poznań: Rys-studio.

2011

Archaismen in der deutsch-polnischen Translation. In: C. Badstübner-Kizik (Hrsg.), Linguistik anwenden (S. 23-40). Frankfurt a.M. u. a.: Peter Lang.

\section{2}

Życzenia jako ekspresywny akt mowny (Wünsche als expressiver Sprechakt). In: A. Kelletat/A. Meger (Hrsg.), Worte und Wendungen. Texte für Erika Worbs mit Dank für zwei Jahrzehnte Germersheim (S. 99-105). Berlin: Saxa. 


\section{6}

O definicji aktu mownego raz jeszcze. Ujęcie antropocentryczne (Noch einmal zur Definition des Sprechakts. Ein anthropozentrischer Zugang). In: M. Aleksandrzak/L. Ciepielewska-Kaczmarek/A. Urban (Hrsg.), Lingwistyka stosowana: doświadczenia $i$ perspektywy (S. 3-10). Poznań: Wydawnictwo Naukowe Uniwersytetu im. Adama Mickiewicza.

\section{8}

Idiolekt pszczelarza - wprowadzenie do projektu badawczego (Der Idiolekt eines Imkers Einführung in ein Forschungsprojekt). In: M. Aleksandrzak (Hrsg.), Języki specjalistyczne $w$ lingwistyce stosowanej: między teoria i praktyka (S. 33-45). Poznań: Wydawnictwo Naukowe Uniwersytetu im. Adama Mickiewicza.

Kilka uwag o kontrastywnej analizie stylu (Einige Bemerkungen zur kontrastiven Stilanalyse). In: Scripta Neophilologica Posnaniensia. Rocznik poświęconyjęzykoznawstwu, literaturoznawstwu i kulturoznawstwu, 18, 207-219.

Kierunki badań nad językami specjalistycznymi w Instytucie Lingwistyki Stosowanej (Richtungen der Fachsprachenforschung am Institut für Angewandte Linguistik), mit J. Kic-Drgas. In: A. Stawikowska-Marcinkowska/B. Grzeszczakowska-Pawlikowska (Hrsg.), Języki specjalistyczne 2. Edukacja-perspektywy-kariera (S. 28-39). Łódź: Wydawnictwo Uniwersytetu Łódzkiego.

Editorial, mit S. Adamczak-Krysztofowicz und A. Szczepaniak-Kozak. Glottodidactica, XLV (2), 7-9.

\section{9}

Erklärende deutsche Grammatik für slawische Muttersprachler, mit O. A. Kostrova. In: $\Gamma$. Б. Воронина/А. В. Анищенко/Е. И. Карпенко/Н. В. Любимова/А. С. Филиппова (Hrsg.): Германистика: nove et nova. Материаль Второй международной научнопрактической конференции (S. 211-214). Москва: Московский государственный лингвистический университет.

\section{0}

Schwerpunkte immer noch schwer? Einige Überlegungen über die deutsche Grammatik für slawische native speakers, mit O. A. Kostrova. Glottodidactica, XLVII (1), 41-52.

Die Problematik des „Wie“ im DaF-Unterricht und ihre Relevanz für Lernende - sprechakttheoretische Überlegungen. In: S. Chudak / M. Thien-Pieklarz (Hrsg.): Die Lernenden in der Forschung zum Lehren und Lernen fremder Sprachen (im Druck). Frankfurt a. M. u. a.: Peter Lang.

Presupozycje w aktach komunikacyjnych (Präsuppositionen in kommunikativen Akten). In: A. Buk / A. Hanus / A. Mac / D. Miller / M. Smykała / I. Szwed (Hrsg.): Księga pamiątkowa dla prof. Zofii Bilut-Homplewicz (im Druck). Rzeszów: Wydawnictwo Uniwersytetu Rzeszowskiego. 


\section{Rezensionen}

\section{5}

Polnisch-deutscher Sprachvergleich I. Arbeitsbuch für Fortgeschrittene. Glottodidactica, XVII, 117-119.

\section{6}

E. Tomiczek (1983). System adresatywny współczesnego języka polskiego i niemieckiego. Socjolingwistyczne studium konfrontatywne. Studia Germanica Posnaniensia, XV, 159-161.

H. Lörcher (1983). Gesprächsanalytische Untersuchungen zur Arzt-Patienten-Kommunikation. Studia Germanica Posnaniensia, XV, 161-164.

\section{9}

C. Fabricius-Hansen (1986). Tempus fugit. Über die Interpretation temporaler Strukturen im Deutschen. Studia Germanica Posnaniensia, XVI, 264-267.

R. Lipczuk (Hrsg.) (1985). Grammatische Studien. Beiträge zur germanistischen Linguistik in Polen. Studia Germanica Posnaniensia, XVI, 249-253.

\section{3}

E. Leupold/Y. Petter (Hrsg.) (1990). Interdisziplinäre Sprachforschung und Sprachlehre. Festschrift für Albert Raasch zum 60. Geburtstag. IDV-Rundbrief, 50, 42-43.

U. O. H. Jung (Hrsg.) (1992). Praktische Handreichung für Fremdsprachenlehrer. IDVRundbrief, 50, 43-45.

\section{4}

A. Frank/K. Maaß/F. Paul/H. Turk (Hrsg.). Übersetzen, Verstehen, Brücken bauen. Geisteswissenschaftliches und literarisches Übersetzen im internationalen Kulturaustausch. Teil 1 und 2. Convivium. Germanistisches Jahrbuch Polen, 267-271.

A. Regales Serna (Hrsg.) (1992). Germanistik und Deutschunterricht. Materialien des V. Symposiums des Spanischen Deutschlehrerverbandes, Valladolid 6.-10. November 1989. Glottodidactica, XXII, 211-212.

\section{5}

J. Israel (1990). Sprache und Erkenntnis. Zur logischen Tiefenstruktur der Alltagssprache. Studia Germanica Posnaniensia, XXI, 113-114.

G. Antos/H. P. Krings (Hrsg.) (1989). Textproduktion. Ein interdisziplinärer Forschungsüberblick. Studia Germanica Posnaniensia, XXI, 115-117. 
W. Franke (1990). Elementare Dialogstrukturen. Darstellung, Analyse, Diskussion. Studia Germanica Posnaniensia, XXI, 117-119.

\section{Konferenzberichte}

\section{6}

Nachwuchswissenschaftler-Konferenz Sprachwissenschaft, November 1985. Skamandros. Germanistisches Jahrbuch DDR-VR Polen 1986, 192-196.

\section{7}

Nachwuchswissenschaftler-Konferenz - Sprachwissenschaft. Deutsch als Fremdsprache, 24/1, 49-50.

\section{0}

I Międzynarodowa Konferencja w ramach projektu badawczego "Język mówiony“ - Gesprochene Sprache (1. Internationale Konferenz im Rahmen des Forschungsprojekts "Język mówiony“ - Gesprochene Sprache), UAM, Poznań, czerwiec 1988. Przegląd Glottodydaktyczny, 11, 109-112.

\section{5}

Internationales Symposium der Martin-Luther-Universität Halle - Gesprochene Sprache II, Lochmühle, 4.-8.06.1990. Studia Germanica Posnaniensia, XXI, 109-111.

\section{Übersetzungen}

\section{3}

P. Weiss (1971). Pod stertą prosa (Unter dem Hirseberg). In: Cz. Karolak (Hrsg.), Niemcyo sobie. Naród - państwo - „charakter narodowy“ w oczach intelektualistów niemieckich (S. 95-98). Poznań: Instytut Zachodni.

K. Sontheimer (1972). Wyniki badań empirycznych (Ergebnisse empirischer Forschungen). In: Cz. Karolak (Hrsg.), Niemcy o sobie. Naród - państwo - „charakter narodowy“ w oczach intelektualistów niemieckich (S. 147-150). Poznań: Instytut Zachodni.

K. Staeck (1979). Co drugi Niemiec ma nadwagę (Jeder zweite Deutsche hat Übergewicht). In: Cz. Karolak (Hrsg.), Niemcy o sobie. Naród - państwo - „charakter narodowy“ $w$ oczach intelektualistów niemieckich (S. 181-186). Poznań: Instytut Zachodni. 


\section{Andere Publikationen}

2000

Hohe deutsche Auszeichnung für Waldemar Pfeiffer. Glottodidactica, XXVII, 122. 


\section{Neue Herausforderungen und aktuelle Konzepte der Angewandten Linguistik - einleitende Bemerkungen}

„Man muss den Leuten aufs Maul sehen, wie sie (durch Sprache) handeln und danach forschen" - diese zweckgebundene Variation der bekannten Worte Martin Luthers aus seinem „Sendbrief vom Dolmetschen“ scheint dem wissenschaftlichen Lebenswerk von Frau Professor Izabela Prokop, der die vorliegende Festschrift zugeeignet ist, eine passende Beschreibungs- und Würdigungsform zu verleihen. Die Jubilarin hat konsequent diesen Forschungsweg beschritten, der heutzutage als eine Via regia der Angewandten Linguistik gilt. Mit dem vorliegenden Band unter dem Titel „Angewandte Linguistik - neue Herausforderungen und Konzepte" würdigen die AutorInnen und HerausgeberInnen die Wissenschaftlerin Professor Izabela Prokop für ihre vielseitigen Tätigkeitsfelder und fruchtbaren Initiativen im Bereich der Sprachwissenschaft sowie für die Entwicklung des Instituts für Angewandte Linguistik an der Adam-MickiewiczUniversität in Poznań (Polen). 24 theoretische und praxisbezogene Beiträge von namhaften WissenschaftlerInnen sowohl aus Deutschland und Österreich als auch aus Polen dokumentieren das breite Spektrum an Perspektiven auf aktuelle Forschungsfragen und -ansätze der Angewandten Linguistik und richten sich somit an einen breiten Kreis von LinguistenInnen, ÜbersetzerInnen und FremdsprachendidaktikerInnen, insbesondere an Dozierende im Bereich der Germanistik und Anglistik, anderer moderner Fremdsprachenphilologien und angrenzender Fächer sowie Studierende dieser Studienfächer.

Die in den weiteren sieben Teilen des Bandes präsentierten Beiträge wurden von den HerausgeberInnen den folgenden thematischen Schwerpunkten zugeordnet:

I. Pragma- und Soziolinguistikstudien

II. Kontrastive Studien

III. Interkulturalität

IV. Spracherwerbs- und Sprachlehrforschung

V. Fachsprachen

VI. Übersetzen und Dolmetschen

VII. Linguistic landscapes 
Die vielfältigen Beiträge bringen eine perspektivisch differenzierte und mehrdimensionale Auseinandersetzung mit dem breiten Spektrum der wissenschaftlichen Interessenschwerpunkte der Angewandten Linguistik und knüpfen an die Forschungsinteressen der Jubilarin an, die sich wichtigen Fragen der kontrastiven Linguistik (Prokop 2019), Pragmalinguistik (Prokop 2010 und 2016), Fremdsprachendidaktik (Prokop \& Wiśniewska 2002) und Übersetzungswissenschaft widmete.

Den vorliegenden Sammelband eröffnet der Aufsatz, der sich mit pragmalinguistischen und soziopragmatischen Studien befasst, die den Kern der akademischen Tätigkeit von Izabela Prokop bilden. Der einleitende Aufsatz von Barbara Kryk-Kastovsky („Impoliteness as a result of power asymmetry in selected social contexts") thematisiert das Problem der Unhöflichkeit, die durch Machtasymmetrie bedingt ist und für verschiedene soziale Kontexte charakteristisch ist. Die Autorin versucht zu ergründen, auf welche Art und Weise unterschiedliche Unhöflichkeitsgrade im Diskurs beobachtet werden können. In dem Beitrag werden (neben verschiedenen bereits in der Literatur beschriebenen Interaktionsarten wie Arzt-Patient, Lehrer-Schüler oder Polizist-Täter) auch andere Machtkontexte berücksichtigt, z. B. militärische oder administrative. Da sich alle diese Interaktionsarten aufgrund der unterschiedlichen sozialen Kontexte qualitativ unterscheiden, argumentiert die Autorin, dass es zwischen ihnen ebenso quantitative Unterschiede gibt, z. B. hinsichtlich des Grades der Unhöflichkeit.

Anna Szczepaniak-Kozak („Pragmatic accent: Sociolinguistic and pragmalinguistic examples") nimmt eine andere Stellung ein und befasst sich mit soziopragmatischen und pragmalinguistischen Unterschieden in der interlinguistischen Pragmatik von Fremdsprachenlernenden, die durch interkulturelle Differenzen untermauert werden (vgl. Szczepaniak-Kozak 2018). Sie vertritt die These, dass ausgewählte pragmatische Merkmale bei solchen Sprachbenutzern bei der Ausführung von Kommunikationsfunktionen in der Zielsprache systematisch auftreten. Szczepaniak-Kozak fasst solche Merkmale unter dem Begriff „pragmatischer Akzent" zusammen. In der Spezifikation dieses Begriffs bezieht sie sich auf zwei verwandte Konzeptualisierungen, eine von Scarcella (1992) und die andere von Yule (1996). Um das Konzept zu begründen, präsentiert sie eine Zusammenfassung der pragmatischen Kompetenzforschung zwischen den Sprachen, die sie unter polnischen Lernenden des Englischen als Fremdsprache durchgeführt hat. Die Studie zeigt, dass selbst fortgeschrittene Lerner des Englischen sich dem pragmatischen Modell der Muttersprachler nicht vollständig annähern und stattdessen eine Reihe von Merkmalen in ihrer pragmatischen Produktion beibehalten, die ihre einfache Identifizierung als Nicht-Muttersprachler ermöglichen. 
In der Problematik der linguistischen Pragmatik ist auch der Beitrag von Anna Pieczyńska-Sulik („Ikonische Dimensionen der Sprachpragmatik am Beispiel von Repräsentativa“) verankert, die das Thema der linguistischen Ikonizität aus der pragmatischen Perspektive aufgreift. Die Autorin präsentiert semiotische, sprachgrammatische und textlinguistische Terminologie in Bezug auf bekannte Zeichenmodelle und verweist auf mögliche Typologien der linguistischen Ikonizität (vgl. u.a. Hausendorf \& Kesselheim 2008). Den Kern des Beitrags bildet eine kurze Präsentation ausgewählter Beispiele von Repräsentativa in Bezug auf ikonische Lokutionen und Perlokution.

Mit der pragmatischen Problematik (diesmal in Bezug auf moderne Kommunikationstechnologien) befasst sich ebenso der Beitrag von Paweł Rybszleger („Pragmatik von Hashtags am Beispiel von Twitter als multimodales Diskurssystem“). Der Autor analysiert Elemente, die in der Online-Kommunikation (hier im multimodalen Diskurssystem Twitter) oft verwendet werden und zu sog. semiotischen Operatoren gehören. Hashtags werden in dem Beitrag einerseits als intuitive Mittel der Online-Kategorisierung beschrieben, andererseits u.a. als Online-Instrumente, die (handlungsbezogen) bestimmte Inhaltsbeschreibungen ermöglichen und von anderen UserInnen entsprechend interpretiert werden können. In Bezug auf das referentielle Modell von Kommunikation von Wilson \& Sperber (2006) wird präsentiert (am Beispiel von 100 ausgewählten deutschsprachigen Online-Beiträgen - sog. Tweets), auf welche Weise Hashtags zur Inferenz von einfach ausgedrückten Propositionen, Explikaturen auf höherer Ebene und Implikaturen verhelfen können.

Der zweite thematische Teil des Bandes („Kontrastive Studien“) beginnt mit dem Aufsatz von Norbert Nübler („Der polnische Verbalaspekt im Fremdsprachenunterricht"), der sich auf mögliche Übersetzungs- und didaktische Probleme mit der grammatischen Kategorie des Verbalaspekts im Polnischen bezieht. Laut dem Autor genießt diese Kategorie besonders unter polnischen Grammatikern kaum Beachtung. Aus diesem Grund wird in dem Text ein Versuch unternommen, den Verbalaspekt aus didaktischer Sicht zu präsentieren, damit verschiedene Entstehungsmechanismen des Verbalaspekts (mit Ausnahmen) dem deutschen Lerner verständlicher werden.

Ebenso kontrastiv angelegt ist der Aufsatz von Magdalena Jurewicz, Martyna Gabrych und Justyna Sobańska („Sprachliches Weltbild in ausgewählten gegenwärtigen polnischen und deutschen parallelen Gebrauchshypertexten“). Die Autorinnen blicken in ihrem Beitrag auf Kontaktanzeigen und Todesanzeigen in Deutschland, Österreich und Polen, die als Beispiel für besonders stark sozialund kulturgeprägte Textsorten in allen drei Ländern stehen können. Jurewicz, Gabrych und Sobańska bemerken zu Recht, dass das alltägliche Bild der Welt sich nicht nur in Überzeugungen einer Gemeinschaft widerspiegelt, sondern auch in gewissen Vorstellungen und Erwartungen, die u. a. durch die Sprache modelliert 
werden. Die Autorinnen heben in ihrem Beitrag ebenso die Wichtigkeit der Paralleltextanalyse für die Didaktik im Rahmen der Angewandten Linguistik hervor.

Elżbieta Dziurewicz („Idiome im deutsch-polnischen Kontrast als Herausforderung für Studierende der Angewandten Linguistik. Ergebnisse einer Umfrage und Postulate für den Einsatz im universitären Unterricht") thematisiert in ihrem praxisbezogenen Beitrag die Problematik der universitären Didaktik im Bereich der kontrastiven Phraseologie und stellt fest, dass gerade das Erlernen von Idiomen für viele Studierende eine besondere Herausforderung darstellt. In einem ersten Schritt liefert die Autorin zahlreiche Argumente für die kontrastive Analyse von Idiomen im DaF-Unterricht. In dem analytischen Teil ihres Beitrags macht sie den Leser mit Ergebnissen der mehrstufigen Studie vertraut, die unter Studierenden der Angewandten Linguistik an der Adam-Mickiewicz-Universität in Poznań durchgeführt wurde. Schließlich formuliert die Autorin konkrete Postulate für die universitäre Didaktik von Phraseologismen.

Der dritte thematische Schwerpunkt des Bandes „Interkulturalität“ ist vertreten durch zwei Beiträge, die sich mit diversen Aspekten des interkulturellen Lernens beschäftigen. Zunächst greift der Beitrag von Sylwia Adamczak-Krysztofowicz und Krystyna Mihułka („Interkulturelle Kommunikation und Angewandte Linguistik: Zur Positionierung der beiden Disziplinen und ihrer Studienschwerpunkte unter Berücksichtigung der neuen Reform im polnischen Hochschulwesen") die Kategorie der Interkulturalität im Studium der Angewandten Linguistik an polnischen Universitäten auf, wobei der Schwerpunkt auf der Verortung Interkultureller Kommunikation im Studium der Angewandten Linguistik nach der neuen Reform im polnischen Hochschulwesen liegt (vgl. u. a. Mihułka et al. 2016). Die Autorinnen führen dabei die wichtigsten Lernergebnisse beim Studienfach „Interkulturelle Kommunikation“ an, die anhand der Deskriptoren des Polnischen Qualifikationsrahmens für Hochschulwesen formuliert und mit den Zielsetzungen zur Vermittlung der interkulturellen Kompetenz bei angehenden Fremdsprachenlehrenden und ÜbersetzerInnen sowie DolmetscherInnen in Verbindung gebracht werden.

Im zweiten und abschließenden Artikel dieses Teils („Fremdes und Eigenes als „Fremdes verstehen“ - Neuere anwendungsbezogene fremdkulturwissenschaftliche Konzepte in Zeichen und Zeiten der Krise") geht Stephan Wolting auf zwei innovative Ansätze fremdkulturwissenschaftlicher Forschung ein, in Hinblick auf ihre Anwendung auf den fremdkulturellen Kontext. In diesem $\mathrm{Zu}$ sammenhang skizziert der Forscher einerseits eine fremd- oder antihermeneutische Position und andererseits die aus der Kognitionspsychologie und Pädagogik bekannten Anti-Bias-Studien. Dabei werden in dem Beitrag Überlegungen angestellt, ob und wie Krisenempfinden in Zeiten der Pandemie mit der Wei- 
terentwicklung eines kulturwissenschaftlichen Forschungsbereichs verbunden werden kann.

Im vierten thematischen Schwerpunkt der Publikation finden sich fünf Aufsätze, die systematisierende Überlegungen zu ausgewählten Erkenntnissen und potentiellen Forschungsdesiderata der Spracherwerbs- und Sprachlehrforschung anstellen. Der Beitrag von Aldona Sopata und Aleksandra Putowska („Polnisch als Herkunftssprache in Deutschland - Einfluss der Familiensprachenpolitik auf die Sprachentwicklung der Kinder") thematisiert die Sprachentwicklung des Polnischen als Herkunftssprache in Deutschland. Nach der Diskussion von internen und externen Faktoren bei der Herkunftssprachentwicklung (vgl. u.a. Hufeisen et al. 2018) werden die wichtigsten Ergebnisse einer eigenen empirischen Studie zur Feststellung des Einflusses der Familiensprachenpolitik auf die Entwicklung des Polnischen als Herkunftssprache bei den Kindern aus den Familien mit polnischem Migrationshintergrund in Deutschland vorgestellt.

Małgorzata Bielicka beleuchtet in ihrem Beitrag („Immersion als eine innovative Methode der Fremdsprachenvermittlung auf der Elementar- und Primarstufe in Polen") den Stellenwert der Immersion als einer innovativen Methode für bilinguale Kindergärten und Grundschulen in Polen (vgl. Bielicka 2017). Bei der Darstellung der Immersionsmethode erwägt die Autorin folgende drei Indikatoren: 1 . historische Perspektive, 2. Auftreten von neuen Eigenschaften im Vergleich mit konventionellen Sprachvermittlungsmethoden und 3. Effizienz der Immersion in Bezug auf die fremdsprachlichen Leistungen der Schüler. Auf der Grundlage von Ergebnissen aus eigener Untersuchung belegt die Forscherin, dass die Immersion weiterhin als innovative Methode fungieren kann.

Den vierten thematischen Bereich runden drei Beiträge $a b$, in denen die Lernerperspektive beim Fremdsprachenlernen diskutiert wird. Der Fokus des Beitrags von Teresa Siek-Piskozub und Aleksandra Wach („EFL Learner agency from the perspective of learners' autobiographies“) richtet sich auf die Bewertung der hermeneutischen Phänomenologie als einer qualitativen Forschungsrichtung, die die Untersuchung der Lerner-Agentur im Prozess des Erwerbs von Sprachkompetenz auf der Basis von Daten aus Autobiographien ermöglicht und sich dabei mit Elementen deduktiver Forschung kombinieren lässt (vgl. u.a. Larsen-Freeman 2019). Das Thema des Lernenden als eines aktiven Akteurs im Prozess des Sprachenlernens wird auch im Beitrag von Magdalena Aleksandrzak („Developing speaking skills at advanced level - the perspective of language department students and the implications for language teaching“) fortgesetzt. Die Autorin behandelt dabei eine wichtige Fragestellung aus dem Bereich der universitären Fremdsprachendidaktik - die Entfaltung der Sprechfertigkeit. In diesem Zusammenhang stellt sie relevante Ergebnisse ihres Forschungsprojekts vor, in dessen Mittelpunkt Wahrnehmungen und Meinungen von fortgeschrittenen Sprachlernenden zu ausgewählten Aspekten beim Sprechen stehen. 
Der Beitrag von Katarzyna Bieniecka-Drzymała („Fragen, die mehr können. Zum Potential von Coaching-Fragen für die Förderung und Entwicklung der Selbstreflexivität bei Fremdsprachenlernenden") wendet sich schließlich dem Potential vom sog. Sprachlern-Coaching bei der Entwicklung der Sprachlernbewusstheit und der Lernerautonomie von Fremdsprachenlernenden zu und bietet die Informationen darüber, wie das Konzept der Sprachlernberatung mit spezifischen Gesprächsführungstechniken und Fragestellungsmethoden zur Optimierung der didaktischen Praxis von Fremdsprachenlehrenden im Hochschulbereich beitragen kann.

Den fünften thematischen Schwerpunkt des Bandes eröffnet der Beitrag von Klaus-Dieter Baumann („Thought patterns in natural scientific and technical scientific communication"). Das weit ausgebaute Projekt zielt auf die interdisziplinäre Analyse von fachsprachlichen Texten ab. Die Hauptannahme des Textes lautet, dass die immense Komplexität einer solchen Kommunikation eine Schwerpunktverlagerung dieser Forschungsrichtung erforderlich macht. Baumann spricht sich für die These aus, dass Fachsprachen als Teil des kognitiven Systems interpretiert werden sollten. In näherer Betrachtung schlägt der Autor vor, dass alle kognitiven Fähigkeiten mit verschiedenen Kommunikationsmitteln in Wechselwirkung stehen und einen Informationsfluss erzeugen, der als dynamische mentale Zustände des Autors/der Autoren und des Empfängers/der Empfänger während der fachsprachlichen Textproduktion und ihres Verständnisses begriffen werden kann. Darüber hinaus beschreibt der Autor (auf der Grundlage empirischer Studien verschiedener englischer und deutscher Texte) die komplexen Prozesse, die Denkmuster und Sprache zusammentragen und anschließend in eine bestimmte Textform integriert werden. Klaus-Dieter Baumann argumentiert überzeugend, dass hoch interaktive kognitiv-kommunikative Module, die auf verschiedenen Ebenen von fachsprachlichen Textformen realisiert werden, fruchtbare Ansätze für die Analyse der Äquivalenz von Denkmustern und Sprachstrukturen sein können.

In seinem Beitrag befasst sich Paweł Kubiak („Politische Begriffe als Herausforderung für die Didaktik der Angewandten Linguistik“) mit der Problematik der universitären Didaktik von politischen Begriffen, die für viele Studierende (besonders an linguistischen Fakultäten) eine richtige Hürde sein können. Aus diesem Grund formuliert der Autor einige mögliche Anforderungen, die an die diesbezügliche Didaktik gestellt werden können. Sie beziehen sich auf den Ausbau von politiksemantischen, aber auch von wörterbuch- und lexikanutzungsbezogenen Teilkompetenzen, die mit den ersteren in engem $\mathrm{Zu}$ sammenhang stehen.

Die Situation der Fachsprachendidaktik an den Hochschulen und Universitäten in Europa und insbesondere in Polen nimmt auch der Beitrag von Joanna Kic-Drgas und Joanna Woźniak („Fachsprachendidaktik an europäischen 
Hochschulen - Stand und Perspektiven“) zum Anlass, um über vorhandene Definitionen sowie Entwicklungsetappen der Fachsprachendidaktik zu reflektieren. Darüber hinaus wird in dem Artikel auf relevante Ergebnisse einer eigenen Studie zu curricularen sowie institutionellen Entwicklungen im Bereich der Fachsprachendidaktik auf dem tertiären Bildungsniveau in Europa fokussiert.

Der sechste Teil „Übersetzen und Dolmetschen“ beginnt mit dem Aufsatz von Joanna Kubaszczyk („Zur Textur des Originalwerkes und der Übersetzung - über die lautlichen und graphematischen Herausforderungen der Übersetzung“). In ihrem Beitrag verweist die Autorin auf die literarische Übersetzung als lautliche und graphematische Herausforderung für ÜbersetzerInnen im Deutschen und Polnischen. Kubaszczyk bespricht in ihrem Text die wichtigsten Figuren der Wiederholung auf der lautlichen und graphematischen Ebene sowie führt Beispiele für die sog. Reliefbildung vor. In einem zweiten Schritt werden spezifische Übersetzungsprobleme aufgezeigt sowie konkrete Übersetzungsmöglichkeiten vorgeschlagen. Dadurch werden (besonders angehende) ÜbersetzerInnen für die poetische Funktion der Sprache in literarischen Texten sensibilisiert.

Hanka Błaszkowska befasst sich dagegen in ihrem Beitrag („Translation und Transkreation: Zu Differenzen der Begriffssystematik zwischen Theorie und Praxis“) mit einem bisher wenig beachteten Thema bei der Übersetzungs- und Translationswissenschaft, nämlich der Transkreation, die ein neues translatorisches Phänomen im Bereich der Marketingpraxis darstellt. Dabei diskutiert die Autorin die Begriffe Translation und Transkreation aus der Sicht der translatorischen Forschung und der professionellen Berufspraxis im Hinblick auf begriffliche Differenzen und Kommunikationsprobleme (vgl. Spinzi \& Rizzo 2018).

Im Fokus der Betrachtung von Alicja Sakaguchi („Die Kunst des Verstehens und Übersetzens sakraler Texte. Ein kritischer Blick auf die Wiedergabe einiger Schlüsselinhalte auf der Grundlage der deutschen, polnischen und EsperantoBibelübersetzungen") steht die Übersetzungskritik sakraler Texte bezüglich der Wiedergabe von bestimmten Schlüsselinhalten. Die Autorin präsentiert Beispiele aus diversen Bibelübersetzungen (im Deutschen, Polnischen und Esperanto) und stellt dabei u.a. fest, dass viele Sichtweisen der ÜbersetzerInnen selbst zu erstarrten Übersetzungsverfahren führen können, was nicht selten in Form von v. a. ideologisch motivierten Verformungen $\mathrm{zu}$ Tage tritt.

Ebenso praxisbezogen ist der Beitrag von Lucyna Krenz-Brzozowska („Postulate hinsichtlich der Entwicklung der Fähigkeit zur Selbstbewertung eigener Dolmetschleistungen - Vorschlag einer Feldübung für Studierende der Angewandten Linguistik mit dem Schwerpunkt Übersetzen/Dolmetschen"), die in ihrem Text auf die Problematik der Bewertung der Qualität von Leistungen im Dolmetschen als einen der wichtigsten Aspekte des Übersetzungsprozesses hinweist. Dazu präsentiert die Autorin einzelne Phasen und Ergebnisse einer Feldübung, die unter Studierenden des Aufbaustudiums zur Ausbildung von 
Übersetzern und Dolmetschern der deutschen Sprache an der Adam-Mickiewicz-Universität in Poznań durchgeführt wurde. Darauf aufbauend werden konkrete Postulate formuliert.

Abschließend präsentiert Augustyn Surdyk in seinem Artikel („Culture in translation: A comparative analysis of English originals and Polish dubbed versions of computer-animated film productions") eine Vielzahl kulturbezogener Referenzen beim englisch-polnischen audiovisuellen Übersetzen. Der Autor untermauert seine Argumentation anhand von Referenzen aus der polnischen synchronisierten Version des amerikanischen Blockbusters „Shrek 2“. Die erkannten und analysierten kulturbezogenen Fragmente wurden in vier Kategorien unterteilt (linguistische Referenzen: Zitate oder Paraphrasen; kulturelle Bezüge; Alltagssprache und schließlich persönliche Hinweise auf bekannte Polen, Prominente und fiktive Figuren). Als Ergebnis seiner detaillierten Analyse hat der Autor 90 kulturbezogene Skriptfragmente entdeckt.

Die zwei Beiträge des siebten und zugleich letzten thematischen Bereiches stellen das Phänomen der Sprachlandschaft (Linguistic Landscape) dar und bieten einen interessanten Blick auf dieses neue Forschungsobjekt der Angewandten Linguistik. Zunächst greift der Artikel von Grzegorz Lisek („Neue Herausforderungen für Angewandte Linguistik - Sprachlandschaften? Visuelle Mehrsprachigkeit in den Linguistic Landscapes der deutsch-polnischen Grenzregion") relevante Ähnlichkeiten und Unterschiede zwischen der schriftbasierten Kommunikation in zwei polnischen Grenzstädten Świnoujście und Słubice, deren Linguistic Landscape aus sprachwissenschaftlicher Sicht analysiert und den möglichen Diskurstypen zugeordnet wird.

Den Teil des Sammelbandes schließt der Beitrag von Danuta Wiśniewska („Linguistic landscape, murals and language learning“) ab, in dem die Autorin das Potential des Einsatzes von Wandmalereien für die Entfaltung aller Sprachfertigkeiten sowohl im Fremdsprachenunterricht im Klassenzimmer als auch außerhalb des Unterrichtsraumes diskutiert und dabei die didaktische Perspektive bei der Auseinandersetzung mit Linguistic Landscapes in den Vordergrund stellt.

Zusammenfassend geben die versammelten Beiträge einen Einblick sowohl in aktuelle wissenschaftliche Fragestellungen und Konzepte als auch neue wissenschaftliche Erkenntnisse der Angewandten Linguistik und bearbeiten dabei viele Aspekte interdisziplinär und in einem neuen Licht.

Als HerausgeberInnen hoffen wir, dass die Aufsätze des vorliegenden Bandes mit ihrem reichen Themenspektrum bei der Leserschaft aus verschiedenen Ländern auf Interesse stoßen. Wir wünschen in diesem Sinne allen LeserInnen eine inspirierende Lektüre, aus der eventuell auch weitere Ideen für die Erforschung von den in diesem Band vertretenen Subdisziplinen der Angewandten Linguistik resultieren. 
Unser Dank gilt in diesem Sinne den AutorInnen des vorliegenden Bandes, die mit ihren Beiträgen und Forschungsdesiderata aktuelle wissenschaftliche Erkenntnisse für die Weiterentwicklung der Angewandten Linguistik darlegen und somit das Werk von Frau Professor Izabela Prokop würdigen und ihr ganz herzlich zum 70. Geburtstag gratulieren.

Hanka Błaszkowska, Magdalena Jaszczyk-Grzyb, Magdalena Jurewicz, Paweł Kubiak, Lucyna Krenz-Brzozowska, Johann Görzen und Joanna Ziemska sei für ihre Unterstützung herzlich gedankt.

Nicht zuletzt gebühren unsere Dankesworte der Adam-Mickiewicz-Universität Poznań (namentlich Frau Professor Katarzyna Dziubalska-Kołaczyk, Frau Professor Aldona Sopata und Frau Professor Beata Mikołajczyk) für die finanzielle Unterstützung der Drucklegung.

Sylwia Adamczak-Krysztofowicz, Anna Szczepaniak-Kozak \& Paweł Rybszleger im Juli 2020

\section{Literaturverzeichnis}

Bielicka, Małgorzata (2017). Efektywność nauczania języka niemieckiego na poziomie przedszkolnym i wczesnoszkolnym $w$ dwujęzycznych placówkach edukacyjnych $w$ Polsce. Poznań: Wydawnictwo Naukowe Uniwersytetu im. Adama Mickiewicza.

Hausendorf, Heiko/Kesselheim, Wolfgang (2008). Textlinguistik fürs Examen. Göttingen: Vandenhoeck \& Ruprecht.

Hufeisen, Britta/Knorr, Dagmar/Rosenberg, Peter/Schroeder, Christoph/Sopata, Aldona/ Wicherkiewicz, Tomasz (Hrsg.) 2018. Sprachbildung und Sprachkontakt im deutschpolnischen Kontext. Frankfurt a.M.: Peter Lang.

Larsen-Freeman, Diane (2019). On language learner agency: A complex dynamic systems theory perspective. The Modern Language Journal, 103, 61-79.

Mihułka, Krystyna/Bąk, Paweł/Chojnacka-Gärtner, Joanna (Hrsg.) (2016). Interkulturalität in Theorie und Praxis der Glottodidaktik und Translatorik. Rzeszów: Wydawnictwo Uniwersytetu Rzeszowskiego.

Prokop, Izabela/Wiśniewska, Danuta (2002). Metodologia i narzędzia badawcze zastosowane w projekcie. In: W. Wilczyńska (Hrsg.), Autonomizacja $w$ dydaktyce języków obcych. Doskonalenie się w komunikacji ustnej (S. 109-128). Poznań: Wydawnictwo Naukowe Uniwersytetu im. Adama Mickiewicza.

Prokop, Izabela (2010). Aspekty analizy pragmalingwistycznej. Poznań: Wydawnictwo Naukowe Uniwersytetu im. Adama Mickiewicza.

Prokop, Izabela (2016). Pragmalingwistyka antropocentryczna. Poznań: Wydawnictwo Naukowe Uniwersytetu im. Adama Mickiewicza.

Prokop, Izabela (2019). Kilka uwag o kontrastywnej analizie stylu. Scripta Neophilologica Posnaniensia, 18, 207-219. 
Scarcella, Robin C. (1992). Interethnic conversation and second language acquisition: Discourse accent revisited. In: Susan Gass/Larry Selinker (eds.), Language transfer in language learning (pp. 109-137). Amsterdam: John Benjamins.

Spinzi, Cinzia/Rizzo, Alessandra (ed.)(2018). Translation or transcreation? Discourses, texts and visuals. Cambridge: Cambridge Scholars Publishing.

Szczepaniak-Kozak, Anna (2018). Interlanguage pragmatic competence. A longitudinal study of ,pragmatic accent' in learning EFL. Poznań: Wydawnictwo Naukowe Uniwersytetu im. Adama Mickiewicza.

Wilson, Deirdre/Sperber, Dan (2006). Relevance theory. In: L. L. Horn/G. Ward (eds.), The handbook of pragmatics (S. 607-632). Malden u. a.: Blackwell Publishing.

Yule, George (1996). Pragmatics. Oxford: Oxford University Press. 


\section{Teil 1: Pragma- und Soziolinguistikstudien}


Open-Access-Publikation im Sinne der CC-Lizenz BY-NC-ND 4.0

(C) 2020, Vandenhoeck \& Ruprecht GmbH \& Co. KG, Göttingen ISBN Print: 9783847111863 - ISBN E-Lib: 9783737011860 


\title{
Barbara Kryk-Kastovsky (Universität Wien)
}

\section{Impoliteness as a result of power asymmetry in selected social contexts}

\begin{abstract}
The paper studies the relation between the phenomenon of impoliteness (as defined in Culpeper et al 2003, modified in Kryk-Kastovsky 2006, and finally put into a wider perspective by Locher 2004 and Bousfield \& Locher 2008) and the degree of power exercised in various social contexts. The methodological question addressed here is how one can account for the different degrees of impoliteness observable in the discourse between the representatives of social roles defined by the $+/-$ power dichotomy. Apart from the different types of interaction described in the literature, like doctor-patient, teacher-student or counsel (policeman)-culprit interaction, other contexts involving power will also be taken into account, e.g. military or administrative ones. Since all these types of interaction differ qualitatively due to the different social contexts, my hypothesis is that they also differ quantitatively, as to the degree of impoliteness imposed by the type of power relation (and who was impolite to whom). The different degrees of impoliteness can be represented on a scale, an analytical tool compatible with the view of socio-pragmatics as a discipline which employs scalar rather than binary constructs to study its objects of interest.
\end{abstract}

\section{Keywords}

communication at work, dinner conversation, discourse (doctor-patient/legal/military/ teacher-student), impoliteness, power

\section{Introduction}

The goal of this study is to investigate how the power imbalance between discourse participants determines their impolite behaviour. For the purposes of the present analysis, I first explore a variety of contexts where the direction of the power relation between discourse participants is presupposed due to their social roles. These include the traditionally power-laden institutions like the military, the courtroom, medical practice, and family. Moreover, I will analyse a few social contexts where the notion of power is transient due to its context-dependence. Thus, in the case of workplace discourse or dinner conversations power is not 
presupposed but asserted by particular discourse participants for particular communicative purposes.

My hypothesis to be corroborated in the course of what is intended as a qualitative study is that while impoliteness is more overt in the case of presupposed power, it is much more covert (i. e. subject to inferencing) in the case of asserted power, where the discourse participants negotiate the power relations in contexts like encounters among colleagues or dinner conversations. Consequently, instead of a simple +/- IMPOLITE dichotomy, a more unified account is proposed which includes the socio-pragmatic information and the classification along the $+/-$ impolite scale in terms of its overtness vs. covertness.

\section{Theoretical background}

Politeness has been subject of extensive debates among linguists ever since Lakoff's (1973) seminal article and Leech's Politeness Principle (1983), which pointed to the insufficiency of the Gricean Cooperative Principle and its maxims. Thus, politeness came to be believed to act as the crucial factor in human communication, to the point of a universal status, cf. Brown and Levinson (1987). The discussion of the criticism of Brown and Levinson's system is beyond the scope of this paper, but see, e.g. Meier (1992, 1995), Watts (1992), Kopytko (1993).

The growing awareness of the importance of politeness in human communication put it into the foreground, whereas the opposite phenomenon, i.e. impoliteness, was neglected and, if noticed at all, has long been considered a default case. The necessity of acknowledging impoliteness as a notion in its own right has been emphasized by Culpeper (1996: 354), who points out that even though politeness remains a norm, impoliteness can also be observed in everyday communication. This is especially true of social contexts which involve an imbalance of power, like courtroom discourse. Drawing on Leech's Politeness Principle (Leech 1983: 104ff), Culpeper juxtaposes inherent impoliteness (obviously offensive acts) and mock impoliteness (banter, irony, sarcasm). Culpeper's system further incorporates Brown and Levinson's notion of face, but in contradistinction to Brown and Levinson's politeness strategies as a means of enhancing face, Culpeper's impoliteness strategies are a means of attacking face, e.g. bald-on-record impoliteness, positive impoliteness, negative impoliteness, mock impoliteness, and the strategy of withholding politeness. The system has been refined in Culpeper et al. (2003: 1556) where the impoliteness strategies are complemented by variables like the notion of context and speaker's intentions.

Following the above-sketched line of thought, I am going to analyse the degree of impoliteness which occurs between discourse participants representing imbalance of power in various social contexts. My approach differs from Locher's 
(2004) analysis of power and politeness in that she focuses on contexts like: family discourse, business meetings and political debates, whereas my study touches upon a wider spectrum of power-laden situations, like courtroom, the military, doctor-patient interaction, etc. Notice that the dichotomy between presupposed power and asserted power introduced here might prove a useful analytical tool in teasing out contexts where the degree of impoliteness cannot be assigned unambiguously, but is subject of negotiation, like in the case of a workplace discourse, where the relation of power between the participants is highly context-dependent. Thus, in the relation between the boss and his/her dependents power is presupposed, whereas among colleagues occupying parallel posts, i. e. being on a socially equal footing, power is only asserted by one of them.

It is worth noting that my distinction between presupposed power and asserted power is parallel to the oppositions hitherto recognised in the study of meaning. The classical Gricean dichotomy of natural-meaning vs. non-natural meaning (meaning- $n n$ ) results in a plethora of semantic (context-independent) vs. pragmatic (context-dependent) realisations of major semantic-pragmatic concepts. Thus, utterance meaning can be contrasted with speaker meaning, semantic presupposition with assertion, and the traditional categories of person/ place/time deixis with social or discourse deixis. Before looking at the (probably more frequent) phenomenon of asserted politeness, my analysis below will first address the social contexts where impoliteness is more likely to occur due to the power imbalance being presupposed.

\section{Impoliteness in presupposed power relations}

The most semantically transparent instances of presupposed power imbalance are encountered in institutions where the power of one party over another party is presupposed because it is socially sanctioned for socio-historical reasons. These include, among others, the contexts of the military, doctor-patient interaction, the law-enforcing institutions, school, and the family, some of which will be discussed below.

\subsection{Military discourse}

The typical power-related context that suggests itself in connection with (written) military discourse is that of instruction. According to Szczepaniak-Kozak who has studied NATO documents in terms of the structure of military discourse "[...] instructions appear in texts as a series of steps in a narrative or a numbered list written in the order in which the steps should be performed" (Szczepaniak- 
Kozak 2004: 132). She shows that, interestingly, most of the military instructions are indirect in that they do not use imperatives, but rather are expressed in the Simple Present tense, they use modals, or are in the passive form, cf. (1), (2), (3), respectively:

(1) Training team and training support team depart.

(2) That is to say, the concept must meet the mission, be flexible enough to facilitate a variety of national approaches, take advantage of national strengths and clearly indicate that it is beneficial to both the CN's and NATO authorities.

(3) Finance missions are divided into two general areas: services provided to organizations and services provided to individuals (ALP 1995: 11-2, in SzczepaniakKozak 2004: 131f).

Consequently, it may seem that in military instructions the question of power, let alone (im)politeness, does not arise since they are formulated in an impersonal, i. e. neutral way, probably due to their written form. However, as has been shown by Culpeper (1996), the situation is markedly different in spoken military discourse. He quotes a documentary on an army training camp where a female recruit private Alves (PA) is interviewed by three sergeants after she performed badly in a training program and showed signs of insubordination. In the excerpt below, one of the sergeants (S) abuses the private in a series of acts of negative impoliteness, like "frighten, condescend, scorn, and ridicule" (cf. Culpeper 1996: 358). These strategies, along with the use of obvious overt markers of condescension and scorn (like taboo terms including invective) constitute an unquestionable case of what I call overt impoliteness. Notice also that the private is deprived of any possibility of defending herself, while the sergeant continues to abuse her in consecutive turns (for the purposes of this paper, the offensive terms have been put into bold type), Culpeper (1996:360):

(4) S: you're going to mess up one of my squad leaders PA:

S: any way you can how about it $=$ don't

PA: $=$ I $=$

S: bullshit me now Alves you want to jump you want to

PA:

S: jump on somebody $=$ JUMP ON ME then...

PA: $\quad=$ no=

S: shut up Alves you're the one who is

PA: said the sergeant

S: running your little mouth again you're the one

PA:

S: intimidating and threatening my squad leaders

PA:

S: bullshit tell that god damn lie to someone 
PA: I didn't sergeant

S: that believes your ass private you've already been

PA:

S: proven to be a damn habitual liar

It is remarkable how the sergeant employs both lexical and grammatical means to abuse the private. As follows from the above, military discourse seems to be a rich source of overt negative impoliteness acts, probably more so than another of power-laden contexts, i. e. medical discourse.

\subsection{The medical scene (Doctor-patient interaction)}

Consider the following examples of an obvious, socially-sanctioned imbalance of power between medical personnel and their patients. In (5) below, taken from Lakoff (1990), the patient is subject to a power game exercised by the receptionist, who tells him/her to wait and in order to emphasize the imbalance of power and the importance of the physician, she uses the term doctor. According to Lakoff (1990: 20), referring to persons by their titles is very unusual in American culture, whereas titles are customarily used as honorific forms of address. ${ }^{1}$

(5) A: I have an appointment with Dr. Snarf at eleven forty-five.

$\mathrm{B}$ : Oh, doctor is running late. Please have a seat.

However, notice that apart from the use of the referring expression doctor the receptionist exhibits her power rather indirectly. On the syntactic level, she refrains from the use of overt exponents of impoliteness and employs what could be taken as politeness markers instead. Rather than using a direct statement refusing cooperation, she opts for indirectness, followed by the speech act of invitation whose inherent politeness is enhanced by the politeness marker please. Nevertheless, the power exercised on the patient obviously follows from the somewhat brief answer on the receptionist's part, lacking any apology or expression of regret. Consequently, (5) is an instance of what I will label covert impoliteness, whose degree of impoliteness is culturally- and contextually-conditioned and has to be inferred by the addressee.

1 For more information on the use of forms of address and titles in American English, cf. Brown and Levinson (1987). The use of titles is obviously idiosyncratic in every language and culture, so that, for example, in Polish professional titles are much more frequently used than in English, cf. Wierzbicka (1991: 104ff; 1992: 316ff). However, it is the speakers of Austrian German who probably use professional titles (including the names of positions in various institutions like Herr Sektionschef [Eng.: Mr. head of the department]) most frequently. Interestingly, these would not only be used in the appropriate contexts of workplace, but it is customary to address/refer to a person with his/her title in any other context if one possesses the relevant information, cf. e.g. Braun (1988). 
In contrast, consider (6), an instance of doctor-patient interaction in Southwestern Nigeria, cf. Odebunmi (2006: 31):

(6) Patient: Doctor, will I be admitted? I want to be under medical care in the hospital. Money is not my problem.

Doctor: Just go and stay outside and relax. I will send you to the laboratory first before any treatment. (To the orderly) Take this card to the laboratory head; I have written some laboratory investigations that are to be carried out on the patient including 3'3'3' screening.

The exchange is an intriguing case of a discrepancy between form and content of the doctor's utterance. On the one hand, the imperative used by the doctor in reply to the patient's request could qualify as a structural exponent of impoliteness, especially since it is reinforced by the brevity of the utterance. On the other hand, from a semantic point of view, the utterance maximizes the addressee's (patient's) benefit in accordance with Leech's Maxim of Tact ("Minimize cost to other, maximize benefit to other", cf. Leech 1983: 132). The patient's benefit is especially apparent in his precarious position since he will have to be tested for HIV/AIDS. The doctor tries to avoid revealing this fact to him by using medical jargon rather than plain words. As a result, the clash between form and content of the utterance leads to a neutralisation of +/-IMPOLITE dichotomy, where the doctor's professional ethics overrides his position of power.

Finally, consider the following exchange between a Polish doctor and his (potential) patient, cf. Weidner (2005: 24):

(7) Doctor: Najlepsze rozwiązanie, jeśli chodzi o stopy, będziemy musieli zrobić nacięcie w okolicy brzusznej, aby dotrzeć do zakończeń nerwowych biegnących wzdłuż nóg i prowadzących do stóp= Umieścimy to tu i - O mój Boże!!! [Eng.: The best solution, in the case of your feet, is that we will have to make an incision in the abdominal area in order to get to the nerve endings running along the legs and leading to the feet. We'll put it here and - Oh, my God!]

Patient: O co chodzi? [Eng.: What's wrong?]

Doctor: O mój Boże! Ależ Pani jest tłusta! [Oh my God! Aren’t you fat!]

In contrast to (6), neutral with respect to its degree of (im)politeness, (7) is an obvious case of overt impoliteness, both on the structural and semantic levels. Thus, the form of (7) is impolite due to the use of an exclamation (Oh, my God!) regarded as a taboo term (both in Polish and in English) because of its religious connotations. Moreover, the use of the taboo term followed by a very direct and unfavourable attribute describing the patient's looks, qualifies (7) as an extreme case of overt impoliteness both on the level of form and content. A question arises at this point whether the extreme degree of impoliteness of (7) is due to the higher 
degree of directness used and tolerated by speakers of Polish in comparison with speakers of other languages, especially English. ${ }^{2}$

\subsection{The legal scene}

It can be expected that in the case of legal contexts (law courts, police precincts), the possibility of impolite (verbal and non-verbal) behaviour is relatively high. As I have pointed out elsewhere on the basis of data from selected Early Modern English court trial records, impolite behaviour of the judges and other interrogators was a norm in the 17th century England, cf. Kryk-Kastovsky (2002: $234 \mathrm{ff}$ ). It was usually manifested overtly both by lexical means (the use of invective and foul language) as well as structurally (the form of the questions used in the interrogation process). Although the judge is still very powerful in the modern Anglo-Saxon adversative legal system, the use of invectives is no longer possible in court (cf. Lakoff 1990: $87 \mathrm{ff}$ ). This does not mean, however, that one cannot observe various degrees of (more or less covert) impoliteness in modern courtroom discourse. Consider the following exchanges between the prosecutor Pertocelli and O.J. Simpson (henceforth OJS) during the civil murder trial when Pertocelli, by using strategies impolite both on the structural and lexical levels, tried to create the impression that O. J. Simpson was lying, cf. Janney (1999). The structural means of threatening the defendant's face included leading questions ${ }^{3}$, which employed question tags, as in (8) and (9), or direct statements, as in (10). On the lexical level, the use of the openly accusatory term lie in (8) and (9) constitutes an instance of overt impoliteness which immediately put OJS on the defensive. Similarly, the expression a different story in (10) might be considered impolite, but only after it is subject to inferencing, thus it can be classified as a case of covert impoliteness:

(8) Q: That was a lie, wasn't it, sir?

A: I think it was morally dishonest of me, yes. I don't know if I would call it a lie.

2 According to Wierzbicka, speakers of Polish are much more direct than speakers of English; however she warns against a biased analysis of the directness/indirectness dichotomy, since it often involves an absolute/relative comparison between extreme cultures, cf. Wierzbicka (1991: $25 \mathrm{ff}$ ). Compare also Hofstede's study of cultural differences in value systems of 40 countries where he investigates the following areas: social inequality, the relationship between an individual and a group, masculinity and femininity, and ways dealing with uncertainty, especially the expression of emotions. As a result, Hofstede postulates four dimensions along which cultures can be differentiated: power distance, individualism, masculinity, and uncertainty avoidance, cf. Hofstede $(1984,1991)$ and the discussion in Clyne (1994: 29ff).

3 On leading questions and their effectiveness as an interrogation technique, cf. Danet (1980), Hiltunen (1996), Luchjenbroers (1997), and Doty and Hiltunen (2002). 
(9) Q: Okay. And when you were trying to protect yourself, you lied, right?

A: I think I may have minimalized the situation.

(10) Q: You have a different story now. It's different now, isn't it?

A: I think it's more accurate now

An interesting case is the prosecutor's meta-comment (referring back to OJS's statement), which is an instance of overt negative impoliteness employing one of Culpeper's negative impoliteness output strategies, i.e. "condescend", cf. Culpeper (1996: 358). Notice how the prosecutor condescendingly reminds OJS that it is him who holds the power and can therefore question the formulation of the defendant's statements:

(11) Q: Are you sure about that?

A: I think everybody knows that

Q: I'm not asking about everybody

As follows from the above examples, the power relation holding between the interrogator and the interrogated in the context of law courts can result in a spectrum of impoliteness strategies, mainly cases of overt impoliteness which range from overtly accusing the defendant of lying to meta-comments questioning the defendant's formulations.

Having discussed a few social contexts in which power is presupposed and, as a result, impoliteness is often overt and easy to detect, I will now pass on to cases where power is not a constant but a transient value. As will be shown below, if power depends on the context, it is not presupposed but simply asserted, i.e. claimed by the discourse participants at a particular point during their exchange. As was the case before, impoliteness occurs there either overtly or covertly (whereupon it has to be inferred).

\section{Impoliteness in asserted power relations}

\subsection{Spontaneous conversations}

Despite a solidarity relation which should normally hold between discourse participants sharing parallel social roles, e.g. colleagues at work place, students, etc. ${ }^{4}$ in some contexts a power relation can be asserted by one of them. The decisive factors can range over many sociolinguistic variables like age, higher professional position, more experience in a particular field, or can be subject to an instantaneous negotiation. Consider a selection of data from A corpus of

4 On the distinction between power and solidarity, cf. Brown and Gilman (1960) and Brown and Levinson (1987). On its application to workplace contexts, cf. Clyne (1994: 56f). 
English conversation (Svartvik \& Quirk 1980) containing spontaneous conversations of educated British speakers. The first example below (12) is a conversation between A (a male academic, age 60), b (male businessman, age 52), and c (female academic, age 40), cf. Svartvik and Quirk (1980: 352ff):

(12) A: and suddenly one of us started saying well didn't we have a lovely time in the County Lough last year

c: (3 sylls)

A: and within a matter of seconds the Irish people were coming over and saying do come and have a drink with us and they said we we didn't want to to we didn't want to spoil your holiday by thinking about the Irish problem but the way you said just now well that it was it was it was like it was a godsend to hear b: ah

A: and they were educated Irish people from the South with plenty of money

c: $\mathrm{m}$

A: but Roman Catholics

b: yes

A: and they just were so absolutely disgusted

b: oh we've all had lovely holidays in Ireland it's a lovely place

c: $\mathrm{m} \mathrm{m}$

A: Yes yes Chrissie's mother says I I like the country but I don't like the people.

It is remarkable how despite the same profession (thus probably a comparable social status) of the three discourse participants and a rather minimal age differences between them, the direction of power assertion is absolutely transparent. $\mathrm{A}$, as the oldest (thus probably occupying the highest position in the academic hierarchy) usurps power by taking most of the turns, which are also the longest. In contrast, the other two participants hardly utter any sound apart from monosyllabic back-channelling. Predictably, it is A who produces some covertly impolite utterances by which he ironically juxtaposes the level of education and wealth of the Irish people with their religion. Apart from being impolite on the semantic level, A also constantly interrupts the others so that they have no chance to contribute to the conversation, and when B finally manages to take a full turn, his remark is dismissed by an ironical comment. Concluding, this short excerpt demonstrates how among discourse participants of an equal social position, power can be negotiated and usurped so that as a result (both covertly and overtly) impolite utterances can occur.

In contrast, consider the following conversation between $\mathbf{A}$ (male academic, age 26), B (female academic, age 48), cf. Svartvik and Quirk (1980: 505):

(13) A: I I I feel as though I've done something wrong you see /3sylls/ I think I haven't B: well you shouldn't - no of course you haven't

A: well he's trying to make me feel as though I have 
B: he's a silly ass he was embarrassed by the situation and that was the way he behaved and it's very stupid.

In this excerpt, it is the older female academic who assumes the position of power in the conversation, thus the gender and age factors are playing a role. However, she is not exercising her power on the interlocutor, i. e. her younger colleague, but is trying to console him by offering an explanation of the older colleague's behaviour. By doing so, she employs offensive terms referring to their boss, which can be classified as obvious instances of overt impoliteness (on the lexical level).

Another context worth studying at this point is an interaction involving an obvious power play between a, a male academic (age 40) and A, a female prospective undergraduate (age ca. 20), cf. Svartvik and Quirk (1980: 776):

(14) a: what is it about literature that you find so attractive

A: because literature is the in some cases the product of the imagination isn't it and and of man's minds and it is the imagination and the mind of man that I'm interested in I think one must know the mind of men why they do things why they say things why they are as they are

a: (coughs)

A: and this is manifested in the works of art

a: if it were only imagination you might get quite a lot out of a fine art course

A: yes but it's not just imagination it's character of men and the actions of men

a: yes $[\mathrm{m}]$

A: that I'm interested in

a: yes I think that's a good reason for wanting to read English

The power play between the older academic and the much younger prospective undergraduate results in some instances of impoliteness, both non-verbal (the cough as a sign of scepticism/disapproval after an unacceptable answer of the candidate), and verbal ones. Notice how the first ironical comment of the examiner ("if it were only imagination you might get quite a lot out of a fine art course") is semantically transparent, i. e. also relatively overt, therefore easy to process. In contrast, his last comment ("yes, I think that's a good reason for wanting to read English") is an instance of irony. If taken literally, i.e. from the point of view of utterance meaning, it would be a perfectly positive comment, whereas if the candidate looks into the speaker meaning and performs some inferencing, she will be able to draw the (conversational) implicature opposite to the utterance meaning ("I don't think that's a good reason for wanting to read English").

At this point, it might be interesting to leave the academic scene and see how power can be negotiated at work place and what kind of communication strategies can ensue to mark impoliteness. 


\subsection{Communication at work}

Consider the following example of a dialogue from Clyne (1994: 64) where Tim is complaining to Lee about dining-room guests and trying to make him reproach them at the same time:

(15) Tim: see what they're doing, they're washing the bloody spoons in the coffee machine eh Lee Lee tell tell this one here

Lee: yes, mate

Tim: come come I'm very sick an' tired oh she's coming there

The two discourse participants in this exchange are sharing the same social role (colleagues in a catering business), but the power relations are very clearly defined. It is Tim who assumes a power position in the discourse and uses it to issue directives at Lee. He is very direct, but not impolite, in his instructions. However, he employs some overt impoliteness markers (both lexical, i.e. the expletive bloody, and phrasal, i.e. the expression sick and tired) when referring to the behaviour of some of the guests.

Another example from the same source (Clyne 1994: 52f) concerns complaints of the colleagues Frank and Ray at their work place about the inefficiency of the work of another colleague, Lola, who tends to misunderstand instructions and perform very slowly:

(16) Frank: no you know why, because I don't want construction there, that's why I Ray: yeah

Frank: want the system running how it should be

Ray: and I said look. I have done about

Ray: three bloody door trims and I go on the cutting table and another

Ray: one is coming. fucking hell. it's not hard for me just four seconds

Frank: even I said many

Ray: for me to do that one like that wack wack wack

Frank: times even one day said so my so my daughter is coming they get upset

Frank: but we are not behind we are actually we are ahead.

Notice that in (16) the question of power does not arise, since it is two colleagues sharing a solidarity relation, who talk about a third one, Lola. However, in the course of the exchange it is Ray who assumes the leading position by performing most of the turns and complaining about Lola's performance in very explicit terms (his overt impoliteness markers include an adjective bloody and a taboo exclamation fucking hell). Thus, it follows from the analysis of (15) that in work place discourse impoliteness is also possible among colleagues enjoying an equal social position if a conflict situation arises. 


\subsection{Dinner conversations}

Another social context which might involve a negotiation of power and potentially lead to a conflict and the use of impoliteness strategies is a family dinner conversation. Consider the following data from a study on non-observance of Gricean maxims in dinner table conversations of Swedish families with children between the ages four and seventeen years, cf. Brumark (2006). (17) is an exchange between the child and its parents who negotiate power (i.e. establish the power relation) in different ways, hence they also differ in their use of impoliteness strategies. While the mother is very direct in her explicit denial (a token of overt impoliteness), the father produces a non-conventional observation by using an expression from the sports domain (a token of covert impoliteness to be inferred by the child):

(17) Child: mamma får ja rapa? (Eng.: mum, can I burp?)

Father: hörru du (Eng.: what was that?)

Mother: nä de får du inte (Eng.: no, you can't)

Father: snacka om att du kommer att ligga på minus (Eng.: boy are you ever going to lose points on this!)

Notice that despite the power relation between the parents and the child, it is the child who uses its position to be overtly impolite, i.e. asking permission to perform an unacceptable act (of burping). Consequently, in the contexts of family relations one may claim that the crucial question about impoliteness is not a dichotomous one, along the $+/$ - POLITE scale, but rather it is about a more complex question asked at the beginning of this paper, viz. "Who is doing what to whom?".

At this point, objections might arise that in family dinner conversations the position of power might be (at least to some extent) presupposed, of course depending on how traditional the family is. Therefore, a look at dinner conversations among friends/acquaintances is in order. In the following four-party dinner conversation between three women FA, FD, FB and a man MC conducted in Canadian French and translated into English by the author, cf. Berrier (1997: $331 \mathrm{ff}$ ), all the four participants cooperate to make the conversation run smoothly. However, as the author points out, the two female participants FA and FD assume the position of power and, as a result emerge, as leaders:

(18) MC: Yeah, that's right(?)

FA: Theory and empirical research, y'know

MC: (?)

FD: Huh?

MC: (?)

FA: But, I had this really hard management course that I was sure to flunk 
FD: Well, now(?)

MC: Ah, yeah

FD: (?)

FA: My roomie, she told me she'd climb up the stairs of the St. Joseph Oratory on her knees when she passed

MC: that class?

FD: I'm missing two (?)

FA: In the end, she passed, just barely, except she got (?) C,

MC: saw yesterday(?)

FA: an' I got an A-

FD: Are you serious? How?

A more detailed look at the conversation reveals how FA assumes most of the power by monopolizing the conversation, which FD and MC clearly dislike. As a result, FD passes an ironical comment, doubting FA's achievement, a clear sign of overt impoliteness. The power play between friends continues when FA does not seem to realize the impoliteness of FD's comment and goes on bragging, whereupon her friends decide to break her monopolising of the conversation and come up with what Berrier (1997: 332) calls a schism. Thus, by ridiculing the selfcriticism of FA's good results FD and MC perform a subtle form of impoliteness, which seems to go unnoticed by FA:

(19) MC: You got what? You got 82.

FA: Yeah, an' it was (laughter)

MC: It was bad y'know. It was bad.

FA: It was the worst. But I got A minus. I was so pissed off, y'know. A minus.

MC: Ohhhhhhh!

FA: No, no but it makes me mad. A minus. Y'know, that's not good

FD: It's awful

FA: It's average

MC: It's terrible

FA: A minus

(pause)

MC: That's about right

FA: That's right. No, it's average

In conclusion, negotiating the power position in a multi-party dinner conversation among friends can lead to various instances of both covert and overt impoliteness. 


\section{Towards a solution}

A question arises at this point how the cases of power interplay described above and resulting in various degrees of impoliteness could be described in a systematic manner. A possible solution would be an analytical tool frequently employed in socio-pragmatic studies, i.e. a scale of impoliteness encompassing all the relevant variables. This should include the distinction between presupposed vs. asserted power relation holding between the participants in various social contexts. Power is presupposed between discourse participants in historically and socially-sanctioned contexts like the military/medical/legal scene, whereas asserted power, which is subject to context-dependant negotiations, is the domain of spontaneous conversation and (to a large extent) communication at work. The holders of power can exercise various impoliteness strategies, whose realisations cut across the form vs. meaning dichotomy. As to form, impoliteness can be realised on the lexical level (invective, taboo terms), or on the structural level (syntactic constructions, e.g. leading questions). As to meaning, the realisations of impoliteness as ways of attacking face can be overt (i.e. understood literally) or covert (i. e. subject to inferencing).

The solution offered here is a modest proposal concerning the possible ways of handling the notion of impoliteness in contemporary spoken English. As has been mentioned in the introduction, numerous other social contexts might prove to be valuable alleys to explore, e.g. teacher-student interaction, shop-assistantcustomer dialogue, etc. All of this could also be done on a cross-linguistic and cross-cultural scale which might lead to insightful observations. While looking at the problem from this wider perspective, we might get closer to answering the question which many researchers have been trying to answer: How impolite is impolite?

\section{References}

Berrier, Astrid (1997). Four party conversation and gender. Pragmatics, 7(3), 325-366.

Bousfield, Derek/Miriam Locher (eds.) (2008). Impoliteness in language. Berlin: Mouton de Gruyter.

Braun, Frederike (1988). Terms of address: Problems of patterns and usage in various languages and cultures. Berlin: Mouton de Gruyter.

Brown, Roger/Albert Gilman (1960). The pronouns of power and solidarity. In: Thomas Sebeok (ed.), Style in language (pp. 253-276). Cambridge, Mass.: MIT Press.

Brown, Penelope/Stephen Levinson (1987). Politeness. Some universals in language usage. Cambridge: Cambridge University Press.

Brumark, Åsa (2006). Regulatory talk and politeness at the family dinner table. Pragmatics, $16(2 / 3), 171-211$. 
Clyne, Michael (1994). Intercultural communication at work. Cultural values in discourse. Cambridge: Cambridge University Press.

Culpeper, Jonathan (1996). Towards and anatomy of impoliteness. Journal of Pragmatics, $25,349-367$.

Culpeper, Jonathan/Derek Bousfield/Anne Wichmann (2003). Impoliteness revisited: with special reference to dynamic and prosodic aspects. Journal of Pragmatics, 35, 15451579.

Danet, Brenda (1980). Language in the legal process. Law and Society Review, 14, 445-464.

Doty, Kathleen/Risto Hiltunen (2002). 'I will tell, I will tell': Confessional patterns in the Salem Witchcraft Trials, 1692. Journal of Historical Pragmatics, 3(2), 299-335.

Hiltunen, Risto (1996). 'Tell me, be you a witch?': Questions in the Salem Witchcraft Trials of 1692. International Journal for the Semiotics of Law, IX, 17-37.

Hofstede, Geert (1984). Culture's consequences. New York: Sage.

Hofstede, Geert (1991). Cultures and organizations. London: McGrew-Hill.

Janney, Richard (1999). The whole truth and nothing but the truth. Linguistic avoidance in the O. J. Simpson transcripts. In: Wolfgang Falkner/Hans-Jörg Schmid (eds.). Words, lexemes, concepts - approaches to the lexicon. Studies in honour of Leonhard Lipka (pp. 259-272). Tübingen: Gunter Narr.

Kopytko, Roman (1993). Polite discourse in Shakespeare's English. Poznań: Wydawnictwo Naukowe Uniwersytetu im. Adama Mickiewicza.

Kryk-Kastovsky, Barbara (2002). Synchronic and diachronic investigations in pragmatics. Poznań: Motivex.

Kryk-Kastovsky, Barbara (2006). Impoliteness in Early Modern English courtroom discourse. Journal of Historical Pragmatics, 7(2), Special Issue: Historical courtroom discourse, ed. by Barbara Kryk-Kastovsky, 213-243.

Lakoff, Robin (1973). The logic of politeness; or, minding your p's and q's. Papers from the Ninth Regional Meeting Regional Meeting of the Chicago Linguistic Society, 292-305.

Lakoff, Robin (1990). Talking power. The politics of language. New York: Basic Books. Leech, Geoffrey (1983). Principles of pragmatics. London: Longman.

Locher, Miriam (2004). Power and politeness in action. Disagreements in oral communication. Berlin: Mouton de Gruyter.

Luchjenbroers, June (1997). 'In your own words...': Questions and answers in a Supreme Court trial. Journal of Pragmatics, 27(4), 477-503.

Meier, Ardith (1992). Brown and Levinson's legacy of politeness. Vienna English Working Papers (VIEWS), 1(1), 15-35.

Meier, Ardith (1995). Passages of politeness. Journal of Pragmatics, 24(4), 381-392.

Odebunmi, Akin (2006). Locutions in medical discourse in Southwestern Nigeria. Pragmatics, 16(1), 25-41.

Szczepaniak-Kozak, Anna (2004). Introduction to military discourse. Warszawa: Elipsa.

Svartvik, Jan/Randolph Quirk (eds.) (1980). A corpus of English conversation. Lund: CWK Gleerup.

Watts, Richard (1992). Linguistic politeness and politic verbal behaviour: Reconsidering claims for universality. In: Richard Watts/Sachiko Ide/Konrad Ehlich (eds.), Politeness in language. Studies in its history, theory and practice (pp. 43-69). Berlin: Mouton de Gruyter. 
Weidner, Matylda (2005). The pragmatics of doctor-patient interaction. Unpublished M.A. thesis. Adam Mickiewicz University, Poznań.

Wierzbicka, Anna (1991). Cross-cultural pragmatics. Berlin: Mouton de Gruyter.

Wierzbicka, Anna (1992). Semantics, culture and cognition. Oxford: Oxford University Press. 


\title{
Anna Szczepaniak-Kozak \\ (Uniwersytet im. Adama Mickiewicza w Poznaniu)
}

\section{Pragmatic accent: Sociolinguistic and pragmalinguistic examples $^{1}$}

\begin{abstract}
In this chapter, it is postulated that foreign language users develop a pragmatic accent, which refers to the systematic occurrence of selected pragmatic features in their performance of communication functions in the target language. In the specification of this term, I refer to two related conceptualizations, one by Scarcella (1992) and the other by Yule (1996). I apply the expression coined by Yule to refer to a phenomenon which is parallel to Scarcella's discourse accent. In order to substantiate this concept, in later sections of this chapter, first a summary of the interlanguage pragmatic competence research which I conducted is presented. This empirical endeavour showed that even advanced Polish EFL speakers do not fully approximate the native speaker model and instead retain in their pragmatic output a set of pragmatic features which enables their easy identification as nonnative users. In the subsequent sections, the title concept is further exemplified by means of evidence coming from research reports offered by other researchers or coming from other languages.
\end{abstract}

\section{Keywords}

pragmatic accent, pragmatic overgeneralization, pragmatic transfer, estimation of future social distance, camaraderie

\section{Introduction}

Early research on interlanguage (IL) focused on errors conditioned by differences between the mother tongue of the learner and the target language (L2) he or she is learning, based on the Contrastive Analysis Hypothesis (Lado 1957). In general, initially IL literature treated learner language as a deficient but necessary makeshift system on its way to an idealized native speaker (NS) baseline. Seen from this perspective, students' mother tongue (L1) was perceived as detrimental to their L2 full attainment. Recently, however, with the shift towards the learner

1 I wish to express my thanks for prof. Karen Glaser (University of Leipzig) for reviwing the draft of this chapter. Her ideas helped me to improve the text to a considerable degree. 
as the focal agent in the process of foreign language teaching, the value and potential of the learner's language system is being re-evaluated. More and more researchers see it an effective means of communication, an approximative dialect, which is a natural outcome of learning and which should not be the object of scorn (Gomez-Laich 2016; Odlin 2016; Wach 2018).

This shift in the evaluation of IL has been taking place simultaneously with the re-formulation of the general attitude to bilingualism and multilingualism. While bilingual or multilingual speakers are increasing in number around the globe, monolingual native speakers are decreasingly often presented as the only norm-bearer, including in the area of pragmatics. Assuming that nowadays we "have to look to bilingual or multilingual speakers for a more appropriate norm in interlanguage pragmatics" (House \& Kasper 2000: 102), in this chapter, it is postulated that foreign language users develop a pragmatic accent which refers to the systematic occurrence of selected pragmatic features in their performance of communication functions in the target language. However, one caveat is necessary. While some distortions from the NS baseline may be attributed to their insufficient knowledge of the target language and its communicative practices, or students' insufficient exposure to authentic language, this chapter argues that some of these differences are shared across larger groups of non-native speakers (NNS) and they cannot be explained on the grounds of lacking proficiency or exposure only. Instead, it is suggested that such features or preferences could be subsumed under the umbrella term pragmatic accent.

In the specification of the term pragmatic accent, which will be presented in the first section, I refer to two related conceptualizations, one by Scarcella (1992) and the other by Yule (1996). In detail, I apply the expression coined by Yule to refer to a phenomenon which is parallel to Scarcella's discourse accent. In order to substantiate this concept, in later sections of this chapter, first I present a summary of the IL pragmatic competence research which I conducted, and which showed that even advanced Polish speakers of English as a foreign language (EFL) do not fully approximate the native speaker model and instead "they display highly predictable, internally consistent patterns which recur systematically in the data" (Scarcella 1992: 128). In the subsequent sections, I exemplify the title concept by means of evidence coming from research reports offered by other researchers or coming from other languages.

\section{Pragmatic accent defined}

In linguistics, accent is a term which usually refers to pronunciation and means "the cumulative auditory effect of those features of a person's pronunciation which identify where he is from" (Crystal 1980: 7). Phonological accents can be 
regional (e.g. rural, urban), national (e.g. Australian English), educational (e.g. the RP accent in Great Britain), or representing the impression of other languages (foreign accent) (Crystal 1980: 7).

It was Yule (1996) who coined the term pragmatic accent. However, he uses it to refer to items/portions of meaning which are not verbalized. He states that "we all speak with what might be called a pragmatic accent, that is, aspects of our talk that indicate what we assume is communicated without being said" (Yule 1996: 88). He coins this term to signal that, despite the fact that he is in favor of universal pragmatic principles, he provides for "a different approach to the relationship between the maxims of quality and quantity in a more comprehensive pragmatics" (Yule 1996: 88). He illustrates his stance by evoking differences in the reactions to compliments between native American Indians and other inhabitants of the USA: "The typical American English style of complimenting creates great embarrassment for some Native American Indian receivers (it's perceived as excessive), and can elicit a reaction similar to apologizing from some Japanese receivers (it's perceived as impossible to accept)". He concludes his discussion of pragmatic accent by underscoring that speech act realizations in particular cultures do not overlap: "it is unlikely that the division one cultural group makes between any two social actions such as 'thanking' or 'apologizing' will be matched precisely within another culture" (ibid.).

As can be seen above, Yule (1996) uses the term to talk about intra-language communication and perlocution, and/or a cross-pragmatic understanding of the term. My understanding of the phenomenon is closer to Scarcella's $(1983,1992)$ concept of the discourse accent, which she uses with reference to extremely proficient Spanish EFL learners' transfer of their L1 conversational management strategies into the TL. She posits that a group's discourse accent is recognized by characteristic language features such as syntax (sentence structure), grammar, lexicon and phonology. It can include members' topic choices, style of talking and conduct of particular speech acts. In writing, it can appear as a distinct pattern of cohesive devices or thought organization, including paragraph organization. Discourse accent has also been included by Claire Kramsch (2003) in her concept of 'the third place' in which learners of foreign languages find themselves. She suggests that it is "a speaking or writing style that bears the mark of a discourse community's ways of using language", which would embrace topic selection, information presentation, interaction style (Kramsch 2003: 7). Such clusters of features enable identification of a particular speech community and, when made conscious, may constitute a source of "strength and pride" for its members (Kramsch 2003: 65). By analogy, I apply the expression pragmatic accent to substantiate a set of pragmatic features which FL speakers reveal to a considerable degree, and which enables their easy identification as non-native users. 
It is necessary to acknowledge that transfer is not the only process which shapes the pragmatic accent of a particular group of non-native speakers, with overgeneralisation playing a similarly significant role. (cf. Szczepaniak-Kozak 2018: 68-75). Some variations in the PC of foreign language learners may also be due to the contexts of L2 learning and individual differences (including learner agency, motivation, character traits, past learning history) (cf. the concept of pragmalect proposed by Thomas 1983). And some learners may prefer not to change their L1 use patterns and, either consciously or not, transfer "their L1 sentiments into the L2" (Wyner \& Cohen 2015: 528). A point in case, GomezLaich (2016: 252-256) postulates that learners' different levels of pragmatic attainment may signal their autonomy and the desire to accentuate their linguistic self. She reaches this conclusion on the basis of a synthesis study conducted in a corpus of eleven refereed papers, whose aim was to discover "the reasons why learners opt to resist certain L2 norms" in the area of pragmatics (ibid.). The same author stresses that learners may react differently to target language pragmatic norms. Some of them "may adopt or resist those norms to index an identity that fits their L1 cultural values" (Gomez-Laich 2016: 256). Some others continue to rely on their L1 pragmatic system when they find TL practices inconsistent with their L1 values. Finally, patterns discovered in groups taught by the same teacher, or in the same instructional settings may be due to the instruction provided and instructional materials.

\section{Concepts underlying pragmatic accent}

What pragmatic accent shares with phonological accent is that non-native speakers' pragmatic systems are partly conditioned by pragmalinguistic and sociopragmatic transfer. In this sense, what Major (2014: 31), applying Trubetzkoy's (1939/1958) work on L2 phonology, claims to be characteristic of foreign accents is characteristic of foreign pragmatics as well: in general "L2 perception is 'filtered' through the 'sieve' of the L1 and the result of this filtering on linguistic production is most noticeable when one is said to have a language $\mathrm{x}$ accent, as it is largely recognizable due to L1 transfer". Major (2014: 30-31) posits that when learning something new "the tendency is to transfer similar patterns already acquired to the new situation".

Although this understanding of the term itself is relatively new, the assumptions which underlie the concept of pragmatic accent are based on general pragmatic principles. First of all, the existence of 'pragmatic accent' could be treated as a natural consequence of the existence of pragmatics of communication. Pragmatics is namely this branch of linguistics which studies differences in language performance due to the language users and the contexts in which 
they speak. The differences can be captured under the term "contemporary pragmatic variation", which Schneider (2017:319) defines as differences between languages or differences across varieties of the same language. This shift in the interpretation of pragmatic universalism was sparked off by Wierzbicka's famous paper "Different cultures, different languages, different speech acts" published in 1985. Although the existence of pragmatic universals is undeniable, nowadays it is generally accepted that there are significant cross-cultural differences in pragmatic production among speakers coming from different socio-cultural groups (e.g. Cenoz 2003; House 2005; Ogiermann 2009). Following this train of thought, what the idea of pragmatic accent underscores is that the differences continue to be expressed when speakers use a foreign language. In this sense, pragmatic accent touches upon the way culture-specific pragmatic features are visible in a foreign tongue.

Wierzbicka $(1985,1991 / 2003)$ was one of the very first to oppose universal rules of politeness or conversation, based on her observations concerning how cultural values shape linguistic interactions, particularly with regard to linguistic data from Australian English and Polish. Wierzbicka challenged Brown and Levinson's (1987) strongly Anglo-centric emphasis on the value of personal independence and freedom from imposition and claimed that it is appreciated only in some world cultures (Wierzbicka 1991/2003: 29). She posited that "specific differences between languages in the area of indirect speech acts are motivated to a considerable degree by differences in cultural norms and cultural assumptions" (Wierzbicka 2003: 67), e.g. the significance of objectivism and affection in interaction. To illustrate this, when we want to understand the way English and Polish requests are formed, we need to take into account, the "autonomy of the individual and anti-dogmatism in Anglo-Saxon culture or cordiality and warmth in Polish culture" (Wierzbicka 2003: 64). Our understanding of the way locution is uttered in an IL pragmatic system can be heightened by Wilkon's (2002: 102) idea of ethnic presuppositions of the contextual-pragmatic type. He posits that, due to the acculturation process, individual speakers have tacit assumptions about speech acts and their felicity conditions which they share with other speakers coming from the same ethnic or national groups. According to Prokop (2000: 30-32), such assumptions are relatively stable and include, for example, shared values, knowledge of the world and systems of conversation rules, and how these underlie communication.

As mentioned above, one of the reasons why there are pragmatic accents is that, due to acculturation, members of a particular culture share mental sets, schemata and scripts, sociocultural norms, speech events, and linguistic etiquette which rely on the evaluation of sociocultural variables (Pohl 2004: 4, cf. Prokop 2010). These discourse-oriented macropragmatic elements govern, in an implicit way, speakers' behavior in interactions or, more generally, in discourse 
(Schneider 2017: 317). They also determine one's evaluation of the contextual factors involved in a given communication act. These elements are shared across a given community and are not totally dependent on the context. To illustrate this, different cultures treat differently a parent's request for an unexpected favor of babysitting rendered to his or her neighbor. In some cultures, asking for such a favor is considered impossible (due to a shared mental set). If such a request can be considered appropriate in a given community, most probably rendering it would take place during a (telephone) chat with a neighbor, which would follow an intuitively activated script. As far as the act of requesting itself is concerned, there are unwritten norms for the speech event involving requesting, e. g. how much explanation is necessary or how polite the request needs to be according to social conventions (knowledge of the speech event). Members of a particular culture also know whom they can ask for such a favor, judging not only the social distance between the persons involved (if the neighbors are on good terms) but also the degree of imposition which is attributed to babysitting. Furthermore, if the parent in need has recently performed a favor for the neighbor, his request would be treated not only as an act of goodwill but also as an act of paying back the favor (the ranking of rights and obligations, Thomas 1983). Finally, following the rules of linguistic etiquette would involve the evaluation of these sociopragmatic variables, jointly with other macro-social pragmatic factors like age, gender, social class.

Most language users are not aware that these sociopragmatic factors underlie communication and adjust their language production, following them in an unconscious and intuitive way (cf. House 2005). The cultural matrix, or to use Prokop's (2010) term 'ethnic presuppositions', which these rules of social conduct compose, is usually unnoticed. However, differences in culturally determined preferences usually appear in interactions between people who do not share the same culture, which often leads to inter-cultural pragmatic failure. Some evidence supporting this stance comes from researchers studying English as a lingua franca. To illustrate, House (2010: 365) notices that "Localized, regionalized or otherwise appropriated varieties - whose linguistic surface is English, but whose speakers creatively conduct pragmatic shifts in their use of this auxiliary language - are taking over the linguistic landscape".

The last issue worth mentioning is whether it is necessary to do away with one's pragmatic accent. Riley (1989: 234) puts forward: "Pragmatic errors are the result of an interactant imposing the social rules of one culture on his communicative behavior in a situation where the social rules of another culture would be more appropriate". Back in 1989, pragmatic infelicities were negatively perceived, which is expressed by the verb 'impose' in the quote by Riley. However, already by 1996 Kasper and Schmidt proposed that "total convergence with NS norm may not be desirable" (1996: 156). Nowadays, it is widely accepted that the aban- 
doning of "the native speaker as the yardstick" principle is an option for some learners (Jenkins 2007: 175). Indeed, currently, we can observe a paradigm shift from the native speaker as a norm-bearer to the expert multilingual user (House 2010: 382) and a greater tolerance of divergent communicative practices. In fact, we can agree that the outcome of communication is not dependent on convergence with the target norm but rather on the ability to establish common ground and to seek consensus, related to the conversational adjustment and maintaining the sense of good spirit (House \& Kasper 2000: 106). All in all, the expansion of English as a lingua franca is nowadays leading to greater acceptance of non-native speaker behavior in a foreign language, which renders total pragmatic convergence a rather idealistic, unattainable and unrealistic goal.

Even if some researchers prefer to assume a non-evaluative approach to the pragmatic output of EFL learners, it is reasonable to expect that their divergent pragmatic systems may cause difficulties in interactions between people representing different cultures. On one side there are native speakers who, as Schneider (2017: 319) claims, "are much less tolerant towards pragmatic errors, tacitly assuming that all humans share the same ideas about e.g. directness, politeness and appropriateness". Native speakers tend to interpret pragmatic errors as rude behaviour, attributing them "to character flaws, rather than to pragmatic differences between languages and cultures". On the other side there are non-native speakers who, as Kasper and Schmidt (1996: 156) advance, may opt for maintaining certain distance from the TL culture and use "pragmatic distinctiveness (sometimes, always, or depending on context) as a strategy of identity assertion". Interestingly enough, "some differences between NS norms and L2 performance may result in negative stereotyping by NS message recipients, whereas others may be heard as somewhat different but perfectly appropriate alternatives" (Kasper \& Schmidt 1996: 156). In an attempt to find the middle ground, we may postulate that while some indicators of our mother tongue pragmatics can be considered tokens of one's national identity, it is optimal convergence, or "pragmatic fluency" (House 2010: 383), that is recommended and desirable (Kasper \& Schmidt 1996: 156), with optimal standing for responding to learners' needs.

\section{First indicators of pragmatic accent: IL requests rendered by Poles}

In the years 2012-2017, I conducted a longitudinal mixed-method study of the pragmatic competence (PC) of 206 Poles learning EFL at an advanced level (Szczepaniak-Kozak 2018). Its aim was to outline their route of PC development 
and assess the impact of predominantly implicit teaching in EFL learning settings on learners' $\mathrm{PC}$, operationalized as uttering requests. The participants' pragmatic performance was measured by means of a discourse completion task (DCT). The DCT scenarios differed in terms of the values ascribed to the classic independent variables influencing pragmalinguistic realizations of requests: power distance, social distance, imposition, the ranking of the speaker's right to render the request and the hearer's obligation to render the service.

My study indicated that the changes in the cohort's PC were relatively modest, both from a longitudinal and cross-sectional perspective. Interestingly, some of the fossilized pragmatic forms recorded are shared with other/non-Polish learners of EFL. More target-like strategies (need statements, conventionalized expressions) are displayed in the IL requests rendered in low-imposition scenarios (e.g. asking for a notepaper during a business meeting) than in highimposition ones (e.g., borrowing a computer from a roommate who has an essay submission deadline a bit later than the speaker or asking a president of a university for an interview despite his or her tight schedule). A similar conclusion is drawn by Hudson (2001: 297) for Japanese learners of English and by Liu (2006: 160) for Chinese EFL students. However, some of the transferred and overgeneralized forms could be labeled as typical of Poles only, for instance, the systematic absence of expressions of gratitude in contexts featuring the large speaker's right and a large power distance between interlocutors. Most characteristically, throughout the years of the investigation the following pragmalinguistic characteristics of rendering requests came to the fore (Szczepaniak-Kozak 2018: 314, 346-348):

- the four most frequent requestive strategies remained the same and they were ordered in the same ranking of top frequencies: the query preparatory, mood derivable (imperative), the permission question, the mitigated preparatory question,

- the students preferred to render head acts by means of the query preparatory and apply it across all contexts,

- they used maybe in suggestory formulas more often than native speakers of English,

- they inserted the politeness marker please in an unembedded sentence position,

- they also used characteristic external and internal mitigation devices, e.g. 'stressing the importance or urgency of the request' and the camaraderie we.

A few comments on the above list are due. Wierzbicka (1985) proposes that Poles treat 'please' as the equivalent of the Polish word prosze (lit. 'I ask'), which is a performative verb. This leads Polish EFL users to produce utterances such as: 'Please! Sit! Sit!' which are somewhat awkward in English (Bhatti \& Žegarac 2012: 
281). Wierzbicka posits that this pragmalinguistic realization cannot be explained by pragmatic universals but rather by the culture specific use of the Polish performative form proszę. Namely, in Polish it is a token of pragmatic force or intended politeness, rather than a conventional politeness marker (Szczepaniak-Kozak 2018: 350).

The IL data also indicates that Poles apply a characteristic external mitigation strategy called 'stressing the importance or urgency of the request', e. g., it's really important/urgent, which has not been identified in the existing research reports. Instead of interpreting such sentences as providing reasons for the request proper (grounders) (cf. Blum-Kulka et al. 1989), I prefer to treat them separately because they are indicators of the cultural and pragmalinguistic difference between Polish and English. In informal contexts and when asking a relative or friend for a favor, Poles are less concerned about the requestive imposition and are less hesitant to express the importance or urgency of the speech act (Szczepaniak-Kozak 2018: 150-151). This behavior may be related to Poles being more easily available to help others in public, especially when some repair action is necessary, including a linguistic one (cf. Szczepaniak-Kozak 2018: 184).

Apart from the pragmalinguistic features discussed above, in my research there also appeared sociopragmatic characteristics of Polish IL in EFL. Most importantly, it turned out that apart from the classic independent variables governing the use of requests in interactions (power distance, social distance, imposition, ranging of rights and obligations), there may be at least one more factor which influences the pragmatic output of Poles. In detail, there were two scenarios in the research instrument which were identical in terms of the sociopragmatic variables taken into consideration. In one Scenario, called Loud music (LM), one student asks his neighbor to turn the music down. The other scenario, called Blocking the view (BV), takes place during a basketball match. During this event, one man asks a person sitting in front of him to move to one side and thus stop blocking his view. Both LM and BV are interactions between two peers in which the speaker's request, actually a demand, is a reaction to the possibly annoying behavior of the hearer. Both these scenarios feature the same values of the independent variables mentioned above, and yet the requestive output elicited in them differs considerably. This allows me to posit that there is some other independent variable which differentiates them. It could be the subjective evaluation of the chances of striking up a longer social interaction with the hearer, which I tentatively call 'the estimation of future social distance' $(F)$ (SzczepaniakKozak 2018: 278; Szczepaniak-Kozak et al. 2020). $F$ is higher with the neighbor than the fellow spectator. The statistical validity of this proposal is positively tested in Szczepaniak-Kozak et al. (2020). The analysis carried out by means of multinomial logistic modeling established highly significant values of $p$ for $F$. 
To sum up, the majority of the pragmatic infelicities in the corpus collected by me are either systematically overgeneralized forms or instances of negative pragmatic transfer - or, to use Hasselgren's (1994: 237) metaphor, the pragmatic 'teddy bears' of these students. Hasselgren (1994: 252) defines learner preferences in the area of lexicon as words or phrases of perceived equal meaning, out of which one spreads its use to the contexts in which normally the other word or phrase would be used in the TL. 'Teddy bears' in the IL pragmatic competence of Polish EFL speakers are safe, all-purpose pragmatic features, which they use regardless of context, and which give them away as foreigners.

\section{Pragmatic accent: examples from other studies}

It is argued throughout this chapter that the interference errors which are characteristic of larger groups of NNS from a particular culture cannot be treated as accidental, but rather as pattern-indicating. It seems that an element which could be treated as a litmus paper for pragmatic accent could be the evaluation of sociopragmatic features which govern the use of honorific titles, the frequency of their use and understanding of their social connotation. In Polish, German, Italian and Spanish, honorifics are more extensively used than, for example, in British or American English. Woodward-Smith's (1997: 172-187) study of greetings in English indicates that, in fact, although English does not render the $\mathrm{T}-\mathrm{V}$ distinction ( $t u$-vous) by means of pronouns or verb forms, it features other items which convey the degree of formality between interlocutors. Specifically, English people use first and last names, titles, honorifics and more formal/ educated vocabulary to mark the distance. At the same time, the same pragmatic features used to express formality may have a wider or narrower scope of situated meaning. For example, in Polish culture honorifics, apart from rendering the power distance or social distance between interlocutors, can also be used to convey respect politely. Therefore, some Poles remain on formal terms even when they know each other well and they are on good terms. For example, even if I have known my gardener for many years and we are very friendly to each other, I continue to call him by the honorific title Mr. (Pol.: Pan) to show him my respect and appreciation of his services. In this sense, being on V-terms in Poland does not always mean that the interlocutors are distant in hierarchy or social network. Relatedly, the availability of V-and T-forms in Polish makes Poles "more aware of and attentive to status differences in everyday interaction than are British people" (Ogiermann 2009: 33; cf. Jakubowska 1999: 47). This indicates that English NSs and Poles assess interpersonal relationships in different ways, which is enacted also when Poles speak English. In particular, Poles "assign divergent 
values to the variables of $\mathrm{P}$ [ower distance] and $\mathrm{D}$ [social distance]" when compared to English speakers (Ogiermann 2009: 34).

Because Poles are very class- and status-conscious, there exists an intricate system of honorific titles in their mother language and sociopragmatic rules of its use. For example, throughout all levels of the Polish educational system, students are obliged to call their teachers by their formal, professional titles or formal terms of address (e.g., miss, mister, professor). What may puzzle foreigners is that teachers are often referred to by higher titles than they actually hold. For example, all high school teachers are called professors, even if the overwhelming majority of them are M.A. degree holders. This discourse practice is transferred to English classes in Polish schools. Polish teachers extremely rarely are called by their first names, or simply the typical English address form 'teacher'. The lesson observation study offered by Majer and Salski (2004) corroborates my assumption. The scholars notice that Poles learning English often use a honorific title followed by the teacher's first name, e.g., Panie Adamie (Eng.: Mr. Adam), please go on, or Mr. Tomasz, what is the next answer? (Majer \& Salski 2004: 59). These examples include an address term which is typical of Polish and transferred literally into English. Hence, students prefer to express sociopragmatic nuances by means of pragmalinguistic means typical of their mother language, e.g., honorific titles. On top of that, it is a frequent practice in Polish to address an adult man by the honorific title Mr., followed by the name of the occupation and the degree he holds. For example, in professional contexts, a male engineer who has completed a post-bachelor degree can be called and, in fact, frequently is: Mr. MSc. Engineer Nowak (Pol.: Pan Magister Inżynier Nowak). This is why sometimes Poles in English wrongly address others: Mr. Engineer Smith.

Furthermore, being on first-name terms implies different linguistic behavior in Polish and English, but Poles learning EFL do not necessarily realize that. In Polish, being on first-name terms is considered a token of close social distance, Poles wrongly interpret being on first-name terms as nullifying power distance. When speaking English, Polish EFL speakers tend to use (unmitigated) direct requestive strategies because directness is a marker of closeness/familiarity for them. In other words, unconsciously they attach greater attention to the positive politeness typical of Polish. NSs of English may consider this language use overfamiliar and/or inappropriate due to the violation of the right to be free from (more directly expressed) imposition and a greater attention paid to the right for privacy typical of negative-politeness cultures. They continue to use mitigated indirect strategies in such interactions because terms of address do not overlap with directness strategies in the way they do in Polish.

Another feature of ILP of Poles is the use of the camaraderie we, a type of external mitigators in requests. Poles use this pronoun in requests to create inclusivity and solidarity or to create an out-group consisting of the speaker and 
others that are not present in the conversation (Szczepaniak-Kozak 2018: 147). This strategy appears in Blum-Kulka et al.'s (1989) investigation of AuE and BrE as both (1) a politeness strategy: 'Equality or camaraderie' (Act as though you and the addressee were equal or friends/make him feel good; Lakoff 1977: 103), and (2) a means of claiming common ground/membership in a group (Brown \& Levinson 1987: 103). However, these scholars do not treat this pronoun use as a separate category of external modification. A somewhat similar extension, under the name 'bonding', was proposed by Glaser (2014: 272) in her study of disagreements conducted among German EFL students. Camaraderie was also noticed in the study offered by García (2009), which delves into how female speakers respond to requests rendered by English NSs and Venezuelan EFL speakers. García (2009: 387) was able to identify an interesting occurrence: in the same situations, speakers coming from different sociocultural backgrounds behaved differently. Native English speakers preferred deference, while Venezuelans opted for camaraderie, which caused that their interactions were disharmonious.

Despite the fact that so far I have mostly underscored characteristics of the pragmatic accent of Poles in EFL, evidence of accents in other languages can be found as well. For example, in Shively's (2011) investigation of the data collected during service encounters, in which seven US students of Spanish took part. It turned out that they avoided using the turn "Can you give me X" typical of NS conversation initiation. Instead, they formulated service requests by means of imperative sentences or elliptical constructions. Despite their realization that other forms are more frequent in Peninsular Spanish, they continued to initiate the exchange by means of the how-are-you-inquiry and formulated their requests as want statements, which are more standard pragmalinguistic practices in American English.

Traces of pragmatic accent can even be found in more formulaic speech acts, e.g. compliments, expressions of gratitude and responses to thanks. Evidence for this stance comes from Bhatti and Žegarac (2012: 288), who offer a contrastive Polish-English investigation of compliments and refusals. Their findings reveal significant systematic cross-cultural differences relating to refusals, while the differences relating to compliments are fewer and more subtle (Bhatti \& Žegarac 2012: 279). With reference to refusals, the researchers posit that Polish participants' responses to refusals were more negative and the refusals they made were more elaborate than those of English participants. Poles were also more verbose in Polish refusals - so it is not only their feature of interlanguage, but perhaps a feature of Poles when communicating in general (Bhatti \& Žegarac 2012: 293). Apart from this conclusion, their study indicates one more aspect characteristic of pragmatic production by Poles. They postulate that, in comparison to the 
English, Poles display a higher concern for solidarity. Bhatti and Žegarac postulate (2012: 295):

Polish culture seems to place a rather high value on others' needs and wants (in the types of everyday situations described in our DCT scenarios). It seems that in Poland acting in a way which is desirable to others in these types of situation has a comparatively higher weight than in England, where an expression of regret and giving a rational justification for refusing is adequate. Polish respondents' emotional reactions to refusals and their verbal responses to refusals did not provide evidence that they followed conventional culture-specific strategies. Their responses were similar in the comparatively high level of concern for the affective emotional impact of refusing on the hearer, and can be explained in general pragmatic terms, without positing special strategies for refusing.

Finally, I would like to refer to House's (2005) argument that English and German differ in their degree of 'formulaicity', with English having more formulas and expected realization patterns than German. In other words, languages can even differ with regard to whether there are routine formulas in a particular situation, which means that characterizing a certain speech act as 'formulaic' is also language-bound (e.g. here Anglocentric). This tendency can further be transfered into a foreign language and make the accent more pronounced.

We are still far from indexing features which are characteristic of a particular group's pragmatic accent. However, it seems that certain sociopragmatic features can be nominated as relatively possible. We can include here the sociopragmatic elements which Kasper and Schmidt (1996: 156-157) list as those which language learners tap into: "learners' assessment of use of situation and the contextual variables in it, their assessment of whether it is appropriate to carry out a certain speech act" and preferences for pragmalinguistic realizations of particular speech acts.

\section{Final remarks}

From my own research, it is clear that pragmatic accent is a hybrid system relying on transfer and overgeneralization which Poles have developed as their 'third way' in-between the pragmatics systems of English and Polish. The existence of pragmatic accent should not be surprising: just as Poles as a national group speak English with a phonological accent, we may safely assume that the pragmatic level of their performance may be 'tinted' with Polish characteristics as well. It is undeniable that conversation conventions differ across cultures, and a set of such conventions which is characteristic of a given culture, when revealed in a foreign language, can be called pragmatic accent. The fact that some of the forms characteristic of pragmatic accent are overgeneralized tokens originating in the 
TL can also be related to preferences originating in the MT. This, however, has not been proven and definitely requires further investigation.

Two issues could be the subject of further research with reference to pragmatic accent. First of all, it is worth investigating what learning conditions enable losing one's pragmatic accent, for those speakers who wish to do so. It seems that this requires higher levels of pragmatic awareness and some proficiency in the orchestration of pragmalinguistic forms, Gricean maxims, semantic strategies, sociocultural conventions, and situational requirements (cf. Taguchi 2012). What is more, demonstrating such expertise in an ongoing interaction demands the expenditure of mental processing effort, especially higher-order inferential processing and operational control (cf. Bialystok 1993; Timpe-Laughlin 2017). The fact remains that even if language learners have a considerable stock of formal knowledge of the TL and are metacognitively mature, they are very often not capable of using it spontaneously, and need more time to develop control over their cognitive processes. The other aspect worth further investigation could be whether one's pragmatic accent can influence the comprehension of pragmatic output by speakers coming from other cultures. According to Bhatti and Žegarac (2012: 283), "relevance theory predicts that relatively small differences in culture-specific factors which systematically influence communication will also influence the production and the interpretation of particular types of speech act systematically and significantly". The data collected in my longitudinal research (Szczepaniak-Kozak 2018: 352-356) indicates that pragmatic accent may influence the comprehension of pragmatic input because pragmatic data collected in a multiple choice test (that is, reception questionnaire) did not indicate a greater pragmatic attainment than data collected in the discourse completion test (that is, a production questionnaire). Pragmatic comprehension tends to be lower in EFL learning contextes, with very limited possibilities for language contact, and consequently very few opportunities for testing hypotheses pertaining to the pragmatics of the foreign language. Corroborating or contradicting this prediction, i.e. testing the effect of pragmatic accent on the comprehension of pragmatic output, could be the aim of further studies.

\section{References}

Bhatti Joanna/Žegarac, Vladimir (2012). Compliments and refusals in Poland and England. Research in Language, 10(3). DOI 10.2478/v10015-011-0025-x.

Bialystok, Ellen (1993). Symbolic representation and attentional control in pragmatic competence. In: Gabriele Kasper/Shoshana Blum-Kulka (eds.), Interlanguage pragmatics (pp. 43-59). New York: Oxford University Press. 
Blum-Kulka, Shoshana/House, Juliane/Kasper, Gabriele (eds.) (1989). Cross-cultural pragmatics: Requests and apologies. Norwood, NJ: Ablex.

Brown, Penelope/Levinson, Stephen C. (1987). Politeness: Some universals in language use. Cambridge: Cambridge University Press.

Cenoz, Jasone (2003). The intercultural style hypothesis: L1 and L2 interaction in requesting behavior. In: Vivian Cook (ed.), Effects of the second language on the first (pp. 62-80). Clevedon: Multilingual Matters.

Crystal, David (2007). A first dictionary of linguistics and phonetics. Boulder: Westview Press.

García, Carmen (2009). Responses to a request by native and non-native English speakers: Deference vs. camaraderie Multilingua, 11(4), 387-406. https://doi.org/10.1515/mult. 1992.11.4.387.

Glaser, Karen (2014). Inductive or deductive? The impact of method of instruction on the acquisition of pragmatic competence in EFL. Newcastle upon Tyne: Cambridge Scholars Publishing.

Gomez-Laich, Maria Pia (2016). Second language learners' divergence from target language pragmatic norms. Studies in Second Language Learning and Teaching, 6(2), 249-269.

Hasselgren, Angela (1994). Lexical teddy bears and advanced learners: A study into the ways Norwegian students cope with English vocabulary. International Journal of Applied Linguistics, 4(2), 237-258.

House, Juliane (2005). Politeness in Germany: Politeness in Germany? In: Leo Hickey/ Miranda Stewart (eds.), Politeness in Europe (pp. 13-28). Clevedon: Multilingual Matters.

House, Juliane (2010). The pragmatics of English as a lingua franca. In: Anna Trosborg (ed.), Pragmatics across languages and cultures (Vol. 7, pp. 363-390). Berlin: De Gruyter Mouton.

House Juliane/Kasper, Gabriele (2000). How to remain a non-native speaker. In: Claudia Riemer (ed.), Kognitive Aspekte des Lehrens und Lernens von Fremdsprachen. Festschrift fuer Willis J. Edmondson zum 60. Geburstag (pp. 101-118). Tübingen: Narr.

Hudson, Thom (2001). Indicators for pragmatic instruction: Some quantitative tools. In: Kenneth R. Rose/Gabriele Kasper (eds.), Pragmatics in language teaching (pp. 283-300). Cambridge: Cambridge University Press.

Jakubowska, Ewa (1999). Cross-cultural dimensions of politeness in the case of Polish and English. Katowice: Wydawnictwo Uniwersytetu Śląskiego.

Jenkins, Jennifer (2007). English as a lingua franca: Attitude and identity. Oxford: Oxford University Press.

Kasper Gabriele/Schmidt, Richard (1996). Developmental issues in interlanguage pragmatics. Studies in Second Language Acquisition, 18(2), 149-169.

Kramsch, Claire (1998/2003). Language and culture. Oxford: Oxford University Press.

Lado, Robert (1957). Linguistics across cultures. Ann Arbor: University of Michigan Press.

Lakoff, Robin (1977). What you do with words: Politeness, pragmatics and performatives. In: Andy Rogers/Bob Wall/John P. Murphy (eds.), Proceedings of the Texas Conference on performatives, presuppositions and implicatures (pp. 94-120). Arlington, VI: Center for Applied Linguistics.

Liu, Jianda (2006). Measuring interlanguage pragmatic knowledge of EFL learners. Frankfurt a.M.: Peter Lang. 
Majer, Jan/Salski, Łukasz (2004). The pragmatics of classroom discourse: Requests and commands. In: Janusz Arabski (ed.), Pragmatics and language learning (pp. 51-67). Kraków: Universitas.

Major, Roy C. (2014). Foreign accent: The ontogeny and phylogeny of second language phonology. Hove: Taylor \& Francis Group.

Ogiermann, Eva (2009). On apologising in negative and positive politeness cultures. Amsterdam: John Benjamins Publishing.

Pohl, Gabriela (2004). Cross-cultural pragmatic failure and implications for language teaching. SLLT, 4, https://www.researchgate.net/publication/255665019.

Prokop, Izabela (2010). Aspekty analizy pragmalingwistycznej. Poznań: Wydawnictwo Naukowe Uniwersytetu im. Adama Mickiewicza.

Riley, Philip (1989). Well don't blame me! - On the interpretation of pragmatic errors. In: Władysław Oleksy (ed.), Contrastive pragmatics (pp. 231-249). Amsterdam: John Benjamins.

Scarcella, Robin C. (1983). Discourse accent in second language performance. In: Susan Gass/Larry Selinker (eds.), Language transfer in language learning (pp. 306-326). Rowley, MA: Newbury House.

Scarcella, Robin C. (1992). Interethnic conversation and second language acquisition: Discourse accent revisited. In: Susan Gass/Larry Selinker (eds.), Language transfer in language learning (pp. 109-137). Amsterdam: John Benjamins.

Schneider, Klaus (2017). Pragmatic competence and pragmatic variation In: Giora Rachel/ Michael Haugh (eds.), Doing pragmatics interculturally. Cognitive, philosophical, and sociopragmatic perspectives (pp. 315-334). Berlin: De Gruyter Mouton. DOI: https://doi. org/10.1515/9783110546095-017.

Shively, Rachel L. (2011). L2 pragmatic development in study abroad: A longitudinal study of Spanish service encounters. Journal of Pragmatics, 43, 1818-1835.

Szczepaniak-Kozak, Anna (2018). Interlanguage pragmatic competence. A longitudinal study of 'pragmatic accent' in learning EFL. Poznań: Wydawnictwo Naukowe Uniwersytetu im. Adama Mickiewicza.

Szczepaniak-Kozak, Anna/Bakinowska, Ewa/Strani, Katerina (2020). Measuring change in longitudinal research on pragmatic competence: A multinomial logistic model. Biometrical Letters, 57(2).

Taguchi, Naoko (2012). Context, individual differences and pragmatic competence. Bristol: Multilingual Matters.

Tarone, Elaine (1998). Interlanguage. In: Jacob L. Mey (ed.), Concise encyclopedia of pragmatics (pp. 389-395). Amsterdam: Elsevier.

Thomas, Jenny (1983). Cross-cultural pragmatic failure. Applied Linguistics, 4(2), 91-112.

Timpe-Laughlin, Veronika (2017). Adult learners' acquisitional patterns in L2 pragmatics: What do we know? Applied Linguistics Review; 8(1), 101-129. DOI 10.1515/applirev2015-2005.

Trubetzkoy, Nikolaj (1939/1958). Grundzüge der Phonologie [Fundamentals of Phonology]. Gottingen: Vandenhoek \& Ruprecht.

Wach, Aleksandra (2018). Trilingual learners' awareness of the role of L1 in learning target language grammar. In: Mirosław Pawlak/Anna Mystkowska-Wiertelak (eds.), Challenges of second and foreign language education in a globalized world (pp. 209-226). Cham: Springer. 
Wierzbicka, Anna (1985). Different cultures, different languages, different speech acts: Polish vs. English. Journal of Pragmatics, 9(2-3), 145-178.

Wierzbicka, Anna (1991/2003). Cross-cultural pragmatics: The semantics of human interaction (2nd ed.). The Hague: De Gruyter Mouton.

Wilkoń, Aleksander (2002). Spójność i struktura tekstu. Kraków: Universitas.

Woodward-Smith, Elizabeth (1997). Lo formal y familiar en Espanol e Ingles. La Corunna: La Corunna University Press.

Wyner, Lauren/Cohen, Andrew D. (2015). Second language pragmatic ability: Individual differences according to environment. Studies in Second Language Learning and Teaching, 5(4), 519-556.

Yule, George (1996). Pragmatics. Oxford: Oxford University Press. 
Open-Access-Publikation im Sinne der CC-Lizenz BY-NC-ND 4.0

(C) 2020, Vandenhoeck \& Ruprecht GmbH \& Co. KG, Göttingen ISBN Print: 9783847111863 - ISBN E-Lib: 9783737011860 


\author{
Anna Pieczyńska-Sulik \\ (Uniwersytet im. Adama Mickiewicza w Poznaniu)
}

\title{
Ikonische Dimensionen der Sprachpragmatik am Beispiel von Repräsentativa
}

\begin{abstract}
Iconic dimensions of language pragmatics on the example of representatives

The purpose of this chapter is to present the issue of linguistic iconicity from the point of view of pragmatics. Semiotics, from which iconicity originates, and pragmatics are connected by the trilaterality of categories. Therefore, in what follows the main possible uses of different types of linguistic iconicity and its limitations are demonstrated on the example of representative speech acts.
\end{abstract}

\section{Keywords}

language pragmatics, (ill)locution, perlocution, representatives, (imagic, diagrammatical) iconicity, constructional iconism

\section{Einleitung}

Das Thema des vorliegenden Beitrags ist an der Kreuzstelle von Semiotik und Pragmatik situiert. Da Pragmatik als Schwester von Semantik und Syntaktik und zusammen mit ihnen als Töchter der Semiotik (vgl. Morris 1938) mit den genannten Subdisziplinen verwandt ist, kann man erwarten, dass ihr auch die Frage der sprachlichen Ikonizität nicht fremd ist. Unter sprachlicher Ikonizität ist eine Ähnlichkeitsbeziehung zu verstehen, die sich zwischen einer oder mehreren Eigenschaften des Repräsentamens (Zeichenträgers) und seinem Objekt und eventuell seinem Interpretanten abspielt. Als sprachphilosophische Frage bereits in der Antike aufgegriffen (thesei-physei), beim Namen erst Ende des 19. Jhts. von Peirce (1885) gerufen (icon) und ein wenig später endgültig von Morris (1946) getauft (iconicity), wandert die ikonische Frage durch die Zeiten und Sprachtheorien bis dato. Obwohl strukturalistisch vor allem von Jakobson (1992) gut bilateral aufgehoben, fühlt sich Ikonizität erst im trilateralen Zeichenmodell wie ein Fisch im Wasser. Und von hier aus ist es nur ein Katzensprung zur Pragmatik. Während Peirce, der Urvater der Zeichenlehre, Semiotik als pragmatisch versteht, ergänzt sein Nachfolger, Charles W. Morris, das semiotische Dreieck um 
die Figur des Zeichenbenutzers, der dann im Sinne der sprachlichen Pragmatik der 60er Jahre des 20. Jhts. zur sprachlichen Handlung fähig ist.

\section{Das Peirce'sche Zeichenmodell}

Das Peirce'sche Zeichenmodell schließt den Zeichenbenutzer nicht direkt ein, seine Kategorie des Interpretanten impliziert jedoch weitführende mentale, sprachliche sowie nichtsprachliche Folgeprozesse (d.h. weitere Zeichen in der Semiosekette in Form von Gefühlen, Handlungen, Schließen). In dem Zeichenmodell resultiert die (nicht nur) sprachliche ikonische Relation aus dem Objektbezug, wobei auch der Interpretantenbezug in einem pansemiotischen Zyklus durchaus denkbar ist.

\section{Sign (1)}

Qualizeichen (1)

Sinzeichen (2)

Legizeichen (3)
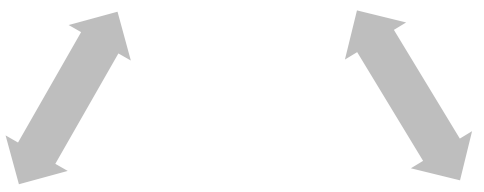

Object (2)

[unmittelbar, dynamisch]

Ikon (1)

Index (2)

Symbol (3)
Interpretant (3)

[unmittelbar, dynamisch, logisch]

Rhema (1)

Dicizeichen (2)

Argument (3)

Abb. 1: Die Struktur des Peirceschen Zeichenbegriffs nach Nagl (1992:43)

Auch der Pragmatik ist Trilateralität nicht fremd (vgl. Prokop 2016: 12) (vgl. Illokution, Lokution, Perlokution (Austin 1962, 1976); Äußerungsakt, propositionaler Akt, illokutionärer Akt (Searle 1971: 40), wobei - erinnerungsgemäß Lokution/Äußerungsakt als „Äußerung von Wörtern (Morphemen, Sätzen)“ (Searle 1971: 40), Illokution/illokutionärer Akt als „Handlungszweck der Äußerung eines lokutiven Akts“ (Ehlich 2000: 279), Proposition als „de[r] eigent- 
liche[-] Satzinhalt“, darunter Referenz und Präposition (Ebd.: 538) und Perlokution als „Folgewirkungen einer Lokution“ (Ebd.: 502) verstanden wird.

Setzt man die drei Arten von Sprechakten beider führenden Sprachpragmatiker (Austin 1962, 1976, Searle 1969, 1971) nicht nur mit den Bestandteilen des Peirce'schen Zeichenmodells, sondern auch mit den textlinguistischen Kategorien (vgl. Hausmann \& Kesselheim 2008) und mit eigens (A.P.-S.) ein wenig modifizierter Nomenklatur zusammen, so erweisen sich die Grenzen zwischen den einzelnen Kategorien - aufeinander bezogen - als verschoben und manifestieren sich wie in der Tabelle präsentiert. Damit wird illustriert, dass nicht nur Semiotik und Pragmatik nicht weit auseinander liegen, sondern dass auch die Textlinguistik - auch wenn in einer anderen „Sprache“ (Nomenklatur) - von gleichen Sprach- und Texterscheinungen handelt. Im Folgenden wird von den terminologischen Errungenschaften aller drei Disziplinen Gebrauch gemacht.

Tabelle 1: Semiotische, sprachpragmatische und textlinguistische Terminologie in vertikaler Entsprechungsordnung

\begin{tabular}{|c|c|c|c|c|c|c|c|}
\hline Name & \multicolumn{7}{|c|}{ Gleichrangige Kategorien in vertikaler Entsprechungsordnung } \\
\hline \multirow[t]{2}{*}{ Peirce } & \multicolumn{2}{|c|}{ Repräsentamen } & \multicolumn{2}{|c|}{ Objekt } & \multicolumn{3}{|c|}{ Interpretant } \\
\hline & & & unmittelbar & dynamisch & 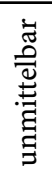 & 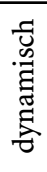 & 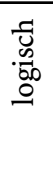 \\
\hline \multirow[t]{2}{*}{ Austin } & \multicolumn{2}{|l|}{ Lokution } & \multicolumn{2}{|l|}{ Illokution } & \multicolumn{3}{|c|}{ Perlokution } \\
\hline & $\begin{array}{l}\text { phone- } \\
\text { tischer Akt }\end{array}$ & $\begin{array}{l}\text { phatischer } \\
\text { Akt }\end{array}$ & $\begin{array}{l}\text { rhetischer } \\
\text { Akt }\end{array}$ & $\begin{array}{l}\text { propositio- } \\
\text { naler Akt }\end{array}$ & \multicolumn{3}{|l|}{-} \\
\hline Searle & \multicolumn{2}{|c|}{ Äußerungsakt } & $\begin{array}{l}\text { illokutio- } \\
\text { närer Akt }\end{array}$ & $\begin{array}{l}\text { propositio- } \\
\text { naler Akt }\end{array}$ & \multicolumn{3}{|l|}{-} \\
\hline $\begin{array}{l}\text { Hausendorf/ } \\
\text { Kesselheim }\end{array}$ & \multicolumn{2}{|c|}{$\begin{array}{l}\text { Textsorte, Sprach- } \\
\text { u.Texthinweise }\end{array}$} & \multicolumn{2}{|c|}{$\begin{array}{l}\text { Textfunktion } \\
\text { Grundfunktion, } \\
\text { Nebenfunktion }\end{array}$} & \multicolumn{3}{|c|}{ Texthandlung } \\
\hline A.P.-S. & \multicolumn{2}{|l|}{ Lokution } & \multicolumn{2}{|c|}{$\begin{array}{l}\text { Proposition } \\
\text { Superillokution, } \\
\text { Grundillokution, } \\
\text { Subillokution }\end{array}$} & \multicolumn{3}{|c|}{ Perlokution } \\
\hline
\end{tabular}

In der Sprachpragmatik werden zuerst (abhängig von den einzelnen Autoren) generelle Illokutionen (weiterhin als Grundillokutionen) festgelegt, die im nächsten Schritt zu einzelnen Subillokutionen (Nebenfunktionen, Textfunktionen) werden. Dazu gehören: 
- Repräsentativa/Assertiva: „Klasse von Sprechakten (Feststellungen, Behauptungen usw.), deren Charakteristikum ist, dass der Sprecher auf die Wahroder Falschheit der in der Äußerung zum Ausdruck gebrachten Proposition festgelegt wird“ (Fries 2000: 65):

- Subillokution (Texthandlung): Berichten, Benachrichtigen,

- Textsorte: Bericht, Nachricht.

- Direktiva: „Aufforderungen, als Sprechakttyp klassifizierte Handlungseinheit, deren Hauptcharakteristikum darin besteht, den Adressaten einer Äußerung $\mathrm{zu}$ einer bestimmten Handlung oder zur Unterlassung einer Handlung zu veranlassen“" (vgl. ebd.: 71, 153):

- Subillokution (Texthandlung): Befehl, Bitte,

- Textsorte: Befehl, Bittschreiben.

- Kommissiva: „Klasse von Sprechhandlungen, deren Charakteristikum es ist, dass sie den Sprecher auf eine zukünftige Handlung festlegen. [...] Ihr propositionaler Gehalt betrifft eine zukünftige Handlung (i.S. von Searle 1979: Welt-auf-Wort-Ausrichtung. Verben, die Elemente dieser Klasse bezeichnen, sind z.B. versprechen und ankündigen" (Fries 2000: 342), d.h. performative Verben. (Ebd.: 193):

- Subillokution (Texthandlung): Versprechen, Drohung, Angebot, Ankündigung,

- Textsorte: z.B. Drohanruf, Angebotsausschreibung, Versprechen.

- Expressiva: „Klasse von Sprechhandlungen, deren Charakteristikum es ist, den in der betreffenden Aufrichtigkeitsbedingung angebbaren psychischen Zustand des Sprechers zum Ausdruck zu bringen: dieser ist auf den in der Proposition denotierten Sachverhalt gerichtet. Deutsche Verben, die Elemente dieser Klasse zum Ausdruck bringen können, sind z.B. danken, gratulieren, bedauern". (Ebd.):

- Subillokution (Texthandlung): Danken, Gratulieren, Bedauern,

- Textsorte: z.B. Gruß(karte), Gratulationsbrief, Beglückwünschung.

- Deklarativa: „Klasse von Sprechhandlungen, deren Charakteristikum ist, eine Korrespondenz von propositionalem Gehalt und außersprachlicher Welt zu erzeugen, z. B.: Ich eröffne hiermit die Sitzung; Sie sind entlassen!“ (Ebd.:133):

- Subillokution (Texthandlung): Taufen, Eröffnen,

- Textsorte: Taufformel, Eröffnungsrede.

Die Illokution als solche schließt dabei nicht nur Grund- und Subillokutionen ein, sondern auch übergeordnete (weiterhin Superillokutionen genannt), oft im engen Zusammenhang stehende Illokutionen, die sowohl auf die Lokutionen, als auch im nächsten Schritt auf die Perlokutionen (Interpretanten) Einfluss nehmen, die aus Grund- und Subillokutionen resultieren. Zu Superillokutionen, die 
alle anderen hierarchischen Lokutionsarten beeinflussen, gehören folgende Kategorien:

- Gesellschaftlicher Funktionsbereich von Texten, d.h. Gegenstand, über den man redet (vgl. Hausendorf \& Kesselheim 2008: 141),

- Höflichkeit: „Qualifizierung sozialen - insbesondere auch sprachlichen Handelns, bei der ein diesem Handeln zugrunde gelegter Maßstab des Entgegenkommens in Bezug auf die Bedürfnisse des/der anderen angewendet wird“ (Ehlich 2000: 270) (Höflichkeit vs. Unhöflichkeit),

- Register: „Funktionsspezifische, d.h. für einen bestimmten Kommunikationsbereich (Institution) charakteristische Sprech- oder Schreibweise, z. B. die eines Pfarrers bei der Predigt, der Eltern gegenüber dem Kind, der Angestellten gegenüber Vorgesetzten“ (Bußmann 1990: 637) (nach dem Grad der Formalität (formal vs. nicht formal), des Fachwissens (fachlich, teil-, nicht fachlich), nach dem Alter (Jugendsprache vs. Elternsprache),

- Proxemik: „Studium von Kommunikationsverhalten im Hinblick auf die Variable Raum (Raumwahrnehmung, körperliche Positionierung im gegebenen Raum, kinetisches (Körperbewegungen betreffendes) Verhalten in unterschiedlichen Kulturen nach Parametern wie soziale Distanz, Geschlechtszugehörigkeit, Alter u.a.)“ (Glück 2000: 541) (nah oder fern sein).

Jede der drei letzten Superillokutionen kann jeweils ins Umgekehrte umschlagen (Höflichkeit in Unhöflichkeit, Formales in Nicht-Formales, Nahes in Fernes). Sie stehen auch - da „Textfunktionen [...] in der Regel in einem hierarchischen Verhältnis der Über- und Unterordnung zueinander stehen“ (Hausendorf \& Kesselheim 2008: 141) in einer nahen Beziehung zueinander. Sollte man die drei letzten Superfunktionen weiterhin hierarchisieren, so stehen jeweils die übrigen zwei immer der einen untergeordnet (Register und Proxemik der Höflichkeit oder Höflichkeit und Proxemik dem Register oder zum Schluss Höflichkeit und Register der Proxemik).

Der Anstoß zur ikonischen Gestaltung der Lokution kommt seitens der Illokution (Super-, Grund-, Subillokution) und Proposition. Die Sache verkompliziert sich einerseits, da einzelne Äußerungen nicht unbedingt nur eine Textfunktion aufweisen. Auch mit den Textsorten gehen Textfunktionen nicht immer - wie üblich - Hand in Hand (vgl. ebd.: 140). Außerdem spaltet sich das Peirce'sche Objekt, wie dem Modell zu entnehmen ist, in ein unmittelbares und dynamisches Objekt. Von diesem kommen die ersten Wahrnehmungseindrücke, bevor sie als jenes verinnerlicht werden. Wenn das Objekt ausschließlich mental ist, fallen die beiden Objekte zusammen. Interpretanten dafür, die auch Ikonizität aufweisen können, gliedern sich in unmittelbare, dynamische (Handlungen: sprachliche und nichtsprachliche) und logische (Schlussfolgerungen) (vgl. Nagl 1992: 43). 


\section{Typologie der Ikonizität}

Wie die Zeichensubklassen vermehren sich auch die Ikonizitätstypen. Die sprachliche Ikonizität kann nach verschiedenen Kriterien gegliedert werden, darunter:

- nach der Systemzugehörigkeit: systeminterne/primäre (im System der Einzelsprache verankerte, z. B. wau wau) vs. systemexterne/sekundäre I. (textuelle, z. B. Bildgedichte der Barockzeit),

- nach der Subebene der Sprache: phonologisch-phonetische (z.B. rattern), morphologische (z.B. Ururvater), syntaktische (z. B. Er erwachte um 7 Uhr, dann ging er ins Badezimmer. (Abfolge), Ich schreibe dir. vs. Ich schreibe an dich. (Abstand), Ich warte und warte. (Wiederholung) und lexikalische I. (z. B. Die Flinte ins Korn werfen),

- nach dem Medium: mündliche/gesprochene (z.B. Krach) vs. graphische/ schriftliche I. (z.B. Lokomotive: $o$ rund wie Räder, Mond: o rund wie Vollmond),

- nach der Ähnlichkeitsart: bildhafte (eine einfache Eigenschaft wird nachgebildet, z. B. grunzen) vs. diagrammatische (relationale, es handelt sich hier um die Relation der Analogie, z. B. er und sie) vs. semantische I. (metaphorische, z. B. Schwarzfahrer, Angsthase),

- nach der Bezugsrichtung: exophorische (objektsprachlich bezogene, z.B. Kuckuck) vs. endophorische (metasprachlich, metatextuell bezogene, z.B. wenn, und nur wenn) I.,

- nach der Ähnlichkeitsart: Isomorphismus (eine Eins-zu-Eins-Beziehung zwischen Form und Bedeutung (vgl. Haiman 1980: 515), z. B. ich für die/den Sprechenden vs. Nicht-Isomorphismus (eine Viel-zu-Eins-Beziehung zwischen Form und Bedeutung, z. B. er, der Mann, jener... für die/den Besprochene(n),

- nach dem Natürlichkeitsgrad/nach der Merkmalhaftigkeit grammatischer Strukturen; „Das Prinzip des konstruktionellen Ikonismus begründet, weshalb in natürlichen Sprachen sem>-Kategorien vorzugsweise merkmalhafter kodiert werden als Basiskategorien. Intuitiver Hintergrund: Was semantisch ,mehr' ist, sollte auch konstruktionell ,mehr' sein“ (Mayerthaler 1981:25), z. B. Pluralendungen im Vergleich zu Singularformen der Nomina: Hund - Hunde.

\section{Lokution}

$\mathrm{Zu}$ lokutiven Sprechakten (Repräsentamen) werden die Illokutionen (partielle mentale Objekte) explizit mit (nicht) performativen (taufen, eröffnen) oder quasi-performativen (hoffen, versprechen) Verben oder implizit mit anderen 
sprachlichen Mitteln (wie etwa Abtönungspartikeln, Modus, Wortstellung usw.), direkt oder indirekt realisiert. Dazu kommen jeweils auch Perlokutionen.

In jedem lokutionären Sprechakt lassen sich - so Hausendorf und Kesselheim (2008: 9) - bestimmte Hinweise (Abgrenzungs-, Gliederungs-, Abschluss-, Ganzheits-, Thema- Intertextualitätshinweise) erkennen, die als all das verstanden werden, „worauf man bei der Analyse achten sollte“. Ohne die Hinweise „wird die Bildung von Lektüreeinheiten irritiert und [...] Textualität [kann] auch in der alltäglichen Lektürepraxis zu einem Problem werden“ (Ebd.: 39). Man kann fragen, ob die alle ikonisch sind. Sicherlich nicht. Deikonisierungsprozesse sind doch im Gange. Aus der ikonischen Perspektive betrachtet lassen sich allerdings bestimmte Eigenschaften von Illokutionen nachweisen, die lokutiv mit ikonischen Mitteln zum Ausdruck gebracht werden und worauf im Weiteren aus Platzgründen nur am Beispiel von Repräsentativa/Assertiva ein wenig detaillierter eingegangen wird.

\section{Ikonische Lokutionen am Beispiel von Repräsentativa}

„Mit der Darstellungsfunktion berühren wir eine Art kommunikative Nützlichkeit, bei der die Lektüre ganz im Zeichen der Erfahrung von Welt steht - als würde der Text gleichsam transitiv auf Welt durchgreifen können! Etwas zugespitzt gesagt: Der Text suggeriert, dass er nur von Welt handelt und nichts anderes tut, als Welt sichtbar und erfahrbar zu machen“. (Hausendorf \& Kesselheim 2008: 146; kursiv von der Autorin).

Als Darstellungshinweise (vgl. ebd.) kommen in Frage:

- Dominanz der Referenzformen ohne Formen der Markierung der Schreiberund Leserrolle, d.h. diejenigen der 3. Person Singular und Plural,

Das Verschwinden der 1. und 2. Person ist wie das Verschwinden von realen Personen, die hier nicht direkt als Kommunikationsbeteiligte stehen. In Wirklichkeit repräsentieren die fehlenden Pronomina metonymisch (also semantisch) nur ein mangelndes Gespräch (pars pro toto), weil de facto sowohl ein Sender, als auch ein Empfänger irgendwo im Hintergrund eines gesprochenen oder geschriebenen Textes stehen. Im Sinne des konstruktionellen Ikonismus handelt es sich bei der 3. Person (vor allem) des Indikativs Präsens um eine merkmalhafte, d.h. morphologisch nicht natürliche Form: „Was semantisch ,mehr' ist, sollte auch konstruktionell ,mehr‘ sein.“ (Mayerthaler 1981: 25). 
- deskriptive und explanatorische Hinweise: z. B. Bezug auf ein Lebewesen und seine Eigenschaften (Größe) und Verortung (Meer), klassifizierende Einordnung,

Deskription bietet eine ganze Palette von Möglichkeiten. Die Autoren Hausendorf und Kesselheim (2008) nennen hier zuerst die Größe, eine der Dimensionen neben der Länge, Breite, z.B. winzig, gering (phonologische [artikulatorische] Ikonizität, da bei der Aussprache von [I] der Abstand zwischen der Zunge und dem Gaumen gering ist und „[k]leiner Kieferwinkel, d.h. hohe bzw. palatale Artikulation [...] mit Kleinheit der Designate korreliert [wird]" (Mayerthaler 1981: 102), GROSS (visuelle Größe), b r e i t (graphische Ikonizität mittels gespreizter Schrift), laaaaange Linie (phonetisch und/oder graphische Ikonizität mit der akustisch-visuellen Verlängerung des Wortes). Eine Einschränkung stellen hier aber Subillokutionen, vor allem das Register, das in dem Falle der Höflichkeit untergeordnet ist. Nur wenige Fälle der vorgestellten Ikonizität sind in der formalen oder Fachsprache richtig am Platze.

- narrative Hinweise im Sinne des Berichtens [...]: Redewiedergabe,

Die Redewiedergabe kann mit dem Bezug auf die mal geäußerte direkte Rede (Diskurs) einen endophorisch-ikonischen Charakter aufweisen. Ähnlich verhält es sich mit allerlei Intertextualitätshinweisen (direkten, indirekten Zitaten, Anspielungen, Wortspielen usw.).

- Dominanz von Aussage- bzw. Deklarativsätzen mit Verben im Indikativ (Ausnahme: Konjunktiv in der Redewiedergabe) und Dominanz des Präsens unterbrochen durch wenige Formen des Präteritums (narrative Hinweise),

„Wir halten fest: Die Basiskategorien ,Singular, Positiv, Indikativ, Präsens, Nominativ, Maskulinum' tendieren zu merkmalloser Kodierung" (Mayerthaler 1981: 27f.), sind also im Sinne des konstruktionellen Ikonismus Basiskategorien. Jede Abweichung (z.B. Konjunktiv, Präteritum) verstärkt den Eindruck eines höheren Natürlichkeitsgrades von ihnen.

- Modalisierung im Sinne der Hervorhebung einer epistemischen Einstellung (Modalisierung des Wahrheitsanspruches, z.B. durch Geltungsadverbien wie tatsächlich, womöglich und epistemische Verben wie gelten),

Modalisierung des Wahrheitsanspruches bedeutet nichts weniger als eine InFrage-Stellung des Wahrheitswertes von Darstellungen. Damit tritt die Grund- 
funktion der Sprechakte ein wenig in den Hintergrund. Die generierte Unsicherheit wird durch ihre Merkmalhaftigkeit konstruktionell ikonisch.

- Bezugnahmen auf Wissenssysteme durch Fachsprachlichkeit,

Damit wird eine Superillokution ikonisch motiviert: der gesellschaftliche Funktionsbereich von Texten, d.h. Gegenstand, über den man redet. Je nach dem Gegenstand kann die (Fach)lexik mehr oder weniger ikonisiert auftreten. Am deutlichsten sind hier zahlreiche, für die deutsche Fachsprache typische Nominalkomposita, von denen die längsten aus der Rechts- und Verwaltungssprache stammen, z.B. Rinderkennzeichnungsfleischetikettierungsüberwachungsaufgabenübertragungsgesetz, die Bezeichnung für ein nicht mehr existentes Gesetz ${ }^{1}$. Der auch hier zu erkennende konstruktionelle Ikonismus zeigt analog eine für Otto Normalverbraucher all zu abschreckende Komplexität des Rechts- und Verwaltungswesens. Die Verdichtung von fachsprachlichen Komposita, Simplizia und auch von Ableitungen aus einem jeweiligen Wissensbereich in einem Wort, Text, Diskurs diagrammatisiert in verkleinertem Maßstab den Fachbereich.

- ausgeprägte thematische Hinweise (Themaeinführung, -beibehaltung und -entwicklung),

Es handelt sich hier um die Pflege von thematischen Strängen, von Referenz (vgl. Hausendorf \& Kesselheim 2008: 106ff.) mittels der Fokus- und metakommunikativen Hinweise, des Titels und der Überschriften, die letzten aber lediglich in geschriebenen Repräsentativa. Die lexikalischen Einheiten bilden in so einem Fachbereich Sachgruppen, deren Bestandteile die Kookkurrenz bestimmter Fachelemente wiedergeben. Im Gesprochenen dafür, die die Autoren nicht mitberücksichtigen, muss anfangs doch eine Anrede formuliert werden. Und hier eröffnet sich ein großer Raum für die mögliche (Un)Höflichkeit, der sich das Register und die Proxemik unterordnen. Für eine Weile kommen also die 1., vor allem aber die 2. Person Sg. oder Pl. aus dem Versteck. Abhängig von mehreren Faktoren, der Situation, die formale oder weniger formale Sprache fordert, von der hierarchischen Beziehung zwischen der/dem Sprechenden und Hörenden wird die Ansprache länger oder kürzer (näher an der/am Sprechenden (z.B. Hallo euch allen!) oder weiter von ihr/ihm entfernt (z. B. Sehr geehrte Damen und Herren!). Eine enorm wichtige Rolle spielen auch grammatisch-lexikalische Entscheidungen. Ob sie dem Register, der Hierarchie entsprechend getroffen wurden. Abtönungspartikeln, die im Dienste der Höflichkeit oder Unhöflichkeit

$1 \mathrm{https} / /$ www.duden.de/sprachwissen/sprachratgeber/Die-langsten-Worter-im-Dudenkorpus (Stand 07.05.2020). 
stehen, schneiden eine Äußerung an gewissen Stellen an, um da die Grundfunktion um die Nebenfunktionen, wie etwa des Emotionsausdrucks, zu ergänzen.

\section{Perlokution (Interpretant(en))}

All die angeführten lokutiven Belege zeigen ansatzweise, dass das ikonischpragmatische Potential besteht. Erst aber die Kategorie der Perlokution (des Interpretanten) bestätigt evident, dass die semiotische Handlungskette weiter geht, dass sie nicht aufzuhalten ist, weshalb die Semiosphäre, in der wir eingetaucht bleiben, als Pansemiose abzulesen ist. Sprachliche Interpretanten können wie alle übrigen Lokutionen ikonische Merkmale tragen.

Als besonders pregnant erweisen sich hier möglicherweise andere Typen von Sprechakten, vor allem Direktiva, jedoch auch unter Repräsentativen finden wir ikonische Interpretanten. Für ein Beispiel eignen sich - wie oben - Merhfachkomposita aus dem juristischen oder Verwaltungsbereich. Wenn ein Laie (unser Otto Normalverbraucher) ein Bandwurmwort wie etwa Rinderkennzeichnungsfleischetikettierungsüberwachungsaufgabenübertragungsgesetz hört, bricht er sich nicht nur die Zunge (Zungenbrecher), sondern auch seine Gedanken. Oben wurde gesagt, dass so eine komplizierte Nominationseinheit die Verkomplizierung des Verwaltungs- und rechtlichen Bereichs neben der perlokutiven Bedeutung (ein konkretes Gesetz) nachbildet. Das Gefühl oder eine Reproduktion des Wortes (auch wenn in Gedanken) zählt beispielsweise zu der Interpretantenmenge.

\section{Zusammenfassung}

Im vorliegenden Beitrag wurde versucht, die Ikonizitätsfrage von der pragmatischen Sicht her zu beleuchten, ein ziemlich halsbrecherisches Vorhaben, vor allem wenn man nicht aus den Augen verlieren möchte, dass Pragmatik - obwohl als linguistische Subdisziplin - den ganzen Sprachbereich umfasst. Das Ziel lässt sich nur durch die Bemühung erklären, dem wissenschaftlichen Interesse der Jubilarin mit eigenen wissenschaftlichen Vorlieben entgegenzukommen. Daher gab es keine andere Wahl, als die beiden Feminina in der ikonischen Repräsentation von gemeinten Personen zusammentreffen zu lassen, nur kurz, beinahe im Vorübergehen, aber immerhin.

Die präsentierten Belege zeigen, dass der konstruktionelle, d.h. diagrammatische Ikonismus innerhalb der ikonischen Typen Oberhand gewinnt, was auch als eine generelle Regel in Bezug auf die sprachliche Ikonizität anzunehmen ist. 
Gibt man sich mehr Zeit und Platz, so erweist sich jedoch, dass alle Ikonizitätstypen in verschiedenen Textsorten Gebrauch finden und eine pragmatische Wirkung erreichen. Hoffentlich wird sich eines Tages eine Gelegenheit bieten, dies nachzuweisen.

\section{Literaturverzeichnis}

Austin, John L. (1976). Zur Theorie der Sprechakte. Stuttgart: Reclam.

Austin, John L. (1962). How to do things with words. London: Oxford University Press. Bußmann, Hadumod (1990). Lexikon der Sprachwissenschaft. Stuttgart: Alfred Kröner Verlag.

Ehlich, Konrad (2000). Illokution. In: H. Glück (Hrsg.), Metzler Lexikon Sprache (S. 279). Stuttgart, Weimar: J.B. Metzler.

Ehlich, Konrad (2000). Lokution. In: H. Glück (Hrsg.), Metzler Lexikon Sprache (S. 407). Stuttgart, Weimar: J.B. Metzler.

Ehlich, Konrad (2000). Perlokution. In: H. Glück (Hrsg.), Metzler Lexikon Sprache (S. 502). Stuttgart, Weimar: J.B. Metzler.

Fries, Norbert (2000). Assertive. In: H. Glück (Hrsg.), Metzler Lexikon Sprache (S. 65). Stuttgart, Weimar: J.B. Metzler.

Fries, Norbert (2000). Aufforderung. In: H. Glück (Hrsg.), Metzler Lexikon Sprache (S. 71). Stuttgart, Weimar: J.B. Metzler.

Fries, Norbert (2000). Deklarative. In: H. Glück (Hrsg.), Metzler Lexikon Sprache (S. 133). Stuttgart, Weimar: J.B. Metzler.

Fries, Norbert (2000). Direktive. In: H. Glück (Hrsg.), Metzler Lexikon Sprache (S. 153). Stuttgart, Weimar: J.B. Metzler.

Glück, Helmut (Hrsg.) (2000). Metzler Lexikon Sprache. Stuttgart, Weimar: J.B. Metzler. Glück, Helmut (2000). Proxemik. In: H. Glück (Hrsg.), Metzler Lexikon Sprache (S. 541). Stuttgart, Weimar: J.B. Metzler.

Haiman, John (1980). The iconicity of grammar: Isomorphism and motivation. Language, $56,515-540$.

Hausendorf, Heiko/Kesselheim, Wolfgang (2008). Textlinguistik fürs Examen. Göttingen: Vandenhoeck \& Ruprecht.

Jakobson, Roman (1992). Semiotik. Ausgewählte Texte 1919-1982. Frankfurt a.M.: Suhrkamp.

Lotman, Jurij M. (1972). Die Struktur literarischer Texte. [übersetzt von Rolf-Dietrich Keil]. München: Fink.

Mayerthaler, Willi (1981). Morphologische Natürlichkeit. Wiesbaden: Akademische Verlagsgesellschaft Althenaion.

Morris, Charles W. (1938). Foundations of the theory of signs. Chicago: Chicago University Press.

Morris, Charles W. (1946). Signs, language and behavior. New York: Braziller.

Nagl, Ludwig (1992). Charles Sanders Peirce. Franfurt, New York: Campus Verlag.

Peirce, Charles Sanders (1885). One, two, three: Fundamental categories of thought and of nature. W 5: 242-247. 
Prokop, Izabela (2016). Pragmalingwistyka antropocentryczna. Poznań: Wydawnictwo Naukowe Uniwersytetu im. Adama Mickiewicza.

Searle, John R. (1971). Sprechakte: Ein sprachphilosphischer Essay. Frankfurt a.M.: Suhrkamp. 


\title{
Pragmatik von Hashtags am Beispiel von Twitter als multimodales Diskurssystem ${ }^{1}$
}

\begin{abstract}
Pragmatics of hashtags using the example of Twitter as a multimodal discourse system The aim of the article is hashtags' contribution to pragmatic meaning in the online multimodal discourse system Twitter, by serving as the so-called, semiotic operators' in this context. From the perspective of the relevance theory of Wilson and Sperber, I argue that, being pragmatic and linguistic phenomena, hashtags not only contribute to relevance by adding a layer of activation to certain contextual assumptions of other users but also guide users' inferential processes. More precisely, hashtags include information which may guide the user towards a greter understanding of both explicity and implicity communicated meaning. They may also have some stylistic consequences (usually adding to the informal/ casual style of the communicated content). These assumptions are exempified by findings coming from a pragmalinguistic analysis of a dataset of 100 selected tweets in German coming from the hashtag \#coronadeutschland.
\end{abstract}

\section{Keywords}

sharing, social tagging, hashtags, Twitter, explicatures, relevance, speech acts, inference

\section{Hashtags: mehr als intuitive Mittel der Online-Kategorisierung}

Hashtags gehören, neben partizipativen Formen der Bereitstellung und OnlinePublizieren (wie interpersonale Anschlusskommunikation und User-GeneratedContent bzw. Social-Media-Content ${ }^{2}$ ) zu der Gruppe der technisch bedingten Formen der Selektion und Anhäufung von (persönlich relevanten) Informatio-

1 Der vorliegende Beitrag, in Würdigung der sprachwissenschaftlichen und organisatorischen Arbeit von Frau Professor Izabela Prokop, knüpft an ihre wichtigsten Werke zur Pragmalinguistik an: Prokop (1995 sowie 2010).

2 Zur Anschlusskommunikation vgl. Neuernbergk (2014), Sommer und Porten-Cheé (2018); zum User-generated-content/Social-media-content vgl. Goderbauer-Marchner und Büsching (2015) sowie Bruns (2008) mit sog. „Produtzung“ (Engl. Produsage) von Inhalten von Internetnutzern. 
nen. In der linguistischen Fachliteratur werden sie als semiotische (bzw. als Kommunikations-) Operatoren bezeichnet (vgl. Thimm \& Dang-Anh \& Einspänner 2011; Androutsopoulos \& Weidenhöffer 2015). Die Idee solcher „Operationsvorschriften" bezog sich ursprünglich ausschließlich auf das Diskurssystem Twitter, fand aber bald ihre Anwendung auch auf anderen Social-MediaPlattformen, v. a. Instagram, YouTube und Facebook. Der Einsatz von diversen Operatoren bedeutet zugleich gewisse Veränderungen sowohl auf der Text-als auch auf der Handlungsebene (vgl. Dang-Anh \& Einspänner \& Thimm 2013: 139f.) $)^{3}$. In Bezug auf die Text- und Handlungsebene unterscheide ich zwei Gruppen der Kommunikationsoperatoren:

1. Operatoren des Typs Teilen/Sharing: Dazu gehören einerseits Hyperlinks mit entsprechenden Verweismöglichkeiten und sog. Redistributionsoperatoren, die das Weiterleiten von existierenden Postings anderer UserInnen ermöglichen. Auf der Textebene bedeuten erstere v.a. URL-Adressen von diversen Webseiten sowie interne und externe Links, die sich meistens je nach technischen Möglichkeiten der gegebenen Plattform in Form von konkreten Bildern oder Icons von geteilten Videos zeigen. Redistributionsoperatoren sind dagegen, textuell gesehen, verschiedene Textzitate oder Kommentierungen Dritter. Auf der Handlungsebene beziehen sich solche Operatoren v. a. auf Informationsverteilung, Argumentation, Referenzierung, Sequenzierung sowie multimodale Weiterführung von Online-Content, der in einem nächsten Schritt in neue Kontexte eingebettet werden kann ${ }^{4}$.

2. Operatoren des Typs Social Tagging: $\mathrm{Zu}$ solchen Operatoren gehören prinzipiell zwei Elemente. Zum einen ist es das @-Zeichen (Adressierungsoperator), das die Einbeziehung von anderen Personen in einzelne Postings ermöglicht und sich auf der Textebene auf einen bestimmten Accountnamen bezieht bzw. Teil einer E-Mailadresse ist. Zum anderen gehört hierher das indexierende und themensetzende Doppelkreuz, also Hashtag (in Postings durch Rautezeichen \# markiert), das textuell gesehen ein Lexem, ein Okkasionalismus, ein Schlagwort oder sogar ein Akronym bzw. eine Abkürzung sein kann (vgl. ebd.: 140) ${ }^{5}$. Mit dem @-Zeichen lassen sich auf der Handlungsebene vor allem Personen referenzieren; darüber hinaus dienen sie auch

3 Ich bediene mich dabei des sog. funktionalen Ebenenmodells der Kommunikation in Social Media von Dang-Anh et al. (2013: 139f.). Zwar beziehen die AutorInnen ihr Modell ausschließlich auf Twitter, aber es lässt sich nach Anpassungen in allen anderen multimodalen Online-Systemen einsetzen.

4 Sowohl Teilen als auch soziales Taggen sind mit unterschiedlichen (Re-)Kontextualisierungsprozessen der Online-Inhalte verbunden (vgl. Dang-Anh et al. 2013).

5 Je nach Anwendung können solche Operatoren noch um die Kategorie von GPS-Operatoren ergänzt werden. Meistens kann nämlich der entsprechende Ort getaggt (markiert) werden. Es ist allerdings möglich, auf beliebige Orte hinzuweisen, unabhängig davon, ob sich der User/die Userin tatsächlich an ihnen befindet oder nicht (vgl. Szyszko 2017). 
der Aufmerksamkeitserzeugung, Kontaktaufnahme und Responsivität sowie $\mathrm{zu}$ bestimmten intertextuellen Bezügen. Hashtags ${ }^{6}$ dagegen ermöglichen solche kommunikativen Handlungen wie Diskursorganisation, thematische Referenzierung, Indexierung/Verschlagwortung, Markierung von (geteilten) Inhalten sowie die gerade angesprochene (Re-)Kontextualisierung von Online-Inhalten.

Darüber hinaus ermöglichen sie, im Gegensatz zu vorgegebenen, hierarchischen Klassifizierungspraktiken (z. B. in analogen Informationssystemen bzw. diversen Taxonomien), beliebige Kategorisierung mit frei erfindbaren/wählbaren Stichworten (vgl. Musser \& O'Reilly 2007; Sonnberger 2012).

Social Tagging, besonders mittels Hashtags, stellt also eine Art der kollaborativen Aktivität ${ }^{7}$ dar „of making shared online content with keywords or tags as a way to organize content for future navigation, filtering or search" (Stephens 2010: 204). Darüber hinaus ermöglichen Hashtags (handlungsbezogen) bestimmte Inhaltsbeschreibungen. Man versieht also bestimmte Schüsselwörter mit Hashtags, oder sie wirken konnotativ ${ }^{8}$ und können dann von anderen UserInnen entsprechend gedeutet werden, was in Bezug auf das im Beitrag formulierte Thema besonders interessant ist. Beides bringt technisch gesehen $u$. a. eine entsprechende Veränderung von Metadaten mit sich. Im Endeffekt führt ein solcher Prozess zu besserer Vernetzung von diversen Websites. Bestimmte Inhalte können daher auch einfacher gefunden werden (vgl. Sonnberger 2012: 93) und einzelne Threads lassen sich zu bestimmten (gemeinsamen) Themen bündeln, nach denen in Social Media besonders gerne gesucht wird (vgl. Autenrieth \& Herwig 2011: 221). All das führt noch zu einer anderen sozialen und kommunikativen Erscheinung: Bestimmte Schlüsselwörter (meistens mit Hashtags versehen) lassen ebenso unterschiedlich große ad-hoc-Gemeinschaften (bzw. Öffentlichkeiten) oder sogar (zeitweilige) Online-Diskursgemeinschaften entstehen, deren Akteure sich um einen bestimmten Themenbereich sammeln, der in weiteren Schritten entsprechend ausgehandelt werden kann (vgl. Rybszleger 2016: 94f.). Typisch sind dabei besonders politische oder soziale Themen, die

6 Hashtags können eventuell noch um sog. Trackbacks ergänzt werden, d. h. die Funktion der Bezugnahme in Blogs, mit der Blogger Informationen über Backlinks in Form von Reaktionen bzw. Kommentaren durch einen automatischen Benachrichtigungsdienst untereinander austauschen können. Trackbacks werden in dem Beitrag nicht weiter thematisiert.

7 Man kann zwar seine eigenen Hashtags ,erfinden', meistens aber werden die schon existierenden Hashtags vom gegebenen Diskurssystem ,zugeflüstert' (es betrifft besonders Instagram) oder man übernimmt den entsprechenden Hashtag von seinen Vorrednern.

8 Autenrieth und Herwig (2011: 221) sprechen vom typischen „konnotativen Hashtaggen“: „wenn [...] durch das Vergeben des Tags der Nachricht zusätzliche Bedeutungsebenen bzw. Nebenbedeutungen hinzugefügt werden." 
meistens auf der Agenda der Mainstream-Media stehen ${ }^{9}$ und weiter aufgegriffen werden. Gleichwohl gibt es immer mehr Situationen, in denen gerade BloggerInnen oder Netzaktivisten/Netzaktivistinnen bestimmte Themen „hochpuschen" und Licht auf verschiedene, insbes. soziale oder sozial-politische Probleme werfen, die erst dann von anderen Medien thematisiert werden. Ein solcher Prozess wäre natürlich ohne Markierung durch entsprechende Hashtags kaum möglich ${ }^{10}$. In einem nächsten Schritt wird auf technische und kommunikative Affordances und Constraints des Diskurssystems Twitter eingegangen.

\section{Twitter: Kommunikative Möglichkeiten und Einschränkungen}

Das im vorliegenden Beitrag thematisierte multimodale ${ }^{11}$ Diskurssystem Twitter gehört zu den meistbenutzten sozialmedialen Plattformen und Micro-BloggingDiensten weltweit. Nach den Angaben des deutschen Online-Portals „Statista“ betrug die Anzahl der aktiven Twitter-UserInnen im vierten Quartal 2019 152 Millionen $^{12}$. So wie andere Social-Media-Typen verfügt Twitter neben bestimmten semiotischen Ressourcen (v. a. Texten, Bildern, Videos, sowie statischen und dynamischen Grafiken) über andere Handlungsoptionen (mittels der gerade angesprochenen Kommunikationsoperatoren, u. a. Hashtags). Dazu kommen unkomplizierte Abonnementsmöglichkeiten ${ }^{13}$, eine einfache Kontaktmodellierung nach dem Muster „A folgt B. B folgt A (nicht unbedingt)“ (vgl. Herwig 2011: 196) sowie Modi-Konstellationen des Typs „One-to-Many“ (seltener "One-to-One“). Dies alles bedeutet, dass die sog. Follower (Abonnenten) von Micro-Blogs problemlos sog. „Teil-Öffentlichkeiten“ bilden können (vgl.

9 Neuberger, Langenohl und Nuernbergk (2014) bemerken in ihrer Studie, dass es nach wie vor hauptsächlich große mediale (kommerzielle) Anbieter gibt, die entscheiden, welche Bereiche und Ereignisse thematisiert werden. Nichtsdestotrotz ändert sich diese Situation zugunsten der kleineren Akteure (besonders Blogger und unabhängigen Online-Journalisten).

10 Es wird deutlich, dass solche Operatoren besonders in mittleren und kleineren OnlineÖffentlichkeiten (u. a. von einfachen UserInnen, unabhängigen JournalistInnen, AktivistInnen oder BloggerInnen) gerne eingesetzt werden, weil ihr Einsatz effektiv ist, bestimmte Inhalte (als Stichwörter) schnell gefunden werden und sich schnell verbreiten können. Unter größeren medialen Anbietern dagegen (bspw. Online-Zeitungen, Fernsehsendern oder Informationsportalen) werden sie deutlich seltener und eher als Stichwörter oder Indexierung bzw. thematische Referenzierung verwendet.

11 In dem Text nehme ich an, dass die Kommunikation dadurch multimodal wird, dass sie „mittels unterschiedlicher Zeichenressourcen bzw. -typen realisiert“ wird (Fraas \& Meier \& Pentzold 2013: 15f.).

$12 \mathrm{Mehr}$ Informationen unter https://www.statista.com/topics/737/twitter/\#dossierSummary_ chapter4 (25.03.2020).

13 Die abonnierten Twitter-Accounts werden den UserInnen automatisch zugestellt (vgl. Goderbauer-Marchner \& Büsching 2015: 28). 
Kirchhoff \& Lombardo \& Urhahn 2015: 232). Kurze Beiträge (sog. Tweets) erscheinen dann im eigenen Newsticker in der Reihenfolge ihrer Erscheinung (vgl. Primbs 2016: 30f.). Darüber hinaus lassen sie sich relativ leicht weiter vernetzen (v. a. durch Links); somit können sich kommunikative Online-Kontinuitäten bilden (v. a. auf diversen sozialmedialen Plattformen und Nachrichtenseiten) ${ }^{14}$.

Trotz der breiten Palette an kommunikativen multimodalen Möglichkeiten, weist das Kommunikationssystem Twitter bestimmte Mängel auf. Eine seiner möglichen Einschränkungen (sog. „medial constraints“, vgl. Hodkinson 2011: 31) ist die Zeichenbegrenzung, die seit November 2017 genau 280 UnicodeZeichen beträgt ${ }^{15}$. Aus diesem Grund sind die UserInnen auf eher kurze Mitteilungen angewiesen, was eine Reihe von möglichen Optimierungstechniken bzw. kommunikativen Strategien nach sich zieht. Scott (2015: 9f.) spricht dabei von einem spezifischen conversational style (d.h. einem konversationellen Stil) jedes Internetbenutzers, der sich meistens bemüht, möglichst viele Informationen in einen Tweet zu zwängen, wie im folgenden Beispiel von einer Userin (@FrolleinSchmit1) vom 30. März 2020 ${ }^{16}$ :

Sohn macht Homework. Ich muss einkaufen - mit Maske aus Männerslip.

Sohn: du siehst aus, wie Darth Mudda.

Das ist die Mudda von Darth Vadder.

\#Coronakrise

\#maskeauf

$\mathrm{Zu}$ typischen kommunikativen Strategien bei Twitter gehören, wie aus dem gerade präsentierten Beispiel ersichtlich, u.a. Abkürzungen (hier Ellipse), Neigung zu Akronymen und Bildung von bestimmten Schlagwörtern oder Schlagwortkombinationen. Darüber hinaus ist der Stil (besonders in Tweets von privaten UserInnen) oft salopp und informell, was an die Charakteristik der gesprochenen Sprache erinnert (vgl. Androutsopoulos \& Weidenhöffer 2015). In textuellen Realisierungen von Tweets gibt es auch viele Okkasionalismen und einzelne Lexeme, die oft in Form von Hashtags erscheinen.

An ausgewählten Beispielen möchte ich die pragmatische Bedeutung von Hashtags zeigen. Dabei wird Folgendes angenommen:

14 Dies erklärt auch, warum Tweets so häufig problemlos in viele online-mediale Berichte und Texte eingewoben werden (besonders von Bloggern, Politikern oder Journalisten), ohne dass für die meisten Empfänger die Notwendigkeit besteht, selbst ein Account zu besitzen. $\mathrm{Zu}$ Tweets wird häufig auch in der gedruckten Presse sowie in Fernsehprogrammen (z. B. Nachrichten) gegriffen.

15 Früher waren es genau 140 Zeichen (inklusive Leerstellen). Jetzt kann jeder Tweet in maximal zwei Teilnachrichten zerlegt werden.

16 Auf dem Bild, das dem Tweet beigefügt wurde, sieht man eine junge Frau in einer provisorischen Maske, die sie selbst, wohl aus Unterwäsche, gemacht hat. In dem Tweet bezieht sich die Userin auf die weltweite Coronavirus-Epidemie. 
Erstens: Durch Hashtags werden in Social Media (außer der typischen thematischen Referenzierung und Indexierung) bestimmte Schlüsselwörter hervorgehoben, die für den Autor/die Autorin von Bedeutung sind ${ }^{17}$. Derselbe durch Hashtags markierte Content ist gleichzeitig für andere potentielle Diskursteilnehmer ersichtlich und kann von ihnen entsprechend gedeutet werden.

Zweitens: Der Einsatz von Hashtags trägt zur Aktivierung von bestimmten kontextuellen Annahmen bei, was im Endeffekt unterschiedliche Schlussfolgerungsprozesse bei den potentiellen Empfängern in die Wege leiten kann. Die Bedeutung kann dabei explizit oder implizit mitgeteilt werden. Trotz ihrer potentiellen Multimodalität beruht die Online-Kommunikation nach wie vor auf Texten, in denen (wie $u$. a. wegen der genannten Zeichenbegrenzung) nicht alle Inhalte explizit ausgedrückt werden können. Dies erfordert selbstverständlich mehr Vertrauen seitens des jeweiligen Textproduzenten zu seinen potentiellen Empfängern. Er/sie muss nämlich annehmen, dass sie imstande sind, die in der Nachricht kodierten Hintergrundinformationen zu ergänzen und somit den Tweet so zu interpretieren, wie es intendiert war (vgl. Scott 2015: 10).

Bevor die Ergebnisse der Analyse präsentiert werden, wird in einem nächsten Schritt die pragmatisch-semantische Perspektive der Erforschung von Hashtags vorgestellt ${ }^{18}$.

\section{Relevanztheorie}

Im vorliegenden Beitrag wird von einem der zentralsten pragmatischen (und kognitiven) Konzepte der letzten 20 Jahre - der sog. Relevanztheorie von Sperber \& Wilson (1995) ausgegangen. Die Autoren basieren ihr referential model of communication (Wilson \& Sperber 2006: 607) auf der Annahme, dass eines der wichtigsten Merkmale der zwischenmenschlichen Kommunikation Ausdruck und Erkennung von Intentionen des Sprechers ist. Ein solches Modell hat also zum Ziel „to explain how the hearer infers the speaker's meaning on the basis of the evidence provided.“ (ebd.). Darüber hinaus wird von den Autoren ange-

17 In diesem Sinne gehören Hashtags nicht nur zu typischen Social-Web-Praktiken des Informationsmanagements, sondern auch zu zahlreichen Handlungskomponenten von Identitätsmanagement (ggf. Beziehungsmanagement), die sich in diversen Social-Web-Praktiken äußern. Besonders ersichtlich ist es in mehr oder weniger emotionalen Botschaften vieler UserInnen, wenn die Autorin/der Autor ihre/seine Aufmerksamkeit auf (für sich) wichtige Themen lenkt und somit ihre/seine Einstellung dem Thema gegenüber schildern möchte (vgl. Wagner 2019: 86). Das allgemeine Schema von Handlungskomponenten von Social-WebPraktiken vgl. Schmidt (2011 sowie 2013).

18 In der Fachliteratur gibt es bereits mehrere Versuche, Hashtags aus einer pragmatischen Perspektive zu erforschen (vgl. u. a. Dang-Anh et al. 2013; Scott 2015; Wagner 2019). 
nommen, dass jede Äußerung eine Erwartung weckt, die den jeweiligen Hörer zu der vom Sprecher intendierten Bedeutung führt:

When paying attention to an ordinary phenomenon, the individual may have hopes of relevance. What makes these hopes reasonable is that humans have a number of heuristics, some of them innate, others developed through experience, aimed at picking out relevant phenomena. (Sperber \& Wilson 1998: 97).

Für die Autoren hat jegliche Kommunikation zwei Seiten. Zum einen ist es Inferenz (Eng. Inference), d. h. ein logischer Schlussfolgerungsprozess, „mit dem ein Hörer die Bedeutung ableitet“ (Meibauer 2008: 121) oder eine Hypothese des Hörers darüber, was der Sprecher wahrscheinlich meint. Zum anderen ist es die sog. Ostension. Ostensive Kommunikation bedeutet ,zeigende' bzw. , anschaulich machende' kommunikative Akte. Mit anderen Worten ist sie als Signal zu verstehen, dass der Sprecher etwas mitzuteilen hat, was für ihn wichtig ist (vgl. ebd.). In ihrem Relevanzprinzip stellen Sperber und Wilson (1998: 98) Folgendes fest: „every act of ostensive communication communicates the presumption of its own optimal relevance“. Darüber hinaus bedarf die ostensiv-inferentielle Kommunikation eines ostensiven Stimulus (Eng. ostensive stimulus), der konstruiert wird, um die Aufmerksamkeit des Empfängers (der Empfänger) auf sich zu ziehen und sich auf die Ansicht des Produzenten zu konzentrieren (vgl. Wilson \& Sperber 2006: 611). Mit einem solchen Anreiz hat der potentielle Adressat nicht nur Hoffnung, sondern ganz genaue Erwartungen hinsichtlich der Relevanz (vgl. Sperber \& Wilson 1998: 98). Gleichzeitig bemerken die Autoren, dass „people will pay attention to a phenomenon only if it seems relevant to them" (ebd.).

Für den potentiellen Empfänger entstehen bei praktisch jeder Äußerungsinterpretation zwei Aufgaben. Erstens muss er ermitteln, welche Proposition ausgedrückt wurde; zweitens ist er dazu gezwungen, eventuell eine zusätzliche Menge von Propositionen zu ermitteln, die mit dem relevanten Kontext identifiziert werden (vgl. Meibauer 2008: 122). Die Proposition (Aussage) ist in der linguistischen Semantik nichts anderes als logische, kontextunabhängige Struktur/logische Form (vgl. Baum 2010: 206) bzw. als Inhalt eines Satzes zu verstehen, der „der Wahrheitsbewertung unterliegt“ (ebd.: 37), oder einen Wahrheitswert annimmt, d. h. wahr oder falsch sein kann (vgl. Pafel \& Reich 2016: 11). Mit anderen Worten ist sie die wörtliche Bedeutung eines Satzes ${ }^{19}$. Propositionen sind in der Regel unterspezifiziert; dies bedeutet in der Relevanztheorie, dass sie durch diverse Schlussprozesse angereichert werden müssen. Im Gegensatz zur Proposition als

19 Diese wörtliche Bedeutung eines Satzes kann allerdings eine andere illokutive Rolle haben ( je nach dem Sprechakt), jedoch die Referenz (hier: Katrin) und die Prädikation (fleißig arbeiten) bleiben unverändert, z. B. in der Feststellung „Katrin arbeitet fleißig“, in der Frage „Arbeitet Katrin fleißig?““ oder in der Aufforderung „Katrin, arbeite fleißig!“ (alle Beispiele nach Homberger 2003: 510). 
logische Form sind für die Autoren der Relevanztheorie Explikaturen und Implikaturen kontextabhängig „sowie mittels konversationaler Schlußmechanismen ableitbar und annullierbar" (Baum 2010: 206).

Eine Anreicherung einer Äußerung mit Information bzw. eine Vollendung eines vorgegebenen Sprachfragments wird in der Relevanztheorie als Explikatur bezeichnet. Darüber hinaus stellen Explikaturen (im Gegensatz zu Implikaturen) eine Art Entwicklung der logischen Form=Proposition dar, was bedeutet, dass „die logische Form in der Explikatur enthalten und erkennbar sein muß“ (ebd.: 207). Dies wiederum setzt voraus, dass die Explikatur aus solchen kontextuellen Bedeutungen besteht, „die Füllungen von Leerstellen in der Satzbedeutung bzw. eine Art (Neben-)Produkt der Referenzbestimmung bilden.“(ebd.) ${ }^{20}$. Explikaturen sind demnach Inferenzen, die unmittelbar aus der konventionellen Bedeutung eines sprachlichen Zeichens ableitbar sind. Eine genaue Definition der Explikatur präsentiert Carston (2002: 377) und stellt Folgendes fest: eine Explikatur ist „an ostensively communicated assumption that is inferentially developed from one of the incomplete conceptual representations (logical forms) encoded by the utterance“. In ihrem Konzept glauben nämlich Wilson und Sperber, dass praktisch jede Äußerung einige Explikaturen besitzt, die sich auf unterschiedlichen Ebenen befinden können. Die propositionale Form als solche drückt meistens eine Explikatur auf unterer Ebene aus (Eng. lower-level explicatures), die ebenso als zentrale Explikatur bezeichnet werden kann. Zum Beispiel in dem vorliegenden Satz (vgl. Feng 2010: 65) „Mary to Peter: Sadly, I cannot help you to find a job. ${ }^{21 “}$ wäre die Explikatur auf unterer Ebene einfach die ausgedrückte Proposition, wie etwa „Mary kann Peter nicht helfen, einen neuen Job zu finden." Eine Explikatur auf höherer Ebene (Eng. higher-level explicature) dagegen könnte im Falle einer solchen Äußerung folgendermaßen ausgedrückt werden: „Mary ist traurig, weil sie Peter nicht helfen kann, einen neuen Job zu finden“. Explikaturen auf höherer Ebene sind demnach „the result of embedding propositional forms (lower-level explicatures) in propositional attitude descriptions, speech acts descriptions and certain other comments on the em-

20 Im Gegensatz zu Grice (1975), der den Sprecher in den Vordergrund stellt, geht die Relevanztheorie vom Hörer aus und beschreibt seinen Verstehensprozess als inferentiellen (bzw. Inferenz-basierten) Vorgang. Die Theorie versucht auch das schwierige Verhältnis zwischen dem "Gesagten“ und „Gemeinten“ zu klären. Das „Gesagte“ bei Grice wird in der Relevanztheorie durch sprachliche Form und Inferenz von Explikaturen ersetzt; die (konversationellen) Implikaturen von Grice sind hier Inferenz von Implikaturen.

21 Besonders Explikaturen auf höherer Ebene werden (intuitiv) sichergestellt durch die Mischung von Dekodieren und Inferenz auf der Basis von unterschiedlichen linguistischen und nicht linguistischen Hinweisen (wie etwa Reihenfolge, Indikatoren der Stimmung im Gesprochenen, Ton, Gesichtsausdruck usw.), vgl. Wilson und Sperber (2006: 623). 
bedded propositions“ (Feng 2010: 64f.) und werden vom Sprecher auf gleiche Weise hergeleitet wie die anderen Inhalte der Kommunikation ${ }^{22}$.

Im Gegensatz zu Explikaturen sind Implikaturen für „weitergehende Andeutungen ohne direkten Bezug zur logischen Form reserviert.“ (Baum 2010: 207), d. h. bei einer Implikatur einer sprachlichen Äußerung handelt es sich um einen Bedeutungsaspekt, der durch diese Äußerung zwar kommuniziert wird, aber von dem jeweiligen Sprecher lediglich angedeutet wird. Folglich bedeutet es, dass die Implikatur „eine auf der Basis von Explikatur und kontextuellen Informationen konstruierte Prämisse oder Deduktion [darstellt], die die propositionale Einstellung des Sprechers zum Ausdruck bringt“ (Baum 2010: 207f.).

\section{Forschungsdesign und Beispielanalyse (\#coronadeutschland)}

Der pragmatisch-semantischen Analyse unterliegen 100 ausgewählte deutschsprachige Tweets, die in der Zeitspanne 27. - 30. März 2020 veröffentlicht wurden und sich auf den Ausbruch der COVID19-Pandemie (bzw. der CoronavirusPandemie) in der Welt und in Deutschland beziehen. Das gemeinsame Element für alle Beiträge ist der Gebrauch eines der häufigsten deutschen Hashtags im Frühjahr 2020 und zwar \#coronadeutschland. Im nächsten Schritt werden einige besonders interessante Beispiele präsentiert und linguistisch beschrieben. Der besondere Fokus liegt auf der Analyse des textuellen Teils der einzelnen Tweets in pragmatisch-semantischer Hinsicht. In einigen Beispielen verweise ich allerdings auch auf eventuelle außersprachliche Elemente (Grafiken, Videos) bzw. auf Links, die manchen Tweets beigefügt worden sind. Anschließend werden die quantitativen Ergebnisse der Analyse präsentiert und mit entsprechenden kommunikativen Funktionen auf der Handlungsebene von Hashtags in Verbindung gebracht.

Bezüglich der Relevanztheorie unterscheide ich zwischen Hashtags, die lediglich zur Inferenz von zentralen Explikaturen (d. h. einfach ausgedrückten Propositionen) und solchen, die zur Erschließung von den meisten Explikaturen auf höherer Ebene sowie Implikaturen verhelfen.

22 Dabei kann es mit Hilfe der linguistischen Form der Äußerung darauf hingewiesen werden, dass der jeweilige Sprecher intendiert, dass er eine bestimmte Einstellung gegenüber der Proposition übermittelt. Gerade diese Einstellung gegenüber der Wahrheit der Proposition bestimmt zum großen Teil die Explikaturen auf höherer Ebene, was u. a. in der OnlineKommunikation (besonders mit beschränkter Zeichenanzahl wie bei Twitter) mittels Hashtags ausgedrückt werden kann. 


\section{Zentrale Explikaturen}

Die kleinste Gruppe unter analysierten Hashtags bilden solche, die die Inferenz von zentralen Explikaturen ermöglichen. Hashtags haben in solchen Beiträgen vor allem eine informationsbezogene Filterungs- bzw. Verschlagwortungsfunktion und durch ihren Einsatz kann der für den User wichtige Inhalt schnell aufgefunden und rezipiert werden ${ }^{23}$. Darüber hinaus erleichtern sie den notwendigen Kontext zu erschließen, wie in dem assertiven Sprechakt (mit deklarativen Elementen) in (1) oder in der rein informativen Aussage (2), in der ein beigefügter Link zu einer Online-Zeitung die Inferenz der zentralen Explikatur zusätzlich erleichtert.

(1)@ErdKaya3

\#coronadeutschland \#Covid_19 liebe medizinisches Personal, Ärzt/in, Krankenschwester, Krankenpfleger/in, die Intensivpfleger des Personals, wir sind neben ihnen. Natürlich gibt es kein ewiges Atemgerät, deswegen \#StayAtHome Bitte \#wirbleibenzuhause und an dem Leben für sie und dich

(2) @keklumpp

Wolfsburg ist der erste Kreis in Deutschland mit „italienischen Verhältnissen“. 115 Corona-Infizierte pro 100 Tsd. Einwohner. Sterblichkeit 10,6\%. Klinikum verhängt Aufnahmestopp. \#coronadeutschland

[Beigefügt: ein Link zu einer lokalen Online-Zeitung]

\section{Explikaturen auf höherer Ebene}

In den meisten analysierten Tweets wird durch Hashtags die Inferenz von Explikaturen auf höherer Ebene erleichtert. In über 80 \% von solchen Postings im Textkorpus wird durch den hinzugefügten Hashtag die Einstellung des Textproduzenten (ggf. seine Gefühle) zum Gesagten ausgedrückt. Es passiert oft mittels anderer Hashtags in demselben Tweet, die zwangsläufig mit dem eventuellen zentralen Hashtag in Verbindung gebracht werden. Dadurch können andere Propositionen derselben Aussage ermittelt werden, da ein und derselbe Hashtag sogar zur ihrer Rekontextualisierung beitragen kann. In (3) bezieht sich der TweetAutor auf die Diskussion im deutschen Bundestag vom 26. März 2020 über das Maßnahmenpaket der Bundesregierung und kritisiert die Worte des Chefs der Freien Demokratischen Partei (FDP) Christian Lindner, der in einem Interview

23 Selbstverständlich muss man an der Stelle annehmen, dass die jeweiligen Textproduzenten von ihren potentiellen Empfängern erwarten, dass sie alle Stichwortkombinationen mit „Corona“ richtig interpretieren können. Somit kann der Hashtag (in diesem Fall \#coronadeutschland) als der erste Wegweiser und einer der ostensiven Stimuli begriffen werden. 
behauptete, dass jeder Ausnahmezustand der menschlichen Natur widerspreche und zu einer offenen Gesellschaft nicht passe ${ }^{24}$, was für den Autor bedeuten könne, die FDP möchte sich allzu sehr in die deutsche soziale Politik einmischen und ihr eventuell schaden. Die rein informativen Hashtags \#coronakrise und \#coronadeutschland (Inferenz von der zentralen Explikatur) werden um Hashtags \#fdpkannweg und \#seidIhralleDumm ${ }^{25}$ ergänzt, die offensichtlich die negative und kritische Einstellung des Textproduzenten (vgl. die Worte „sie müssten an der Demokratie rütteln!") hervorheben. Die AutorInnen von (4) und (5) konzentrieren sich dagegen auf Kommentare zu nicht sprachlichen Teilen ihrer Tweets. In (4) fügt der Textproduzent ein Foto von einem wohl selbstgemachten Schild in einem Supermarkt mit dem Schriftzug „Einkaufen ist kein Familienausflug“ bei. Durch die Phrase „Bin a bissl \#beeindruckt“, die mit einem Hashtag versehen wurde, wird die Einstellung des Sprechenden zu der geschilderten Situation ausgedrückt. Gleichzeitig erleichtert der Hashtag die Inferenz der Explikatur auf höherer Ebene (z. B. ,dem Textproduzenten gefällt, wie das Personal des Supermarkts in der Zeit der Pandemie zurechtkommt und sich um die Sicherheit der Kunden kümmert ${ }^{\prime}$ ). Die Autorin von (5) bezieht sich auf das Foto, das wohl von ihren Enkelkindern aus einem Fenster im Erdgeschoss gemacht wurde. Die Autorin steht auf der Straße und winkt lächelnd den Kindern zu. Zwar kann man die zwei Hashtags in dem Beitrag lediglich als einfache Schlagwörter bzw. als thematische Referenzierung betrachten, dank denen sich zusätzliche ad-hoc-Gemeinschaften herausbilden können, aber der Hashtag \#SocialDistancing kann auch (gerade in einem so gestalteten Text-Bild-Gefüge) als ostensiver Stimulus dienen - die Textproduzentin verstärkt damit die durch das Bildliche vermittelte Botschaft (z. B. , für die Autorin ist die Aktion der sozialen Distanzierung während der Corona-Pandemie von Bedeutung, was sie auch durch das beigefügte Foto bestätigt' ${ }^{\prime}$ ).

(3)@NiemalsD

2 Wochen \#Coronakrise in Deutschland und einige meinen, sie müssten an der Demokratie rütteln!

Ich fasse es nicht. Werft die Kasper der FDP aus dem Bundestag! \#fdpkannweg \#coronadeutschland \#seidIhralleDumm

(4) @PremiumSaft

Der Edeka in meiner Heimatstadt rüstet Woche für Woche auf. Am Ende des Weges werden schon länger die Hände der Kunden und die Einkaufswagen desinfiziert.

$24 \mathrm{Mehr}$ u. a. unter https://www.liberale.de/content/muessen-schnellstmoeglich-freiheit-zuru eckkehren-koennen (3.04.2020).

25 An diesem Beispiel zeigt sich deutlich eine andere Tendenz vieler Twitter-UserInnen. Im Falle von längeren Hashtags, die aus mehreren Wörtern bestehen, werden oft Anfangsbuchtstaben großgeschrieben. Obwohl eine solche Schreibweise keinen Einfluss auf die Ergebnisse eventueller Suchanfragen hat, trägt sie zu ihrem schnelleren und besseren Verständnis bei. 
Wurst an der Wursttheke kommt via selbstgebauter Rutsche zum Kunden. Bin a bissl \#beeindruckt \#coronadeutschland \#coronavirus

(5)@MAStrackZi

Ich wünsche allen einen guten Wochenstart \& hoffe, dass alle trotz der aktuellen Situationen das Wochenende genießen konnten. Mit angemessenen Sicherheitsabstand konnte ich wenigstens kurz meine Enkel vor dem heimischen Fenster wiedersehen. \#coronadeutschland \#SocialDistancing

\section{Implikaturen}

Die zweitgrößte Gruppe von Hashtags bilden in dem analysierten Textkorpus solche, die den potentiellen Empfängern helfen, gewisse Andeutungen des Textproduzenten zu verstehen - auf der Basis von mitgelieferten Explikaturen und kontextuellen Informationen. Dazu kommen in fast allen Beispielen von Tweets beigefügte (meist ironische oder scherzhafte) multimodale Elemente. Dem Tweet (6) ist ein Kurzfilm beigefügt, auf dem zwei junge Frauen in ihren Händen eine entrollte Toilettenpapierrolle halten. Auf den einzelnen Teilen kann man die Buchstaben einer handgeschriebenen Parole entziffern: „Leave No One Behind“. Das Stichwort wird ebenso in Form eines Hashtags benutzt. Während der Ausgangshashtag als der wichtigste ostensive Stimulus dient, verhilft \#LeaveNoOneBehind zur Inferenz von Explikaturen auf höherer Ebene (bspw. es handelt sich wohl um mehr als nur ein Stichwort in englischer Sprache; es heißt „niemand sollte zurückgelassen werden“26). Der Hashtag \#Klopapier erleichtert dagegen eine weitere Inferenz von versteckten Implikaturen und dem bereits erschlossenen Kontext der Aussage (z. B. ,die Autorinnen beziehen sich auf Toilettenpapier, das in der letzten Zeit in vielen deutschen Supermärkten ausverkauft ist, obwohl kein Risiko besteht, dass Artikel der Körperpflege nicht mehr erhältlich sind. Dazu sollte niemand alleine gelassen werden; wir sind alle bedürftig ${ }^{67}$ ). In (7) dagegen wird die durch das Bild vermittelte Botschaft (anscheinend einer rein informativen Natur in Form von einem expressiven Sprechakt - einer Werbung von einem Blumenladen) dank der drei eingesetzten

26 In den meisten Fällen klicken viele UserInnen auf den entsprechenden Hashtag, um zu prüfen (oder sich zu vergewissern) welche Bedeutung er hat und in welchem Kontext er benutzt wird.

27 Der Hashtag und der Name \#LeaveNoOneBehind beziehen sich ursprünglich darauf, dass angesichts von Covid-19 die Aufmerksamkeit für die schwierige Lage in Flüchtlingslagern an EU-Außengrenzen noch mehr abnimmt. Vor allem steht das Lager Moria auf der griechischen Insel Lesbos beispielhaft dafür (vgl. https://leavenoonebehind2020.org/de/, 30.03. 2020). 
Hashtags rekontextualisiert. Ein solcher Schlussfolgerungsprozess könnte bei dem potentiellen Empfänger folgendermaßen aussehen:

- zentrale Explikatur: Der Textproduzent stellt eine Frage, die Wiedereröffnung eines gewissen Blumenladens in der Nähe betrifft (Hauptinformationen in dem textuellen Teil des Tweets und das beigefügte Bild),

- Inferenz von Explikaturen auf höherer Ebene (teilweise durch den Hashtag \#coronadeutschland verstärkt): Der Textproduzent ist verärgert, weil ein Laden wieder geöffnet hat, obwohl einige Tage zuvor deutschlandweit eine Ausgangssperre und Notschließung von Institutionen und den meisten Geschäften angeordnet wurde,

- Inferenz von Implikaturen (ohne direkten Bezug zur logischen Form, allerdings auf der Basis von mitgelieferten Explikaturen und kontextuellen Informationen; durch weitere Hashtags \#systemrelevant und \#essen vereinfacht): Der Autor suggeriert mit viel Ironie, dass die Blumen so wichtig für die deutsche Wirtschaft seien, dass man ein Blumengeschäft öffnen kann. Dazu scherzt er darüber, dass man Blumen essen kann, so wie normale Lebensmittel.

(6) @_Seebruecke_

Keine Panik, es ist genug für alle da! \#Klopapier \#LeaveNoOneBehind \#coronadeutschland

(7) @farbfunkelnd

wieso darf ein Blumenladen in Dorsten wieder öffnen? Ja Leute, die Blumen sind doch wirklich \#systemrelevant.. und man kann sie \#essen, nicht? \#coronadeutschland

\section{Ergebnisse der Analyse und Schlussfolgerungen}

Ein genauer Einblick in das Textkorpus zeigt deutlich, dass außer der typischen Erschließung von meist einfachen und offensichtlichen Informationen (zentralen Explikaturen), zu deren Inferenz über $9 \%$ der analysierten Hashtags beitragen, sind die meisten Hashtags auf der Handlungsebene nicht nur als kommunikative Operatoren des Social Tagging zu betrachten, die lediglich einer Indexierung, Kategorisierung oder Verschlagwortung, bzw. Markierung von geteilten Inhalten dienen ${ }^{28}$. Die durchgeführte Analyse des Textkorpus zeigt deutlich, dass beinahe $60 \%$ von eingesetzten Hashtags eben zur Schlussfolgerung

28 Die Praxis der Online-Kommunikation zeigt auch, dass entsprechende Hashtagkombinationen eine Suche bei Suchanfragen mit ähnlichen Suchbegriffen erleichtern sowie besseres Sortieren und Filtern von Inhalten ermöglichen. Darüber hinaus können bestimmte Standort- und Veranstaltungsdaten (z. B. durch das Angeben von Ortsnamen) bekanntgegeben werden. 
von Explikaturen auf höherer Ebene verhelfen. Es liegt m. E. an der Tatsache, dass gerade das Anfang 2020 viel diskutierte vielschichtige Problem der CoronavirusPandemie für starke Emotionen und unterschiedliche Meinungen sorgt. Der Einsatz von Hashtags muss selbstverständlich nicht bedeuten, dass alle sprachlichen Elemente, die zum Gesagten bzw. Gemeinten der gegebenen Aussage gehören, nur mit Hilfe von Hashtags erschlossen werden können. Eine „richtige“ Inferenz von Explikaturen auf höherer Ebene, der in einem nächsten Schritt das Erschließen von eventuellen Implikaturen folgt, ist in rein textuellen Beiträgen online in vielen Fällen beschränkt. Die meisten AutoriInnen greifen aus diesem Grund zu einer breiten Palette von anderen semiotischen Ressourcen. Außer Links sind es Grafiken, Emoticons, sog. GIFs (animierte Bilder) und (z.T. selbstgemachte) Fotos oder Videos. Nichtsdestotrotz verhelfen die eingesetzten Hashtags in $31 \%$ der analysierten Tweets zur Inferenz von Implikaturen. Hashtags, die die Inferenz von Explikaturen auf höherer Ebene sowie Implikaturen erleichtern, dienen auf der Handlungsebene v. a. einer bestimmten Kontextualisierung, Positionierung des Autors zu dem Thema (bzw. Bekenntnis oder Aufruf zu einer im Hashtag benannten sozialen oder politischen Aktion) und schließlich Zuweisung zu einer Eigenschaft bzw. einem Verhalten. Darüber hinaus führt der Einsatz von bestimmten Hashtags zu einer Art Themenbündelung und demzufolge zu einer Diskursorganisation, die es den Vertretern von unterschiedlichen Öffentlichkeiten ermöglicht, den Diskurs entsprechend weiter $\mathrm{zu}$ entwickeln.

\section{Literaturverzeichnis}

Androutsopoulos, Jannis K./Weidenhöffer, Jessica (2015). Zuschauer-Engagement auf Twitter: Handlungskategorien der rezeptionsbegleitenden Kommunikation am Beispiel von \#tatort. Zeitschrift für angewandte Linguistik, 62(1), 23-59.

Autenrieth, Ulla P./Herwig, Jana (2011). Zwischen begrenzten Mitteln und komplexen Strukturen: gemeinschaftsorientierte Kommunikation und Interaktion auf Microblogging-Plattformen am Beispiel Twitter. In: K. Neumann-Braun/U. P. Autenrieth (Hrsg.), Freundschaft und Gemeinschaft im Social Web. Bildbezogenes Handeln und Peergroup-Kommunikation auf Facebook \& Co (S. 211-232). Baden-Baden: Nomos.

Baum, Friederike (2010). Pragmatische Verstehensprozesse sprachlicher Äußerungen. Möglichkeiten und Grenzen der Theorie Generalisierter Konversationsimplikaturen im Vergleich zur Relevanztheorie (Reihe: Kriterium. Arbeiten zur Sprachtheorie und Kommunikationspraxis. Band 2). Berlin: LIT Verlag Dr. W. Hopf.

Carston, Robyn (2002). Thoughts and utterances: The pragmatics of explicit communication. Malden u. a.: Blackwell Publishing. 
Bruns, Axel (2008). The active audience: Transforming journalism from gatekeeping to gatewatching. In: C. Paterson/D. Domingo (ed.), Making online news: The ethnography of new media production (S. 171-184). New York: Peter Lang.

Dang-Anh, Mark/Einspänner, Jessica/Thimm, Caja (2013). Kontextualisierung durch Hashtags: die Mediatisierung des politischen Sprachgebrauchs im Internet. In: H. Diekmannshenke/T. Niehr (Hrsg.), Öffentliche Wörter: Analysen zum öffentlich-medialen Sprachgebrauch (S. 137-159). Stuttgart: ibidem.

Feng, Guangwu (2010). A theory of conventional implicature and pragmatic markers in Chinese. Bingley: Brill.

Fraas, Claudia/Meier, Stefan/Pentzold, Christian (2013). Zur Einführung: Perspektiven einer interdisziplinären transmedialen Diskursforschung. In: C. Fraas/S. Meier/C. Pentzold, (Hrsg.), Online-Diskurse. Theorien und Methoden transmedialer OnlineDiskursforschung (S. 7-34). Köln: Herbert von Halem Verlag.

Goderbauer-Marchner, Gabriele/Büsching, Thilo (2015). Social-media-content. Konstanz u. a.: UVK Verlagsgesellschaft mbH.

Grice, Herbert Paul (1975). Logic and conversation. In: P. Cole/J. L. Morgan (ed.), Syntax and semantics 3: Speech acts (S. 41-58). New York: Academic Press.

Herwig, Jana (2011). Die 140-Zeichen-Frage: Microblogging, Twitter und die liminoide Verhandlung des Sozialen im Web 2.0. In: K. Neumann-Braun/U. P. Autenrieth (Hrsg.), Freundschaft und Gemeinschaft im Social Web. Bildbezogenes Handeln und PeergroupKommunikation auf Facebook \& Co (S.195-209). Baden-Baden: Nomos Vrelagsgesellschaft.

Hodkinson, Paul (2011). Media, culture and society. An introduction. Los Angeles u.a.: Sage.

Homberger, Dietrich (2003). Sachwörterbuch zur Sprachwissenschaft. Stuttgart: Reclam.

Kirchhoff, Sabine/Lombardo, Paolo/Urhahn, Lars (2015). Ausgewählte Plattformen, Tools und Technologien. In: S. Kirchhoff (Hrsg.), Online-Kommunikation im Social Web. Mythen, Theorien und Praxisbeispiele (S. 225-236). Opladen u. a.: Verlag Barbara Budrich.

Meibauer, Jörg (2008). Pragmatik. Zweite, verbesserte Auflage. Tübingen: Stauffenburg Verlag.

Musser, John/O’Reilly Tim (2007). Web 2.0 principles and best practices. Sebastopol: O'Reilly Media.

Neuberger, Christoph./Langenohl, Susanne/Nuernbergk, Christian (2014). Social media und journalismus. In: LfM-Dokumentation, Band 50. https://www.medienanstalt-nrw. de/fileadmin/lfm-nrw/Publikationen-Download/Social-Media-und-Journalismus-LfMDoku-Bd-50-web.pdf.

Pafel, Jürgen/Reich, Ingo (2016). Einführung in die Semantik. Grundlagen - Analysen Theorien. Stuttgart: J.B. Metzler.

Primbs, Stefan (2016). Social Media für Journalisten. Redaktionell arbeiten mit Facebook, Twitter \& Co. Wiesbaden: Springer VS.

Prokop, Izabela (1995). Erotetische Sprechakte im Deutschen und im Polnischen anhand natürlicher Gespräche. Poznań: Wydawnictwo Naukowe Uniwersytetu im. Adama Mickiewicza.

Prokop, Izabela (2010). Aspekty analizy pragmalingwistycznej. Poznań: Wydawnictwo Naukowe Uniwersytetu im. Adama Mickiewicza. 
Rybszleger, Paweł (2016). Diskursive Konstituierung von Wissen in onlinebasierten Social Media Plattformen. tekst i dyskurs - text und diskurs, 9, 85-102.

Sommer, Denise/Porten-Cheé, Pablo (2018). Anschlusskommunikation als Medienwirkung. Der Einfluss von Relevanz und Qualität von Medieninhalten auf das Gesprächsverhalten. Publizistik, 63, 159-161.

Neuernbergk, Christian (2014). Partizipation durch Anschlusskommunikation in der Blogosphäre. Ergebnisse einer Inhalts- und Netzwerkanalyse. In: J. Einspänner-Pflock/ M. Dang-Anh/C. Thimm (Hrsg.), Digitale Gesellschaft - Partizipationsstrukturen im Netz (S. 190-207). Berlin: LIT Verlag Dr. W. Hopf.

Schmidt, Jan (2011). Das neue Netz. Merkmale, Praktiken und Folgen des Web 2.0 (2., überarbeitete Auflage). Konstanz: UVK Verlagsgesellschaft mbH.

Schmidt, Jan-Hinrik (2013). Onlinebasierte Öffentlichkeiten: Praktiken, Arenen und Strukturen. In: C. Fraas/S. Meier/C. Pentzold (Hrsg.), Online-Diskurse. Theorien und Methoden transmedialer Online-Diskursforschung (S. 35-56). Köln: Herbert von Halem Verlag.

Scott, Kate (2015). The pragmatics of hashtags: Interference and conversational style on Twitter. Journal of Pragmatics, 81, 8-20.

Sonnberger, Roman (2012). Facebook im Kontext medialer Umbrüche. Eine theoretische und empirische Annäherung. Boizenburg: Verlag Werner Hülsbusch.

Sperber, Dan/Wilson, Deirdre (1995). Relevance: Communication and cognition. 3rd ed. (with postface). Blackwell: Oxford.

Sperber, Dan/Wilson Deirdre (1998). Précis of relevance: Communication and cognition. In: A. Kasher (ed.), Pragmatics. Critical concepts (S. 82-115). London u. a: Routledge. Stephens, Todd R. (2010). Integrating Web 2.0 technologies within the Enterprise. In: A. Tatnall (ed.), Web technologies: Concepts, methodologies, tools and applications. Vol. I. Information science reference (S. 201-218). New York: Hershey.

Szyszko, Agata (2017). Zastosowanie znaczników z informacją o lokalizacji oraz danych GPS przez media społecznościowe. Studia Ekonomiczne. Zeszyty Naukowe Uniwersytetu Ekonomicznego w Katowicach, 342, 148-165.

Thimm, Caja/Dang-Anh, Mark/Einspänner, Jessica (2011). Diskurssystem Twitter: Semiotische und handlungstheoretische Perspektiven. In: M. Anastasiadis/C. Thimm (Hrsg.), Social Media. Theorie und Praxis digitaler Sozialität (S. 265-285). Frankfurt a.M. u. a.: Peter Lang.

Wagner, Elke (2019). Intimisierte Öffentlichkeiten: Pöbeleien, Shitstorms und Emotionen auf Facebook (Sozialtheorie). Bielefeld: transcript Verlag.

Wilson, Deirdre/Sperber, Dan (2006). Relevance theory. In: L. L. Horn/G. Ward (eds.), The handbook of pragmatics (S. 607-632). Malden u. a.: Blackwell Publishing. 


\section{Teil 2: Kontrastive Studien}


Open-Access-Publikation im Sinne der CC-Lizenz BY-NC-ND 4.0

(C) 2020, Vandenhoeck \& Ruprecht GmbH \& Co. KG, Göttingen ISBN Print: 9783847111863 - ISBN E-Lib: 9783737011860 


\title{
Norbert Nübler (Christian-Albrechts-Universität zu Kiel)
}

\section{Der polnische Verbalaspekt im Fremdsprachenunterricht}

\begin{abstract}
Teaching verbal aspect in Polish as a foreign language

Verbal aspect is commonly accepted as a grammatical category typical of the Polish verb, which causes serious problems in translation as well as in teaching Polish as a foreign language. Surprisingly, Polish grammars (whether written for Polish native speakers or for German learners of Polish) do not pay considerable attention to this problematic category. Taking a didactic point of view, it is argued in this chapter it could be useful to introduce the aspect as an inflectional category of its own kind early in the curriculum, explaining the mechanisms of regular aspect change (prefixation, suffixation etc.) and pointing out exceptions in order to enable (German) students of Polish to identify a given verb form as imperfective or perfective.
\end{abstract}

\section{Keywords}

verbal aspect, grammatical category, mechanisms of aspect change, inflection of the Polish verb, Polish as a foreign language

\section{Aspekt als strukturelle Divergenz zwischen dem Polnischen und Deutschen}

Eines der schwer lösbaren Probleme bei der Übersetzung polnischer Texte in das Deutsche und umgekehrt beruht auf einem strukturellen Unterschied der beiden Sprachen ${ }^{1}$. Im Verbalsystem des Polnischen findet sich mit dem Aspekt eine grammatische Kategorie, für die das Deutsche kein Äquivalent aufweist. Ohne weiteren Kontext entsprechen deshalb dem deutschen Satz (1) die beiden polnischen Sätze (2) und (3):

1 Die folgenden Ausführungen nehmen vorwiegend den Blickwinkel eines Lerners des Polnischen als L2 mit dem Deutschen als Vermittlungs- und Umgebungssprache (L1) ein. Anmerkungen zur sprachlichen Bildung polnischer Muttersprachler lassen sich jedoch nicht völlig vermeiden. 
(1) Mein Freund schrieb einen Brief

(2) Mój przyjaciel pisał list.

(3) Mój przyjaciel napisał list.

Der Unterschied zwischen den polnischen Sätzen (2) und (3) besteht ausschließlich in der Wahl des Verbs. Im Polnischen gibt es also ganz offensichtlich für deutsche Verben mindestens zwei Übersetzungsmöglichkeiten. Das Verb im Satz (2) wird als imperfektiv bezeichnet, das Verb im Satz (3) hingegen als perfektiv. Imperfektivität und Perfektivität können als Subkategorien der Opposition gesehen werden, für die sowohl in der polnischen als auch in der deutschen Grammatikschreibung der Begriff „Aspekt“ verwendet wird. Inwiefern diese Wahl bei allen deutschen Verben zur Verfügung steht, mag hier zunächst offen bleiben. Der Gegensatz zwischen imperfektiven Verben (wie pisać) und perfektiven Verben (wie napisać) impliziert jedenfalls, dass die Paradigmen der beiden Verben semantisch nicht vollständig äquivalent sind. Demzufolge sind auch die beiden polnischen Übersetzungen des deutschen Satzes (1) semantisch nicht vollkommen gleichwertig. Damit stellt sich für Lehrkräfte im Fremdsprachenunterricht das Problem einer Erklärung dieses dem deutschen Lerner zunächst völlig fremden Gegensatzes, der auch nicht durch das bei den Lernern vorauszusetzende Wissen um die deutsche Grammatik expliziert werden kann.

In der Regel erfahren Lernende also zunächst, dass es im Polnischen imperfektive Verben gibt - wie eben pisać „schreiben“ oder auch robić „machen, tun“, pić „trinken“, sedzieć „sitzen“ u.v.m. Diesen stehen perfektive Verben gegenüber - also etwa napisać „schreiben“, wypić „austrinken“, wstać „aufstehen“ u.v.m. Eine Erklärung, worin der Unterschied zwischen diesen beiden Verbarten besteht, oder auch nur eine Anleitung, wie bei einem gegebenen Verb die Imperfektivität oder Perfektivität zu erkennen ist, wird meist gar nicht oder nur sehr unvollständig geliefert. Zur weiteren Verwirrung der Lernenden trägt bei, dass sowohl in deutschsprachigen als auch in polnischsprachigen Lehrwerken, Grammatiken und Nachlagewerken die aus dem 19. Jahrhundert tradierten und inhaltlich irreführenden Ausdrücke „unvollendet“ (niedokonany) für imperfektiv und „vollendet“ (dokonany) für perfektiv verwendet werden ${ }^{2}$. Spätestens dann, wenn Lerner mit der Tatsache konfrontiert werden, dass Verben wie zaczać „anfangen“, rozbiec się „loslaufen“ oder posiedzieć „eine Weile sitzen“ vollendet sein sollen, erweist sich die Inadäquatheit dieser Bezeichnungen.

Als Konsequenz aus solch irreführenden Erklärungen kann es insbesondere bei der Übertragung von Fachtexten zu erheblichen Schwierigkeiten oder Miss-

2 Hier sei nur kurz auf die „Lerngrammatik“ von Madelska (2007: 25) verwiesen, wo die Bezeichnungen „unvollendet“ und „vollendet“ praktisch als Erklärungen für „imperfektiv“ und „perfektiv“ eingesetzt werden. 
verständnissen kommen. Das gilt auch für Übersetzungen aus dem Polnischen in das Deutsche. Nimmt man die Bezeichnung „unvollendet“ für das polnische Verb pisać ernst, so ergeben sich für den polnischen Satz (2) im Deutschen mehrere Übersetzungsmöglicheiten - das können etwa die Sätze (4) und (5) sein:

(4) Mein Freund schrieb an einem Brief.

(5) Mein Freund war dabei, einen Brief zu schreiben.

Nur in diesen Übersetzungen ist tatsächlich die „Unvollendetheit“ auch im Deutschen klar ausgedrückt. Der polnische Satz (2) kann aber darüber hinaus auch die bloße Feststellung, dass jemand einen Brief verfasst hat, bezeichnen, also wie in Satz (1) übersetzt werden. Er lässt dabei offen, ob dieser Brief jemals vollendet wurde. Tatsächlich muss das Schreiben hier also keineswegs „unvollendet" sein. In diesem Zusammenhang sei darauf hingewiesen, dass es in manchen Bereichen von Fachsprachen - insbesondere im juristischen Bereich ein wesentliches Kriterium für die Beurteilung einer Handlung (oder auch eines Prozesses) darstellt, ob diese sicher vollendet wurde, wie in Satz (6) mit dem perfektiven $u k r a s ́ c ́$ „stehlen“, oder aber, ob der Erfolg der Handlung offen bleibt wie in (7) mit dem imperfektiven kraść „stehlen“:

(6) Obwiniony ukradł książkę.

(7) Obwiniony kradł książkę.

Je nach Kontext kann der Satz (7) den bloßen Versuch kennzeichnen und offen lassen, ob das Stehlen erfolgreich war. Der Satz (6) hingegen setzt zweifellos die vollständige und erfolgreiche Durchführung einer Straftat voraus. Insbesondere bei der Formulierung von Gesetzestexten, aber auch im täglichen juristischen Betrieb ist diese im Deutschen nur schwer nachvollziehbare Unterscheidung von eminenter Bedeutung.

Die für manche Fachsprache relevante „Unschärfe“ bei der Übertragung von einer Sprache in die andere ist aber nicht das einzige Problem, das das polnische Verbsystem und darin insbesondere die Aspektkategorie aufwirft, wenn man das Deutsche zum Vergleich heranzieht. Im Präteritum sind - wie die Verwendungsbeispiele (1) - (3) zeigen - für das deutsche Verb schreiben ganz offensichtlich zwei polnische Übersetungsmöglichkeiten (pisać und napisać) möglich. Setzt man aber den obigen deutschen Beispielsatz (1) in das aktuelle Präsens, so ist jedoch nur eine polnische Übersetzung denkbar:

(8) Mein Freund schreibt (jetzt gerade) einen Brief

(9) Mój przyjaciel (teraz) pisze list.

Die Verwendung von napisać ist hier ausgeschlossen. Dessen Präsensform ist allerdings wieder im Futur möglich: 
(10) Mein Freund wird einen Brief schreiben.

(11) Mój przyjaciel będzie pisał list.

(12) Mój przyjaciel napisze list.

Ähnlich wie im Präteritum sind aber auch im Futur die beiden Übersetzungsmöglichkeiten des deutschen schreiben semantisch nicht völlig äquivalent. Besonders für die Lernenden des Polnischen ist dies ein besonders schwieriges Problem. Die Präsensform von napisać zeigt, wie praktisch alle perfektiven Verben, Futurbedeutung ${ }^{3}$. Andererseits ist die für imperfektive Verben mit einem Auxiliar (also mit będę usw.) gebildete „echte" Futurform bei diesem Verb nicht möglich. Es muss betont werden, dass diese Art der Interaktion zwischen den Kategorien Tempus und Aspekt selbst innerhalb der slavischen Sprachen keine Selbstverständlichkeit darstellt. Man vgl. dazu etwa das Futur im Slovenischen und den anderen südslavischen Sprachen, wo sowohl die imperfektiven wie auch die perfektiven Verben ein „echtes“ Futur mit Hilfe des Auxiliars bilden - etwa im Slovenischen pisal bom und napisal bom „ich werde schreiben“4.

Bereits diese kurze Skizze einiger Übersetzungs- und Lernerprobleme, die mit dem polnischen Verbalaspekt verknüpft sind, zeigt, dass diese Kategorie mehr Aufmerksamkeit verdient, als ihr in der polnischen Sprachwissenschaft und in den Sprachlehrwerken meist zuteil wird. Selbst wenn man davon ausgeht, dass Muttersprachler des Polnischen die Aspektwahl bei selbst formulierten Texten intuitiv und korrekt treffen, so stellt sich dennoch die Frage, wie diese Wahl bei einem vorgegebenen fremdsprachigen Text und deren Übersetzung in das Polnische getroffen werden soll oder muss. Für den Lernenden des Polnischen oder auch für den Übersetzer eines polnischen Textes in das Deutsche sind insbesondere die Frage der Auswahl oder Wiedergabe von imperfektiven bzw. perfektiven Verben und das Problem der spezifischen Interaktion der jeweiligen Verben mit dem Tempussystem äußerst schwierige Hindernisse.

\section{Der linguistische Status der Aspektkategorie}

Aufgrund der Komplexität der Aspektkategorie des Polnischen stünde zu erwarten, dass in den Grammatiken und Nachschlagewerken - seien diese nun für ausländische Lernende oder für polnische Muttersprachler bestimmt - den

3 Das Präsens perfektiver Verben stellt somit den klassischen Fall eines Non-Past dar (vgl. Löbner 2015: 182).

4 Selbst im dem Polnischen geographisch und sprachgeschichtlich näher stehenden Obersorbisch sind analytisch gebildete Futurformen von perfektiven Verben keine Seltenheit; vgl. dazu Faßke und Michalk (1980): 253. 
aspektuellen Eigenschaften der Verben besondere Aufmerksamkeit gewidmet wird. Leider ist dies häufig nicht der Fall. Besonders auffallend sind die teils weder für einen Polen noch für einen deutschen Lernenden nachvollziehbaren, manchmal sogar widersprüchlichen Aussagen, die sich bei der Darstellung des Aspekts in entsprechenden Werken finden. So trifft man etwa im Kapitel „Odmiana czasowników“ (also „Flexion der Verben“) bei Wróbel (2001: 137) folgende Aussage:

Kategoria aspektu obejmuje wszystkie polskie czasowniki i dzieli je na dwa wielkie zbiory - czasowniki niedokonane i dokonane. Ma więc charakter słownikowy $[\ldots] .^{5}$

Selbst für einen sprachwissenschaftlich nur durchschnittlich gebildeten Leser stellt sich sofort die Frage, warum der Aspekt, dem an dieser Stelle „lexikalischer Charakter" zugeschrieben wird, im Kapitel zur Flexion des Verbs auftaucht. Eine gewisse Erklärung für diese zunächst widersprüchlich erscheinende Aussage findet man am Ende des entsprechenden Abschnitts, wenn der Aspekt als „klassifizierende“ Kategorie bezeichnet wird (vgl. Wróbel 2001: 138), eine Auffassung, die in der gegenwärtigen Polonistik sehr verbreitet ist (vgl. z.B. auch Laskowski in Urbańczyk \& Kucała 1999: 26-27, Gruszczyński \& Bralczyk 2002: 21, mit gewissen Vorbehalten auch Bańko 2002: 98). Als „klassifizierend“ wird dabei eine grammatische Kategorie bezeichnet, die eine unveränderliche Eigenschaft eines Lexems darstellt. Als typisches Beispiel kann das Genus der Substantive gelten. Das polnische Substantiv dom „Haus“ ist ein Maskulinum, während książka „Buch" ein Feminum oder okno „Fenster" ein Neutrum darstellt. Das Genus ist ein unveränderlicher Bestandteil eines jeden Substantivs. Die Kategorie begründet kein eigenes Flexionsparadigma. In dieser Auffassung stellt demzufolge pisać „schreiben“ ein Lexem dar, das in allen seinen Formen imperfektiv ist, napisać „,schreiben“ wäre dann ein zweites Lexem, das in all seinen Formen perfektiv ist. Die beiden Lexeme wären dann weitgehend, aber nicht vollständig synonym. In diesem Falle müsste man allerdings von einer ungeheuren Ausweitung des Phänomens der Synonymie im Polnischen sprechen, da wohl die Mehrheit der Verben eine perfektive Form und eine imperfektive Form aufweist. Es ergäbe sich daraus eine weite Teile des polnischen Verbalsystems umfassende, systematische Synonymie. Selbst Linguisten, die die Auffassung von der klassifizierenden Kategorie vertreten, können nicht leugnen, dass sich im Polnischen sehr viele Verben zu Paaren ordnen lassen, die sich semantisch sehr nahe stehen, aber verschiedenen Subaspekten angehören:

5 Die Kategorie des Aspekts umfasst sämtliche polnischen Verben und teilt sie in zwei große Ansammlungen - die imperfektiven und die perfektiven Verben. Sie hat folglich lexikalischen Charakter [...]. (Deutsche Übersetzung - N.N.) 
czasowniki teliczne tworzą pary aspektowe, złożone z dwu czasowników o identycznym znaczeniu leksykalnym, ale różniących się a[spektem] (Laskowski in Urbańczyk \& Kucała 1999: 27).

Es soll hier dahingestellt bleiben, ob das Auftreten lexikalisch identischer Aspektpaare auf telische Verben eingeschränkt werden kann ${ }^{6}$. Grundlegend ist aber die Feststellung, dass es lexikalisch identische Paare gibt. Zwei Formen mit identischer lexikalischer Semantik müssen aber demselben Lexem zugerechnet werden: „Different forms of the same lexeme will generally, though not necessarily, differ in meaning: they will share the same lexical meaning, but differ in respect of their grammatical meaning" (Lyons 1995: 52).

Für das Paar pisać (ip) „schreiben“ versus napisać „schreiben“ oder auch für das Paar podpisać (pf) versus podpisywać (ip) „unterschreiben“ trifft sicherlich $\mathrm{zu}$, dass die beiden Verben lexikalisch identisch sind. Daher entspricht beiden polnischen Verben nur ein deutsches Äquivalent. Die von Lyons zitierte Eingrenzung des Lexembegriffes trifft bei Aspektpaaren also zu: Sie unterscheiden sich nicht in der lexikalischen Semantik, wohl aber in der Semantik der (grammatischen) Aspektkategorie. Wenn die grammatische Semantik ohne Veränderung der lexikalischen Semantik veränderlich ist, dann bleibt aber nur die Schlussfolgerung, dass der Aspekt keine „klassifizierende“ Kategorie ist, sondern zu den flektivischen Kategorien gezählt werden muss. Aus sprachwissenschaftlichen Gesichtspunkten spricht somit alles gegen den klassifizierenden Charakter der Aspektopposition. Auch der Gegensatz zwischen den unbestritten grammatischen Formen der 1. und 2. Person wie pisze und piszesz, czytam und czytasz, mówię und mówisz usw. lässt sich als eine systematische Synonymie auffassen. Die Formen der 1. und der 2. Person des Singulars sind semantisch nicht identisch. Sie haben aber einen Teil ihrer Bedeutung gemeinsam. Auch diese Gegebenheiten kann man als eine systematische partielle Synonymie auffassen. Eine solche systematische Synonymie ist daher ein deutliches Indiz für den flektivischen Charakter der Kategorie. Wird der Aspekt als flektivische Kategorie eingestuft, so bilden die Glieder eines Aspektpaares Formen ein und desselben Lexems. Dieser Auffassung ist auch Nagórko, wenn sie für das Polnische fünf Tempora konstatiert (Nagórko 2010: 144, weitere Argumente für den flektivischen Charakter bei Nübler 2016: 165-169). Fremdsprachendidaktisch

6 Man wird Laskowski wohl weitgehend zustimmen können, wenn man Telizität wie bei Pafel und Reich (2016: 85) eingrenzt: „Ein Ereignis heißt telisch, wenn seine (vollständige) Realisierung notwendig zu einer Zustandsveränderung führt, ansonsten heißt es atelisch. "Wie oben schon gezeigt wurde, ist die „vollständige Realisierung“ bei Verben des imperfektiven Subaspekts allerdings problematisch. Zudem weist Löbner (2015: 161) darauf hin, dass auch bei vollständiger Realisierung (telische) Accomplishment-Verben - je nach syntaktischem Kontext - auch atelische Prozessbedeutung annehmen können. Der Begriff „telisch“ scheint daher nicht ausreichend geklärt. 
gesehen wäre es also sinnvoll und linguistisch gut begründet, beide Infinitive des Lexems gleichzeitig zu erlernen.

Dies stößt aber auf eine ganze Reihe von Vermittlungsproblemen. Im Gegensatz zu anderen Kategorien des Verbs finden bei der Aspektänderung nämlich Verfahren Anwendung, die üblicherweise in der Wortbildung verortet werden, in erster Linie Präfigierung und Suffigierung. Dies bedeutet, dass neben den parallelen Reihen der finiten Formen im Rahmen des Lexems auch zwei Infinitive vorhanden sind. Lernende des Polnischen sind in der Regel nicht daran gewöhnt, dass ein Lexem über zwei Infinitive verfügen kann. Dies gilt auch für Muttersprachler des Polnischen. Insgesamt ist die traditionelle polnische Grammatik auf dem Prinzip aufgebaut, dass ein verbales Lexem einem Infinitiv entspricht. Man verdeutliche sich etwa, dass in der Regel für das Polnische drei Tempora genannt werden (vgl. z. B. Skibicki 2007: 323 oder Wróbel 2001: 139 u.a.). Betrachtet man aber die Glieder eines Aspektpaares als Formen eines einzigen Lexems ergeben sich auf diese Art fünf Tempora (imperfektives Präteritum, perfektives Präteritum, Präsens, imperfektives Futur und perfektives Futur - vgl. Nagórko 2010: 144). Auch hier müsste als Konsequenz eine Veränderung der Vermittlungsverfahren in Betracht gezogen werden.

\section{Aspektpaare}

Als weiteres Problem kommt hinzu, dass es der Sprachwissenschaft bis heute nicht gelungen ist, eine allgemein akzeptierte und didaktisch gut anwendbare Erklärung für den semantischen Gehalt des Verbalaspekts im Polnischen (und anderen slavischen Sprachen) zu präsentieren. Dies wäre jedoch umso nötiger, als - im Gegensatz zu grammatischen Kategorien wie Person, Numerus u. a. - der Aspekt den Lernern des Polnischen aus ihrer Herkunftssprache meist nicht bekannt ist. Deswegen beschränken sich Grammatiken und sprachliche Nachschlagewerke für Lernende auf mehr oder minder detaillierte Beschreibungen der Aspektverhältnisse.

Bei Skibicki (2007: 285) findet sich zunächst der Hinweis, dass im Polnischen eine große Zahl von Verben in beiden Subkategorien der Aspektopposition vorkommen. Als Beispiel wird u.a. rzucić (pf): rzuczać (ip) „werfen“ benannt”. Weitere Verben kommen nur in einer der beiden Subkategorien vor, entweder in der imperfektiven - man spricht dann von Imperfektiva tantum wie mieć (ip) „haben“ - oder nur in der perfektiven Subkategorie - in letzterem Fall spricht man von Perfektiva tantum wie oniemieć (pf) „verstummen“. Als dritte Gruppe

7 Hier und im Weiteren werden die Abkürzungen ip = imperfektiv und pf = perfektiv zur Kennzeichnung der aspektuellen Subkategorie des jeweiligen polnischen Verbs verwendet. 
werden schließlich aspektuell indifferente Verben benannt - als Beispiel fungiert u.a. mianować (ip + pf) „ernennen“8. Als vierte Gruppe, evtl. als besondere Untergruppe der in beiden aspektuellen Subkategorien vorkommenden Verben, wären die erst auf der folgenden Seite (Skibicki 2007: 286) benannten suppletiven Aspektpartner des Typs brać (ip) : wziaćc (pf) „nehmen“ aufzuführen.

Bleibt man auf der Ebene der Beschreibung der Aspektverhältnisse im Polnischen, so lässt sich zunächst konstatieren, dass die weit überwiegende Anzahl der Verben Formen in beiden Subkategorien der Aspektopposition aufweist. Man spricht hier in der Regel von Aspektpaaren. Diese Formen, die - wie oben gezeigt - als Repräsentanten eines einzigen Lexems aufgefasst werden müssen, können voneinander abgeleitet sein (podpisać (pf) $\rightarrow$ podpisywać (ip) „unterschreiben“), keinen formalen Zusammenhang aufweisen (brać (ip) : wziaćc (pf) „nehmen“) oder homonyme Formen für beide Aspekte haben ( ${ }^{1}$ mianować (ip) : ${ }^{2}$ mianować (pf) „ernennen“). Eine vergleichsweise kleinere Gruppe von Verben kommt nur in jeweils einer der beiden Subkategorien vor.

Allerdings trägt eine weitere Differenzierung zu den Schwierigkeiten der Darstellung bei. Skibicki zählt nämlich zu der Gruppe der Aspektpaare - neben den formal nicht differenzierten Verben und den wenigen suppletiven Paaren - ausschließlich die durch Suffigierung gebildeten Paare des Typs rzucić (pf) $\rightarrow$ rzuczać (ip) „werfen“ oder podpisać (pf) $\rightarrow$ podpisywać (ip) „unterschreiben“. Durch Präfigierung gebildete Paare des Typs pisać (ip) $\rightarrow$ napisać (pf) „schreiben“ oder robić (ip) „machen, tun“ $\rightarrow$ zrobić (pf) „machen, tun“ lehnt sie explizit ab:

$\mathrm{Zu}$ den imperfektiven Simplizia gibt es keine bedeutungsgleichen Aspektpartner, denn die durch Präfigierung gebildeten perfektiven Formen weisen z.T. beträchtliche Bedeutungsunterschiede auf [...]. (Skibicki 2007: 287)

Man wird hier wohl insofern zustimmen können, als die durch Präfigierung gebildeten Verben in der Mehrzahl aller Fälle neue Lexeme darstellen - also etwa das von pisać (ip) „schreiben“ derivierte podpisać (pf) „unterschreiben“. Für den deutschsprachigen Lerner des Polnischen ist allerdings nicht einsichtig, warum der Unterschied zwischen dem perfektiven podpisać (pf) „unterschreiben“ und dem genau gleich zu übersetzenden imperfektiven podpisywać (ip) „unterschreiben" anders zu bewerten sein soll als der Unterschied zwischen dem perfektiven napisać (pf) „schreiben“ und dem ebenfalls gleich zu übersetzenden

8 Schon bei Skibicki lässt sich auch eine in weiten Bereichen der Aspektforschung zu beobachtende terminologische Verwirrung beobachten. Skibicki bezeichnet Verben des Typs rzucić (pf) : rzuczać (ip) „werfen“, die also formal differenzierte Formen in beiden Subkategorien aufweisen, als „biaspektuell“. In der Literatur zur Aspektforschung wird dieser Terminus allerdings auf Verben des Typs mianować (ip + pf) „ernennen“ angewandt. Man vgl. z.B. Laskowski in Urbańczyk und Kucała (1999: 27) oder Saloni (2002: 29) - jeweils in der polnischen Form czasownik dwuaspektowy. 
imperfektiven pisać (ip) „schreiben“. Dabei ist es nicht hilfreich, dass der von Skibicki (2007: 287) konstruierte Bedeutungsunterschied beim Paar pisać (ip) : napisać (pf) inakzeptabel ist. Eine deutsche Übersetzung des präfigierten napisać (pf) mit „aufschreiben“ ist nicht möglich. Würde man den obigen Beispielsatz (3), der hier als (13) wiederholt wird, entsprechend übersetzen, ergäbe sich eine fehlerhafte deutsche Konstruktion:

(13) Mój przyjaciel napisał list.

(14) ${ }^{\star}$ Mein Freund schrieb einen Brief auf.

Dem deutschen aufschreiben entspricht im Polnischen am ehesten das Aspektpaar zapisać (pf): zapisywać (ip), man vgl.:

(15) Jemandes Telefonnummer aufschreiben.

(16) Zapisać/zapisywać czyjś numer telefonu.

Tatsächlich unterscheiden sich durch Präfigierung gebildete Aspektpaare funktional nicht von den durch Suffigierung gebildeten Paaren. Das Verhältnis von pisać (ip) zu napisać (pf) entspricht genau dem Verhältnis von podpisywać (ip) zu podpisać (pf). Der einzige Grund, durch Präfigierung gebildete Aspektpaare aus der grammatischen Korrelation auszuschließen, ist offenbar die bei der Präfigierung weitaus häufiger als bei der Suffigierung auftretende Veränderung der lexikalischen Bedeutung. Von einem Simplex wie pisać (ip) können zahlreiche Präfixbildungen abgeleitet werden: dopisać (pf) „hinzuschreiben“, napisać (pf) „schreiben“, odpisać (pf) „abschreiben“, opisać (pf) „beschreiben“, podpisać (pf) „unterschreiben“, zapisać (pf) „aufschreiben, notieren“ u.a. Nur eine dieser Präfixbildungen - nämlich napisać - fungiert im Rahmen eines Aspektpaares, also innerhalb eines Lexems, als perfektiver Partner zum imperfektiven pisać. Alle anderen Präfixbildungen weisen lexikalische Veränderungen im Vergleich zum Imperfektivum auf und konstituieren dadurch je ein neues Lexem. Die Tatsache allein, dass das Verfahren überwiegend lexikalische Funktionen besitzt, ist jedoch kein Grund, durch Präfigierung gebildete Aspektpaare aus den Aspektkorrelationen auszuschließen. Deshalb führt auch z.B. Saloni (2002: 29) durch Präfigierung gebildete Paare ebenso wie durch Suffigierung gebildete Paare auf. An dieser Stelle sei zudem darauf hingewiesen, dass auch die Suffigierung lexikalische Funktion haben kann - man vgl. z. B. poboleć (pf) „eine Zeit lang schmerzen“ $\rightarrow$ pobolewać (ip) „ab und zu ein wenig schmerzen“, popłakać (pf) „ein Weilchen weinen“ $\rightarrow$ popłakiwać (ip) „ab und zu ein wenig weinen“. Allerdings ist die lexikalische Funktion der Suffigierung seltener als die lexikalische Funktion der Präfigierung.

Man muss also im Hinblick auf die Aspektkategorie des Polnischen die eingefahrenen Denkschemata der traditionellen Grammatik verlassen, was für jeden 
Sprachvermittler ebenso wie für die Lernenden eine Herausforderung darstellt. In der europäischen Grammatologie besitzt ein flektierbares Lexem eine Grundoder Nennform, die für alle bildbaren Formen steht. Bei Substantiven ist das immer der Nominativ des Singulars (bei Pluralia tantum des Plurals), bei Adjektiven die maskuline Form des Nominativs Singular und bei Verben eben der Infinitiv. Erkennt man den Aspekt aber als flektivische Kategorie an, so besitzt die Mehrzahl der verbalen Lexem zwei Infinitive. Lediglich bei Imperfektiva tantum und Perfektiva tantum ist nur ein Infinitiv vorhanden. Man sollte sich deshalb darüber im Klaren sein, dass der Infinitiv eben nur eine spezifische Verbform mit charakteristischen Funktionen ist. Er muss nicht vorhanden sein, wie das moderne Bulgarisch oder Makedonisch zeigt. Dort fungiert die 1.Person des Singulars als Nenn- und Wörterbuchform. Dementsprechend kann umgekehrt auch die Existenz mehrerer Infinitive kein Argument gegen deren Zugehörigkeit zu einem einzigen Lexem sein. Dies gilt analog auch für die weiteren Konjugationsparadigmata, zumal diese sich semantisch ergänzen. Wie bereits erwähnt, erwecken die Grammatiken des Polnischen in der Regel den Eindruck, es gäbe nur ein Präteritum und nur ein Futur. Tatsächlich sind aber das imperfektive und das perfektive Präteritum bzw. das imperfektive und das perfektive Futur semantisch und funktional zu unterscheiden. Andernfalls müssten die entsprechenden Formen in allen Kontexten austauschbar sein, was sie nicht sind:

(17) Już pisze list i dzisiaj go jeszcze napisze.

(18) ${ }^{*} J u z ̇$ pisze list i dzisiaj go jeszcze będzie pisał.

(19) Codziennie pisał list.

(20) ${ }^{\star}$ Codziennie napisał list.

Gerade in der Ablehnung der Präfigierung als eines rein aspektändernden Verfahrens zeigt sich in den Ausführungen Skibickis das Problem einer Vermischung von (grammatischer) Aspektsemantik und (lexikalischer) Wortsemantik. Akzeptiert man die Voraussetzung, dass der Verbalaspekt eine flektivischgrammatische Kategorie des polnischen Verbs darstellt, so muss die lexikalische Semantik des Lexems von der grammatischen Bedeutung des Aspekts streng getrennt werden. Dieses Unterfangen soll im Folgenden ansatzweise demonstriert werden. Dabei beschränken sich die folgenden Ausführungen auf die Mechanismen der Aspektänderung. Das Problem einer eventuellen lexikalischen Veränderung muss hier schon aus Platzgründen weitgehend ausgespart bleiben. 


\section{Die Mechanismen der Aspektänderung}

Für jeden Lerner des Polnischen ist es im Hinblick auf die Aspektkategorie notwendig, das Funktionieren des Systems zu begreifen. Um dieses zu erfassen, scheint es zunächst nicht vordergründig sinnvoll, über konkrete Aspektpaare zu sprechen. Vielmehr müssen die relevanten Einheiten im morphologischen System des Polnischen bekannt sein. Den Ausgangspunkt kann dabei die bekannte Tatsache bilden, dass der weit überwiegende Teil der polnischen Simplex-Verben zum imperfektiven Subaspekt gehört ${ }^{9}$. Als Beispiele mögen nur einige wenige Verben benannt werden: bić (ip) „schlagen“, czytać (ip) „lesen“, grać (ip) „spielen“, pisać (ip) „schreiben“, sypać (ip) „schütten“. Tritt an ein solches Simplex-Verb ein eigensprachliches Präfix, so ändert sich zunächst einmal der Aspekt. Das aus der Präfigierung resultierende Verb ist perfektiv. Es sei hier nochmals betont, dass diese Aspektänderung noch keinerlei Aussage zur lexikalischen Semantik des präfigierten Verbs beinhaltet, also auch nichts über Aspektpaare verrät. Für die benannten fünf polnischen Verben lässt sich also feststellen: bić (ip) „schlagen“ $\rightarrow$ rozbić (pf) „zerschlagen“, czytać (ip) „lesen“ $\rightarrow$ doczytać (pf) „zu Ende lesen“, grać (ip) „spielen“ $\rightarrow$ wygrać (pf) „gewinnen“, pisać (ip) „schreiben“ $\rightarrow$ zapisać (pf) „aufschreiben, notieren“, sypać (ip) „schütten“ $\rightarrow$ przesypać „um-, überschütten“. Das Präfix ändert in all diesen Fällen die aspektuelle Subkategorie von imperfektiv zu perfektiv.

Aus didaktischen Gründen ist es daher bereits recht früh sinnvoll, die aspektrelevanten Präfixe in den Polnischunterricht einzuführen. Nicht jedes beliebige verbale Präfix ändert den Aspekt des Basisverbs - man vergleiche Fälle wie montować (ip) „montieren“ $\rightarrow$ demontować (ip) „demontieren“ oder cytować (ip) „zitieren“ $\rightarrow$ recytować (ip) „rezitieren“. Bei den aspektrelevanten Präfixen handelt es sich um eigensprachliche, aus dem Urslavischen ererbte Präfixe. Im heutigen Polnische sind dies (mit einem jeweiligen Beispiel): do- (dopisać (pf) „zu Ende schreiben; hinzuschreiben“), na- (napetnić (pf) „auffüllen“) nad-/nade(nadgryźć (pf) „anbeißen, annagen“, naderwać (pf) „an- einreißen“), o- (okraść (pf) „bestehlen“), ob-/obe- (obmalować (pf) „bemalen“, obełgać (pf) „belügen“), od-/ode- (odwalić (pf) „wegwälzen“, odegrać (pf) „,abspielen“), po- (pobudzić (pf) „hervorrufen“), pod-Ipode- (podpisać (pf) „unterschreiben“, podeprzeć (pf) „stützen“), prze- (przemienić (pf) „verwandeln“), przy- (przylepić (pf) „ankleben“), roz-/roze- (rozbić (pf) „zerschlagen“, rozesłać (pf) „versenden“), $u$ (umówić (pf) „verabreden“), w-/we- (wkroplić (pf) „einträufeln“, wejść (pf) „hineingehen“), wz-/ws-/wez-/wes- (wzniecić (pf) „,anfachen (Feuer)“, wskazać (pf) „zeigen, hinweisen“, wezbrać (pf) „steigen, anschwellen“, wesprzeć (pf)

9 Der Begriff „Simplex“ bzw. „Simplex-Verb“ ist nicht einfach einzugrenzen. Er wird hier im Sinne von Verben verwendet, die nicht deverbal deriviert sind. 
„unterstützen“), wy- (wybrudzić (pf) „,beschmutzen“), z-/ze-/s-/ś- (zgryźć (pf) „zerbeißen“, zerwać (pf) „herunter-, zerreißen“, spisać (pf) „,zusammenschreiben“, ściemnić (pf) „dunkler werden“), za- (zatopić (pf) „versenken“).

Nur eine sehr begrenzte Anzahl von Simplizia gehört zum perfektiven Subaspekt. Es handelt sich im Wesentlichen um die folgenden Verben: dać (pf) „geben“, darować (pf) „schenken“, chwycić (pf) „(er)greifen“, jać (pf) „(alt, buchspr.) anfangen“, kupić (pf) „kaufen“, lec (pf) „(buchspr.) sich (hin)legen“, paść (pf) „fallen“, puścić (pf) „loslassen, fallen lassen“, ruszyć (pf) „rühren, bewegen“, $r z e c$ (pf) „,(buchspr.) sagen“, rzucić (pf) „werfen“, siąść (pf) „,sich setzen“, skoczyć (pf) „springen“, stawić się (pf) „sich melden (zum Dienst)“, stąicić (pf) „schreiten“, trącić (pf) „leicht stoßen“, trącić się (pf) „anstoßen (mit Gläsern)“, wrócić (pf) „zurückkehren“. Unter didaktischen Gesichtspunkten ist diese relativ kleine Gruppe von perfektiven Simplizia problemlos als „Ausnahme“ zu vermitteln. Auch deren präfixale Ableitungen gehören selbstverständlich dem perfektiven Subaspekt an: dać (pf) „geben“ $\rightarrow z d a c$, „übergeben, übertragen“, kupić (pf) „kaufen“ $\rightarrow$ nakupić (pf) „einkaufen“ usw. Unter linguistischen Gesichtspunkten bleibt noch zu klären, aus welchen Gründen die unpräfigierten Verben zum perfektiven Subaspekt gehören ${ }^{10}$.

Neben diesen wenigen perfektiven Simplizia sind alle präfigierten Verben im Polnischen perfektiv. Perfektive Verben können mit Hilfe eines eigensprachlichen Suffixes wiederum imperfektiviert werden: rozbić (pf) „zerschlagen“ $\rightarrow$ rozbijać (ip) „zerschlagen“, doczytać (pf) $\rightarrow$ doczytywać (ip) „zu Ende lesen“, wygrać (pf) „gewinnen“ $\rightarrow$ wygrywać (ip) „gewinnen“, zapisać „aufschreiben, notieren“ $\rightarrow$ zapisywać „aufschreiben, notieren“, przesypać (pf) „um-, überschütten“ $\rightarrow$ przesypywać „um-, überschütten“. Auch für die überwiegende Mehrzahl der nichtpräfigierten Perfektiva gibt es die Möglichkeit, sie durch Suffigierung zu imperfektivieren: dać (pf) „geben“ $\rightarrow$ dawać (ip) „geben“, kupić (pf) „kaufen“ $\rightarrow$ kupować (ip) „kaufen“ usw. Die Anzahl der für die Imperfektivierung zur Verfügung stehenden Suffixe ist weitaus geringer als die Anzahl der Präfixe. Im heutigen Polnisch findet man folgende Imperfektivierungssuffixe: -ywa-I-iwa- (pokazać (pf) $\rightarrow$ pokazywać (ip) „zeigen“, zapukać (pf) $\rightarrow$ zapukiwać (ip) „anklopfen“), -owa- (nakupić (pf) $\rightarrow$ nakupować (ip) „einkaufen“), -wa$($ zakryć $(\mathrm{pf}) \rightarrow$ zakrywać (ip) ,verdecken“), -a-l-ja -(zaznaczyć (pf) $\rightarrow$ zaznaczać (ip) „markieren“, rozbić (pf) $\rightarrow$ rozbijać (ip) „zerschlagen“). Auch diese Suffixe sollte ein Lerner des Polnischen bereits recht früh kennenlernen.

Eine gewisse Ausnahmestellung besitzt im modernen Polnisch das Suffix $n a-$, das in der Präsenskonjugation nur durch $-n$ - vertreten ist. Grundsätzlich lassen sich Verben mit diesem Suffix feststellen, die dem imperfektiven Aspekt

10 Die Gründe sind zweifellos in der Semantik dieser Verben zu suchen. Es gibt keinen formalmorphologischen Grund, warum diese Simplizia nicht imperfektiv sein sollten. 
angehören: cieknaćc (ip) „fließen“, moknąć „,nass werden“, więdną́ „(ver)welken“ u. a. Fast alle diese imperfektiven Verben mit dem Suffix -na- stellen jedoch Simplizia dar. Sie sind nicht deverbal deriviert. Eine Ausnahme bilden unter den imperfektiven Verben mit dem Suffix - ną- lediglich einige wenige determinierte Bewegungsverben, die als Derivate der entsprechenden indeterminierten Verben interpretiert werden können. Konkret handelt es sich um das determinierte biegnąć (ip) „laufen“ im Gegensatz zum indeterminierten biegać (ip) „laufen“, determiniertes pelznąc (ip) „kriechen“ im Gegensatz zum indeterminierten pelzać (ip) „kriechen“ und determiniertes płynać (ip) „schwimmen; mit dem Schiff fahren“ im Gegensatz zum indeterminierten pływać (ip) „schwimmen; mit dem Schiff fahren“, evtl. auch indeterminiertes sunać (ip) „schieben“ zu indeterminiertem suwać (ip) „schieben“ und determiniertes ciagnać (ip) „ziehen, schleppen“ zu indeterminiertem ciagać (ip) „ziehen, schleppen“. Der in der polnischen Grammatik kaum beachtete Determiniertheitsgegensatz (auch unter der Bezeichnung „Verben der Bewegung“ subsumiert) darf nicht mit der Aspektopposition verwechselt werden. Beide Glieder eines Determiniertheitspaares gehören zum imperfektiven Subaspekt ${ }^{11}$. Es sei hier lediglich darauf hingewiesen, dass im Rahmen der Aspektänderung eine Reihe von Besonderheiten auftreten: Insbesondere findet sich bei Verben, die dem Determiniertheitsgegensatz unterliegen, häufig die Wurzelsuppletion im Aspektparadigma ${ }^{12}$.

Die überwiegende Mehrheit der mit -nq- suffigierten Verben gehört jedoch zum perfektiven Subaspekt. In diesen Fällen handelt es sich um deverbale Ableitungen: klapnać (pf) „(einmal) klappern“ $\leftarrow$ klapać (ip) „klappern“, puknać (pf) „(einmal) klopfen“ $\leftarrow$ pukać (ip) „klopfen“, krzyknać (pf) „(kurz) aufschreien“ $\leftarrow$ krzyczeć (ip) „schreien“. Diese Art der Ableitung und Perfektivierung ist im modernen Polnisch durchaus produktiv.

11 Zum Determiniertheitsgegensatz im Polnischen vgl. Włodarczyk (1980).

12 So wird meist das determinierte Bewegungsverb präfigiert und damit perfektiviert: $i s ́ c ́(i p) \rightarrow$ wejść (pf) „hineingehen“. Die Imperfektivierung erfolgt jedoch häufig nicht durch Suffigierung, sondern durch die Suppletion der determinierten Wurzel durch die indeterminierte Wurzel: wejść (pf) $\rightarrow$ wchodzić (ip) „hineingehen“. Wie die Zugehörigkeit zum imperfektiven Subaspekt zeigt, handelt es sich bei wchodzić nicht um eine Präfigierung des indeterminierten chodzić (ip) „gehen“, sondern um das Resultat des Imperfektivierungsprozesses von wejść. Präfigierungen von indeterminierten Bewegungsverben sind selten. Sie resultieren aber ebenfalls immer in einem perfektiven Verb: chodzić (ip) $\rightarrow$ pochodzić (pf) „eine Weile (umher)gehen“. 


\section{Resümee}

Durch die beiden gegenläufigen Verfahren, also durch die Perfektivierung durch ein Präfix oder durch das Suffix -nq- und durch die Imperfektivierung durch eines der genannten Suffixe ergibt sich das Grundmuster der Aspektänderung im Polnischen: imperfektives Simplex $\rightarrow$ perfektive Präfixbildung (ggf. perfektives Verb mit dem Suffix $-n a-) \rightarrow$ imperfektive Suffixbildung. Vor dem Hintergrund dieses Schemas können die „Ausnahmen“ identifiziert werden, die jeweils ganz bestimmte Gruppen von Verben betreffen (also etwa die oben benannten perfektiven Simplizia oder die biaspektuellen Verben des Typs mianować). Auf dem Hintergrund dieses Grundschemas kann auch die Eingrenzung von Aspektpaaren gelingen. Aspektpaare entstehen durch die „reine“ Aspektänderung, also ohne Veränderung oder Modifizierung der lexikalischen Semantik. Dabei ist aber nicht zu übersehen, dass im Bereich der Semantik des Aspektgegensatzes noch linguistische Forschung erforderlich ist, um die eingangs zitierten „Unschärfen" in den Übersetzungsmöglichkeiten zu erklären. Selbstverständlich muss das angedeutete Grundschema im weiteren Lernprozess verfeinert werden. Darzustellen bleiben insbesondere Mehrfachpräfigierungen (po-z-bawić (pf) „(nach und nach) wegnehmen“) oder andere Kombinationen von Affixen. Eine besondere Lehreinheit erfordert der oben nur kurz skizzierte Determiniertheitsgegensatz, bei dem auch die formalen Besonderheiten in der Aspektänderung behandelt werden sollten. Ungeachtet dessen können Lerner des Polnischen auf dem Hintergrund der dargestellten Aspektänderungsmechanismen in der weit überwiegenden Zahl der Fälle die Zugehörigkeit eines bisher nicht bekannten Verbs zum imperfektiven oder zum perfektiven Subaspekt erkennen, was bereits einen wichtigen Schritt in der Fremdsprachenkompetenz bedeutet.

\section{Literaturverzeichnis}

Bańko, Mirosław (2002). Wykłady z polskiej fleksji. Warszawa: Wydawnictwo Naukowe PWN.

Faßke, Helmut/Michalk, Siegfried (1980). Grammatik der obersorbischen Schriftsprache der Gegenwart. Morphologie. Bautzen: Domowina.

Gruszczyński, Włodzimierz/Bralczyk, Jerzy (Hrsg.) (2002). Słownik gramatyki języka polskiego. Warszawa: WSiP.

Löbner, Sebastian (2015). Semantik. Eine Einführung. (2., aktualisierte und stark erweiterte Auflage). Berlin/Boston: De Gruyter.

Lyons, John (1995). Linguistic semantics. An introduction. Cambridge: Cambridge University Press.

Madelska, Liliana (2007). Hurra!!! Polnisch entdecken. Eine Lerngrammatik. Kraków: Prolog Publishing. 
Nagórko, Alicja (2010). Podręczna gramatyka języka polskiego. Warszawa: Wydawnictwo Naukowe PWN.

Nübler, Norbert (2016). Zur Morphologie und Semantik des Aspekts im Polnischen. Anzeiger für Slavische Philologie, XLIII, 157-173.

Pafel, Jürgen/Reich, Ingo (2016). Einführung in die Semantik. Grundlagen - Analysen Theorien. Stuttgart: J.B. Metzler.

Saloni, Zygmunt (2002). Czasownik polski. Odmiana. Słownik. (Wydanie II zmienione). Warszawa: Wiedza Powszechna.

Skibicki, Monika (2007). Polnische Grammatik. Hamburg: Buske Helmut Verlag.

Urbańczyk, Stanisław/Kucała, Marian (Hrsg.) (1999): Encyklopedia języka polskiego. Wydanie trzecie poprawione i uzupełnione. Wrocław/Warszawa/Kraków: Ossolineum.

Włodarczyk, Hélène (1980). Czasowniki ruchu a kategoria aspektu w języku polskim. Polonica, VI, 49-56.

Wróbel, Henryk (2001). Gramatyka języka polskiego. Podręcznik akademicki. Kraków: Spółka Wydawnicza „Od Nowa“. 
Open-Access-Publikation im Sinne der CC-Lizenz BY-NC-ND 4.0

(C) 2020, Vandenhoeck \& Ruprecht GmbH \& Co. KG, Göttingen ISBN Print: 9783847111863 - ISBN E-Lib: 9783737011860 


\title{
Magdalena Jurewicz / Martyna Gabrych / Justyna Sobańska (Uniwersytet im. Adama Mickiewicza w Poznaniu)
}

\section{Sprachliches Weltbild in ausgewählten gegenwärtigen polnischen und deutschen parallelen Gebrauchshypertexten}

\begin{abstract}
Selected differences and similarities in the linguistic picture of the world in Poland and Germany: A corpus linguistics study

This chapter presents selected differences and similarities between the linguistic picture of the world in Poland and Germany in the first and second decade of the 21st century, based on data coming from a corpus of hypertexts. The dataset includes advertisements on dating sites and obituaries. These parallel texts were analyzed by students of applied linguistics at Adam Mickiewicz University in their bachelor seminars in 2016 and 2018. The analysis of the texts allowed conclusions to be drawn about the perceived differences in verbalization, e.g. with regard to the expectations of the partner in ads on dating sites, or in visual aspects which supplements the text in obituaries.
\end{abstract}

Keywords

hypertext, linguistic picture of the world, parallel text, German, Polish

\section{Einleitung}

Der Artikel untersucht ausgewählte Unterschiede und Gemeinsamkeiten im sprachlichen Weltbild ${ }^{1}$ in Polen und Deutschland im ersten und zweiten Jahrzehnt des 21. Jahrhunderts, die anhand eines Korpus von Gebrauchstexten veranschaulicht werden. $\mathrm{Zu}$ dem Korpus der analysierten Texte gehören Anzeigen auf Partnerbörsen und Todesanzeigen. Die Analyse der Texte erlaubte es, Rückschlüsse auf die wahrgenommenen Unterschiede in der Verbalisierung zu ziehen, z. B. in Bezug auf Erwartungen an den Partner in Anzeigen auf Partnerbörsen oder die visuelle Sphäre, die den Text in den Todesanzeigen vervollständigt. Eine solche Analyse erweist ein großes didaktisches Potenzial, denn authentische Texte, die online zugänglich sind, sind für die meisten Studierenden

1 Der Begriff wird hier nach Bartmiński und Tokarski (1986) und Grzegorczykowa (1999) verstanden. 
interessant, was ihre Motivation zum Selbststudium steigert. Und eben die Relevanz der Autonomie der Lernenden im Fremdsprachenunterricht hat Frau Professor Izabela Prokop unterstrichen, die die Rolle der Lehrer und Lehrerinnen u. a. im Bereich der Entwicklung der Selbständigkeit der Schüler und Schülerinnen sah (vgl. Prokop 2002 und 2003). Umso mehr ist die Idee der Autonomisierung der Didaktik im akademischen Unterricht zu propagieren, deren Entwicklung hier am Beispiel der selbständig von Studierenden durchgeführten Analysen dargestellt wird. Diese Analysen dienten nicht nur dem Erwerb der Fähigkeit einer Paralleltextanalyse, sondern ermöglichten es den Studierenden auch, Unterschiede und Gemeinsamkeiten in der Verbalisierung des gegenwärtigen Weltbilds, einerseits des deutschen und österreichischen und andererseits des polnischen, selbst festzustellen.

\section{Gebrauchshypertexte}

In ihrem Artikel, in dem sie die Einführung der parallelen Analyse von Gebrauchstexten ${ }^{2}$ im Unterricht mit Studierenden der Angewandten Linguistik postuliert, motiviert Czesława Schatte (2009) dieses Postulat mit der Notwendigkeit, Studierende, die heutzutage selten mit Textmustern konfrontiert werden, dazu zu ermutigen, über die verschiedenen Arten der Formulierung konkreter Inhalte in zwei verschiedenen Sprachen gründlich nachzudenken. Eine solche vertiefte Analyse dient zum einen der Erweiterung der sprachlichen Kompetenz in einer Fremdsprache und zum anderen der Entwicklung einer für die Analyse komplexerer Fachtexte nützlichen Analysemethode.

Dieser wertvolle Kommentar, der mit den Postulaten von Adamzik (2001) polemisiert, die wiederum die Notwendigkeit der Analyse von Gebrauchstexten als stark konventionalisierten Texten negiert, weil solche Texte (ihrer Meinung nach) gewöhnlich auch nicht übersetzt werden, kann insofern ergänzt werden,

2 Als Gebrauchstext wird hier ein Text verstanden, dessen Nutzen im Allgemeinen unmittelbar und kurzfristig ist und der dazu dient, Informationen allgemeiner Art oder in einem bestimmten Bereich zu vermitteln. Die ästhetische Seite eines solchen Textes hat eine sekundäre Bedeutung (vgl. Tomaszkiewicz 2004: 97). Als klare Unterscheidungsmerkmale der Gebrauchstexte von sogenannten literarästhetischen Texten nimmt Stechman die zugrunde liegende Intention der Texte an. Gebrauchstexte dienen im Allgemeinen einem bestimmten Zweck und weisen einen unmittelbaren Bezug zur Realität auf, was von anderen Texten als Intention nicht behauptet werden kann, selbst wenn sich diese Merkmale bei der Rezeption einstellen sollten (vgl. Stechman 2003: 3). Zu den Gebrauchstexten werden zum Beispiel Sachbücher, Lexika, Zeitungen, Zeitschriften, Gebrauchsanweisungen, Bedienungsanleitungen sowie Rundfunk- und Fernsehprogramme gerechnet. Als weitere Möglichkeiten werden zudem Kochrezepte, Anleitungen jeglicher Art, Gesetzestexte, Katalogtexte, Reiseprospekte und Werbeanzeigen aufgeführt (vgl. Stechman 2003: 4). 
dass die parallele ${ }^{3}$ Gegenüberstellung von Gebrauchstexten nicht nur das Erlernen von Sprachstrukturen ermöglicht, sondern Studenten und Studentinnen auch mit einer anderen Auswahl des Inhalts dieser Texte konfrontiert, die sich aus der Fokussierung der jeweiligen Gesellschaft auf bestimmte Themen innerhalb einer bestimmten Textgattung ergibt. Man kann Adamzik (2001) auch nicht zustimmen, dass Gebrauchstexte in der Regel nicht übersetzt werden, denn im sich vereinigenden Europa ist es manchmal notwendig, auch solche Texte $\mathrm{zu}$ übersetzen, die früher im streng konventionellen Rahmen schriftlicher Texte für den internen gesellschaftlichen Gebrauch existierten. Heute liegen solche Texte immer häufiger in Form von Hypertexten ${ }^{4}$ vor, deren allgemeine Verfügbarkeit sie anfällig für häufige Musterwechsel macht, was die Studenten und Studentinnen zusätzlich frustrieren kann, da es unmöglich ist, sich auf ein bestimmtes Muster zu beziehen. Nehmen wir als Beispiel Informationen über die Zubereitung eines bestimmten Gerichts, die heute in Form von Videoblogs, Hypertexten mit Bildern oder in Form von Bildunterschriften erscheinen können (vgl. Hanus \& Kaczmarek 2019).

Ein Fremdsprachennutzer und ein Übersetzer haben also ständig mit solchen Texten zu tun; besonders im Zeitalter der Allgegenwart des Internets und der Gewohnheit, es täglich zu nutzen. Dies macht es notwendig, sich selbst in einer fremden Realität wiederzufinden oder Kunden der Übersetzer näher an eine fremde Kultur heranzuführen.

3 „Unter Paralleltexten sind hierbei verschiedensprachige Texte zu verstehen, die originär in ihrer jeweiligen Sprache - am besten von kompetenten Muttersprachlern - erstellt wurden, die also keine Übersetzungen voneinander sind, aber ein möglichst ähnliches Thema behandeln und sich in ihrer kommunikativen Funktion entsprechen [...]“ (Göpferich 1998: 184).

4 Wenn wir mit einem auf einer Webseite gefundenen Text zu tun haben, arbeiten wir an einem Hypertext. Diese Textsorte charakterisieren laut Burger und Luginbühl (2005: 232) Multimedialität, nicht-lineare Gestaltung und elektronische Möglichkeit der Veröffentlichung. Man kann auch solche Merkmale wie Interaktivität, Assoziativität, Intertextualität und Vielfalt von Rezeptionsweisen nennen (vgl. Fleischer et al. 2001: 511). Hypertexte gehören zu World Wide Web (WWW). Man kann Hypertexte wesentlich schneller als gedruckte Texte benutzen; fast alles lässt sich per Mausklick finden. Nach Maciejewski (2009: 17) ist Hypertext eine interaktive Präsentation, die kohärente Informationseinheiten miteinander verknüpft. Hypertexte können immer weiter entwickelt werden. Die Texte sind dynamisch und werden ständig aktualisiert. Sie besitzen keinen festen Anfang und brauchen nicht vervollständigt/ergänzt zu werden. Der Textempfänger kann die Rolle des Textabsenders übernehmen und den Text weiter verfassen. Der Zugang ist leichter, man braucht weniger Zeit und man muss sich weniger Mühe geben, um ein gewisses Wissen zu gewinnen. Hypertexte sind heutzutage im Internet allgegenwärtig. Sie sind oft mit Bildern, Videomaterialien und Audiodateien vernetzt. „Die Grundidee von Hypertext besteht darin, dass informationelle Einheiten, in denen Objekte und Vorgänge des einschlägigen Weltausschnitts auf textuelle, graphische oder audiovisuelle Weise dargestellt werden, flexibel über Verknüpfungen manipuliert werden können.“ (Fleischer et al. 2001:510). Der Leser fasst selbst den Entschluss, wie tief er in ein Thema eintauchen will. Die Verknüpfungen (links) sind nicht-linear organisiert, die Reihenfolge des Textlesens ist vom Textempfänger abhängig. 
Für einen sprachlich unvorbereiteten Studenten bietet jedoch das Internet aus unterschiedlichen Sprachen übersetzte und nicht immer korrekt lokalisierte Gebrauchstexte an, z.B. Webseiten internationaler Unternehmen (vgl. StarbucksMenü), sowie viele verschiedene Versionen von Textgattung-Mustern (z.B. eine Vorlage eines Wohnungsmietangebotes auf verschiedenen Portalen). Daher postuliere ich im Unterricht mit den Studierenden die Notwendigkeit, jedes Mal die Definition eines Gebrauchstextes zu betonen, der aus einer bestimmten Sprachgemeinschaft stammt (z.B. der deutschsprechenden Gemeinschaft in Deutschland ${ }^{5}$ und der polnischsprechenden Gemeinschaft in Polen), denn nur dann kann man erwarten, dass die Verwendung einer bestimmten Phrase in der direkten Kommunikation nicht den Verfremdungseffekt hervorruft und in der Übersetzung nicht zu Fehlern in der Verwendung der Kollokationen führt. Obwohl die CatTools und andere Werkzeuge, die die Arbeit eines Übersetzers unterstützen, z.B. der Onlinedienst Deepl, eine immer korrektere Wahl der Lexik und der Sprachstrukturen ermöglichen, kann die Unkenntnis ganzer Textmuster dazu führen, dass bestimmte Inhalte, die in einer bestimmten Kultur nicht vorkommen, in einer unreflektierten Übersetzung eingeführt werden, oder umgekehrt, dass bestimmte für eine Gemeinschaft spezifische Inhalte nicht berücksichtigt werden, z. B. bei Wohnungsmietangeboten in Deutschland sind Energiesparparameter einer bestimmten Wohnung angegeben, was in Polen noch nicht in diesem Umfang funktioniert. In Gebrauchstexten spiegelt sich nämlich ganz konkret das sprachliche Weltbild der jeweiligen Gemeinschaft wider.

\section{Sprachliches Weltbild}

Das Konzept eines sprachlichen Weltbildes (linguistic view (picture) of the world, Übersetzung von MJ.), entstand aufgrund der anthropologisch-kulturellen, auf die Gemeinschaft ausgerichteten Sprachwissenschaft, die sich an der Grenze zwischen Ethnologie und Ethnolinguistik bewegt. Man geht davon aus, dass seine Ursprünge direkt aus den Theorien von J. G. Herders und Wilhelm von Humboldts Theorien und der Theorie amerikanischer Ethnolinguisten, Edward Sapir und Benjamin Lee Whorf, die die bekannte Hypothese des Sprachrelativismus aufgestellt haben, abgeleitet sind.

Humboldt behauptete, ohne Worte gäbe es keinen Gedanken, daher sei Sprache notwendig, um der umgebenden Wirklichkeit „Konturen und Form“ zu geben - um sie und ihre Teile benennen zu können: „so liegt in jeder Sprache eine

5 Texte, die auf Deutsch geschrieben, aber in anderen deutschsprachigen Ländern produziert werden, können erhebliche Unterschiede aufweisen. 
eigentümliche Weltansicht“ (Humboldt 1907: 60, vgl. Anusiewicz, Dąbrowska \& Fleischer 2000: 25).

Auf die bereits erwähnte Frage der Abhängigkeit des Denkens vom Sprechen wurde auch von den amerikanischen Kulturanthropologen, die sich mit der Linguistik beschäftigten, und den Pionieren der Ethnolinguistik - Edward Sapir und Benjamin Lee Whorf - hingewiesen. Der Prozess der Gedankenformulierung sei nicht unabhängig und rational im traditionellen Sinne, sondern Teil einer bestimmten Grammatik und weist je nach dieser mehr oder weniger große Abweichungen auf.

Eine der ersten Definitionen von dem sprachlichen Weltbild auf dem Gebiet der polnischen Sprachwissenschaft stammt aus dem Werk von Bartmiński und Tokarski „Językowy obraz świata a spójność tekstu“ (Das sprachliche Weltbild und die Kohärenz des Textes) aus dem Jahre 1986. Demnach ist das sprachliche Weltbild eine bestimmte Reihe von mehr oder weniger sprachlich fixierten Urteilen, die in Bedeutungen von Wörtern enthalten oder durch diese Bedeutungen impliziert sind und die die Merkmale und Existenzweisen der Objekte der außersprachlichen Welt beschreiben (ebd.: 72).

In Fortsetzung dieses Gedankens stellt Bartmiński (2006: 76f.) fest, dass die Sprache eine Art Interpretation der Realität enthält, die als eine Reihe von Urteilen über die Welt ausgedrückt werden kann. Solche Urteile sind vor allem in der Struktur der Sprache festgelegt: in grammatischen Formen, Wortschatz und Sprichwörtern und Phraseologismen.

Gegenwärtig wird „Językowy obraz świata“ (JOS; das sprachliche Weltbild) am häufigsten als eine konzeptuelle Struktur definiert, die im System einer bestimmten Sprache fixiert ist (vgl. Grzegorczykowa 1999).

Das sprachliche Bild unserer Welt ist eine Aufzeichnung der Interaktionen zwischen dem Menschen und der Realität: die Welt, die uns umgibt, wird priorisiert, geordnet und klassifiziert. Die Forschung über das sprachliche Weltbild ist demnach die Beobachtung der Beteiligung der Sprache am kognitiven Prozess. Man soll die Beziehungen zwischen Sprache und Kognition untersuchen, eine Antwort auf die Frage zu finden, welches Bild der Welt (der außersprachlichen Realität) in der jeweiligen Sprache enthalten ist und woraus diese Welt besteht. Es ist auch relevant zu ergründen, welches Wissen, welche Erfahrungen und Werte, die von der Gemeinschaft, die eine bestimmte Sprache verwendet, akzeptiert und anerkannt werden, in ihr gesammelt, gespeichert und an die nächsten Generationen weitergegeben werden (vgl. Kopińska 2009: 56, in Anlehnung an Anusiewicz 1999).

In einer globalisierten Welt wird es jedoch immer schwieriger, „reine“ sprachliche Weltbilder zu finden, d.h. solche, die sich nur in einer bestimmten Sprachgemeinschaft herauskristallisieren. Die Strukturen einer Sprache werden immer mehr durch Englisch, Internationalität usw. unterwandert, was auf eine 
größere Fremdsprachenkompetenz, aber auch auf vermehrte Reisen und den Erfahrungsaustausch mit anderen Kulturen zurückzuführen ist.

Ein anderes Thema ist die Existenz von globalen Ideen, deren Quellen nicht immer bekannt sind, wie z.B. die Idee des Naturschutzes, die Idee der nachhaltigen Entwicklung, die Toleranz gegenüber Unterschieden, z. B. im Bereich der Moral, usw. Diese Ideen durchdringen einzelne Kulturen, wobei nicht immer sprachspezifische Strukturen erzeugt werden, sondern eher Bilder, in der Regel in Form von Metaphern, verwendet werden, die für den gesamten Kulturkreis (z.B. europäisch) verständlich sind. Für den Übersetzer ergeben sich daraus zusätzliche Probleme, denn eine in einer bestimmten Sprache (z.B. im Englischen) geformte Metapher erlaubt nicht immer die korrekte Verwendung nach Wortbildungsmustern oder phonetischen Prinzipien in der Zielsprache. Man kann hier z. B. das schwer auszusprechende Konzept ,frankensteinizacja prawa ${ }^{\text {“ }}$ in Polen anführen.

Wir werden hier zwei Situationen in Betracht ziehen, in denen die Notwendigkeit der Übersetzung eines bestimmten Gebrauchstextes durchaus vorstellbar ist. Dabei muss man als Übersetzer nicht nur charakteristische Sprachstrukturen erkennen, sondern auch bestimmte Inhalte berücksichtigen oder weglassen, die nicht in ein bestimmtes, für die polnische und deutsche Sprache charakteristisches Textmuster passen.

\section{Analyse anhand ausgewählter Texte}

Im Rahmen der von mir seit 2013 geführten Bachelorseminare haben sich Studierende des sechsten Semesters in ihren Bachelorarbeiten mit dem Vergleich von Parallelsachtexten beschäftigt. Unter die Lupe genommen wurden u. a. deutsche und polnische Mietverträge, Immobilienanzeigen, Arbeitsvertragsmuster für Lehrer, Arbeitsverträge für Fahrer im Güterkraftverkehr, tiermedizinische Packungsbeilagen, Krankenhausentlassungsberichte oder Bedienungsanleitungen. Anhand der zugänglichen Modelle der Ausgangstextanalyse, die entsprechend modifiziert wurden, bestimmten die Studierenden, wo die meisten Unterschiede liegen, und zwar nicht nur in der sprachlichen Form der verglichenen Texte, sondern auch in der Makrostruktur der Texte und in dem suprasegmentalen Bereich. Nachfolgend werden beispielhafte Analysen von zwei Textsorten dargestellt.

6 Vgl. https://www.polityka.pl/tygodnikpolityka/swiat/1940242,1,frankensteinizacja-prawapolska-objeta-monitoringiem-rady-europy.read. 


\subsection{Kontaktanzeigen}

Beide hier besprochenen Textsorten wurden von Studentinnen des 5. und 6. Semesters der Angewandten Linguistik im Rahmen ihrer von mir betreuten Bachelorarbeit analysiert.

Die erste dargestellte Textgattung ist die Kontaktanzeige.

Unter einer Kontaktanzeige ist eine Annonce zu verstehen, die man in der Presse oder auf einer Singlebörse finden kann. Das Ziel der Anzeige ist das Schließen einer Bekanntschaft, Freundschaft oder Liebesbeziehung. Die Hauptfunktion der Kontaktanzeigen ist, für Textverfasser (Inserenten) einen potentiellen Partner zu finden, den sie in der realen Welt nie treffen könnten. Außerdem besteht hier ein konkreter Wunsch, eine andere Person kennen zu lernen (vgl. Riemann 1999: 47). Inserenten sind motiviert und sich ihres Handelns bewusst und beschreiben sich, ihre Wünsche und Hoffnungen, Stärken, Schwächen, Erwartungen und alles, worüber sie dem potentiellen Partner Bescheid geben möchten. Der Inserent trifft die Entscheidung, was er bekannt machen und was er verheimlichen möchte, wobei er eine Reaktion erwartet und die Empfänger zum Handeln ermuntert, weil er versucht, von möglichst vielen Lesern Aufmerksamkeit zu bekommen. Kontaktanzeigen sind demnach monologische Texte mit informierendem, aber auch appellativem Charakter ${ }^{7}$. Man kann eine informative Funktion unterscheiden, weil der Inserent in seiner Kontaktanzeige sowohl seine Person als auch die Person des gesuchten Partners darstellt. Es wird dabei auch eine Selektivfunktion erkannt (vgl. Rutkowski 2003: 12): nur ein Teil der Empfänger, der sich angesprochen fühlt, sollte auf die gelesene Kontaktanzeige reagieren und nach dem Kontakt mit dem Inserenten streben. Die Kommunikation verläuft mittels eines Massenmediums. Kontaktanzeigen können sowohl in einer gedruckten, als auch in einer digitalen Form im Internet vorkommen. Die Autoren von Anzeigen haben das Ziel, von vielen Menschen gelesen $\mathrm{zu}$ werden, wobei Kommunikationspartner zeitlich und räumlich getrennt sind.

7 Insofern erinnern sie auch an Werbetexte. Klüver (2009: 10) schreibt, dass als Werbetext im weitesten Sinne jede schriftliche oder mündliche Botschaft genannt wird, die das Ziel hat, auf das Verhalten des Empfängers einzuwirken. Außerdem vermitteln Werbetexte Informationen und verursachen konkrete Handlungen. Es sollte eine Beziehung zwischen Absender und Empfänger des Textes entstehen. In dem hier besprochenen Fall sollen sich Rezipienten von den Inserenten angesprochen fühlen. Die in so einer Werbung wie Kontaktanzeige vorhandenen Informationen sollen interessant und unterhaltsam dargestellt werden. Dieser Aspekt stimmt auch mit dem Hauptzweck der Werbesprache überein, die Bedürfnisse wecken und eine Aktion hervorrufen soll. 
Die einzige Bedingung ist, dass man sich auf einer Partnerbörse registrieren soll. Die Benutzung von Dating-Seiten kann kostenpflichtig sein, so kann sie nicht jeder benutzen.

Die erste bekannte Kontaktanzeige wurde bereits am 19. Juli 1695 in England in einer Zeitung veröffentlicht (vgl. Kaminsky 2017). Seitdem haben sich die Kontaktanzeigen auch geändert. Obwohl heutzutage Zeitungen noch eine bedeutsame Informationsquelle sind, verloren sie an Bedeutung.

Die Sprache der Kontaktanzeigen ändert sich je nach Gesellschaftsgruppe (Jugendsprache, Schichtensprache). Die entscheidenden Faktoren sind auch Geschlecht und Alter.

Kontaktanzeigen als Forschungsbereich erfreuen sich keiner größeren Beliebtheit. Sie bleiben als Textsorte in vielen Fällen unbemerkt, obwohl sie ein interessantes Untersuchungsgebiet bilden, weil in diesen Texten auf einer relativ begrenzten Fläche der Mensch und seine Persönlichkeit, Begabungen, Neigungen, Schwächen, Träume, Wünsche, Vorschläge, Hoffnungen und Vorstellungen beschrieben werden. Die Wahl von sprachlichen Mitteln soll vor allem verständlich und ehrlich sein. Es sollte hier auch keinen Platz für Lügen und Unehrlichkeit geben, sonst kann der Textersteller ein gegenläufiges Ziel erwarten und erreichen. Die Zielgruppe soll sich überzeugt und ernst betrachtet fühlen.

Im Kontext des Artikels war die Frage interessant, wie sich die Deutschen und Polen der ersten Jahrzehnte des 21. Jahrhunderts vorstellen und welche Unterschiede es zwischen den Kontaktanzeigen in beiden Ländern gibt. Es wurden die Kontaktanzeigen von Männern analysiert, die in der zweiten Hälfte des Jahres 2017 auf polnischen und deutschen Singlebörsen erschienen sind. Die Anzeigen betreffen verschiedene Altersgruppen, damit verschiedene Trends in Verwendung von Wörtern und Strukturen beobachtet und eine größere und vollere Einsicht in diese Textsorte gewonnen werden kann, was bei einer Inhaltsanalyse unentbehrlich ist.

In den analysierten Kontaktanzeigen werden charakteristische Begriffe und Wendungen benutzt, die für eine Zielgruppe typisch sind und sie wirksam beeinflussen können. Es wurden zwei deutsche Partnerbörsen (Parship.de und LoveScout24.de) und zwei polnische Dating-Seiten (Swatka.pl und Sympatia.pl) gewählt, die sich in dem jeweiligen Land größter Beliebtheit erfreuen. Außerdem ermöglichen die Portale den Inserenten große Freiheit beim Verfassen des Textes. Es gibt kein festes Muster und keine Einschränkungen bei der Anzeigeveröffentlichung.

Es wurden 15 deutsche und 15 polnische Kontaktanzeigen untersucht, wobei wegen des begrenzten Umfangs einer Bachelorarbeit die Entscheidung getroffen wurde, nur die von Männern verfassten Texte zu analysieren. Um die aktuellsten Annoncen zu untersuchen, wurden nur die Anzeigen berücksichtigt, die in der zweiten Hälfte des Jahres 2017 veröffentlicht wurden. 
Einer der wichtigsten Teile der Kontaktanzeige ist die Selbstdarstellung. Der Empfänger des Textes lenkt seine Aufmerksamkeit immer auf die Selbstbezeichnung des Textabsenders. Jeder hat Vorstellungen und Erwartungen dem Partner gegenüber und bevor er die Entscheidung trifft, eine Anzeige zu beantworten, will er möglichst viel über die Person wissen. In vielen Fällen veröffentlichen die Inserenten nur die Basisinformationen oder wollen überhaupt nichts über sich selbst erzählen.

Die meisten deutschen und polnischen Inserenten stellen sich mit dem Vornamen vor. Es gibt auch eine Gruppe, die zu diesem Zweck einen Spitznamen benutzt, beispielweise Hönigbär, Goldhase, Angel. Diese Art und Weise erfreut sich aber größerer Beliebtheit in Deutschland. In fast allen Kontaktanzeigen gibt es eine Angabe vom Alter und Wohnort, wobei in den analysierten Anzeigen der deutschen Benutzer die Städte genau genannt werden. Die Polen bezeichnen dagegen nur die Woiwodschaft oder die Umgebung von einer Großstadt. Die deutschen Inserenten fassen oft den Entschluss, die Information über ihr Geschlecht in ihrer Kontaktanzeige bekannt zu geben, z. B. ein $100 \%$ Mann sucht... oder in abgekürzter Form bin $M, 42 \ldots$ Die polnischen Inserenten nennen dagegen oft den Familienstand ledig (wolny), Junggeselle (kawaler). Das Thema der Größe ist beliebter in polnischen Kontaktanzeigen: Polen geben häufiger ihre Körpergröße und ihr Gewicht an. Die Deutschen verzichten darauf. In den polnischen Annoncen wird seltener über Hobby geschrieben als in den deutschen Anzeigen. Die Informationen über Konfession, Ausbildung und Beruf sind sowohl in den polnischen als auch in den deutschen Texten nicht so oft anzutreffen.

Aus der durchgeführten Analyse resultieren folgende Schlussfolgerungen:

- man kann feststellen, dass in den deutschen Kontaktanzeigen größerer Wert auf Selbstbeschreibung und in den polnischen Annoncen auf Beschreibung der Wunschpartnerin gelegt wird.

- in den polnischen Anzeigen wurden Erwartungen der Partnerin gegenüber direkt verbalisiert, und in den deutschen dient eher die Selbstdarstellung indirekt der Suche nach einer Partnerin: auf diese Art und Weise werden nur solche Frauen zum Kontakt eingeladen, die Interesse an dem sich so und nicht anders selbstbeschreiben Partner haben. Die Taktik des Handelns von deutschen und polnischen Inserenten ist also anders.

- nach der Analyse der Selbstdarstellung kam heraus, dass die Deutschen häufiger Spitznamen verwenden, was davon zeugen kann, dass sie anonym bleiben wollen oder durch den Spitznamen einen zusätzlichen Inhalt ausdrücken möchten.

- die wichtigste Information in der Selbstbeschreibung scheint das Alter zu sein, weil es in fast allen Kontaktanzeigen angegeben wird.

- die Textabsender geben auch ihren Wohnort oder die Umgebung an, in der sie eine Partnerin suchen. Die Deutschen schreiben meistens den konkreten 
Ortsnamen. Die Polen bevorzugen es dagegen nur die Gegend, z. B. Woiwodschaft anzugeben. Es gibt auch Inserenten, für die die Entfernung keine Rolle spielt.

- die polnischen Inserenten legen einen großen Wert aufs Aussehen. Die Informationen über Körpergröße und Gewicht sind in den polnischen Kontaktanzeigen oft anzutreffen. Die Deutschen verzichten darauf. Sie schreiben häufiger über Hobbys und Leidenschaften in ihren Annoncen.

- Ähnliche Schlussfolgerungen kann man aus der Analyse der Wunschpartnerbeschreibung ziehen.

- für die polnischen Inserenten spielt das Äußere eine größere Rolle als für die Deutschen. Sie nennen oft eine konkrete Altersgruppe einer Partnerin. Für die deutschen Inserenten ist das Alter nicht so bedeutsam; sie vermeiden oft diese Angabe.

- In polnischen Anzeigen ist auch oft eine Information über Kinder anzutreffen.

- In den deutschen Texten wird häufiger die Konfession der potenziellen Partnerin direkt bezeichnet. So eine Information kommt in den polnischen Anzeigen nicht vor.

Der Gebrauch von Adjektiven zum Zweck der Selbstbeschreibung und Wunschpartnerbeschreibung in den polnischen und deutschen Kontaktanzeigen ist auch interessant. Sowohl die Deutschen als auch die Polen verwenden ähnliche Adjektive, um sich selbst zu beschreiben, nämlich solche, die positive Konnotationen wecken. Wenn es um Adjektive zur Wunschpartnerbeschreibung geht, verwenden die Polen mehr Adjektive, die mit dem Aussehen verbunden sind. Für die Deutschen sind die Charaktereigenschaften wichtiger. Es gibt aber ein paar Adjektive, die in den deutschen und polnischen Texten genauso oft vorkommen. Sie bezeichnen Eigenschaften, die in jeder Beziehung als Grundlage betrachtet werden.

Wenn es um Satzkonstruktionen geht, beobachtet man, dass die polnischen Inserenten mehr Imperativsätze verwenden. Dadurch scheinen ihre Kontaktanzeigen direkter zu sein. Auf diese Art und Weise wollen sie die eventuelle Partnerin überzeugen. Der überwiegende Teil der Informationen wird explizit ausgedrückt. In den deutschen Kontaktanzeigen sind dagegen häufiger Fragesätze anzutreffen. Sie ermutigen indirekt, also implizit zum Kontakt, weil man den Eindruck haben kann, dass jemand von dem Rezipienten Antwort erwartet.

Dank der beobachteten Tendenzen und Unterschiede zwischen deutschen und polnischen Kontaktanzeigen kann man schlussfolgern, dass sie problematisch fürs Übersetzen sein können. Die Polen und die Deutschen halten andere Elemente in den Annoncen für bedeutsam. Um das Ziel eines solchen Textes zu erreichen, sollte man ihn an die Bedingungen in dem konkreten Land anpassen. In den Anzeigen, die auf dem polnischen Markt erscheinen, werden die Informationen meistens direkt übermittelt. Der Übersetzer sollte also dementspre- 
chend auf Wortspiele und dichterische Elemente verzichten. In den Annoncen, die dagegen in Deutschland veröffentlicht werden, sollten die Informationen über das Äußere nicht vorkommen, weil für die deutschen Empfänger die Charakterbeschreibung eine größere Rolle spielt. Die deutschen Anzeigetexte sollen auch nicht zu direkt sein.

\subsection{Todesanzeigen}

Eine andere problematische Textgattung sind Todesanzeigen, die ein traditioneller Bestandteil der Regional- und Tageszeitungen sind. Es sind private offene Anzeigen, die für Verwandte, Freunde, Arbeits- und Teamkollegen, Vereinsmitglieder oder Sportkameraden aufgegeben werden. Eine Todesanzeige kann entweder als Einzeldrucksache in Kartenform oder in einer Zeitschrift oder Zeitung erscheinen.

Die Todesnachricht kennt man aus allen Epochen und in allen Ländern. Nur selten sind alle, die vom Tod eines bestimmten Menschen erfahren sollten, während der Sterbephase anwesend, und so entsteht ein Bedürfnis, andere über den Sterbefall zu unterrichten. Im Laufe der Geschichte übernahmen verschiedenartigste Medien diese kommunikative Funktion (vgl. Haus 2007: 43). Die Todesanzeigen haben eine Darstellungsfunktion (sie informieren über den Tod), Appellfunktion (Hinterbliebene werden zu der Bestattung eingeladen) und auch Ausdrucksfunktion (Hinterbliebene geben Ausdruck ihrer Traurigkeit).

Es wurde die Vergleichsanalyse der polnischen und der österreichischen Todesanzeigen aus den Seiten www.enekrolog.pl und www.trauerhilfe.at durchgeführt., wobei acht polnische und acht österreichische online Todesanzeigen gewählt wurden.

Todesanzeigen, die im Internet veröffentlicht werden, sind anders als solche, die in den Zeitungen veröffentlicht werden. Sie geben zum Beispiel sehr oft die Todesursache an. Man spricht im Internet zum Beispiel über Krebs, und in Zeitungen ist das bis zu einem gewissen Grade immer noch ein Tabuthema. Man sollte auch erwähnen, dass solche Informationen wie die Todesursache öfter in österreichischen als in polnischen Todesanzeigen veröffentlicht werden.

Die österreichischen Todesanzeigen werden am häufigsten in vier Teile geteilt: Einleitung, Ort, Adresse und Datum, Mitteilungsteil und Abschlussteil mit organisatorischen Hinweisen. Die polnischen Todesanzeigen dagegen haben zum Beispiel keine Einleitung.

Andere Elemente, die in Todesanzeigen beobachtet werden (aber eine Todesanzeige muss nicht alle diese Elemente enthalten), sind: Symbole, Mottos, Daten über den Verstorbenen, Hinterbliebene, Todesursache, Nachruf, Informationen über die Bestattung und Seelenmesse oder Fotos etc. Für Todesan- 
zeigen sind Euphemismen also „beschönigende, verhüllende, mildernde Umschreibung für ein anstößiges oder unangenehmes Wort" (http://www.duden.de/ rechtschreibung/Euphemismus) typisch. Paradoxerweise schreibt niemand in den Todesanzeigen über den Tod als Tod. Am meisten treten solche Ausdrücke wie zum Beispiel „letzte Reise“ auf.

Die analysierten österreichischen Todesanzeigen sagen mehr über den Verstorbenen. Alle Hinterbliebenen werden namentlich genannt. Das zeigt, wer sie für den Verstorbenen waren. Man findet in den österreichischen Todesanzeigen auch eine kurze Information, wie diese Person war. Die polnischen Todesanzeigen informieren nur, dass der Verstorbene zum Beispiel eine Tochter oder einen Bruder hatte.

Es gibt auch Unterschiede in Layouts der analysierten polnischen und österreichischen Todesanzeigen. Die österreichischen haben oft einen Hintergrund mit Bildern von Meer, Wald, Gebirge usw. und ein Foto des Verstorbenen, die polnischen haben es nicht, sie haben aber eine Abbildung der religiösen Art, wie die Gestalt von Christus oder der heiligen Maria.

Wenn es um den Inhalt geht, sind die polnischen Todesanzeigen ${ }^{8}$ eindeutig ernsthafter und in einem förmlichen Ton. Wenn man die österreichischen Todesanzeigen ${ }^{9}$ liest, empfindet man dagegen mehr Hoffnung.

In den Todesanzeigen wird noch ein weiterer kultureller Unterschied sichtbar, nämlich der Aspekt der Visualisierung von Religiosität ${ }^{10}$. Man kann nicht leugnen, dass beide Texte mit dem katholischen Begräbnisritual verbunden sind, aber in den österreichischen Anzeigen ist das eher erst im Text sichtbar, wo man an katholische Feiertage erinnert und das Layout ist hier eher allgemein christlich gehalten, was sich an dem dargestellten Kreuz ablesen lässt, und nicht rein katholisch. In den polnischen Texten finden wir dagegen sehr oft z. B. die Figur der Heiligen Maria, wodurch die religiöse Zugehörigkeit sofort ersichtlich wird.

Die Abbildung von Verstorbenen mit lächelnden Gesichtern in den österreichischen Anzeigen lässt zweierlei Schlüsse zu: einerseits sind die Anzeigen dadurch personalisierter und die Familie fühlt sich dadurch stärker angesprochen. Das Foto der geliebten Person, die lächelt, was, der Meinung des Verfassers nach, vom Glück im Jenseits zeugen könnte, soll den Schmerz der Hinterbliebenen

8 Beispiel einer polnischen Todesanzeige: www.enekrolog.pl/nekrologi/MALOPOLSKIE/bo chenski/kazimiera-mirska-kowalska/.

9 Beispiel einer österreichischen Todesanzeige: https://www.trauerhilfe.at/todesanzeigen/de tail/uid/54357/verstorbener/128156/.

10 Das polnische sprachliche Weltbild wurde auch vom Christentum geprägt, genauer gesagt von dem Katholizismus. Diese Klarstellung erscheint notwendig, da der Ausdruck „heiliger Glaube“ bezeichnet in Polen den katholischen Glauben, den die Gottesmutter symbolisiert, die von Jan Kazimierz den Titel „Königin der Polnischen Krone“ erhalten hat. Seitdem hat die Kombination „Pole/Polin - Katholik/Katholikin“ den Status einer parametrisierten Beziehung erlangt (vgl. Pajdzińska 2001: 45). 
lindern. Andererseits sticht der religiöse Aspekt in den polnischen Anzeigen im Vergleich zu den österreichischen mehr ins Auge, weil die Gemälde von Christus oder der heiligen Maria in den polnischen Todesanzeigen eine wichtigere Rolle zu spielen scheinen als der Verstorbene selbst.

Todesanzeigen sind keine typischen Texte. Sie werden auch selten übersetzt, was jedoch die Notwendigkeit ihrer Übersetzung nicht ausschließt. Wenn zum Beispiel eine Person aus Österreich in Polen arbeitete (oder umgekehrt), wird deren Todesanzeige wahrscheinlich sowohl in Österreich als auch in Polen veröffentlicht. Da es derart große Diskrepanzen in der Gestaltung der Todesanzeigen gibt, sollte die Übersetzung einer Todesanzeige jeweils an die Zielkultur angepasst werden, um den Verfremdungseffekt und, damit verbunden, eine mögliche Verwirrung der Hinterbliebenen zu vermeiden.

\section{Desiderat}

Das elementare alltägliche Bild der Welt spiegelt sich nicht nur in den Überzeugungen einer Gemeinschaft, sondern auch in den nicht vollständig bewussten Vorstellungen und Erwartungen, die weitgehend durch die Sprache geformt werden. Das Gefühl der Gemeinschaft wird durch den Kontakt mit dem Fremden entdeckt: das Eigentümliche und Andere erlaubt es, das Gemeinsame zu erkennen. Welche Faktoren diese Vertrautheit und Identifizierung bestimmen, wird aber in der Regel nicht explizit ausgedrückt. Man reagiert, auch sprachlich, nicht auf seine eigenen Charakteristika, sondern eher auf fremde Merkmale (vgl. Pajdzińska 2001: 44f).

Aufgrund der oben dargestellten Überlegungen zur Bedeutung der Paralleltextanalyse für die Didaktik in der Angewandten Linguistik lassen sich folgende Schlüsse ziehen:

Erstens: Im Rahmen des Seminars hatten die Studierenden die Möglichkeit, Analysen von gegenwärtigen Hypertexten durchzuführen und selbst zu beobachten, wie sich in solchen Texten Unterschiede in der Wahrnehmung der Welt zwischen Polen und Deutschen oder Österreichern zeigen, nicht nur in Bezug auf die sprachliche Gestaltung, sondern auch auf die Wahl der angesprochenen Themen oder der nonverbalen Elemente in den Texten, wie z.B. Fotos. Die von ihnen gezogenen Schlüsse waren insofern interessant, dass solche Texte einerseits das gegenwärtige sprachliche Weltbild von Gesellschaften zeigen und andererseits eine Übersetzung solcher Texte potenziell möglich ist.

Zweitens: Eine solche Analyse von Paralleltexten hat ein großes didaktisches Potenzial, denn authentische Texte, die online zugänglich sind, sind nicht nur leichter verfügbar, sondern auch interessanter für die meisten Studierenden, was deren Motivation enorm steigert. Nach der selbstständig durchgeführten 
Analyse sind die Studierenden dann oft im Stande, auch andere Paralleltexte mit der bereits bekannten Methode zu analysieren, was sehr hilfreich für ihre Übersetzungspraxis sein kann.

Auf diesen Ergebnissen basierend lässt sich ein Postulat der Einführung einer solchen Analyse in das Programm des Studiums der Angewandten Linguistik aufstellen, und zwar bereits im vierten Semester als eine Art Vorbereitung auf die Bachelorarbeit.

Ein anderes Postulat ist, dass man nicht nur Sachtexte einer solchen vergleichenden Analyse unterziehen sollte. Und tatsächlich haben wir im Rahmen meines Bachelorseminars in den Jahren 2018 und 2020 auch Texte aus dem Bereich Politik analysiert, u. a. Programme der Rechts- und Linksparteien. Die Ergebnisse ermöglichten den Studierenden die selbständige Arbeit an authentischen politischen Texten, was ihnen z. B. dabei geholfen hat, über die Rolle und das Ausmaß der sprachlichen Manipulation und über kulturelle Unterschiede in diesem Bereich zwischen Polen, Deutschland und Österreich zu reflektieren.

Das Bild der Welt, eingraviert in die Sprache einer bestimmten Gemeinschaft, ist aus der Sicht ihrer Mitglieder „natürlich“, also im gewissen Masse auch verpflichtend. Das bedeutet natürlich nicht, dass die Sprache uns vollständig bestimmt, sondern, dass man ihre Implikationen überwinden muss, um anders zu denken und zu handeln. Wenn man sich mit der Problematik der eigenen Identität befasst, lohnt es sich zu fragen: Welche Erfahrungen in der Textsortengestaltung die anderen Mitglieder einer Gesellschaft haben. Die Antwort darauf erlaubt es bestimmte soziale, darunter auch sprachliche Verhaltensweisen, zu verstehen, was nicht nur für sprachliche Mittler, sondern für alle sich mit einer fremden Kultur befassenden Menschen relevant ist.

\section{Literaturverzeichnis}

Adamzik, Kirsten (2001). Die Zukunft der Text(sorten)linguistik - Textsortennetze, Textsortenfelder, Textsorten im Verbund. In: U. Fix/S. Habscheid/J. Klein (Hrsg.), Zur Kulturspezifik von Textsorten, Bd.3 (S. 15-30). Tübingen: Stauffenburg.

Anusiewicz, Janusz/Dąbrowska, Anna/Fleischer, Michael (2000). Językowy obraz świata $i$ kultura. Projekt koncepcji badawczej. Wrocław: Wydawnictwo Uniwersytetu Wrocławskiego.

Anusiewicz, Janusz (1999). Problematyka językowego obrazu świata w poglądach niektórych językoznawców i filozofów niemieckich XX wieku. In: J. Bartmiński (Hrsg.), Językowy obraz świata (S. 261-289). Lublin: Wydawnictwo Uniwersytetu Marii CurieSkłodowskiej.

Bartmiński, Jerzy/Tokarski, Ryszard (1986). Językowy obraz świata a spójność tekstu. In: T. Dobrzyńska (Hrsg.), Teoria tekstu. Zbiór studiów (S. 65-81). Wrocław: Ossolineum. 
Bartmiński, Jerzy (2006). Językowe podstawy obrazu świata. Lublin: Wydawnictwo Uniwersytetu Marii Curie-Skłodowskiej.

Harald Burger/Martin Luginbühl (Hrsg.) (2005). Mediensprache. Eine Einführung in Sprache und Kommunikationsformen der Massenmedien. Berlin: De Gruyter.

Fleischer, Wolfgang/Helbig, Gerhard/Lerchner, Gotthard (Hrsg.): Kleine Enzyklopädie Deutsche Sprache. Frankfurt a.M.: Peter Lang.

Gabrych, Martyna (2018). Vergleichende Analyse der deutschen und polnischen Kontaktanzeigen. Eine unveröffentlichte Bachelorarbeit (Instytut Lingwistyki Stosowanej UAM).

Göpferich, Susanne (1998). Paralleltexte. In: M. Snell-Hornby/H.G. Hönig/P. Kußmaul/ P.A. Schmitt (Hrsg.), Handbuch Translation (S. 184-186). Tübingen: Stauffenburg.

Grzegorczykowa, Renata (1999). Pojęcie językowego obrazu świata. In: J. Bartmiński (Hrsg.), Jezzkowy obraz świata (S. 34-46). Lublin: Wydawnictwo Uniwersytetu Marii Curie-Skłodowskiej.

Hanus, Anna/Kaczmarek, Dorota (2019). Das Kochrezept im digitalen Raum. Immer noch eine Alltagsselbstverständlichkeit? Text- und medienlinguistische Überlegungen. tekst $i$ dyskurs - text und diskurs, 12, 213-231.

Haus, Adrian (2007). Todesanzeigen in Ost-und Westdeutschland. Ein sprach- und kulturwissenschaftlicher Vergleich. Todesanzeigen aus der Leipziger Volkszeitung und der Frankfurter Neuen Presse 1976 bis 2004. Frankfurt a.M.: Peter Lang.

Klüver, Nathalie (2009). Werbesprache als Spiegel der Gesellschaft? Hamburg: Diplomica Verlag.

Humboldt, Wilhelm von (1907). Über die Verschiedenheit des menschlichen Sprachbaues und ihren Einfluss auf die geistige Entwicklung des Menschengeschlechts (1835). In: W. von Humboldt, Gesammelte Schriften, Bd.VII Berlin: Behrl's Verlag.

Kaminsky, Ralph (2017). Kontaktanzeigen im Wandel der Zeit. https://www.migrosmaga zin.ch/archiv/kontaktanzeigen-im-wandel-der-zeit.

Kopińska, Marta (2009). Język jako narzędzie interpretacji rzeczywistości - językowy obraz świata. Mundu bat begirada anitz. Un mundo michas miradas, 2, 53-76.

Maciejewski, Marcin (2009). Gatunki hipertekstu w perspektywie tekstologicznej. Analiza na przykładzie internetowych prezentacji przedsiębiorstw. Poznań: Wydawnictwo Naukowe Uniwersytetu im. Adama Mickiewicza.

Pajdzińska, Anna (2001). My to znaczy...(z językowego obrazu świata). Teksty Drugie 2001, $1,33-53$.

Prokop, Izabela (2003). Skuteczna szkoła - mrzonka to czy nadzieja? In: P. Hostyński (Hrsg.), Szukamy nowych dróg. Materialy II meetingu glottodydaktycznego zorganizowanego w ramach Podyplomowego Studium Glottodydaktyki 2000/2001 (S. 23-30). Poznań: Rys Studio.

Prokop, Izabela (2002). Typologia interakcji dydaktycznych, In: W. Wilczyńska (Hrsg.), Autonomizacja $w$ dydaktyce języków obcych. Doskonalenie się w komunikacji ustnej (S. 159-174). Poznań: Wydawnictwo Naukowe Uniwersytetu im. Adama Mickiewicza.

Sapir, Edward (1978). Kultura, język, osobowość. Warszawa: Państwowy Instytut Wydawniczy.

Schatte, Czesława (2009). Teksty użytkowe jako element programu lingwistyki stosowanej. Refleksja praktyczna. Lingwistyka Stosowana/Applied Linguistics, 27, 203-212. 
Sobańska, Justyna (2016). Polnische und österreichische Todesanzeigen im Vergleich. Eine Paralleltextanalyse anhand Online-Todesanzeigen. Eine unveröffentlichte Bachelorarbeit (Instytut Lingwistyki Stosowanej UAM).

Riemann, Viola (1999). Kontaktanzeigen im Wandel der Zeit. Eine Inhaltsanalyse. Opladen, Wiesbaden: Westdeutscher Verlag GmbH.

Rutkowski, Nicole (2003). Kontaktanzeigen im intermedialen Vergleich. Universität Duisburg-Essen, Linguistik-Server Essen: Essen.

Stechman, Ingo (2003). Gebrauchstexte und ihre Funktion beim Erwerb der Lesekompetenz. Eine Studienarbeit, Norderstedt: GRIN Publishing.

Tomaszkiewicz, Teresa (2004). Terminologia tłumaczenia. Poznań: Wydawnictwo Naukowe Uniwersytetu im. Adama Mickiewicza.

Whorf, Benjamin Lee (1982). Język, myśl i rzeczywistość. Warszawa: Państwowy Instytut Wydawniczy. 


\title{
Elżbieta Dziurewicz \\ (Uniwersytet im. Adama Mickiewicza w Poznaniu)
}

\section{Idiome im deutsch-polnischen Kontrast als Herausforderung für Studierende der Angewandten Linguistik. Ergebnisse einer Umfrage und Postulate für den Einsatz im universitären Unterricht}

\begin{abstract}
Idioms in German-Polish contrast as a challenge for students of applied linguistics. Results of a survey and postulates for use in university teaching

The aim of this chapter is to test German-Polish phraseological competence on the example of idioms, as evidenced in students of applied linguistics at Adam Mickiewicz University in Poznań. First, idioms as a phraseological category are described as featuring inherent characteristics. Subsequently, the question of to what extent learners of German can benefit from a contrastive German-Polish analysis of idioms is examined. The main part of this contribution presents the findings of a study carried out in 2020. It was aimed to investigate whether students had already dealt with idioms in the German-Polish contrast and whether it was difficult for them to solve contrasting tasks in this context. Finally, postulates for a more intensive and successive treatment of idioms are formulated within the framework of university courses (i.e. German and Applied Linguistics).
\end{abstract}

Keywords

idioms, phraseological competence, contrastive analysis, German, Polish

\section{Einleitung}

Unter phraseologischer Kompetenz wird laut Ehrhardt (2007: 258) verstanden „die Bedeutung von Phrasemen zu kennen und diese dann kontextangemessen verwenden zu können“. Als Phraseme werden in Anlehnung an Burger (2015: 11) Einheiten aufgefasst, die sich durch die Merkmale Polylexikalität und relative Festigkeit auszeichnen. Während erstere Eigenschaft meint, dass Phraseme eine Einheit von mindestens zwei Komponenten bilden, impliziert die zweite, dass Deutschsprechende sie genau in dieser Kombination kennen und verwenden (vgl. ebd.). In diesem weiten Verständnis ${ }^{1}$ umfasst Phraseologie eine Reihe von unterschiedlichen Strukturen, wie z.B. Idiome, Zwillingsformeln, geflügelte

1 Zum Terminus Phraseologie im engeren und weiteren Sinne vgl. Palm (1995: 2) sowie Fleischer (1982: 72f.). 
Worte, phraseologische Vergleiche, Kollokationen, Funktionsverbgefüge und Sprichwörter. Zu der Phraseologie im engeren Sinne werden dagegen Verbindungen gezählt, die neben den zwei genannten Charakteristika mit der Idiomatizität noch ein weiteres Merkmal aufweisen. Mit der Idiomatizität ist gemeint, dass die Bedeutung einer idiomatischen Einheit sich nicht aus der Summe der Einzelbedeutungen aller Bestandteile ableiten lässt, sondern außerhalb der Wendung liegt.

Hierzu gehören Idiome, die als typische Vertreter der Phraseologie fungieren und demnach im Zentrum-Peripherie-Modell zentral anzusiedeln sind. ${ }^{2}$ Was sie außer ihrer übertragenen Bedeutung von den peripheren Einheiten wie Kollokationen noch unterscheidet, ist ihre Bildhaftigkeit und Expressivität. Idiome werden als bildhaft bezeichnet, weil sie konkrete visuelle Vorstellungen hervorrufen bzw. hervorrufen können (vgl. Burger 2015: 81). Das Merkmal der Expressivität - von Burger (ebd.: 77) auch als konnotativer Mehrwert bezeichnet bezieht sich auf die stilistische Markierung zahlreicher Idiome. In der Phraseologieforschung wird davon ausgegangen, dass Idiome, gegenüber ihren nichtphraseologischen Pendants als ausdrucksstärker fungieren (vgl. Koller 2007). ${ }^{3}$

Aufgrund dieses Eigenschaftenbündels bilden Idiome - insbesondere im interlingualen Vergleich, wo diese Merkmale je nach der Ausgangs- bzw. Zielsprache unterschiedlich ausgeprägt sind - einen äußerst interessanten Untersuchungsgegenstand. Im Folgenden wird aufgezeigt, welchen Nutzen die kontrastive Analyse von Idiomen hat und warum sie in den DaF-Unterricht einzubeziehen ist. Es werden insgesamt sechs Argumente präsentiert, die für ihre Einbindung in den fremdsprachlichen Unterricht plädieren. Im Hauptteil werden die Ergebnisse einer 2020 durchgeführten Untersuchung präsentiert, deren Ziel es war, zu überprüfen, ob die Studierenden der Angewandten Linguistik bereits Idiome behandelt hatten und inwiefern ihnen das Lösen von kontrastiven (deutsch-polnischen) Aufgaben zu ausgewählten Idiomen Schwierigkeiten bereitet. Als Resümee werden Postulate für eine intensivere und systematische Behandlung von Idiomen im Rahmen der universitären Lehrveranstaltungen (im Falle der Germanistik und Angewandten Linguistik) formuliert.

2 Ausführlich zur Zentrum-Peripherie-Konzeption, vgl. Lüger (2019).

3 Im Gegensatz dazu konstatiert Schmale (2010) anhand einer Korpusanalyse und einer unter deutschen Muttersprachlern durchgeführten Umfrage, dass zahlreiche Idiome weniger expressiv sind als ihre nicht-phraseologischen Entsprechungen. 


\section{Argumente für die kontrastive Analyse deutsch-polnischer Idiome im DaF-Unterricht}

Heutzutage herrscht weitgehend Einigkeit darüber, dass Phraseme im gesamten Deutschunterricht auf allen Sprachstufen Beachtung verdienen (vgl. stellvertretend Ettinger 2011, Jesenšek 2006, Hallsteinsdøttir 2001, Kühn 1992). Dabei sollen allerdings einige Phrasemgruppen wie z. B. Kollokationen und pragmatische Phraseme Priorität haben, während Idiome eher bei fortgeschrittenen Lernenden intensiv zu behandeln sind (vgl. Lüger 2019). ${ }^{4}$

Die Argumente für die Einbindung der Phraseme in den DaF-Unterricht wurden in der Forschungsliteratur bereits hinreichend diskutiert (vgl. Ehrhardt 2014, Jesenšek 2006). Als einer der wichtigsten Gründe für ihre Thematisierung wird ihre hohe Frequenz im Schriftlichen und Mündlichen genannt. Jesenšek (2006: 138) betont, dass unsere Redeweise phraseologisch geprägt ist und sie „ein Normalfall der geschriebenen und gesprochenen Sprachverwendung [ist]“. Aus diesem quantitativen Argument ergibt sich das qualitative, welches besagt, dass ihre Kenntnis unentbehrlich ist, um zielsprachige Texte zu verstehen. Des Weiteren wird von Ehrhardt (2014) sowie Földes und Kühnert (1990) der Motivationsfaktor hervorgehoben. Die Auseinandersetzung mit festen Wendungen macht Spaß und Lernen mit Vergnügen ist in der Didaktik anzustreben, weil es zu Erfolgen im Lernprozess führen kann.

Im Folgenden soll aufgezeigt werden, inwiefern Deutschlernende von der kontrastiven Analyse deutscher und polnischer Idiome profitieren können.

\subsection{Sensibilisierung der Lernenden für interlinguale Gemeinsamkeiten und} Unterschiede

Bei der Kontrastierung deutscher Idiome mit ihren polnischen Äquivalenten lassen sich zahlreiche Gemeinsamkeiten und Unterschiede erkennen. Das Wissen darüber ist für alle Lernenden von eminenter Bedeutung, weil dadurch Interferenzfehler vermieden werden können. Koller (2007) entwickelte speziell für Phraseme eine Klassifikation in Voll-, Teil-, Substitutions- und Nulläquivalenz.

Im ersten Fall, bei Volläquivalenz, haben Lernende keine Verstehensschwierigkeiten, weil in ihrer Muttersprache ein exakt deckungsgleiches Idiom vorhanden ist. Es handelt sich dabei im Regelfall um Interphraseme mit einer ge-

4 Gemeint ist dabei eine intensive und systematische Behandlung von Idiomen. Natürlich können vereinzelte Einheiten bereits in den Anfängerstufen als eine Art Interessewecker eingeführt werden. 
meinsamen Quelle in der Mythologie, Geschichte oder in der Bibel (z.B. dt.: das schwarze Schaf/pol.: czarna owca).

Bei partieller Äquivalenz liegen geringfügige Unterschiede im Bereich der Morphosyntax bzw. der Lexik vor. Lernende können in diesem Falle Vergleiche anstellen, über ihre Mutter- und über die Fremdsprache reflektieren und werden mit den Abweichungen vertraut gemacht, um das Fehlerrisiko zu minimieren. Als Beispiel für eine partielle Äquivalenz gilt das deutsche Idiom auf den Hund kommen und seine polnische Entsprechung zejść na psy, bei dem die nominale Komponente im Deutschen im Singular und im Polnischen im Plural auftritt und sich das Verb unterscheidet.

Mit Substitutions-Äquivalenz ist gemeint, dass das Ausgangsphrasem durch ein anderes Phrasem in der Zielsprache ersetzt wird, wie dies bei der Einheit $z w e i$ Fliegen mit einer Klappe schlagen (pol.: upiec dwie pieczenie na jednym ogniu) der Fall ist. Obwohl beide Idiome verschiedene lexikalische Komponenten aufweisen und somit unterschiedliche Bilder hervorrufen, haben sie die gleiche Bedeutung.

Bei der Null-Äquivalenz (Ersatzäquivalenz) besteht eine phraseologische Lücke in der Zielsprache, weshalb das Phrasem an dieser Stelle mithilfe einer monolexikalen Struktur oder einer Paraphrase ausgedrückt wird. Als Beispiel hierfür fungiert das deutsche Idiom etw. auf die Beine stellen, das im Polnischen mithilfe des Einzellexems organizować coś wiedergegeben wird.

Als ein Sonderfall bei der Kontrastierung sind schließlich phraseologische falsche Freunde zu betrachten. Es handelt sich dabei um Phraseme „deren wörtliche Bedeutung und folglich die syntaktische Struktur identisch ist, deren phraseologische Bedeutung differiert" (Kahl 2015: 130). Diese scheinbare Gleichheit bzw. Ähnlichkeit kann Fehler verursachen. ${ }^{5}$ Aus diesem Grund sollte diesen Phrasemen im Rahmen universitärer Lehrveranstaltungen besondere Aufmerksamkeit geschenkt werden.

\subsection{Bewusstmachung der Expressivität und der Bildhaftigkeit der Sprache(n)}

Bei der Beschäftigung mit Idiomen erfahren die Lernenden, dass viele von ihnen nicht als neutral, sondern stilistisch markiert fungieren. Demnach gibt es in jeder Sprache eine breite Palette von idiomatischen Einheiten, die einer umgangssprachlichen, saloppen, derben bzw. vulgären Ausdrucksweise zuzurechnen sind.

Im Familien- und Freundeskreis werden von Muttersprachlerinnen und Muttersprachlern in der Regel häufiger Einheiten gebraucht, die zur Umgangs-

5 Mit phraseologischen falschen Freunden im deutsch-polnischen Kontrast befasst sich ausführlich Guławska-Gawkowska (2012). 
sprache oder saloppen Sprache tendieren, während diese in offiziellen Gesprächen eher vermieden werden. Auch in der Fremdsprache sollten sich die Lernenden Einheiten bedienen, die in einem konkreten situativen Kontext stilistisch gesehen angebracht sind. Demnach gilt das Idiom den Mund halten als umgangssprachlich, während die Fresse/das Maul halten von den meisten Deutschsprachigen als salopp bzw. vulgär wahrgenommen wird.

Zudem bringt die Reflexion über die in beiden Sprachen existierende Bildhaftigkeit Vorteile mit sich. Lüger (1997: 84) weist darauf hin, dass diese beim Memorieren helfen kann. Darüber hinaus kann die Konfrontation von übertragener und wörtlicher Bedeutung in der Fremdsprache bei polnischen Lernenden ein Schmunzeln auslösen, da sie häufig absurd oder komisch ist, was sich vorteilhaft auf den Lernprozess auswirkt. So klingt die wörtliche Übersetzung des Idioms Sei kein Frosch! (pol.: Nie badź żabą!) lustig und ruft positive Emotionen hervor und dürfte daher leichter erinnert werden.

\subsection{Entwicklung aller Sprachfertigkeiten}

Durch die Vermittlung von Idiomen können sowohl rezeptive (Hören und Lesen) als auch produktive (Sprechen und Schreiben) Fertigkeiten geschult werden. Die Palette an möglichen Übungen dazu ist äußerst umfangreich (vgl. LorenzBourjot \& Lüger 2001, Dziurewicz 2018).

Um rezeptive Kompetenzen zu entwickeln, können deutschsprachige Filme und Serien eingesetzt werden, in denen Phraseme (darunter auch Idiome) zahlreich auftreten. Empfehlenswert ist hier beispielsweise ein Vergleich der im Original gebrauchten Idiome mit denen in der polnischen Wiedergabe. Dadurch lässt sich sowohl die phraseologische Kompetenz im Deutschen und im Polnischen als auch die allgemeine Sprachkompetenz verbessern. ${ }^{6}$

Idiome mit ihrer Herkunft, Motiviertheit, ihren möglichen Übertragungen in die Zielsprache bieten ebenfalls einen sehr guten Anlass zur Diskussion, bei der wiederum das Sprechen gefördert wird. Eine unter Studierenden der Angewandten Linguistik an der Adam-Mickiewicz-Universität in Poznań beliebte Übungsform besteht darin, dass sie ein polnisches Idiom losen und versuchen, seine Bedeutung auf Deutsch zu erklären, während die Kommilitoninnen und Kommilitonen erraten, um welchen Ausdruck es sich dabei handelt. Anschließend wird über die interlingualen Differenzen und Analogien zwischen den Idiomen in den beiden Sprachen diskutiert.

6 Einige phraseologische Übungen, die neue Medien in den Lernprozess miteinbeziehen werden in Dziurewicz und Woźniak (2020) vorgeschlagen. 


\subsection{Erweiterung des fremdsprachlichen und (eventuell auch} muttersprachlichen) Wortschatzes

Bei der kontrastiven Beschäftigung mit Idiomen kann in erster Linie die fremdsprachliche aber bisweilen auch die muttersprachliche phraseologische Kompetenz erweitert werden. ${ }^{7}$ Da der Phrasembestand jeder einzelnen Sprache sehr umfassend ist, sind Muttersprachlerinnen und Muttersprachler selbst nicht imstande alle Phraseme zu kennen. Basierend auf meiner fünfjährigen Erfahrung im Unterrichten der kontrastiven deutsch-polnischen Phraseologie kann ich feststellen, dass die Studierenden in diesem Zusammenhang auch viel voneinander lernen. Dieser Austausch ist äußerst gewinnbringend, denn gelegentlich werden auch polnische Idiome behandelt, die nicht zum Inventar der Studierenden gehören. Dies erfordert eine Reflexion über muttersprachliche phraseologische Kompetenz und kann Anlass dazu geben, die Studierenden auf ein lebenslanges Lernen sowohl im Kontext der Fremd- als auch der Muttersprache vorzubereiten.

Beim Erlernen der deutschen Idiome ist es empfehlenswert, Lernhilfsmittel wie eine Kartei oder ein Plakat zu erstellen, welche möglichst viele Informationen (darunter die Bedeutung auf Deutsch, stilistische Markierungen, die Entsprechung auf Polnisch, Synonyme, Antonyme und Beispielsätze) zu den verschiedenen Idiomen zusammenführen. ${ }^{8}$ Sinnvoll sind außerdem Korpusrecherchen, die verdeutlichen, in welcher lexikalischen Umgebung, in welchem Kontext und mit welcher Sprechereinstellung das behandelte Idiom bevorzugt gebraucht wird. All diese Informationen ermöglichen laut Mellado Blanco (2009: 7), Idiome adäquat anzuwenden. ${ }^{9}$ Je mehr Informationen zu einem Idiom zusammengetragen werden und je länger sich die Lernenden damit beschäftigen, desto schneller prägen sie es sich ein und seine korrekte Anwendung wird wahrscheinlicher.

7 Vertiefend zur muttersprachlichen und fremdsprachlichen phraseologischen Kompetenz, vgl. Hallsteinsdøttir (2001).

8 Nicht zu unterschätzen sind verschiedene Applikationen wie Quizlet, die unter Studierenden häufig eingesetzt werden und zum wiederholten Lernen ebenfalls nützlich sein können.

9 Im Zusammenhang mit der lexikographischen Beschreibung von Idiomen betont Mellado Blanco: „Die Kombinatorik ist überhaupt eine der relevantesten und gleichzeitig komplexesten Fragen bei der lexikografischen Phrasembeschreibung. Damit ein Sprechakt erfolgreich wird, müssen die das Phrasem umgebenden Elemente in ihrer Semantik mit den Semen und Semenen des gegebenen Phrasems kompatibel sein, sie müssen mit ihm distributionell die Syntaxregel einhalten und pragmatisch, d. h. situationsangemessen, adäquat sein." (Mellado Blanco 2009: 7) 


\section{5 Öffnung für die eigene und die andere Kultur}

In ihrem in Kürze erscheinenden Band „Phraseologie des Deutschen für polnische Deutschlernende“ betonen Dziurewicz und Woźniak (2020): „[D]ie kontrastive Darstellung der Phraseme kann auch als Anlass zur Diskussion über Gemeinsamkeiten und Unterschiede in dem sprachlichen Weltbild der beiden Sprachkulturen gelten“. In zahlreichen deutschen Idiomen spiegeln sich die Vorlieben der Deutschsprachigen, ihre Tradition, ihre Gewohnheiten und ihre Denkweise wider. Schatte (1995: 94) machte bereits vor 25 Jahren darauf aufmerksam, dass „Phraseologismen eine in dieser Dichte in der Sprache sonst seltene Verflechtung von Sprache, Kultur und Soziokultur, von historischen und gesellschaftlichen Traditionen und Faktoren, von Leben und Dichtung“ darstellten. Aus diesem Grund kann durch die Beschäftigung mit Idiomen im deutsch-polnischen Kontrast das interkulturelle Lernen unterstützt werden. Als Beispiel hierfür kann die Einheit Schwein haben mit der Bedeutung Glück haben (pol.: mieć farta) dienen. Obgleich Deutsch und Polnisch benachbarte Sprachen darstellen, sind die der phraseologischen Bedeutung zugrundeliegenden Bildmotive nicht immer gleich (vgl. Schatte 2006: 145). Demnach ruft das Lexem Schwein im Polnischen ausschließlich pejorative Assoziationen hervor, während es im Deutschen sowohl positive (als Symbol des Glücks) als auch negative Konnotationen besitzt. Eine anfangs als rein kontrastiv gedachte Übungsform könnte an dieser Stelle im nächsten Schritt in eine Diskussion über deutsche und polnische Glücksbringer übergehen.

\subsection{Vertrautwerden mit Hilfsmitteln wie zweisprachige Wörterbücher und Korpora}

Laut Ehrhardt (2014) lassen sich durch die Vermittlung von Phrasemen zahlreiche Lernstrategien trainieren. Bei einer kontrastiven Vorgehensweise sollte insbesondere die Arbeit mit zweisprachigen (allgemeinen und phraseologischen) Wörterbüchern und mit Referenzkorpora des Deutschen und Polnischen Vorrang haben. Auf der Suche nach Äquivalenten gelten Wörterbücher als wichtigste Hilfsmittel für Lernende. Dabei benutzen diese, den Untersuchungen von Hollós (2018) und Janus (2017) zufolge, lieber Online- als Printwörterbücher.

Bei der Suche nach einer angemessenen Wiedergabe eines Idioms in der Zielsprache können zudem Korpora behilflich sein. Laut Dobrovolskij und Piirainen (2009: 161) weisen auch Einheiten, die auf den ersten Blick als völlig äquivalent zu sein scheinen, bei genauer Betrachtung Differenzen hinsichtlich der Pragmatik und Frequenz auf. Dies lässt sich am Beispiel der deutschen Einheit jmdm ein Dorn im Auge sein veranschaulichen, die in den deutsch- 
polnischen Großwörterbüchern PWN und PONS mit być komuś sola w oku wiedergegeben wird. Obgleich beide Idiome als analog zu betrachten sind, lässt sich mithilfe der Referenzkorpora ermitteln, dass sie signifikante Unterschiede bezüglich der Auftretenshäufigkeit aufweisen. Das deutsche Idiom hat 36.400 Vorkommen in DeReKo und gilt damit als relativ frequent, die polnische idiomatische Einheit tritt hingegen mit 152 Treffern im Narodowy Korpus Języka Polskiego äußerst selten auf. ${ }^{10}$

\section{Studie zu Idiomen im deutsch-polnischen Kontrast}

\subsection{Ziele und Forschungsfragen}

Im Jahre 2020 wurde unter den Studierenden der Angewandten Linguistik an der Adam-Mickiewicz-Universität in Poznań eine Untersuchung durchgeführt. Das Hauptziel der Studie bestand darin, zu überprüfen, ob die Analyse von Idiomen im deutsch-polnischen Kontrast eine Herausforderung für die Studierenden darstellt. Dabei wurde auf folgende Fragen eingegangen:

- Erkennen die Studierenden Unterschiede und Gemeinsamkeiten, die in deutschen und polnischen Idiomen auftreten?

- Welchen Einfluss hat die Äquivalenzart auf das Verstehen und Anwenden von Idiomen?

- Wie beeinflusst die Muttersprache die fremdsprachliche phraseologische Kompetenz?

- Steigt die idiomatische Kompetenz während des Studiums?

- Welche Aufgaben gelten als besonders motivierend?

- Kennen die Studierenden polnische Entsprechungen frequenter deutscher Idiome?

\subsection{Aufbau des Fragebogens}

Der Fragebogen ${ }^{11}$ bestand aus drei Teilen:

- Teil 1 umfasste allgemeine Fragen zum Alter, Studienjahr, Sprachniveau und zu der geschätzten Anzahl der geläufigen deutschen Idiome. Außerdem wurde

10 Auch wenn man die absolute Zahl der Tokens berücksichtigt, ist der Unterschied auffällig. Im polnischen Korpus gibt es 240.192.461 Tokens. 152 Treffer machen 0,00005 \% des Korpus aus. Das DeReKo umfasst dahingegen 9.840.692.470 Tokens, der Prozentanteil für das Idiom beträgt $0,004 \%$ (Stand 16.03.2020).

11 Aus Platzgründen wurden der Fragebogen und die Aufgaben an diesen Beitrag nicht angehängt. Sie können allerdings unter edziu@amu.edu.pl angefordert werden. 
danach gefragt, wie lange die Befragten bereits Deutsch gelernt haben und ob sie sich mit Idiomen ${ }^{12}$ in der Schule, an der Universität oder selbstständig beschäftigt haben. Eine Frage bezog sich schließlich darauf, ob sie dieser sprachlichen Erscheinung mehr Lernzeit als bisher widmen würden und wenn ja warum.

- Teil 2 enthielt insgesamt neun Übungen, die progressiv - beginnend bei einfachen Zuordnungs- und Ergänzungsübungen bis hin zu anspruchsvolleren offeneren Übungen am Ende - geordnet wurden. In der ersten Übung befanden sich allein Interphraseme, d.h. volläquivalente, analoge Einheiten. In den restlichen Übungen wurden Vertreter aller vier Äquivalenzarten nach Koller (2007) berücksichtigt. Zahlreiche der in den Aufgaben erfassten Idiome wurden der phraseologischen Optimumliste ${ }^{13}$ von Hallsteinsdóttir et al. (2006) entnommen, einige Übungen stammten von Dziurewicz und Woźniak (2020).

- In Teil 3 wurden die Studierenden gebeten, einzuschätzen, welche Übungen ihrer Meinung nach am schwierigsten waren und welche ihnen am besten gefallen haben. An dieser Stelle gab es auch die Möglichkeit, Kommentare zu schreiben. Nachstehend werden die Ergebnisse jedes einzelnen Teils besprochen, wobei der zweite, analytische Teil am intensivsten behandelt wird.

\subsection{Ergebnisse der Studie - Teil 1}

An der anonymen Untersuchung haben insgesamt 121 Studierende der Angewandten Linguistik an der Adam-Mickiewicz-Universität in Poznań teilgenommen. ${ }^{14}$ Der Fragebogen wurde unter Studierenden aller fünf Studienjahre (dreijähriger Bachelor- und zweijähriger Masterstudiengang) durchgeführt. Es nahmen 25 Personen aus dem ersten, 29 aus dem zweiten und 22 aus dem dritten Studienjahr des Bachelorstudiengangs sowie 18 aus dem ersten und 27 aus dem zweiten Studienjahr des Masterstudiengangs an der Studie teil.

$35 \%$ der Studierenden haben sich mit den Idiomen bisher nur an der Universität, $12 \%$ allein in der Schule, $5 \%$ nur selbstständig beschäftigt. $20 \%$ haben alle drei Antworten (in der Schule, an der Universität und selbstständig) markiert. $28 \%$ der Studierenden haben bisher noch nie Idiome behandelt.

12 Eine Definition des Idioms wurde zusammen mit einem Beispiel im Fragebogen angeführt.

13 Mit der phraseologischen Kompetenz von Germanistikstudenten befasst sich ausführlich Janus (2014), die untersucht, ob Germanistikstudenten die 142 Phraseologismen aus der Optimumliste von Hallsteinsdóttir et al. (2006) verstehen und anwenden.

14 Im Institut für Angewandte Linguistik an der Adam-Mickiewicz-Universität in Poznań gibt es zwei mögliche Studiengänge (mit Schwerpunkt Deutsch und Englisch). An der Untersuchung haben nur diejenigen mit Schwerpunkt Deutsch teilgenommen, die am Anfang des Studiums bereits über Deutschkenntnisse auf dem Niveau B1 verfügen. 
Was die geschätzte Anzahl der geläufigen Idiome anbelangt, so hat beinahe die Hälfte zwischen 10 und 50 Einheiten markiert, $30 \%$ entfallen auf 50-100 Idiome, $15 \%$ haben die Antwort weniger als 10 angekreuzt, $5 \%$ meinen, dass sie mehr als 100 Idiome kennen. Die nächste Frage bezog sich darauf, ob Studierende Idiomen mehr Lernzeit widmen würden. In diesem Zusammenhang hat sich bestätigt, dass ein Fragebogen „eine wertvolle Informationsquelle“ (Prokop \& Wiśniewska 2002: 121, übers. von ED) über Einschätzung einer bestimmten Forschungsfrage fungieren kann. ${ }^{15} 90 \%$ der Studierenden haben hierbei die Antwort $j a$ angegeben, $7 \%$ schwer zu sagen, $3 \%$ nein. Als Begründung haben die Studierenden mehrheitlich hervorgehoben, dass sie ihren Wortschatz um gängige Idiome erweitern wollen, da diese einen wichtigen Teil der Sprache bildeten. ${ }^{16}$

\subsection{Ergebnisse der Studie - Teil 2 und 3}

a) Die erste Aufgabe bestand in der Ergänzung der nominalen Komponenten in deutschen und polnischen Idiomen, bei denen allein Interphraseme mit Körperteilbezeichnungen vorhanden waren. $92 \%$ aller beteiligten Studierenden haben die Übung fehlerfrei gelöst. 8 \% (im ersten Bachelorstudienjahr) haben in 14 Beispielen zwei bis vier Fehler gemacht. Fehlerfrei wurden folgende Idiome ergänzt: aus erster Hand, den Kopf in den Sand stecken,jmds. rechte Hand sein, alle/beide Hände voll zu tun haben. Bei den Einheiten jmdm. freie Hand lassen, von Kopf bis Fuß, mit dem Kopf durch die Wand gehen/rennen tauchten hingegen vereinzelt Fehler auf.

b) Bei der zweiten Übung sollten die Studierenden die richtige polnische Entsprechung eines deutschen Idioms unter drei angegebenen Varianten finden. Es zeichnete sich die Tendenz ab, dass sie im Falle der volläquivalenten Einheiten kaum Schwierigkeiten hatten, die richtige Antwort anzukreuzen (90\% der Studierenden wählten bei jmdm. auf die Nerven gehen die richtige Antwort działać komuś na nerwy). Problematischer waren diejenigen Fälle, bei denen divergierende Bildlichkeit vorhanden war, z. B. reinen Wein einschenken (45\% der richtigen Antworten). Die meisten Fehler liegen bei den Idiomen vor, die keine idiomatische Entsprechung im Polnischen haben wie

15 Prokop und Wiśniewska (2002, S. 121 f.) verweisen in ihrem Beitrag nicht nur auf die Vorteile eines Fragebogens, sondern befassen sich auch mit seinen Nachteilen.

16 Zur Bestätigung werden hierbei vier Aussagen angeführt: 1 . „Weil dadurch die Art und Weise wie ich mich ausdrücke abwechslungsreicher ist“; 2 . „Idiome sind wichtig, Deutsche nutzen sie oft“; 3. „Weil ich denke, dass sie in der deutschen Sprache oft benutzt werden“. 4. „Idiome werden in der Umgangssprache oft verwendet“. 
etw. auf die Beine stellen, sich ins Zeug legen, rote Zahlen schreiben. Hierbei wählte lediglich ein Drittel der Studierenden die richtige Antwort.

c) Die dritte Aufgabe setzte sich aus drei Teilen zusammen. Zuerst sollten die Studierenden ein passendes Bild zu einem vorgegebenen Idiom finden, dann dessen Bedeutung erkennen und zuletzt dessen polnische Entsprechung angeben. Während mit den ersten beiden Teilen die meisten Teilnehmenden (89 \%) keine Probleme hatten, erwies sich der dritte Teil als problematisch, insbesondere für Studierende im ersten und zweiten Bachelorstudienjahr. Unter den sechs deutschen Idiomen waren lediglich die zwei folgenden unproblematisch: Bei Schlange stehen nannten $87 \%$ die richtige polnische Entsprechung (stać $w$ kolejce). Das Idiom Schwein haben erkannten $82 \%$ korrekt, wobei die Wendung am häufigsten mit mieć fart(a) (90\%) wiedergegeben wurde, gefolgt von być w czepku urodzonym (8\%) und wygrać los na loterii (2\%). Die Frage danach, warum die beiden keine Schwierigkeiten bereiteten, lässt sich nur schwer beantworten. Möglicherweise ist dies auf ihre hohe Frequenz im Deutschen zurückzuführen. Das Idiom mit jmdm. Katz und Maus spielen wurde ebenfalls von vielen Studierenden (78\%) korrekt übertragen. Dies war höchstwahrscheinlich unproblematisch, weil im Polnischen eine analoge Einheit (bawić się z kimś w kotka i myszkę) existiert. Die mehrheitliche Kenntnis (71 \%) der Einheit aus einer Mücke einen Elefanten machen ergibt sich womöglich aus der nachvollziehbaren Motiviertheit. $\mathrm{Zu}$ den Problemfällen in dieser Aufgabe gehörten zwei Idiome: Sei kein Frosch! und für die Katz sein. Nur ca. ein Fünftel der Studierenden im ersten Bachelorstudienjahr hat in diesem Fall die richtige Entsprechung ergänzt. Die Masterstudierenden schnitten je um ca. 10 und 15 Prozentpunkte besser ab. Der häufigste Fehler lag in der wörtlichen Übersetzung einzelner Komponenten (nie badźżabą und być dla kota). ${ }^{17}$ Bei den korrekten Entsprechungen wurden im Falle der Einheit für die Katz sein sowohl bildhafte psu na budę als auch nicht übertragene Wiedergaben wie być zbędnym genannt.

d) In der vierten Aufgabe wurden die Studierenden gebeten für neun deutsche Idiome polnische Entsprechungen anzugeben und Bestandteile $\mathrm{zu}$ unterstreichen, die sich in beiden Sprachen wiederholen. Zwei analoge Idiome die Katze im Sack kaufen und grünes Licht für etw. geben waren für $92 \%$ aller Studierenden unproblematisch. Auch im Falle der Einheit auf den Hund kommen, die eine geringfügige morphosyntaktische Differenz aufweist, hatten die meisten Studierenden (76 \%) keine Schwierigkeiten.

Unter neun Beispielen gab es zwei Idiome, zwei Fliegen mit einer Klappe

$17 \mathrm{Ob}$ das Bild, das die wortwörtliche Bedeutung illustriert für einige Lernende irreführend sein kann, bedarf einer eingehenden Analyse. Auf Grundlage einer Aufgabe lässt sich das nicht schlussfolgern. 
schlagen und ein Haar in der Suppe finden, die sich durch SubstitutionsÄquivalenz auszeichnen und demnach durch eine divergente Bildhaftigkeit gekennzeichnet sind. Während mit der ersten Einheit die Mehrheit (66 \%) keine Probleme hatte, stellte die zweite für ca. die Hälfte (48 \%) ein Problem dar.

Als äußerst problematisch können die Idiome in den sauren Apfel beißen und das beste Pferd im Stall sein gelten, die über keine idiomatische Entsprechung im Polnischen verfügen und bei denen nur ein Fünftel aller Studierenden (davon entfallen beinahe $90 \%$ auf die Studierenden im Master) eine korrekte Wiedergabe im Polnischen vorgeschlagen hat.

Die schwierigsten Einheiten sind ohne Zweifel die falschen Freunde unter der Hand und ein Auge auf jmdn. werfen, die von $98 \%$ fehlerhaft wiedergegeben oder gar nicht ergänzt wurden. Nur drei Studierende des zweiten Masterstudienjahres haben sie richtig erkannt. Das beruht sicherlich darauf, dass das polnische Idiom rzucić okiem (dt.: ein Auge werfen) hochfrequent und seine Struktur der deutschen ähnlich ist, weshalb die polnische Bedeutung des Idioms fehlerhaft auf das Deutsche übertragen wird. ${ }^{18}$ Dasselbe passierte im Falle des Somatismus unter der Hand. Im Polnischen bedeutet pod ręką: nah bzw. erreichbar, während es im Deutschen mit heimlich oder inoffiziell eine völlig andere Bedeutung hat. Die hohe Fehlerquote im Falle der falschen Freunde beweist, dass man dieser sprachlichen Erscheinung mehr Aufmerksamkeit im Studium schenken sollte.

e) In der fünften Aufgabe sollten die Studierenden die Äquivalenz zwischen deutschen idiomatischen bzw. teilidiomatischen Vergleichen und ihren polnischen Pendants bestimmen. Bei den teiläquivalenten Ausdrücken sollten die Studierenden zusätzlich erklären, worin die Teiläquivalenz besteht. Um die Aufgabe zu erleichtern, wurde ein Beispiel für diese kontrastive Analyse gegeben. Unter acht Vergleichen waren sechs als teiläquivalent einzustufen. Dies wurde von $38 \%$ (davon entfielen $70 \%$ auf die Masterstudierenden) richtig erkannt. $32 \%$ machten im ersten Teil der Übung einen bis zwei Fehler, $28 \%$ drei oder mehr.

Der zweite Teil dieser Aufgabe bereitete beinahe der Hälfte aller Studierenden (49 \%) Probleme. Viele Lücken wurden nicht ergänzt. Als relativ unproblematisch galten Vergleiche wie: jmd. lügt wie gedruckt, etw. passt wie die Faust aufs Auge und jmd. redet wie ein Wasserfall, bei denen die Unterschiede mehrheitlich richtig erklärt wurden. Überraschenderweise hatten die meisten Studierenden Probleme mit dem Vergleich etw. wie seinen Augapfel hüten. Dies lässt sich möglicherweise damit begründen, dass ihnen das Verb hüten und das Substantiv Augapfel nicht geläufig waren. Ebenfalls die Einheiten

18 Weiterführendes zur Interferenz bei polnischen Germanistikstudenten vgl. Podgórni (2010). 
jmd. lebt wie die Made im Speck und jmd. ist jmdm. wie aus dem Gesicht geschnitten wurden selten ( $23 \%)$ korrekt mit ihren polnischen Entsprechungen assoziiert.

f) In der sechsten Aufgabe sollten die Studierenden ausgewählte derbe und vulgäre Idiome ihren polnischen Entsprechungen gegenüberstellen, um Gemeinsamkeiten und Unterschiede zu finden. Nur $30 \%$ der Studierenden versuchten, diese Aufgabe zu lösen. Am häufigsten wurde von Masterstudierenden unterstrichen, dass die Komponente Arsch sich in beiden Sprachen wiederholt. Außerdem hatte $15 \%$ der Studierenden den Eindruck, dass die polnischen Entsprechungen vulgärer als die deutschen Ausgangseinheiten klingen.

g) In der siebten Aufgabe sollten die Studierenden polnische Sätze ins Deutsche übersetzen und dabei ein Idiom benutzen, dessen nominale Komponente in Klammern angegeben wurde. Die Übersetzungsaufgaben (d.h. die 7. und 8. Aufgabe) wurden von den meisten Studierenden (78\%) als schwierigste Aufgaben bewertet. Obwohl der erste Satz das volläquivalente Idiom schwarzes Schaf enthielt, versuchten $76 \%$ des ersten und zweiten Bachelorstudienjahres überhaupt nicht, ihn zu übersetzen. Eine korrekte Übersetzung haben nur $18 \%$ der Studierenden geliefert (davon $90 \%$ Masterstudierende). Der zweite Satz enthielt das teiläquivalente Idiom jmdm. die Daumen drücken und wurde ebenfalls mehrheitlich entweder fehlerhaft oder gar nicht übersetzt $(83 \%)$. Die meisten Fehler gingen auf die Muttersprache zurück: die Studierenden wählten das falsche Verb (halten statt drücken). Der dritte Satz (Ledwo wiąża koniec $z$ końcem) mit dem Idiom (sich über Wasser halten) der Substitutions-Äquivalenz angehört, haben nur $13 \%$ zu ergänzen versucht, wobei davon ein Viertel erfolgreich war.

h) In der achten Aufgabe sollten die Studierenden die deutschen Sätze ins Polnische übersetzen. Mit dem ersten Satz, der das in beiden Sprachen gebräuchliche Idiom Mir sind die Hände gebunden enthielt, hatte die Mehrheit der Studierenden (73 \%) keine Übersetzungsprobleme. Auch der zweite Somatismus sich etw. zu Herzen nehmen bereitete den meisten (69\%) keine Schwierigkeiten. Als das komplizierteste Idiom in dieser Aufgabe erwies sich das dritte, etw. unter einen Hut bringen, für das keine idiomatische Entsprechung im Polnischen zur Verfügung steht und das sich mithilfe einer monolexikalen Struktur pogodzić coś ze soba wiedergeben lässt. Dies haben lediglich $13 \%$ der Studierenden gewusst.

i) In der letzten, der neunten Aufgabe handelte sich um eine offene Übungsform, bei der die Studierenden deutsche und polnische Beispiele für Idiome zu bestimmten thematischen Gruppen (darunter z.B. Dummheit, Sterben) angeben sollten. Danach sollten sie die genannten Einheiten hinsichtlich der Analogien und Kontraste analysieren. Lediglich $20 \%$ haben versucht, in 
mindestens einer Kategorie Idiome zu nennen. Bei dem Thema Dummheit wurde im Deutschen dumme Gans zwölfmal und im Polnischen der Vergleich głupi jak but 14-mal genannt. Im thematischen Bereich Sterben wurde im Deutschen vereinzelt ins Gras beißen (fünfmal), im Polnischen kopnać w kalendarz (20-mal), wachać kwiatki od spodu (siebenmal) angegeben.

Generell ist anzumerken, dass einige Studierende polnische Idiome fehlerhaft zitierten. Vereinzelt haben sie das falsche Verb uderzyć statt kopnać in der Einheit kopnaćc w kalendarz eingesetzt. Dies ist auf Defizite im Bereich der muttersprachlichen phraseologischen Kompetenz zurückzuführen

Den letzten, dritten Teil des Fragebogens stellte die Selbsteinschätzung dar. Als schwierigste Aufgaben nannten Studierende die neunte, gefolgt von der achten und der siebten Aufgabe. Einige Studierende (12\%) schrieben, dass alle Übungen schwierig waren. Am besten gefielen ihnen die Aufgaben: 3 (80\%), 2 (70\%) und 5 $(60 \%) .^{19}$

\section{Schlussfolgerungen und Postulate}

Für die universitäre Didaktik im Bereich der kontrastiven Phraseologie im engeren Sinne ergeben sich aus dem bereits Dargestellten folgende Schlussfolgerungen:

- im Falle der volläquivalenten Idiome bereitet die kontrastive Analyse den meisten Studierenden keine allzu großen Schwierigkeiten. Als Problemfälle erwiesen sich Einheiten, bei denen es entweder im Polnischen kein analoges Idiom mit dem gleichen Bild gibt (Substitutions-Äquivalenz), oder keine idiomatische Entsprechung vorhanden ist (Nulläquivalenz).

- die muttersprachliche phraseologische Kompetenz beeinflusst die fremdsprachliche. Dies ist im Falle der Interphraseme positiv zu bewerten, bei teilidiomatischen Einheiten dagegen kann es zu einem negativen Transfer führen (vgl. dazu den häufig auftretenden Interferenzfehler, der jmdm. die Daumen halten). Die eindeutig höchste Fehlerquote lag bei den falschen Freunden: scheinbare Ähnlichkeiten mit den polnischen Idiomen wurden auf die deutschen Einheiten übertragen.

- was den Fortschritt im Laufe des Studiums anbelangt, konnte man zwischen Studierenden im ersten und zweiten Bachelorstudienjahr keine signifikanten Unterschiede beobachten, während sie beim Vergleich von Bachelor- und Masterstudierenden auffällig waren. Die Durchschnittswerte im Test zeigten einen kontinuierlichen Fortschritt, wobei Masterstudierenden mit Abstand die

19 Disproportionen ergeben sich daraus, dass hierbei Mehrfachnennungen möglich waren. 
besten Resultate erzielten (Durchschnittlich lösten sie $67 \%$ der Aufgaben richtig, Studierende des ersten Bachelorstudienjahres hingegen lediglich $23 \%$ ).

- als besonders motivierend galten die Übung mit Bildern und Multiple-ChoiceÜbung, als schwierig erwiesen sich dagegen kontrastive Übersetzungsübungen und offene Übungsformen.

- es stellte sich ebenfalls heraus, dass Studierende bisweilen mit muttersprachlichen Idiomen Probleme haben. An einigen Stellen haben sie falsche Verben benutzt. Außerdem waren sie mehrheitlich nicht imstande, polnische Idiome im Zusammenhang mit jemandes Dummheit oder Tod anzugeben, was auf klare Defizite im Bereich der muttersprachlichen phraseologischen Kompetenz hinweist.

Zusammenfassend lässt sich feststellen, dass eine kontrastive (deutsch-polnische) Analyse von Idiomen eine Herausforderung für die meisten Studierenden der Angewandten Linguistik an der Adam-Mickiewicz-Universität in Poznań darstellt. Um diesem Problem entgegenzuwirken, werden abschließend einige Postulate für universitäre Didaktik (im Falle der Germanistik und Angewandten Linguistik) formuliert:

\subsection{Sukzessiver Aufbau phraseologischer Kompetenz im Rahmen der universitären Didaktik}

Angesichts dieser Problematik ist Bergerova (2009: 75) zuzustimmen, die feststellt: „Phraseologismen sollten nicht gelegentlich in speziell dafür vorgesehenen Einzelstunden behandelt werden, sondern bei jeder sich bietenden Gelegenheit: Wenn ein Text/eine Kommunikationssituation dafür einen günstigen Ansatz liefert". Deshalb sollten Phraseme nicht nur während des Seminars zur Phraseologie, sondern auch in Veranstaltungen zur Übersetzung und im allgemeinen Sprachunterricht behandelt werden. Ich plädiere dafür, frequente und geläufige Idiome ab dem Niveau B2 (am besten von Anfang an kontrastiv ${ }^{20}$ ) in alle universitäre Lehrveranstaltungen, die für Studierende der Germanistik und der Angewandten Linguistik angeboten werden, einzubeziehen. Die Auswahl von Übungen und Aufgaben sowie ihre Anzahl hängt von vielen Faktoren ab (wie z. B. Thema, Bedürfnisse der Lernenden, Zeit, die zur Verfügung steht).

20 Dadurch wird die interkulturelle Kommunikation, die heutzutage als eine äußerst relevante Kompetenz in der Didaktik angesehen wird, gefördert (Mackiewicz et al. 2019). 


\subsection{Vorbereitung auf selbstständiges und lebenslanges Lernen}

Im Rahmen des universitären DaF-Unterrichts sollten Studierende mit unterschiedlichen Hilfsmitteln (wie Wörterbücher, Lehrwerke zur Phraseologie, Online-Plattformen ${ }^{21}$ ) vertraut gemacht werden, die dabei helfen, Phraseme eigenständig zu erlernen. Zu Recht verweisen Lüger und Zenderowska-Korpus (2018: 15) darauf, dass „das gesteuerte Lernen immer auch der Ergänzung und der Fortsetzung durch selbständiges Weiterlernen [bedarf]“. Außerdem sollten die Lernenden dafür sensibilisiert werden, dass Phraseologie ein äußerst heterogenes Feld ist und sie sich intensiv mit dieser Thematik beschäftigen sollten, um ihr individuelles Idiominventar nach und nach zu erweitern.

\subsection{Erstellung innovativer und abwechslungsreicher (darunter auch kontrastiver) phraseologischen Materialien ${ }^{22}$}

Didaktische Konzepte zur Einbindung von Phrasemen in den DaF-Unterricht sind nur in beschränktem Ausmaß vorhanden. Diesem Problem wird für das Sprachenpaar Deutsch und Polnisch in Dziurewicz und Woźniak (2020) Rechnung getragen. Bei der Erstellung phraseodidaktischer (darunter auch kontrastiver) Materialien sollten neue Medien integriert werden. Des Weiteren hat die durchgeführte Analyse bestätigt, dass Bilder als Motivationsfaktor fungieren. Idiome sollten schließlich durch authentische Texte (Artikel in der Presse, Werbung, Lieder, Filme) vermittelt werden. Erstens erfahren Deutschlernende dadurch, dass sie einen integralen Teil der Sprache bilden und zweitens spiegeln sie im Gegensatz zu selbstkonstruierten Texten den realen Sprachgebrauch wider.

\subsection{Zielgruppen- und sprachniveau-orientierte Vorgehensweise}

Je nach Zielgruppe und Sprachniveau ergeben sich jeweils andere Prioritäten bei der Vermittlung von Idiomen innerhalb der universitären Didaktik. Während im ersten Studienjahr des Bachelors Idiome nicht so intensiv behandelt werden müssen, können ab dem zweiten Studienjahr nach und nach mehr Übungen angeboten werden. Bei den stärker interessierten Lernenden bzw. Gruppen ist es

21 Gemeint sind in erster Linie Ephras (http://demo.ephras.org/uebung.html) und SprichwortPlattform (http://www.sprichwort-plattform.org/sp/\%C3\%9Cbungen).

22 Dieses Postulat knüpft an eines der von Hallsteindøttir (2011) genannten Desiderate innerhalb der Phraseodidaktik an. 
empfehlenswert, von Anfang an idiomatische Kompetenz systematisch und intensiv zu entwickeln.

Es wird davon ausgegangen, dass bei Beachtung und Einhaltung dieser vier Postulate die phraseologische Kompetenz im Polnischen und Deutschen dauerhaft gestärkt wird und in der Folge auch langfristig Fortschritte zu beobachten sein werden.

\section{Literaturverzeichnis}

Bergerová, Hana (2009). Wie viel Phraseologie brauchen die künftigen Deutschlehrenden? In: L. Spáčilová/L. Vaňková (Hrsg.), Germanistische Linguistik und die neuen Herausforderungen in Forschung und Lehre in Tschechien (S. 71-80). Brno: Academicus.

Burger, Harald (2015): Phraseologie. Eine Einführung am Beispiel des Deutschen. Berlin: Erich Schmidt.

Dobrovol'skij, Dmitrij/Piirainen, Elisabeth (2009). Zur Theorie der Phraseologie. Kognitive und kulturelle Aspekte. Tübingen: Stauffenburg.

Dziurewicz, Elżbieta (2018). Didaktisierung von Phraseologismen im DaF-Unterricht Probleme, Übungsvorschläge, Anforderungen. In: A. Gondek/A. Jurasz/J. Szczęk (Hrsg.), Einblicke und Rückblicke: Beiträge zur deutschen Phraseologie und Parömiologie aus intra- und interlingualer Sicht Bd. II (= Phraseologie und Parömiologie, Band 34) (S. 307-321). Baltmannsweiler: Schneider.

Dziurewicz, Elżbieta/Woźniak, Joanna (2020): Phraseologie des Deutschen für polnische Deutschlernende. Warszawa: Wydawnictwo Naukowe PWN.

Ehrhardt, Claus (2014). Idiomatische Kompetenz: Phraseme und Phraseologie im DaFUnterricht. http://www.gfl-journal.de/1-2014/Ehrhardt.pdf.

Erhardt, Claus (2007). Phraseme in der Jugendsprache. In: H. Burger/D. Dobrovol'skij/P. Kühn/N.R. Norrick (Hrsg.), Phraseologie: Ein internationales Handbuch der zeitgenössischen Forschung (S. 253-264). Berlin, New York: De Gruyter.

Ettinger, Stefan (2011). Einige kritische Fragen zum gegenwärtigen Forschungsstand der Phraseodidaktik. In:. P. Schäfer/C. Schowalter (Hrsg.), In mediam linguam. Mediensprache - Redewendungen - Sprachvermittlung. Festschrift für Heinz-Helmut Lüger (S. 231-250). Landau: Empirische Pädagogik.

Fleischer, Wolfgang (1982). Phraseologie der deutschen Gegenwartssprache. Tübingen: De Gruyter.

Földes, Csaba/Kühnert, Helmut (1990). Hand- und Übungsbuch zur deutschen Phraseologie. Budapest: Tankönyvkiadó.

Guławska-Gawkowska, Małgorzata (2012). Aspekte im Vergleich von deutschen und polnischen Phraseologismen. In: G. Pawłowski/M. Olpińska-Szkiełko (Hrsg.), MenschSprachen-Kulturen. Beiträge und Materialen der internationalen wissenschaftlichen Jahrestagung des Verbandes Polnischer Germanisten, 25.-27. Mai 2012 (S. 81-101). Warszawa: Euro Edukacja. 
Hallsteinsdóttir, Erla (2011). Aktuelle Forschungsfragen der deutschsprachigen Phraseodidaktik. Linguistik online 47, 2011. 3-31. http://www.linguistik-online.de/47_11/hall steinsdottir.html.

Hallsteinsdóttir, Erla/Šajánoková, Monika/Quasthoff, Uwe (2006). Phraseologisches Optimum für Deutsch als Fremdsprache. Ein Vorschlag auf der Basis von Frequenz- und Geläufigkeitsuntersuchungen. Linguistik online 27, 2006. 117-136. https://www.linguis tik-online.net/27_06/hallsteinsdottir_et_al.pdf.

Hallsteinsdóttir, Erla (2001). Das Verstehen idiomatischer Phraseologismen in der Fremdsprache Deutsch. Hamburg: Kovač.

Hollós, Zita (2018). Wörterbuchkritik - phraseologische Wörterbücher. In: M. Bielińska/ S. Schierholz (Hrsg.), Wörterbuchkritik. Dictionary criticism (S. 323-352). Berlin/New York: De Gruyter.

Jesenšek, Vida (2006). Phraseologie und Fremdsprachenlernen. Zur Problematik einer angemessenen phraseodidaktischen Umsetzung. Linguistik online 27, 2006. 137-147. http://www.linguistik-online.de/27_06/jerensek.html.

Janus Dominika (2017). ,Erlernen, wo nachzuschlagen ist ${ }^{`}$ - einige Bemerkungen zur phraseologischen Kompetenz polnischer Germanistikstudenten. In: Z. Berdychowska/ H.H. Lüger/Cz. Schatte/G. Zenderowska-Korpus (Hrsg.), Phraseologie als Schnittstelle von Sprache und Kultur II. Öffentlicher Raum - Medien - Phraseodidaktik (S. 185-200). Frankfurt a.M.: Peter Lang.

Janus, Dominika (2014). Zur phraseologischen Kompetenz polnischer Germanistikstudierender. In: K. Lukas/I. Olszewska (Hrsg.), Deutsch im Kontakt und Kontrast. Festschrift für Prof. Andrzej Kątny zum 65. Geburtstag (Z. 313-326). Frankfurt a.M.: Peter Lang.

Kahl, Stefanie (2015). Kontrastive Analyse zu phraseologischen Somatismen im Deutschen und Italienischen. Bamberg: University of Bamberg Press.

Koller, Werner (2007). Probleme der Übersetzung von Phrasemen. In: H. Burger/D. Dobrovol'skij/P. Kühn/N.R. Norrick (Hrsg.), Phraseologie. Ein internationales Handbuch zur zeitgenössischen Forschung. Bd. 1 (S. 601-613). Berlin [u. a.]: De Gruyter.

Kühn, Peter (1992). Phraseodidaktik. Entwicklungen, Probleme und Überlegungen für den Muttersprachenunterricht und den Unterricht Deutsch als Fremdsprache Fremdsprachen Lehren und Lernen 21, 1992, 169-186.

Lorenz-Bourjot, Martine/Lüger, Heinz-Helmut (2001). Phraseologie und Phraseodidaktik. Wien: Praesens.

Lüger, Heinz-Helmut (1997). Anregungen zur Phraseodidaktik. Beiträge zur Fremdsprachenvermittlung, 32, 69-120.

Lüger, Heinz-Helmut (2019). Phraseologische Forschungsfelder. Impulse, Entwicklungen und Probleme aus germanistischer Sicht. Beiträge zur Fremdsprachenvermittlung, 61, 51-82.

Lüger, Heinz-Helmut/Zenderowska-Korpus, Grażyna (2017). Phraseologie - Fragen des Gebrauchs und der Vermittlung. In: Z. Berdychowska/H.-H. Lüger/Cz. Schatte/G. Zenderowska-Korpus (Hrsg.), Phraseologie als Schnittstelle von Sprache und Kultur IAbgrenzungen - sprach- und textvergleichende Zugänge (S. 7-19). Frankfurt a.M.: Peter Lang. 
Mackiewicz, Maciej/Wilczyńska, Weronika/Krajka, Jarosław (2019). Komunikacja interkulturowa. Wprowadzenie. Poznań: Wydawnictwo Naukowe Uniwersytetu im. Adama Mickiewicza.

Mellado Blanco, Carmen (2009). Theorie und Praxis der idiomatischen Wörterbücher. Tübingen: Max Niemeyer.

Palm, Christine (1997). Phraseologie. Eine Einführung. Tübingen: Gunter Narr.

Podgórni, Halina (2010). Interferenzbedingte Sprachfehler im lexikalischen und grammatischen Subsystem des Deutschen bei polnischen Germanistikstudenten. Kraków: Wydawnictwo Uniwersytetu Jagiellońskiego.

Prokop, Izabela/Wiśniewska, Danuta (2002). Metodologia i narzędzia badawcze zastosowane w projekcie. In: W. Wilczyńska (Hrsg.), Autonomizacja $w$ dydaktyce języków obcych. Doskonalenie się w komunikacji ustnej (S. 109-128). Poznań: Wydawnictwo Naukowe Uniwersytetu im. Adama Mickiewicza.

Schatte, Czesława (1995). Kriterien für die Auswahl von Phraseologismen für den Unterricht Deutsch als Fremdsprache. In: J. Iluk (Hrsg.), Aspekte der Wortschatzbeschreibung für Zwecke des Fremdsprachenunterrichts (S. 93-105). Katowice: Wydawnictwo Uniwersytetu Śląskiego.

Schatte, Czesława (2006), Zu Interphraseologismen in der Mehrsprachigkeitsdidaktik. Studia Germanica Gedanensia 14, 143-156.

Schmale, Günter (2010). Ist ein idiomatischer Ausdruck immer expressiv? Korpusbasierte und fragebogengestützte Beobachtungen zu einer verbreiteten Prämisse. In: K. Kuiper (Hrsg.), Yearbook of Phraseology I (S. 97-124). Berlin/New York: De Gruyter. 
Open-Access-Publikation im Sinne der CC-Lizenz BY-NC-ND 4.0

(C) 2020, Vandenhoeck \& Ruprecht GmbH \& Co. KG, Göttingen ISBN Print: 9783847111863 - ISBN E-Lib: 9783737011860 


\section{Teil 3: Interkulturalität}


Open-Access-Publikation im Sinne der CC-Lizenz BY-NC-ND 4.0

(C) 2020, Vandenhoeck \& Ruprecht GmbH \& Co. KG, Göttingen ISBN Print: 9783847111863 - ISBN E-Lib: 9783737011860 


\title{
Interkulturelle Kommunikation und Angewandte Linguistik: Zur Positionierung der beiden Disziplinen und ihrer Studienschwerpunkte unter Berücksichtigung der neuen Reform im polnischen Hochschulwesen
}

\begin{abstract}
Intercultural communication and applied linguistics - On the parallels between the areas of study in the light of the latest reform in Polish higher education

The article aims to discuss the problems of introducing intercultural issues in the study programmes of applied linguistics at Polish universities. The first part presents a systematic reflection on the key concepts relevant to the fields of intercultural communication and applied linguistics. The second part focuses on the intended learning outcomes in the academic subject Intercultural Communication. They are presented with the use of descriptors suggested in the Polish qualification framework for higher education (the seventh level) and confronted with the objectives developed by the present authors for teaching of the subject Intercultural Communication at university. Finally, some conclusions are drawn regarding the importance of intercultural communication in the studies of applied linguistics, particularly in the light of the latest reform in the Polish higher education system.
\end{abstract}

\section{Keywords}

intercultural communication, applied linguistics, descriptors used in the Polish qualification framework for higher education, learning outcomes

\section{Einleitung}

Infolge des interkulturellen Booms der 1990er Jahre und der daraus resultierenden nicht immer wissenschaftlichen, sondern eher „euphorischen“ und populistischen Behandlung der interkulturellen Problematik, neigte man dazu,

1 Der Beitrag ist eine Würdigung der sprachwissenschaftlichen Leistungen und des universitären Lebenswerks (d. h. eines dynamischen personellen und wissenschaftlichen Ausbaus vom Institut für Angewandte Linguistik an der Adam-Mickiewicz-Universität in Poznań) von Izabela Prokop, der wir aus Anlass ihres 70. Geburtstags viel Gesundheit und Lebensfreude sowie weiterhin viel Schaffenskraft wünschen. 
Interkulturalität, darunter auch Interkulturelle Kommunikation ${ }^{2}$ als ein Modewort/einen Modebegriff zu bezeichnen. Der Fall des Eisernen Vorhangs, der den Beginn einer Veränderung der politischen Landschaft in Europa mit sich brachte, die allmähliche Ausdehnung der Europäischen Union und des Schengen-Raums auf weitere Mitgliedstaaten, neue Wirtschaftsbündnisse zwischen den Ländern Europas und der Welt ermöglichten verstärkte Kontakte zwischen Vertretern verschiedener Kulturen. Mit dem Aufeinanderprallen unterschiedlicher Kulturen wurden Menschen in verschiedenen Ländern nicht nur im Zuge der friedlichen Veränderungen konfrontiert, sondern auch der krisenhaften Ereignisse der letzten Dekade, von denen beispielsweise die angespannte Situation in der Ukraine und der Bürgerkrieg in Syrien zu erwähnen sind, die zu einem Zustrom von Einwanderern, Flüchtlingen und Asylsuchenden in die EU-Mitgliedsstaaten beitrugen. Da die Verständigung zwischen Menschen mit unterschiedlichen kulturellen Wurzeln heutzutage ein unausweichlicher Prozess ist und viele Lebensbereiche ${ }^{3}$ betrifft, ist „die Verwendung und Bedeutung des Begriffs Interkulturelle Kommunikation jedoch nicht einfach ein Modewort" (BroszinskySchwabe 2011: 11). Inzwischen ist dieser Terminus zu einem festen Bestandteil vieler wissenschaftlicher Disziplinen geworden, darunter auch einer breit gefassten modernen Sprachwissenschaft. Interkulturelle Kommunikation als Fach nimmt einen besonderen Platz auch im Studium der angewandten Linguistik ein, das zum Ziel hat, angehende Fremdsprachenlehrer/innen und Übersetzer/innen möglichst professionell auszubilden. Damit ist die gründliche Vorbereitung nicht nur im Bereich der Sprach- und Fachkompetenz gemeint, sondern auch im Bereich der interkulturellen Kompetenz, weil sowohl Fremdsprachenlehrer/ innen als auch Übersetzer/innen Vermittler/innen zwischen zumindest zwei Kulturen sind. Von ihrer Vorbereitung auf diese Berufe hängt die Qualität der von ihnen in der Zukunft ausgeführten Tätigkeit ab.

Dieser Beitrag verfolgt entsprechend das Ziel, sich der Problematik der Kategorie Interkulturalität im Studium der Angewandten Linguistik an polnischen Universitäten anzunähern. In den nächsten zwei Abschnitten, die in die gesamte Thematik einführen sollen, werden systematisierende Überlegungen $\mathrm{zu}$ den Schlüsselbegriffen des Beitrags Interkulturelle Kommunikation und Angewandte Linguistik angestellt. Nach der Bestimmung der Fachtermini werden im vierten Teil die wichtigsten Lernergebnisse beim Studienfach „Interkulturelle Kommu-

2 Im vorliegenden Beitrag werden die Begriffe Interkulturelle Kommunikation und Interkulturelle Interaktion synonymisch verwendet, ohne auf die Unterschiede zwischen Kommunikation und Interaktion einzugehen. In diesem Artikel werden die Namen der Interkulturellen Kommunikation und der Angewandten Linguistik mit Absicht groß geschrieben.

$3 \mathrm{Zu}$ den Praxisfeldern der Interkulturellen Kommunikation gehören z. B.: Management, Entwicklungshilfe, akademischer Bereich, Schule, Sozialarbeit, Personalberatung, (Außen)Politik und Tourismus (vgl. Maletzke 1996: 9-12; Lüsebrink 2005: 5; Erll \& Gymnich 2007: 5). 
nikation" erfasst, die anhand der Deskriptoren des Polnischen Qualifikationsrahmens für Hochschulwesen formuliert und mit den von den Autorinnen herausgearbeiteten Zielsetzungen zur Vermittlung der interkulturellen Kompetenz bei angehenden Fremdsprachenlehrenden und Übersetzer/innen sowie Dolmetscher/innen verglichen wurden. Abschließend wird ein Fazit in Bezug auf die Verortung und Stellung Interkultureller Kommunikation im Studium der angewandten Linguistik nach der neuen Reform im polnischen Hochschulwesen gezogen.

\section{Interkulturelle Kommunikation: Erfassungsversuch}

Interkulturelle Kommunikation ist ein uraltes und zugleich ein relativ neues Phänomen. Sie ist etwas, was wohl alle Erdenbewohner in ihrem Leben zwar erlebt haben, sie sind aber oft nicht imstande, es zu erläutern. Begegnungen zwischen Menschen mit unterschiedlichem kulturellen Hintergrund gab es immer, obwohl diese Kontakte erst vor kurzem mit der Bezeichnung Interkulturelle Kommunikation versehen wurden. Dieser Begriff erschien erstmals Ende der 1950er Jahre in den Vereinigten Staaten dank der ethnologischen Studie „The Silent Language“ von Edward T. Hall (1959), der zum „Stammvater“ (vgl. Broszinsky-Schwabe 2011: 16) der Interkulturellen Kommunikation erklärt wurde. In den kommenden Jahren fand Interkulturelle Kommunikation Eingang in die öffentliche Diskussion, um schließlich zum Gegenstand eines eigenständigen sich rasch entwickelnden Forschungszweiges und zur Teildisziplin vieler Wissenschaftsdisziplinen zu werden. Es sei darauf verwiesen, dass die inter- und multikulturellen Alltagserfahrungen der Menschen die Basis der neuen Disziplin bildeten, die sich zum Ziel gesetzt hat, den Verlauf der Interkulturellen Kommunikation wissenschaftlich zu erforschen, die Gründe der möglichen Spannungen und Missverständnisse $\mathrm{e}^{4} \mathrm{zu}$ diagnostizieren, darzulegen und an Beispielen $\mathrm{zu}$ veranschaulichen sowie die Hinweise des effektiven Umgangs mit ihnen (die wirkungsvollen Konfliktlösungsstrategien) zusammenzustellen.

Da Interkulturelle Kommunikation als „ein Fall unter vielen im Bereich zwischenmenschlicher Kommunikation betrachtet werden kann" (Feuerstein 2001: 203), wird zuerst der Versuch unternommen, die Kommunikation im Allgemeinen zu beschreiben, ohne auf die kulturellen Unterschiede der Kommunikationspartner explizit einzugehen. Anhand ausgewählter eng gefasster

4 Aleksandrowicz-Pędich (2005: 15-16) stellt die Liste der am meisten auftretenden, „typischen“ interkulturellen Missverständnisse dar und betont dabei, dass diese Liste nicht vollständig und abgeschlossen ist und dass sie mit weiteren Problemfeldern ergänzt werden kann. 
Definitionen von Kommunikation ${ }^{5}$ wird auf ihre für weitere Ausführungen wichtigen Merkmale verwiesen, die sich drei Hauptkategorien zuordnen lassen: 1. Kommunikationsteilnehmer - zumindest zwei Personen; 2. Code - Sprache und andere Zeichensysteme; 3. Intentionalität - beabsichtigte Beeinflussung des Kommunikationspartners. Der letztgenannte Faktor scheint fraglich zu sein. Es ist umstritten, ob jede Übertragung einer Nachricht einen Einfluss auf den Empfänger impliziert (mehr dazu Wilczyńska et al. 2019: 319). Außerdem macht die vertiefte Analyse des ersten Axioms der Kommunikation Man kann nicht nicht kommunizieren (vgl. Watzlawick, Beavin \& Jackson 1969/1990: 23) deutlich, dass Mitteilungen auch unbeabsichtigt oder sogar unbewusst weitergegeben werden können. Gemeint sind grundsätzlich nonverbale Signale, von denen der Sender gar nicht weiß. ${ }^{6}$ Demnach kann die Intentionalität nicht als ein fester Bestandteil jeder zwischenmenschlichen sich im interkulturellen Kontext abspielenden Kommunikation gelten.

In der einschlägigen Literatur findet man nicht nur definitorische Auffassungen der Kommunikation, sondern auch zahlreiche Kommunikationsmodelle, deren Autoren den Verlauf der zwischenmenschlichen Interaktion graphisch darstellen. Im Prinzip wird zwischen klassischen/ traditionellen/ formalen „Transportmodellen“ und konstruktivistischen Modellen bzw. Theorien unterschieden. Die erstgenannten, in denen die interpersonale Kommunikation nach dem Muster der Nachrichtentechnik gegliedert wird, zeichnen sich durch eine lineare Übertragung der Nachricht von einem Sender durch einen Kommunikationskanal zu einem Empfänger aus, wobei die Kommunikationspartner abwechselnd die Rolle des Senders und des Empfängers einnehmen. Als Beispiele sind hierbei Kommunikationsmodelle von Karl Bühler aus den 1930er, von Claude E. Shannon und Warren Weaver aus den 1940er sowie von Roman Jakobson aus den 1960er Jahren zu nennen. Im Gegensatz zu den traditionellen fokussieren die konstruktivistischen Ansätze, denen die kognitive Autonomie des Menschen zugrunde liegt, auf die Annahme, dass "nicht die Informationen oder Bedeutungen von einem Sender zu einem Empfänger übertragen werden, sondern allenfalls Signale“ (Nünning \& Zierold 2010: 134), denen der Rezipient eine Bedeutung aufgrund seines Vorwissens, seiner Interessen, Bedürfnisse, kulturellen Muster etc. zuschreibt. Zu den Kommunikationsmodellen, in denen Kommunikation als eine wechselseitige Konstruktion von Bedeutungen darge-

5 Dabei wurden die Definitionen von Kommunikation folgender Autoren einer Analyse unterzogen: Knapp und Knapp-Potthoff (1990: 66), Donec (1991: 223), Keller (1994: 104), Szopski (2005: 18-19), Dakowska (2008: 59-60), Nünning und Zierold (2010: 134).

6 Ausführlicher über das Wesen und die Wichtigkeit des nonverbalen Codes in der interpersonalen Kommunikation berichten in ihren Arbeiten u. a.: Oksaar (1988), Klammer (1989: 18), Argyle (1999: 40-60, 2013), Erll und Gymnich (2007: 111-119), Heringer (2007: 81-104), Broszinsky-Schwabe (2011: 121-139), Nagel (2012: 24) oder Mihułka (2015). 
stellt wird, gehört z. B. das Kommunikationsquadrat von Friedmann Schulz von Thun aus den 1980er Jahren, in dem vier Felder einer sprachlichen Äußerung (Sachinhalt, Selbstkundgabe, Beziehung und Appell) differenziert werden ${ }^{7}$, deren Gewichtung je nach der Äußerung unterschiedlich ausfallen kann. Dabei wird hervorgehoben, dass sich die vier Botschaften des Senders von den vier Botschaften des Empfängers mehr oder weniger unterscheiden können. Im Falle der am Schnittpunkt zweier oder mehrerer Kulturen verlaufenden Kommunikation ist noch mit der Schwierigkeit zu rechnen, dass „verschiedene Kulturen die vier Seiten höchst unterschiedlich gestalten "8 (Kumbier \& Schulz von Thun 2009: 12-13). Erwähnenswert ist in diesem Kontext auch das Kommunikationsfaktorenmodell von Pavel Donec (1991: 223), in dem die Aufmerksamkeit nicht nur auf unterschiedliche Zeichenreservoirs der beiden Kommunikationspartner, sondern auch auf ihr unterschiedliches kulturelles Umfeld gelenkt wird, dem bei der Interpretation einer Nachricht eine wichtige Rolle zukommt. ${ }^{9}$

Die im vorherigen Abschnitt präsentierten konstruktivistischen Modelle eignen sich sehr gut für die Beschreibung der Interkulturellen Interaktion. Wie kompliziert die Kommunikation zwischen Menschen mit unterschiedlichem kulturellen Hintergrund sein kann, offenbaren die bildhaften Darstellungen einiger Autoren. Für Broszinsky-Schwabe (2011: 20) ist Interkulturelle Kommunikation wie „eine Straße mit Schlaglöchern voller Missverständnisse, auf der andere ungewohnte Verkehrsregeln gelten.“ Aleksandrowicz-Pędich (2014) vergleicht Interkulturelle Kommunikation mit dem Gehen auf einem Seil. Dabei sind die Kommunikationspartner mit zusätzlichem Gepäck, d. h. mit Koffern, Reisetaschen, Rücksäcken etc., ausgestattet, die kulturelle Muster und individuumsspezifische Elemente symbolisieren. Diese zwei Ebenen „der mentalen Programmierung des Menschen“ (Hofstede 1993: 19-20) determinieren das Denken, Fühlen und Handeln der beiden Agierenden. In den meisten Definitionen der Interkulturellen Kommunikation verweisen die Autoren ${ }^{10}$ auch auf die kulturelle Andersheit der Begegnungspartner als Hauptfaktor dieser Kommunikationsart. In manchen, vorwiegend aus den vorherigen Dekaden stam-

7 Es ist anzumerken, dass der Sach- (Inhalts-) und Beziehungsaspekt schon von Watzlawick unterschieden und in dem zweiten Axiom der Kommunikation beschrieben wurden (Watzlawick, Beavin \& Jackson 1969/1990: 23ff.).

8 Anhand des Kommunikationsquadrats werden ein deutsch-chinesisches Missverständnis von Wang (2009: 189-191) und ein deutsch-türkisches Missverständnis von Holzbrecher (2004: 33-34) erklärt.

9 Eine ausführliche Besprechung der einzelnen Elemente des Faktorenmodells von Donec in Bezug auf den interkulturellen Kontext der zwischenmenschlichen Begegnung findet sich in Mihułka (2011: 233-235).

10 Siehe z. B. Maletzke (1996: 37), Schugk (2004: 52), Mikułowski-Pomorski (2003: 11-12), Lüsebrink (2005: 7), Erll und Gymnich (2007: 77-78), Broszinsky-Schwabe (2011: 21) sowie Wilczyńska et al. (2019: 322). 
menden Auffassungen von Interkultureller Kommunikation ${ }^{11}$ steht der Aspekt der Sprachlichkeit im Mittelpunkt der Betrachtung, oft ohne dabei präzisiert zu haben, ob sich die beiden Interaktionsteilnehmer der fremden bzw. zweiten Sprache bedienen müssen oder lediglich einer von ihnen. Für Ehrhardt (2003: 193, in: Erll \& Gymnich 2007: 79) ist das Kriterium der Verwendung von Fremdsprachen ein grundlegendes Merkmal, um Kommunikation als Interkulturelle Kommunikation zu qualifizieren. Knapp \& Knapp-Potthoff (1990: 66) fügen noch hinzu, dass die zweite oder fremde Sprache, mittels der ein Kommunikationsteilnehmer seine Gedanken zum Ausdruck bringt, keine Varietät seiner eigenen Sprache (Muttersprache) sein soll. Feuerstein (2001: 207) äußert sich dazu nicht so orthodox, indem er feststellt, dass nur im Standardfall Interkultureller Kommunikation mindestens einer der Beteiligten ein „fremdes“ Zeichensystem benutzen muss. ${ }^{12}$ Das Betrachten des Fremdsprachlichkeitsaspekts als eines der entscheidenden Merkmale der Interkulturellen Kommunikation kann jedoch manchmal irreführend sein. Wie soll man also die sich beispielsweise zwischen einem Amerikaner und einem Engländer, zwischen einem Spanier und einem Argentinier, zwischen einem Deutschen und einem Schweizer vollziehende Kommunikation bezeichnen, wenn beide sich ihrer Muttersprache bedienen? In allen obigen Fällen handelt es sich ohne Zweifel um Interkulturelle Kommunikation. Es soll aber zugegeben werden, dass bei dieser Art der Interaktion sehr häufig Fremdsprachen verwendet werden. Wie schon oben angedeutet, sollte nicht die Sprache als Kommunikationsmittel, sondern die unterschiedliche kulturelle Zugehörigkeit der Interaktionsteilnehmer, ihr Bewusstsein über die Andersheit des jeweils anderen sowie ihre wechselseitige Wahrnehmung als „fremd“ (Maletzke 1996: 37) zu den wesentlichen Faktoren werden, die eine Kommunikation zu einer interkulturellen Kommunikation machen.

In allen, in diesem Beitrag vorgestellten Auffassungen, Theorien und Modellen erscheint die Interkulturelle Kommunikation als unmittelbare Interaktion zwischen Angehörigen verschiedener Kulturen, die mittels der sprachlichen und nicht-sprachlichen Zeichen miteinander kommunizieren. Laut Lüsebrink (2005:

11 Siehe z. B. Knapp und Knapp-Potthoff (1990: 66), Ehrhardt (2003: 193, in: Erll und Gymnich 2007: 79), Riehle und Seifert (2001: 16) sowie die Besprechung ausgewählter Definitionen von Schröer (2009: 8-9).

12 Als einer der Aspekte, anhand dessen zwischen der intrakulturellen und interkulturellen Kommunikation differenziert wird, wird der Sprachlichkeitsaspekt betrachtet. Je nachdem, ob die Verständigung zwischen Kommunikationspartnern mittels der Mutter- oder Fremdsprache verläuft, ist die Rede entsprechend von einer oder anderer Art der Kommunikation. Schröer (2009: 8) verweist mit Recht darauf, dass „diese scharfe Abgrenzung [...] zunehmend in Frage gestellt wurde" und erklärt seine Feststellung auf der Basis wissenschaftlicher Ausführungen und empirischer Befunde. Andere Merkmale der intra- und interkulturellen Kommunikation (auch wenn sie anders benannt werden) besprechen u. a. Knapp und KnappPothoff (1990: 66), Mikułowski-Pomorski (2003: 11-12) und Wilczyńska et al. (2019: 322). 
8) beschränkt sich der Begriff der Interkulturellen Kommunikation nicht nur auf interpersonale, Face-to-Face-Kommunikationsprozesse, denn er umfasst auch die mediatisierte Dimension dieser Kommunikationsart in ihren verschiedenen Facetten. Demzufolge schlägt der Autor vor, Interkulturelle Kommunikation nicht nur eng auf der interaktiven Ebene, sondern auch weiter auf der mediatisierten Ebene zu analysieren und zu erläutern. Auf eng und weiter gefasste Interkulturelle Kommunikation richten ihr (genaues) Augenmerk zahlreiche Wissenschaftsdisziplinen, wie z. B. Wirtschaftslehre, Speech Kommunikation, Soziologie, Kulturwissenschaft, Geschichte, Literaturwissenschaft, Anthropologie, Pädagogik, Psychologie, Philosophie und last but not least Linguistik (vgl. Lüsebrink 2005: 5, Erll \& Gymnich 2007: 5), die versuchen, das Wesen der Interkulturellen Kommunikation je nach ihrem Forschungsgegenstand wissenschaftlich zu erkunden und dann die Forschungsergebnisse in die praktische Ausbildung von Fachleuten zu integrieren.

\section{Interkulturelle Kommunikation und (Angewandte) Linguistik: Zur Beziehung zwischen den Disziplinen}

Das Ziel des vorliegenden Abschnitts ist es, die Interkulturelle Kommunikation und Angewandte Linguistik im Verhältnis zueinander zu positionieren und erste Schlussfolgerungen für die interkulturell orientierte Ausrichtung des Studiums der angewandten Linguistik in Polen zu ziehen. Dieses Vorhaben ist trotz einer langen Tradition der Verbindung von Sprache und Kultur nicht einfach. Es wird auch durch die Tatsache erschwert, dass die Wissenschaftskonzepte zur Konturierung und Erforschung allein der Allgemeinen und Angewandten Linguistik ${ }^{13}$ in England, den USA, Deutschland und Polen nicht deckungsgleich sind. Dies verursacht, dass Gegenstandsbeschreibungen der Angewandten Linguistik im internationalen Vergleich unterschiedlich definiert werden. Während die Auffassung im angelsächsischen Raum auf die Analyse der Sprachverwendung in konkreten Sprachsituationen und die Anwendung linguistischer Theorien und Modelle für den Sprachunterricht fokussiert, wird Angewandte Linguistik in den zeitgemäßen Definitionen im deutschsprachigen Raum (vgl. dazu u.a. Spillner 2003: 31-32, Knapp 2004a: XVIII, Graefen \& Liedke 2008: 297) als Disziplin konzipiert, die sich mit der Beschreibung, Erklärung und Lösung von lebens- und gesellschaftspraktischen Problemen in den Bereichen Sprache und Kommunikation befasst. Ihr Fokus auf das Problemlösen trägt dazu bei, dass „die Angewandte Linguistik oft

13 Die nicht einfache und auch schwer vorzunehmende Abgrenzung zwischen der Allgemeinen und der Angewandten Linguistik wird in diesem Beitrag nicht vorgenommen. Wir verweisen in diesem Zusammenhang auf zahlreiche Publikationen von F. Grucza (vgl. u. a. 1976, 1983, 2017). 
über den vorhandenen linguistischen Forschungsstand hinausgehen und gegenstandsbezogene neue Methoden und theoretische Konzepte entwickeln und dabei auch auf andere Disziplinen Bezug nehmen muss" (vgl. Knapp 2004a: XVIII). Ihre Arbeitsfelder umfassen in der deutschsprachigen Fachliteratur: Sprachvermittlung, mündliche und schriftliche Kommunikation, mediale, öffentliche und mehrsprachige Kommunikation, Diagnostizieren und Therapieren. Die genannten Arbeitsbereiche werden zum Objekt der Analyse vielfältiger Subdisziplinen, zu denen Sprachdidaktik, Sprachberatung, Schreibtraining, technische Kommunikation, Gesprächsanalyse, Gesprächsberatung und Gesprächstraining, Terminologiearbeit, Sprachplanung, Sprachdiagnostik, klinische Linguistik, forensische Linguistik und interkulturelle Kommunikation gehören (vgl. dazu die Einführung in die wichtigsten Arbeitsfelder der Angewandten Linguistik bei Knapp u. a. 2004 sowie bei Graefen \& Liedke 2008: 297-313).

Die gerade aufgelisteten inhaltlichen Arbeitsschwerpunkte in der deutschsprachigen Angewandten Linguistik, welche allerdings angesichts der raschen Entwicklung der Sprachwissenschaft an die aktuellen Entwicklungen und Erfordernisse erneut angepasst werden mussten, sind heutzutage in den folgenden Sektionen der Gesellschaft für Angewandte Linguistik repräsentiert: Fachkommunikation, Gesprächsforschung, interkulturelle Kommunikation, Lexikographie, Mediendidaktik und Medienkompetenz, Medienkommunikation, Mehrsprachigkeit, Migrationslinguistik, Phonetik und Sprechwissenschaft, Schreibwissenschaft, Soziolinguistik, Sprachdidaktik, Sprachkritik, Textlinguistik und Stilistik, Übersetzungs- und Dolmetschwissenschaft. ${ }^{14}$

In der polnischsprachigen Fachliteratur werden konzeptionelle Diskussionen um die Entwicklungsgeschichte und fachliche Verortung der Angewandten Linguistik auch seit vielen Jahrzehnten geführt (vgl. hierzu den Beitrag von I. Prokop 2000 sowie den genauen geschichtlichen Überblick und die Charakteristik der wichtigsten Arbeitsfelder der Angewandten Linguistik aus polnischer Perspektive bei F. Grucza 2017). Erst in den letzten drei Jahrzehnten wird allerdings auf ihre gemeinsamen Interessenareale mit den Kulturwissenschaften/ Cultural Studies verwiesen und dabei auf Grundpositionen fokussiert, welche die Relation von Interkultureller Kommunikation und Angewandter Linguistik unter besonderer Berücksichtigung der Glottodidaktik und Translatorik in der deutsch- und polnischsprachigen Forschungsliteratur bestimmen (vgl. u. a. die Beiträge von F. Grucza 2000, W. Pfeiffer 2003, Adamczak-Krysztofowicz 2005, Neuland 2013 sowie die von Adamczak-Krysztofowicz et al. 2009, AdamczakKrysztofowicz \& Stork 2012 sowie Mihułka et al. 2016 herausgegebenen Sam-

14 https://gal-ev.de/sektionen/. 
melbände ${ }^{15}$ ). In diesem Zusammenhang werden unterschiedliche Erkenntnisse Interkultureller Kommunikation bei der Erarbeitung von zentralen curricularen Komponenten universitärer Ausbildung in neuphilologischen Studienrichtungen, insbesondere aber im Studium der angewandten Linguistik, mit unterschiedlichen Ausrichtungen (linguistisch, didaktisch-methodisch, kulturwissenschaftlich, literaturwissenschaftlich) tiefgründig und umfassend reflektiert.

Folglich ist der Beitrag der Interkulturellen Kommunikation als anwendungsorientierter autonomer Bezugsdisziplin, die das Ziel verfolgt „Auswirkungen kultureller Unterschiede zu identifizieren, deren Ursachen aufzudecken und durch geeignete Formen von Beratung, Ausbildung oder Training ihr Auftreten zu vermeiden oder ihre Folgen zu minimieren“ (Knapp 2004b: 410), bei der Formulierung der Lernziele, der Auswahl des Lernstoffes und der Lehr-/Lernverfahren sowie der Entwicklung der Curricula und Lernmaterialien für die einzelnen Studienfächer im Studium der Angewandten Linguistik kaum zu überschätzen:

Linguistische Zugänge zu interkultureller Kommunikation befassen sich einerseits mit den kommunikativen Voraussetzungen, die Teilnehmer in interkulturelle Kontaktsituationen einbringen, andererseits mit den tatsächlichen Auswirkungen dieser Voraussetzungen in konkreten Interaktionssituationen. Erstere sind Gegenstand kontrastiver Arbeiten, die kulturrelevante Unterschiede zwischen Sprachen auf allen deskriptiven Ebenen (verbal, paraverbal, nonverbal) beschreiben, letztere stehen im Fokus von empirischen Studien, die authentische interkulturelle Interaktionen mit Mitteln der Gesprächsanalyse untersuchen (Knapp 2004b: 411).

$\mathrm{Zu}$ den Arbeitsfeldern der Angewandten Linguistik, die sich mit Interkultureller Kommunikation beschäftigen und zugleich Lehr-/Lerninhalte jedes neuphilologischen Studiums bilden, gehören seit langem kontrastive Linguistik und kontrastive Pragmatik ${ }^{16}$, die sich mit kulturbedingten Unterschieden zwischen Sprachen auseinandersetzen sowie die Ethnographie der Kommunikation, die Kommunikationsereignisse in unterschiedlichen kulturellen oder ethnischen Gemeinschaften analysieren, um Handlungsmuster der sprachlichen Interaktion und deren situationsabhängige Variationen zu beschreiben. Eine vergleichsweise junge Forschungsrichtung der Linguistik ist darüber hinaus die interpretative

15 Der erste von ihnen, dessen Herausgeber/innen Mitarbeiter/innen des Posener Instituts für Angewandte Linguistik sind, dokumentiert die wissenschaftliche Konferenz „Aktuelle Probleme der Angewandten Linguistik. Interkulturalität als Schlüsselkompetenz für Fremdsprachenlehrer, Übersetzer und Mediatoren“, die 2009 stattgefunden hat. In dem zweiten, dritten und vierten Sammelband werden ebenfalls Beiträge präsentiert, die eine enge Verzahnung von Theorie und Praxis auf dem Feld einer breit verstandenen linguistisch fundierten und anwendungsorientierten Forschung zu Fragen der Interkulturalität sichtbar machen.

16 Vgl. hierzu die zahlreichen Arbeiten der Jubilarin, darunter I. Prokop (1992 und 1995). 
Soziolinguistik ${ }^{17}$, die aufdeckt, „ob und wieweit Gesprächsbeteiligte mit unterschiedlichem kulturellem Hintergrund sprachliche Mittel und Merkmale des interaktiven Kontextes" (Knapp 2004b: 411) anders interpretieren. ${ }^{18}$

Die bereits erwähnten relevanten Forschungsergebnisse der weit gefassten Kategorie „Interkulturalität“ werden verstärkt in die curricularen Aufgabenkataloge der Neuphilologien, darunter auch des Studiums der Angewandten Linguistik, aufgenommen. Die Aufmerksamkeit wird dabei vor allem auf die Rolle der Sprache in interkulturellen Kontakten und Kontexten, die Fremdheitswahrnehmung und ihre xenologische Untersuchung, kulturell bedingte Schwierigkeiten beim Dolmetschen und Übersetzen, interkulturelle Erziehung und interkulturelles Lernen ${ }^{19}$, kulturspezifische Prägung der Fachkommunikation sowie „Potenziale der schönen Künste in der Vermittlung zwischen den Kulturen“ (Hess-Lüttich 2010: 138) gerichtet.

Im Folgenden soll herausgefunden werden, inwiefern die wichtigsten Erkenntnisse zur Relation zwischen Interkultureller Kommunikation und Angewandter Linguistik in den neuen Richtlinien des Polnischen Qualifikationsrahmens für Hochschulwesen berücksichtigt werden und wie sie sich den interkulturellen Zielsetzungen des auf der Grundlage der wissenschaftlichen Erkenntnisse von den Autorinnen modellierten Studienfaches „Interkulturelle Kommunikation" zuordnen lassen.

17 Interpretative Soziolinguistik und die Ethnographie der Kommunikation haben zur linguistischen Konzeptualisierung von Kultur und Kommunikation beigetragen sowie die Vorstellungen vom interkulturellen Kommunikationsprozess wesentlich beeinflusst.

18 Auf die bereits in der Linguistik beschriebene Vielfalt kulturspezifischer Unterschiede in der sprachlichen Kommunikation (wie z. B. fehlende Übersetzungsäquivalente im Bereich des Lexikons, kulturell variierende Unterschiede bei Sprechakten, Sprechaktsequenzen und Argumentationsstrukturen sowie größeren kommunikativen Handlungsmustern), insbesondere in der Makro- (bei erwarteten Inhalten, Funktionen und formalen Abläufen) und Mikrostruktur (beim Sprecherwechsel) von Gesprächen, unterschiedliche interkulturell bedingte Kommunikationsprobleme in der lernersprachlichen Kommunikation, DolmetschKommunikation und lingua-franca-Kommunikation) kann im Folgenden nicht eingegangen werden. Vgl. hierzu den kurzen Überblick bei Knapp (2004b: 415-420).

19 Die Kategorie Interkulturalität als Inbegriff der Kommunikation zwischen Menschen aus unterschiedlichen Kulturtraditionen weckt auch das Interesse der Fremdsprachendidaktik, deren Vertreter/innen nach der Rezeption von relevanten linguistischen, literaturästhetischen, historischen, medialen, komparatistischen und didaktischen Forschungserkenntnissen Theorie und Praxis interkultureller Landeskunde und interkultureller Literatur zum Thema ihrer Untersuchungen machen und interkulturelle (kommunikative) Kompetenz, Stärkung von Kultur- und Sprachbewusstheit, Förderung des Eigen- und Fremdverstehens, den produktiven Umgang mit Alterität sowie Vorurteilen- und Stereotypenrelativierung (vgl. hierzu Adamczak-Krysztofowicz 2005, Neuland 2013, Mihułka 2016) als wichtige Lernziele für den (universitären) Sprachunterricht erklären. 


\section{Charakteristik des Studienfaches „Interkulturelle Kommunikation" auf der Grundlage von Deskriptoren des Polnischen Qualifikationsrahmens für Hochschulwesen}

Mit der Verordnung des Ministers für Wissenschaft und Hochschulwesen (MNiSW) vom 14. November 2018 wurde der neue Polnische Qualifikationsrahmen (PQR ${ }^{20}$ eingeführt (Dz. U. 2018 poz. 2218). Seit dem 29. November 2018 soll die Organisation, Planung und Durchführung der Bildung an polnischen Hochschulen diesem Dokument gemäß verlaufen. Der PQR enthält eine genaue Beschreibung der Qualifikationen für die Niveaus 6 bis 8, durch die sich die Studierenden des Bachelor- und Masterstudiums sowie Doktoranden nach dem Abschluss des Studiums auszeichnen sollen. Dabei werden im Rahmen jedes Niveaus allgemeine Deskriptoren für Umfang und Komplexität des Wissens, der Fertigkeiten und Sozialkompetenzen unterschieden, die wiederum einer Unterteilung in weitere Matrixfelder unterliegen.

Aus Platzgründen wird im vorliegenden Beitrag nur das siebte Niveau in Bezug auf den Verlauf des Lernens im Rahmen des Faches „Interkulturelle Kommunikation“ im Studium der angewandten Linguistik diskutiert und tabellarisch zusammengestellt. Der Inhalt der drei ersten Spalten (Hauptkategorien, beschreibende Kategorien, grundlegende Aspekte) der Tabelle 1 wurde dem ministerialen Dokument ${ }^{21}$ direkt entnommen und von den Autorinnen ins Deutsche übersetzt. Die vierte Spalte (Interkulturelle Kommunikation - Lernergebnisse formuliert anhand der Deskriptoren des PQR für das siebte Niveau) beinhaltet die Lernergebnisse, die mit Fokus auf das Fach „Interkulturelle Kommunikation“ von den Autorinnen präzisiert und ergänzt wurden. In der letzten Spalte (Interkulturelle Kommunikation - Zielsetzung) der Tabelle 1, die von den Autorinnen des Beitrags konzipiert wurde, befinden sich die fachspezifischen Ziele, die die Studierenden der angewandten Linguistik in Polen nach dem Abschluss dieser Lehrveranstaltung erzielen sollen. Die von uns erstellte ausführliche Liste von Zielsetzungen für den interkulturellen Kompetenzerwerb im kognitiven, pragmatischen und sozialen Bereich am Beispiel eines modell-

20 Der Polnische Qualifikationsrahmen wurde auf der Grundlage des Europäischen Qualifikationsrahmens für lebenslanges Lernen - EQR erarbeitet. Der EQR wurde als ein MetaRahmen konzipiert, in dem allgemeine Deskriptoren (als Ausgangspunkte) differenziert wurden, die in den einzelnen EU-Mitgliedstaaten je nach Bedarf aktualisiert, ergänzt etc. werden können (vgl. Europäische Kommission 2008: 9). Die erste, im Jahr 2008 angekündigte Version des EQR wurde nach elf Jahren (2017) modifiziert, verbessert und vervollständigt. Ausführlicher dazu siehe Dz. U. UE 2017/C 189/03; vgl. auch https://prk.men.gov.pl/de/polni scher-qualifikationsrahmen-pqr-und-europaeischer-qualifikationsrahmen-eqr/ (07.03. 2020), http://www.kwalifikacje.gov.pl/polska-rama-kwalifikacji (07.03.2020).

21 Online: http://www.kwalifikacje.gov.pl/images/downloads/tabele_PRK/PRK_tab5.pdf (07.03. 2020). 
haften Studienfaches „Interkulturelle Kommunikation“ zeichnet sich dadurch aus, dass die formulierten Ziele den Hauptkategorien (Wissen, Fertigkeiten, Sozialkompetenzen) und Teilkategorien des ministerialen Dokuments entsprechend zugeordnet wurden. Bei unserer Modellierung des Faches reflektieren wir nur die neue Reform im polnischen Hochschulwesen, ohne die verschiedenen Angebote des Studiums der angewandten Linguistik an polnischen Universitäten zu diskutieren.

Die tabellarische Form der Zusammenstellung kann in Zukunft als Grundlage für die Analyse interkultureller Bildung bei angehenden Fremdsprachenlehrenden sowie Übersetzer/innen und Dolmetscher/innen an polnischen Universitäten und Hilfestellung bei der Anpassung der interkulturell orientierten Studienfächer an die neue Reform im polnischen Hochschulwesen dienen. 


\begin{tabular}{|c|c|}
\hline 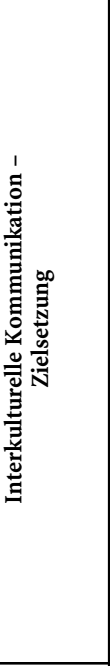 & 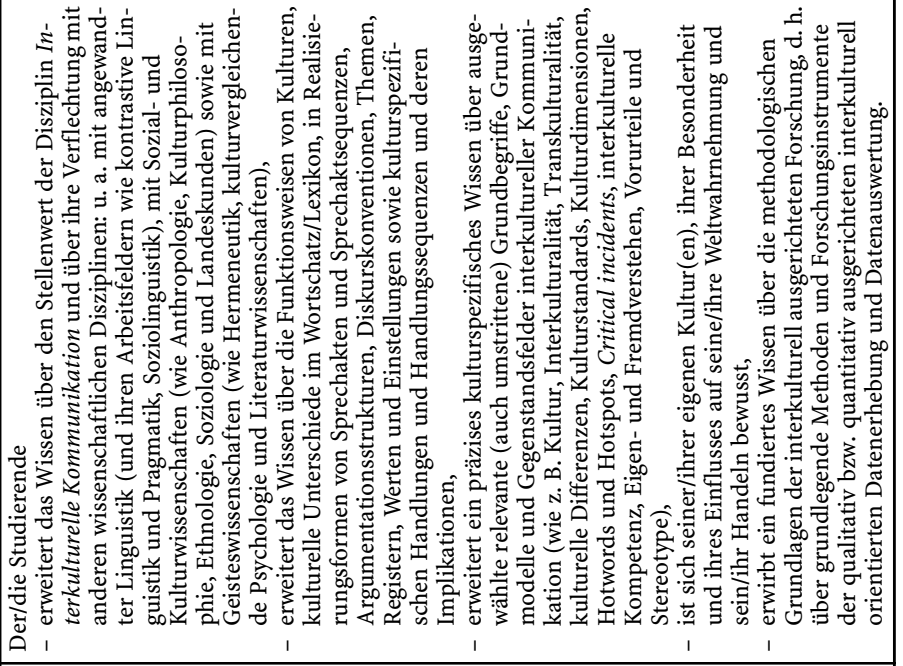 \\
\hline 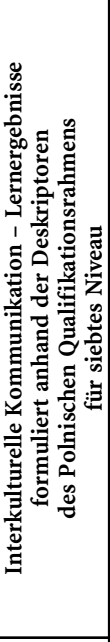 & 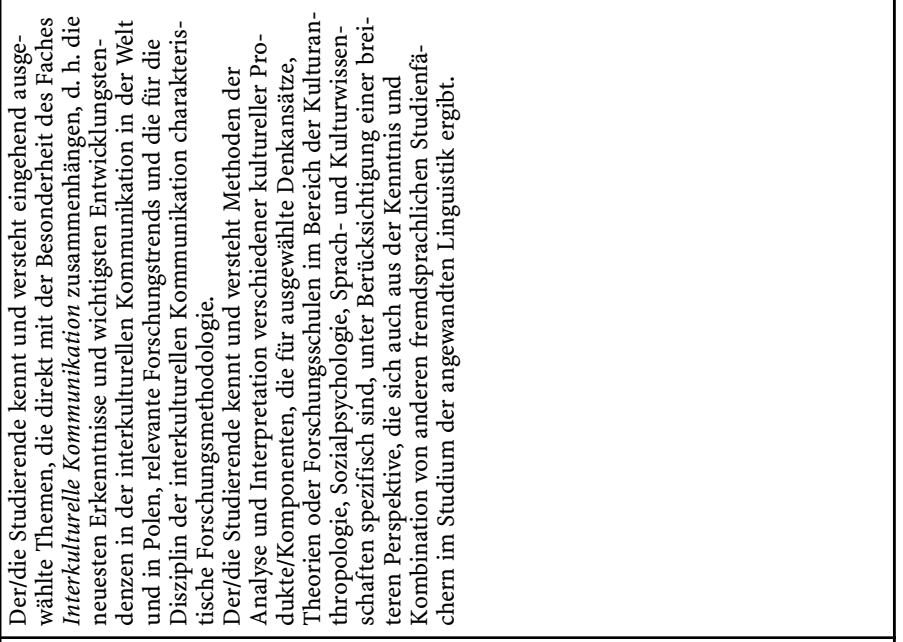 \\
\hline 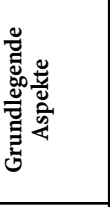 & 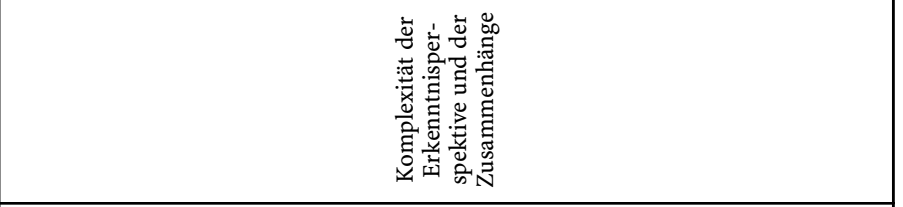 \\
\hline 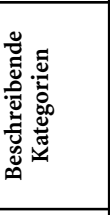 & 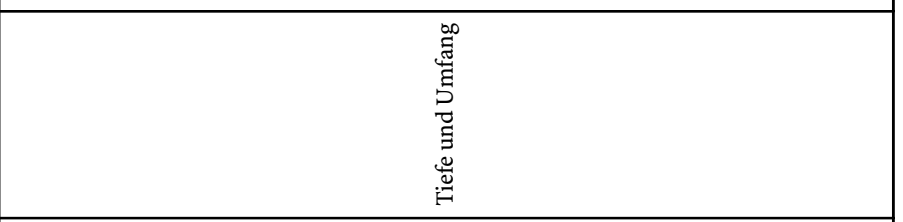 \\
\hline & NGSSIM \\
\hline
\end{tabular}




\begin{tabular}{|c|c|c|}
\hline 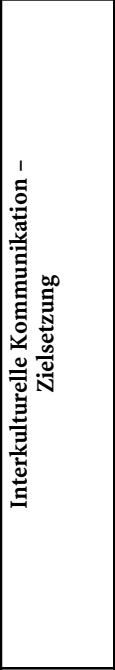 & 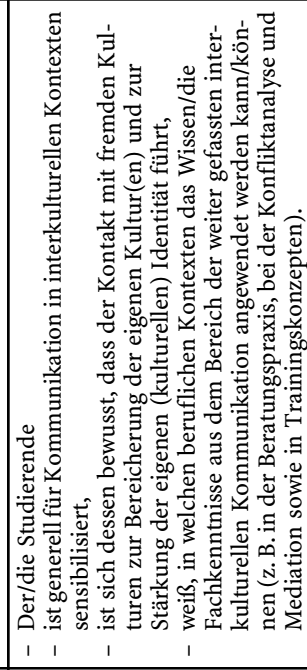 & 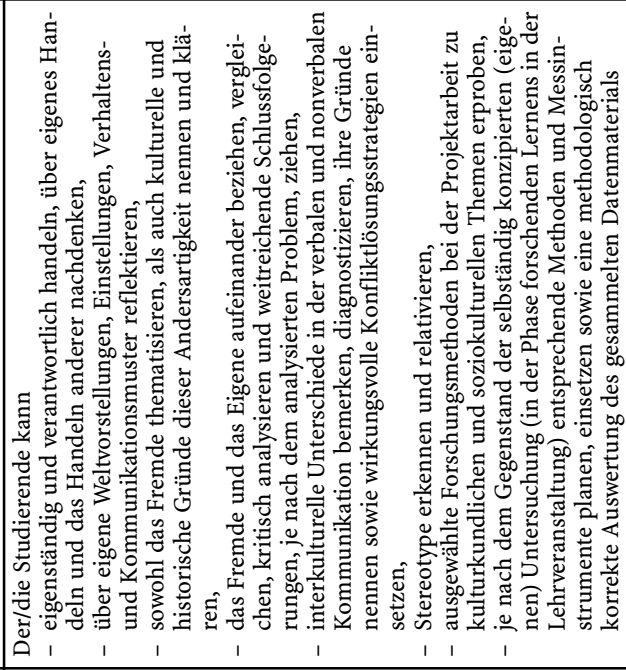 \\
\hline 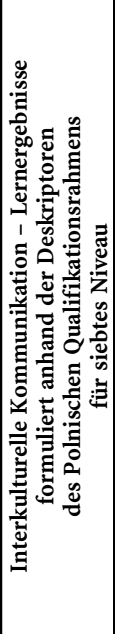 & 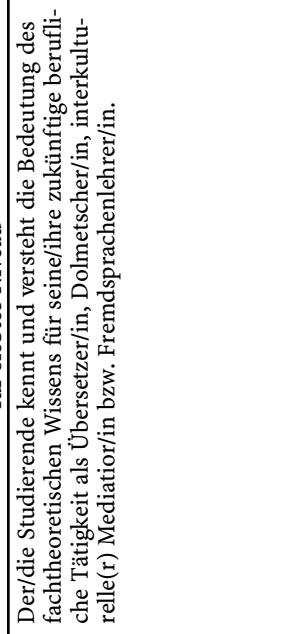 & 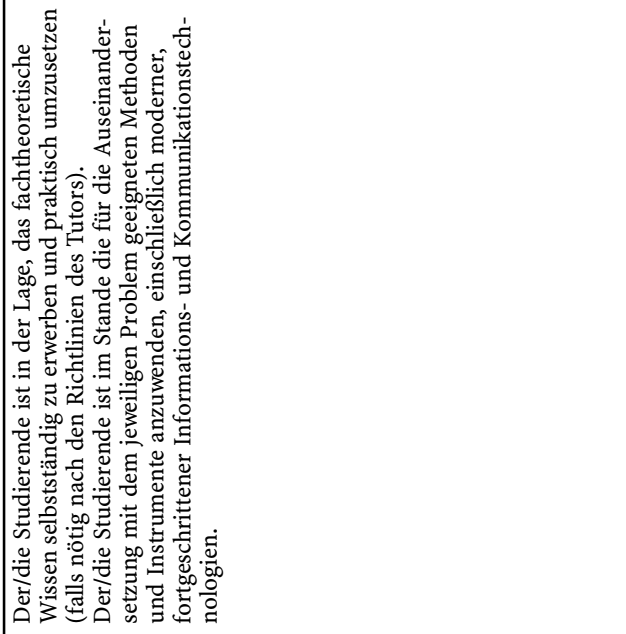 \\
\hline 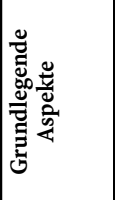 & 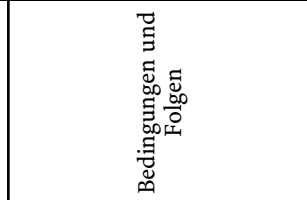 & 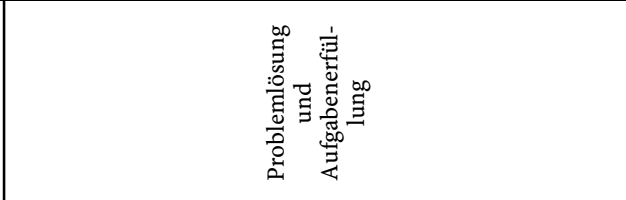 \\
\hline 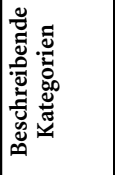 & 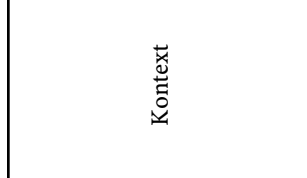 & 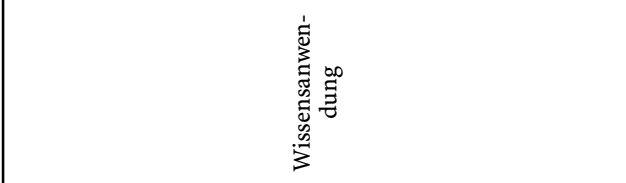 \\
\hline & & NALIGY9ILYAG \\
\hline
\end{tabular}




\begin{tabular}{|c|c|c|c|c|c|}
\hline 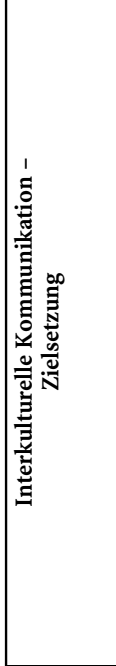 & 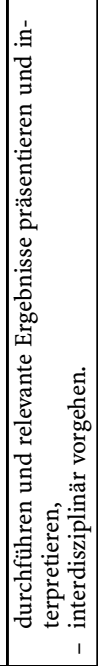 & 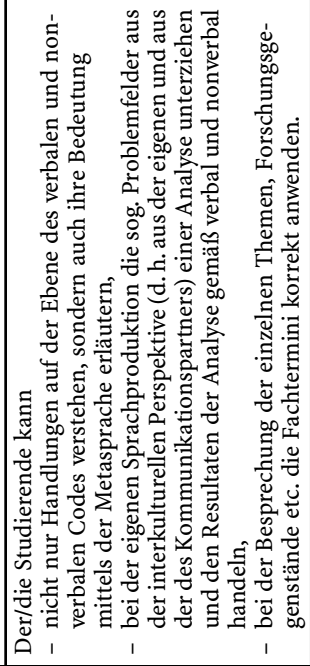 & 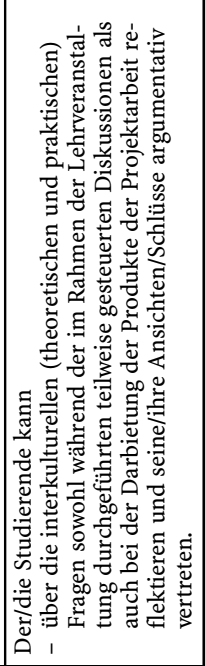 & 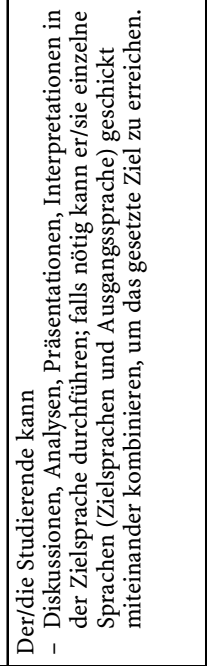 & 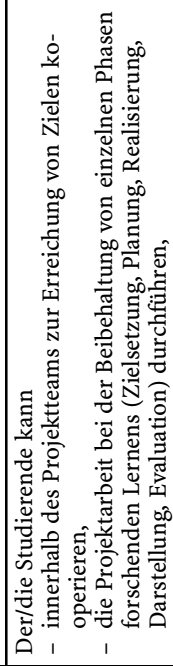 \\
\hline 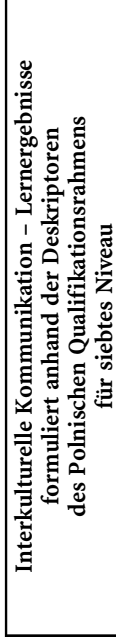 & & 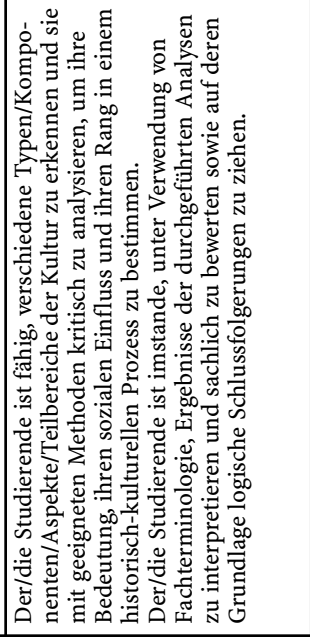 & 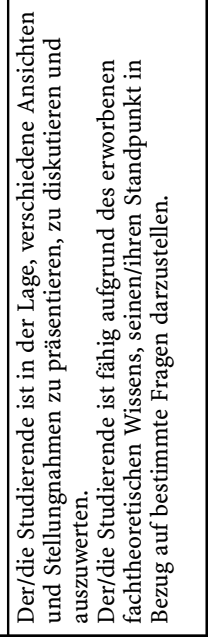 & 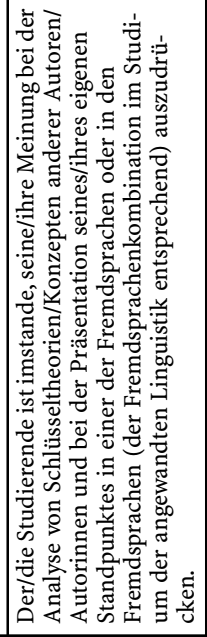 & 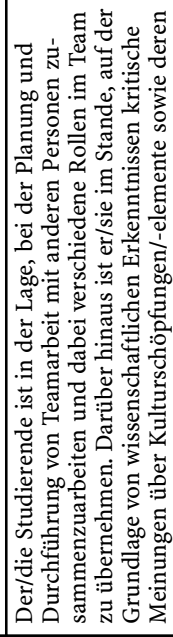 \\
\hline 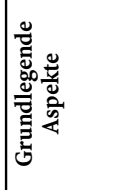 & & 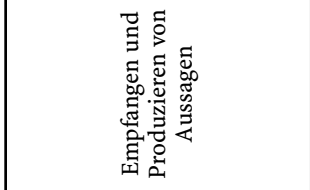 & 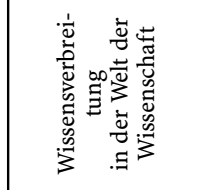 & 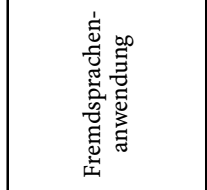 & 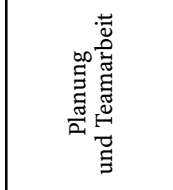 \\
\hline 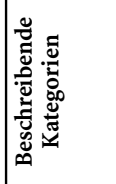 & & & 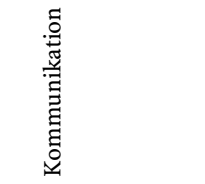 & & 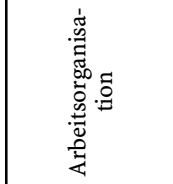 \\
\hline
\end{tabular}




\begin{tabular}{|c|c|c|c|c|c|}
\hline 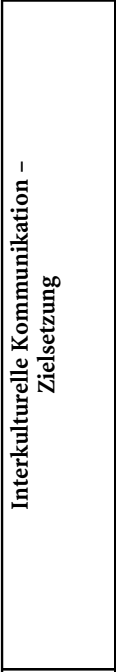 & 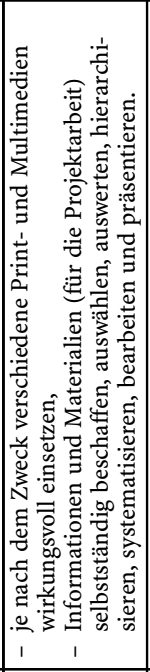 & 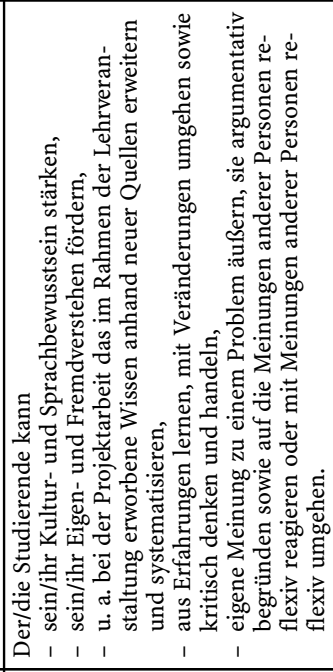 & 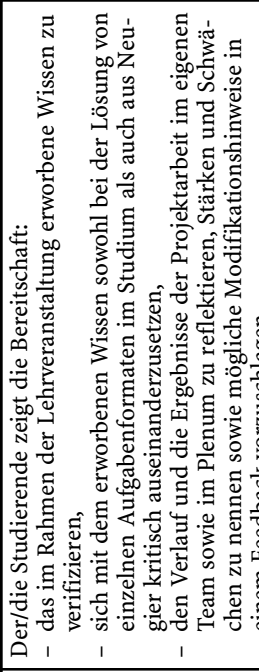 & 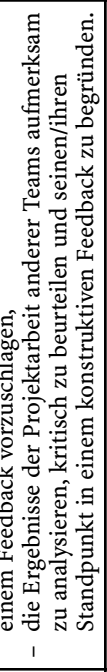 & 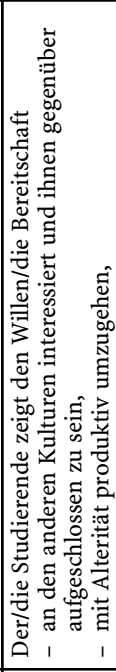 \\
\hline 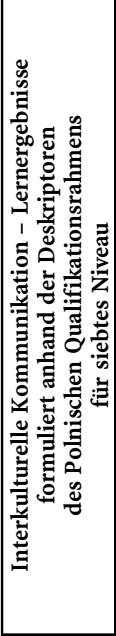 & 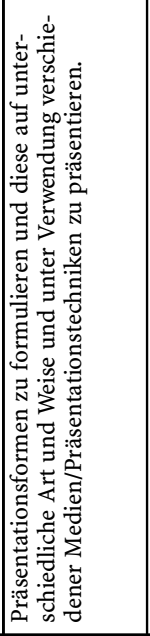 & 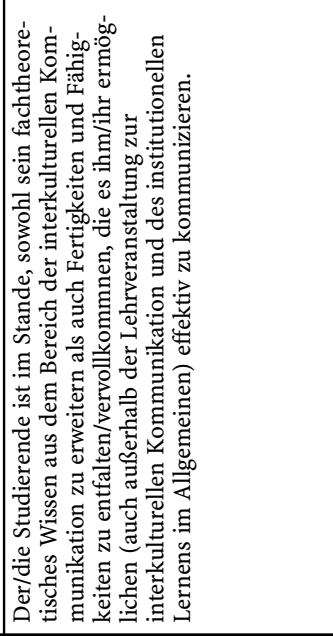 & 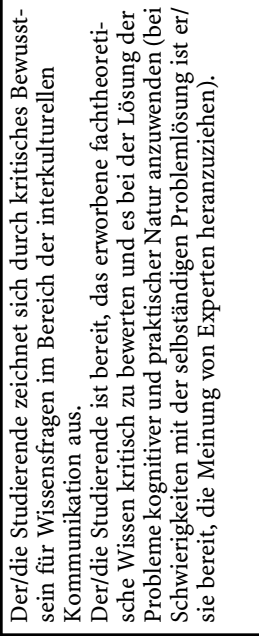 & & 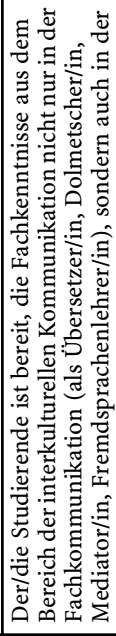 \\
\hline 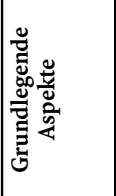 & & 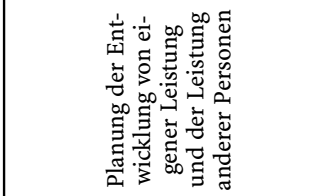 & 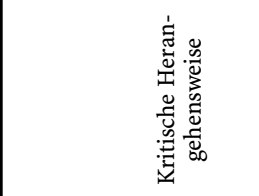 & & 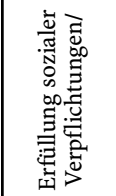 \\
\hline 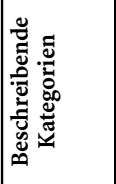 & & 志 & 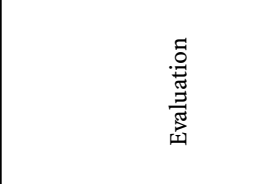 & & 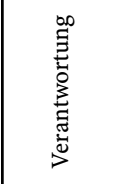 \\
\hline & & & \multicolumn{3}{|c|}{ NAZNGLAdWOYTVIZOS } \\
\hline
\end{tabular}




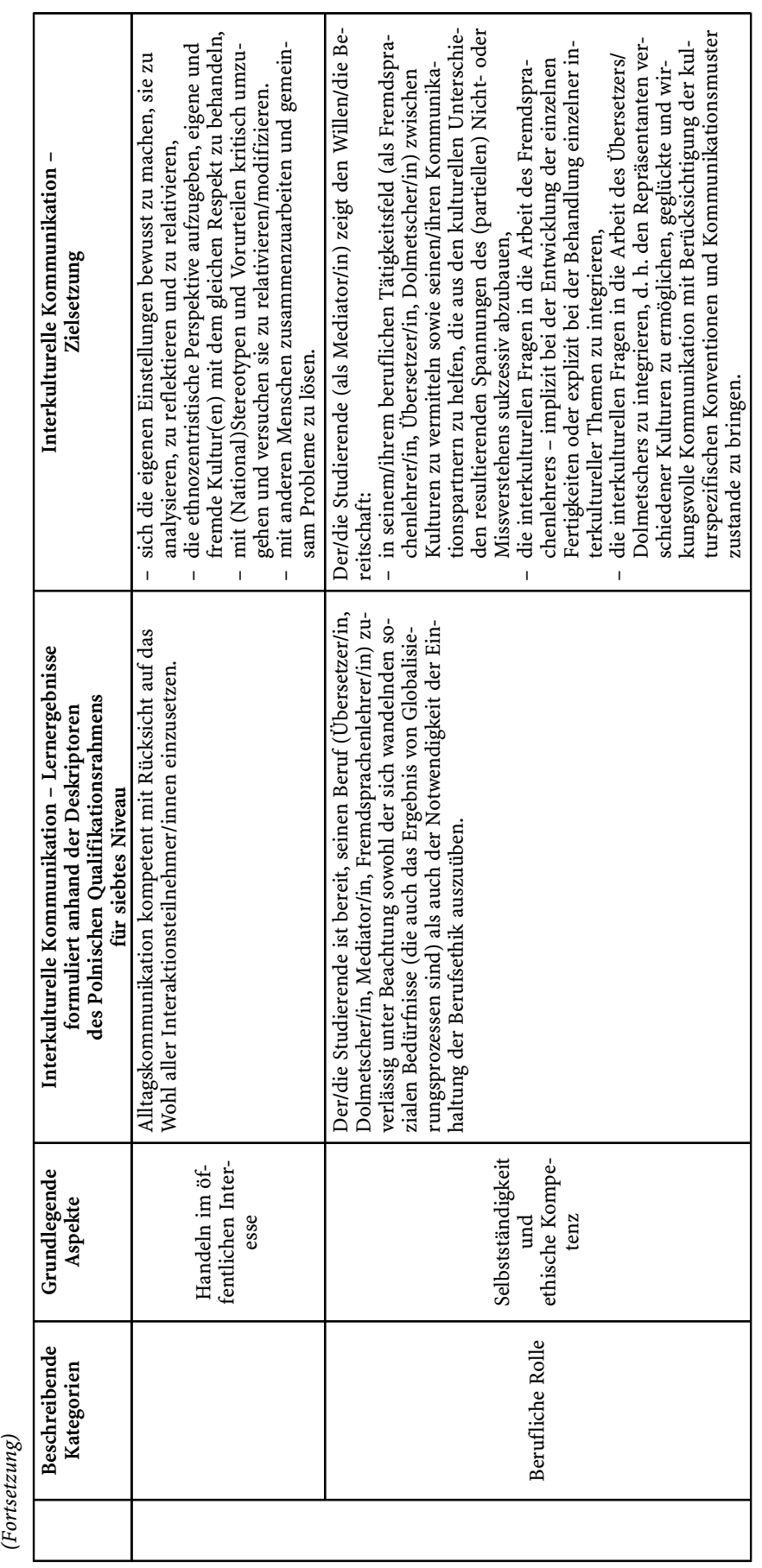




\section{Zusammenfassung und Ausblick}

Der vorliegende Beitrag vermittelt Einblicke in Trends des weit verstandenen Gebiets der Angewandten Linguistik aus der neuphilologischen Perspektive, die neben einem fremdsprachendidaktischen und einem übersetzungswissenschaftlichen Schwerpunkt auch Forschungen und Lehre zur Interkulturellen Kommunikation umfasst. Obwohl die verschiedenen Angebote des Studiums der angewandten Linguistik wegen der Interdisziplinarität der interkulturellen Kommunikation und vielfältiger Erwartungen von Zielgruppen unterschiedlich ausgerichtet sind, ist die Kategorie Interkulturalität ein integraler Bestandteil in der akademischen Bildung der Fremdsprachenlehrer/innen, Mediatoren und Mediatorinnen sowie Übersetzer/innen und Dolmetscher/innen an jeder Hochschule. Nichtdestotrotz sollten die aktuellen Studienfächer für interkulturellen Kompetenzerwerb im Studium der angewandten Linguistik an polnischen Universitäten einer Analyse unterzogen werden. Interessant wäre es z. B. herauszufinden, inwiefern und wie die nach der neuen Reform des polnischen Hochschulwesens aktualisierten Zielsetzungen mit ihren Inhalten in ausgewählten interkulturell orientierten Lehrveranstaltungen theoretische und empirische Zugriffe auf Fremdheit, multikulturelle Identitäten und Mehrsprachigkeit, Rezeption der interkulturellen Literatur, korpus- und gesprächslinguistische Analysen interkultureller Kommunikationsprozesse und critical incidents, kulturwissenschaftliche Erschließungen landeskundlicher Inhalte sowie die Vermittlung interkultureller Kompetenzen im Sprachunterricht (Umgang mit Stereotypen und Vorurteilen, Didaktik des Fremdverstehens, kulturell beeinflusste Interpretationen literarischer Texte, Filme, Comics und Bilder als Anlass interkulturellen Lernens) einbeziehen. Als Analyseraster könnte dabei die von uns in tabellarischer Form konzipierte Zusammenstellung der aktuellen Lernergebnisse und Zielsetzungen im Studienfach „Interkulturelle Kommunikation“ dienen, die bereits an die neuen Deskriptoren des Polnischen Qualifikationsrahmens für Hochschulwesen angepasst worden ist.

\section{Literaturverzeichnis}

Adamczak-Krysztofowicz, Sylwia (2005). Kooperatives Miteinander statt nebeneinander. Zur Beziehung zwischen der interkulturellen Fremdsprachendidaktik und den Kulturwissenschaften. Glottodidactica, XXX/XXXI, 5-11.

Adamczak-Krysztofowicz, Sylwia/Kowalonek-Janczarek, Monika/Maciejewski, Maciej/ Sopata, Aldona (Hrsg.) (2009). Aktuelle Probleme der Angewandten Linguistik. Interkulturalität als Schlüsselkompetenz für Fremdsprachenlehrer, Übersetzer und Mediatoren. Poznań: Wydawnictwo Naukowe Uniwersytetu im. Adama Mickiewicza. 
Adamczak-Krysztofowicz, Sylwia/Stork, Antje (Hrsg.) (2012). Multikompetent - multimedial - multikulturell? Aktuelle Tendenzen in der DaF-Lehrerausbildung. Band 2. Frankfurt a.M.: Peter Lang.

Aleksandrowicz-Pędich, Lucyna (2005). Międzykulturowość na lekcjach języków obcych. Białystok: Wydawnictwo Uniwersytetu w Białymstoku.

Aleksandrowicz-Pędich, Lucyna (2014). Rozwijanie kompetencji interkulturowych $w$ kształceniu językowym. Plenarvortrag, der am 23.09.2014 in Warschau während der internationalen Konferenz anlässlich des Europäischen Tages der Sprachen Kompetencje interkulturowe w edukacji językowej gehalten wurde. Warszawa.

Argyle, Michael (1999). Psychologia stosunków międzyludzkich. Warszawa: Wydawnictwo Naukowe PWN.

Argyle, Michael (2013). Körpersprache \& Kommunikation. Nonverbaler Ausdruck und soziale Interaktion. 10. Auflage. Paderborn: Junfermann Verlag. (übersetzt aus dem Englischen von Karsten Petersen).

Broszinsky-Schwabe, Edith (2011). Interkulturelle Kommunikation. Missverständnisse Verständigung. Wiesbaden: VS Verlag für Sozialwissenschaften/Springer Fachmedien.

Dakowska, Maria (2008). Psycholingwistyczne podstawy dydaktyki jezzyków obcych. Warszawa: Wydawnictwo Naukowe PWN.

Donec, Pavel (1991). Landeskunde und Linguolandeskunde in der interkulturellen Kommunikation. Deutsch als Fremdsprache 28(4), 222-226.

Erll, Astrid/Gymnich, Marion (2007). Interkulturelle Kompetenzen. Erfolgreich kommunizieren zwischen Kulturen. Stuttgart: Klett Lernen und Wissen.

Feuerstein, Heinz-Joachim (2001). Interkulturelle Kommunikation und Verwaltungshandeln. In: E. Riehle (Hrsg.), Interkulturelle Kompetenz in der Verwaltung? Kommunikationsprobleme zwischen Migranten und Behörden (S. 195-236). Wiesbaden: Springer Fachmedien.

Graefen, Gabriele/Liedke, Martina (2008). Germanistische Sprachwissenschaft. Deutsch als Erst-, Zweit- oder Fremdsprache. Mit CD-ROM. Tübingen/Basel: Francke UTB.

Grucza, Franciszek (1976). Dziedzina lingwistyki stosowanej. In: F. Grucza (Hrsg.), Lingwistyka stosowana i glottodydaktyka (S. 7-22). Warszawa: Wydawnictwa Uniwersytetu Warszawskiego.

Grucza, Franciszek (1983). Zagadnienia metalingwistyki. Lingwistyka - jej przedmiot, lingwistyka stosowana. Warszawa: Państwowe Wydawnictwo Naukowe.

Grucza, Franciszek (2000). Kultur aus der Sicht der angewandten Linguistik. In: H.-D. Schlosser (Hrsg.), Sprache und Kultur (S. 17-29). Frankfurt a.M.: Peter Lang.

Grucza, Franciszek (2017). Lingwistyka Stosowana. Historia - Zadania - Osiagnięcia. Dzieła zebrane. Tom 9. Warszawa: Wydawnictwo Naukowe Instytutu Komunikacji Specjalistycznej i Interkulturowej Uniwersytet Warszawski.

Hess-Lüttich, Ernest W.-B. (2010). Interkulturalität, die/interkulturell. In: H. Barkowski/ H.-J. Krumm (Hrsg.), Fachlexikon Deutsch als Fremd- und Zweitsprache (S. 137-138). Tübingen/Basel: Francke.

Heringer, Hans Jürgen (2007). Interkulturelle Kompetenz. Tübingen/Basel: A. Francke Verlag.

Hall, Edward T. (1959). The silent language. New York: Doubleday \& Company, INC., Garden City. 
Hofstede, Geert (1993). Kulturen-Organisationen-Management. Interkulturelle Zusammenarbeit. Wiesbaden: Gabler.

Holzbrecher, Alfred (2004). Interkulturelle Pädagogik. Berlin: Cornelsen.

Klammer, Marion (1989). Nonverbale Kommunikation beim Verkauf. Heidelberg: PhysicaVerlag.

Knapp, Karlfried/Knapp-Potthoff, Annelie (1990). Interkulturelle Kommunikation. Zeitschrift für Fremdsprachenforschung, 1, 62-93.

Knapp, Karlfried (2004a). Vorwort. In: K. Knapp u. a. (Hrsg.), Angewandte Linguistik. Ein Lehrbuch (S. XVII-XX). Tübingen/Basel: Francke UTB.

Knapp, Karlfried (2004b). Interkulturelle Kommunikation. In: K. Knapp u. a. (Hrsg.), Angewandte Linguistik. Ein Lehrbuch (S. 409-430). Tübingen/Basel: Francke UTB.

Knapp, Karlfried u. a. (Hrsg.) (2004). Angewandte Linguistik. Ein Lehrbuch. Tübingen/ Basel: Francke UTB.

Kumbier, Dagmar/Schulz von Thun, Friedemann (2009). Interkulturelle Kommunikation aus kommunikationspsychologischer Perspektive. In: D. Kumbier/F. Schulz von Thun (Hrsg.), Interkulturelle Kommunikation: Methoden, Modelle, Beispiele (S. 9-27). Reinbek bei Hamburg: Rowohlt Taschenbuch Verlag.

Lüsebrik, Hans Jürgen (2005). Interkulturelle Kommunikation. Interaktion, Fremdwahrnehmung, Kulturtransfer. Stuttgart/Weimar: Metzler.

Maletzke, Gerhard (1996). Interkulturelle Kommunikation. Zur Interaktion zwischen Menschen verschiedener Kulturen. Opladen: Westdeutscher Verlag.

Mihułka, Krystyna (2011). Interkulturelle Kommunikation - obligatorisches Fach philologischer Studienrichtungen. Studia Germanica Resoviensia, 9 (67), 233-250.

Mihułka, Krystyna (2015). „Man kann nicht nicht kommunizieren“ - zur Bedeutung des nonverbalen Codes in der interpersonalen Kommunikation. In: P. Bąk/K. Mihułka/B. Rolek (Hrsg.), Sprache und Kommunikation (S. 33-48). Rzeszów: Wydawnictwo Uniwersytetu Rzeszowskiego.

Mihułka, Krystyna (2016). Interkulturalität in der philologischen Bildung. Curriculare Vorgaben, ihre Realisierung und Evaluation anhand der Hinweise der Germanistikstudenten. In: K. Mihułka/P. Bąk/J. Chojnacka-Gärtner (Hrsg.), Interkulturalität in Theorie und Praxis der Glottodidaktik und Translatorik (S. 21-37). Rzeszów: Wydawnictwo Uniwersytetu Rzeszowskiego.

Mihułka, Krystyna/Bąk, Paweł/Chojnacka-Gärtner, Joanna (Hrsg.) (2016). Interkulturalität in Theorie und Praxis der Glottodidaktik und Translatorik. Rzeszów: Wydawnictwo Uniwersytetu Rzeszowskiego.

Mikułowski-Pomorski, Jerzy (2003). Komunikacja interkulturowa. Wprowadzenie. Kraków: Wydawnictwo Akademii Ekonomicznej w Krakowie.

Nagel, Friederike (2012). Die Wirkung verbaler und nonverbaler Kommunikation in TVDuellen. Eine Untersuchung am Beispiel von Gerhard Schröder und Angela Merkel. Wiesbaden: Verlag für Sozialwissenschaften/Springer Fachmedien.

Neuland, Eva (2013). Interkulturalität - immer noch eine Herausforderung für Linguistik und Deutsch als Fremdsprache. ZVPG. Zeitschrift des Verbandes Polnischer Germanisten. Czasopismo Stowarzyszenia Germanistów Polskich 2013/2, 161-177, verfügbar über http://www.ejournals.eu/ZVPG/Tom-2(2013)/Zeszyt_2_(2013)/art/2645/. 
Nünning, Ansgar/Zierold, Martin (2010). Kommunikation. In: C. Surkamp (Hrsg.), Metzler Lexikon Fremdsprachendidaktik. Ansätze - Methoden - Grundbegriffe (S. 134-135). Stuttgart/Weimar: Verlag J.B. Metzler.

Oksaar, Els (1988). Kulturemtheorie. Ein Beitrag zur Sprachverwendungsforschung. Hamburg: J. Jugius-Gesellschaft der Wissenschaften.

Pfeiffer, Waldemar (2003). Von der linguistisch bezogenen Fremdsprachendidaktik zur interkulturellen Fremdsprachenpädagogik. Ein Essay. Linguistik Online, 13 (1), verfügbar über http://www.linguistik-online.de/13_01/pfeiffer.html.

Prokop, Izabela (1992). Zum Begriff Höflichkeit. In: J. Papiór (Hrsg.). Untersuchungen zur polnisch-deutschen Kulturkontrastivik (S. 189-196). Poznań: Wydawnictwo Naukowe Uniwersytetu im. Adama Mickiewicza.

Prokop Izabela (1995). Fehlschläge in der interkulturellen Kommunikation zwischen Deutschen und Polen. In: W. Pfeiffer (Hrsg.), Wissenschaftseinrichtungen und Strukturentwicklung in der Grenzregion. Modellfall Europa-Universität Viadrina Frankfurt (Oder) (S. 165-172). Poznań: Wydawnictwo Naukowe Uniwersytetu im. Adama Mickiewicza.

Prokop Izabela (2000). W poszukiwaniu lingwistyki (do)stosowanej. Scripta Neophilologica Posnaniensia. Rocznik poświęcony językoznawstwu, literaturoznawstwu $i$ kulturoznawstwu, II, 259-274.

Riehlel, Eckart/Seifert Michael (2001). Stolpersteine interkultureller Verwaltungskommunikation. In: E. Riehle (Hrsg.), Interkulturelle Kompetenz in der Verwaltung? Kommunikationsprobleme zwischen Migranten und Behörden (S. 11-35). Wiesbaden: Springer Fachmedien.

Schröer, Norbert (2009). Interkulturelle Kommunikation. Einführung. Essen: Oldib.

Schugk, Michael (2004). Interkulturelle Kommunikation. Kulturbedingte Unterschiede in Verkauf und Werbung. München: Vahlen.

Spillner, Bernd (2003). Angewandte Linguistik. In: K.-R. Bausch/H. Christ/H.-J. Krumm (Hrsg.), Handbuch Fremdsprachenunterricht. 4. Aufl. (S. 31-37). Tübingen/Basel: A. Francke.

Szopski, Marek (2005). Komunikowanie międzykulturowe. Warszawa: WSiP.

Watzlawick, Paul/Beavin, Jannet H./Jackson, Don D. (1969/1990). Menschliche Kommunikation: Formen, Störungen, Paradoxien. 8. Auflage. Bern/Stuttgart/Toronto: Huber.

Wang, Lei (2009). Wenn Konfuzius Schulz von Thun trifft ... Kommunikationspsychologie aus Sicht einer Chinesin. In: D. Kumbier/F. Schulz von Thun (Hrsg.), Interkulturelle Kommunikation: Methoden, Modelle, Beispiele (S. 187-205). Reinbek bei Hamburg: Rowohlt Taschenbuch Verlag.

Wilczyńska, Weronika/Mackiewicz, Maciej/Krajka, Jarosław (2019). Komunikacja interkulturowa. Wprowadzenie. Poznań: Wydawnictwo Naukowe Uniwersytetu im. Adama Mickiewicza. 


\section{Internetquellen}

Dziennik Ustaw: Rozporządzenie Ministra i Szkolnictwa Wyższego z dnia 14 listopada 2018 roku w sprawie charakterystyk drugiego stopnia efektów uczenia się dla kwalifikacji na poziomach 6-8 Polskiej Ramy Kwalifikacji (Dz. U. 2018 poz. 2218), 2018, verfügbar unter: http://prawo.sejm.gov.pl/isap.nsf/DocDetails.xsp?id=WDU20180002218.

Dziennik Urzędowy Unii Europejskiej: Zalecenie Rady z dnia 22 maja 2017 r. w sprawie europejskich ram kwalifikacji dla uczenia się przez całe życie i uchylające zalecenie Parlamentu Europejskiego i Rady z dnia 23 kwietnia 2008 r. w sprawie ustanowienia europejskich ram kwalifikacji dla uczenia się przez całe życie (Dz. U. UE 2017/C 189/03), 2017, verfügbar unter: https://ec.europa.eu/ploteus/sites/eac-eqf/files/pl.pdf.

Europäische Kommission: Der europäische Qualifikationsrahmen für lebenslanges Lernen. Luxemburg: Amt für amtliche Veröffentlichungen der Europäischen Gemeinschaften, 2008, verfügbar unter https://ec.europa.eu/ploteus/sites/eac-eqf/files/broc hexp_de.pdf.

Ministerstwo Nauki i Szkolnictwa Wyższego: Charakterystyki II stopnia Polskiej Ramy Kwalifikacji typowe dla kwalifikacji uzyskiwanych w ramach szkolnictwa wyższego poziomy 6-8 Zapisy - wiedza, umiejętnos'ci, kompetencje społeczne - należy odnosic' do uczenia się w okres'lonym zakresie wyższego wykształcenia. verfügbar unter : http:// www.kwalifikacje.gov.pl/images/downloads/tabele_PRK/PRK_tab5.pdf.

Ministerstwo Nauki i Szkolnictwa Wyższego: Polnischer Qualifikationsrahmen (PQR) und Europäischer Qualifikationsrahmen (EQR). verfügbar unter: https://prk.men.gov.pl/ de/polnischer-qualifikationsrahmen-pqr-und-europaeischer-qualifikationsrahmeneqr/.

Ministerstwo Nauki i Szkolnictwa Wyższego: Polska Rama Kwalifikacji (PRK). verfügbar unter: http://www.kwalifikacje.gov.pl/polska-rama-kwalifikacji. 


\title{
Fremdes und Eigenes als „Fremdes verstehen“- Neuere anwendungsbezogene fremdkulturwissenschaftliche Konzepte in Zeichen und Zeiten der Krise
}

\begin{abstract}
Understanding of the other and of one's own as "understanding of otherness“- New applied concepts of cultural aera studies in times and signs of crisis

The present chapter provides an overview of new theoretical approaches to the cultural phenomena of otherness, alteration and alienation. Its aim is to point out the limits of different ways of understanding of the other, of oneself and of the world around in a sense of „solastalgia, a new concept in human health and identity“ (Albrecht 2007). Facing the current situation of the coronavirus crisis and the lack of understanding of the desease or its desciption as „the Chinese desease“ (Trump) or „the white man desease“ (Africans), it is getting more and more important "to understand the own non-understanding“ from an (anti-) and the "other" point of view and the recognition of no understanding to some extent. Therefore, the cultural area studies have to confront theses new challenges. During this process, on the one hand. it is going to change oneself and, on the other hand, in a reciprocal sense, it is going to impact the everyday life or the world. The chapter also explores some of the connections that have been made in recent years between cultural area studies and cultural xenology. Furthermore, the author also intends to highlight anti-biasconcepts and anti-hermeneutic conspects which focus on categories such as boundaries, alienation and strangeness. To this effect, it constitutes an attempt to show how applied science could impact cultural and social constructions in the contemporary world which faces the current challenges.
\end{abstract}

\section{Keywords}

cultural area studies, new studies in xenology, coronavirus crisis, otherness, alienation, anti-bias approaches, anti-hermeneutic concepts, solastalgia 


\section{Einführung: Krise als Chance: Zur Neuaufstellung xenologischer Forschungsbereiche}

„Ganz begreifen werden wir uns nie, aber wir werden und können uns weit mehr als begreifen. "

(Novalis: Blütenstaub)

In dem Beitrag wird vor dem Hintergrund der aktuellen globalen Krisensituation $^{2}$ versucht, zwei innovative Ansätze fremdkulturwissenschaftlicher Forschung in Hinblick auf die Anwendung kulturellen Wissens (Biebert \& Schetsche 2016: 99) neu zu justieren. Dabei handelt es sich zum einen um eine fremd- oder antihermeneutische Position sowie zum anderen um aus der Kognitionspsychologie und Pädagogik bekannte Anti-Bias-Studien und ihre Anwendung auf den fremdkulturellen Kontext. Die Verbindung von Krisenempfinden und Weiterentwicklung eines kulturwissenschaftlichen Forschungsbereichs mag auf den ersten Blick nicht unmittelbar evident erscheinen. Doch wie auf gesellschaftlich-kulturellen Gebieten oder naturwissenschaftlichen Forschungsbereichen, an denen der Einfluss der Pandemie offenkundig und direkt ablesbar ist, scheinen sich zumindest mittelbar auch in dieser Fachdisziplin, neue Impulse zu ergeben bzw. Entwicklungen bereits feststellbarer Tendenzen dieser wissenschaftlichen Provenienz zu beschleunigen. Die Krise könnte somit als Chance zur Weiterführung eines ins Stocken geratenen wissenschaftlichen Diskurses begriffen werden. ${ }^{3}$

\section{Anknüpfung an einen unterbrochenen Wissenschaftsdiskurs}

Zum versiegten „Verständnis der Fremde“ oder eine Art „hermeneutischer Selbstauflösung" Eo ipso wird versucht, die potenzielle Weiterentwicklung einer Disziplin zu skizzieren, die sich trotz einiger Gegenbeispiele (Wierlacher 1993,

1 Novalis: Athenäums-Fragmente. http://www.zeno.org/Literatur/M/Novalis/Fragmentensamm lung/Blüthenstaub. [10.04.2020].

2 Das ursprünglich griechische Wort xpíıı krísis wird im Sinne von Trennung, Unterscheidung, Meinung, Behauptung, Zuspitzung und als Wendepunkt gebraucht und ist etymologisch mit Kritik verbunden. Kritik ist spätestens seit Kants drei Kritiken auch immer Hinterfragung, in diesem Falle eines Wissenschaftsbereichs. Es geht innerhalb des Beitrags letztendlich auch um Zuspitzung von kulturellen und gesellschaftlichen Tendenzen im Sinne der Fremdheit, die in ihrer Überzogenheit neue konstruktive Ansätze von Weltbewältigung bieten.

3 Wie schnell selbst nicht primär naturwissenschaftliche Wissenschaftsdisziplinen auf die Krise reagieren, lässt sich beispielsweise an einer internationalen Konferenz: Multidisciplinary studies: „Resilience for survival“), die von der Universität Cambridge organisiert wurde. 
Leskovec 2011, Pabis 2014, Müller-Funk 2016) ${ }^{4}$ seit ihrer Begründung in den 70er Jahren und einer Hochphase bis Ende der 80er Jahre bzw. Anfang der 90er Jahre, über Jahre hinaus in Stagnation befand und sich forschungsmäßig nicht wirklich weiter entwickelte. ${ }^{5}$ Der Grund hierzu ist nicht allein in der Unterbrechung eines wissenschaftlichen Diskurses bzw. in dessen punktueller, „sich hinschleppender" Fortsetzung zu suchen, sondern könnte ebenso im Widerspruch von „Verstehen“ als epistemologischer Grundvoraussetzung und der Opazität des Gegenstandsbereichs der „Fremde“ selbst liegen. In diesem Zusammenhang spricht Waldenfels (2006: 125) von einer hermeneutischen und phänomenologischen „Selbstaufhebung des Wissenschaftsbereichs“, weil jeder „interpretatorische Zugriff auf das Fremde seinen Gegenstand auflöst“, was als das Paradoxon der Xenologie überhaupt anzusehen ist. Fremdheit wird durch „Auslegung beseitigt“ (Pabis 2014: 25), d. h. die Entwicklung des Fachbereichs Fremdheitswissenschaft wurde durch zunehmende „Selbstaufhebung“ erkauft (Waldenfels 2006: 125). Es handelt sich hierbei um „Aneignungsbemühungen“ des Fremden, die zugleich „Abwehrmechanismen“ darstellen (Pabis 2014: 9).

Durch das aneignende Verfahren (Pabis 2014: 15) wird Fremdes dem Eigenen sozusagen „einverleibt“ und das Eigene durch das Fremde nicht in Frage gestellt. Von den Anfängen der Fremdheitsforschung ${ }^{6}$ an bis heute ist gegen diese Art der Aneignung des Fremden Widerstand geleistet und versucht worden, Fremdes komplementär zur traditionellen Hermeneutik um den Aspekt kultureller Alterität ${ }^{7} \mathrm{zu}$ erweitern, wenn man etwa an die Studien von Said $\left({ }^{4} 2014 / 1979\right)$, Bhabha (1994) oder Reinhard ( $\left.{ }^{2} 2016\right)$ denkt. Außerdem nimmt etwa eine fremdkulturelle Germanistik Anleihen bei der Konzeption der „Alterität“ und entwickelt eine „Hermeneutik der Distanz“ (Wierlacher 1980), eine „Kultur der Fremdheit“ (Röttgers 2007: 97/2011: 11) bzw. eine „Hermeneutik der Alterität“

4 Interessanter Weise kommen die Impulse vor allem von fremdkulturellen Germanistinnen und Germanisten, um den unseligen Ausdruck „Auslandsgermanistik“ zu vermeiden, der beispielsweise auf dem IVG-Kongress im Jahre 2015 in Shanghai stark kritisiert wurde. Es existiert auch keine „Auslandsromanistik oder Auslandsanglistik“.

5 Als Begründung für diesen Sachverhalt wird u. a. herangezogen, dass der Begriff „Fremde“ ähnlich wie der Begriff der „Interkulturalität“, „unscharfen Definitionen und Abnutzungen“ unterliegt (vgl. Leskovec 2011: 9). In der Tat finden sich viele Veröffentlichungen (oftmals Sammelbände), die den Begriff nicht definieren oder zumindest ausweisen, auf welchen „Fremdheitsbegriff“ sie sich beziehen.

6 Es sei daran erinnert, dass über lange Zeit sich der Begriff „Fremde“ einer regelrechten Missachtung „erfreute“. So tauchte der Begriff trotz seiner Bedeutung für die griechische Philosophie, z. B. der Xenos etwa bei Platon, im berühmten philosophischen Wörterbuch von Ritter lange Zeit nicht auf. Inzwischen gibt es einen Artikel von Bernhard Waldenfels zu „Xenologie, Wissenschaft vom Fremden“. Waldenfels beklagt im Übrigen diesen Umstand ebenso im erwähnten Artikel.

7 Die Beziehung von Fremde und Alterität hat Harald Weinrich (1993: 131) mit seiner bekannten Formulierung der „Fremdheit als Interpretament der Andersheit“ geprägt. 
(Böhler 1985: 236). Ähnlich wie Waldenfels hatten vor allem Wierlacher (1985) und Krusche (1980) die Hermeneutik Gadamers und insbesondere seinen über verschiedene Forschungsbereiche hinausreichenden Begriff der „Horizontverschmelzung“ (Vgl. u. a. Gadamer 41975: 308ff.) kritisiert, weil dieser letztendlich eine Auflösung des „Fremden im Eigenen“ bedeuten würde. (vgl. Wierlacher 1985: 10).

\section{3. „Neue Komplementarität“ durch Alteritätskonzepte und die Forderung nach einer Entwicklung von Fremdheitskompetenz}

Etwas zugespitzt ließe sich behaupten, dass die Xenologie vor allem deshalb begründet wurde, um diesem Dilemma Abhilfe zu schaffen. ${ }^{8}$ Der zunächst eher eng gefasste ethnologische Begriff war in Anbetracht der Herausforderung der modernen bzw. spätmodernen Welt so nicht mehr weiter aufrechtzuhalten und wurde u. a. etwa um „internationale Diversitätskompetenz“ erweitert (Krewer \& Uhlmann 2014). ${ }^{9}$ Konkret handelt es sich um die Herausbildung einer geforderten spezifischen Art von „Fremdheitskompetenz“ (Leskovec 2011: 9), die sich um diverse Konzepte der Relation des Eigenen und des Fremden dreht und anwendungsorientiert sowohl auf den didaktischen wie gesellschaftlichen Prozess auszustrahlen vermag.

Operational geht es letztendlich um zwei Verfahren „angewandter Verstehenswissenschaft“: zum einen um den Versuch, das Fremde als Fremdes in der Differenz im Sinne eines anti- oder fremdhermeneutischen Ansatzes bestehen zu lassen ${ }^{10}$ und zum anderen um einen durch kognitions- und psychologische Untersuchungen beeinflussten Ansatz (etwa bei Freud 1940 oder Erdheim 1986), der der amerikanischen und deutschen Pädagogik (Derman-Sparks 1989, Schmidt 2009/2010, Gramelt 2010) bzw. Kognitionspsychologie (Pohl ${ }^{2} 2017$ ) entstammt. Bei letzterem wird von der Voraussetzung der Erkenntnis des

8 Mit „Fremdkulturwissenschaft“ bzw. „Xenologie“ ist jener Forschungsbereich gemeint, der ursprünglich von dem Kameruner Politologen, Soziologen und Ethnologen Léopold-Joseph Bonny Duala-M'Bedy bereits im Jahre 1977 begründet. (Vgl. Munasu Duala-M'Bedy 1977) und in der Zwischenzeit um "Theorien des Fremden“ (Müller-Funk 2016: 17) in Hinsicht auf historische, soziologische, philosophische, psychologische u. ä. Erkenntnisse erweitert wurde.

9 Im Modell des Wertschätzenden Vergleichs (Krewer \& Uhlmann 2014) werden fünf Punkte der Wahrnehmung der Fremde herausgestellt: von kultureller Unbewusstheit über die Erziehung zu kulturellem Bewusstsein, zu Perspektivenwechsel, Systemvergleich und schließlich zur Co-Konstruktion, um zu einem besseren Fremdverständnis zu kommen, in dem Sinne wie auch die GIZ (Gesellschaft für internationale Zusammenarbeit) ihr didaktisches Konzept der Lernlandschaften mit dem Ziel der „internationalen Diversitätskompetenz“ aufgebaut hat.

10 Was nicht synonym zu verstehen ist. 
„Fremden in uns allen“ (Gruen ${ }^{6} 2002$, Kristeva 1990) oder der eigenen erkenntnismäßigen Vorprägungen oder gar Verzerrungen (Anti-Bias-Ansätze) ausgegangen. Gerade in Zeiten der Krise scheint diesem letzteren Ansatz in Anbetracht der momentanen Pandemie als „unverfügbare Fremde“ (Rosa 2019) eine besondere Rolle zukommen: im Hinblick auf die Veränderung einer Fremdheitslehre und deren Bedeutungsgewinn und reziprok in ihrer Rückwirkung auf Kulturen und Gesellschaften.

\section{Die hermeneutische Unverfügbarkeit einer „fremden“ Welt}

Ohne konkrete Ergebnisse des Beitrags bereits vorweg zu nehmen, ließe sich mit Ricoeur $(1972 / 1973)^{11}$ von einer wissenschaftlich fundierten prinzipiellen Unerkennbarkeit des Fremden ausgehen, aber im Sinne einer „hermeneutischen Spirale $^{\text {“12 }}$, was eine potentielle Annäherung ohne vollständige Erfassung des Phänomens meint. Auf diese Weise bildet sich ein Einverständnis des hermeneutischen Entziehens der Welt bei gleichzeitiger Konstatierung der partiellen Fremdheit des eigenen Erkenntnisstandpunkts heraus. Diesem Paradoxon ist nicht zu entgehen, wie gerade aktuelle Veröffentlichungen zum „Unverständnis der Krise" belegen (Brock 2020 oder Giordano 2020). In Anlehnung an den Begriff Adornos \& Horkheimers lässt sich von einer „Dialektik der Hermeneutik“ oder einer „negativen Hermeneutik“ sprechen Vgl. Schurz (1995). An anderer Stelle wird auch von „Post-Hermeneutik“ gesprochen (Meier \& Westerkamp 2016: 3).

Gerade eine solche Position der hermeneutischen Unverfügbarkeit der Fremde wird zum Katalysator eines innovatorischen Konzepts von Fremdkulturwissenschaft. Paradox ausgedrückt:

Je mehr die Unverfügbarkeit der Welt (wie des Eigenen und des Anderen) „erkannt“ und als Grundvoraussetzung ausgegangen wird, umso mehr eröffnen sich konkrete handlungsorientierte und kommunikationsstrategische Möglichkeiten im Hinblick auf die Entwicklung und Anwendung der erwähnten Fremdheitskompetenz. Diese wird in bestimmten Zeitphasen und Epochen besonders virulent, so wie Schurz schreibt, dass es

11 Ricoeur (1996: 11ff, 143ff, 173ff.) hat zudem einen unersetzlichen Beitrag für die Fremdheitsforschung im Hinblick auf die Unterscheidung der Identität geleistet: Zum einen stellt er in seinem Werk „Soi-même comme un autre“ die komplizierte Relationen von Selbst und (geschlechtsneutral) Anderem heraus, zum anderen differenziert er das Selbst oder das Eigene im Hinblick auf idem (Selbigkeit, englisch: sameness, mit unveränderlichem Kern) und ipse (Selbstheit, englisch: selfhood, veränderlich in Relation zu dem Anderen). Vgl. auch Müller-Funk (2016: 11ff).

12 Als Weiterführung des Hermeneutischen Zirkels vgl. Bolten (1985). 
in Angesicht existentieller Krisen, Krankheiten und vor allem des Todes, „Momente gibt, in denen das Nicht-Verstehen seinen festen (rituellen) Platz hat" (Schurz 1995: 7).

\section{Die Zeit der Krise als Katalysator fremdkulturwissenschaftlicher Erkenntnisse}

Wie in vielen anderen wissenschaftlichen und gesellschaftliche Bereichen könnte sich die Corona-Krise also auch als konstruktiv für den inkriminierten Wissenschaftsbereich erweisen: Die Wirkungsweise des Corona-Virus' wird im Sinne der Entwicklung eines Impfstoffes oder eines Therapeutikums momentan noch nicht „verstanden“, zeichnet sich auf eine besondere Art und Weise durch hermeneutische Unzugänglichkeit und „Fremdheit“ aus und zwingt nicht nur Naturwissenschaften, sondern auch „Verstehens- oder Textwissenschaften“ (Dilthey 1964) dazu, sich mit ihren eigenen Grundlagen und Paradigmata auseinander zu setzen.

Die sich daraus ergebenden, andrängenden, sich verändernden Aufgaben einer angewandten Fremdkulturwissenschaft in Zeiten der Krise erfolgt auf der Basis der Betrachtung aktueller gesellschaftlicher bzw. kulturkundlicher Tendenzen oder soziologischer wie politischer „Befunde“ der Bundesrepublik Deutschland ${ }^{13}$ und unter Berücksichtigung der erwähnten komplizierten Relation von Eigenem und Fremden. Dieses Verhältnis lässt sich mit Schneider (2018) wie folgt fassen:

Fremdheit ist ein Begriff, vermittels dessen eine bestimmte Form von Differenz markiert wird, sei es die als Entfremdung bezeichnete Differenzerfahrung gegenüber der eigenen Person, der eigenen Gruppe, Kultur und Gesellschaft oder im Sinne von Fremdheit gegenüber anderen Subjekten und deren jeweiligen Lebensweisen. Fremdheit lässt sich daher nur relational begreifen, wobei das Fremde meist die negativ konnotierte oder zumindest problematisierte Seite eines asymmetrischen Gegenbegriffspaars bezeichnet (Schneider 2018: 53).

In den Fokus rückt darüber hinaus die Frage, inwieweit eine so verstandene Konzeption „Angewandter (Fremd-) Kulturwissenschaft ${ }^{\text {“14 }}$ Orientierung in einer

13 In Hinblick auf die Einschätzung der Bundesrepublik vor der Pandemie erscheinen Veröffentlichungen signifikant, die in einer Art Zeit- oder Zeitgeistdiagnose von einer "Gesellschaft der Angst“ (Bude 2013), einer „aufgeregten Gesellschaft“ (Hübl 2019), „einer erschöpften Gesellschaft“ (Grünwald 2013) oder gar „psychotischen Gesellschaft“ (von Schirach 2019) etc. sprechen oder eine „große Gereiztheit in der öffentlichen Diskussion“ (Pörksen \& von Thun 2020, Pörksen 2019) feststellten. Peter Sloterdijk (2020) spricht von „medialen Erregungsräumen“.

14 Der Begriff „Angewandte Fremdkulturwissenschaft“ wird vor allem in dem Handbuch „Interkulturelle Germanistik" von Andrea Bogner und Alois Wierlacher (2003: 8f, 24, 32, 292, 549f., 552f. und 626). Vgl. auch Pabis (2014). 
zunehmend Besorgnis erregenden, „fremden“Welt geben kann. Schon lange vor Ausbruch der Corona-Pandemie erhebt Heinrichs (2005) die Forderung nach einem „Engagement“ der Kulturwissenschaften im weiten Sinne, denn ihnen „wächst mehr denn je die Aufgabe zu, ihr historisches Wissen und ihr strukturelles Verstehen in den Dienst eines dringend notwendig gewordenen anthropologisch/ethnologischen Begreifens der heutigen weltpolitischen Lage zu stellen“ (Heinrichs 2005: 9).

\section{Wirklichkeitskrise und die neue Fremdheitskonzeption der „Solastalgie“}

Gerade im Hinblick auf das „historische Wissen“ und das „strukturelle Verstehen“, werden im Weiteren zwei innovatorische Modelle „kulturwissenschaftlichen Fremdstellens" diskutiert, die den Anwendungsbereich einer Fremdkulturwissenschaft verändern und erweitern können. Dabei handelt es sich um spezifische Arten von „Fremdstellen“ der eigenen Wahrnehmung, der Perzeption des Anderen sowie der Einschätzung des Kommunikationsprozesses. Zudem wird mit Berger und Luckmann (2013: 1) von der „sozialen Konstruktion der Wirklichkeit“ ausgegangen:

Für unsere Zwecke genügt es, ,Wirklichkeit' als Qualität von Phänomenen zu definieren, die ungeachtet unseres Wollens vorhanden sind - wir können sie ver- aber nicht wegwünschen. ,Wissen' definieren wir als die Gewißheit, daß Phänomene wirklich sind und bestimmbare Eigenschaften haben.

Außerdem bildet die Prämisse einer „Wirklichkeitskrise“ oder „epistemischen Krise" den Ausgangspunkt der Überlegungen:

Wirklichkeitskrisen können im Einzelfall recht unterschiedlich ausfallen, sind aber meist dann besonders folgenreich, wenn mit ihnen individuelle oder kollektive Grenzerfahrungen verbunden sind: Epidemien und Unglücksfälle, Kriege und Naturkatastrophen, Verbrechen und Wahnsinn - alles Ereignisse, bei denen eine nicht übersehbare Diskrepanz zu dem aufscheint, was unter vertrauten Bedingungen als ,wahr', ,normal', ,richtig' oder, vernünftig' gilt. Kulturell besonders problematisch sind dabei jene Krisen, in denen grundlegende Überzeugungen hinsichtlich der Beschaffenheit der Welt infrage gestellt werden, [...] was wir hier epistemische Krisen nennen möchten, um die generelle Beschaffenheit unserer natürlichen Umwelt, der sozialen Ordnung und vielleicht sogar der, ganzen Welt' in einem metaphysischen Sinne (Biebert \& Schetsche 2016: 3).

Mit dieser Infragestellung „grundlegender Überzeugungen in Bezug auf die Beschaffenheit der Welt" beschäftigt sich auch ein relativ junger Bereich innerhalb der Fremdheitsforschung, die "Solastalgie“ in Hinblick auf eine neue Be- 
grifflichkeit von „Fremde“. In einer globalisierten und sich immer stärker vernetzenden Welt mutiert die eigene Umgebung selbst zur Fremde. Das Krisenempfinden vieler Menschen wird dadurch bestärkt, dass sie die eigene Welt nicht mehr wieder erkennen. ${ }^{15}$ Der französische Philosoph Baptiste Morizot (2019) bezeichnet dieses Gefühl des „Heimwehs ohne Exil“ als „Solastalgie“. Er markiert diesen Zustand mit einem Begriff, der auf den Philosophen Glenn Albrecht (2003) zurück geht. Dieser benutzte den Begriff allerdings noch zur psychologischen und medizinischen Beschreibung von Burn-Outs, Stresszuständen oder von psychischen und existentiellen Ängsten, hervorgerufen durch den Verlust der vertrauten Welt. Morizot versteht jedoch den Begriff etwas anders im Sinne der Unbehaustheit des modernen Menschen in der Welt, die über die „transzendentale Obdachlosigkeit“ Lukacs $\left({ }^{2} 1963\right)$ oder die Gott-ist-tot-Philosophie Nietzsches (1954) hinausgeht, ein „Gefühl im Exil zu sein, ohne fortgegangen“ zu sein, wie er betont:

Es geht um den Verlust von Halt und Trost (engl. solace), den Verlust des Zuhauses. Ohne sich dabei jedoch vom Fleck zu rühren - ein Verlust, der durch den globalen Klimawandel hervorgerufen wird. [...] Ich verstehe hier Solastalgie als zeitgenössische Lebenssituation, hervorgerufen durch Umweltveränderungen, die niemanden verschonen werden, und die in einigen Jahrzehnen wahrscheinlich weltweit verbreitet sein wird. Sie lässt selbst die beständigsten und sesshaftesten Menschen unter uns den Verlust und die Verwirrung spüren, die von Migranten besungen wurde. Es ist die Sehnsucht nach einem Zuhause, das zwar präsent ist, aber einem direkt unter den Füßen hinweg, entgleitet, ohne dass man es einen Moment verlassen hätte. [...] Uns kommt die Welt abhanden-, die Welt, wie Moderne, industrielle Revolution und Naturwissenschaften sie begriffen und zugerichtet haben. Was sich verändert, ist der Raum, in dem wir leben, seine Beständigkeit, seine Unwandelbarkeit und seine vorhersehbaren, zyklisch wiederkehrenden Gewohnheiten (Morizot 2020: 58f.).

Diese Tendenzen werden in Zeiten der Krise noch einmal potenziert. Insofern schien es angesichts dieser Entwicklung geboten, den „Fremdheitsbegriff“ neu zu justieren und das Verhältnis von Eigenem und Fremdem auszuloten ${ }^{16}$. Dabei geht es nicht zuletzt um ein „Fremdstellen “17 eigener wissenschaftlicher Positionen

15 Auf gewisse Weise argumentiert auch Hübl (2019: 148) in Bezug auf die DDR-Identität in diese Richtung, wenngleich mit einem anderen Impetus: „Während sich der bewegliche, neophile Teil der Ostdeutschen schnell an die Kultur des Westens assimilierte, haben paradoxerweise vor allem diejenigen Ostdeutschen, die in ihren Heimatorten geblieben sind, eine Art Migrationserfahrung gemacht“.

16 Dazu steht eine größere Arbeit des Verfassers zur Neubewertung verschiedener Arten der „Fremde“ unter dem Titel: „Fremdheitskonstruktionen in der deutschsprachigen Gegenwartsliteratur" kurz vor ihrem Abschluss.

17 Ganz bewusst ist hier versucht worden, einen neuen Begriff einzuführen, der zum Teil synonym zu „verfremden“ zu verstehen ist. Das Problem mit letzterem besteht darin, dass dieser Begriff bereits auf eine lange Tradition zurückblicken kann, etwa bei Brecht der V-Effekt, was aber dann etwas ganz anderes als das hier Intendierte meint. 
bzw. von zuvor scheinbar in Stein gemeißelten Paradigmata des eigenen Fachbereichs.

\section{Kleiner Exkurs: Fremdkulturelle Zuschreibungen der Krise und die „Konfrontation mit unlösbaren Problemen“}

Wenn Menschen in Zukunft überhaupt noch etwas gemeinsam haben werden, dann sind es nicht Illusionen kultureller Identität - wie gemeinsame Sprache, Religionen, Tischsitten - sondern die Konfrontation aller mit nicht lösbaren Problemen

(Brock 2020)

Schon auf den ersten Blick gerät der phänomenologisch fremdhermeneutische oder fremdkulturelle Aspekt mit in den Fokus der Krisen-Diskussion bzw. jenes Diskurses. ${ }^{18}$ In Zeiten einer großen Krise scheinen die Menschen zunächst auf einfache kulturelle Schuldzuweisungen, Sündenbocktheorie (Girard 1998) oder „konservative Lösungen“ wie es Hübl (2019: 105) bezeichnet ${ }^{19}$, verfallen zu wollen.

Eine erweiterte Aufgabe (fremd-) kulturwissenschaftlicher Forschung könnte nun darin bestehen, über (anti-) hermeneutische und kognitionspsychologische oder kommunikationswissenschaftliche Verfahren solche Fehlzuweisungen oder Fehlzuschreibungen aufzudecken und die „falschen Geister“ auszutreiben, um im Bild der „Geisteswissenschaften“ zu bleiben, und die kantianisch gesprochen „Bedingungen der Möglichkeit“ von Eigen- und Fremdwahrnehmung aufzudecken. In einer Situation, wo es um besonnenes Handeln und Gelassenheit als Haltung geht, können fremdkulturelle oder xenologische Studien als Orientierung in der „Einübung des Verstehens des Unverständlichen“, bzw. Analyse der kommunizierten eigenen Position etwa in der Einschätzung der Differenz zwischen wirklichen Gefahren oder nur vermeintlichen Ängste hilfreich sein.

Dabei soll keineswegs der Eindruck entstehen, auf komplizierte naturwissenschaftliche, politische, soziale Fragen abschließende Antworten geben zu können, gerade in einer Zeit und Situation, in der es kaum verbindliche Ant-

18 In einer Zeit, in der alle Grenzen in Europa geschlossen werden und der österreichische Bundeskanzler Kurz die Ansicht äußert, dass Europa angesichts der Corona-Krise versagt hätte bzw. in einer Phase, in der Verschwörungstheorien lanciert werden oder wo der amerikanische Präsident den Corona-Virus einen „Chinese-Virus“ nennt, was wiederum umgekehrt von chinesischer Seite die Entstehung des Viruses ohne wirkliche Belege zu nennen, daraufhin als ,amerikanische Erfindung“ bezeichnet wird. Vgl.: Der Tagesspiegel vom 23.03. 2020. https://www.msn.com/de-de/nachrichten/coronavirus/streit-der-weltmächte-ver schärft-sich-china-behauptet-coronavirus-könnte-in-usa-entstanden-sein/ar-BB11Amtz? ocid=spartanntp, Zugriff am 23.03.2020.

19 Hübl (2019: 133ff) verweist auch auf das Parasiten-Modell des amerikanischen Evolutionsbiologen Randy Thornhill, wonach Menschen im Angesicht von Seuchen und Epidemien „konservativer" würden. Vgl. Thornhill (2009: 131). 
worten $\mathrm{zu}$ geben scheint ${ }^{20}$ Was aber versucht werden kann, ist Evidenz dafür herzustellen, dass sich Basis geistes- oder kulturwissenschaftlicher Grundlagen und Erkenntnissen der hier inkriminierte Fachbereich der gesellschaftlichen und (europäisch-) politischen Verantwortung nicht entziehen kann.

Was Menschen letztendlich als Kulturträger (ver-)eint, ist die Konfrontation mit „unlösbaren Problemen“ (Brock 2020, 27) sowie mit unverständlichen Entwicklungen existentiellen Bedrohungsphase lässt sich nicht nur mit der empirischen Beschreibung anthropologischer Ängste, Aggressionen etc. begegnen, sondern zugleich in der wissenschaftlichen Begründung von Werten wie Solidarität (Bude 2019) oder dem Engagement für andere, die Menschen im philosophisch ethischen wie moralischen Sinne zum, gemeinsamen „Überleben “21 benötigen.

\section{Desiderat: Der „fremde Blick“ als hermeneutisch operatives Verfahren}

Zur Bewahrung der Fremdheit des Anderen und die Erkenntnis der Fremdheit des Eigenen kommt dem Selbst- und Weltverstehen oder der (Kultur-) Vermittlung hier im Besonderen, aber auch im allgemeineren, prinzipiellen Sinne eine besondere Rolle zu, worin zugleich eine Aufgabe einer modernen (Fremd-) Kulturwissenschaft liegen kann. Dies soll im Folgenden an zwei anwendungsbezogenen Modellen im Hinblick auf eine hermeneutische Verminderung des „Unverständnisses“ des prinzipiell Anderen sowie der Darstellung von Fremdheit der eigenen Wahrnehmung konkretisiert werden.

\subsection{Modell A: Das Verstehen des Anderen als „Fremde“: \\ Methodische Anleihen bei der (Fremd- und Anti-) Hermeneutik}

Wenn die Corona-Pandemie einen bislang (irgend-) einen noch so verborgenen, „fremden Sinn“ hat, dann besteht dieser in der Ahnung, Erkenntnis oder Anerkennung des Nicht-Verstehens. Bislang sind sich alle Experten darin einig, dass wir noch zu wenig über die Gegenmittel gegen die Krankheit wissen. Das heißt im hegelianischen Sinne, dass wir zunächst viele Dinge noch "glauben“ oder an-

20 In diesem Zusammenhang sei an das im angelsächsischen Raum entwickelte Konzept einer „post-normal science“ erinnert, die Forderung zum interdisziplinären Zusammenschluss von Wissenschaftlern unterschiedlicher Provenienzen in einer Welt in der „facts uncertain, values in dispute, stakes high and decisions urgent" geworden sind. Vgl. Funtowicz undJerome Ravetz (1993: 755).

21 Denn darum geht es jetzt, nicht mehr allein um den Einzelnen. 
nehmen müssen (Hegel 1979). ${ }^{22}$ Es lassen sich zwei signifikante Tendenzen innerhalb der Krise festhalten: Zum einen die Renaissance der (Natur-) Wissenschaft in Öffentlichkeit und Medien, zum anderen aber die „Anerkennung“ des (Noch-) Nicht-Wissens der Wissenschaftler.

Das betrifft zum einen die Sphäre der Empirie und der objektiven Fakten, zum anderen bezieht es sich auf den Vermittlungsaspekt: Wir wissen, dass wir (noch) nichts wissen und wir wissen gleichermaßen um unser Nichtwissen und um jenes der anderen. Diese Zuspitzung lässt sich auf den hermeneutischen Aspekt insgesamt übertragen. Ist an einer anderen Stelle das gegenseitige Unverständnis behauptet worden, in Bezug auf einen Satz von Schleiermacher und diesen umformend: „Wir wissen, dass wir uns nicht verstehen, und das müssen wir besser verstehen“ (Schleiermacher 2012, 17) ${ }^{23}$, so geht es nun darum, den Satz wie folgt zu formulieren: Wir wissen, dass wir nichts wissen, das müssen wir besser wissen bzw. verstehen.

Damit gerät die eigene Perspektive, Prägung, Wahrnehmung, Vorurteils- oder Verzerrungsfähigkeit in den Fokus der Betrachtung. Denn wie andere Forscher sind Kulturwissenschaftlerinnen und Kulturwissenschaftler in „freihändigen Kulturdiagnosen“ ebenso wenig vor einer Art von Berufskrankheit gefeit, die Hübl (2019) u.a. den „Bestätigungsirrtum“ nennt: „[...] Wir alle neigen nämlich dazu, eher nach Hinweisen zu suchen, die unsere eigenen Thesen bestätigen, als nach solchen, die ihnen widersprechen“ (Hübl 2019: 19). ${ }^{24}$

Diese „bittere Erkenntnis“ leitet über zur Erkenntnis oder Wahrnehmung der eigenen Bias oder analog zum zuvor Gesagten, zum „eigenen Fremden “25, „um sich aus der Blase der eigenen Vorurteile zu befreien [... “ (Pörksen 2019: 191). In der klassischen soziologischen Handlungstheorie (namentlich bei Weber 2011

22 Vgl. auch: Walser (2012: 34): „Unsere Glaubensfähigkeit ist viel ausgeprägter als unsere Wissensfähigkeit.“

23 Als Begründer der Hermeneutik im deutschsprachigen Bereich gelten neben Gadamer $\left({ }^{4} 1975\right)$ vor allem Friedrich Schleiermacher und Wilhelm Dilthey, von der französischen Philosophie ist zudem Paul Ricoeur zu nennen. Schleiermacher, der Urvater der „Hermeneutik“, war sich im Übrigen der Begrenzung dieser durchaus bewusst. Auf gewisse Weise ließe sich folgende Aussage eigentlich schon der Anti-Hermeneutik oder der „negativen“ Hermeneutik zurechnen, wo er schreibt: „[...] daß sich das Mißverstehen von selbst ergibt und das Verstehen auf jedem Punkt muß gewollt und gesucht werden“ Vgl. Schleiermacher (1993: 92). Schleiermacher ist der erste, der die Hermeneutik aus ihrem Korsett als „Textauslegungslehre“ befreit und den Begriff weiter im Sinne der „Auslegung von Selbst und Welt" begreift, Dilthey (1964) dagegen derjenige, der zwischen den erklärenden Wissenschaften (den Naturwissenschaften) und den „verstehenden Wissenschaften (den Geisteswissenschaften)" unterscheidet.

24 Vgl. zum „Bestätigungsirrtum“ - „Confirmation-Bias“ Wason (2007) und Kahnemann (2012). Hübl (2019: 19) weist etwa darauf hin: „Psychologen und Evolutionsbiologen überschätzen tendenziell den Einfluss der Natur, Geisteswissenschaftler den der Kultur."

25 Im Sinne von Däublers (1919: 65) „Feind als unsere eigene Frage als Gestalt“. 
und Schütz 1993) dient dies geradezu die Voraussetzung für „deutendes Verstehen“ bzw. „verstehendes Erklären“ innerhalb einer Kultur:

Der Sinn, den ein Mensch seinem Handeln zuweist, kann von anderen Menschen deutend verstanden werden. Menschen können auch die Motive des Handelns verstehen, solange es sich entweder um rationale Motive handelt oder um irrationale Motive, die dem Beobachter selbst kognitiv-emotional zugänglich sind. [...] Voraussetzungen für die unterstellte Kongruenz von Motiven sind eine angenommene strukturelle Gleichheit von Bewusstseinsverläufen und eine Erlebnisnähe zwischen Alter und Ego: An Stelle des Anderen würde ich aus denselben Motiven handeln (Schetsche et al. 2009: 476f.).

Das Verstehen des Anderen als anderen respektive Fremden und das Erkennen der eigenen Bias-Anfälligkeit, ist zum einen in Form der Betrachtung des Verstehensprozesses in Kommunikation zu untersuchen (Pörksen \& Schulz von Thun 2020), zum anderen kann aber der eigene „Erkenntnisapparat“, selbst zum Gegenstand der Betrachtung gemacht werden, wie es in der Bias- und Anti-BiasForschung versucht wird. In der Kombination des hermeneutischen und des didaktischen pädagogischen bzw. kognitionspsychologischen Ansatzes liegt nach Auffassung des Verfassers die einzige Möglichkeit, um der eigenen „Verständnisblindheit“ wie der Vorurteilsgeprägtheit, wenn schon nicht zu entkommen, so diese doch wenigstens abzumildern.

\subsection{Modell B: Das Eigene als das Fremde: Anti-Bias-Ansätze}

Innerhalb der in den USA entwickelten Anti-Bias-Forschung wurden im Laufe der Zeit mindestens achtzehn Erkenntnisverzerrungen (Biasse) herausgestellt: von bekannten kognitiven Fehlleistungen wie déformación professionelle, Bestätigungsfehler ${ }^{26}$ oder Attributionsfehler ${ }^{27}$, über Status-Quo-Verzerrungen ${ }^{28}$ bis hin $\mathrm{zu}$ weniger bekannten wie dem Impact Bias ${ }^{29}$ o.ä.

Ziel dieser Forschungsrichtung ist es, demjenigen, der insbesondere im geisteswissenschaftlichen Bereich nach wissenschaftlicher Erkenntnis strebt,

26 Dieser taucht auch in der deutschsprachigen Fachliteratur als confirmation bias auf und bedeutet die Neigung, Informationen so auszuwählen und zu interpretieren, dass sie die eigenen Erwartungen erfüllen.

27 Auch dieses Phänomen wird in der einschlägigen Literatur mit dem englischen Begriff correspondence bias bezeichnet und meint die Neigung, die Ursache für ein beobachtetes Verhalten zu oft in (feststehenden) „Charaktereigenschaften“ der handelnden Person und zu selten in den (variablen) Merkmalen der jeweiligen Situation zu suchen. Vgl. auch dazu Kahnemann (2012).

28 Das meint die Tendenz der Bevorzugung des Status Quo gegenüber Veränderungen.

29 Die psychischen Auswirkungen eines vorgestellten negativen Ereignisses wie Verlust des Arbeitsplatzes oder Trennung vom Partner werden in Dauer und Tiefe systematisch zu stark erwartet. 
seine ihm je eigene Fehleranfälligkeit aufgrund subjektiv gesetzter Grundannahmen vorzuführen bzw. ihn über seine „Vorurteilsanfälligkeit“ (selbst-) aufzuklären. Pörksen nennt dies in nuce eine „Irrtumswissenschaft“ (Pörksen 2018: 207), die der Philosoph Edgar Morin (2001: 12) wie folgt beschreibt:

Wir müssen die kulturellen, intellektuellen und zerebralen Eigenschaften des menschlichen Verstands, seine Prozesse und Modalitäten sowie die psychologischen und kulturellen Veranlagungen, die uns anfällig für Fehler und Illusionen machen, einbeziehen und ihre Erforschung vertiefen [...] Das Wissen um dieses Wissen sollte die entscheidende Voraussetzung bilden, um unseren Geist auf die ständige Bedrohung durch den Irrtum und die Täuschung vorzubereiten, der er ausgesetzt ist. Es geht darum, den Geist im Entscheidungskampf, um Klarheit zu bewaffnen.

Es nimmt Wunder, dass diese Richtung nicht stärker Eingang in fremdkulturelle oder inter- bzw.- transkulturelle Studien gefunden hat. Anti-Bias-Forschung entwickelte sich zuerst in der Kognitionspsychologie und macht uns auf unsere zum Teil unhaltbaren Grundannahmen aufmerksam, die psychologisch, aber auch linguistisch, soziologisch, kulturell, oder in Bezug auf unser Leben oder unseren Beruf, existent sein können. Immer handelt es sich dabei um eigene „Deutungsphänomene“, die sich unserem Bewusstsein zunächst entziehen. Allerdings würde es den Umfang dieses Artikels überschreiten, auf gezielt jeden der Biasse einzugehen (siehe oben). Nichtsdestoweniger es sei darauf verwiesen, dass hierzu schon an Unterrichtsmaterialien und konkreten didaktischen Übungen gearbeitet wird, inwieweit diese Biasse für die jeweils einzelne Betrachtung oder Wahrnehmung transparent gemacht werden können, gerade in Zeichen und Zeiten der Krise.

\section{Desiderate für eine (Fremd-) Kulturwissenschaft der Zukunft - xenologische Wege aufzeigen in einer als fremd erfahrenen Welt}

Die Krise macht uns im fremdhermeneutischen Sinne also vor allem auf zwei Aspekte aufmerksam: Zum einen darauf, dass sich und mit unserem hermeneutischen Erkenntniszugriff sowohl das Verständnis von Welt wie jenes der Gesprächs- oder Dialogpartner uns immer mehr entzogen wird, zum anderen macht sie auf das „Fremde in uns“ aufmerksam, dass wir an jedem Verständigungsprozess bzw. Verständigungsversuch nur auf unsere eigene „mentale Infrastruktur“ (Welzer 2011), unsere habituelle Prägung (Bourdieu 1987) zurückgreifen können. Damit sind jeder eigenen Wahrnehmung von vorne herein „natürliche Grenzen“ gesetzt: Diese wirkt von vorne herein „verzerrt“. Nun lässt sich aber versuchen, der eigenen Fremde, dem Verständnis des Anderen wie der 
Fremdheit der Welt bis zu einem gewissen Grad auf die Schliche zu kommen, indem wir durch Operationen der Fremdkulturforschungen der Pädagogik, Kognitionspsychologie oder der Hermeneutik das Verstehen selbst zum Gegenstand der Betrachtung machen und auf diese Weise eine Annäherung an den eigenen Verständnisprozess versuchen. Im Sinne des Eingangszitats von Novalis: „Begreifen werden wir uns nie, aber wir können mehr als begreifen“, lassen sich die Bedingungen der Möglichkeit des Verstehens, des Anderen, des Fremden wie der „Konstruktion der fremden Welt“ in ihrer phänomenologischen o.a. Dimension beschreiben, in einer Art von „Kritik der reinen Hermeneutik“ als kleinster gemeinsamer Nenner.

So ergibt sich die Aufgabe für eine Angewandte Fremdkulturwissenschaft der näheren Zukunft, über die Anerkennung der Fremde der bzw. des Anderen, das nie abgeschlossene und nie völlig vollzogene Verstehen des Anderen sowie das Erkennen der eigenen Bias-Anfälligkeit, in Form einer dialogischen Metakommunikation, darzustellen. Im besten Falle handelt es sich um ein „Aushandeln“ fremdkultureller kommunikativer Situationen im Habermaschen Sinne (1981. Bd.1: 271), um über die Feststellung dieser beiden unterschiedlichen fremden bzw. anderen Positionen zu einem „Verstehen in der Distanz“ zu kommen. (Pörske \& Schulz von Thun 2020) Insofern können fremdkulturelle Ansätze sui generis wichtige Impulse für ein besseres „Selbst-, Fremd- und Weltverstehen“ liefern und auf diese Weise im Sinne einer Angewandten Fremd-, „Irrtums“- oder Kulturwissenschaft wertvolle Verständnishilfen für die Zeit während, aber vor allem nach der Krise liefern. Dazu könnten auf der Basis des hier Exponierten weitere Einzeluntersuchungen oder -fallstudien weiteres konkretes Material liefern, wie es beispielsweise schon, bezogen auf den internationalen Hochschulbereich, konkret an anderer Stelle versucht worden ist (Wolting 2017).

\section{Literaturverzeichnis}

Albrecht, Glenn (2010). Solastalgia: A new concept in human health and identity. PAN, 3, 41-55.

Albrecht, Glenn (2007). Solastalgia: The distress caused by environmental change. Australian Psychiatry, 15, 95-98.

Appiah, Kwame Anthony (2019). Identitäten - Die Fiktionen der Zugehörigkeit. Übersetzt von Michael Bischoff. Berlin: Hanser.

Bhabha, Homi (2000). The location of culture. 1994. Deutsche Ausgabe: Die Verortung der Kultur. Aus dem Englischen übersetzt von M. Schiffmann und J. Freudl. Tübingen: Stauffenburg Verlag.

Bargatzy, Thomas (1993). Die Ethnologie und der Begriff der kulturellen Fremde. In: A. Wierlacher Alois (Hrsg.), Kulturthema Fremdheit. Leitbegriffe und Problemfelder kulturwissenschaftlicher Fremdheitsforschung (S. 219-234). München: iudicium. 
Benthien, Claudia/Velten, Hans Rudolf (2002). Germanistik als Kulturwissenschaft. Eine Einführung in neue Theoriekonzepte. Reinbek bei Hamburg: Rowohlt.

Berger, Peter L./Luckmann, Thomas $\left({ }^{25} 2013\right)$. Die gesellschaftliche Konstruktion der Wirklichkeit. Eine Theorie der Wissenssoziologie. Frankfurt a.M.: Fischer.

Biebert, Martina F./Schetsche, Michael T. (2016). Theorie kultureller Abjekte. Zum gesellschaftlichen Umgang mit dauerhaft unintegrierbarem Wissen. In: BEHEMOTHA Journal on Civilisation, 9(2), 96-123 https://www.google.de/searchsxsrf=ALeKk02M focjNz8wUYmM5EVRLfOWnai5hQ:1585388007089\&q=Theorie+kultureller+Objekte +Zum+gesellschaftlichen+Umgang+mit+dauerhaft+unintegrierbaremWissen\&spell= $1 \& s a=X \& v e d=2 a h U K E w j G m \_n H 7 r z o A h X C-a Q K H Y 5 p C 3 I Q B S g A e g Q I B x A t \& b i w=136$ $6 \& \mathrm{bih}=473 \#[28.03 .2020]$.

Böhler, Michael (1985). Deutsche Literatur im kulturellen Spannungsfeld von Eigenem und Fremdem in der Schweiz. In: A. Wierlacher (Hrsg.), Das Fremde und das Eigene. Prolegomena zu einer interkulturellen Germanistik (S. 234-261). München: Iudicium Verlag.

Bolten, Jürgen (1985). Die Hermeneutische Spirale. Überlegungen zu einer integrativen Literaturtheorie. Poetica, 1 (3/4).

Bourdieu, Pierre (1993). Über einige Eigenschaften von Feldern. In: P. Bourdieu (Hrsg.), Soziologische Fragen (S. 107-114). Frankfurt a.M.: Suhrkamp.

Bourdieu, Pierre (2001). Das politische Feld: Zur Kritik der politischen Vernunft. Frankfurt a.M.: Suhrkamp.

Brock, Bazon (2020). Das Leben nach dem Virus. Die Welt, 30.03. 2020. (PdF-Datei). https:// www.welt.de/kultur/plus206779101/Bazon-Brocks-Essay-ueber-die-Corona-Krise-Lo gik-der-Dummheit.html (Stand 08.04.2020). Vgl. dazu auch: https://www.br.de/media thek/podcast/aktuelle-interviews/was-kommt-nach-corona-bazon-brock-professorfuer-kulturvermittlung/1794987 (Stand 08.04.2020).

Bazon, Brock (2020). Probleme, die unlösbar sind: Wie gehen wir damit um? Ursachen, Folgewirkungen, Konsequenzen. https://bazonbrock.de/werke/detail/?id=2821 (Stand 15.04.2020).

Bude, Heinz (2014). Gesellschaft der Angst. Hamburg: Hamburger Edition.

Bude, Heinz (2019). Solidarität. Die Zukunft einer großen Idee. München: Hanser.

Däubler, Theodor (1919). Sang an Palermo. In: Ders., Hymne an Italien (S. 65). Leipzig: Reclam.

Derman-Sparks, Louise and the A.B.C. Task Force (1989). Anti-bias-curriculum: Tools for empowering young children. Washington D.C.: National Association for the Education of Young Children.

Dilthey, Wilhelm (1964). Die Entstehung der Hermeneutik (1900). „Zusätze aus den Handschriften“. In: W. Dilthey (Hrsg.), Gesammelte Schriften, Bd. V (S. 317-331, 332338). Stuttgart und Göttingen: Teubner und Vandenhoek \& Ruprecht. https://www.lern helfer.de/sites/default/files/lexicon/pdf/BWS-DEU2-0931-04.pdf (Stand 16.04.2020).

Duala-M’Bedy, Munasu (1977). Xenologie. Die Wissenschaft vom Fremden und die Verdrängung der Humanität in der Anthropologie. Freiburg (Breisgau)/München: Verlag Karl Alber.

Erdheim, Mario (1982). Die gesellschaftliche Produktion von Unbewußtheit. Eine Einführung in den ethnopsychoanalytischen Prozess. Frankfurt a.M.: Suhrkamp. 
Erdheim, Mario (1986). Psychoanalyse, Wissenschaft und Kultur. Frankfurt a.M.: Suhrkamp.

Erdheim, Mario (1988). Psychoanalyse und Unbewußtheit in der Kultur - Aufsätze 19801987. Frankfurt a.M.: Suhrkamp.

Freud, Sigmund (1940). Das Unheimliche. In: Sigmund Freud: Gesammelte Werke. Hrsg. v. Anna Freud u. a. Band XII (S. 229-268). Frankfurt a.M.: Fischer.

Funtowicz, Silvio/Jerome Ravetz (1993). Science for the post-normal age. Futures, 31(7), 735-755.

Gadamer, Hans Georg $\left({ }^{4} 1975\right)$. Wahrheit und Methode. Grundzüge einer philosophischen Hermeneutik. Tübingen: J.C.B. Mohr (Paul Siebeck).

Giordano, Paolo (2020). In Zeiten der Ansteckung. Hamburg: Rowohlt.

Girard, René (1998). Der Sündenbock. Einsiedeln: Benzinger.

Gramelt, Katja (2010). Der Anti-Bias-Ansatz. Zu Konzept und Praxis einer Pädagogik für den Umgang mit (kultureller) Vielfalt. Wiesbaden: Verlag für Sozialwissenschaften.

Grün, Arno ( $\left.{ }^{6} 2002\right)$. Das Fremde in uns. Stuttgart. Klett-Cotta.

Grünewald, Stephan (2013). Die erschöpfte Gesellschaft. Warum Deutschland neu träumen muss. Frankfurt a.M./New York. Campus.

Habermas, Jürgen (1981). Theorie des kommunikativen Handelns. Frankfurt a.M.: Suhrkamp.

Hegel, Georg Friedrich Wilhelm (1979). Glauben und Wissen oder die Reflexionsphilosophie der Subjektivität in der Vollständigkeit ihrer Formen als Kantische, Jacobische und Fichtesche Philosophie. www.zeno.org/Philosophie/M/Hegel+Georg+Wilhelm +Friedrich/Aufsätze+aus+dem+Kritischen+Journal+der+Philphie/Glauben+und+Wis sen+oder+die+Reflexionsphilosophie+der+Subjektivität+in+der+Vollständigkeit+ih rer+Formen+als+Kantische,+Jacobische+und+Fichtesche+Philosophie (Stand 25.03. 2020).

Heinrichs, Heinz Jürgen (2005). Expeditionen ins Innere Ausland. Freud. Morgenthaler. Lévi-Strauss. Kerényi. Das Unbewußte im modernen Denken. Gießen: Psychosozial Verlag.

Hübl, Philipp (2019). Die aufgeregte Gesellschaft. Wie Emotionen unsere Moral prägen. Gütersloh: C. Bertelsmann Verlag.

Kahnemann, Daniel (2012). Schnelles Denken, langsames Denken. München: Siedler.

Krewer, Bernd/Uhlmann, Adelheid (2014). Modelle zur internationalen Kompetenzentwicklung. Das Didaktik-Konzept der Akademie für Internationale Zusammenarbeit. Bonn: Deutsche Gesellschaft für internationale Zusammenarbeit (GIZ). https://www. giz.de/akademie/de/downloads/AIZ-Didaktikkonzept_D_150217_SCREEN.pdf (Stand 25.01.2016).

Kristeva, Julia (1990). Fremde sind wir uns selbst. Frankfurt a.M: Suhrkamp Edition.

Krusche, Dietrich (1980). Die Kategorie der Fremde. Eine Problemskizze. In: A. Wierlacher (Hrsg.), Fremdsprache Deutsch, Heft 1 (S. 46-56). München: iudicium. http://gepesk onyv.btk.elte.hu/adatok/Germanisztika/111Balk\%E1nyi/Horv\%E1thPabis/25-DieKate gorie....pdf. (hier: 1981, sic! S. 126-133) (Stand 16.04.2020).

Krusche, Dietrich ( $\left.{ }^{2} 1993\right)$. Literatur und Fremde: Zur Hermeneutik kulturräumlicher Distanz. München: iudicium.

Krusche, Dietrich/Wierlacher, Alois (Hrsg.) (1990). Hermeneutik der Fremde. München: iudicium. 
Leskovec, Andrea (2011): Einführung in die Interkulturelle Literaturwissenschaft. Darmstadt: Wissenschaftliche Buchgesellschaft.

Lukács, Georg ( $\left.{ }^{2} 1963\right)$. Die Theorie des Romans. Ein geschichtsphilosophischer Versuch über die Formen der großen Epik. Zweite um ein Vorwort vermehrte Auflage. Neuwied am Rhein und Berlin-Spandau: Luchterhand 1963. Zuerst in: Zeitschrift für Ästhetik und Allgemeine Kunstwissenschaft, herausgegeben von Max Dessoir, 1916. Als Buch Berlin: Paul Cassirer 1920. Neuauflage: Bielefeld: Aisthesis-Verlag 2009.

Meier, Albert/Westerkamp, Dirk (2014): Negative Hermeneutik - Formen und Leistungen des Nichtverstehens. www.collegiumphilosophicum.uni-kiel.de/de/projektkollegien/ab geschlossene-projektkollegien/projektkolleg-formen-und-leistungen-des-nichtverste hens-1/nichtverstehen-projektskizze.

Morin, Edgar (2001). Seven complex lessons in education for the future. Paris: UNESCO Publishing.

Morizot, Baptiste (2019). Ce mal de Pays sans exil. Les affects du mauvais temps qui vient. Revue Critique, 1, 860-861.

Morizot, Baptiste (2020). Heimweh ohne Exil. Wie können wir mit der Verunsicherung umgehen, die eine sich immer schneller verändernde Welt hervorruft? Philosophie Magazin Edition Januar, 2020, 56-61.

Müller-Funk, Wolfgang (2016). Theorien des Fremden. Eine Einführung. Unter Mitarbeit von Johanna Chovanec. Tübingen: A. Francke Verlag.

Nietzsche, Friedrich (1954). Der tolle Mensch. Die fröhliche Wissenschaft. In: Friedrich Nietzsche, Werke in drei Bänden, Band 2 (S. 125, 126-128, 348, 477). München: http:// www.zeno.org/Philosophie/M/Nietzsche,+Friedrich/Die+fröhliche+Wissenschaft/Drit tes+Buch/125.+Der+tolle+Mensch (Stand 29.03.2020).

Pabis, Eszter (2014). Fremde, Fremderfahrung und Verfremdung. Grundbegriffe und Probleme literaturwissenschaftlicher Fremdheitsforschung. Werkstatt, 9, 12-30 (Stand 02.04.2020].

Pörksen, Bernhard/Schulz von Thun, Friedemann (2020). Die Kunst des MiteinanderRedens: Über den Dialog in Gesellschaft und Politik. München: Carl Hanser Verlag.

Pörksen, Bernhard (2018). Die große Gereiztheit: Wege aus der kollektiven Erregung. München: Carl Hanser Verlag.

Pohl, Rüdiger F. (22017). Cognitive illusions: Intriguing phenomena in thinking, judgement and memory. London: Routledge.

Reckwitz, Andreas (2017). Gesellschaft der Singularitäten. Vom Strukturwandel der Moderne. Frankfurt a.M.: Suhrkamp.

Reinhard, Wolfgang (2016). Die Unterwerfung der Welt. Globalgeschichte der Europäischen Expansion 1415-2015. München: C.H. Beck.

Ricoeur, Paul (1972). Der Text als Modell: hermeneutisches Verstehen. In: W. L. Bühl (Hrsg.). Verstehende Soziologie. Grundzüge und Entwicklungstendenzen (franz. 1971) (S. 252-283). München: Nymphenburger Verlag.

Ricoeur, Paul (1973). Hermeneutik und Strukturalismus. Der Konflikt der Interpretation I. Übers. v. J. Rutsche (franz. 1969). München: Nymphenburger Verlag.

Ricoeur, Paul (1996). Das Selbst als ein Anderer. München: Fink.

Röttgers, Kurt (2011). Fremdheit. In: A. Wildfeuer/P. Kolmer (Hrsg.), Neues Handbuch philosophischer Grundbegriffe (S. 818-832). Freiburg/München: Karl Albers.

Röttgers, Kurt/Schmitz-Emans, Monika (Hrsg.) (2007). Die Fremde. Essen: Die Blaue Eule. 
Rosa, Hartmut (2016). Resonanz. Frankfurt a.M.: Suhrkamp.

Rosa, Hartmut (2019). Unverfügbarkeit. Wien/Salzburg: Residenz Verlag.

Said, Edward ( $\left.{ }^{4} 2014\right)$. Orientalismus. Frankfurt: Fischer. Engl. Orientalism. 1st ed. 1979.

Schirach, Ariadne von (2019). Die psychotische Gesellschaft: Wie wir Angst und Ohnmacht überwinden. Stuttgart: Klett-Cotta.

Schleiermacher, Friedrich (2012). Vorlesungen zur Hermeneutik (1819). In: Friedrich Schleiermacher, Kritische Gesamtausgabe. Hrsg. Von Günter Meckenstock, Andreas Arndt, Ulrich Barth, Lutz Käppel und Notger Slenczka. Bd. 4 (S. 57). Berlin: de Gruyter. https://www.theol.uni-kiel.de/de/professuren/st-munzinger/team/munzinger/pdf/ schleiermacher-hermeneutik (Stand 27.03.2020).

Schleiermacher, Friedrich (1993). Hermeneutik und Kritik. Frankfurt a.M.: Suhrkamp.

Schmidt, Bettina (2010). Den Anti-Bias-Ansatz zu Diskussion stellen. Beitrag zur Klärung theoretischer Grundlagen in der Anti-Bias-Arbeit. Oldenburg: BIS-Verlag der Carl von Ossietzky Universität Oldenburg.

Schmidt, Bettina/Dietrich, Katharina/Herdel, Shantala (2009). Anti-Bias-Arbeit in Theorie und Praxis - kritische Betrachtungen eines Antidiskriminierungsansatzes. In: W. Scharathow/R. Leiprecht (Hrsg.), Rassismuskritik. Band 2: Rassismuskritische Bildungsarbeit (S. 154-170). Schwalbach im Taunus: Wochenschau Verlag.

Schmidt, Bettina (2009). Theorie und Praxis der Anti-Bias-Arbeit, Internalisierung von Machtverhältnissen. In: Institut zur Interkulturellen Öffnung: Dokumentation der Fachtagung „Wir behandeln alle gleich, alle sind willkommen“? Der Anti-Bias-Ansatz: Chancengleichheit durch unterschiedsbewusstes Handeln (S. 8-19). Köln. www.susannebourgeois.de/index.php?id=20 (Stand 01.07.2012).

Schmitz-Emans, Monika (2011). Fremde und Verfremdung. Einführende Überlegungen zu Modellen des Lesens. https://d-nb.info/1134963750/34 (Stand 10.04.2020).

Schütz, Alfred/Luckmann, Thomas (2003). Strukturen der Lebenswelt. Konstanz: UVK.

Schütz, Alfred (1993). Der sinnhafte Aufbau der sozialen Welt. Eine Einleitung in die verstehende Soziologie. Frankfurt a.M.: Suhrkamp.

Schurz, Robert (1995). Negative Hermeneutik. Zur sozialen Anthropologie des Nicht-Verstehens. Wiesbaden: Springer Fachmedien.

Simmel, Georg (2013). Soziologie. Untersuchungen über die Formen der Vergesellschaftung. (Erstveröffentlichung 1908). Frankfurt a.M.: Suhrkamp.

Sloterdijk, Peter (2020). Für Übertreibungen ist kein Platz mehr. Der Philosoph Peter Sloterdijk über die Rolle der Medien in der Corona-Krise, staatlichen Rigorismus, europäische Solidarität und die Frage, warum der symptomfreie Virenträger eine bleibende Figur sein wird. Interview mit Adam Soboczynski. Die ZEIT, 07.04.2020. https://www.zeit.de/2020/16/peter-sloterdijk-corona-krise-gesundheitspolitik (08.04. 2020).

Thornvill, Randy (2009). Parasites, Democratisation, and the liberation of values across contemporary countries. Biological Review, 84, 113-131.

Walser, Martin (2012). Über Rechtfertigung. Eine Versuchung. Reinbek bei Hamburg: Rowohlt Verlag GmbH.

Wason, Peter C. (1968). Reasoning about a rule. Quarterly Journal of Experimental Psychology, 20(3), 273-281.

Weber, Max (2011). Wissenschaft als Beruf. Berlin: Matthes \& Seitz. 
Welzer. Harald (2011). Mentale Infrastrukturen: wie das Wachstum in die Welt und in die Seelen kam. Heinrich-Böll-Stiftung. Die grüne politische Stiftung. file:///Endf_Mentale_Infrastrukturen.pdf (Stand 08.04.2020).

Weinrich, Harald (1993). Fremdsprachen als fremde Sprachen. In: Wierlacher, Alois (Hrsg.), Kulturthema Fremdheit. Leitbegriffe und Problemfelder kulturwissenschaftlicher Fremdheitsforschung (S. 129-152). München: iudicium.

Wierlacher, Alois (Hrsg.) (1993). Kulturthema Fremdheit. Leitbegriffe und Problemfelder kulturwissenschaftlicher Fremdheitsforschung. München: iudicium.

Wierlacher, Alois/Bogner, Andrea (Hrsg.) (2003). Handbuch interkulturelle Germanistik. Berlin: Springer Verlag.

Wolting, Stephan (2016). Kulturelle MittlerInnen - Überlegungen zum Aufgabenprofil interkultureller Wissens- und Dienstleistungsberufe. Berliner Debatte, Initial, 50-57.

Wolting, Stephan (2017). Akademische Kulturen im Vergleich - Anmerkungen auf der Basis einer Online-Ringvorlesung innerhalb des Intercultural Campus. In: Brons-Albert, Ruth/Adamczak, Sylwia u.a. (Hrsg.), Perspektiven Deutsch als Fremdsprache, $B d$. 34. Hochschulen international vernetzen: Internationale Lehrkooperationen in der Germanistik und in Deutsch als Fremdsprache (Perspektiven Deutsch als Fremdsprache (S. 157-169). Baltmannsweiler: Schneider Verlag Hohengehren.

Wundt, Wilhelm (1912). Elemente der Völkerpsychologie. Grundlinien einer psychologischen Entwicklungsgeschichte der Menschheit. Leipzig: Kröner. 
Open-Access-Publikation im Sinne der CC-Lizenz BY-NC-ND 4.0

(C) 2020, Vandenhoeck \& Ruprecht GmbH \& Co. KG, Göttingen ISBN Print: 9783847111863 - ISBN E-Lib: 9783737011860 
Teil 4: Spracherwebs- und Sprachlehrforschung 
Open-Access-Publikation im Sinne der CC-Lizenz BY-NC-ND 4.0

(C) 2020, Vandenhoeck \& Ruprecht GmbH \& Co. KG, Göttingen ISBN Print: 9783847111863 - ISBN E-Lib: 9783737011860 


\title{
Polnisch als Herkunftssprache in Deutschland - Einfluss der Familiensprachenpolitik auf die Sprachentwicklung der Kinder
}

\begin{abstract}
Polish as a heritage language in Germany: The influence of family language policy on children's language development

The aim of this chapter is to investigate the influence of family language policy on the heritage language development of Polish children living in Germany. We argue that the age of onset of the second language, German, and the family interactions are important factors which bear influence on the heritage language development. We support our arguments with the findings from a study carried out with the participation of 29 Polish children from kindergarten and primary school. The research focused on the children's elicited speech production and our findings were compared with information about parent-child interactions and their language policy.
\end{abstract}

Keywords

heritage language, family language policy, age of onset, parent-child interactions

\section{Polnisch in Deutschland}

Der Anteil der Bewohner mit polnischem Hintergrund in Deutschland zählt zu den größten Gruppen, die eine andere Sprache als Deutsch zu Hause sprechen. Die Gruppe der Polnischsprecher ${ }^{2}$ in Deutschland beträgt ca. 2 Millionen, und steht damit an zweiter Stelle nach der Bevölkerung mit türkischem Migrationshintergrund. Befragungen unter Grundschülern in Hamburg ergaben, dass ein Drittel mit polnischem Hintergrund mit beiden Eltern zu Hause Polnisch sprechen (Fürstenau, Gogolin \& Yagmur 2003). Die erste Generation spricht überwiegend bzw. nur Polnisch zu Hause, bei der zweiten Generation ist dies bei einem Drittel der Fall (Hufeisen et al. 2018). In den Familien, in denen nur ein

1 Die Forschung ist im Rahmen des Projekts 2014/15/G/HS6/04521 an Aldona Sopata vom Nationalen Forschungszentrum (Narodowe Centrum Nauki) in Polen finanziert worden.

2 In dem vorliegenden Artikel wird der Begriff Polnischsprecher als Oberbegriff sowohl für Sprecher als auch für Sprecherinnen verwendet. 
Partner eine polnische Familienbiographie hat, ist aber häufig Deutsch die Partnersprache. Die Partnersprache unterscheidet sich oft in diesen Situationen von der Familiensprache, weil jene Personen mit polnischem Hintergrund oft mit ihren Kindern Polnisch sprechen, auch, wenn sie das Deutsche als Kommunikationssprache mit den Ehepartnern gewählt haben (Jańczak 2013). Angesichts dessen kann es nur verwundern, warum die Sprachentwicklung des Polnischen als Herkunftssprache in Deutschland so selten zum Untersuchungsgegenstand der Spracherwerbsforscher wird. Diese Lücke will der vorliegende Beitrag zumindest in einem geringen Ausmaß füllen.

\section{Erstsprache, Zweitsprache, Herkunftssprache (HS) und Umgebungssprache (US)}

Die zeitliche Reihenfolge des Spracherwerbs bestimmt die Begriffe der Erstsprache und der Zweitsprache. Unter der Erstsprache wird jene Sprache verstanden, die als erste vom Kind erworben wird. Sie wird als Muttersprache bezeichnet, aber sie muss natürlich nicht immer „die Sprache der Mutter“ sein. Werden in früher Kindheit zwei Sprachen gleichzeitig erworben, so haben wir es mit einem bilingualen Spracherwerb zu tun. Beide Sprachen gelten in dem Fall als Erstsprachen des zweisprachigen Kindes. Bei einer mehrsprachigen Erziehung in der Kindheit haben wir es sogar mit drei oder mehreren Erstsprachen zu tun. In der Praxis führt der simultane Erwerb von zwei oder mehr Erstsprachen manchmal zur Entwicklung einer stärkeren Sprache und einer oder zwei schwächeren Sprachen.

Der Spracherwerb kann in der Kindheit aber auch sukzessiv verlaufen. Mit einem sukzessiven Spracherwerb haben wir es zu tun, wenn eine Sprache zuerst erworben wird, und erst, wenn ihr Erwerb relativ fortgeschritten ist, tritt das Kind in Kontakt mit der zweiten Sprache. Liegt der Erwerb der zweiten Sprache in der ganz frühen Kindheit, kann sie noch als Erstsprache bezeichnet werden. Wenn der Erwerb der zweiten Sprache erst nach 3-4 Jahren beginnt, wird sie von vielen Forschern schon als frühe Zweitsprache aufgefasst (Meisel 2011). Als sukzessiver Zweitspracherwerb im Kindesalter wird also ein Prozess verstanden, bei dem die zweite Sprache erworben wird, nachdem die Grundzüge der Erstsprache vom Kind schon angeeignet worden sind.

Die meisten Definitionen der Herkunftssprache (HS) differenzieren nicht nach dem Zeitpunkt des Erwerbsbeginns. Als HS wird die Sprache einer demografischen Minderheit oder einer Immigrantengruppe bezeichnet, die in der Kindheit zu Hause erworben wird. Bilinguale Sprecher erwerben meistens die HS als L1 und simultan oder sukzessiv die Umgebungssprache (US), also die Sprache 
der demografischen Mehrheit, als zweite Erstsprache oder frühe Zweitsprache. In den meisten Fällen wird die US zur dominanten Sprache der bilingualen Kinder, und die HS zur schwächeren Sprache (Benmamoun, Montrul \& Polinsky 2013, Brehmer \& Mehlhorn 2015).

\section{Interne und externe Faktoren, welche die Herkunftssprachentwicklung beeinflussen}

Bilingualer simultaner Erwerb von zwei Sprachen sowie sukzessiver Erwerb von zwei Sprachen im Kleinkindalter stellt für Kinder prinzipiell kein Problem dar. Der Erwerbsverlauf bei simultan bilingualen Kindern unterscheidet sich im Wesentlichen nicht vom Verlauf des Erstspracherwerbs (Meisel 2004). Der sukzessive Erwerb von zwei Sprachen im Kleinkindalter führt auch oft zum Spracherwerbserfolg, trotz einiger Unterschiede im Verlauf (Sopata 2009). Gleichzeitig kommt es aber durchaus vor, dass der bilinguale Spracherwerb im Kindesalter nicht zu einem aktiven Gebrauch beider Sprachen führt (De Houwer 1995). Mehrere Untersuchungen haben gezeigt, dass die Fähigkeiten der Kinder in der HS auf einem Kontinuum anzusiedeln sind, das von völliger Entsprechung den Fähigkeiten monolingualer Kinder im gleichen Alter, bis hin zu fast nicht vorhandenen aktiven Sprachfähigkeiten reicht (z. B. Anstatt 2009).

Eine Vielzahl von Faktoren beeinflusst den Spracherwerb. Zu den externen Faktoren, die von der Umgebung bestimmt sind, und mit der Ebene der HSGemeinschaft zusammenhängen, gehören die demographischen Eigenschaften der HS-Gemeinschaft, z. B. der Grad der Offenheit und die Größe der Gemeinschaft, die Einstellung gegenüber der Zielsprachengemeinschaft, und die Sprachenpolitik. Den Spracherwerb beeinflussen auch soziale Faktoren, wie die Einstellung der Eltern der Mehrsprachigkeit gegenüber, das Streben der Familie nach der Bewahrung der ethnischen und kulturellen Identität und die intendierte Länge des Aufenthalts im Zielsprachenland.

Bei den individuellen Faktoren handelt es sich einerseits um Eigenschaften des Kindes, wie z.B. sein Alter bei L2-Erwerbsbeginn, und andererseits um emotionale Faktoren, wie Selbsteinschätzung, Identität oder Assimilationsbestreben bei älteren Kindern.

Als besonders wichtiger Faktor für die HS-Entwicklung wird in der einschlägigen Literatur das Alter der Kinder betrachtet, in dem sie in Kontakt mit der zweiten Sprache, also mit der US oder Mehrheitssprache treten. Einige Forscher, wie zum Beispiel Montrul (2008) behaupten, dass HS-Sprecher, die beide Sprachen simultan von Geburt an erwerben, oft schlechter in der HS abschneiden, als solche, die mit dem Erwerb der zweiten Sprache erst nach einigen Jahren beginnen, d.h. 
die zweite Sprache sukzessiv erwerben. Viele Forscher räumen in diesem Zusammenhang ein, dass es sich bei den simultan-bilingualen Kindern lediglich um einen verzögerten Erwerb handelt, weil ab dem Alter von 12 Jahren eine zunehmende Konvergenz von HS mit monolingualen Gleichaltrigen durch mehrere Untersuchungen gezeigt worden ist (Flores, Rinke \& Azevedo 2017).

Der andere Faktor, der für die HS-Entwicklung besonders wichtig zu sein scheint, ist der HS-Input. Dabei wird in der Forschung vor allem eine wichtige Rolle der Konsequenz in der Familiensprachenwahl und Inputsqualität, d.h. der Komplexität sowie der Vielfalt der Eltern-Kind-Interaktionen, beigemessen (Blom 2010). Wie in den meisten Fällen des bilingualen Spracherwerbs, ist der Input in der HS meistens geringer im Vergleich zum monolingualen Spracherwerb. Das muss dazu führen, dass Kinder mehr Zeit benötigen, um positive Evidenzen für bestimmte Strukturen im HS- und L2-Input zu akkumulieren (Flores 2015).

Die Input-Situation bilingualer Kinder kann unterschiedlich aussehen und hängt vor allem mit der Familiensprachenpolitik zusammen, also mit der Sprachenwahl in der Alltagskommunikation innerhalb der Familie. Mehrere Szenarien sind möglich: Die Alltagskommunikation in der Familie kann gänzlich in der HS erfolgen, was als Modell „non-dominant home language without community support“ gelten kann (Romaine 1995). Dieses Szenario der Familienkommunikation kann von den Eltern gewählt worden sein, weil sie beide die Umgebungssprache/Mehrheitssprache nicht sprechen oder ein Elternteil sie nicht bzw. nicht gut genug spricht. Dieser Typ der Familiensprachenpolitik kann aber auch aus der Überzeugung der Eltern resultieren, dass sie durch den exklusiven Gebrauch der HS zu Hause den Erhalt der HS und der Herkunftsidentität bei ihren Kindern fördern.

Das zweite Szenario der Familiensprachenpolitik ist mit dem Prinzip „one person, one language“ verbunden, nach dem ein Elternteil eine Sprache und der andere Elternteil die andere Sprache gegenüber dem Kind konsequent verwenden (Romaine 1995). Mehrere Forscher unterstreichen, dass in beiden oben genannten Szenarien typisch ist, dass die Eltern wenigstens gelegentlich nicht nur in der HS sondern auch in der US mit dem Kind kommunizieren (Anstatt 2009).

Das andere Szenario in der Familiensprachenpolitik beruht darauf, dass beide Eltern mit den Kindern in der HS sprechen, aber nicht erwarten, dass ihre Kinder ihnen in der HS antworten. Das Ziel scheint in diesem Szenario die Entwicklung von rezeptiven Sprachfähigkeiten in der HS bei den Kindern, ein sog. passiver Bilingualismus, zu sein (Perez-Leroux, Cuza \& Thomas 2011).

Eine weitere Möglichkeit der Familiensprachenpolitik ist mit der Entscheidung jener bilingualer Eltern verbunden, die die HS in der Familie gar nicht oder sehr begrenzt gebrauchen (Lambert 2008). Die Kinder können dann immer noch gelegentlich in einer Sprachschule oder während der Ferien im Herkunftsland in 
Kontakt mit der HS treten, was jedoch normalerweise ein sehr eingeschränkter Input ist.

\section{Aufbau der empirischen Studie}

\subsection{Forschungsfragen}

Das Ziel der vorliegenden empirischen Studie ist die Feststellung des Einflusses der Familiensprachenpolitik auf die HS-Entwicklung der Kinder. Insbesondere werden als mögliche Einflussfaktoren unterschiedliche Familiensprachenszenarien und das Alter der Kinder beim Erwerb der Umgebungssprache betrachtet. Die Forschungsfragen lauten:

1. Welchen Einfluss hat das Alter der Kinder beim Beginn des Erwerbs der US auf die HS-Entwicklung der Kinder?

2. Welchen Einfluss hat die HS-Wahl in der Interaktion zwischen beiden Eltern und dem Kind auf die HS-Entwicklung der Kinder?

3. Welchen Einfluss hat die HS-Wahl in der Interaktion zwischen der Mutter und dem Kind auf die HS-Entwicklung des Kindes?

4. Welchen Einfluss hat die HS-Wahl in der Interaktion zwischen dem Vater und dem Kind auf die HS-Entwicklung der Kinder?

5. Welchen Einfluss hat das HS-Land als Ferienort auf die HS-Entwicklung der Kinder?

6. Was ist das häufigste Familiensprachenszenario in der Gruppe der Kinder,

a. die die HS sehr gut beherrschen?

b. die die HS gut beherrschen?

c. die die HS weniger gut beherrschen?

\subsection{Methodologie}

Der Stand der HS-Entwicklung wurde anhand einer Aufgabe zur elizitierten Sprachproduktion untersucht. Den Kindern wurde die Figur eines kleinen Jungen - Peter - zuerst vorgestellt. Dann sahen die Kinder 18 kurze Bildgeschichten, in denen Peter unterschiedliche Handlungen durchführt. Der Forscher erzählte eine kurze Geschichte zu jeder Bildfolge, stellte die Hintergründe jeder Situation vor, und stellte am Ende eine Frage zu jeder Bildgeschichte. Der das Experiment durchführende Forscher wiederholte die Frage, falls das gefragte Kind es wollte. Die Reihenfolge der Bilder war bei jedem Teilnehmer gleich. Die Äußerungen der Kinder wurden mithilfe eines Diktiergeräts aufgenommen und dann transkribiert. 
Die Familiensprachenpolitik und die sprachlichen Interaktionen in den Familien wurden anhand umfangreicher Fragebögen untersucht. Für jedes Kind wurde seine Sprachbiographie im Einvernehmen mit den Eltern erstellt. Die Fragebögen enthielten Informationen über das Kind (u. a. das Alter, in dem es in Kontakt mit der zweiten Sprache getreten ist, die Art und Weise des Kontakts, Geschwister, Input in HS und Input in der zweiten Sprache) und über die Eltern bzw. Betreuer (u. a. Geburtsland, seit wie vielen Jahren sie in Deutschland wohnen, ob sie die deutsche Schule besucht haben).

\subsection{Probanden}

Alle an dem Experiment teilnehmenden Kinder wohnen in Deutschland im Bundesland Hessen. Die Studie wurde in einem bilingualen deutsch-polnischen Kindergarten und in einer Grundschule durchgeführt. Der Altersbereich der Kinder beträgt 4-11 Jahre mit dem Durchschnittsalter - 7,03. Insgesamt haben 34 Kinder an dem Experiment teilgenommen, davon 12 Kinder aus dem Kindergarten und 22 aus der Grundschule. Zusammen gab es 22 Mädchen und 12 Jungen. 25 Kinder sind in Deutschland geboren, 5 Kinder sind innerhalb von einem Jahr nach ihrer Geburt nach Deutschland gekommen, 1 Kind ist im Alter von 1,5 und 3 Kinder im Alter von 4 und 5 nach Deutschland gekommen. Die Sprachenpolitik zu Hause hängt mit der Herkunft der Eltern zusammen. In der untersuchten Gruppe sind bei 18 Kindern beide Eltern Polen, bei 12 Kindern ist die Mutter Polin und der Vater Deutscher, bei einer Person kommt die Mutter aus Deutschland und der Vater aus Polen, bei 2 Personen kommt die Mutter aus Polen und der Vater aus Tschechien oder aus China. Ausgeschlossen aus der weiteren Analyse wurde ein Kind, dessen Angaben zur Familiensprachenpolitik lückenhaft waren. Bei der weiteren Auswertung der Daten wurden die Daten von vier Kindern nicht berücksichtigt, die auf keine Frage des Forschers geantwortet haben. Die Gesamtzahl der Kinder, die in der weiteren Analyse berücksichtigt wird, beträgt daher 29.

\section{Ergebnisse der Untersuchung}

Bei der Auswertung der Daten werden alle Äußerungen der Kinder berücksichtigt, die die Kinder als Antwort auf die Fragen des Forschers gegeben haben. Als korrekt gelten Antworten, in denen kein grammatischer oder lexikalischer Fehler vorkommt. Als syntaktisch korrekte Sätze werden solche ausgewertet, die keinen syntaktischen Fehler beinhalten und als morphologisch korrekte Sätze gelten in der Untersuchung solche, die keinen morphologischen Fehler beinhalten. 
Um die Antwort auf die erste Forschungsfrage zu finden, wurde das Alter der Kinder beim Beginn des Erwerbs der Umgebungssprache zuerst untersucht. Das Alter der untersuchten Kinder beim Beginn des Erwerbs des Deutschen als US ist unterschiedlich. Die meisten Kinder, 25, sind in Deutschland geboren oder nach Deutschland vor ihrem dritten Lebensjahr gekommen. Das Alter ihres ersten Kontakts zum Deutschen ist also im Zeitraum von 0-3 Jahren anzusetzen. Vier Kinder sind aber erst im Alter von 4 oder 5 Jahren nach Deutschland gekommen. Wenn man den Stand der HS-Entwicklung, also in der vorliegenden Untersuchung die Korrektheit der elizitierten Sprachproduktion, zwischen den beiden Gruppen vergleicht, sieht man einen deutlichen Unterschied. Die Kinder, die später ihren Kontakt mit der US Deutsch begonnen haben, erreichen eine höhere Korrektheit in der HS, also im Polnischen. Ihr Korrektheitswert liegt bei 70,8 \% im Vergleich zu 56 \% im Falle der Kinder, die den Erwerb des Deutschen von Geburt an oder in den drei ersten Lebensjahren begonnen haben.

Um Antworten auf die Forschungsfragen 2-5 zu finden, wurden mithilfe von den Fragebögen viele Informationen bezüglich der Interkationen in der Familie gesammelt. ${ }^{3}$ In der Tabelle 1 sind Korrektheitswerte bei den elizitierten Äußerungen im Polnischen (HS) als Prozentzahlen für bestimmte nach den unten beschriebenen Kriterien definierten Gruppen dargestellt.

Tabelle 1: Korrektheit in der HS in Relation zur Häufigkeit der Interaktionen in der HS in der Familie und in den Ferien

\begin{tabular}{|l|l|l|}
\hline \multirow{2}{*}{} & \multicolumn{2}{|l|}{ Häufigkeit der Interaktion } \\
\cline { 2 - 3 } & immer & manchmal, selten, niemals \\
\hline Eltern-Kind & $67,4 \%$ & $39,8 \%$ \\
\hline Mutter-Kind & $63,9 \%$ & $38,9 \%$ \\
\hline Vater-Kind & $67,4 \%$ & $50 \%$ \\
\hline Polen als Ferienort & $64,2 \%$ & $52,4 \%$ \\
\hline
\end{tabular}

Wenn es sich um die Interaktionen zwischen den Eltern und dem Kind handelt, hat die Untersuchung gezeigt, dass acht Kinder mit ihren Eltern immer Polnisch und umgekehrt die Eltern mit den Kindern immer Polnisch sprechen. Die durchschnittliche Korrektheit für die Gruppe von Kindern, die immer Polnisch mit den Eltern und umgekehrt sprechen, beträgt 67,4\%. Sechs Kinder sprechen mit den Eltern manchmal, selten oder niemals Polnisch und ebenso die Eltern mit den

3 Die Frage nach Interaktionen umfasst zwei Aspekten, einerseits wie oft die jeweilige Person mit dem Kind Polnisch spricht, andererseits wie oft das Kind mit der jeweiligen Person Polnisch spricht. Die Personen, nach denen gefragt wurde, sind: die Mutter, der Vater, Brüder und Schwestern, Großeltern und andere. In diesem Artikel werden die Interaktionen mit den Großeltern und Geschwistern nicht berücksichtigt. 
Kindern. Die durchschnittliche Korrektheit für diese Gruppe beträgt 39,8 \% (siehe Tabelle 1).

In Hinsicht auf die dritte Forschungsfrage werden die Interaktionen zwischen der Mutter und dem Kind untersucht. Sechzehn Kinder sprechen mit der Mutter immer Polnisch und erreichen eine durchschnittliche Korrektheit von 63,9\%. Sieben Kinder sprechen mit ihrer Mutter manchmal, selten oder niemals Polnisch und erreichen eine durchschnittliche Korrektheit von 38,9 \% (siehe Tabelle $1)$.

In der Untersuchung der Familiensprachenpolitik wurden aber auch die Interaktionen in der HS zwischen dem Kind und dem Vater untersucht. In der untersuchten Gruppe sprechen acht Kinder mit dem Vater immer Polnisch und ihre durchschnittliche Korrektheit in der HS beträgt 67,4\%. Im Gegensatz dazu sprechen zwölf Kinder mit dem Vater nur manchmal, selten oder niemals Polnisch. Die durchschnittliche Korrektheit in der HS dieser Gruppe beträgt nur $50 \%$ (siehe Tabelle 1).

In der vorliegenden Untersuchung wurde angenommen, dass nicht nur die Interaktionen in der HS in der Familie, sondern auch die Interaktionen in der HS außerhalb der Familie eine Rolle bei der Entwicklung der HS spielen können. Die Inputquelle für die HS außerhalb der Familie ist häufig die weitere Familie, die in den Ferien im HS-Land vom Kind besucht wird. In den Fragebögen wurden daher Fragen nach dem Ferienort der Kinder gestellt. Die Untersuchung hat gezeigt, dass neun Kinder die Ferien immer in Polen verbringen. Im Gegensatz dazu fahren vierzehn Kinder in den Ferien nach Polen nur manchmal, ein paar Mal oder niemals. Die durchschnittliche Korrektheit in der HS beträgt $64,2 \%$ für diese Kinder, die immer ihre Ferien im HS-Land verbringen, und 52,4 \%, für die Kinder, die manchmal, selten oder niemals nach Polen in die Ferien fahren (siehe Tabelle 1).

Im weiteren Schritt der Analyse wurde die Sprachproduktion der einzelnen Kinder in der HS im Hinblick auf ihre allgemeine, syntaktische und morphologische Korrektheit ausgewertet. Die Kinder wurden auch aufgrund des Korrektheitswertes in der HS in drei Gruppen eingeteilt. Der Maximalwert bei diesem Experiment beträgt 18. Die erste Gruppe besteht aus Kindern, bei denen der Korrektheitswert im Bereich 13-18 enthalten ist. Sie besteht aus 10 Kindern, bei denen die durchschnittliche Korrektheit bei 81,1 \% liegt. Die zweite Gruppe bilden Kinder, die den Korrektheitswert aus dem Bereich 7-12 erreicht haben. Sie besteht aus 14 Kindern mit der durchschnittlichen Korrektheit in der HS bei $56,3 \%$. Die dritte Gruppe umfasst Kinder, die den Korrektheitswert aus dem Bereich 0-6 erzielt haben. Sie besteht aus 5 Kindern, bei denen die durchschnittliche Korrektheit in HS bei $16,7 \%$ liegt.

Die Kinder aus der ersten Gruppe, die ihre HS, Polnisch, sehr gut beherrschen, bilden meistens morphologisch korrekte Sätze. Vier Kinder haben alle 18 Ant- 
worten in Form von morphologisch korrekten Sätzen gegeben, bei den anderen beträgt die Anzahl der morphologisch korrekten Sätze 15-17, sie ist also auch hoch. Die Kinder aus der ersten Gruppe bilden auch viele syntaktisch korrekte Sätze. Ihre Anzahl beläuft sich auf 14-17. Bei vier Kindern aus der ersten Gruppe ist die Mehrheit der Sätze komplex (9-15 Sätze). Sechs Kinder bilden eher einfache Sätze. Die Anzahl der komplexen Sätze ist bei ihnen kleiner (3-8 Sätze).

Das Durchschnittsalter für die erste Gruppe beträgt 6,7. Sechs Kinder aus dieser Gruppe sind in Deutschland geboren. Vier Kinder sind im Alter von 1, 4 oder 5 Jahren nach Deutschland gekommen. Die Kinder unterschieden sich also bezüglich des Erwerbsalters der US. Zur ersten Gruppe, die die Kinder mit dem höchsten Korrektheitswert in der HS umfasst, gehören zwei Kinder, die am spätesten nach Deutschland, d.h. im Alter von 4 und 5 Jahren, gekommen waren. Man kann annehmen, dass ihr Kontakt zum Deutschen eben zu diesem Zeitpunkt erst angefangen hat. Die erste Gruppe umfasst aber auch sechs Kinder, die in Deutschland geboren sind, und zwei, die im ersten Lebensjahr nach Deutschland gekommen sind. Sie haben also höchstwahrscheinlich einen sehr frühen Beginn des Erwerbs des Deutschen als US.

Sechs Kinder aus der ersten Gruppe verbringen immer oder fast immer ihre Ferien in Polen. Die übrigen wählen Polen manchmal als Ferienort. Wenn man die Daten zu den Kindern der ersten Gruppe in Hinsicht auf die Herkunft ihrer Eltern untersucht, dann kann man feststellen, dass bei fünf Kindern die Mutter Polin und der Vater Deutscher ist. Bei fünf anderen Kindern der ersten Gruppe sind beide Eltern Polen.

Die häufigsten Familiensprachenszenarien in der ersten Gruppe sind:

- beide Eltern sprechen mit dem Kind und das Kind mit den Eltern immer Polnisch (drei Kinder),

- die Mutter spricht mit dem Kind immer oder fast immer Polnisch, der Vater seltener oder niemals (fünf Kinder),

- Kinder kommunizieren mit keinem Elternteil häufig auf Polnisch, sie sprechen aber häufig Polnisch in den Ferien (zwei Kinder).

In der ersten Gruppe gilt zu beachten, dass $90 \%$ der Kinder eine polnische Schule in Deutschland besuchen. Nur ein Kind aus der ersten Gruppe besucht keine polnische Schule in Deutschland, drei Kinder haben gerade angefangen, sechs Kinder besuchen eine polnische Schule seit 1-3 Jahren.

Die zweite Gruppe besteht aus vierzehn Kindern, die Polnisch gut beherrschen. Sie bilden viele, morphologisch korrekte, Sätze. Ein Kind bildet nur 9 morphologisch korrekte Sätze. Bei den anderen Kindern aus dieser Gruppe beträgt die Anzahl der morphologisch korrekten Sätze 13 bis 18. Die Anzahl der syntaktisch korrekten Sätze ist niedriger, aber auch relativ hoch. Es sind 7-8 syntaktisch korrekte Sätze bei zwei Kindern, und 10-14 syntaktisch korrekte 
Sätze bei elf Kindern. Ein Kind aus der zweiten Gruppe hat sogar 17 syntaktisch korrekte Sätze gebildet. Drei Kinder aus der zweiten Gruppe bilden meistens komplexe Sätze. Es handelt sich bei ihnen um 9-11 komplexe Sätze (in 18 Antworten). Bei den anderen ist die Anzahl der komplexen Sätze kleiner und beträgt von 0 bis 7 .

In der zweiten Gruppe, die Polnisch als HS gut beherrscht, sind neun Kinder, die in Deutschland geboren, und vier die innerhalb des ersten Lebensjahres nach Deutschland gekommen sind. Ein Kind dieser Gruppe ist im Alter von 4,5 Jahren nach Deutschland gekommen. Es überwiegen also in der zweiten Gruppe Kinder, die höchstwahrscheinlich sehr früh Kontakt zur Umgangssprache Deutsch aufgenommen haben.

Drei Kinder aus der zweiten Gruppe kommen aus einer deutsch-polnischen Familie, wo die Mutter Polin und der Vater Deutscher ist. Bei neun Kindern sind beide Eltern Polen, bei zwei Kindern ist die Mutter Polin und der Vater kommt aus Tschechien oder aus China. Das Durchschnittsalter in dieser Gruppe beträgt 6,9.

Acht Kinder verbringen ihre Ferien immer oder fast immer in Polen, sechs Kinder manchmal oder selten. Fünf Kinder besuchen keine polnische Schule in Deutschland, vier Kinder haben gerade angefangen und fünf Kinder besuchen eine polnische Schule seit einem oder zwei Jahren.

Die häufigsten Familiensprachenszenarien in der zweiten Gruppe sind folgende:

- beide Eltern sprechen mit dem Kind immer Polnisch und das Kind spricht mit den Eltern immer oder oft Polnisch (sechs Kinder),

- die Mutter spricht mit dem Kind immer oder fast immer Polnisch, der Vater manchmal, selten oder niemals (sieben Kinder),

- ein Kind aus der zweiten Gruppe spricht mit keinem Elternteil häufiger Polnisch, es spricht aber Polnisch in den Ferien.

Die dritte Gruppe, die ihre HS Polnisch nicht gut beherrscht, besteht aus fünf Kindern. In dieser Gruppe haben immerhin zwei Kinder mehrere syntaktisch korrekte Sätze gebildet (12 und 14 Sätze). Außerdem bilden die Kinder aus der dritten Gruppe auch morphologisch korrekte Sätze. Ihre Anzahl ist aber relativ niedrig (1-9). Nur zwei Kinder haben einige komplexe Sätze während der Untersuchung gebildet (3-4 Sätze). Vereinzelte semantische und lexikalische Fehler konnten beobachtet werden. Das Durchschnittsalter ist 7,4. Vier Kinder aus der dritten Gruppe sind in Deutschland geboren und ein Kind ist mit 18 Monaten nach Deutschland gekommen. Alle Kinder der dritten Gruppe haben also höchstwahrscheinlich sehr früh den Erwerb des Deutschen als US angefangen.

Es gibt zwei deutsch-polnische Familien, wo die Mutter Polin und der Vater Deutscher ist, bei drei Kindern sind beide Eltern Polen. Ein Kind verbringt die Ferien immer in Polen, vier Kinder manchmal oder ein paar Mal. Drei Kinder 
besuchen keine polnische Schule in Deutschland, ein Kind besucht eine solche Schule seit einem Jahr, und ein Kind seit zwei Monaten. Die Familiensprachenszenarien in der dritten Gruppe sind folgende:

- die Mutter und das Kind sprechen miteinander manchmal oder selten Polnisch und der Vater und das Kind sprechen miteinander niemals Polnisch (zwei Kinder),

- die Mutter und das Kind sprechen miteinander immer Polnisch, der Vater und das Kind sprechen miteinander oft Polnisch (ein Kind),

- die Mutter und das Kind sprechen miteinander manchmal Polnisch, der Vater und das Kind sprechen miteinander oft Polnisch (ein Kind),

- die Eltern sprechen mit dem Kind manchmal oder selten Polnisch, das Kind spricht mit den Eltern niemals Polnisch (ein Kind).

\section{Schlussfolgerungen}

Die Erforschung des Zustands der Entwicklung des Polnischen als HS bei Kindern in den Familien mit Migrationshintergrund in Deutschland im Hinblick auf die Familiensprachenpolitik hat gezeigt, dass die sprachlichen Interaktionen in der Familie einen gravierenden Einfluss auf die HS-Entwicklung der Kinder haben (Mehlhorn 2014).

Das Alter beim Beginn des Erwerbs der US scheint einen deutlichen Einfluss auf den Korrektheitswert in HS zu haben (Frage 1). Bei den Kindern, die nach dem 3. Lebensjahr nach Deutschland gekommen sind, liegt der Wert ca. $15 \%$ höher. Da die Gruppe der Kinder, die später den Erwerb der US begonnen haben, nicht groß ist, kann die Antwort auf die erste Forschungsfrage jedoch schlecht verallgemeinert werden.

Die Antworten auf die Forschungsfragen zwei bis vier sind ein klares Ja. Je häufiger die Kinder mit beiden Eltern oder mit einem Elternteil Polnisch sprechen, desto höhere Korrektheit erreichen sie. Der Unterschied in der HS-Kompetenz beim Vergleich der Häufigkeit der Interaktionen in der HS, immer vs. manchmal/selten/niemals, ist im Falle der HS-Interaktionen zwischen dem Kind und beiden Eltern und zwischen dem Kind und der Mutter besonders groß. Der HS-Korrektheitswert fällt um 27,6 \%, wenn die Kinder nicht immer, sondern manchmal, selten oder niemals mit beiden Eltern in der HS kommunizieren (siehe Tabelle 1). Der Wert fällt um 25,1 \%, wenn die Kinder mit der Mutter statt immer nur manchmal, selten oder niemals in der HS sprechen (siehe Tabelle 1). Der Rückgang ist etwas geringer (17,4 \%), wenn der Unterschied die Vater-Kind Interaktionen in der HS betrifft.

Die vorliegende Studie hat eindeutig gezeigt, dass die HS-Interaktionen außerhalb der engen Familie auch eine wichtige Rolle spielen. Je häufiger die Kinder 
ihre Ferien im HS-Land verbringen, desto höhere HS-Korrektheit erreichen sie (Forschungsfrage 5). Der Rückgang des HS-Korrektheitswertes liegt bei 11,8 \%, wenn die Kinder nicht immer, sondern seltener Polen als Ferienort angegeben haben (siehe Tabelle 1).

Die Untersuchung der häufigsten Familiensprachenszenarien hat bewiesen, dass, um sehr gute oder gute HS-Kompetenz bei den Kindern zu erreichen, die beste Familiensprachenpolitik auf den sehr häufigen gegenseitigen HS-Interaktionen zwischen beiden Eltern und dem Kind (non-dominant home language without community support) bzw. zumindest zwischen einem Elternteil und dem Kind (one person, one language) beruhen soll (Forschungsfrage 6). In der vorliegenden Studie war es in allen Fällen die Mutter, die mit dem Kind in der HS kommuniziert hat, wenn es nur ein Elternteil war, der in der HS mit dem Kind gesprochen hat.

Zusammenfasend muss man sagen, dass der Einfluss der Familiensprachenpolitik auf die Entwicklung des Polnischen als Herkunftssprache bei den Kindern aus den Familien mit polnischem Migrationshintergrund in Deutschland von großem Belang ist. Die vorliegende Studie lässt die Feststellung zu, dass die Häufigkeit der Herkunftssprache-Interaktionen in der Familie die Herkunftssprachentwicklung der Kinder determiniert.

\section{Literaturverzeichnis}

Anstatt, Tanja (2009). Der Erwerb der Familiensprache: Zur Entwicklung des Russischen bei bilingualen Kindern in Deutschland. In: I. Gogolin/U. Neumann (Hrsg.), Streitfall Zweisprachigkeit - The bilingualism controversy (S. 111-131). Wiesbaden: Springer Verlag.

Benmamoun, Elabbas/Montrul, Silvina/Polinsky, Maria (2013). Heritage languages and their speakers: Opportunities and challenges for linguistics. Theoretical Linguistics, 39 (3-4), $129-181$.

Blom, Elma (2010). Effects of input on the early grammatical development of bilingual children. International Journal of Bilingualism, 14(4), 1-26.

Brehmer, Bernhard/Mehlhorn, Grit (2015). Russisch als Herkunftssprache in Deutschland. Ein holistischer Ansatz zur Erforschung des Potenzials von Herkunftssprachen. Zeitschrift für Fremdsprachenforschung, 26(1), 85-123.

De Houwer, Annick (1995). Bilingual language acquisition. In: P. Fletcher/B. MacWhinney (Hrsg.), The handbook of child language (S. 219-250). Oxford: Blackwell.

Flores, Cristina (2015). Understanding heritage language acquisition. Some contributions from the research on heritage speakers of European Portuguese. Lingua, 164, 251-265.

Flores, Cristina/Rinke, Esther/Azevedo, Cecilia (2017). Object realisation across generations. A closer look on the spontaneous speech of Portuguese first and second generation migrants. In: E. Di Domenico (Hrsg.), Complexity in acquisition. (S. 178-205). Newcastle: Cambridge Scholars Publishing. 
Fürstenau, Sarah/Gogolin, Ingrid/Yagmur, Kutlay (2003). Mehrsprachigkeit in Hamburg. Ergebnisse einer Sprachenerhebung an den Grundschulen in Hamburg. Münster: Waxmann.

Hufeisen, Britta/ Knorr, Dagmar/ Rosenberg, Peter/ Schroeder, Christoph/ Sopata, Aldona/ Wicherkiewicz, Tomasz (2018). Sprachbildung und Sprachkontakt im deutsch-polnischen Kontext. Frankfurt a.M.: Peter Lang.

Jańczak, Barbara (2013). Deutsch-polnische Familien: Ihre Sprachen und Familienkulturen in Deutschland und in Polen. Frankfurt a.M.: Peter Lang.

Lambert, Brigitte (2008). Family language transmission: Actors, issues, outcomes. Frankfurt a.M.: Peter Lang.

Mehlhorn, Grit (2014). Wpływ rodziny i szkoły na posługiwanie się językiem polskim jako językiem pochodzenia przez uczniów dwujęzycznych. Polnisch in Deutschland. Zeitschrift der Bundesvereinigung der Polnischlehrkräfte/Polski w Niemczech. Pismo Federalnego Nauczycieli Jezzyka polskiego, 2, 101-111.

Meisel, Jürgen M. (2011). First and second language acquisition: Parallels and differences. Cambridge: Cambridge University Press.

Meisel, Jürgen M. (2004). The bilingual child. In: T. K. Bhatia/W. C. Ritchie (Hrsg.), The handbook of bilingualism and multilingualism (S. 91-113). Malden, MA: Wiley Blackwell.

Montrul, Silvina (2008). Incomplete acquisition in bilingualism. Re-examining the age factor. Amsterdam: Benjamins.

Perez-Leroux, Ana/Cuza, Alejandro/Thomas, Danielle (2011). From parental attitudes to input conditions. In: K. Potowski/J. Rothman (Hrsg.), Bilingual youth: Spanish in English-speaking societies (S. 149-176). Amsterdam: Benjamins.

Romaine, Suzanne (1995). Bilingualism. Oxford: Blackwell.

Sopata, Aldona (2009). Erwerbstheoretische und glottodidaktische Aspekte des frühen Zweitspracherwerbs. Sprachentwicklung der Kinder im natürlichen und schulischeniii Kontext. Poznań: Wydawnictwo Naukowe Uniwersytetu im. Adama Mickiewicza. 
Open-Access-Publikation im Sinne der CC-Lizenz BY-NC-ND 4.0

(C) 2020, Vandenhoeck \& Ruprecht GmbH \& Co. KG, Göttingen ISBN Print: 9783847111863 - ISBN E-Lib: 9783737011860 


\title{
Małgorzata Bielicka \\ (Uniwersytet im. Adama Mickiewicza w Poznaniu)
}

\section{Immersion als eine innovative Methode der Fremdsprachenvermittlung auf der Elementar- und Primarstufe in Polen ${ }^{1}$}

\begin{abstract}
Immersion as an innovative method of teaching a foreign language at preschool and early primary school level

The purpose of this chapter is to address the question whether the immersion method can now be considered as an innovative method of teaching a foreign language at the preschool and early primary school level. Responding to this question, three parameters of innovation were discussed. The first one was the parameter of novelty viewed from the historical perspective, the second one was the parameter of the difference of immersion in relation to the traditional model of language teaching, and the third one, after Coyle and Bartholemy (2018: 19), was the parameter of effectiveness. The undertaken discussion showed that although the immersion method is not new in historical terms, it can still be considered an innovative method in Poland. Compared to the traditional model, the innovativeness of the immersion method is manifested by different objectives and teaching processes, as well as in its effectiveness, visible in the level of linguistic competence and the level of students' subject knowledge.
\end{abstract}

\section{Keywords}

early foreign language learning, effectiveness of foreign language learning, immersion, innovation, multilingualism

\section{Zum Begriff der Immersion}

Die Immersion bezeichnet Schulprogramme für Schüler einer Mehrheitensprache, in denen ein substantieller Teil des Curriculums für einige Schuljahre in einer Zweit-/Fremdsprache unterrichtet wird (Burmeister 2009: 386). Bei der Immersion fungiert die L2 als Vehikel zum Transport der Inhalte, wobei die

1 Mit diesem Beitrag möchte ich an das von Frau Professor Prokop häufig formulierte Postulat nach den innovativen Lösungen für den Fremdsprachenunterricht anknüpfen (Prokop 2003). Die Inspirationen für die von mir betriebe Forschung zur Immersion habe ich zweifelsohne in der modernen Denkweise der Jubilarin gefunden. 
Auswahl der sprachlichen Mittel sich direkt aus der jeweiligen Thematik ergibt (Burmeister 2006: 202). In Kanada galten nur diese Programme als Immersionsprogramme, wenn mindestens $50 \%$ der Unterrichtszeit auf das Vermitteln von Fachinhalten in der L2 verwendet wurden (Genesee 1987: 1). Nach Swain und Johnson (1997: 6-8) sind immersive Programme durch folgende zentrale Merkmale gekennzeichnet: die Verwendung der L2 als Unterrichtssprache, die Orientierung des Immersionsprogramms an dem Lehrplan des regulären Unterrichts, die gezielte Unterstützung der Muttersprache der Schüler, die Ausrichtung des schulischen Programms auf die additive Zweisprachigkeit, die Begrenzung der L2 auf den Klassenraum, das vergleichbare und in der Regel beschränkte L2-Niveau der Schüler zu Beginn des Programms, Zweisprachigkeit der Lehrer und die Prägung der Schulkultur durch die Kultur der lokalen Sprachgemeinschaft.

Das Ziel des vorliegenden Beitrags ist es, zu untersuchen, ob die Immersion als innovative Methode bezeichnet werden kann.

\section{Immersion als innovative Methode}

Die Diskussion der Immersion als einer innovativen Methode wird mit der Erklärung des Begriffs Innovation eröffnet. Im Duden steht Innovation für:

1. a) geplante und kontrollierte Veränderung, Neuerung in einem sozialen System durch Anwendung neuer Ideen und Techniken, b) Einführung von etwas Neuem; Neuerung; Reform, 2. Realisierung einer neuartigen, fortschrittlichen Lösung für ein bestimmtes Problem, besonders die Einführung eines neuen Produkts oder die Anwendung eines neuen Verfahrens (Duden - online) ${ }^{2}$.

In demselben Sinne definiert den Terminus Innovation das Fremdwörterbuch eines anerkannten polnischen Wissenschaftsverlags „Słownik wyrazów obcych“ (Sobol 2000: 476). Dort bezeichnet die Innovation 1. die Einführung von etwas Neuem, Neuerung oder 2. eine neu eingeführte Sache, Neuartigkeit (Übersetzung von mir).

Vor dem didaktischen $\mathrm{CLIL}^{3}$-Hintergrund, der uns am meisten interessiert, diskutiert den Aspekt der Innovation die Koryphäe der CLIL-Didaktik Do Coyle: "If it is successful and when learners engage in the experiences and develop the skills which evolve into them becoming plurilingual pluricultural citizens, then this is ,innovative“" (Coyle \& Bartholemy 2018: 19).

2 https://www.duden.de (Stand 05.03.2020).

3 CLIL steht für Content and Language Integrated Learning (Integriertes Sachfach- und Fremdsprachenlernen). 
Die angeführten Zitate lassen drei Dimensionen des Begriffes Innovation hervorheben. Die erste Dimension betont die diachronische Perspektive, das heißt die Antwort auf die Frage, ob es in der Geschichte der Menschheit schon ähnliche Lösungen bzw. Verfahren gab. Die zweite Dimension beinhaltet den Aspekt der Neuerung, also die Antwort auf die Frage, ob ein Phänomen durch neue Eigenschaften beschrieben werden kann, und die dritte Dimension nach Coyle (Coyle \& Bartholemy 2018: 19) legt Gewicht auf die Effizienz des Lehrprozesses, der sich in der multisprachlichen Leistung der Schüler bemerkbar machen sollte.

In den folgenden Abschnitten wird eine Diskussion der immersiven Methode in Bezug auf die drei oben erwähnten Indikatoren geführt.

\subsection{Immersion als eine historisch neue Methode? - diachronische Perspektive}

Historisch gesehen ist zweisprachiges Lehren kein modernes Verfahren. Die Wurzeln des zweisprachigen Unterrichts reichen in die Zeiten 3000 v. Chr.. Die ersten Belege für zweisprachiges Lehren findet man bereits auf Tontäfelchen, die Textfragmente in der sumerischen Keilschrift beinhalten. Aus diesen Texten geht hervor, dass Schülern mit akkadischer Erstsprache Sumerisch als Fremdsprache beigebracht wurde, aber auch Geografie und Mathematik wurden auf Sumerisch unterrichtet. Belege für mehrsprachige Bildung findet man fernerhin auf den altertümlichen Schrifttafeln in Ägypten. Die Bildung junger Römer war ebenfalls von Anfang an eine zweisprachige Bildung in Griechisch und Latein. Im altertümlichen Rom wurde auf der höchsten Bildungsstufe Rechtswesen auf Latein und Philosophie, Naturwissenschaften und Medizin auf Griechisch (Möller 2013: 15) unterrichtet.

Auf polnischem Boden findet man ebenso genug Belege für mehrsprachige Erziehung. Erwähnenswert sind vor allem zwei Bildungsinstitutionen aus den Zeiten der Aufklärung: das Collegium Nobilium und die von Stanislaus August Poniatowski eingerichtete Ritterschule. Der Gründer des Collegium Nobilium, Stanisław Konarski, betonte das Nützlichkeitsziel der Fremdsprachen als Schlüssel zum Aufschließen des Wissens. Aus diesem Grunde haben Schüler Fremdsprachen mit Bediensteten, im Unterricht, in den Alltagsgesprächen, und auch in den von den Schülern auf Deutsch und auf Französisch vorgeführten Theaterstücken benutzt, deren Ziel die Verbreitung neuer und fortgeschrittener Gedanken war. In der Ritterschule hat man den Fremdsprachen sogar in der 1. Klasse 24 Stunden, in der 2., 3., 4. und 6. Klasse 21 Stunden, in der 5. Klasse 9 Stunden und in der 7. 3 Stunden gewidmet! Die sprachlichen Kompetenzen erwarben die Schüler auf drei Wegen: erstens durch den klassischen Fremd- 
sprachenunterricht, zweitens durch den Unterricht von anderen Schulfächern (wie Geografie, Geschichte, Literatur und Berufsfächer) in den Fremdsprachen und drittens durch Gespräche mit Lehrern, Bediensteten und Schulkameraden (Cieśla 1974: 105f.).

Am Anfang des 20. Jahrhunderts gab es in Australien über 60 zweisprachige Schulen (Geiger-Jaillet 2003: 1), aber die Fundamente für die gegenwärtigen Schulen in Europa soll man eher in Kanada sehen, wo in den 60er Jahren das zweisprachige Bildungsmodell eingeführt wurde.

Das Gesagte lässt den Schluss zu, dass - diachronisch gesehen - das zweisprachige Bildungsmodell so lange existiert, wie lange schon Menschen ihre Kinder durch einen regulären Unterricht zu bilden versuchen. Das Lehren in zwei Sprachen ist somit kein neues Phänomen.

2.2 Immersion versus traditioneller Unterricht in Bezug auf didaktische Ziele und Lehr- und Lernprozesse

Wie bereits erwähnt, beinhaltet der zweite Punkt der Analyse den Aspekt der Neuerung, somit die Antwort auf die Frage, ob ein Phänomen durch neue Eigenschaften beschrieben werden kann. Im folgenden Kapitel wollen wir also untersuchen, ob die Immersion im Vergleich zum konventionellen, am meisten in der Welt verbreiteten, Fremdsprachenunterricht durch völlig neue Eigenschaften beschrieben werden kann. Dabei sind wir aus Platzmangel nicht imstande, ausführlich auf alle Elemente des glottodidaktischen Gefüges ${ }^{4}$ einzugehen, deswegen werden hier nur zwei Bausteine dieses Gefüges, und zwar die didaktischen Ziele und der Unterrichtsprozess, erwogen.

Der fundamentale Unterschied zwischen dem immersiven Unterricht und dem traditionellen Fremdsprachenunterricht ist in der Zielsetzung ersichtlich. Wenn im Fremdsprachenunterricht die L2-Kenntnisse mit Hilfe von Sachfachinhalten vermittelt werden, werden die Sachfachinhalte im immersiven Unterricht mit Hilfe der L2 gelehrt. Aus dieser globalen Zielsetzung ergibt sich die Planung einer immersiven Unterrichtsstunde, die grundsätzlich vom jeweiligen Fachinhalt ausgeht, d.h. die Lehrperson muss zunächst die sachfachlichen Ziele der konkreten Unterrichtseinheit formulieren, und aufgrund dieser sachfachlichen Ziele werden erst die sprachlichen Mittel bestimmt. In diesem Fall muss der Lehrer überlegen, was zur content-obligatory language und was zur contentcompatible language gehört (Baumeister 2009: 388). Zur content-obligatory language gehören Fachtermini und grammatische Strukturen, die für das Ver-

4 Das glottodidaktische Gefüge ist ein Modell, das konstitutive Faktoren des glottodidaktischen Prozesses sowie die Beziehungen zwischen ihnen abbildet (Pfeiffer 2003: 19-22). 
ständnis und die Produktion der sachbezogenen Inhalte unverzichtbar sind, wogegen content-comaptible language jene Termini, Redemittel und Strukturen bezeichnet, die außerhalb des spezifischen Sachfachkontextes gelehrt werden, nicht unbedingt bei der Beherrschung des jeweiligen Sachfachinhaltes verlangt werden und sich eher aus dem Curriculum des Fremdsprachunterrichts ergeben (Snow, Met \& Genesee 1989: 206).

Kurz gesagt: im traditionellen Fremdsprachenunterricht steht der Erwerb der sprachlichen Kompetenz im Vordergrund, während im immersiven Unterricht der Erwerb des Sachfachwissens und der Lernkompetenz Priorität erhalten (Mehisto, Marsh \& Frigols 2008: 12).

Unsere anfänglichen Überlegungen zu Lernprozessen sind jene zur Beschreibung der Spracherwerbsprozesse: zumindest seit Krashen wird in der einschlägigen Literatur zwischen dem gesteuerten und dem ungesteuerten Zweitspracherwerb unterschieden. „Mit gesteuertem Zweitspracherwerb wird der Erwerb unter institutionalisierten Bedingungen verstanden, der im Fremdsprachenunterricht durch verschiedene Arten formaler Lehrverfahren, wie beispielsweise Lehrpläne, Lehrmaterialien und Lehrer, gesteuert, also beeinflusst wird (sog. instructed second language acquisition)" (Sopata 2009: 78). Beim gesteuerten Zweitspracherwerb setzt man voraus, dass die Sprache linear, d.h. nach einer, von Linguisten und Fremdsprachendidaktikern festgelegten, Progression unterrichtet wird. Während in den Zeiten von GÜM und ALM/AVM- Methoden die Grammatik der relevanteste Indikator der Lehrstoffprogression war, nannte das pragmatisch-funktionale Konzept einige weitere Faktoren der Lehrstoffprogression, die zumindest die gleichrangige Rolle wie die Grammatik erfüllten. Dies waren Sprechintentionen, Rollen/Situationen, Themen/Inhalte und Texte (Neuner \& Hunfeld 1993: 97). Den gesteuerten Spracherwerbsprozess beschreibend dürfen wir noch eine wichtige Eigenschaft nicht aus den Augen verlieren: die Bewusstheit der Unterrichtsprozesse, die sich im bewussten Lernen von grammatischen Regeln manifestiert.

Mit dem ungesteuerten oder natürlichen Zweitspracherwerb erfasst man das Lernen von Sprachen außerhalb des Unterrichts ohne Unterweisung (sog. naturalistic second language acquisition) (Sopata 2009: 78). Diese Erwerbsprozesse werden als natürliches, implizites und unbewusstes Lernen beschrieben (Edmondson \& House 2000: 11) $)^{5}$. Beim ungesteuerten Zweitspracherwerb werden die Lernenden in die komplexe Sprache mit all ihren Dimensionen (Form, Inhalt und Pragmatik) und ihrer ganzen hierarchischen Struktur (Sprache, Literatur, Gattung, Text, Kapitel, Abschnitt, Satz, Wendung, Wort, Silbe/Morphem, Laut/

5 Es muss aber darauf hingewiesen werden, dass die präzise Trennung beider Prozesse nicht möglich ist, weil beide Prozesse natürlich sind und beide durch innere Erwerbsmechanismen zu einem gewissen Grad gesteuert werden (Sopata 2009: 78). 
Phonem) eingetaucht (Bleyhl 2000: 10). „In dem Moment, in dem sich jemand sprachlich äußert, ist er gleichzeitig (!) faktisch auf nahezu allen Hierarchieebenen der Sprache und in allen Dimensionen aktiv“ (Bleyhl 2000: 10).

Resümierend kann man sagen, dass die immersive Methode, im Vergleich mit dem traditionellen Unterricht, eher auf dem natürlichen Spracherwerbsmechanismus der Schüler aufbaut, der durch eine interaktions- und damit sprachinputreiche Umgebung aktiviert wird. Durch Curricula, Schulpläne und Stundenentwürfe wird aber der Prozess in einem relativ starken Maße gesteuert. Nichtdestotrotz schafft der immersive Unterricht eine völlig andere und neue Qualität der Lernprozesse als der traditionelle Unterricht. In diesem Sinne kann man sagen, dass die immersive Methode innovativ ist.

\subsection{Effizienz der Immersion auf der Elementar- und Primarstufe}

Laut des dritten Kriteriums der Innovativität, das wie bereits erwähnt von Coyle (Coyle \& Bartholemy 2018: 19) formuliert wurde, wird im folgenden Kapitel überprüft, ob Immersion eine effiziente Methode ist.

Die ersten groß angelegten Untersuchungen wurden in Kanada durchgeführt (Genesee 1987, Lambert \& Tucker 1972, Lambert, Genesee, Holobow \& Chartrand 1993). In diesen Studien wurden viele Bereiche des Sprachlernprozesses untersucht, vor allem aber die Auswirkungen der Immersion auf die Entwicklung der L1 und der L2, die Auswirkungen der Immersion auf die Leistungsfähigkeit in anderen Schulfächern, und die Effizienz der Methode im Bereich allgemeiner kognitiver Leistungen der Schüler. Die Ergebnisse der Untersuchungen in den oben aufgelisteten Kompetenzen waren für die Immersion positiv, denn weder das L1-Niveau noch das Sachfachwissen waren bei Immersionsschülern beeinträchtigt. Am intensivsten war aber die Entwicklung der L2 untersucht worden, und auch auf diesem Gebiet konnte man feststellen, dass Immersionsschüler aus allen Immersionsvarianten beträchtlich besser als konventionell unterrichtete Schüler abgeschnitten hatten (Wode 1995: 79).

Neuere Studien, die sich auf die Elementar- und Primarstufe beziehen, bringen auch eindeutig positive Ergebnisse. In der von Häckel (2013) mithilfe eines Bildertestes durchgeführten Untersuchung rezeptiver lexikalischer Leistungen von Kita-Kindern, erreichten Kinder aus drei Kindergärten mit einer unterschiedlichen Kontaktzeit zur fremdsprachlichen Erzieherin die Identifikationsraten $68 \%, 77 \%$ und $80 \%$ (Häckel 2013: Anhang, Seiten 19, 103 und 70). Damit hat die Forscherin die L2-Leistungen der Kita-Kinder, aber auch die positive Korrelation zwischen der L2-Kontaktzeit und dem Niveau der L2-Kompetenz belegt. Edelenbos und Kubanek (2008: 72ff.) haben ähnlich hohe Werte im Hörverstehenstest erzielt, der das Verstehen einzelner Items nachprüfte, aber in 
den komplexen, situativ angelegten Testformaten (z. B.: stell die Tasse auf den Tisch!) waren die Ergebnisse bedeutend niedriger - hier konnte man den Kindern im Durchschnitt nur 30,3\% korrekte Reaktionen attestieren (Edelenbos und Kubanek 2008: 75). Obwohl der Wortschatz das erste Element ist, auf das Kinder ihre Aufmerksamkeit lenken (Hatch 1983, Singleton 1999), werden von Kindern grammatische Informationen auch wahrgenommen und verarbeitet. Burmeister und Steinlein (2008) betonen, dass Kinder schon nach einem halben Jahr in einer zweisprachigen Kita die ersten Anzeichen grammatischer Kompetenz zeigen, die sich zum Kitaufenthaltsende bedeutend vergrößert. Belege dafür findet man in der ELIAS-Untersuchung, in der Kinder in einem grammatischen Bildertest das hohe Ergebnis von 78,16 \% bekommen haben (Kersten, Rohde, Schelletter \& Steinlein 2010: 80), sowie in der Untersuchung von Häckel, in der Kinder entsprechend der Intensivität der L2-Kontaktzeit die Testergebnisse von 64 \%, 68 \% und $77 \%$ erreicht haben ( Häckel 2013: Anhang, Seiten 23, 106 und 73).

Markante Errungenschaften von bilingual unterrichteten Kindern werden aber erst auf der Primarstufe bescheinigt. Viele Untersuchungen (Wode, Fischer, Pasternak \& Franzen 2003; Wode \& Werlen 2003; Piske 2006; Burmeister \& Pasternak 2004) zeigen, dass Grundschüler sowohl die Alltagssprache als auch die akademische Sprache, einschließlich des Fachwortschatzes und der Diskurskompetenz, entwickeln. Im Rahmen des Fachwissens erzielen sie dieselben Ergebnisse wie monolingual unterrichtete Schüler (Turnbull, Hart \& Lapkin 2001). Manche Untersuchungen zeigen, dass immersive Schüler sogar ein bisschen höhere Ergebnisse als ihre monolingual gelehrten Schulkameraden in den mathematischen Testformaten (Gebauer, Zaunbauer \& Möller 2013, Zaunbauer, Gebauer \& Möller 2013) erreichen. Außerdem ist ihre L1-Kompetenz auch nicht beeinträchtigt (Lambert, Genesee, Holobow \& Chartrand 1993, Wesche 2002, Wode 2009).

In den letzten Jahren hat man am Institut für Angewandte Linguistik an der Adam-Mickiewicz-Universität in Poznań auch eine groß angelegte Untersuchung des Sprachstandes von immersiv gelehrten Kita- und Grundschulkindern durchgeführt (Bielicka 2017). Das ausführliche Ziel dieser Untersuchung war die Ermessung der grammatischen Kompetenz der Kinder in der Rezeption und in der Produktion der Sprache. Um nicht den Rahmen dieses Artikels zu sprengen, werden im Folgenden allerdings nur die Verstehensleistungen der Kinder angeführt.

Probanden waren Kinder aus zwei Bildungseinrichtungen mit Deutsch als Fremdsprache: dem zweisprachigen polnisch-deutschen Kindergarten „Ene due Rabe“ und dem deutschen Schulhort. Der deutsche Schulhort war eine völlig innovative Einrichtung, die von drei Institutionen (dem Institut für Angewandte Linguistik, dem zweisprachigen Kindergarten „Ene due Rabe“ und der TytusDziałyński-Grundschule) als Bildungsprojekt ins Leben gerufen und auch ge- 
tragen wurde. Die Rahmenbedingungen beider Einrichtungen wurden in Bielicka (2017: 144-148) präsentiert. An dieser Stelle sollte nur erwähnt werden, dass die wöchentliche Kontaktzeit der Kinder zum Deutschen im Kindergarten ungefähr 25 Stunden und im Hort 10 Stunden betrug. Deutsch war auch fast zu $100 \%$ die Kommunikationssprache der Lehrerinnen. Untersucht wurden 3 Gruppen von Kindern:

- Gruppe 1: Kinder aus der zweisprachigen Kita, am Ende ihres Aufenthaltes im Kindergarten,

- Gruppe 2: Grundschulkinder ohne frühere zweisprachige Erfahrungen in der Kita, am Ende ihres Aufenthaltes in dem deutschen Schulhort,

- Gruppe 3: Grundschulkinder, die früher die zweisprachige Kita besucht haben und weiter im Hort Deutsch gelernt haben, am Ende ihres Aufenthaltes im deutschen Schulhort.

Die Untersuchung wurde mit Hilfe des Bildertests aus der ELIAS-Untersuchung (Kersten, Rohde, Schelletter \& Steinlein 2010) durchgeführt, allerdings mussten die untersuchten Strukturen und die Bilder, die sie veranschaulichten, an die deutsche Sprache angepasst werden. Man hat auch eine völlig neue Struktur, das Perfekt, eingeführt. Die Liste der Strukturen hat endgültig folgend ausgesehen:

SVO - Identifikation des Subjekts, Prädikats und Objekts in einem einfachen Satz,

$\mathrm{SgPl} \quad$ - Identifikation des Singulars und des Plurals der Nomen,

PersPro - Identifikation des männlichen und femininen Pronomens er und sie,

PronDekl - Identifikation des deklinierten männlichen und femininen Pronomens ihn und sie,

PossPro - Identifikation der Possesivpronomen sein und ihr,

Neg - Identifikation eines verneinten/affirmativen Satzes,

Verb - Konjugation der thematischen Verben,

Vcop - Konjugation des Kopulaverbs sein,

Tem - Identifikation eines Satzes im Präsens und im Perfekt.

Im Bildertest haben oben erwähnte Kindergruppen wie folgt abgeschnitten:

Tabelle 1: Ergebnisse des Grammatiktests

\begin{tabular}{|l|c|c|c|}
\hline & Gruppe 1 & Gruppe 2 & Gruppe 3 \\
\hline Gruppendurchschnittsergebnisse [\%] & 59,42 & 65,5 & 85,6 \\
\hline \multicolumn{4}{|c|}{ Gruppendurchschnittsergebnisse in Bezug auf grammatische Strukturen [\%] } \\
\hline SVO & 73,9 & 89,2 & 100 \\
\hline SgPl/Ball, Bälle & 60,9 & 83,3 & 88,9 \\
\hline PersPron/er, sie & 55,8 & 55 & 88,9 \\
\hline PronDekl/ihn, sie & 41,3 & 55 & 66,7 \\
\hline
\end{tabular}


(Fortsetzung)

\begin{tabular}{|l|c|c|c|}
\hline & Gruppe 1 & Gruppe 2 & Gruppe 3 \\
\hline PossPron/sein, ihr & 31,9 & 40,8 & 55,6 \\
\hline Neg/schläft, schläft nicht & 79,7 & 91,7 & 100 \\
\hline Verb/springen, springt & 62,3 & 50 & 88,9 \\
\hline Vcop/ist, sind & 56,5 & 51,7 & 81,5 \\
\hline Tem/zieht an, hat angezogen & 72,5 & 72,5 & 100 \\
\hline
\end{tabular}

Aus diesen Daten ist ersichtlich, dass alle Kinder viel gelernt haben, aber die Lernenden mit der längsten Kontaktdauer zu L2 (Gruppe 3) erzielten die besten, man kann sogar sagen, hervorragende, Ergebnisse. Im Rahmen von drei Strukturen hat diese Gruppe der Kinder das Ergebnis von 100 \%, im Rahmen weiterer vier Strukturen das Ergebnis über $80 \%$, und nur in Bezug auf das Verständnis der Possesivpronomen sein und $i h r$, sowie der deklinierten Personalpronomen ihn und sie haben die Kinder die Ergebnisse von 55,6 \% und 66,7 \% erreicht. Damit wurde bewiesen, dass die immersive Methode eine sehr effiziente Methode der Sprachvermittlung ist.

\section{Fazit}

Bei der Diskussion der Immersion als einer innovativen Methode hat man drei Indikatoren berücksichtigt: 1. historische Perspektive, 2. Auftreten von neuen Eigenschaften im Vergleich mit konventionellen Sprachvermittlungsmethoden und 3. Effizienz der Immersion in Bezug auf die fremdsprachlichen Leistungen der Schüler. Aus der Diskussion ergibt sich, dass die Immersion weiterhin als innovative Methode fungieren kann, obwohl sie in der Geschichte seit langem bekannt ist. Völlig neu im Vergleich mit konventionellem Unterricht sind in dieser Methode vor allem didaktische Zielsetzungen, die die Vermittlung der Sachfachziele hervorheben, sowie die Qualität des Unterrichtsprozesses, der in bedeutend größerem Maße als das im konventionellen Unterricht der Fall ist, auf dem Aktivieren des natürlichen Erwerbsmechanismus der Schüler aufbaut. Schließlich, wie dies das Posener Projekt gezeigt hat, können auch immer wieder neue, innovative Konzepte und Arbeitsweisen der immersiven Einrichtungen entwickelt werden. Wegen ihres innovativen Charakters wird aber empfohlen, die dort in Gang gesetzten glottodidaktischen Prozesse in Zusammenarbeit mit Fremdsprachenforschern empirisch zu untersuchen, um den Schülern die besten Bedingungen für das Fremdsprachenlernen zu gewährleisten. 


\section{Literaturverzeichnis}

Bielicka, Małgorzata (2017). Efektywność nauczania języka niemieckiego na poziomie przedszkolnym i wczesnoszkolnym $w$ dwujęzycznych placówkach edukacyjnych $w$ Polsce. Poznań: Wydawnictwo Naukowe Uniwersytetu im. Adama Mickiewicza.

Bleyhl, Werner (2020). Fremdsprachen in der Grundschule. Grundlagen und Praxisbeispiele. Hannover: Schroedel.

Burmeister, Petra (2009). Frühbeginnende Immersion. In: U. O.-H. Jung (Hrsg.), Praktische Handreichung für Fremdsprachenlehrer, Bayreuther Beiträge zur Glottodidaktik Band 2 (S. 385-391). Frankfurt a.M.: Peter Lang.

Burmeister, Petra (2006). Immersion und Sprachunterricht im Vergleich. In: M. Pienemann/J.-U. Keßler/E. Roos (Hrsg.), Englischerwerb in der Grundschule. Ein Studienund Arbeitsbuch (S. 197-216). Paderborn: Ferdinand Schöningh.

Burmeister, Petra/Pasternak, Ruth (2004). Früh und intensiv: Englische Immersion in der Grundschule am Beispiel dr Claus-Rixen-Grundschule in Altenholz. https://www.fmks. eu (Stand 5.11.2016).

Burmeister, Petra/Steinlein, Anja K. (2008). Sprachstandserhebungen in bilinguallen Kindertagesstätten. In: G. Bell/R. Kupetz (Hrsg.), Fremdsprachenlehren und -lernen. Prozesse und Reformen (S. 129-146). Frankfurt a.M.: Peter Lang.

Cieśla, Michał (1974). Dzieje nauki języków obcych w zarysie. Warszawa: PWN.

Coyle, Do/Bartholemy, Claudia (2000). An interview with Do Coyle. BABYLONIA, 2, 18-20.

Edmonson, Willis/House, Juliane (2000). Einführung in die Sprachlehrforschung. Tübingen und Basel: A. Francke Verlag.

Gebauer, Sandra Kristina/Zaunbauer, Anna C. M./Möller, Jens (2013). Englischer Immersionsunterricht in der Grundschule: Effekte und vermittelnde Prozesse. https://www.iaa.unirostock.de/storages/uni-rostock/Alle_PHF/IAA/Projekt_Immersion/Vortrag_Gebauer_ Zaunbauer_Moeller.pdf (Stand 11.03.2020).

Geiger-Jaillet, Anemone (2003). Zur Typologie bilingualer Unterrichtsmodelle: Gedanken zu einer möglichen Systematik, gefolgt von Beispielen aus der Praxis. www.cebip.com/ download.asp?file=/elementi/www/...5_geiger (Stand 24.08.2016).

Geiger-Jaillet, Anemone (2003). Zur Typologie bilingualer Unterrichtsmodelle: Gedanken zu einer möglichen Systematik, gefolgt von Beispielen aus der Praxis. EDUCATION ET SOCIÉTÉS PLURILINGUES, 15, 43-58.

Genesee, Fred (1987). Learning through two languages: Studies of immersion and bilingual education. Cambridge, Mass.: Newbury House Publishers.

Häckel, Alexandra (2013). Bilinguale Kita-Einrichtungen. Untersuchungen zur sprachlichen Entwicklung deutsch-englisch bilingual betreuter Kita-Kinder. Baltmannsweiler: Schneider Verlag Hohengehren.

Hatch, Evelyn Marcussen (1983). Psycholinguistics: A second language perspective. Rowley, Mass.: Newbury House Publishers.

Kersten, Kristin/Rohde, Andreas/Schelletter, Christina/Steinlein Anja K. (2010). Bilingual preschools, vol. 1, Learning and development. Trier: Wissenschaftlicher Verlag.

Lambert, Wallace E./Tucker, G. Richard (1972). Bilingual education of children: The St. Lambert experiment. Rowley: Newbury House Publishers. 
Lambert, Wallace. E./Genesee, Fred/Holobow, Naomi/Chartrand, Louise (1993). Bilingual education for majority English-Speaking children. European Journal of Psychology of Education, 8(1), 3-22.

Mehisto, Peter/Marsh, David/Frigols, Maria Jesús (2008). Content and language integrated learning in bilingual and multilingual education. London: Macmillan Education.

Möller, Christine (2013). Zur Geschichte und Zukunft des bilingualen Unterrichts. In: A. Steinlein/A. Rohde (Hrsg.), Mehrsprachigkeit in bilingualen Kindertagesstätten und Schulen. Voraussetzungen - Methoden - Erfolge (S. 14-30). Berlin: dVb.

Neuner, Gerhard/Hunfeld, Hans (1993). Methoden des fremdsprachlichen Deutschunterrichts. Berlin: Langenscheidt.

Pfeiffer, Waldemar (2001). Nauka języków obcych. Od praktyki do praktyki. Poznań: Wagros.

Piske, Thorsten (2006). Ergebnisse aus der bilingualen Grundschule. www.fmks-online.de/_ wd_showdoc.php?pic=546 (Stand 5.11.2016).

Prokop, Izabela (2003). Skuteczna szkoła - mrzonka to czy nadzieja? In: P. Hostyński (Hrsg.), Szukamy nowych dróg, Materiały II meetingu glottodydaktycznego zorganizowanego $w$ ramach podyplomowego stadium glottodydaktyki 2001/2002 (S. 23-29). Poznań: Rys-Studio.

Singleton, David (1999). Exploring the second language mental lexicon. Cambridge: CUP. Snow, Marguerite Ann/Met, Myriam/Genesee, Fred (1989). A conceptual framework for the integration of language and content in second/foreign language instruction. TESOL Quarterly, 23(2), 201-217.

Sobol, Elżbieta (Hrsg.) (2000). Słownik wyrazów obcych. Warszawa: PWN.

Sopata, Aldona (2009). Erwerbstheoretische und glottodidaktische Apekte des frühen Zweitspracherwerbs. Sprachentwicklung der Kinder im natürlichen und schulischen Kontext. Poznań: Wydawnictwo Naukowe Uniwersytetu im. Adama Mickiewicza.

Swain, Merrill/Johnson, Robert Keith (1997). Immersion education, A category within bilingual education. In: R. K. Johnson/M. Swain (Hrsg.), Immersion education: International perspectives (S. 1-16). Cambridge: CUP.

Turnbull, Miles/Hart, Doug/Lapkin, Sharon (2001). Grade three immersion students' performance in literacy and mathematics: Province-wide results from Ontario (199899). The Canadian Modern Language Review, 58(1), 9-26.

Wesche, Mariorie Bingham (2002). Early French immersion. How has the original Canadian model stood the test of time? In: P. Burmeister/T. Piske/A. Rohde (Hrsg.), An integrated view of language development. Papers in honor of Henning Wode (S. 357379). Trier: Wissenschaftlicher Verlag.

Wode, Henning (2009). Frühes Fremdsprachenlernen in bilingualen Kindergärten und Grundschule. Braunschweig: Westermann.

Wode, Henning/Fischer, Uta/Pasternak, Ruth/Franzen V. (2003). Frühes Englisch lernen im Altenholzer Verbund von Kita und Grundschule: Erfahrungen aus Praxis und Forschung zum Ende der 4. Klasse. www.fmks-online.de (Stand 16.03.2016).

Wode, Henning/Werlen, Erika (2003). Was Kinder können können. Englisch als Unterrichtssprache? Grundschulmagazin Englisch, 6, 6-9.

Zaunbauer, Anna Ch. R./Gebauer, S. Kristina/Möller, Jens (2013). Bilinguale Grundschulen: Auswirkungen auf das Sachfachwissen am Beispiel Deutsch und Mathematik. In: 
A. K. Steinlein/A. Rohde (Hrsg.), Mehrsprachigkeit in bilingualen Kindertagesstätten und Schulen. Voraussetzungen- Methoden- Erfolge (S. 96-106). Berlin: dVb.

Internetquellen

Duden. https://www.duden.de (Stand 05.03.2020). 


\title{
Teresa Siek-Piskozub / Aleksandra Wach \\ (Uniwersytet im. Adama Mickiewicza w Poznaniu)
}

\section{EFL learner agency from the perspective of learners' autobiographies}

\begin{abstract}
EFL learner agency from the perspective of learners' autobiographies

The phenomenon of agency in the educational context has recently attracted researchers' attention. This interest has been accompanied by the influence of hermeneutic phenomenology as a qualitative research methodology in educational research, and by the shift from exclusively deductive research to an acknowledgement of inductive exploration as an understanding of particular learning and teaching practices. In the article, the specificity of the hermeneutic phenomenology in the context of education will be discussed, followed by an explanation of the construct of agency and the chordal triad model for studying it. The study is aimed to investigate learner agency in the process of acquiring language competence from the perspective of the lived experience of the participants, who were two English majors at a Polish university. The data came from the autobiographic narratives on the participants' way to competence in English as a foreign language and were analysed through the chordal triad model.
\end{abstract}

Keywords

learner agency; hermeneutic phenomenology; EFL learning; autobiography

\section{Introduction}

The last few decades of the past century marked the beginning of a growing interest in qualitative methods of research in education, of which hermeneutic phenomenology is a notable example. As defined by Creswell (2014: 14), the essence of phenomenological research is that it explores the "lived experiences of individuals" on the basis of their own descriptions. Empirical data for such research come from individuals who have participated in the same kind of phenomenon. In a more extensive definition, van Manen (2016: 26) explains the nature of hermeneutic phenomenology in the following way:

Hermeneutic phenomenology is a method of abstemious reflection on the basic structures of the lived experience of human existence. The term method refers to the way or attitude 
of approaching a phenomenon. Abstemious means that reflecting on experience aims to abstain from theoretical, polemical, suppositional, and emotional intoxications. Hermeneutic means that reflecting on experience must aim for discursive language and sensitive interpretive devices that make phenomenological analysis, explication, and description possible and intelligible. Lived experience means that phenomenology reflects on the prereflective or prepredicative life of human existence as living through it.

Although hermeneutic phenomenology has its roots in philosophy, the empirical and analytical methods which could lead to an insightful study of a phenomenon are also used in social sciences. They include, for example, biographies, diaries or interviews. For a phenomenological analysis, genuine experience is reflected upon and meaning is attached to it. As van Manen (2016: 38) claims, "[a]ny and every possible human experience (event, happening, incident, occurrence, object, relation, situation, thought, feeling, and so on) may become a topic for phenomenological inquiry". Agency, thus, can be viewed as a phenomenon which can become an object of reflection.

Emirbayer and Mische (1998) and Biesta and Tedder (2007) observe that relatively little attention has been paid to a systematic analysis of the phenomenon of agency, despite the growing interest in lifelong learning, as revealed, for example, in the learning biographies of adults. Emirbayer and Mische believe that one of the reasons is that "in the struggle to demonstrate the interpenetration of agency and structure, many theorists have failed to distinguish agency as an analytical category in its own right" (1998: 962-963).

Depending on epistemological goals of research on a given phenomenon, a single case can be studied if one wants to understand relationships between the described elements in an event (Creswell 2014: 14). However, to generalise an interpretation, a bigger sample is needed. In the chapter, we will define the phenomenon of agency and introduce a chordal triad model (Emirbayer \& Mische 1998) for studying it, because it provided a framework for the data analysis in the present study. The study aimed to explore learner agency through two selected autobiographical narratives entitled My way to English, written by the BA programme students at the English Faculty in Poznań.

\section{Agency and the chordal triad model}

Recent challenges of globalization and modernization processes force people to become more agentic, and it is believed that their own prior experience helps them to further enhance their agency. Emirbayer and Mische (1998: 972) criticize traditional theories of agency which tended to focus on routine, or purpose, or judgement, for their one-sidedness, whereas, in their opinion, agency is an 
overarching category which encompasses a dynamic interplay between these three dimensions. They define agency as:

a temporally embedded process of social engagement, informed by the past (in its habitual aspect), oriented toward the future (as a capacity to imagine alternative possibilities) and 'acted out' in the present (as a capacity to contextualize past habits and future projects with the contingencies of the moment) (Emirbayer \& Mische 1998: 963).

In accordance with this definition, the authors propose a model for studying agency which they have named the chordal triad of agency. The chordal triad model is built of three dimensions which affect an individual's ability to act. The authors also posit that the interplay of these dimensions may vary within different contexts. The first dimension of the chordal triad of agency is its iterational element, which encompasses people's life and professional histories. It includes an individual's personal life story, thought patterns and ways of acting which are incorporated into the current situation. Such a reactivation gives stability to identities, interactions and institutions over time. The second dimension is of a practical-evaluative character and includes the individual's capacity to make practical and normative judgments by choosing among different ways to act in relation to a particular situation, its demands as well as dilemmas. This dimension refers to cultural aspects (i.e., ideas, values, beliefs, discourses and languages), structural aspects (i.e., social structures, relationships, roles, power, trust), and material aspects (i.e., resources and the physical environment within which the individual acts). Finally, there is a projective element of agency (both for the short and long term), as decisions concerning a current situation are also influenced by the individual's imaginative ability, such as thoughts, hopes and/or fears, to see a future that differs from the past and present (Emirbayer \& Mische 1998: 971).

Van Lier (2010) sets agency within an ecological perspective on learning, in which it is incorporated into the dynamic actions performed by teachers and learners as an element of a multi-layered, complex system of interaction and language use. He refers to agency as movement (i.e., a change of state or direction), or even a lack of movement when a person makes a conscious decision not to act. Discussing the relationship among agency and other notions, he believes that "autonomy, motivation and investment are in a sense products (or manifestations) of a person's agency", but agency is a more profound concept and a principal component of a learning process (Van Lier 2010: 4).

Vitanova, Miller, Gao and Deters (2018: 4) discuss agency in L2 learning as a "socially mediated process", as it usually emerges and evolves through interpersonal interactions and negotiations of social positions. As such, it is a crucial element of the development of language competence, but also of learner identity. In L2 learning, agency is closely related to self-regulation, but not necessarily 
with active participation in class activities. In fact, sometimes resisting active participation in classroom tasks may be a demonstration of one's agency. Muramatsu's (2018:5) definition also stresses agentic learners' relationships with the social environment, through which they try to make their own choices on their way to opportunities for self-transformation. Agency is the driving force behind their pursuits, which stimulates learners' imagination and creative activity, and makes them accept certain situations, rejecting and refusing others.

Larsen-Freeman (2019) analyses learner agency within the perspective of the complex dynamic systems theory, in this way presuming that agency is an element in a system whose parts are connected through mutual interactions and interrelationships. Agency is always tied up to its context, and is influenced by one's relationship with the environment. These relations, following Emirbayer and Mische (1998), are always spatially and temporarily situated. Agency can thus fluctuate over time, and can be achieved by a learner through an interplay of his/her effort and various environmental factors. Larsen-Freeman (2019: 67) underscores the multidimensional nature of agency: it is manifested not only by one's behaviour, but also by the ability to evaluate events, to distinguish what is relevant, to assign personal meanings to things, etc.

Commenting on the recent educational practices, Larsen-Freeman (2018) notes that despite the challenges of our times, learners are often categorised and assumed to need customized input, as well as to follow similar learning trajectories, while what, in fact, is needed is promoting learner agency. LarsenFreeman (2019) generally objects to categorizing learners, and argues that

Given the complicated histories that individuals bring with them to learning and using a language, it is not hard to understand how by placing them in categories, we miss out on all the intra-category differences that make them unique (Larsen-Freeman 2019: 70).

Optimizing conditions for language learning and helping students enhance their agency are thus a core responsibility of teachers.

In relation to the educational context, a related concept, teacher agency, has been discussed by Priestley, Biesta and Robinson (2013) with reference to the use of the model proposed by Emirbayer and Mische (1998). The researchers (Priestley et al. 2013) claim that all the dimensions can hinder or enable teachers' agencies. How teachers act in a given situation may be due to their beliefs and values, relationships in schools, between schools and also relationships with surrounding environments. Other aspects which are important are the teachers' desires for a change in the short and long term, as well as their life histories and education. The authors emphasize the need for a professional discourse that consists of teachers' own thoughts and reflections to achieve agency so that they are not restricted in their profession because of political influence or educational tradition. In a study investigating L2 learner agency, Gao (2013) examined 
learners' reflective narratives in order to find out how agency guided their efforts, motivations and planning in choosing the right path of learning. It was revealed that agency allowed the learners to undertake some efforts to learn, but further successful learning was conditioned by a number of contextual factors (such as interactions, institutional settings, and a macro-context of ideology and economic and political conditions). The study thus showed that "it is important to perceive learners' autonomy or learners' capacity to control the learning process as socioculturally mediated and context-situated" (Gao 2013: 235). Along similar lines, Mercer (2012) embedded her case study research on a single tertiary-level learner in the complex dynamic systems theory, finding that the learner's agency was deeply situated in sociocultural and educational contexts. It was also interpersonally, temporarily, and intrapersonally situated. Siek-Piskozub (2019) applied the chordal triad model in the analysis of the qualitative data on learner agency elicited through a case study of two successful learners of English. The results of the analysis showed that the model is applicable to studying foreign language learning processes, as the participants' reflections embraced past experiences and present behaviours which were assumed to help them achieve their projected goals. The students were aware of the interrelations of these elements in the process of building their L2 competence.

The study reported in the following section aimed to trace EFL learners' agency with the application of the chordal triad model. Our assumption was that the temporal view of agency in this model can capture the influence of the temporal dimension on learners' way to acquiring English. Contextualised narratives are considered to yield valuable qualitative data, whose analysis can lead to a better understanding of the language learning process (e.g., Benson \& Nunan 2004; Oxford \& Cuéllar 2014; Oxford 2014; Siek-Piskozub \& Jankowska 2018).

\section{The study on EFL learners' agency}

\subsection{The aims of the study}

The decision to study learner agency in the context of English as a foreign language (EFL) learning emerged as a result of a prior study of learners' way of acquiring EFL in Poland, as revealed in their essays entitled My way to English. The essays had already been analysed with regard to the role of the teacher in the students' learning experience as a person who had an important impact on the learners' process of developing EFL competence, both in the negative and positive sense (Siek-Piskozub \& Jankowska 2018). During the previous analysis, however, it was also observed that the process of EFL learning should be treated as a 
dynamic system where different factors, not all of them related to the institutional setting, interplay with each other and influence each other. Therefore, a decision was made to try to get a deeper insight into the EFL learning process with a special focus on learner agency. The present analysis thus aimed to explore learner agency evident in EFL learners' autobiographies. More specifically, the study looked at the three elements of agency in accordance with the chordal triad model by Emirbayer and Mische (1998). Consequently, the following research questions were formulated:

- How did the past events influence the participants' agency?

- What judgments and beliefs do they express about their learning?

- What decisions and hopes for the future emerge from the narratives?

\subsection{Participants}

Two learners' narratives were analyzed. Both participants were final-year students in the 3-year BA program at the Faculty of English at a university, with a minor in teaching English as a foreign language. Both were female, and their proficiency in English was at the B2/C1 level. For anonymity purposes, pseudonyms were used in the analysis of the data: Barbara and Zuzanna.

\subsection{Methodology}

The data for the analysis came from autobiographic narratives entitled My way to English. Altogether, nine narratives were collected, out of which two were selected for the present analysis. The material underwent content analysis with regard to the study aims and research questions. The data were thus coded according to the most representative and relevant themes, and subsequently categorized into three main sections consistent with Emirbayer and Mische's model (1998): (1) the iterational element, (2) the practical-evaluative element, and (3) the projective element. The essays were originally written in the students' native language, Polish, and summarised in English by the researchers. The direct quotations of the data are a direct translation from Polish into English with occasional additional information from the researchers placed in square brackets. 


\section{Results}

\subsection{Barbara}

The iterational element of agency

Barbara had her first contact with English when she was six - in the first grade of primary school. She writes that she was not good at language learning, just managed to pass from grade to grade. She recalls an event which initially seemed not to have any immediate impact on her decision concerning English as a foreign language learning; however, the fact that she still remembers it shows that somehow it was important. She writes:

I remember how one day an American preacher visiting our parish, who was an acquaintance of my grandfather and my mother, called in on us. I was about eight and could not understand a word [in English], however, I was impressed seeing my mother talking with our guest; and even my father who at that time understood very little in English somehow was communicating [with our guest]. When he left, my mother encouraged me to learn the language by saying that it was satisfying to be able to understand what one talks to us and be able to answer.

Still, in the following years she did not put forth much effort into learning any foreign languages, although in grade four, apart from English, she had to study German. She puts the blame on the constant change of teachers who had different teaching approaches, and would also change coursebooks.

It was in middle school that Barbara's attitude to English changed, despite the fact that in three years again she had three different English teachers. It was then that she became interested in English pop-music and this was her motivating force. American soap-operas became another source of English for her. She tried to adjust the overheard words to the Polish subtitles. She also participated in a summer vacation camp with American students and felt happy when they could understand her, despite her English being incorrect and vocabulary limited. All this helped her to develop her competence and she proudly reports scoring almost 100 per cent on her final middle school test. She writes that she could not wait for beginning learning English in secondary school. The secondary school she attended ensured one teacher for the three-year English course, which was a pleasant surprise to Barbara after her earlier experiences. She became a successful learner then, and the high score (over $90 \%$ ) on the Matura test ${ }^{1}$ was a factor encouraging her to start English Philology studies at university.

1 Matura is the final state test at the end of secondary school in Poland. Candidates may take a basic test and/or advanced test. 
The practical-evaluative element of agency

It is evident that Barbara makes evaluative comments about the quality of instruction and the teachers she had at school. She notes that she did not particularly appreciate her earliest contacts with English in the first grades of primary school, and she declares openly that she did not like any of her teachers at that time. The evaluation of her later experiences is much more positive. She liked the teacher and activities they had to engage in (she writes, "Learning English was fun for me"). However, the only activities she did not like were grammar exercises. She explains, "Having learnt English from songs and films I did not grasp many grammar rules, and this is my problem until today". In this way, she makes a judgment of her weakest area in her English competence, and links it with the previous learning practice.

She also offers an evaluation of the materials for learning in accordance with her values. At school, she liked to listen to popular songs and learn from them; however, being aware that such songs may transfer undesirable messages or use vulgar language, she got involved in searching for translations of the lyrics, and only if she approved of the message and language did she memorise the text and sing along with the band.

Barbara also shares with us her recent happiness of having been able to meet her long-awaited dream of flying to the United States for vacations. She recalls again what her mother had once said about the feeling of satisfaction when people understand you and you can participate in conversations with speakers from another culture. She felt the same, and was very happy when Americans complimented her on her level of English and on the appropriate accent. At the same time, her formal knowledge of the student of English philology cautioned her against being too confident with her English language competence. For example, she felt ashamed each time she had made a mistake or did not know the word. Although at the same time she thinks that it was an unnecessary and inhibiting feeling.

The projective element of agency

In Barbara's account, there is no explicit statement about her plan, decisions, or hopes for the future. On the basis of the evaluative comments about the learning environments and experiences so far, it can be inferred that seeking naturalistic communicative situations in which she can practice her oral interactions skills, and overcoming inhibitions resulting from imperfect accuracy are her future goals and projections. 


\subsection{Zuzanna}

The iterational element of agency

Zuzanna started learning English at the age of five, in the form of extra classes in nursery school. English was not an obligatory school subject in grades 1-3 when she was beginning primary school, but her parents enrolled her at private paid classes. She recalls making use of the Internet resources to support her self-study for various school subjects at home, as well as getting access to English-language popular music. She writes, "[because of] listening to songs and singing karaoke, I had the opportunity to learn English quite unconsciously". The early start and the extra practice made her the best learner at English in her class. This, however, and the low level of instruction in later grades of primary school accommodated to weaker students, appeared to be demotivating factors. She explains this in the following way:

While I was able to communicate at a basic level, most of my classmates still struggled to pronounce simple words, such as "but" and "well". I think this made me rest on my laurels. I still did very well without any effort, so I didn't see the need to join extra classes.

At secondary school level, she joined a class with extended curriculum in mathematics and was one of the best students in the school, achieving high in various subjects. Choosing her tertiary education major was a hard decision. She applied to architecture, civil engineering, and English philology. Having failed a drawing test required as an entrance procedure at the architecture department, she selected English studies, as she wanted to further develop her English skills and was interested in the culture of English-speaking countries.

Studying English, especially in the first year, required hard work and considerable perseverance from her, as her initial level of English competence was not too high. However, her efforts paid off and with time she became a good student.

\section{The practical-evaluative element of agency}

Zuzanna evaluates her earliest instruction in grades 1-3 as very effective, and admits that it gave her an advantage over her peers in grade 4, when obligatory English lessons started. In higher grades, however, she perceived the teachers and the didactic procedures they used as boring, which contributed to her abandoning the efforts to progress.

She clearly appreciates the opportunities to enhance her communicative competence created by the Internet, which started to be commonplace at people's homes at the time she was in primary school. As a learner, she benefitted from the 
online learning opportunities created by the Internet, which she evaluates as an important breakthrough in her L2 development.

When choosing her major, she had to stand up against the pressure from her family, who argued that studying civil engineering was a better option. Majoring in English was thus her autonomous decision. She evaluates her studying as a struggle to survive, because, as she admits, she had not worked hard enough on her English since the third grade of primary school, and her English was not at a sufficient level. She writes:

In the first year, I felt I did not belong here. It was a considerable change for me, because having always been a very good student who scored high with no effort, I suddenly encountered difficulties [...] Surviving the first year took a lot of effort and hard work, but now I know it was worth it.

She contends that, although her English competence is still not perfect, she has made a huge progress. She finds her studies really interesting and rewarding. Although at the beginning of her studies her grades were rather poor, in the third year she was one of the best students in her group.

\section{The projective element of agency}

Thinking about the future, Zuzanna highlights that her choice of the studies was a good one, with promising prospects for the future. She is aware of the fact that she still needs to keep working on her English competence, and is convinced that her future will be connected with English.

\section{Discussion}

It can be seen from both narratives that the participants displayed considerable levels of agency at various stages of their education. Agentic behaviour and mindsets can be traced within all dimensions of the chordal triad model.

Discussing the iterational element, which concerns their learning histories, it can be concluded that both participants were aware of the impact that their past experiences had on their attitudes and performance. Barbara, describing her struggle to learn English, recalls a very early experience with the language, the encounter with the American priest, which appeared to be an important experience on her way to English. Although it did not have an immediate impact on her engagement to learn the language, with time and more experience, she recognized the value of the lesson her mother once gave her: "that it is a very satisfying feeling to be able to interact with someone from a different culture. 
This lesson and the message it carried was a recurrent stimulus for her at later stages of her English experience."

Zuzanna referred to her early enthusiasm about English and her outstanding successes as a factor shaping her future engagement with English education. She openly judged her later involvement as unsatisfactory, and admits that it resulted from a compilation of contextual and inner factors. Much later, being a university student, she had to commit considerable effort to compensating for the earlier neglect in learning.

At the evaluative level, both participants were fully aware of the importance of their own commitment to learn and practice English, despite the fact that at times both of them were not very happy with what the educational institutions were offering. Both recalled teachers, materials, and type of instruction that were not stimulating enough, or even simply demotivating. At the same time, the participants admitted that their occasional or more permanent problems with learning (e.g., accuracy) resulted mainly from the ways they used to learn the language. Both mentioned self-study practices (e.g., learning through songs, using online resources, viewing films with subtitles, interacting with native speakers) that had helped them develop their L2 competence. Moreover, both were able to evaluate their own competence and involvement and tried to seek an explanation for them. It is also interesting to note how both participants' agentic behaviour and attitudes were influenced by their social relationships. Naturally, their teachers played major roles in their L2 learning. Apart from them, both students mentioned their families (Barbara's mother gave her the important lesson and $\mathrm{Zu}$ zanna resisted her family's pressure to convince her into studying engineering), and their peers (both compared themselves with their peer groups). This confirms that agency is socially-embedded, as indicated by the literature (e.g., Priestley et al. 2013; Vitanova et al. 2018).

The history of learning and the evaluation of one's capacities lead to certain prospects for the future. It was evident from the narratives that both participants were deeply involved in their learning and their future hopes were connected with the English language. Zuzanna stated it explicitly; it can also be assumed from Barbara's account of her experience that her decisions were similar.

\section{Conclusion}

The analysis of Barbara's and Zuzanna's autobiographic narratives shows an interplay of elements of the past, the present situation (including its evaluation), and prospects for the future. Past events are stored in memory and may be activated when needed to justify present decisions, while the observation of the present engagement leads to the anticipation of the future decisions to achieve a 
desirable outcome. Barbara used all possible resources available in her immediate environment (Internet, TV, an international language camp) and further in the target language natural environment (a visit to the USA), and demonstrated a willingness to communicate with the native speakers, despite being aware of some limitations in her competence. Zuzanna's early success, followed by a decline in motivation and a plateau stage at which she did not progress, stimulated her to pursue studying at an English department and overcome the initial difficulty by working hard and not giving up.

Beside exploring the concept of learner agency in the narratives produced by two learners of English, the study also shows the applicability of the chordal triad model for studying learner agency, confirming Siek-Piskozub's (2019) earlier presumption. However, the very limited number of participants (only two) in this investigation constitutes a serious limitation of this study. More research is needed to verify whether the chordal triad model can give more insight into the process of developing foreign language competence, both in the educational institutions and the environment in which learners have a chance to function, if only temporarily.

However, even small-scale research can lead to certain didactic implications. The impact of the environment other than school is commonly neglected by teachers who, as observed by Larsen-Freeman (2018), often categorise their learners basing on how they perform exposed to customized input which may be below or above their needs and interests. For example, teachers in Poland do not fully appreciate the motivating function of English pop-songs, despite the fact that foreign learners generally reveal in their surveys their commitment to this kind of input, also as a source of learning culture (e.g., Derenowski 2015). It was also observed in the previous analysis of learners' autobiographies (Siek-Piskozub \& Jankowska 2018) that teachers seem to pay little attention to the language needs and interests of their students. Research on learner agency is an invaluable source of data on learners' lived experiences, hopes and desires, which need to be respected by their teachers. As asserted by Larsen-Freeman (2019:63), in guiding their students' efforts to learn effectively, teachers "must not overlook the learning paths that individuals take".

\section{References}

Benson, Phil/Nunan, David (eds.) (2004). Learner stories: Difference and diversity in language learning. Cambridge: Cambridge University Press.

Biesta, Gert/Tedder, Michael (2007). Agency and learning in the lifecourse: Towards an ecological perspective. Studies in the Education of Adults, 39 (2), 132-149. 
Creswell, John (2014). Research design. Qualitative, quantitative, and mixed methods approaches. $4^{\text {th }}$ edition. Los Angeles: SAGE Publications.

Derenowski, Marek (2015). Teaching culture in the FL senior high school classroom. Coursebook evaluation and teachers' and learners' views. Poznań: Wydawnictwo Naukowe Universytetu im. Adama Mickiewicza.

Emirbayer, Mustafa/Mische, Ann (1998). What is agency?, American Journal of Sociology, 103 (4), 962-1023.

Gao, Xuesong (Andy) (2013). Reflexive and reflective thinking: A crucial link between agency and autonomy. Innovation in Language Learning and Teaching, 7 (3), 226-237.

Larsen-Freeman, Diane (2018). The times we live in and the agency of language learners, Plenary lecture at the LIF Annual Conference (Language in Focus 2018) held in Thessaloniki, Greece, May 3-5 2018.

Larsen-Freeman, Diane (2019). On language learner agency: A complex dynamic systems theory perspective. The Modern Language Journal, 103 (Supplement 2019), 61-79.

Mercer, Sarah (2012). The complexity of learner agency. Apples - Journal of Applied Language Studies, 6 (2), 41-59.

Muramatsu, Chie (2018). Portraits of second language learners: An L2 learner agency perspective. Bristol: Multilingual Matters.

Oxford, Rebecca (2014). What we can learn about strategies, language learning, and life from two extreme cases: The role of well-being theory. Studies in Second Language Learning and Teaching, 4 (4), 593-615.

Oxford, Rebecca/Cuéllar, Lourdes (2014). Positive psychology in cross-cultural narratives: Mexican students discover themselves while learning Chinese. Studies in Second Language Learning and Teaching, 4 (2), 173-203.

Priestley, Mark/Biesta, Gert/Robinson, Sara (2013). Teachers as agents of change: Teacher agency and emerging models of curriculum. In: Mark Priestley/Gert Biesta (eds.), Reinventing the curriculum: New trends in curriculum policy and practice (pp. 187-206). London: Bloomsbury Publishing.

Siek-Piskozub, Teresa/Jankowska, Aleksandra (2018). Learners' personal narratives on their way to EFL competence: The impact of teachers on learners' development. Glottodidactica, 45 (2), 243-268.

Siek-Piskozub, Teresa (2019). Learner agency in students' EFL learning narratives. Konińskie Studia Językowe, 7 (2), 159-170.

Van Lier, Leo (2010). The ecology of language learning: Practice to theory, theory to practice. Procedia Social and Behavioral Sciences, 3, 2-6.

Van Manen, Max (2016). Phenomenology of practice. Meaning-making methods in phenomenological research and writing. Abingdon: Routledge.

Vitanova, Gergana/Miller, Elizabeth/Gao, Xuesong/Deters, Ping (2018). Introduction. In: Ping Deters/Xuesong (Andy) Gao/Elizabeth Miller/Gergana Vitanova (eds.), Theorizing and analyzing agency in second language learning: Interdisciplinary approaches (pp. 1-13). Bristol: Multilingual Matters. 
Open-Access-Publikation im Sinne der CC-Lizenz BY-NC-ND 4.0

(C) 2020, Vandenhoeck \& Ruprecht GmbH \& Co. KG, Göttingen ISBN Print: 9783847111863 - ISBN E-Lib: 9783737011860 


\author{
Katarzyna Bieniecka-Drzymała \\ (Uniwersytet im. Adama Mickiewicza w Poznaniu)
}

\title{
Fragen, die mehr können? Zum Potential von Coaching-Gesprächen für die Entwicklung der Sprachlernbewusstheit und Lernerautonomie bei Fremdsprachenstudierenden
}

\begin{abstract}
Potential of coaching dialogues in supporting and developing self-reflection and autonomy with philology students

The author discusses the metacognitive awareness and self-reflection in foreign language studying, especially their role in developing learner self-awareness and autonomy. She perceives the teacher as a key agent in such a development, whose role is transforming from a person providing knowledge into a facilitator of the language learning process, encouraging students to start self-reflection, leading them throughout the process and thus empowering them for taking responsibility for their own learning process both in the classroom and outside. Although there are many ways of developing self-reflection for the purpose of improving foreign language acquisition, an individual dialogue with a student preceding or accompanying the learning process, where deep reasoning questions can be asked and issues important from the student's perspective discussed, seems not to enjoy much interest in the methodological literature. To fulfill these needs, the author presents the potential of coaching and coaching questions for supporting foreign language teaching in a broader context including both the activation of learner's self-reflection and providing teachers with a useful tool for their new, challenging role as coaches/facilitators.
\end{abstract}

Keywords

metacognitive awareness, learner autonomy, coaching in FLT

\section{Einführung}

Die Fähigkeit, sich des eigenen Lernprozesses bewusst zu werden und dabei die individuellen Voraussetzungen, Motive und Bedürfnisse zu erkennen, gehört zu einer autonomen Haltung dem Fremdsprachenlernprozess gegenüber und hilft den Lernenden konkrete, realisierbare Lernziele eigenständig zu identifizieren und zu verfolgen. Die Ausbildung solcher Haltung gewinnt an Bedeutung, besonders im Kontext von allgemein zugänglicher Bildung, größeren Klassengrößen sowie on-line und digitalen Technologien, die heutzutage immer öfter faceto-face Unterricht ersetzen (müssen). Auch Lehrkonzepte, wie flipped classroom 
oder blended learning haben zur Folge, dass autonomes Lernen in der Fremdsprachenausbildung, besonders im universitären Kontext, integriert werden muss (vgl. u. a. Moore \& Kearsley 2012).

Bei der Suche nach Methoden von Entwicklung und Förderung der Lernerautonomie und unter Berücksichtigung der evolvierenden Lehrerrolle, wird in dem vorliegenden Beitrag auf Sprachlern-Coaching als besondere Art allgemein konzipierter Sprachlernberatung hingewiesen. Das Konzept der Sprachlernberatung zielt darauf $a b$, Lernende in ihrem Fremdsprachenerwerb in unterschiedlichen Lernumgebungen (z. B. Selbstlernzentren, im Tandem beim Distanzlernen, aber auch als Kursbegleitung) zu unterstützen und ihnen zu helfen, für sich effiziente Lernwege zu finden und Verantwortung für den eigenen Lernprozess zu übernehmen. Dieses an deutschen Hochschulen fest etablierte ${ }^{1}$ Unterstützungskonzept ist in der polnischen Sprachlernwirklichkeit wenig verbreitet und es wäre geboten, es auch bei polnischen Fremdsprachenstudierenden zu erproben (vgl. Nerlicki 2011: 331). Nach einem Überblick über das Konzept von Lernerautonomie als theoretischer Grundlage der Sprachlernberatung, wird in dem vorliegenden Beitrag auf die Sprachlernbewusstheit und Möglichkeiten ihrer Entwicklung sowie auf Förderung der individuellen Lernerreflexion eingegangen. Das Sprachlern-Coaching als personalisierter, strukturierter Dialog über Fremdsprachenlernen zentrierte Themen scheint einen wesentlichen Beitrag zur Entwicklung der Sprachlernbewusstheit und der Lernerautonomie bei Fremdsprachenlernendenden leisten zu können. In dem vorliegenden Artikel wird das Konzept samt seiner Hauptprinzipien und Anwendungsbereiche kurz dargestellt sowie das Potential seiner spezifischen Gesprächsführungstechniken und Fragestellungsmethoden skizziert.

\section{Lernerautonomie und Sprachlernbewusstheit}

Die Lernerautonomie ist im Sinne von dem im didaktischen Kontext häufig zitierten Henri Holec (1985) als eine didaktische Situation definiert, in der der Lernende die Steuerung und Verantwortung für das eigene Lernen übernimmt, Lernziele selbständig definiert, Inhalte und Progression bestimmt, Methoden und Techniken auswählt und den gesamten Lernprozess überwacht und evaluiert. Das Konzept der Lernerautonomie hat seit den 90-er Jahren beim Fremd-

1 In Deutschland hat sich die Community der Sprachlernberater seit ihren Anfängen enorm entwickelt und mit Konzepten, empirischen Untersuchungen (siehe u.a. Berndt \& Deutschmann 2014, Claußen 2009, Kleppin 2003, 2006, Saunders 2014), vor allem aber mit best-practice Beispielen auseinandergesetzt (vgl. auch Themenheft Zeitschrift für Interkulturellen Fremdsprachenunterricht 2006). Es wird mittlerweile in vielen Lernkontexten angeboten und ist an den Institutionen in verschiedenen Settings organisiert. 
sprachenlernen an Bedeutung gewonnen und wird kontinuierlich in Curricula und Lehr- und Lernmaterialien als das zentrale Ziel des Lehr- und Lernprozesses integriert. Die unterschiedlichen Aspekte des Konzepts und seine unterschiedlichen Ausprägungen hinsichtlich des Fremdsprachenlernens, bereiten jedoch zahlreiche definitorische Probleme, die in der Diskussion berücksichtigt werden müssen (Koch \& Saunders 2014, Schmenk 2008, Vogler \& Hoffmann 2011). Nerlicki (2011:321) definiert das Wesen der Autonomie als Fähigkeit eines Menschen, sich als Individuum unterschiedliche Ziele zu setzen, diese reflexiv zu verfolgen und zu verantworten. Eine der notwendigen Bedingungen für die Erreichung der Lernautonomie ist das Bewusstwerden der anzustrebenden Ziele, das sich u. a. aus dem Reflektieren über den eigenen Fremdsprachenlernprozess ergibt. Gleichzeitig weist der Autor auf den prozessualen Charakter der Autonomieentwicklung (ebda: 331) und auf die Selbstwahrnehmungsbereitschaft hin, die dem Prozess zugrunde liegen sollte (ebda: 323). Schmenk (2008: 13) plädiert für einen bildungswissenschaftlich fundierten Autonomiebegriff, der nicht einseitig auf die Individualisierung und Selbstverantwortlichkeit des Sprachlernens anhebt, sondern auch die Verwobenheit der Selbst- und Fremdbestimmung beim Definieren der Autonomie berücksichtigt. Der Übergang von Fremdzur Selbstbestimmung definiert Schmenk (2008: 13) als Autonomisierung. Das Prozess der Autonomisierung von Fremdsprachenlernenden, die zur Lernerautonomie führen sollte, wurde 1999 von Wilczyńska beschrieben. Unter Lernerautonomie versteht die Forscherin eine gewisse Haltung, die im Laufe des Sprachlernprozesses entwickelt und gefördert werden kann und in dem Lernenden wird ein Individuum gesehen, das seine eigene (Sprach)Entwicklung aktiv und absichtlich steuern kann. Das Konzept wurde in einem umfangreichen Projekt zur Autonomisierung der Studierenden am Lehrstuhl für Glottodidaktik und Translatorik entwickelt und mit zahlreichen Untersuchungen untermauert (mehr zu diesem Projekt in Wilczyńska 2002).

Wie aus den oben angeführten Ausführungen hervorgeht, ist für die Entwicklung der autonomen Haltung neben der schrittweisen Übernahme von Verantwortung für den eigenen Lernprozess auch die Förderung der Selbstwahrnehmung und Reflexivität bei den Lernenden von Bedeutung. Hierzu gehören nach Nerlicki (2011: 320) nicht nur die Kenntnisse über bevorzugte Lernund Kommunikationsstile, sondern auch die Auffassungen der Studierenden bezüglich deren Selbstkonzepte, Selbstwertgefühle und Selbstwirksamkeit. Eine Auseinandersetzung mit eigenen sowie fremden Auffassungen evoziert Selbstund Fremdwahrnehmung und in der Konsequenz Sprach-, Lern- und Kommunikationsbewusstheit. Hierzu bemerkt Harting (2011: 8-11), dass Selbststeuerung, die dem lebenslangen, eigenverantwortlichen Lernen zugrunde liegt, ohne eine realistische Selbstwahrnehmung nicht möglich ist. Myczko (2015) diskutiert die Rolle des bewussten Nachdenkens bei der Förderung der Sprachbewusstheit 
und plädiert im Hinblick auf das Fremdsprachenlernen für reflexive, erfahrungsbasierte Auseinandersetzung mit der eigenen Fremdsprachenaneignung, die als „Wahrnehmung eigener kognitiver, affektiver und sozialer Voraussetzungen und die Fähigkeit zum Definieren eigener Lernziele, Überwachung des Lernprozesses und Durchführung der Selbstevaluation definiert werden kann" (Myczko 2015: 48). Die Fähigkeit, eigene Lernprozesse bewusst wahrzunehmen, wird als metakognitive Bewusstheit (metacognitive awareness) definiert. Nerlicki (2011) betont die Schlüsselrolle der Sprachlernbewusstheit bei fremdsprachlichen Lern- und Kommunikationsprozessen ${ }^{2}$ und konstatiert, dass:

menschliche kognitive Prozesse auf der Metaebene durch das Individuum selbstreflexiv eingeschätzt, gesteuert und reguliert werden können und die Entwicklung und Förderung metakognitiver Bewusstheit bei Fremdsprachenstudierenden zu den primären Zielen ihres autonomen Fremdsprachenlernens werden sollte (Nerlicki 2011: 51).

Basiert auf einer eigenen Untersuchung zu Lernererfahrungen und -auffassungen über Fremdsprachenlernen und -Kommunizieren, die unter Germanistikstudierenden durchgeführt wurde, weist der Forscher auf die Entwicklungs- und Förderungsmöglichkeiten von individuellen Reflexionsprozessen bei Fremdsprachenlernenden hin (Nerlicki 2011: 149).

\section{Entwicklung der Sprachlernbewusstheit bei Fremdsprachenlernenden}

In Anlehnung an die Überlegungen, dass die metakognitive Bewusstheit als grundlegend für selbstreflexives Fremdsprachenlernen und -kommunizieren und somit auch für die Entwicklung von Lernerautonomie betrachtet werden kann, sollen in dem folgenden Artikel die Möglichkeiten ihrer Förderung und Entwicklung bei Fremdsprachenlernenden im universitären Kontext zur Diskussion gebracht werden. Die Berücksichtigung der Metakognition im Fremdsprachenlernprozess entspricht neueren Tendenzen, die die Fachdiskussion über die unterrichtliche Vermittlung von Sprache und den Spracherwerb außerhalb des Klassenzimmers seit den 90-er Jahren kennzeichnen (vgl. Saunders 2014). Es entspricht auch den Grundsätzen des Gemeinsamen Europäischen Referenzrahmens für Sprachen (GER) $)^{3}$. Die in Kapitel 5 und 6 dieses Dokuments beschriebenen allgemeinen Kompetenzen umfassen theoretisches Wissen, Fertig-

2 Der Terminus Metakognition soll nach Nerlicki (2011:52-53) sowohl auf das Lernen sowie das Kommunizieren in der Fremdsprache bezogen werden, wobei die beiden nicht als zwei getrennte, sondern ineinandergreifende Prozesse mit gegenseitiger Wechselwirkung zu betrachten sind.

3 Deutsche Version online unter http://www.goethe.de/Z/50/commeuro/i2.htm. 
keiten und prozedurales Wissen sowie persönlichkeitsbezogene Kompetenz (savoir-etre) und die Lernfähigkeit (savoir-apprendre), über die Sprachverwendende/Lernende verfügen müssen, um kommunikative Aktivitäten erfolgreich auszuführen. Laut den Autoren wird die kommunikative Tätigkeit der Sprachverwendenden/Lernenden nicht nur durch ihr Wissen, ihr Verständnis und ihre Fertigkeiten beeinflusst, sondern auch durch individuelle ihre jeweilige Persönlichkeit charakterisierenden Faktoren wie Einstellungen, Motivationen, Werte, Überzeugungen, kognitive Stile und Persönlichkeitstypen, die zu ihrer Identität beitragen. Die persönlichkeitsbezogene allgemeine Kompetenz (savoiretre) bedeutet nach Smuk (2016) nicht nur Kenntnis der oben genannten, individuellen Faktoren, sondern auch die Fähigkeit, diese zum optimalen Fremdsprachenlernen und Kommunizieren entsprechend einzusetzen. Der Autor zeigt anhand einer eigenen Untersuchung, dass es möglich ist, savoir-etre im Bereich des Fremdsprachenlernens als Kompetenz zu betrachten und diese zu entwickeln. Die Selbstlernkompetenz, verstanden als die Fähigkeit eigene Entwicklung einzuschätzen und den Lernweg selbst aktiv zu gestalten, gilt ebenfalls als eine der im Rahmen der Bologna-Reform geforderten Schlüsselkompetenzen (EHEA 2012). Janocha (2016: 76-77) bemerkt, dass viele deutsche Universitäten verbindliche Standards zur Förderung der Metakognition festlegen, um das Bewusstsein der Studenten für die eigenen Fähigkeiten und Stärken zu entwickeln aber auch für Schwächen und Lücken beim Lernen zu sensibilisieren.

Die praktische Umsetzung dieser Anforderungen und die stärkere Berücksichtigung des Selbstwahrnehmungsaspekts in den Ausbildungskonzepten der Fremdsprachendidaktik findet ihren Ausdruck in vielerlei Methoden zur Entwicklung der Selbstreflexivität der Lernenden. Ein wichtiges Instrument zur Förderung der Reflexionsprozesse, das begleitend zum eigentlichen Fremdsprachenunterricht eingesetzt werden kann, bildet das Europäische Sprachenportfolio. Zur Rolle der Portfolioarbeit im Fremdsprachenunterricht vgl. u.a. Ballweg (2015), die empirische Studie zu Schreibportfolios im DaF-Unterricht durchgeführt hat. Die Entwicklung der Selbstreflexivität der Lernenden kann auch in der Form von Gesprächen oder (dialogischen) Lerntagebüchern (vgl. Stork 2017) realisiert werden ${ }^{4}$. Der Lernende wird dabei als ein Individuum betrachtet, das reflexiv handelt und seine Gegebenheiten im Fremdsprachenlernprozess selbst verbalisiert. Diese Auffassung entspricht den Prinzipien des sog. metakognitiv-kontextuellen Ansatzes (vgl. u.a. Wenden 1998). Der metakognitiv-kontextuelle Ansatz setzt voraus, dass die Lerner ihre Erfahrungen,

\footnotetext{
4 Das Werk von Stork (ges. 2016), wurde durch den Fachbereich Erziehungswissenschaften der Philipps-Universität 02.06.2010 angenommen und war die erste Habilitationsschrift in Deutschland zu dialogischen Lerntagebüchern. In der polnischen Forschungslandschaft hat sich Nerlicki intensiv mit dem Thema befasst. (vgl. Nerlicki 2008a, 2008b)
} 
Auffassungen, Probleme und Erfolge im Fremdsprachenunterricht selbst reflexiv erfassen und über sie reden/sprechen können ${ }^{5}$. Das Ziel eines dialogischen Tagebuchs oder eines Gesprächs ist demnach, die Konstruktion eines Gefüges zwischen Lernern und Lehrern/Forschern in dem über das Fremdsprachenlernen reflektiert wird ${ }^{6}$. Der Lernende wird somit als Mitforscher in den Erkenntnisprozess einbezogen, weil er seine Voraussetzungen im Fremdsprachenlernen nicht nur verbalisiert, sondern auch diese zu erklären und zu begründen versucht (vgl. Nerlicki 2014: 143). Die Funktion eines reflexionsfördernden Dialogs mit dem Lernenden wird durch entsprechende Fragen realisiert. Auf differenzierte Funktionen von Fragen im didaktischen Prozess weist auch Iluk (2018) hin, sie sollen u.a. zum Nachdenken und zur Reflexion inspirieren.

Die Notwendigkeit, verschiedene Formen der Selbstreflexionsaktivierung zur Diskussion zu stellen, hängt mit der evolvierenden Rolle des Lehrers zusammen. Neben Bereitstellung von Informationen, die heutzutage zunehmend unabhängig von der Lehrerperson bezogen werden können, bietet er immer öfter auch unterschiedliche Formen der Unterstützung. Auf den Unterstützungsaspekt der Lehrer-Lerner Beziehung wies bereits Prokop (2001) in der von ihr präsentierten alternativen Methode der Suggestopädie hin, nach welcher der Lehrer ein kluger Berater, Koordinator, der sog. Mentor für die Lerner sein soll. Von Kleppin und Spänkuch (2014: 36) wird die Unterstützung als Hilfe bei der Entwicklung von Sprachlernkompetenz verstanden, die als Aktivierung der Reflexion über eigene Lernprozesse oder die Anleitung zu bestimmten Verhaltensweisen realisiert werden kann. Der Lehrer gilt nun als Facilitator, Moderator, Mediator sowie als Berater/Coach, der den Lernenden „strukturierte Hilfe zum Nachdenken“ (Spänkuch 2014: 98) anbietet. Im Kontext der Entwicklung von interkultureller Kompetenz kann der Lehrende die Rolle des interkulturellen Mediators/Coach oder des kulturellen Mittlers übernehmen (Nazarkiewicz \& Krämer 2012, Wolting 2016).

\section{Sprachlern-Coaching als Vorschlag zur Aktivierung der Selbstreflexivität bei Fremdsprachenlernenden}

Sprachlern-Coaching ${ }^{7}$ ist eine Unterstützungsform, deren Menschenbild und Konzept der Gesprächsführung an den nichtdirektiven, personenzentrierten Beratungsansatz nach Rogers (2002) anknüpft. Seine theoretische Grundlage

5 Im Gegensatz dazu steht der normative Ansatz, der den traditionellen Fragebogen, wie z. B. der BALLI Fragebogen (Horowitz 1988) einsetzt.

6 Zum Potential des Dialogs in Untersuchungsbeziehung siehe. u.a. Wiśniewska (2009: 283298).

7 Im Allgemeinen ist Coaching als personenbezogene Beratungsform mit primärem Fokus auf beruflichen Anliegen zu einer festen Institution in der Personalentwicklung und auf dem 
bildet u. a. das Konzept der Lernerautonomie verstanden als Fähigkeit, Verantwortung für das eigene Lernen $\mathrm{zu}$ übernehmen. In diesem Hinblick sehen Kleppin und Spänkuch (2014: 34) die Rolle der Sprachlernberater darin, den Lernenden dabei zu unterstützen, sich den eigenen Lernprozess bewusst $\mathrm{zu}$ machen, seine individuellen Voraussetzungen, Motive und Bedürfnisse zu erkennen sowie konkrete realisierbare Ziele zu identifizieren, bevor er in einem späteren Schritt den Zugriff auf nutzbare Ressourcen, beispielsweise auf solche „Werkzeuge“ wie Lernstrategien, organisiert.

Die konstruktivistischen Rahmenbedingungen für Coaching beinhalten Freiheit (der Lernende kann selbst entscheiden oder wählen, was mit dem Lernthema zusammenhängt) Freiwilligkeit (der Lernende meldet sich freiwillig), Ressourcenverfügung (der Lernende verfügt über Zugriff auf Ressourcen Motive, Werte, Erfahrungen, Kompetenzen, Fähigkeiten und Fertigkeiten) und Selbststeuerung (der Lernende ist in der Lage, die Veränderungsanforderungen selbst zu erkennen und ist fähig, sich selbst so zu steuern, dass er seine Veränderung realisieren kann, vgl. Meier \& Janßen 2011)

\subsection{Systemisch-konstruktivisches Sprachlern-Coaching}

Innerhalb der verschiedenen Sprachlern-Support-Konzepte ${ }^{8}$ etablierte sich das Konzept des systemisch-konstruktivistischen Sprachlern-Coachings. Die Bezeichnung systemisch bedeutet im Sprachlern-Coaching, dass das Denken in Zusammenhängen erfolgt. Ein Problem wird als Geschehen gesehen und es wird nach einem kontextuellen Verständnis des Problems gesucht. Es wird als eine nicht-direktive, lösungs- und ressourcenorientierte Lernbegleitung verstanden, die zum Ziel hat, die Lernenden dabei zu unterstützen, Sprachlernkompetenz (weiter) zu entwickeln, einen höheren Grad der Selbststeuerung ihres Lernens herauszubilden und somit eine größere Nachhaltigkeit anzustreben. Im Folgenden werden diese drei Charakteristika nach dem Verständnis von Spänkuch (2014: 58) erklärt.

Nicht-direktive Lernbegleitung bedeutet, dass die Lösung von dem Lernenden selbst entwickelt wird, der als „Experte für die eigene Person“ gilt und dadurch die Verantwortung für seinen Sprachlernprozess übernehmen kann. Dem Lernenden wird in diesem Verfahren von einem Sprachlern-Coach als „Experten für das eigene Fremdsprachenlernen“ (Kleppin \& Spänkuch 2014: 105) strukturierte

Weiterbildungsmarkt geworden (vgl. Graf 2008: 1). Zur Unterscheidung des SprachlernCoachings von anderen (meist direktiverer) Beratungsformen siehe Kleppin und Spänkuch (2014).

8 Detaillierte Unterscheidung zwischen den verschiedenen Verfahren ist bei Kleppin und Spänkuch (2014: 36-37) zu finden. 
Hilfe zum Nachdenken über ein Lernprojekt angeboten, vor allem durch deduktive Hypothesenbildung und Reflexionsangebote (ausschließlich) auf der Grundlage seiner Daten und Wünsche. Nur der Lernende entscheidet darüber, was zur Sprache kommt, auf welche Aspekte seines Themas er konkret fokussieren und welche Handlungsalternativen er für die Zielerreichung organisieren möchte.

Lösungs- und ressourcenorientierte Vorgehensweise setzt voraus, dass vordringlich nach Ressourcen gefragt wird und danach, was funktioniert oder in der Vergangenheit funktionierte. Systemisch-konstruktivische Vorgehensweise setzt voraus, dass man sich grundsätzlich nicht mit Problemursachen beschäftigt, sondern nach Kompetenzen fragt, auf denen Lernprozesse aufbauen können.

Ein anderer sehr wichtiger Aspekt dieses Ansatzes ist seine Zukunftsorientierung. Das gesamte Gespräch basiert auf der Vorstellung des gewünschten Ergebnisses. Das Konzept der Zukunftsorientierung wurde auch von Dörney und Kubanyiova (2014) bei dem Vision-basierten Motivationstraining für Fremdsprachenlernen eingesetzt und ist nach den Forschern meist Erfolg versprechender als der Abbau vermeintlicher Defizite.

\subsection{Zwischen Fragen und Zuhören}

Nach einem dialogischen Verständnis ist Coaching ein Gespräch oder eine Interaktion, die von TeilnehmerInnen gemeinsam hergestellt und konstituiert wird. Graf und Spranz-Fogasy (2018) situieren Coaching im Rahmen professioneller Gespräche als eine helfende Interaktion, wobei die Hilfe in und durch das Gespräch der Beteiligten realisiert wird. Zu instrumentellen Zwecken dieses Interaktionstyps gehören neben der erwähnten Hilfe-zur-Selbsthilfe auch Beziehungsgestaltung. Das Gespräch dient jedoch einem übergeordneten Ziel, nämlich der gewünschten Veränderung oder dem angestrebten Erkenntnis- und Wissensgewinn auf Seiten des Coachees ${ }^{9}$. Charakteristisch für Coaching ist ein fester Ablauf und ein bestimmtes Technik-Repertoire. Dazu gehören hauptsächlich aktives Zuhören und systemische Fragetechniken (zirkuläre, hypothetische, skalierende Fragen), die in festgelegten Phasen eingesetzt werden.

- aktives Zuhören

Die Rolle des aktiven Zuhörens ist es, einen wohlwollenden und angstfreien Rahmen zu schaffen, in dem sich die Gesprächspartner öffnen und ihre subjektiven Sichtweisen darstellen können. Das Sprechen über das eigene Lernen

9 Als Coachee oder Klient wird die an dem Coachingprozess teilnehmende Person definiert. 
trägt dazu bei, dass dem Lerner selbst vieles klarer wird. Der Coach signalisiert durch aktives Zuhören, dass seine gesamte Aufmerksamkeit dem Sprechenden gewidmet ist; er zeigt Interesse an dem Gesagten und dass er die Äußerungen des Lerners versteht - sowohl verbal als auch durch körpersprachliche Signale. Aktives Zuhören ist im Coaching nicht ausschließlich auf nonverbale Signalisierung der Aufmerksamkeit reduziert. Indem der Coach dem Klienten folgt, erfragt er auch präzise die Bedeutung, die Zusammenhänge und Bedeutsamkeit der Wörter. Auf der inhaltlichen Ebene versucht der Coach die Kernaussagen des Lerners zu erfassen. Aktives Zuhören heißt nicht nur gut zuhören zu können, sondern auch im entscheidenden Moment die richtigen Fragen zu stellen.

\section{- Coachingphasen und -fragen}

Fragen sind die zweite zentrale Intervention des Coachings. Fragen helfen $\mathrm{Zu}$ sammenhänge aufzuzeigen, die Wahrnehmungs- und Beschreibungsfähigkeit des Betroffenen zu erweitern und sie ermöglichen neue Blickwinkel - wie die Wirklichkeit anders gesehen werden könnte (vgl. Spänkuch 2014: 64). Interessant ist, dass Coachingfragen nicht immer Antworten erwarten, sie initiieren Denkprozesse für alternative Lösungen und verschieben die Verantwortung in Richtung dessen, der antwortet. Eine genaue Klassifikation von Coachingfragen bieten Wehrle (2012) sowie Graf und Spranz-Fogasy (2018).

Wie oben erwähnt ist Coaching ein strukturiertes Gespräch mit einem festen Verlauf, das aus spezifischen, zielgerichteten Phasen besteht, die im Folgenden in Anlehnung an Spänkuch (2014) samt ihrer kommunikativen Ziele und Fragebeispiele präsentiert werden.

- Phase 1: Kontakt/Kontrakt. Sie dient hauptsächlich dem Kontaktaufbau und Überprüfung der Motivation, die am häufigsten gestellten Fragen hier sind: Was bringt dir Coaching? Welche Ergebnisse erwartest du?

- Phase 2: Themenerklärung und Zielformulierung. Ihre Rolle beruht auf Auslösung der Wahrnehmungserweiterung und Ausbildung der Fähigkeit, aus den Problembereichen positive, konkrete und selbstrealisierbare Lernziele zu formulieren. $\mathrm{Zu}$ Leitfragen dieser Phase gehören: Welche Bereiche möchtest du bearbeiten? Was willst du am Ende deines Lernprojekts erreicht haben? Woran erkennst du, dass du das Ziel erreicht hast? Warum ist das wichtig?

- Phase 3: Ressourcen-Identifikation. Sie dient der Ausbildung des Ressourcenbewusstseins und der Bewertungsfähigkeit hinsichtlich der Nutzbarkeit von Ressourcen. Es wird hier gefragt: Was hast du schon, um das Ziel zu erreichen? Was und wer steht dir zur Verfügung? Welche Ressourcen müssen neu erworben werden? Welche Auffassungen unterstützen dein Lernvorhaben? Welche nicht? 
- Phase 4: Entwicklung und Auswahl von Handlungsmöglichkeiten (Lernwegen). Sie dient der Wahrnehmung von Handlungsalternativen und dem Aufbau der Fähigkeit, realistische Handlungspläne zu schaffen. Die Leitfragen sind hier: Wie gehst du vor? Wie willst du das erreichen? Was könnte der erste Schritt sein? Was könnte dazwischen kommen?

- Phase 5: Metareflexion. Diese Phase dient direkt der Entwicklung des Selbstbewusstseins und der Selbstwahrnehmung im Reflexionsprozess sowie der Sicherung der Entscheidungsfähigkeit. Hauptfragen sind hier: Was hast du gerade über dich selbst erfahren? Was wurde dir heute hinsichtlich dieses Themas klar?

- Phase 6: Erprobung und Evaluation. Die Erprobung findet außerhalb der eigentlichen Coaching-Session statt. Der Lernende setzt seinen Handlungsplan um und prüft selbst, ob die Handlungen so wie geplant ausgeführt werden und ob sie die erwartete Wirkung haben. Die Leitfragen der Evaluationsphase sind: Was funktioniert (nicht)? Welche Anpassungen müssen vorgenommen werden? Ist dein Ziel immer noch wichtig? Müssen die Ressourcen aktualisiert bzw. angepasst werden?

\section{Erforschung der Sprachlernberatung und des Sprachlern-Coachings}

In der Fachliteratur werden einige Formen der Sprachlernberatung, wie Aussprachelernberatung, Schreibberatung, Paar-Tandemberatung oder Studierstrategien-Beratung beschrieben und teilweise empirisch erforscht (Ballweg 2011 und 2015, Claußen \& Peuschel 2006; Hoffmann 2011, Mehlhorn \& Kleppin 2006). Individuelle Sprachlernberatung und -Coaching stellen ein interessantes Feld der Sprachlernforschung dar, insbesondere in Bezug auf die Untersuchung individuellen Faktoren wie Sprachlernmotive, Attribution von Lernerfolgen, subjektive Lerntheorien, aber auch die Entwicklung von Sprachbewusstheit und Lernbewusstheit (vgl. Mehlhorn \& Kleppin 2006: 7). Einen wichtigen Forschungsmethodischen Rahmen bildet dabei die Aktionsforschung, die Lehrer/ Sprachlernberatende selbst durchführen. Von Vorteil sind hier gute Zugangsmöglichkeiten zum Untersuchungsfeld als Berater und Forscher in einer Person, die die Einbeziehung des Lerners als Untersuchungspartner und die Ergänzung der Beraterperspektive durch die des Lerners (vgl. Schmelter 2004) erlauben. Die Forschung im deutschen Sprachraum erbrachte empirische Arbeiten (u.a. Ballweg 2015, Claußen \& Peuschel 2006, Claußen 2009, Hoffmann 2014, Kleppin 2008, Sedlaczek 2008,), die auf den Mehrwert und zum Teil auch auf die empirisch geprüfte Wirksamkeit des Konzepts verweisen. In Rahmen dieser Forschungs- 
ansätze wurden Fragen nach dem Auftreten und der Anregung von Reflexionsprozessen, sprachlichen, interkulturellen und strategischen Veränderungsund Lernprozessen und ihren Ergebnissen bearbeitet (vgl. Böcker 2014). Nach der von Hoffmann (2014) durchgeführten empirischen Untersuchung zum Potential der Sprachlernberatung beim Aufbau fremdsprachlicher Kompetenz liegt der Einflussbereich der Fremdsprachenlernberatung hauptsächlich auf der metakognitiven Ebene. Sedlaczek (2008, nachfolgend Koch/Saunders 2004: 143) stellt fest, dass Lerner in der Untersuchungszeit autonomer und selbstsicherer geworden sind.

\section{Fazit und Forschungsdesiderata}

Aktuelle Herausforderungen in der Fremdsprachenvermittlung, die sich aus dem Anspruch der Lernerorientierung und dem Ziel der Autonomieförderung ergeben, bedürfen neuer, die Lernenden unterstützender Handlungsweisen (vgl. u.a. Saunders 2014: 69). Das Potential von Sprachlern-Coaching scheint sich in dieser Hinsicht aus der Förderung der Metakognition und Aktivierung der Selbstreflexion bei den Studierenden zu ergeben, die die Fähigkeit entwickeln, über eigene Stärken und Schwächen im Lernprozess zu reflektieren und entsprechend selbstkritisch zu handeln. Dieses erfolgt im Sprachlern-Coaching durch entsprechende Gesprächsführungsmethoden, insbesondere nicht-direktive Fragestellungstechniken. Die Coaching-Fragen können in diesem Sinne vielleicht deswegen mehr, weil sie tiefere, personenbezogene Reflexion auslösen und die Lernenden ermuntern, ihre Selbstwahrnehmung und dadurch auch ihre Fremdsprachenlern- und Kommunikationsbewusstheit $\mathrm{zu}$ erweitern. Auf weiteren Forschungsbedarf in diesem Bereich weisen u. a. Claußen und Peuschel (2006) sowie Schmelter (2004) hin. Um diesen Forschungsanforderungen nachzugehen und das Konzept des Sprachlern-Coachings mit polnischen Fremdsprachenstudierenden auszuprobieren, wurde im Institut für Angewandte Linguistik eine fallbasierte Longitudinalstudie zum Potential von SprachlernCoaching bei der Entwicklung der Sprachlernbewusstheit und Autonomisierung von Fremdsprachenstudierenden durchgeführt (Bieniecka-Drzymala in Vorbereitung). In Bezug auf polnische Studierende könnte u. a. erforscht werden:

- Welche Potenziale und Grenzen besitzt Sprachlern-Coaching als Instrument zur Förderung der metakognitiven Reflexion bei Fremdsprachenstudierenden und inwieweit kann es die fremdsprachlichen Lern- und Kommunikationsprozesse beeinflussen?

- Welche Themen wollen die Studierenden aus der Perspektive eigener fremdsprachlicher Entwicklung einer vertieften Reflexion unterziehen und warum? 
- Wie verläuft der Reflexionsprozess und wie entwickelt sich im Rahmen einzelner Sitzungen und innerhalb des gesamten Prozesses ihre Sprachlernbewusstheit?

- Welche Rolle spielen bei dieser Entwicklung die folgenden Elemente des Sprachlern-Coaching: die Struktur, der Prozess und die Rahmenbedingungen des Gesprächs.

Die Ergebnisse der Forschung zum Sprachlern-Coaching sollen nicht lediglich als Datenquelle betrachtet werden. Die im Laufe der Coaching-Gespräche gemeinsam ausgearbeiteten Lösungen der Probleme sollen zur Optimierung der Lernund Kommunikationsprozesse der Studierenden führen und zur Verbesserung der didaktischen Praxis der Fremdsprachenlehrenden im Hochschulbereich beitragen.

\section{Literaturverzeichnis}

Ballweg, Sandra (2011). Schreibberatung für internationale Studierende. In: S. Vogler/S. Hoffmann (Hrsg.), Sprachlernberatung für DaF (S. 123-136). Berlin: Frank \& Timme.

Ballweg, Sandra (2015). Portfolioarbeit im Fremdsprachenunterricht. Eine empirische Studie zu Schreibportfolios im DaF-Unterricht. Tübingen: Narr.

Berndt, Annette/Deutschmann, Ruth-Ulrike (Hrsg.) (2014). Sprachlernberatung Sprachlerncoaching. Frankfurt a.M.: Peter Lang.

Böcker, Jessica (2014). Die Qualitative Inhaltsanalyse als Instrument zur Beschreibung von Reflexionsprozessen. In: A. Berndt/R.U. Deutschmann (Hrsg.), Sprachlernberatung Sprachlerncoaching (S. 177-192). Frankfurt a.M.: Peter Lang.

Claußen, Tina/Peuschel, Kristina (2006). Zur Wirksamkeit individueller Sprachlernberatungen. Zeitschrift für Interkulturellen Fremdsprachenunterricht. https://tujournals. ulb.tu-darmstadt.de/index.php/zif/article/view/376/364.

Claußen, Tina (2008). Auswirkungen und Evaluation von Lernberatungen. Ergebnisse einer empirischen Fallstudie. In: R. Arntz/B. Kühn (Hrsg.), Fremdsprachen in Lehre und Forschung: Vol. 43. Autonomes Fremdsprachenlernen in Hochschule und Erwachsenenbildung. Erträge des 1. Bremer Symposions zum autonomen Fremdsprachenlernen (S. 119-130). Bochum: AKS.

Claußen, Tina (2009). Strategientraining und Lernberatung. Auswirkungen auf das Kommunikations- und Lernverhalten ausländischer Studierender an deutschen Hochschulen. Tübingen: Stauffenburg.

Dörney, Zoltan/Kubanyiova, Magdalena (2014). Motivating students, motivating teachers. Building vision in the language classroom. Cambridge: Cambridge University Press.

Graf, Eva (2011). Wirksamkeitsforschung und authentische Coaching-Gesprächsdaten: Ist ,Veränderung im Coaching mittels diskursanalytischer Methoden analysierbar? In: E. Graf et al. (Hrsg.), Beratung, Coaching, Supervision (S. 133-148). Wiesbaden: VS Verlag. Online: https://doi.org/10.1007/978-3-531-93341-2. 
Graf, Eva/Spranz-Fogasy, Thomas (2018). Welche Frage, wann und warum? - Eine qualitativ-lingustische Programmatik zur Erforschung von Frage-Sequenzen als zentrale Veränderungspraktik im Coaching. Coaching Theorie \& Praxis, 4 (2018), 17-32. https:// doi.org/10.1365/s40896-018-0021-4.

Harting, Angela (2011). Eigenverantwortliches Lernen. Eine zentrale Aufgabe der Schule in Schulmanagement. Die Fachzeitschrift für Schul- und Unterrichtsentwicklung, Heft 3, Oldenbourg Schulbuchverlag, 8-11. https://www.oldenbourg-klick.de/zeitschriften/ schulmanagement/2011-3/eigenverantwortliches-lernen.

Hoffmann, Sabine (2011). Fremdsprachenlernberatung zur Erhebung von Lernvorhaben. In: S. Vogler/S. Hoffmann (Hrsg.), Sprachlernberatung für DaF (S. 146-156). Berlin: Frank \& Timme.

Hoffmann, Sabine (2014). Sprachlernberatung beim Aufbau von fremdsprachlicher Kompetenz. In A. Berndt/R.U. Deutschmann (Hrsg.), Sprachlernberatung - Sprachlerncoaching (S. 193-212). Frankfurt a.M.: Peter Lang.

Holec, Henri (1985): On autonomy: some elementary concepts. In: Ph. Riley (Hrsg.), Discourse and learning (S. 173-190). London: Longman.

Janocha, Aleksandra (2016). Fremdsprachenlerner als Reflexionspartner beim Aufbau der Feedbackkultur im universitären DaF-Unterricht. Dopiewo: Rys.

Koch, Lennart/Saunders, Constanze (2014). Theoretische und methodische Grundlagen der empirischen Erforschung von Sprachlernberatung. In: A. Berndt/R.U. Deutschmann (Hrsg.), Sprachlernberatung - Sprachlerncoaching. (S. 131-150). Frankfurt a.M.: Peter Lang.

Kleppin, Karin (2003). Sprachlernberatung. Zur Notwendigkeit eines eigenständigen Ausbildungsmoduls. Zeitschrift für Fremdsprachenforschung, 14 (1), 71-85.

Kleppin, Karin (2008). „Eigentlich trifft kein Sprachkurs genau das, was ich brauche.“ Zur Implementierung von Sprachlernberatung in Fremdsprachenzentren. In: R. Arntz/B. Kühn (Hrsg.), Autonomes Fremdsprachenlernen in Hochschule und Erwachsenenbildung. Fremdsprachen in Lehre und Forschung (FLF) 43, 64-73.

Kleppin, Karin/Spänkuch, Elke (2014a) Fremdsprachenlerner beraten/coachen - Was hat das mit Lehren zu tun? Fremdsprachen Lehren und Lernen, 43 (1), 94-108.

Kleppin, Karin/Spänkuch, Elke (2014b). Konzepte und Begriffe im Umfeld der Sprachlernberatung - Aufräumarbeiten im terminologischen Dschungel. In: A. Berndt/R.U. Deutschmann (Hrsg.), Sprachlernberatung - Sprachlerncoaching (S. 31-50). Frankfurt a.M.: Peter Lang.

Iluk, Jan (2018) Methodische Probleme beim Einsatz von Fragen im Fremdsprachenunterricht, Glottodidactica. An International Journal of Applied Linguistics, 45 (2), 151167.

Mehlhorn, Grit/Kleppin, Karin (2006). Sprachlernberatung: Einführung in den Themenschwerpunkt. Zeitschrift für Interkulturellen Fremdsprachenunterricht, 11 (2), 12. https://tujournals.ulb.tu-darmstadt.de/index.php/zif/article/viewFile/365/35.

Meier, Rolf/Janßen, Axel (2011). CoachAusbildung - ein strategisches Curriculum. Hamburg: Wissenschaft und Praxis.

Moore, Michael/Kearsley, Greg (2012). Distance education: A systems view of online learning (3rd ed.). Belmont, CA: Wadsworth.

Myczko, Kazimiera (2015). Wieviel Reflexion braucht der Fremdsprachenlerner. Glottodidactica. An International Journal of Applied Linguistics, 42(2), 45-59. 
Nazarkiewicz, Kirsten/Krämer, Gesa (Hrsg.) (2012). Handbuch Interkulturelles Coaching. Konzepte, Methoden, Kompetenzen kulturreflexiver Begleitung. Göttingen: Vandenhoeck \& Ruprecht.

Nerlicki, Krzysztof (2008a). Dzienniczki i ich rola we wspieraniu świadomości uczeniowej i komunikacyjnej studentów neofilologii - studium przypadku. Przegląd Glottodydaktyczny, 25, 143-161.

Nerlicki, Krzysztof (2008b). Selbstreflexives Fremdsprachenlernen im Philologiestudium mit Hilfe dialogischer Lerntagebücher. In: R. Lipczuk/P. Jackowski (Hrsg), Wörter und Wörterbücher. Übersetzung und Spracherwerb (S. 149-172). Hamburg: Verlag Dr Kovac.

Nerlicki, Krzysztof (2011). Lernerreflexionen im Blick. Polnische Germanistikstudenten über ihre fremdsprachlichen Lern- und Kommunikationserfahrungen. Hamburg: Verlag Dr. Kovac.

Prokop, Izabela (Hrsg.) (2001). Materiały z I Meetingu Glottodydaktycznego. Poznań: Wydawnictwo Naukowe Uniwersytetu im. Adama Mickiewicza.

Rogers, Carl R. (2002). O stawaniu się osobą. Poznań: Rebis.

Saunders, Constanze (2014). Online-Sprachberatung im universitären Kontext: Szenarien auf dem Prüfstand. Eine fallbasierte Longitudinalstudie im Rahmen von Aktionsforschung. Dissertation. Universität Leipzig. http://nbnresolvinde/urn:nbn:de:bsz:15-quc osa-165292.

Schmenk, Barbara (2008). Lernerautonomie. Karriere und Sloganisierung des Autonomiebegriffs. Tübingen: Narr.

Sedlaczek, Bettina (2008). Aussprachelernberatung: eine empirische Untersuchung. unv. Magisterarbeit, Universität Leipzig.

Schmelter, Lars (2004). Selbstgesteuertes oder potenziell expansives Fremdsprachenlernen im Tandem. Tübingen: Narr.

Smuk, Maciej (2016). Od cech osobowości do kompetencji savoir-etre. Rozwijanie samoświadomości w nauce języków obcych. Lublin: Werset.

Spänkuch, Elke (2014). Systemisch-konstruktivistisches Sprachlern-Coaching. In: A. Berndt/R. Deutschmann (Hrsg.), Sprachlernberatung - Sprachlerncoaching (S. 51-81) Frankfurt a.M.: Peter Lang.

Stork, Antje (2017). Lernende im Dialog. Lerntagebücher im Fremdsprachenunterricht. Giessener Fremdsprachendidaktik online 10. http://geb.uni-giessen.de/geb/frontdoor. php?source_opus $=13145 \&$ la $=$ de.

Vogler, Stephanie/Hoffmann, Sabine (Hrsg.) (2010). Sprachlernberatung für DaF. Berlin: Frank \& Timme.

Wehrle, Martin (2012). Die 500 besten Coaching-Fragen. Bonn. Manager-Seminare Verlag. Wenden, Anita (1998). Metacognitive knowledge and language learning. Applied Linguistics, 19(4), December, 515-537. http://doi.org/10.1093/applin/19.4.515.

Wilczyńska, Weronika (Hrsg.) (2002). Autonomizacja w dydaktyce języków obcych. Doskonalenie się w komunikacji ustnej. Poznań: PWN.

Wiśniewska, Danuta (2009). Dialog jako przestrzeń między badaniem, poznaniem a rozwojem nauczyciela. In: J. Nijakowska (red.), Jezyk-Poznanie-Zachowanie. Perspektywy $i$ wyzwania $w$ studiach nad przyswajaniem języka obcego (S. 283-298). Łódź: Wydawnictwo Uniwersytetu Łódzkiego.

Wolting, Stephan (2016). Kulturelle Mittler. Überlegungen zum Aufgabenprofil interkultureller Wissens- und Dienstleistungsberufe. Berliner Debatte Initial, 27, 50-57. 


\title{
Developing speaking skills at advanced level - the perspective of language department students and the implications for language teaching
}

\begin{abstract}
The chapter presents the findings of a research project which centred around the perceptions and opinions of advanced language learners on certain aspects of their development of speaking skills. During the research process, the respondents were finishing their final year of BA studies in a language department (applied linguistics) at Adam Mickiewicz University in Poznań. Their perspective was intended to shed new and interesting light on the process of acquiring oral skills in formal educational settings. The presentation of the research questions and the results is preceded by a discussion of selected theoretical problems connected with developing speaking competence in institutional language teaching and learning. Thus, some leading approaches to formal speaking instruction ${ }^{1}$ are briefly discussed and general problems of the subdiscipline (teaching speaking) are signaled.
\end{abstract}

Keywords

speaking skill, approach, task-based teaching/learning, genre, genre-based methodology, speaking activity, self-assessment

\section{Introduction}

In the opinion of many teachers and researchers, the field of foreign language teaching has been marked by a noticeable discrepancy between the character of the theoretical (academic) debate and the real problems and needs of practitioners. Theoretical discussions, academic research findings and their resultant implications for language teachers are very often criticized as being too detached from the practice of everyday teaching, and, as such, are considered to be of rather limited applicability in typical classroom conditions. In fact, the above observation points to a more general debate concerning the actual status of the

1 Formal instruction is understood here as the kind of instruction which "occurs in classrooms when attempts are made to raise the learners' consciousness about the nature of target language rules in order to aid learning" (Ellis 1995: 297). 
field and reflects the specific position of foreign language teaching, which is treated as a separate academic discipline, the area of practical (educational) activity or both in one. It seems that the very scope of interest of foreign language teaching (FLT) seems to connect the above perspectives to such an extent that the need for practical application of theoretical knowledge and research findings becomes an important and expected factor in research design and evaluation.

Bearing in mind the above observation, it can be claimed that the voice of those who are the recipients of the teaching process may add another important dimension to the research outcomes. Certainly, it allows for getting a more indepth picture of the particular situation/problem/phenomenon being explored and helps in formulating conclusions and pedagogical implications which are relevant to the actual problems and needs of teachers and learners. In fact, advanced language learners who have managed to develop a good insight into the nature of their problems in speaking may seem to be a particularly trustworthy source of first-hand information on the learning process. They are often able to provide their own assessment of the approaches, teaching techniques and materials, forms of practice in the language classroom and beyond, as well as reflect on some individual factors which may play an important role in developing competence in speaking. ${ }^{2}$ Thus, a group of 64 third-year students of the language department (applied linguistics) was invited to take part in the research. As experienced and knowledgeable language students, they were considered to be the most suitable group of language learners to address the questions identified in the project.

Thus, this chapter presents the findings of a project in which the perspective of language learners lies at the centre of the research interest. The presentation of the research problems and the results of the project is, however, preceded by a theoretical introduction which concentrates on the methodological aspects of teaching speaking. In this part, some approaches to formal speaking instruction are briefly discussed and general problems of the field are signaled. Importantly, teaching speaking is interpreted here as a subdiscipline of a broader field of foreign language teaching. In the chapter, the contents and aims of the subdiscipline are characterized with a view to underlining the numerous difficulties involved in the process of learning and teaching speaking in a foreign language.

2 A perspective concentrating on the use of categories and the examination of issues that are meaningful to members of the group under investigation is referred to as an emic perspective (Mackey \& Gass 2016). 


\section{Approaches to teaching speaking}

The domination of the written language in traditional applied linguistics was undoubtedly one of the reasons why spoken language was not given sufficient attention in the early years of FLT development. The lack of recognition of all the complexities involved in the process of developing the speaking skill in a nonnative language contributed to the tendency to adopt the forms and patterns typical of writing to speaking instruction. This, in turn, resulted in a situation in which the written language was generally used as the main point of reference in teaching speaking and the specific mechanisms of spoken language production were not yet properly investigated and understood. One of the consequences of this situation was the lack of effective procedures for teaching spoken language, and relying on methods and techniques used for teaching writing and receptive skills.

Speaking occupied a changing position in the hierarchy of language skills in traditional language teaching methods in the $20^{\text {th }}$ century and before. It was rather ignored in the Grammar-Translation Method, prioritized in the Direct Method and given even more attention in Audiolingualism. Yet, the most noticeable change in the educational practice came with the onset of Communicative Language Teaching (CLT). Without any doubt, this approach added a more realistic dimension to teaching speaking and introduced different forms of interaction to the language classroom. Although CLT is often criticized for ignoring the psycholinguistic dimension of speaking, attaching too much importance to information gap criteria and, in consequence, promoting unrealistic or impractical tasks (Dakowska 2005), the method has drawn the attention of both teachers and learners to the complexities and hardships of teaching spoken language. Nowadays, speaking is generally treated as the fundamental language skill to acquire, yet, at the same time the most difficult one to develop in classroom conditions.

The decision to follow a given teaching procedure, or opt for some type of material design, is usually connected with choosing a particular approach to language teaching. The following approaches can be identified as the main methodological backgrounds behind language courses which prioritize speaking:

- Task-based language teaching - one of the most popular approaches to language teaching nowadays (Ellis 2003; Nunan 2004; Willis \& Willis 2007). According to Thornbury (2007: 119), the approach "was originally motivated by the belief that a language is best learned through using it, rather than learned and then used". Consequently, it is based on the conviction that accuracy comes out of fluency, and tasks should imitate or simulate authentic situations in order to prepare learners for real-life language use beyond the language classroom.

- The situational approach - rooted in behaviourism and structuralism. In it, the choice of language items and structures practiced in the classroom is 
determined by typical situational contexts and characteristic specific speech events (Thornbury 2012). It allows for the clear description of the situational context and relevant language skills.

- The speech act approach - language functions (such as greeting, requesting, explaining, apologizing, etc.) are used as the main point of reference. This approach is frequently used in integrated skills teaching (Cohen 2005; Alcón \& Martinez-Flor 2008; Martinez-Flor \& Usó-Juan 2010).

- The skills and strategies approach - the skill of speaking is taught in steps by introducing subskills (such as starting and closing a conversation, paraphrasing, turn-taking in interaction) and relevant learning and communicative strategies. This approach is also used mostly in integrated skills teaching (Cohen 1998; Cohen \& Macaro 2007).

- The genre-based approach - popular mainly in teaching the written language (Swales 1990; Derewianka 1990; Hyland 2007), but recommended as the source of inspiration and adaptation for teaching speaking (Thornbury 2007, 2012). It focuses on the social purposes of communicative events and associated genres with their typical structures and features. It involves the explicit introduction to a particular genre by identifying its characteristic properties, defining its typical social context, and determining its goals.

- The corpus-informed approach - relies on corpora of spoken language and uses them to identify its specific features (Thornbury 2012). Such corpora should be carefully analysed before they are used for any teaching purposes and appropriately adjusted to the needs of a particular language course (Liu 2003; Grant 2007; Boulton, Carter-Thomas \& Rowley-Jolivet 2012).

- A classroom culture that prioritizes communication - intended to promote the development of speaking skills, independence and cooperation in classroom communication (Thornbury 2012). In it, a high quality of communication is achieved by introducing topics which matter to the learners.

It can be claimed that two approaches, namely task-based language teaching and the genre-based approach, are the most commonly used nowadays as background methodologies in teaching speaking. Both have their strengths and weaknesses. Task-based language teaching is frequently criticized for concentrating on the processes of learning rather than its products, while the genrebased approach is often seen as a methodology which favours the explicit presentation of language behaviours, which may encourage the habit of imitating models among language learners.

In fact, it cannot be denied that choosing a particular approach usually determines several essential aspects of the teaching process. In the case of teaching speaking, this concerns not only such obvious elements as patterns of interaction in the language classroom and types of speaking activities, but also the forms of 
teachers' control of classroom communication (Johnson 1995), evaluation procedures and the scope of autonomy granted to learners in deciding about the character of their learning and language practice in the classroom. However, it is worth underlining that although the actual decision of how and what to teach depends to some extent on a number of institutional specifications, it also reflects the teacher's judgments concerning the specific methodological framework. Whether it is a typical task-based language lesson, a corpus-informed class or a combination of some available approaches, it has to allow for tailoring the syllabus of a language course to the needs of a particular learner group ${ }^{3}$.

As regards the theoretical foundations for a theory of spoken language in applied linguistics, relevant to language pedagogy and teaching speaking skills in particular, several principles were suggested by McCarthy (1998: 47):

- "Spoken language pedagogy cannot simply just proceed from the same assumptions as written language pedagogy with regard to language use [...].

- Spoken language has its own grammar and lexicon [...].

- We can only begin to describe the special lexico-grammar of talk by looking at real data $[. .$.$] .$

- The best data for a pedagogical theory of spoken language is everyday, informal talk [...].

- The text-typologies often used in the description of written language should not be simply transferred to spoken language [...].

- Central to a theory of spoken genre are participant goals and relationships [...].

- Observation of spoken language use by participants in real encounters of various kinds is the first step in building language syllabuses and teaching materials [...]".

Although the author (McCarthy 1998) considers his postulates to be tentative, and stresses the variation typical of speech events, he also points to the fact that they share features which allow linguists to categorise them into genres. Such a description of spoken genres may turn out to be an adequate framework or a point of reference for designing a practical syllabus for teaching speaking in a particular educational context. This opinion is strongly supported by Bhatia (2002: 3), who claims that "[g]enre-analysis can be viewed [...] as a pedagogically effective and convenient tool for the design of language teaching programmes, often situated within simulated contexts of classroom activities". The benefits of genre-based pedagogy are also underlined by other researchers, among them

3 It is argued elsewhere (Aleksandrzak 2018) that a combination of the task-based and genrebased approaches and integrating them into a comprehensive framework may be of benefit to advanced language learners and prove effective in developing their speaking skills. 
Hyland (2007) who enumerates the characteristics of the approach which combine language, context and content into a comprehensive framework. According to Hyland (2007: 150), the main advantages of genre pedagogy can be summarized in the following way:

- It is explicit and systematic - it provides a framework which incorporates language and contexts.

- Content and objectives of a particular course are based on learners' needs.

- It is supportive and empowering - teachers promote learners' learning and creativity and learners are provided with patterns of texts and their variations.

- It is consciousness-raising - teachers become more aware of texts and the differences between them and they are able to offer learners more individualized feedback on their specific problems.

Additionally, as Bhatia (2002: 3) asserts,

instead of using generic descriptions as models for linguistic reproduction of conventional forms to respond to recurring social contexts, as is often the case in many communication based curriculum contexts, they can be used as analytical resource to understand and manipulate complex inter-generic and multicultural realisations of professional discourse.

In the light of this statement, it is worth adding that genre-based methodologies are in fact a common point of reference in the growing fields of ESP (English for specific purposes) and EAP (English for academic purposes). Nowadays, it is also acknowledged that genre-based methodologies allow for taking into account the social and cultural contexts of communication to a larger extent than other approaches (Paltridge 2001).

\section{Introduction to the research project}

The research problems discussed in the present chapter were part of a bigger research project (Aleksandrzak 2018) carried out at Adam Mickiewicz University in the years 2015-2017. The project involved 64 third-year students of applied linguistics and the research was defined as qualitative, although some elements of quantification were employed (Mackey \& Gass 2016). More specifically, the project was classified as an example of exploratory-interpretative research (Nunan 1989), however, at some stages of the research, procedures typical of action research (Burns 1999) were followed. For the purpose of triangulation, the process of data collection comprised different research techniques - the respondents took part in the research by completing some CEFR illustrative scales (Council of Europe 2001) and written questionnaires, answering questions 
during individual face-to-face interviews and participating in language classes observed by the researcher. The discussion of the general aims and findings of the project is beyond the scope of the present article, as it aims to focus on some selected aspects of the informants' speaking skill development, in particular the findings which refer to:

- the students' evaluation of their speaking skills in English, the factors which determine this self-assessment and the aspects which dominate in it,

- the types of tasks and speaking activities and their efficiency as tools for developing speaking abilities as advanced learners of English.

The relevant research questions were formulated in the following way:

1. What is the students' assessment of their speaking abilities according to the selected CEFR illustrative scales?

2. In what way do the students define their strong and weak points in the descriptions of their speaking abilities and how do they account for their individual problems in speaking?

3. Which speaking activities are perceived as the most useful (from the individual perspective of particular learners) and in what way do the students explain their choices?

4. What types of activities (potentially effective) are missing in the language classroom?

Due to the amount of data collected and the fact that it came from a relatively large group of respondents, the discussion in the following section presents only some general results which characterise the investigated group as a whole, and provide the answers to the research questions listed above.

\section{Results and discussion}

\subsection{Discussion - research questions 1-2}

As far as the ability to produce a sustained monologue is concerned, two separate scales were used. The learners self-assessed their skills with regard to the ability to describe experience and the ability to put forward a specific case (present one's own viewpoint or opinion in a debate). The results depend to a large extent on the general level of language proficiency displayed by the student (also defined on the basis of their self-assessments) and are presented below in Table 1. 
Table 1: Self-assessment results - sustained monologue

\begin{tabular}{|c|c|c|}
\hline \multicolumn{3}{|c|}{ Sustained monologue } \\
\hline & Describing experience & Putting a case \\
\hline C1 & $23 \%$ & $17 \%$ \\
\hline B2 & $46 \%$ & $53 \%$ \\
\hline B1 & $31 \%$ & $27 \%$ \\
\hline A2 & - & $3 \%$ \\
\hline
\end{tabular}

The results of another self-assessment procedure indicated the following results as far the three forms of spoken interaction (as different from production according to CEFR interpretation) are concerned (cf. Table 2).

Table 2: Self-assessment results - forms of spoken interaction

\begin{tabular}{|c|c|c|c|}
\hline \multicolumn{4}{|c|}{ Spoken interaction } \\
\hline & Conversation & Informal discussion & Formal discussion \\
\hline C1 & $38 \%$ & $38 \%$ & $14 \%$ \\
\hline B2 & $40 \%$ & $43 \%$ & $38 \%$ \\
\hline B1 & $22 \%$ & $19 \%$ & $48 \%$ \\
\hline
\end{tabular}

The results for the formats referred to as conversation and informal discussion are very similar (or even identical in $\mathrm{C} 1$ group). This is mainly due to the fact that the students perceive them as one and the same form of interaction, or consider the differences between these two formats negligible. Formal discussion is seen as the most difficult of the three formats in practical implementation. This result also indicates that the students are aware of the specific character and the conventions typical of formal discourse. They understand that effective communication within formalised discourse requires a good knowledge of routine language structures and the use of appropriate discursive strategies.

Two scales used in the research concerned the qualitative aspects of spoken language use, namely (propositional) precision and (spoken) fluency (cf. Table 3). The choice of these particular aspects was connected with their increasing importance and relevance for advanced language learners. In other words, fluency and precision, in the opinion of many teachers and higher level students, determine the level of proficiency actually developed in a target language and are often considered as the key descriptors of individual communicative competence at advanced level. A competent user of a foreign language is able to speak fluently and has developed the ability to express the intended meanings in a precise, comprehensible, yet individual and natural manner, in accordance with their communicative intent and social norms. 
Table 3: Self-assessment results - qualitative aspects of spoken language use

\begin{tabular}{|c|c|c|}
\hline \multicolumn{3}{|c|}{ Qualitative aspects of spoken language use } \\
\hline & Fluency & Precision \\
\hline C1 & $22 \%$ & $18 \%$ \\
\hline B2 & $41 \%$ & $31 \%$ \\
\hline B1 & $34 \%$ & $48 \%$ \\
\hline A2 & $3 \%$ & $3 \%$ \\
\hline
\end{tabular}

It seems that both fluency and precision are perceived as difficult aspects of spoken language use by advanced language learners. This is confirmed by the frequent indication of B1 category (in the case of precision, this concerns almost half the research group) even by those learners who estimate their general level in spoken interaction as levels $\mathrm{C} 1$ and $\mathrm{B} 2$.

The above results correspond with the opinions expressed by the students during face-to-face semi-structured interviews, and they may serve the purpose of illustrating some of their findings. Yet these were interviews which made it possible to gain more detailed insight into the personal and subjective perceptions of the respondents' speaking abilities. One of the most striking observations which resulted from the conversations with the learners was the conclusion that they generally tend to interpret self-assessment questions as a form of negative evaluation. When asked about their strong points and weaknesses as regards their speaking abilities, the respondents most often started with enumerating their weak points and problems they encounter while communicating in English. Eliciting from the informant any positive comments on their oral skills usually required additional questions from the researcher, the answer was much shorter than while discussing one's weaknesses, or the respondents claimed that they did not see any strong points in this respect, or were not able to indicate them.

Among the few positive aspects mentioned by the students, the following ones were most common:

- the ability to respond to the interlocutor in conversation,

- the ability to conduct informal, natural conversation,

- fluency in speaking,

- absence of communication anxiety,

- the ability to cope with problems in communication.

As regards the most commonly experienced problems in speaking, the respondents indicated the following areas of difficulty:

- lexical deficiency,

- being unable to use newly learned words and phrases in ongoing interaction, 
- monitoring grammatical structures and the contents of the utterance at the same time,

- problems with using the appropriate register and/or style of communication,

- problems with pronunciation,

- grammatical mistakes and errors while speaking,

- formal language - serious problems with using appropriate language patterns, routine phrases, typical expressions suitable for the context,

- translating parts of the utterance from the native language which results in slow, unnatural pace of speaking and/or too many hesitation gaps.

The respondents indicated several sources of the problems:

- inadequate frequency of speaking opportunities during classes,

- lack of sociocultural knowledge - socially acceptable, natural patterns of communication are not taught explicitly,

- the fact that speaking activities in the classroom do not reflect the whole variety and distribution of real-life communicative genres,

- artificial character of educational discourse,

- restricted opportunities to interact with highly competent users of the target language,

- language anxiety and fear of communication,

- other personality factors, such as high levels of introversion, low self-esteem,

- inhibitions of different kinds - shyness, fear of making mistakes, fear of losing face,

- stressful atmosphere during speaking classes,

- boring topics suggested for classroom discussions,

- domination of some learners in classroom discussions and conversations,

- the conviction that spoken language can be acquired only in natural circumstances, in the target language speaking country, by means of extensive contacts with native users of the language and participating in the life of community.

The character of the above comments shows that the students relate their problems in speaking to the factors connected with their natural predispositions (personality, affective factors), the character and circumstances typical of the particular language classroom (or language course), and, last but not least, the difficulty and complexity of the general task (developing high levels of communicative competence and specific speaking skills in the target language). 


\subsection{Discussion - research questions 3-4}

The respondents agree in their opinions regarding the most effective speaking activities in the language classroom. Conversations in pairs or small groups are generally perceived as most effective and student-friendly tasks, as they allow for reducing the stress and anxiety related to speaking in public. A stress-free atmosphere during speaking practice helps students to develop fluency and discuss all kinds of individually relevant problems and issues. In this way the students experience the state of being authentic and natural in communication. They also have a chance to practice informal or colloquial language and learn how to react naturally to their interlocutors' utterances in a conversation. In other words, the possibility of learning and trying out useful conversational formulas and appropriate communication strategies without being constantly monitored or assessed is appreciated by learners and seen as a chance for meaningful, contextualized language practice. Thus, it may be claimed that such speaking activities should be treated not as the products (results) of learning, but rather as the context in which the process of learning actually occurs (Thornbury 2007). It is worth noticing that the respondents appreciate the possibility of being introduced to such forms of spoken language by means of video or audio recordings of conversations, or small talk on similar topics. It is essential that the students are given the chance of getting acquainted with such samples of communicative events before they actually start producing their own utterances. Such models of real-life authentic interactions provide them with an appropriate repertoire of ready-made patterns, fixed phrases and other expressions which are suitable in a particular social or situational context. In other words, the students stress the importance of formulaic language 4 in speaking. Importantly, it is perceived as difficult to acquire and use, yet a necessary aspect of the target language.

Whole-class discussions are enjoyed and appreciated by a relatively smaller group of students, however, all the respondents are sure that they should be the part of the curriculum for many reasons. First of all, discussions on interesting or controversial topics are able to engage many learners in a real debate. They open up a space for expressing personal opinions, exchanging viewpoints and presenting them to interlocutors who may disagree or object to some arguments. This is a great opportunity to practice the skill of public speaking, learn how to monitor the accuracy of speaking in authentic communication and use structures typical of informal, semi-formal or formal discussion or debate. Yet, at the same time the need to cope with stress related to public speaking, too low self-

4 Formulaic language - fixed or semifixed phrases (often multiwords) which comprise approximately 60 percent of spoken English discourse (Thornbury 2012) or expressions acquired as unanalysable wholes and used on particular occasions (Ellis 1995). 
esteem or the fear of making mistakes prevent many more anxious students from participating in discussions. Some respondents also claim that whole-class discussions are usually dominated by the same group of speakers, while the majority of the group choose the role of passive listener.

Another speaking task, often given by the respondents as an example of a very useful activity, is presentation. It seems to be particularly valued by these learners of English who are aware of the fact that they are likely to use this format in their future professional life. Thus, the possibility of practicing the skill of giving formal or semi-formal presentations in English is treated by them as a chance to practice the language, as well as to learn how to cope with the stress related to public speaking. The students think that the actual task of preparing the presentation enables them to learn topic-specific vocabulary and useful communication strategies. They also develop their ability to talk about specific problems in English and choose the style of delivery which seems most appropriate in a particular social context, including the expectations or the needs of the audience. The students are generally of the opinion that a great advantage of presentations in the language classroom is also the fact that they are often followed by learnertailored feedback (both from the teacher and other students in the group). As the feedback is usually provided right after the presentation, they are able to combine the assessment with their performance, reflect on it and appropriately modify their presentation style in the next task.

Some respondents also mentioned other types of speaking activities, such as monologues, in which they report or comment on current events, review a book or a newspaper article, and spontaneous conversations which create opportunities for authentic communication and informal discourse practice. A surprisingly small number of students indicated role-plays as effective tasks in developing their speaking skills. The students who like this format regard it as helpful in simulating different communicative contexts in which they may perform different social roles, including those which they are likely to take on in real life. The respondents who do not feel comfortable while performing role-plays usually consider them as unnatural or inauthentic tasks which lead to producing artificial discourse. For that reason, they are viewed as not being particularly beneficial in developing their communicative skills.

Interestingly, the respondents found it rather difficult to answer the question concerning activities which are missing during language classes in a straightforward manner. Instead, they referred to the lack of teacher feedback on their performance in different tasks which, in their opinion, should be given more often. 


\section{Conclusions}

The findings of the project show clearly that the students' perspective (particularly the perspective of experienced language learners who have developed a good insight into the nature of their problems) is worth taking into consideration in planning the teaching procedure for a particular group of learners. Learners themselves are often the best observers and assessors of their individual drawbacks and preferences, although they often tend to be very critical of their own abilities and the progress made so far. On the other hand, teaching speaking usually requires employing a more individualized approach on the part of the teacher than in the case of other language skills. It also demands more tailored feedback and a greater variety of tasks and activities in a given group of learners if the teaching process is to be effective and supportive for all learners. Needless to say, such circumstances make the skill of speaking in a foreign language not only the most difficult one to acquire in classroom conditions (as most learners claim), but also the most demanding skill to teach. The problems stem from numerous individual differences between learners in a group, which become particularly noticeable during speaking practice. Individual learners should be allowed to follow their own pace of development, to take advantage of their natural predispositions (for example, certain personality traits ${ }^{5}$ which seem to make speaking easier or more difficult for some students) and find out how to minimize their problems and barriers. Moreover, developing speaking skills means developing an individual style which, nevertheless, should be in accordance with the conventional discursive practices and rules of social behavior typical of the target language community. In other words, it requires combining the social and individual dimensions of oral communication within a variety of communicative situations. Consequently, it seems that the teaching of speaking skills to students at higher levels of language proficiency should aim at developing their sensitivity to a variety of discourse types and genres, and the role of social and cultural context in meaningful interactions. This, in turn, means the necessity of enhancing the learners' ability to observe, analyse and reflect on their own language use in different circumstances, both in the language classroom and beyond.

Another general conclusion coming from the research is that one of the most common problems experienced by higher level students is not the lack of knowledge of the language or about the language, but rather the difficulty of combining their linguistic repertoire with their sociolinguistic knowledge in

5 Personality factors such as anxiety, self-esteem, risk taking, levels of extraversion and introversion are most often discussed in the literature as affecting the process of foreign language learning and developing speaking skills in particular (see for example: Dewaele \& Furnham 1999; Rubio 2007; Piechurska-Kuciel 2011; Dewaele 2012; Cervantes 2013; Dörnyei \& Ryan 2015). 
ongoing interaction. Many students notice that their linguistic knowledge is in fact not properly integrated and, as a result, it is not easily retrievable in natural communication. This situation indicates that contextualized extended speaking practice should be the main point of focus in all forms of teaching which prioritize speaking. Such extensive oral practice can be also seen as a rich source of meaningful input and individualized feedback. Undoubtedly, the unavoidable selection of teaching formats and learning activities should be relevant to learners' needs and the objective of a given language course.

It is worth underlining that the students regard formal communication as more difficult to master, in comparison to conversational skills and styles of everyday informal speaking which they are more likely to acquire by means of social media, contacts with foreign friends and the easy access to popular English language culture. This observation suggests that perhaps a genre-based approach can be an effective solution in case of some types of formal or semi-formal conventional discourse as it involves introducing students to samples of real-life language use and typical patterns.

Last but not least, it seems that there is a list of features that a particular speaking activity has to possess in order to be motivating for students and conducive to effective learning. First of all, it has to be language productive and challenging for learners, yet at the same time they appreciate when the task has a clear purpose and enhances their sense of achievement. Second of all, according to the students, speaking activities should bear some resemblance to real-life circumstances and give them the feeling of participating in authentic communication. Finally, it is helpful when the atmosphere in the classroom is supportive and non-judgmental, yet the feedback on individual performance and progress (or lack of it) is offered regularly.

\section{References}

Alcón Soler, Eva/Martinez-Flor, Alicia (eds.) (2008). Investigating pragmatics in foreign language learning, teaching and testing. Clevedon: Multilingual Matters.

Aleksandrzak, Magdalena (2018). Rozwijanie sprawności mówienia w kształceniu neofilologicznym z perspektywy studentów uczacych sie języka angielskiego jako obcego. Badanie jakościowe. Poznań: Wydawnictwo Naukowe Uniwersytetu im. Adama Mickiewicza.

Bhatia, Vijay K. (2002). Applied genre analysis: a multi-perspective model. IBERICA, 4, 3-19. Boulton, Alex/Carter-Thomas, Shirley/Rowley-Jolivet, Elizabeth (eds.) (2012). Corpus-informed research and learning in ESP. Issues and applications. Amsterdam: John Benjamins Publishing Company.

Burns, Anne (1999). Collaborative action research for English language teachers. Cambridge: Cambridge University Press. 
Cohen, Andrew (1998). Strategies in learning and using a second language. Essex: Longman.

Cohen, Andrew (2005). Strategies for learning and performing L2 speech acts. Intercultural Pragmatics, 2, 275-301.

Cohen, Andrew/Macaro, Ernesto (eds.) (2007). Language learner strategies: 30 years of research and practice. Oxford: Oxford University Press.

Council of Europe (2001). Common European framework of reference for languages: Learning, teaching, assessment. Strasbourg: Council of Europe.

Dakowska, Maria (2005). Teaching English as a foreign language. A guide for professionals. Warszawa: Wydawnictwo Naukowe PWN.

Derewianka, Beverly (1990). Exploring how texts work. Sydney: Primary English Teaching Association.

Ellis, Rod (2003). Task-based language learning and teaching. Oxford: Oxford University Press.

Ellis, Rod (1995). Understanding second language acquisition. Oxford: Oxford University Press.

Grant, Lynn (2007). In a manner of speaking: Assessing frequent spoken figurative idioms to assist ESL/EFL teaching. System, 35, 169-181.

Hyland, Ken (2007). Genre pedagogy: Language, literacy and L2 writing instruction. Journal of Second Language Writing, 16, 148-164.

Johnson, Karen (1995). Understanding communication in second language classrooms. Cambridge: Cambridge University Press.

Liu, Dilin (2003). The most frequently used spoken American English idioms: A corpus analysis and its implications. TESOL Quarterly, 38, 671-700.

Mackey, Alison/Gass, Susan (2016). Second language research: Methodology and design. New York: Routledge.

Martinez-Flor, Alicia/Usó-Juan, Esther (eds.) (2010). Speech act performance. Theoretical, empirical and methodological issues. Amsterdam: John Benjamins Publishing Company.

McCarthy, Michael (1998). Spoken language \& applied linguistics. Cambridge: Cambridge University Press.

Nunan, David (1989). Understanding language classrooms. A guide for teacher-initiated action. London: Prentice Hall.

Nunan, David (2004). Task-based language teaching. Cambridge: Cambridge University Press.

Paltridge, Brian (2001). Genre and the language learning classroom. Ann Arbor: University of Michigan Press.

Swales, John (1990). Genre analysis: English in academic and research settings. Cambridge: Cambridge University Press.

Thornbury, Scott (2007). How to teach speaking. Harlow: Pearson Education Limited.

Thornbury, Scott (2012). Speaking instruction. In: Anne Burns/Jack Richards (eds.), The Cambridge guide to pedagogy and practice in second language teaching (pp. 198-206). Cambridge: Cambridge University Press.

Willis, Dave/Willis, Jane (1997). Doing task-based teaching. Oxford: Oxford University Press. 
Open-Access-Publikation im Sinne der CC-Lizenz BY-NC-ND 4.0

(C) 2020, Vandenhoeck \& Ruprecht GmbH \& Co. KG, Göttingen ISBN Print: 9783847111863 - ISBN E-Lib: 9783737011860 


\section{Teil 5: Fachsprachen}


Open-Access-Publikation im Sinne der CC-Lizenz BY-NC-ND 4.0

(C) 2020, Vandenhoeck \& Ruprecht GmbH \& Co. KG, Göttingen ISBN Print: 9783847111863 - ISBN E-Lib: 9783737011860 


\title{
Klaus-Dieter Baumann (Universität Leipzig)
}

\section{Thought patterns in natural scientific and technical scientific communication}

\begin{abstract}
As a result of the immense complexity of the language/communication for specific purposes, the focus of LSP research on the analysis of their characteristics has changed several times in the last century. The cognitive perspective of modern LSP research holds that LSP is part of the cognitive system: all cognitive abilities interact with various means of communication and generate an information flow which is to be understood in terms of dynamic mental states of the author(s) and recipient(s) during LSP text production and comprehension processes. Furthermore, on the basis of empirical studies of various English and German LSP texts the complex processes will be described that bring together thought patterns and language and integrate them into a specific LSP text form. One of the most fruitful approaches to analyse the equivalence of thought patterns and language structures is to be opened by highly interactive cognitive-communicative modules which are realised at different levels of LSP text forms (e.g. intercultural, social, subject-specific, functional, textual, stylistic, syntactic, semantic level). This work is part of an ambitious project which aims at the interdisciplinary analysis of LSP texts.
\end{abstract}

\section{Keywords}

LSP thought pattern, mind style, communicative-cognitive modules, interdisciplinary approach to LSP communication

\section{Introduction}

Since the mid-1980s, as is generally known, the cognitive expansion of Language for Specific Purposes (LSP) research has led to a shift in its analytical focus (Kalverkämper \& Baumann 1996: 355). Since then, the focus of LSP research has been on the implementation of the mental structures and processes in communication, which form the basis of the production and reception of LSP texts.

Representative LSP text analyses coming from various scientific disciplines, technical chains of action, and particular languages, by examining particularly the dialectic relationship between LSP and LSP-based thought patterns, have provided evidence that the correlation between LSP thinking and LSP can contribute to new 
insights in LSP economy as a cognitive and communicative phenomenon (Baumann 1992: 139; Baumann 1994; Haken \& Haken-Krell 1997; Zimmer 1999) and can lead us to more precise research results. There is a tremendous application potential of possible findings resulting from this multi-faceted investigation. This type of investigation affects the different aspects of linguistic externalisation and internalisation of scientific results and the inherent strategies to transfer mental representations of the LSP-based reality by means of communication (Baumann 2001). From the methodological point of view, this gives rise to the task of analysing the specific influence exerted by a respective object or subject field on LSP (-based thought patterns).

Simultaneously, R. Buhlmann and A. Fearns (2000) have reached wide-ranging results from their foreign language method-oriented analyses, which were aimed at the conveyance of natural/technical science LSP. These findings concern the complex relationship between the respective technical object at hand - LSP-based thought patterns and LSP. The two researchers express the following endpoint:

LSP as a means of communication is a result of socialisation within a certain scientific discipline. It is characterised as such by reflecting certain thought structures that are determined by the interest in findings and research prevailing in the respective field. LSP is important for the communication of technical contents - objects, operations, processes, procedures, theories, etc. - and, from a linguistic point of view, uses the most concise and precise form (Buhlmann \& Fearns 2000: 12-13).

They ultimately draw the fundamental conclusion that: "LSP is therefore linked to the thought elements of the field that the technical terms exist in - the thought structures of the field and the customary communication structures of the discipline” (Buhlmann \& Fearns 2000: 13).

There is an increasing number of data, relating to the history of science and the philosophy of science, pointing out the methodological significance of the interactive relationship between the technical object at hand and LSP-based thought patterns, characteristic of a particular scientific discipline (Grmek 1996; Breuer 2001; Pauen \& Roth 2001; Kromrey 2002). In this respect, one of the crucial challenges for LSP research and other (non-)linguistic disciplines lies in finding a method of differentiating the diverse communicative-cognitive strategies which are uses in an efficient transfer of specialised knowledge.

The category of LSP-based thought patterns has, since the mid-1980s, assumed a central epistemological role in interdisciplinary LSP text analyses (Baumann 1992: 144). It is aimed at systematically analysing the particularities of the knowledge process in a specific technical-professional environment of reality. LSP-based thought patterns are, therefore, perceived as exceedingly complex cognitive operations that are based on analysing and/or regulating the processing of information. 
In the scientific process of investigation, the dominant role befits LSP-based thought patterns, because terminologically predetermined cognitive reflections of technical spheres of objective reality are conveyed through analytic-synthetic thought operations. Depending on the status of such a kind of investigations, the cognitive reflection on the technical contents and processes is of a partial nature, which may be complemented through secondary knowledge processes (Müsseler \& Prinz 2002: 645). By uncovering the driving force, the causes, and the regularities in particular developments in the technical reality, it becomes possible to formulate specific scientific theories, which enable reflecting the object of a given investigation more thoroughly than through mere observation. In doing so, LSP-based thought patterns are viable through the use of the preferred thought methods and thought procedures (Bochenski 1993).

From the perspective of the appropriate analyses, the LSP-based thought process is mainly determined by the following factors (Baumann 2001: 57-101):

- the mental structuring of the scientifically specialised subject field,

- the nature of the mental objects (the homogeneity or heterogeneity of objects that are subject to cognitive processing),

- the quality of the subject or object-specific status of investigation,

- the subjective stance on the object of investigation,

- the allocation of tasks and the inherent cognitive requirements,- the proximity of the object of investigation to the researcher's quotidian scope of experience,

- the practice-orientedness of the knowledge process (fundamental vs. applied sciences),

- notions and schemata as cognitive models of organisation,

- the application of scientific principles of investigation (inductive or deductive reasoning),

- the historic, ideological-philosophical, cultural, social and economic foundations of the knowledge process,

- the mental quality of perception of specialised contents (affirmative/critical/ rational/ emotional orientation),

- the identification of the individual's specific thought patterns (schools of thought),

- the mentally anticipated goal of investigation (quest for rules, practical implementations),

- researchers' motivation determining the knowledge process,

- mental strategies/methods of analysis,

- the ethical neutrality of scientific investigations and

- the intra-, inter-, and transdisciplinary nature of scientific illustrations. 
Representative studies carried out in various academic disciplines and individual languages have indicated that the specific strategies of LSP-based thought patterns constitute the methodological starting point for the analysis of the linguistic transfer of terminologically predetermined reflections of specialised contents. This epistemologically innovative analytical approach enables current LSP research to implement the cognitive shift in an object-specific way. This shift is aimed at developing typologies of LSP-based thought strategies, conductive to the deduction of a typology of communicative regularities that occur when implementing reflections of a subject-specific reality.

On the above-mentioned ground, the first step of communicative-cognitive examinations of LSP-based communication consists in depicting the diversity of individual scientific disciplines in terms of cognitive operations, strategies and processual qualities of mental activity, which determine the way in which specialists orient themselves in their specialised environment and how they intellectually master them (Prim \& Tilmann 1997). The next step will then necessitate a delineation of the multi-faceted communicative constitution of technical thought patterns. This leads to a detachment of the relationship between the mental representations from the specific specialised situation of activity, its generalisation, and the transfer of cognitive performance onto new technical requirements. The third focus consists in forming an analytical connection between the mental representations of the different classes of contents which are customary in the respective field, as well as the mental performance characteristic of certain specialised situations of activity and their structural-functional implementation in the context of LSP-based communication.

The results of LSP-linguistic research carried out in the last few decades confirm that a scientifically reflected differentiation of objective reality goes hand in hand with the multi-faceted differentiation of LSP communication, designed to facilitate the best possible transfer of information. The increasing number of LSPs are characterised by a considerable diversity of structural-functional and/or communicative/cognitive characteristics on varying levels (Hoffmann 1976; Baumann 1994). Relatedly, statistical analyses of language have shown that the number of identical characteristics shared by respective LSPs may vary (Hoffmann \& Piotrowski 1979). In addition to these differences, consequently, the criterion of the homogeneity of LSPs has become a further goal of linguistic analysis (Hoffmann 1984: 53; Fluck 1997). This is the analytic focus of mainly these LSP-linguistic approaches which endeavour to present evidence for a structural-functional kinship between LSPs in scientific fields with LSPs in other academic disciplines linked to them (nature, society, thought and others) (Hoffmann 1978; Satzger 1999).

Three different scientific clusters have emerged regarding the level of the homogeneity or heterogeneity of particular specialised academic disciplines and their LSPs: 
- LSPs and the natural sciences (cf. exact and biological natural sciences, e.g. biochemistry, beekeeping; Pörksen 1986; van Doren 1996),

- LSPs and the humanities (philosophy, cultural and social sciences, political science, economics and law, linguistics, art research, pedagogy, ethnology, anthropology and others) (Skudlik 1990),

- LSPs and the technical sciences (process engineering, biomedical engineering, mechanical engineering, nuclear engineering, biotechnology, environmental technology, traffic technology, mining and others) (Krings 1996; Fischer 2004).

Furthermore, some researchers working within the 'philosophy of science' circle are increasingly interested to extract the cognitive foundations of LSP-based thought patterns in the natural, social, and technical sciences (Gloy 1995; 1996; Lyre 2002). A first focus of these studies consists in implementing the purpose of the sciences and in documenting the respective epistemological basic values, basic norms and perspectives. The other one aims at investigating the question of how the specialised basic concepts and methods customary in one scientific field have to be arranged in order to optimise the acquisition of knowledge.

Taking all the above ruminations into consideration, the central elements of LSPbased thought patterns in the natural sciences can be characterised as follows (Lyre 2002: 214ff.):

1. The acquisition of knowledge oscillates around a complex of animated, as well as lifeless characteristics of nature (including man's nature-relatedness).

2. Depending on the current level of knowledge in society, a theoretically founded and empirically secured differentiation of natural scientific reality shall be assumed.

3. In the specialised "spheres", the acquisition and classification of factual knowledge is in the centre of attention, i.e., deriving physical constants, understanding basic characteristics of matter, deducting equations, algorithms, and models, deductive theories, and the extraction of objective laws of nature. From the epistemological point of view, the principle of causality plays a decisive role here.

4. The systematic investigation of the branches of biology requires an objectoriented system of technical terms, categories, constructs, principles, and methods (Thielmann 1999).

5. The acquisition of knowledge from the knowledge subject to the knowledge object is put into practice with the help of certain analytical means (appliances, substances, experiments etc.) (Lenk 1998).

6. Without establishing measurement parameters and/or exact metering of measurable entities of specialised matter, the acquisition of knowledge on a high level of formality, objectivity, and precision, is impossible (e.g. pattern recognition, development of paradigms and regularities) 
In contrast to the above, LSP-based thought in the field of humanities is characterised by the following factors (Otto et al. 2000: 11-594):

1. The quest for knowledge concentrates on the extraordinarily complex interactive relationship between man and society. Finding evidence for certain regularities of social development is the main interest of investigation.

2. Man and society as objects of investigation are subject to an object-oriented classification into individual scientific disciplines, which deals with the various aspects of this dialectic element of analysis in a target-oriented way. In this context, the unity of theory and practice, and the primacy of thought and existence play a fundamental epistemological role.

3. Basic elements of the knowledge process are the deduction of the goal of investigation, a theory (an intellectual approach or school of thought), the creation of a terminological system, the finding of empirical facts, and the theoretical assessment of specific results (Kromrey 2002: 53ff.). The appropriate thought cycles can be followed back to the cognitive theory-experiment-theory pattern.

4. The self-orientedness of man's intellectual discussion, concentrating on himself as the object of investigation, leads to the utilisation of methods of investigation that interpret, compare, classify, assess, and are open to subjective explanations (Bochenski 1993: 12ff.).

5. The epistemological quality of thought patterns characteristic of the social sciences is mainly dependent on the researcher's point of view, since the knowledge perspective of the individual is influenced by a complex of individual, social, economic, cultural, ideological, and other factors.

6. Emotions as a cognitive assessment of social scientific subject matters are a constituent of specialised thought strategy.

Specialised thought patterns in the only recently-evolved technical sciences mainly feature the following characteristics (Fischer 2004: 180)

1. The acquisition of knowledge focuses on illustrating, designing, and optimising technical procedures, as well as on modelling and using technical systems. Its prerequisites are: the knowledge of the laws of nature, acquaintance with materials and their characteristics, the knowledge of handling methods, the ability to think ahead in a productive manner, and implementing ideas purposefully in order to improve man's practical living conditions.

2. The traditional distinction between different branches of technical sciences is justified on the basis of the practically or industrially connected branches of work processes (mining and metallurgy, electrical engineering, production engineering, construction technology, heating engineering etc.). 
3. The depiction and utilisation of natural events and the laws of nature in technical and technological systems represents the epistemological framework for the specialised thought patterns characteristic of the technical sciences. Both are aimed at the derivation of technical laws, which are in turn based on the dialectic relationship between the system in its entirety and the element, or rather between the necessity and coincidence. Correspondingly, technical laws could be subdivided into anticipating (normative-descriptive, prescriptive-descriptive), universal and representational ones.

4. Thought strategies typical of the technical sciences are coined by the specific handling methods used by the researcher with regard to an object of investigation, by the same token these are closely linked to the characteristics and functions of the respective instruments (appliances, machines, procedures etc.).

5. The cognitive transformation procedures which are to be presented, and which result from applying the laws of nature to their practical spheres of validity, constitute the central element of thought patterns characteristic of the technical sciences.

6. Thought patterns typical of the technical sciences are genuinely interdisciplinary and aim at the activation of man's unlimited development potentials as an unconscious element of the man-machine-system.

The analysis of LSP-based thought patterns characteristic of each of the three scientific disciplines shows that specialised thought strategies contain changing as well as unchanging elements. As a consequence of the interdisciplinary interplay of natural, social and technical scientific communication, however, the respective specialised thought strategies may overlap.

\section{The contribution of rhetorical-stylistic means to LSP-based thought patterns in the natural and technical sciences}

On the basis of LSP text analyses, it has been established that the following levels (in the decreasing order), all bearing on the use of rhetorical-stylistic means, are important for developing strategies of specialised thought patterns in the natural, technical, and social scientific communication spheres. 


\subsection{The culturally specific level}

The importance of culturally specific knowledge for the specialised communication process has been underestimated for a long time. In interlingual comparative analyses of rhetorical-stylistic elements in LSP texts from the three scientific disciplines mentioned above, it has been shown that significant cultural differences exist, especially between LSP texts from the humanities and texts coming from the natural and technical sciences. Some of these differences should be statistically analysed.

In this context, we should mention the existence of different, historically rooted, communication styles. Among these, there are, for instance, in the field of LSP-based communication, the Teutonic, Gallic, Anglo-Saxon and Nipponic Style (Clyne 1987: 211-247). These four communicative styles, which ere postulated based on scientific LSP text analyses carried out in the field of linguistics and sociology by the Australian linguist M. Clyne, are linked to a certain content-related and formal degree of abstraction in LSP-based communication. M. Clyne stresses that the Teutonic and the Gallic Style in LSP-based communication require the highest degree of linguistic articulation. In contrast, the Anglo-Saxon and the Nipponic scientific style are considered to be less elitist. Moreover, M. Clyne has convincingly drawn attention to the fact that, in the fields of linguistics and sociology, German LSP-based communication utilises different textual organisation structures than the ones customary in the English language. In detail, while German LSP-based communication is distinguished by the element of reader responsibility (the reader's duty to understand the LSP text without any communicative aids provided by the author), English LSP-based communication features writer responsibility. This implies that the author assumes strategic responsibility for the comprehensibility of his text. He may, for instance, facilitate its comprehension by means of a higher degree of rhetoricalstylistic means in the technical text. According to M. Clyne, the respective strategic differences in communication can be attributed to varying cultural traditions, customary in the respective scientific communities. Relatedly, he states that "each cultural group has their own expectations of communication, which in turn are rooted in a specific cultural value system" (Clyne 1993: 3).

As a result of interdisciplinary investigations of social, natural, and technical texts, it was confirmed that the culturally specific dimension of the use of rhetorical-stylistic elements is particularly significant in social science texts. In natural and technical science texts, however, the culturally specific dimension is of secondary importance, given the more strongly regulated and conventionalised LSP text structure (Lauren \& Nordman 1996). 


\subsection{The social level}

Analysing the influence of social factors on the choice and use of rhetoricalstylistic means in natural and technical science texts, the following findings have to be considered:

a. Bringing a specialised technical fact or subject matter closer to an interested layman requires great care and attention on the part of the LSP text author in the process of the linguistic composition of explanations and comments. This implies that a greater effort is needed to convey the message of the text to a layman than, for instance, to a work colleague. Varying degrees of previous knowledge on the parts of LSP text author and recipient(s) respectively lead to the integration of socially relevant elements of partner-related redundancy in order to secure the success of the information transfer.

In technical texts directed to laymen, the LSP text author is forced to adapt him/herself to the level of knowledge and expertise of his recipients by adding explanations and illustrations to foster the comprehensibility of the text. Addressing other specialists, however, these pieces of additional information are dispensable. In these partner-oriented constellations, the stylistic means parentheses, parallelism, addenda, and others may be used to foster textual comprehensibility.

b. The analysis of rhetorical-stylistic elements indicates that a partner-related attitude on the part of the author of the LSP text can be put into practice by means of efficiently stylised wording and a loosened sentence structure. By using syntactic stylistic means, like the chiasmus, for instance, the author is able to get the recipient's full attention, emphasise important findings, and/or design the conclusion in an original and memorable manner.

c. The expressivity conveyed through stylistic means represents an important prerequisite for a successful course of LSP-based communication, since it leads to a heightened degree of perception on the part of the LSP text recipient regarding the subject matter at hand. Activating their attention triggers the release of cognitive energy, which favours the subjective processing of information. In this context, the degree of expressivity of grapho-stylistic means (visual code) is an element which facilitates the decryption of natural and technical scientific texts (Riesel \& Schendels 1975).

d. LSP text analyses coming from all three scientific disciplines have confirmed that certain semantic and syntactic stylistic means are particularly suitable to bridge the varying levels of previous knowledge on the part of the communication partners in the case of a socially asymmetrical relationship between them. As a consequence, the LSP text author may vary the linguistic structuring of specialised facts or subject matters by means of repetitions and/or 
synonymy, and thereby helping the recipient to remember a larger share of the pieces of information presented to him.

Choosing and using the appropriate rhetorical-stylistic means in natural and technical scientific texts is, therefore, highly dependent on the communicative partners' social constellation.

\subsection{The cognitive level}

The cognitive level of choosing and using rhetorical-stylistic elements in LSP texts gives rise to the belief that certain stylistic means are particularly suitable to optimise proceedings linked to information processing and information storage (Möller 1983). With regard to this, L. Fleck (1994) has introduced the term "thought styles", which he defines as "directed perception and appropriate mental and factual assimilation of what has been perceived" (Fleck 1994: $109 \mathrm{ff}$.). From a cognitive-linguistic point of view, thought-stylistic means (e.g., amplification, syllogism, isologue, antithesis, simile, allegory, irony, hysteronproteron etc.) constitute theoretical-linguistic elements of information processing strategies, the application of which can be attributed to certain attitudes, previous knowledge, emotional states, assessment standards, and/or emotions on the part of the author (Nischik 1991: $58 \mathrm{ff}$.). In natural and technical scientific LSP-based communication, thought-stylistic means contribute to the implementation of the following functions (Baumann 1992, 139-158):

a. increasing the vigour when designing an LSP text (metaphor, metonymy, epithets etc.),

b. increasing clarity in the illustration of the specialised content (e.g. anaphora+ parallelism as a clearly perceptible theoretical organisation pattern, antithesis, question-answer combination, rhetorical question),

c. striving for clarity (by means of explanatory parenthesis [explication, appendix and/or isolation]) and for precision (images, charts, formulas etc.) as an indication of associative thought patterns on the part of the author, and d. efforts to facilitate the text reception (improving comprehensibility).

In LSP texts, the combination of general, particular, and/or single pieces of information helps to memorise the information and foster new findings. A generalisation of findings without reference to particular or single pieces of information bears the risk of misinterpretation on the part of the recipient. By inserting examples, the recipient is granted a break to think about the information that has been previously mentioned. In order to make the text reception of a complicated complex of thoughts more palatable to the reader, the 
author moreover can repeat facts of the case. Thereby, one thought from the progression of information is emphasised in the LSP text. This emphasis can be achieved by means of (syndetic, asyndetic, polysyndetic) repetition or synonymy.

LSP text research in the fields of the natural and technical sciences confirms that the thought-stylistic means have a knowledge-fostering function. The choice and utilisation of the appropriate means occur on the basis of certain senderrecipient-strategies that facilitate the conveyance of specialised text contents. In this context, it has been observed that due to differing levels of previous knowledge, the diversity and the number of thought stylistic means increase, if the degree of specificity between the communication partners is rather low (Baumann 1994: 122ff.).

\subsection{The content-related fact-based level}

Comparative analyses of different LSP texts from varying scientific disciplines, individual languages and LSP text types particularly indicate three determining factors, which illustrate the significance of the content-related fact-based level's task to choose and use rhetoric-stylistic means in LSP-based communication.

a. The influence of the individual scientific discipline

Rhetoric-scientific similarities between different LSP text forms coming from a single discipline (e. g. historiography, linguistics, psychology) are obviously more significant than the number of matching elements existing in texts belonging to the same LSP text form from different scientific disciplines (Baumann 1992: 74). S. Skudlik (1990) also draws our attention to the connection between the scientific discipline and style: "Undifferentiated prior understanding detects clear differences between the language customary in the natural sciences and that of humanities. The latter seems closer to everyday speech and it is characterised, it seems, by terminologically more strongly determined use of standard language and especially through stylistic usages distinct from the scientific language. The first, however, evokes the idea of an extensive terminological apparatus, offering expressions entirely unknown to a layman, a host of formulas, and/or the formula-like employment of certain linguistic means" (Skudlik 1990: 221). 
b. The relationship between the LSP-communicators and the subject matter at hand

Rhetorical-stylistic means (e.g. captions in technical texts in the field of architecture, automotive engineering etc.) activate important impulses in the text that may foster knowledge (rationality, emotionality), facilitating the partner's analysis of the subject of communication. Thus, rhetorical-stylistic means can contribute to expressing the objective information contained in the text specific meaning. In social science texts, the stylistic means characterise the communicator's attitude to the reflected object. In natural and technical scientific texts, the choice and use of rhetorical-stylistic means are determined by the degree of detail (redundancy vs. restrictions) and precision (e.g. understatement vs. overstatement) with which the author endeavours to linguistically implement the subject matter (Jahr 2000).

c. The correlation between the system of the LSP text with regard to content and object of investigation, and the individual levels of knowledge and expertise of those participating in the act of LSP-based communication (Skyum-Nielsen/Schröder 1994)

In this context, classification of the specialised styles customary in the natural, technical, and social sciences has been postulated. For example:

1. the theoretical scientific technical style,

2. the popular scientific style,

3. the didactic style, and

4. the instructing style (Riesel \& Schendels 1975; Sandig 1986).

To summarise, it can be said that certain specialised contents in specific LSP text/ text forms are preferably conveyed using a set of selected stylistic elements. These interactions facilitate a more efficient communicative implementation of the information transfer.

\subsection{The functional level}

The functional level of rhetorical-stylistic elements in LSP-based communication relates to their mode of application in the LSP text. Together with the stylistic inventory, typical aspects of the facts and processes addressed in the text are thus emphasised. In the field of lexis, this level of rhetorical-stylistic elements is expressed by means of the area of activity of the lexical stylistic means used (foreign words, phraseologisms etc.) and/or by means of their communicative 
connotation (technical and dialectical terms, specialised vocabulary etc.). However, these elements are predominantly used in social science texts. In contrast to this, natural and technical scientific papers are dominated by nomenclatures, abbreviations and formula expressions. On the syntactic level, it is primarily the function-oriented variation of sentence length, the contracted verb forms, and/or the degree of function-oriented changes of word order and sentence structure that determine the functional level of rhetorical-stylistic means. On the textual level, the functional dimension of the rhetorical-stylistic inventory is determined by the percentage of nominal or verbal LSP text constituents (nominal/verbal style), the respective sentence linkage means, the use of certain tenses, grammatical mood, diathesis as functionally determined and stylistically relevant LSP text elements, the stylistic form of mental-linguistic communication and complex procedures, and the functional particularities of the author's communication strategy (the integration of quotes as pieces of evidence, illustrations, theses and/or quotations in order to express one's ironic and critical stance on the subject matter at hand). In natural science and technical LSP texts, the high level of content-related precision and the labelling of the text's inherent logical congruity can cause the text to be dominated by the nominal style, causal types of sentence relations, and passive structures (Lauren \& Nordman 1996).

The functional level of the rhetorical-stylistic potential thus encompasses any linguistic and non-linguistic means that indicate the communicative partner's ability to acquire knowledge and to act, at their present level of knowledge/or degree of specificity.

\subsection{The textual level}

Representative LSP text studies carried out in all three scientific disciplines a provided evidence for the existence of rhetorical-stylistic text layout principles, which have a lasting effect on the absorption and the integration of the pieces of information in the text. In this context, the rhetorical-stylistic text layout principles feature a qualitative-functional dimension, relating to the compulsory and optional stylistic means that are integrated into the LSP text. The frequency, the distribution, and the combination of stylistic means constitute the quantitativestructural side of rhetorical-stylistic text layout principles.

The development of different rhetorical-stylistic text layout principles is primarily based on the following links (Baumann 1992: 69-70):

- The body of stylistic means used in the LSP text has structural and/or functional particularities. They emphasise the lack of symmetry of form and content in the field of LSP communication. 
- The choice and the use of rhetorical-stylistic means are closely linked to the intention, the topic, and the author's communication strategy. During the linguistic implementation of certain contents, these factors are combined to form a text-specific pattern which secures certain communicative effects.

- LSP text forms (essays, scientific magazine articles, and others) distinguish themselves through regularities in the structure and the combination of stylistic means. Apparently, it is possible in these LSP text forms to summarise groups of rhetoric-stylistic means in terms of identical function (e.g. convincing the communication partner) to formulate equivalent rhetorical-stylistic text layout principles (e.g. euphemistic/pessimistic point of view regarding items and processes of specialised reality, forming an affirmative/ critical stance on specialised contents, integration/omission of personal matters, redundant/restricted illustrations etc.)

Investigations on the subject-matter have shown that certain rhetorical-stylistic text layout principles predominate in natural and technical science texts (congruity of statements, omission of personal matters etc.) In conclusion, it can be said that the implementation of stylistic layout principles in LSP texts do not bear on the efficiency of the decoding process of contained information only. They also have an effect on the comprehensibility of the text.

\subsection{The syntactic level}

The complex cognitive structure of (specialised) knowledge which is to be transferred in the communication process among the communicative partners requires applying a particular perspective to their interaction, which includes a determination of form and function regarding the syntactic stylistic means. The syntactic stylistic means focus on organising passages of the LSP text, in such a manner that the individual effort necessary to process the text be minimised, the restricted capacity of one's memory be not overworked, and the subsequent information contained in the text conforms to the expectations of the recipient(s) (Miller 1962: 748-762; Redder \& Rehbein 1999).

Technical text analyses from three different scientific complexes have shown that the following text-syntactic categories are stylistically relevant (Baumann 2001: $57 \mathrm{ff}$.):

a. tense (the historical present tense),

b. grammatical mood (the author's position on the level of validity of the conclusion of the text),

c. functional sentence perspective (FSP, topicalisation and rhematisation),

d. formation of ellipses, 
e. syntactic stereotypes (firmly established emotional "bias") in certain LSPbased texts forms (e.g. user manual),

f. the syntactic combination of LSP text passages and captions (images, diagrams, charts, symbols, formulas etc.),

g. the share of paratactic and hypotactic sentence configurations in the technical text,

h. sentence linkage means (anaphora + parallelism, antitheses and others),

i. reproduction of somebody else's discourse (quotations),

j. emphases by means of inverted word order (stylistic inversion, rhetorical question and others) and

$\mathrm{k}$. the logical structure of the content (question-answer-combination, rhetorical question).

Furthermore, the stylistic analyses by L. Hoffmann and R.G. Piotrowski (1979) have demonstrated that the syntactic stylistic means are of varying significance for the LSP-based communication process. In social scientific texts they are first and foremost aimed at increasing the degree of emotionality and expressivity of the LSP-based text's conclusion. This occurs with the help of the following strategies:

1. Securing accessibility of the relevant pieces of information in the technical text by means of emphasis (presence of consciousness),

2. Increasing the conspicuousness of relevant LSP text contents by means of topicalisation (topic-comment-structure, inversion, emphasis),

3. Stressing LSP text contents by restructuring communicative acts (adopting someone else's perspective, referring to someone else's viewpoints, direct speech as quotation, question-answer-sequences, as well as contrasting and repetition of statements) and

4. Modifying of the LSP text author's position (grammatical mood: subjunctive, imperative).

Syntactic stylistic means offer a wide range of possibilities to express the LSP text author's emotional and/or mental commitment to certain contents in an efficient manner.

\subsection{The semantic-lexical level}

In all scientific disciplines the coordination of conceptual knowledge between the communication partners occurs by means of terminology (Budin 1996). It secures a maximum level of conceptual agreement among the participants of a communicative act. Since the terminological system follows the precepts of the 
respective scientific discipline and represents the highest degree of conceptual abstraction, it facilitates optimal communication between specialists (Kalverkämper 1988: 166). Therefore, technical terms represent the obligatory stylistic means of a technical text. In the field of natural sciences, these do not have an expressive colouring.

In stylistic analyses of lexis and semantics in natural science and technical science texts, it has been established that the share of technical terms in relation to the total word count varies considerably, depending on the level of subjectspecificity. In this context, it was found that a high level of specificity always entailed a high percentage of technical terms (Baumann 1994: $127 \mathrm{ff}$.). Moreover, the number of term repetitions in a technical text, the concentration of terms in certain macro-structural elements of the text, and the number and the nature of those scientific disciplines that contribute to the specialised word count are of special significance ( $\mathrm{Nycz}$ et al. 2016).

In text analyses of natural as well as technical scientific texts, it has been demonstrated by means of statistical surveys on language that the degree of subject-specificity in the text increases along with a higher percentage of specialised vocabulary in comparison to the total word count (Baumann 1994: 128ff.). As a consequence of this, text comprehension would be rendered more difficult for laymen, since they would have to activate several contexts of knowledge in their memories.

In contrast to the obligatory stylistically neutral elements of technical texts, the optional semantic stylistic means indicate individual contributions to the reflection process of the respective specialised reality (rationality vs. emotionality, metaphors, metonymy and others) when defining terms (synonymy, polysemy and others) and/or in the case of a combination of certain characteristics (hyperonym - hyponym).

A comparative analysis of lexical-semantic stylistic means in social, natural, and technical science texts has shown that the percentage and the diversity of optional semantic stylistic means is much higher in social science texts than in the other two academic spheres, where inner logic and mandatory precision during the linguistic implementation of technical contents entail a more economical use of stylistically relevant elements (Baumann \& Kalverkämper 1992, 2004; Kalverkämper \& Baumann 1996).

\section{Summary}

Our recent interdisciplinary empirical analyses of different LSP text forms (scientific article, monograph, students' textbook), from various social (linguistics, history, law, economy), natural scientific (psychology, medicine) and technical 
sciences (technology and materials, audio engineering), from oral, written and electronic media and from German and English languages have led to several new insights, some of which prove to be extremely significant for further interdisciplinary specialist thinking research.

\section{References}

Baumann, Klaus-Dieter (1992). Integrative Textlinguistik. Forum für Fachsprachen-Forschung 18. Tübingen: Gunter Narr Verlag.

Baumann, Klaus-Dieter (1994). Fachlichkeit von Texten. Deutsche Hochschulschriften 1023. Egelsbach, Frankfurt a.M, Washington: Hänsel-Hohenhausen.

Baumann, Klaus-Dieter (2001). Kenntnissysteme im Fachtext. Deutsche Hochschulschriften 1193. Egelsbach, Frankfurt a. M., München, New York: Hänsel-Hohenhausen.

Baumann, Klaus-Dieter/Kalverkämper, Hartwig (eds.) (1992). Kontrastive Fachsprachenforschung. Forum für Fachsprachen-Forschung, 20. Tübingen: Gunter Narr Verlag.

Baumann, Klaus-Dieter/Kalverkämper, Hartwig (eds.) (2004). Pluralität in der Fachsprachenforschung. Forum für Fachsprachen-Forschung, 67. Tübingen: Gunter Narr Verlag.

Bochenski, Joseph Maria (1993). Die zeitgenössischen Denkmethoden. 10th edition. UTB 6. Budin, Gerhard (1996). Wissensorganisation und Terminologie. Forum für FachsprachenForschung, 28. Tübingen: Gunter Narr Verlag.

Buhlmann, Rosemarie/Fearns, Anneliese (2000). Handbuch des Fachsprachenunterrichts. 6th edition. Tübingen: Narr Studienbücher.

Breuer, Ingeborg (2001). Das 20. Jahrhundert-Projekt: Kultur- und Geisteswissenschaften. Hamburg: Rotbuch Verlag.

Clyne, Michael (1987). Cultural differences in the organization of academic texts. Journal of Pragmatics, 11, 217-247.

Clyne, Michael (1993). Pragmatik, Textstruktur und kulturelle Werte. Eine interkulturelle Perspektive. In: Hartmut Schröder (ed.), Fachtextpragmatik. Forum für FachsprachenForschung (pp. 3-18). Tübingen: Gunter Narr Verlag.

Fischer, Peter (2004). Philosophie der Technik. UTB 2504. München: Fink.

Fleck, Ludwik (1994). Entstehung und Entwicklung einer wissenschaftlichen Tatsache. 3. Berlin: Suhrmamp Verlag.

Fluck, Hans-Rüdiger (1997). Fachdeutsch in Naturwissenschaft und Technik. Heidelberg: Groos.

Gloy, Karen (1996). Das Verständnis der Natur. Bd. 2: Die Geschichte des ganzheitlichen Denkens. München: $\mathrm{CH}$ Beck.

Grmek, Mirko D. (ed.) (1997). Die Geschichte des medizinischen Denkens. München: CH Beck.

Haken, Hermann/Haken-Krell, Maria (1997). Gehirn und Verhalten. Stuttgart: DVA.

Hoffmann, Lothar (1976). Kommunikationsmittel Fachsprache. Eine Einführung. Berlin: Akademie-Verlag.

Hoffmann, Lothar (ed.) (1978). Sprache in Wissenschaft und Technik. Leipzig: Enzyklopädie. 
Hoffmann, Lothar (1984). Kommunikationsmittel Fachsprache. Eine Einführung. 2nd revised version. Berlin: Akademie Verlag.

Hoffmann, Lothar/Piotrowski, Rajmund G. (1979). Beiträge zur Sprachstatistik. Leipzig: Enzyklopädie.

Jahr, Silke (2000). Emotionen und Emotionsstrukturen in Sachtexten. Berlin, New York: Mouton de Gruyter.

Kalverkämper, Hartwig (ed.) (1998). Fachsprachen in der Romania. Forum für Fachsprachenforschung 8. Tübingen: Gunter Narr Verlag.

Kalverkämper, Hartwig/Baumann, Klaus-Dieter (eds.) (1996). Fachliche Textsorten. Forum für Fachsprachen-Forschung 25. Tübingen: Gunter Narr Verlag.

Krings, Hans P. (ed.) (1996). Wissenschaftliche Grundlagen der Technischen Kommunikation. Forum für Fachsprachenforschung 32. Tübingen: Gunter Narr Verlag.

Kromrey, Helmut (2002). Empirische Sozialforschung. 10th. edition. UTB 1040. Stuttgart: Springer Verlag.

Lauren, Christer/Nordman, Marianne (1996). Wissenschaftliche Technolekte. Nordeuropäische Beiträge aus den Human- und Gesellschaftswissenschaften 10. Frankfurt a. M.: Peter Lang.

Lyre, Holger (2002). Informationstheorie. UTB 2289. Fink: München.

Miller, G.A. (1962). Some psychological studies of grammar. American Psychologist, 17, 748-762.

Möller, Georg (1983). Warum formuliert man so? Leipzig: Bibliographisches Institut.

Müsseler, Jochen/Prinz, Wolfgang (eds.) (2002). Allgemeine Psychologie. Spektrum: Heidelberg.

Nischik, Reingard. M. (1991). Mentalstilistik. Tübingen: Gunter Narr Verlag.

Nycz, Krzysztof/Baumann, Klaus-Dieter/Kalverkämper, Hartwig (eds.) (2016). Fachsprachenforschung in Polen. Forum für Fachsprachen-Forschung 127. Berlin: Frank \& Timme.

Otto, Jürgen H./Euler, Harald A./Mandl, Heinz (2000). Emotionspsychologie. Weinheim: Psychologie Verlags Union.

Pauen, Michael/Roth, Gerhard (eds.) (2001). Neurowissenschaften und Philosophie. UTB 2208. Wilhelm Fink: München.

Pörksen, Uwe (1986). Deutsche Naturwissenschaftssprachen. Forum für FachsprachenForschung 2. Tübingen: Gunter Narr Verlag.

Prim, Rolf/Tilmann, Heribert (1997). Grundlagen einer kritisch-rationalen Sozialwissenschaft. 7. UTB 221. Wiebeisheim: Quelle \& Meyer Verlag.

Redder, Angelika/Rehbein, Jochen (eds.) (1999). Grammatik und mentale Prozesse. Tübingen: Stauffenburg.

Riesel, Elise/Schendels Josifovna Evgenija (1975). Deutsche Stilistik. Moskow: Verlag Wissenschaft.

Sandig, Barbara (1986). Stilistik der deutschen Sprache. Sammlung Göschen 2229. Berlin, New York: Mouton de Gruyter.

Satzger, Axel (ed.) (1999). Sprache und Technik. Forum Angewandte Linguistik 36. Frankfurt a. M.: Peter Lang.

Skudlik, Sabine (1990). Sprachen in den Wissenschaften. Forum für Fachsprachen-Forschung 10. Tübingen: Gunter Narr Verlag.

Thielmann, Winfried (1999). Fachsprache der Physik als begriffliches Instrumentarium. Arbeiten zur Sprachanalyse 34. Frankfurt a.M: Peter Lang. 
Van Doren, Charles (1996). Geschichte des Wissens. München: Fink.

Zimmer, Dieter E. (1999). So kommt der Mensch zur Sprache. 5. München: Heyne. 
Open-Access-Publikation im Sinne der CC-Lizenz BY-NC-ND 4.0

(C) 2020, Vandenhoeck \& Ruprecht GmbH \& Co. KG, Göttingen ISBN Print: 9783847111863 - ISBN E-Lib: 9783737011860 


\title{
Politische Begriffe als Herausforderung für die Didaktik der Angewandten Linguistik
}

\begin{abstract}
Political concepts as a challenge to the didactics of applied linguistics

The primary purpose of this chapter is to offer a brief presentation of selected challenges which applied linguists, especially those who are also this subject's teachers, face due to the intricacies of the sphere of politics. Secondly, a concise reflection on the means and ways of didactic handling of the concepts in question is intended. Its ultimate goal should be the development of a global concept competence. This contribution also presents the student sub-competences to be targeted, especially competence in political semantics and the competence in the use of lexicographical aids such as special dictionaries.
\end{abstract}

Keywords

political concepts, didactics, concept competence, competence in political semantics, competence in the use of lexicographical sources

\section{Einleitung und Aufbau'}

Der deutsche Philosoph Jakob Sigismund Beck hielt 1794 im Gefolge seines großen Lehrers Immanuel Kant, unter vielem anderen, Folgendes fest: „Unser Verstand ist ein Vermögen der Begriffe, das heißt, der Vorstellungen der Objecte nur durch einige Merkmale" (Beck 1794: 301). Begriffen kommt also ein eminenter Status im kognitiven Apparatus des Menschen zu, weil sie ihm die Auseinandersetzung mit der Komplexität der Welt erleichtern oder gar ermöglichen (vgl. Trimmel 2004: 168). ${ }^{2}$ Ganz allgemein kann die Fähigkeit zur Begriffsbildung als eine Schlüsselkompetenz (Nünning 2008: pass.) in der kognitiven Auseinandersetzung mit der Umwelt (Jannidis \& Winko 2008: 64), unter anderem in

\footnotetext{
1 Es sei an dieser Stelle ein besonders herzliches Dankeswort der immer hilfsbereiten Frau Joanna Ziemska vom Zentrum für Translationswissenschaft der Universität Wien für das Gegenlesen des Typoskripts gewidmet.

2 Trimmel bezieht sich seinerseits auf Lenneberg (1977: 406f.) und Wessells (1994).
} 
Lernprozessen, aber auch beim Verfolgen des in die Sphäre der Begriffsbildung stark eingreifenden sozial resonanzfähigen Geschehens, angesehen werden.

Je mehr man sich die Funktionen von Begriffen (Ordnungsfunktion, Kommunikationsfunktion, Bewertungsfunktion, Appellfunktion; Patzelt 2013: 81f.) vergegenwärtigt, je besser man in sich die Erkenntnis resonieren lässt, dass Begriffe „Fähigkeiten des Unterscheidens von Gegenständen“ darstellen (Ros 1997: o. S.), umso stärker reift in uns höchstwahrscheinlich das Postulat einer angeleiteten und strukturierten Begriffsarbeit in der jeweiligen Fachdidaktik heran, was insbesondere für viele in der Öffentlichkeit mehr oder minder stark echoende Begriffe (wegen ihrer Spezifik, die noch zur Sprache kommt) reklamiert werden kann.

Wohlgebildete Begriffe perforieren also stark die Matrix unserer Wirklichkeitsanschauung und erweitern damit unser Generalisierungs- und Unterscheidungsvermögen (vgl. Trimmel 2004: 168), was einerseits vor kognitiv-emotiver Überforderung schützt (vgl. Wessells 1994, rezipiert bei Trimmel 2004: 168) und andererseits den Grad der individuellen Resistenz gegen manipulative Übergriffe von mit einer gewissen Begriffsbildungsmacht ausgestatteten Akteuren nur zu steigern vermag. Begriffe sind einerseits konsensuell gesehen mentale Gebilde, die an ein kognizierendes Individuum gebunden sind, andererseits schimmert aus den vorangehenden Zeilen ein soziales Gepräge ihrer Konstitution hervor. Ohne sich auf ein längeres Referat dazu einlassen zu können, sei im Schlepptau von Seiler (2012: 11f.) von zwei interdependenten - wie der deutsche Kognitionspsychologe es nennt - Existenzweisen von Begriffen auszugehen, einer personalen und einer konventionellen, und dabei ihre Verzahnung, ihre enge Interrelationalität hervorzuheben. Dabei ist stets der individuelle Beitrag zur Begriffsbildungsleistung gebührend zu berücksichtigen, denn Individuen partizipieren nicht unwesentlich an der Konstitution von konventionellen Begriffen, „indem sie ausgewählte mentale Begriffe oder Bestandteile von ihnen an sprachliche Ausdrücke" andocken und gewöhnlich durch die Interaktion mit den Zeitgenossen einen (unterschiedlich ausgeprägten) Beitrag zu ihrer Standardisierung und Konventionalisierung leisten (ebd.: 11). Zu beachten ist überdies, dass „die konventionelle Bedeutung sprachlicher Ausdrücke ein ideeller Näherungswert ist, dem sich jeder Verwender mehr oder weniger annähert oder den er in seinem Sinn verändert" (ebd.). Womit klar aufgezeigt wird, dass wir es mitunter mit einer merklichen Amplitude an semantischen Approximationswerten zu tun haben können, denen mit einer verstärkten "Sonographie“ der jeweiligen Materialisierungen der Auf-den-Begriff-Bringens-Akte begegnet werden muss. Es liegt für Seiler (ebd.) nahe, „dass Sprachbedeutung im pragmatischen Verwendungskontext gleichzeitig und untrennbar idiosynkratische und konventionelle Aspekte“ aggregiert. Das Zoon politikon lebt folglich sein kognizierendes Wesen im Spannungsfeld zwischen individuellem und suprain- 
dividuellem Wissen aus, oder präziser gesagt zwischen Wissen und Informationen - als „in Zeichen objektivierte[m] Wissen“ (Seiler 2004: 303), welchem vor der Inkorporation in die geistige Welt eines Individuums das Charakteristikum der Virtualität zugeschrieben werden muss (ebd.: 304) - oder noch anders betrachtet: zwischen mentalen Begriffen und semantisierten, i. e. konventionell versprachlichten (Ortner 2017: 290) resp. in eine andere semiotische Form gegossenen Begriffen (auch wenn man sich an der Metaphorizität oder „Exteriorisierung" des vorwiegend konsensuell durch das Merkmal mental konzeptualisierten Begriff-Begriffs stören mag).

Das Hauptaugenmerk des vorliegenden Beitrags liegt, wie im Titel angekündigt, auf Begriffen, die dem Revier des Politischen (prototypisch) entstammen bzw. in ihm prototypisch zirkulieren ${ }^{3}$ (Kilian 2018: 261) und einige Leitbilder der mit gewichtigen Herausforderungen einhergehenden didaktischen Arbeit mit fraglichen Begriffen, im Zuge derer es zu einem produktiven Austausch zwischen idiosynkratischen und konventionalisierten Bedeutungsbildungsstimuli kommen kann.

Eine relativ greifbare Antwort auf die Frage, welche Begriffe nun mit dem Prädikat politisch gleichsam hinaufgeadelt werden können, wird freilich von dem Verständnis von Politik beeinflusst (Spieß 2020: 302f.), bei dem man grundsätzlich in diverse Blickrichtungen schielen kann. Anzupeilen ist daher eine möglichst weitgreifende Auffassung von Politik, auf die in Kap. 2 eingegangen wird. Die uns interessierende Myriade von Begriffen stellt aufgrund ihrer Eigenschaften, von denen in Kap. 3 kursorisch berichtet wird, eine Herausforderung für jede Didaktik dar, im Besonderen für die Fremdsprachendidaktik, wie sie beispielsweise im Studium der Angewandten Linguistik mit einem germanistischen Schwerpunkt ihren Verlauf nimmt und nicht nur mit der (universellen) Natur von politischen Begriffen, so z. B. mit ihrer (restringierenden) Standortgebundenheit, sondern darüber hinaus mit der immanenten Notwendigkeit eines national profilierten kulturellen Vergleichs zu „kämpfen“ hat. Der Nervus Rerum des vorliegenden Beitrags ist mit bestimmten Anforderungen an die Didaktik verbunden, die in Kap. 4 gestreift werden. Vor dem Hintergrund der angerissenen Probleme wird in Kap. 5 über Mittel und Wege nachgedacht, wie man der sich nun abzeichnenden Herausforderung im Hinblick auf die anvisierte Zielgruppe, d. h. nicht-muttersprachliche Studierende der Angewandten Linguistik, realistisch beikommen kann. Eine (aus Platzgründen minimalistische) Ausschau rundet den Artikel ab. Hingewiesen sei noch darauf, dass die in diesem Beitrag zur Diskussion gestellten Überlegungen lediglich einen globalen und konzeptvorbereitenden Charakter haben.

3 Dabei ist ihr stenotopes Vorkommen auch in anderen Kommunikationsbereichen nachweisbar. 


\section{Was ist Politik?}

Die Fülle an Zugängen zum Wesen der Politik einem Überblick zuzuführen, muss das Prärogativ der politologischen Literatur bleiben. In vielen Einführungen in die Politikwissenschaft findet man nämlich eine Auswahl von Politik-Definitionen, z. B. bei Meyer (vgl. 2010: 38) oder Patzelt (vgl. 2013: 20 f.), so dass man sich ein ungefähres Bild davon machen kann, wie bunt der Regenbogen der Definitionen gestaltet werden kann. Wichtig ist jedoch bei alldem mit Meyer (vgl. 2010: 39) zu betonen, dass die anzutreffenden definitorischen Unterschiede eher aus der Perspektivierung von bestimmten Merkmalen oder ihren Präsuppositionen als aus einer gänzlich divergierenden Antwort auf die der Politik geltenden ti-esti-Frage (Wille 2019: 452) resultieren:

Bereits die Durchsicht und der Vergleich [...] berühmter Definitionen des Politischen läßt erkennen, daß die Unterschiede weniger aus unüberbrückbaren Gegensätzen im Kern der Definitionen resultieren. Sie ergeben sich vielmehr daraus, welches ihrer Elemente oder welche ihrer in der Definition selbst unausgesprochenen Voraussetzungen jeweils in besonderer Weise herausgehoben und beleuchtet werden (Meyer 2010: 39).

Ich möchte dennoch auf die von dem heuer von uns gegangenen Sir Roger Scruton, in einem zeitlich nicht fern liegenden Zeitungsinterview dargelegte Fasson von Politik aufmerksam machen:

It's not the imposition of conformity from above by some sanctified elite, which is essentially the liberal position, but it's a form of continuous discussion between diverse and possibly conflicting interests, an attempt to conciliate, to arrive at a solution acceptable to everyone, which requires institutions like parliaments and Congress and so on and a rule of law, committees, and all the rest. So it's about procedure, and it sees politics as aiming to conciliate rival interests rather than to impose conformity (Scruton 2018: o. S.).

Dies ist freilich nicht die einzige hervorhebenswerte, im öffentlichen Disput stehende Auffassung von Politik (immerhin ist sie adversativ formuliert), doch sie scheint ziemlich stark die Rolle einer lösungsorientierten ${ }^{4}$ Streitkultur zu pointieren, die über diverse Modi, Mechanismen und Institutionen über die Bühne geht. Einer Streitkultur, die unausweichlich und auch ratsam erscheint, wenn man sich das Diktum Peter Sloterdijks „Politik ist der Schmerz, der entsteht, wenn andere Leute anderes wollen“ (Sloterdijk 2018: o. S.) zu Herzen nimmt und gleichzeitig mitreflektiert, dass dieser Schmerz in einem beträchtlichen Grad durch Sprache zum Ausdruck kommen muss.

4 In Anbindung an Mantl (1984: 4), der die den Regeln der Demokratie gehorchende Politik als Verständigung und Einigung konzeptualisiert. 


\section{Politische Begriffe als Krux}

Die von Scruton vertretene Auffassung von Politik setzt u. a. das Operieren mit Begriffen voraus, die in eine lebendige Streitkultur eingebunden werden. Begriffe im Metier der Politik stellen „Interpretationsangebote sozialer Wirklichkeit mit impliziten Handlungsfolgen“ dar (Kopperschmidt 1991: 83); sie sind also selbstverständlich keineswegs als sterile, in Laborbedingungen destillierte Deutungen zu konsumieren, sondern als durch politisch motivierte Interessen und andere Determinanten figurierte und somit perspektivierte, sich dennoch durch bestimmte rekurrente Eigenschaften auszeichnende Ensembles an relevanten Mosaiksteinen der Bedeutungsbildung. Gleichzeitig sind sie ob ihrer Knappheit gleichsam begehrenswert, so dass um die Hoheit über sie ein Wettkampf geführt werden kann (Kopperschmidt 1991: 84).

Um die angesprochenen Charakteristika (im Überflug) auszumachen, lauschen wir vorerst einer synoptisch orchestrierten Sinfonie an (vorwiegend) einzelfallbezogenen und doch mehr oder weniger verallgemeinerbaren, mitunter mit einem zeitgeistigen Laut vertonten Aussagen zu politischen Begriffen:

- „Es gibt kaum einen politischen Begriff, dem so unterschiedliche Deutungen gegeben werden wie dem der Demokratie“ (Detjen 2009: 151).

- „[...] heute [werden] - womöglich mit Absicht - viele politische Begriffe auf den Kopf gestellt [...]“ (Wegner 2018: o. S.).

- „Es gibt wohl nur wenige politische Begriffe, die so häufig verwendet und gleichzeitig so unbestimmt sind wie die Mitte" (Jaschke 1992: 55).

- „I doubt that any political term has occasioned more confusion over the last few years than ,populism'. In many ways, it is a word in search of a definition" (Kimball 2018: 4).

- „Kaum ein politischer Begriff ist so umstritten wie der des Terrorismus“ (Daase 2013: 336).

- „Kaum ein Begriff hat sich in der Politikwissenschaft wie in den öffentlichen Diskursen in den letzten Jahren einer solchen Aufmerksamkeit erfreut wie der der Globalisierung“ (Zimmer 2008: 176).

- „Kaum ein Begriff löst derzeit solche gesellschaftlichen und politischen Emotionen aus wie ,Austerität““ (Sturm \& Griebel \& Winkelmann 2017: 1).

- „Wie so viele politische Begriffe steht auch der Begriff der öffentlichen Meinung unter dem Zauber einer langen Tradition“ (Luhmann 2009: 163).

Viele Begriffe scheinen in einem bestimmten Sinne exzeptionelle Entitäten $\mathrm{zu}$ sein, was nahezu phrasenhafte Formulierungen wie die oben angeführten als auch zahlreiche andere dieser Sorte, für die es hier keinen Platz gibt, suggestiv nahelegen. Die Zitate sind generalisierte Aussagen über die durch (Sprach)Wissenschaftler und Nichtwissenschaftler diagnostizierten/reprodu- 
zierten Eigenschaften von politischen bzw., allgemeiner gesagt, in öffentlichen Diskursen zirkulierenden Begriffen. Sie geben insgesamt ein interessantes Bild der politischen Begriffswelt bzw. Sprache in der Politik ab, wobei bei einer tiefer gehenden Einsicht in die ko(n)textuelle Einbettung der Begriffe noch mehr an Strukturen der Konzeptualisierung der Sprache in der Politik und mithin des Politischen insgesamt freigelegt werden könnte. Es lässt sich als eine vorläufige Bilanzierung der obigen (zwangsläufig rudimentären, dennoch repräsentativen) Zitate-Kollektion ein Pool an einschlägigen Prädizierungen über Begriffe in der Domäne der Politik zusammenstellen: (i) Deutungs- und Umdeutungsoffenheit (mitunter als Missbrauchsanfälligkeit interpretierbar), (ii) Vagheit bzw. Ambiguität, (iii) Konfusionsanfälligkeit/Missverständlichkeit, (iv) Kontroversialität, (v) Aufmerksamkeitskanalisierungspotenzial (gepaart mit signifikant hoher Gebrauchsfrequenz), (vi) Emotionalisierungspotenzial, (vii) Geschichts- und Denktraditionsgebundenheit.

Dieser Minikatalog strebt freilich keine Vollständigkeit an Eigenschaften der Begriffe wie auch der Sprache in der Politik an; in der politolinguistischen wie auch politologischen Literatur lassen sich sicher elaboriertere Übersichten finden. Das Spektrum an in Berührung mit Begriffen und Begrifflichkeiten des politischen Universums gewonnenen Eindrücken, wie sie sich in den soeben zitierten Einlassungen abzeichnen, dürfte sich jedoch durch generalisierende Urteile in der politikdidaktischen Literatur im Großen und Ganzen erhärten lassen. So merkt zum Beispiel Juchler (vgl. 2008: 172) an, dass politische Begriffe sich einer Einrasterung in stringent angeordnete Begriffssysteme nicht beugen. Es kann nach Ansicht des Forschers nicht bestritten werden, dass bestimmte Begriffe auf iterative Erscheinungen wie Diktatur, Demokratie oder Außenpolitik verweisen, allerdings verhindern raumzeitlich disparate Determinanten der Politik eine der Systematik und Hierarchisierung verpflichtete begriffliche Ordnung des politikbezogenen Wissensinputs (ebd.). Die politische Welt lässt sich nach Juchler (ebd.) als „schwach strukturierte Domäne“ beschreiben, die auf kein strenges Begriffsgefüge festgenagelt werden kann und der „die Deutungsvarianz der politischen Begriffe" eigen ist. Diese Variabilität lässt sich selbstverständlich im synchronen wie auch im diachronen Schnitt verorten (vgl. ebd.). Eine Herausforderung beim Erwerb von politischen Begriffen mag in den Augen des Gelehrten unter anderem dem semantischen Wandel geschuldet sein, welchen sie infolge von sich ändernden historischen Umständen durchmachen (ebd.). Eine weitere Verkomplizierung stellen für Juchler (ebd.) etwa die Positionsabhängigkeit von Begriffen wie auch ihre ideologische Vereinnahmung dar; man spricht in diesem Zusammenhang in der Politolinguistik nicht von ungefähr von ideologischer Polysemie.

Rohlfes (2005: 70) macht uns auch darauf aufmerksam, dass historische (und ebenfalls politische) Begriffe nicht isolationistisch, sondern immer in Begriffs- 
feldern zu denken sind, deren Unkenntnis eine angemessene Handhabung von Begriffen wesentlich erschwert. Die Vorteile der begriffsfeldsensitiven Perspektivierung liegen auf der Hand. Ein gewichtiger Vorteil liegt, mit den Worten von Rohlfes gesprochen, in der Schärfung des Profils des jeweiligen Begriffs: „Der Begriff ,Revolution' z.B. gewinnt ein deutlicheres Profil, wenn seine Nachbarschaft zu Begriffen wie Revolte, Staatsstreich, Machtwechsel ins Blickfeld tritt“ (ebd.). ${ }^{5}$ Dass der dabei erfolgende Erwerb fachgebundenen Wissens in einen fachexternen Unterricht (i. e. im Studium der Angewandten Linguistik) entsprechend $\mathrm{zu}$ integrieren ist, versteht sich von selbst.

Vor dem Hintergrund des Vorgetragenen ist es wohl keine Übertreibung, in Anlehnung an die Geschichtsdidaktik sogar von Begriffskompetenz zu sprechen, die die Lernenden u. a. in die Lage versetzen sollte, „aus fachspezifischen Begriffen und den dahinter stehenden Kategorien und Konzepten ,semantische Netze [...] zu bilden sowie den Wandel von Begriffen zu erkennen“ (Hellmuth \& Klepp 2010: 129). Und die Auflistung von Teilkompetenzen ließe sich noch erweitern (vgl. Sauer 2015: $8^{6}$ ), etwa um den entlang der kulturellen Trennachse verlaufenden Vergleich von Begriffen. ${ }^{7}$

\section{Anforderungen an die Didaktik}

\subsection{Hin zur Ambiguitätskompetenz}

Juchler (2008: 172f.) sagt mit Blick auf die schulische politische Bildung (was sich ebenfalls auf die DaF-Hochschuldidaktik ausdehnen lässt), dass es ihr darauf ankommt, „die epochal und perspektivisch variierenden Bedeutungen von Begriffen zu thematisieren“, denn es ist gerade die Befassung „mit dem je unterschiedlichen Begriffsgebrauch, die je eigene Interpretation von Texten“, die den Subjekten des Lernens „im hermeneutischen Prozess die Erlangung eines adäquaten Verständnisses des Begriffs“ ermöglichen (ebd.: 173). Aus den vorstehenden Zeilen spricht eine beträchtliche Materialfülle. Hinzu kommt die Schwierigkeit, dass Ambiguität, wie sie z. B. im Dunstkreis des Politischen vor-

5 Behilflich können hierbei Fachlexika sein, die die Möglichkeit haben, Anliegerbegriffe zumindest anzuzeigen. Hierzu ein Beispiel aus dem Wörterbuch von Bayer/Wende (1995: 479) betreffend das Lemmazeichen Revolution: „Abzugrenzen ist der R.sbegriff gegen $\rightarrow$ Evolution als organische Entwicklung, gegen die Revolte als mißlungene R. und gegen die von vorr.nären Kräften getragene Gegenrevolution sowie gegen den $\rightarrow$ Staatsstreich oder $\rightarrow$ Putsch."

6 Sauer zitiert aus Alavi (2004: 41), die ihrerseits aus Grzesik (1992) schöpft.

7 In der interkulturellen Kommunikation zeigen sich beispielsweise bedeutsame Unterschiede zwischen scheinbaren „Europäismen“ wie Liberalismus, Nationalstaat oder Zivilgesellschaft, die zum semantischen Nominalismus verleiten können (Leonhard 2011: 109). 
kommt, leicht den Verwirrungs- und Zerstreuungseffekt mit sich bringen kann, so dass die Didaktik in die Rolle eines Aufmerksamkeitskanalysators zu schlüpfen hat. Mit ihrer reflektierenden, ordnenden, strukturierenden und integrierenden Herangehensweise kann sie dafür sorgen, dass der Kognizierende in dem anströmenden Datenmeer nicht ertrinkt (vgl. Bolz 2010: o. S.), dass die Komplexität begrifflicher Architekturen ihn nicht überwältigt, d. h. statt eines Conceptual Change ist manchmal die Erreichung eines Basisverständnisses ausreichend, das von einem fachlich ausgereiften Konzept durchaus abweichen mag (vgl. Sauer 2015: 8). Doch eine gewisse Irritation und Konfusion kann dem Lerner nicht erspart bleiben, sie ist gar notwendig, um die Ambiguität erlebbar und dadurch integrierbar (also nicht zuletzt „aushaltbar“) zu machen. Mit der in die Sphäre des Politischen eingeschriebenen Mehrdeutigkeit und ihren Implikationen (wie semantischer Dubitativität ${ }^{8}$ ) haben Sprachlerner in einem großen Maße zu kämpfen, daher kann man das von Ulrich (2018) formulierte, der muttersprachlichen Didaktik des Deutschen geltende Desiderat der Priorisierung der Entfaltung von Ambiguitätskompetenz umso mehr auf den DaF-Unterricht herunterbrechen. Ohne auf Komponenten dieser Kompetenz einzugehen - Ulrich (2018: 20) fasst darunter u. a. Ambiguitätstoleranz, Ambiguitätsdeutung und Ambiguitätsauflösung zusammen - sei den Ausführungen des Gelehrten noch die Fähigkeit angefügt, einerseits selbst internalisierte Unterscheidungen und Unterschiede, auf die die ersteren zurückführbar sind, sowie andererseits einem extern „zugespielte“ Unterscheidungen (wie sie beispielsweise in der Publizistik, in Wörterbüchern oder Enzyklopädien fixiert sind) und sie antezedierende Unterschiede nachzuvollziehen oder zu kritisieren. ${ }^{9}$

8 In Anlehnung an die Lexikologie des semantischen Zweifels von Jörg Kilian, die in zahlreichen Beiträgen abgehandelt worden ist (bspw. Kilian 2003).

9 Die Nuancierung in Unterscheidung und Unterschied ist von der Philosophin Katrin Wille (2018: 461) entlehnt worden, für die die Logik dieser Sonderung darin besteht, dass Unterscheidungen, als Bewusstseinsakte (mithin als eine psychologische Kategorie zu verstehen), Unterschiede, als existente Eigenschaften der Wirklichkeit (somit als eine ontologische Kategorie aufzufassen), erkennbar werden lassen. Wille (ebd.) verweist dabei auf die Möglichkeit, dass so manche Unterscheidungen gegebene Unterschiede nicht in Rechnung stellen (oder sie gar verbergen), so dass dann ein Anlass entsteht, diese Unterscheidungen einer Kritik zu unterziehen, die sich eben der Unterscheidung in Unterschied und Unterscheidung bedient. Abgesehen von diesem Fall, nämlich dass Unterscheidungen die einen Unterschiede perspektivieren und die anderen kaschieren (vgl. ebd.: 462), gibt es aber daneben andere Erscheinungsweisen von Unterscheidungen. Manche Unterscheidungen bringen es mit sich, dass wir auf einen Unterschied gestoßen werden, den wir früher nicht wahrgenommen haben (vgl. ebd.: 461). Eine andere Ausprägung von Unterscheidungen, die Wille erwähnt, betrifft die Macht der Gewohnheit, die bestimmten Unterscheidungen inhärent sein kann, so dass man zum Beispiel bei einem Abrücken von diesen Unterscheidungsgewohnheiten den ontologischen Status von Unterschieden mit Zwiespalt zu sehen geneigt sein kann (vgl. ebd.: 462). Und schließlich können Unterscheidungen ihre konstruktive Seite zur Schau stellen, indem sie „Neues in die Welt bringen“ (ebd.: 462). Die Relevanz der Unterscheidungskunst für Lerner, 


\subsection{Ziele, Ressourcen und „Übungsgelände“}

In der Einleitung war von einer realistischen Didaktisierung der angeklungenen Problematik die Rede. Bezogen auf die Realität des Studiums der Angewandten Linguistik hat sich das weitere Räsonnement über das in den Raum gestellte Thema vorerst an drei Leitplanken zu orientieren: (a) Welche Teil- bzw. Subziele können im Lehrbetrieb verfolgt werden?; (b) Welche Ressourcen lassen sich zur Erreichung der anvisierten Ziele ertragreich auswerten?; (c) Welche Lehrveranstaltungen kommen für die vorgeschlagene Thematik in Frage? Freilich ist der relevante Fragenkatalog damit noch nicht erschöpft, mehr kann der vorliegende bescheidene Beitrag jedoch nicht stemmen (auch der Antwortenskopus kann keine große Alternativenbreite aufzeigen).

Zielsetzungen, auf die hingesteuert werden kann bzw. sollte, hängen zum Teil mit didaktischen Ressourcen zusammen, die in den Lehreinheiten zum Einsatz kommen können. Und dies sind probate Medien der Wissenstransmission wie z. B. lexikographische Publikationen und (essayistische) Publizistik oder aber auch diverse Textsorten der politischen Kommunikation. Quellen, die eine umfangreichere Recherche voraussetzen würden, sind zwar immer von Nutzen, müssen allerdings mit dem jeweiligen Lehrveranstaltungskonzept ob eines möglicherweise hohen Zeit- und Arbeitsaufwands akkordiert werden. Würde es sich um ein politolinguistisches Seminar handeln, so empfähle es sich, bei Übernahme eines begriffszentrierten Themas für die als Abschlussleistung vorsehbare Semesterarbeit stets Rückgriff auf extensive Korpora zu nehmen, und zwar nicht nur „zum Drüberstreuen“, sondern zweck- und methodengeleitet. In einem sprachpraktischen Unterricht sollten hingegen Korpora und andere Quellen wie z. B. Publikationen vom Volumen eines Buches allenfalls als komplementäre Fundstellen zur fakultativen Nutzung vorgeschlagen werden. Somit sind wir bei den für die Behandlung der angerissenen Problematik in Frage kommenden Lehreinheiten angelangt. Neben den vorgenannten Lehrveranstaltungen können etwa Seminare zur Semantik, zur Lexikologie und Lexikographie oder u. U. auch zur Landeskunde als gangbare „Übungsparcours“ anvisiert werden, die nun in den Betrachtungsradius der folgenden didaktikrelevanten Überlegungen mit eingehen.

auch in Konfrontation mit den im Reich des Politischen kursierenden Unterscheidungsangeboten ist hoffentlich mit diesem kurzen Exkurs einigermaßen deutlich herausgestellt worden, so dass ihr im Unterricht folglich ein gebührlicher Raum zugewiesen wird. 


\section{Fingerzeige für die Didaktik}

Wenden wir uns nun auf denkbare Seminarziele zurück, aus denen eine schmale Auswahl getroffen werden muss. Lexikologisch-semantische Qualifikationen rezeptiver und produktiver Natur weiterzuentwickeln, schwebt als erstrangiges desiderables Ziel von didaktischen Anstrengungen vor. Dazu ist auch der Erwerb des relevanten Sach- und Fachwissens vonnöten. Da in allen Lehrveranstaltungen Wörterbücher, Lexika und Enzyklopädien Verwendung finden sollten bzw. gar müssen, leuchtet unmittelbar ein, dass man den Ausbau der Wörterbuch- bzw. der lexikographischen Kompetenz als ein weiteres verfolgenswürdiges Ziel am Horizont der Lehrveranstaltungskonzepte aufscheinen lassen kann. Diese so allgemein und verschwommen formulierten Ziele bedürfen freilich einer Präzisierung bzw. Zerlegung in Teilziele, was auch einige Zeilen später geschieht. Angemerkt sei noch ganz generell, dass ein nützliches Arbeitswerkzeug zur Erreichung dieser Zielvorstellungen in Gestalt üblicherweise praktizierter Strategien des Unterscheidens vorliegt. Dass Unterscheiden enorm wichtig ist, insbesondere wenn man begründet annehmen kann, dass das Unterscheidungsvermögen beeinträchtigt ist, wird nicht zuletzt in Platons Sophistes deutlich vor Augen geführt (vgl. Wille 2018: 409). In Anknüpfung an Platons Werk führt Wille (ebd.: 443) vier Praktiken des Unterscheidens auf, nämlich: das Einteilen (ebd.: $444 \mathrm{ff}$.) (oder - wenn man den von Wille primär informell gedachten Praxisbereich verlässt und ins Wissenschaftliche hinüberwechselt - Klassifizieren; ebd.: 445), das Kontrastieren (ebd.: 447ff.), das Bestimmen (ebd.: 452ff.) und schließlich das Differenzieren (ebd.: $454 \mathrm{ff}$.), die hinsichtlich ihres Adaptationspotenzials für analytische Operationen in didaktischen Arrangements auf den Prüfstand zu stellen sind. Vorerst sei festgehalten, dass sie nicht (nur) solitär, sondern (auch) in synergetischem Verbund einzusetzen sind. So kann zum Beispiel die sich in der „ti-est-Frage“ (Wille 2019: 452) verdichtende Technik des Bestimmens (Was ist Populismus?) die Operation des Kontrastierens mit auf den Plan rufen (Populismus vs. Extremismus). ${ }^{10}$

10 Die beiden Techniken sind keineswegs monodimensional zu betrachten. Das Kontrastieren lässt sich nicht nur als Aufstellen von kontradiktorischen oder konträren Gegenbegriffen auffassen, die nicht zuletzt in der Lexikologie gut eingeführt sind, sondern einzubeziehen sind dabei überdies subkonträre Entgegensetzungen, also „solche partikulär bejahenden und partikulär verneinenden Aussagen“, die den Eindruck erwecken, im Widerspruch zueinander zu stehen, die gleichwohl beide parallel zutreffend sein mögen (Wille 2018: 451). Was das Bestimmen angeht, so schärft Wille (ebd.: 452) unser Augenmerk dafür, dass begriffliche Demarkationslinien nicht nur durch Definitionen sichtbar gemacht werden können. Vor allem aus didaktischer Perspektive erscheint es geboten, begriffliche Gemarkungen ebenfalls durch „Ergänzungen oder Entfaltungen von Definitionen mit dem Zweck, Missverständnissen vorzubeugen", abzustecken (ebd.). Eine nicht minder begriffsbildungsstimulierende Möglichkeit liegt in dem Versuch, „die Leistungskraft der Grenzziehungen, die bei Defini- 


\subsection{Politiksemantische Teilkompetenzen}

In Bezug auf die sukzessive Komplementierung der semantischen Tüchtigkeit von Studierenden ist es ratsam, einen unterscheidungs- und unterschiedssensitiven Aufgabenkomplex zu konzipieren, der auf die bi- bzw. multilateral ausgelegte Sensibilisierung für die zeitlich und diskursspezifische Diversität der Semantik der politischen Lexie und damit für die ihnen eigene Komplexität der semasiologischen Struktur (im Anschluss an Kilian 2001: 45) im synchronen wie auch im diachronen Zuschnitt abzielt (vgl. Kubiak 2020: 56). Dabei geht es u. a. um die Weckung des Bewusstseins dafür, „daß die Polysemie sog. ideologischer Ausdrücke [...] durch statische (bzw. synchronische) Gegenüberstellungen oder auch durch merkmalsanalytische Charakterisierungen sprachgeschichtlich nur sehr unzureichend erfaßt wird und daher eine Skizzierung des Argumentationsablaufs für die Erfassung der Geschichte politischen Handelns unabdingbar ist“" (Stötzel 1995: 15; vgl. Kubiak 2020: 56). Unvermeidlich ist im Rahmen des Zielunterrichts die Veranschaulichung des Phänomens von Kampfbegriffen, also von Entitäten, die von bestimmten politischen Gruppierungen gebraucht werden, die „entweder einen wichtigen Stellenwert in der Feindmarkierung haben“ [...] oder politisch umkämpft werden, um „die Verankerung eigener Deutungen in möglichst breiten Kreisen der Gesellschaft" zu erreichen (Gießelmann et al. 2016: 9; vgl. Kubiak 2020: 57). Dass die Unterscheidungsfertigkeit im Spiel ist, braucht wohl nicht eigens betont zu werden.

Die politiksemantischen Forschungen erschöpfen sich manchmal nicht in einzellexematischen Eskapaden, sondern stellen sich im Nachhinein als umgreifende Unternehmungen heraus, die offenlegen, welche Wörter aus dem Kampf mit ihnen und um sie als Sieger hervorgehen, die den Gegner bzw. die Gegner zwar nicht total verbannt, aber auf jeden Fall die Oberhand über ihn und damit einen dominante Positionen auf der öffentlich wirksamen Kampfarena gewonnen haben: „Dominanz erlangen Bezeichnungen und Bedeutungen meist nicht einzelwortspezifisch, sondern im Verbund ganzer politischer Wortfelder“ (Klein 1989: 29). Hier wären Aufgabenstellungen sinnvoll, die die drei in der Politolinguistik usuell unterschiedenen Arten von lexikalisch-semantischen Rivalitäten zwischen Opponenten im politischen Rampenlicht thematisieren, also denotative Lesarten-Konkurrenz, evaluative Lesarten-Konkurrenz und last but not least Nominationskonkurrenz (Girnth 2015: 74ff.).

tionen vorgenommen werden, in Bezug auf naheliegende Verwechslungen zu demonstrieren “ (ebd.). 


\subsection{Wörterbuch- und lexikanutzungsbezogene Teilkompetenzen}

Als Grundlage der Arbeit in diesem Teilbereich können sog. zeitgeschichtliche Wörterbücher und Fachlexika vereinnahmt werden. Die erstere Bezeichnung geht auf Schaeder (2005: 40) zurück, der darunter solche Publikationen versteht, die sich u. a. der Aufgabe annehmen, „die in den allgemeinen Sprachwörterbüchern unzureichend erläuterte politische Lexik semantisch-ideologisch auszuleuchten" (vgl. Kubiak 2020: 51). Solche Nachschlagewerke warten u. a. mit generalisierungs- und unterscheidungsförderlichen Einblicken in repräsentative Ausprägungen der axiologisch-ideologischen Variationsbreite der Semantik von bestimmten Wörtern aus dem öffentlich-politischen Mundus auf, die dann in didaktischen Kontexten entsprechend ausgewertet werden können (vgl. Kubiak 2020: 51). Von daher erscheint es als zweckmäßig zu demonstrieren, wie man mit der in exempli causa aufzugreifenden Publikationen archivierten soziopolitisch relevanten Lexik gewinnbringend (nicht nur gesteuert, sondern auch autodidaktisch) arbeiten kann und welche Kompetenzzugewinne dabei zu erwarten wären. ${ }^{11}$ Es dürfte plausibel sein, dass im Hinblick auf das Ziel der Wörterbuchkompetenz nicht nur an klassische Pfeiler der Wörterbuchdidaktik wie etwa die Schärfung des Sensoriums für die Funktionen der jeweiligen lexikographischen Publikation oder die Vervollkommnung der Fähigkeit zur Identifizierung und Erkundung der Makro- und Mikrostruktur des Wörterbuchs (bzw. eines Lexikons) zu denken ist (Schaeder 2000: 261f. u. 267ff.), sondern auch daran, Studierende durch den Vergleich von Begriffserläuterungen an der Erfahrung teilhaben zu lassen, dass man in lexikographischen Nachschlagewerken unterschiedlichen und/oder unterschiedlich präsentierten Konzeptualisierungen eines bestimmten Begriffs begegnen kann (vgl. Kühberger 2008: 71) und dass „man die meist allgemeinen Erklärungen in Lexika [...] ,wenden', das heißt auf die spezielle Situation, die man gerade bearbeitet, beziehen [muss]“ (ebd.).

Für die Hinzuziehung von Fachlexika spricht die Tatsache, dass sie das konsensual objektivierte Wissen einer Fachsozietät darbieten und damit Wissensstabilisierung und -kanonisierung mit herbeiführen lassen (Horn 2012: 352). In Anbetracht der Voraussetzung, dass „der in einem Wörterbuch gebotene Begriffsapparat mit dem Fachwortschatz einer Diskursgemeinschaft identisch [ist] “ (ebd.), dürfte es möglich und zugleich erstrebenswert sein, Einblicke im die

11 Beispiele für konkrete didaktisch verwertbare, auf eine sich u. a. zeitgeschichtlichen Wörterbüchern widmende, (vorzugsweise spezial-)lexikographische Lehrveranstaltung zugeschnittene und durch Exkurse zur politischen Semantik flankierte (wie auch aufgrund ihrer monolingualen Ausrichtung für die Bedürfnisse einer kontrastiven Betrachtung in einem nichtmuttersprachlichen Studium der Angewandten Linguistik adaptierungsbedürftige) Vorschläge zur Umsetzung der gerade aufgerollten Zielsetzungen finden sich in Kubiak (vgl. Kubiak 2020: $58 \mathrm{ff}$.$) .$ 
Denk- und Erfassungsweisen einer bestimmten Wissens- und Handlungsgemeinschaft zu gewinnen, womit man Zugang zu einem konventionalisierten/ konsensualisierten Begriffssubstrat bekäme, von dem man als Individuum (im Hinblick auf mentale Begriffsbildung, d. h. Conceptual Change oder Conceptual Enrichment; vgl. Sauer 2019: 14f.) schöpfen kann und muss. Die hineinprojizierbare Konsensualiät darf freilich nicht den Blick dafür verstellen, dass die soziale Größe „Wissens- und Handlungsgemeinschaft“ durchaus kein unisono „singendes“ Kollektiv sein kann und abgesehen davon pluralfähig ist.

Es dürfte im Zusammenhang mit der skizzierten Zielsetzung für Studierende von Belang sein, zu eruieren, wie bestimmte Nachschlagewerke besonders heikle, diskursiv sensitivierte oder nahezu zu einem Politikum herangeschwollene Wörter beschreiben, d. h. welche Informationen über ihren Gebrauchswert (Kilian 2017: 27) dem Wörterbuch- und Lexikanutzer in lexikographischen Publikationen angeboten werden und inwiefern sie seine präsupponierten Nachschlagebedürfnisse saturieren mögen (vgl. Kubiak i. E., in Anknüpfung an Kilian 2003a: 113 u. 123, 2003b: o. S., und Kilian 2017: 27).

\section{Ausschau}

Didaktische Konfigurationen, in die sich politische Begriffe einflechten lassen, d.h. auf konkrete Lehrveranstaltungen im Studienprogrammangebot abgestimmte Thematisierungssettings und Arbeitstechniken, auf die hier nicht eingegangen werden kann und deren Erörterung einem anderen Beitrag vorbehalten bleiben muss, mögen variieren, sie können jedoch alle aus den knapp skizzierten Handreichungen schöpfen. Die allgemein gehaltenen und methodischer Umsetzungskonzepte harrenden Hinweise stellen zumindest einige kognitive Profite in Aussicht, die es sich durch geeignete Arrangements und Ressourcen herauszuschlagen lohnt, denn mit ihnen ausgerüstet lässt es sich auf dem Wege zur Entfaltung der für angehende Lehrer und Übersetzer so erstrebenswerten Begriffskompetenz im kritisch-sensiblen Bereich der politischen Kommunikation leichter voranschreiten.

\section{Literaturverzeichnis}

Alavi, Bettina (2004). Begriffsbildung im Geschichtsunterricht. Problemstellungen und Befunde. In: U. Uffelmann/M. Seidenfuß (Hrsg.), Verstehen und Vermitteln. Armin Reese zum 65. Geburtstag (S. 39-61). Idstein: Schulz-Kirchner.

Bayer, Erich/Wende, Frank (1995). Wörterbuch zur Geschichte. Begriffe und Fachausdrücke. Stuttgart: Kröner. 
Beck, Jacob Sigismund (1794). Erläuternder Auszug aus den critischen Schriften des Herrn Prof. Kant auf Anrathen desselben. Zweiter Band. Riga: Hartknoch.

Bolz, Norbert (2010). Journalismus: Orientierung ist gefragt. Kölner Stadt-Anzeiger 11.05. 2010. https://www.ksta.de/journalismus-orientierung-ist-gefragt-12224498.

Daase, Christopher (2014). Terrorismus. In: B. Enzmann (Hrsg.), Handbuch Politische Gewalt. Formen - Ursachen - Legitimation - Begrenzung (S. 335-348). Wiesbaden: Springer Fachmedien.

Detjen, Joachim (2009). Die Werteordnung des Grundgesetzes. Wiesbaden: VS Verlag für Sozialwissenschaften.

Gießelmann, Bente/Kerst, Benjamin/Suermann, Lenard/Virchow, Fabian (Hrsg.) (2016). Handwörterbuch rechtsextremer Kampfbegriffe. Schwalbach/Ts.: Wochenschau.

Girnth, Heiko (2015). Sprache und Sprachverwendung in der Politik. Eine Einführung in die linguistische Analyse öffentlich-politischer Kommunikation. Berlin u. a.: de Gruyter.

Grzesik, Jürgen (1992). Begriffe lernen und lehren. Psychologische Grundlage: Operative Lerntheorie. Unterrichtsmethoden: Typische Phasen. Unterrichtspraxis: Kommentierte Unterrichtsprotokolle. Stuttgart u. a.: Klett.

Hellmuth, Thomas/Klepp, Cornelia (2010): Politische Bildung. Geschichte - Modelle Praxisbeispiele. Wien u. a.: Böhlau.

Horn, Klaus-Peter (2012). Erziehungswissenschaftliche Lexika. In: K.-P. Horn/H. Kemnitz/ W. Marotzki/U. Sandfuchs (Hrsg.), Klinkhardt Lexikon Erziehungswissenschaft. Bd. 1 (S. 352-353). Bad Heilbrunn: Klinkhardt.

Jannidis, Fotis/Winko, Simone (2008). Begriffsbildung. In: V. Nünning (Hrsg.), Schlüsselkompetenzen: Qualifikationen für Studium und Beruf(S. 64-77). Stuttgart: J.B. Metzler.

Jaschke, Hans-Gerd (1992). Politische Richtungsbegriffe im Wandel: Neue Linke, Neue Rechte - Gibt es auch eine Neue Mitte? In: K. Guggenberger/K. Hansen (Hrsg.), Die Mitte (S. 55-73). Wiesbaden: Springer Fachmedien.

Juchler, Ingo (2008). Politische Begriffe der Außenpolitik. Konstituenten von Fachkonzepten und Political Literacy. In: G. Weißeno (Hrsg.), Politikkompetenz. Was Unterricht zu leisten hat (S. 169-183). Wiesbaden: VS Verlag für Sozialwissenschaften.

Kilian, Jörg (2001). Kritische Semantik. Für eine wissenschaftliche Sprachkritik im Spannungsfeld von Sprachtheorie, Sprachnorm, Sprachpraxis. Zeitschrift für Germanistische Linguistik, 29, 293-318.

Kilian, Jörg (2003a). Vom Elend in der deutschen Sprachnorm. Zum lexikographischen Umgang mit Menschen aus dem Ausland. Prolegomena für eine kritische Semantik im Wörterbuch. Unveröffentlichtes Manuskript, 109-141.

Kilian, Jörg (2003b). Wörter im Zweifel. Ansätze einer linguistisch begründeten kritischen Semantik. Linguistik online, 16 (4). http://www.linguistik-online.de/16_03/kilian.html.

Kilian, Jörg (2017). Nationale Stereotype und Ethnosemantica im Wörterbuch. In: A. Burkhardt/T. Niehr/J. Zhao/D.U. Kim (Hrsg.), Sprachpflege und Sprachkritik als gesellschaftliche Aufgaben (S. 27-32). Frankfurt a. M. u. a.: Peter Lang.

Kilian, Jörg (2018). Politische Semantik, interkulturelle „Hotwords“ und didaktische Sprachkritik. In: A. Fábián/I. Trost (Hrsg.), Sprachgebrauch in der Politik. Grammatische, lexikalische, pragmatische, kulturelle und dialektologische Perspektiven (S. 261277). Berlin u. a.: de Gruyter.

Kimball, Roger (2018). Introduction: Populism and its critics. The New Criterion, 36(5), 4. https://newcriterion.com//issues/2018/1/introduction-populism-its-critics. 
Klein, Josef (1989). Wortschatz, Wortkampf, Wortfelder in der Politik. In: J. Klein (Hrsg.), Politische Semantik. Bedeutungsanalytische und sprachkritische Beiträge zur politischen Sprachverwendung (S. 3-50). Opladen: Westdeutscher Verlag.

Kopperschmidt, Josef (1991). Soll man um Worte streiten? Historische und systematische Anmerkungen zur politischen Sprache. In: F. Liedtke/M. Wengeler/K. Böke (Hrsg.), Begriffe besetzen. Strategien des Sprachgebrauchs in der Politik (S. 70-88). Opladen: Westdeutscher Verlag.

Kubiak, Paweł (2020). Fachdidaktische Vorschläge zur Auswertung von Allbüchern mit zeitgeschichtlicher Ausrichtung im Studium Deutsch als Fremdsprache. Zielsprache Deutsch, 47 (3), 51-67.

Kühberger, Christoph (2008). Basiskonzepte der Politischen Bildung positionieren. Informationen zur Politischen Bildung, 29, 69-73.

Lenneberg, Eric H. (1977). Biologische Grundlagen der Sprache. Frankfurt a.M.: Suhrkamp. Leonhard, Jörn (2011). Politisches Sprechen im Zeitalter der Extreme. Überlegungen zu einer Erfahrungsgeschichte der Moderne. In: M. Sabrow (Hrsg.), Zeiträume. Potsdamer Almanach des Zentrums für Zeithistorische Forschung, 2010 (S. 107-126). Göttingen: Wallstein.

Luhmann, Niklas (2009). Soziologische Aufklärung 5. Konstruktivistische Perspektiven. Wiesbaden: VS Verlag für Sozialwissenschaften.

Mantl, Wolfgang (1984). Demokratische Verständigung. politicum, 5(22), 4-5.

Meyer, Thomas (2010). Was ist Politik? Wiesbaden: VS Verlag für Sozialwissenschaften.

Nünning, Vera (2008). Einleitung. In: V. Nünning (Hrsg.), Schlüsselkompetenzen: Qualifikationen für Studium und Beruf (S. 1-19). Stuttgart: J.B. Metzler.

Ortner, Hanspeter (2017). Semiotisierung und Semantisierung von Erfahrung, Weltsicht und Wissen in literarischen Texten. In: A. Betten/U. Fix/B. Wanning (Hrsg.), Handbuch Sprache in der Literatur (S. 290-309). Berlin u. a.: de Gruyter.

Patzelt, Werner J. (2013). Einführung in die Politikwissenschaft. Grundriss des Faches und studiumbegleitende Orientierung. Passau: Richard Rothe.

Rohlfes, Joachim (2005). Geschichte und ihre Didaktik. Göttingen: Kohlhammer.

Ros, Arno (1997). Was ist Philosophie? Vortrag in Leipzig am 12. Dezember 1997. Leipzig 1997. http://jrjs.bplaced.net/www/Anderes/Arno\%20Ros/content/t97a.htm.

Sauer, Michael (2015). Begriffsarbeit im Geschichtsunterricht. Geschichte lernen 2015/28 (168), 2-11.

Sauer, Michael (2019). Begriffslernen und Begriffsarbeit im Geschichtsunterricht. Frankfurt/ M.: Wochenschau.

Schaeder, Burkhard (2005). Studienbibliographie germanistische Linguistik. Frankfurt a. M. u. a.: Peter Lang.

Scruton, Roger (2018). „Maoist students“vs „terrified policemen“- „I found myself on the side of the latter" (Interview geführt von Hugo Gurdon). Washington Examiner, 23. Okt., https://www.washingtonexaminer.com.

Seiler, Thomas Bernhard (2004). Wissen und Wissensverarbeitung aus humanwissenschaftlicher Perspektive. In: G. Jüttemann (Hrsg.), Psychologie als Humanwissenschaft. Ein Handbuch (S. 302-317). Göttingen: Vandenhoeck \& Ruprecht.

Seiler, Thomas Bernhard (2012). Evolution des Wissens. Band II: Evolution der Begriffe. Münster: LIT. 
Sloterdijk, Peter (2018). Die Sitten verwildern, die Gerechtigkeit ist obdachlos (Interview geführt von René Scheu). Neue Zürcher Zeitung, 30. März. https://www.nzz.ch/feuille ton/wir-erleben-ein-grosses-gleiten-ld.1370201. 


\title{
Joanna Kic-Drgas / Joanna Woźniak (Uniwersytet im. Adama Mickiewicza w Poznaniu)
}

\section{Fachsprachendidaktik an europäischen Hochschulen - Stand und Perspektiven ${ }^{\top}$}

\begin{abstract}
Languages for specific purposes at European universities - Current situation and perspectives

This chapter discusses the status and developmental trends in the didactics of languages for specific purposes at European colleges and universities. It presents the findings of the European project „LSP Teacher Training Summer School“ (TRAILs), which aims to develop an innovative LSP teacher training curriculum. The chapter is divided into two parts: theoretical and empirical. First of all, the aim, the scope and the history of the development of didactics of languages for specific purposes are presented. Subsequently, the data collected by means of a survey conducted in 25 European countries are analysed. The presented findings indicate a lack of standardisation of the forms and contents of didactics of languages for specific purposes at European universities.
\end{abstract}

\section{Keywords}

didactics of languages for specific purposes, LSP-didactics, tertiary education, languages for specific purposes, Europa, curriculum

\section{Einführung ${ }^{2}$}

Angesichts der fortschreitenden Spezialisierung der Wirtschaftsbranchen sowie der Intensivierung der internationalen Zusammenarbeit im beruflichen Umfeld gewinnt die Kenntnis von Fremdsprachen immer mehr an Bedeutung. Sowohl

1 Der Beitrag entstand als Folge der im Rahmen des Projekt TRAILs durchgeführten Analysen der Studienprogramme zur Feststellung des Status der Fachsprachendidaktik an europäischen Hochschulen und Universitäten. Die im Rahmen des Projekts geführten Forschungsaktivitäten sind im Rahmen des Programms Erasmus+ der Europäischen Union (Re: 2018-1-FR01KA203-048085) gefördert.

2 Den Beitrag widmen wir Frau Professor Izabela Prokop und überbringen somit unseren herzlichen Glückwunsch zu Ihrem Geburtstag. Wir wünschen viel Gesundheit und Genuss, weiterhin viel Schaffenskraft, Erfolg und Freude. Mit dem projektorientierten Beitrag möchten 
auf beruflicher als auch zunehmend auf privater Ebene reicht dabei die Beherrschung der allgemeinen Fremdsprache nicht mehr aus. Vor diesem Hintergrund entsteht ein erhöhter Bedarf an Fachfremdsprachenlehrkräften, welche die entsprechenden fachbezogenen Sprachkenntnisse vermitteln.

Um dieser sich herauskristallisierenden Nachfrage angemessen zu entgegnen, etablierte sich im akademischen Kontext ein neuer praxisorientierter Bereich die Fachsprachendidaktik. Im vorliegenden Beitrag wird die Situation der Fachsprachendidaktik an den Hochschulen und Universitäten in Europa mit einem besonderen Fokus auf Polen skizziert. Zudem sollen vorhandene Tendenzen sowie Perspektiven der Entwicklung der Fachsprachendidaktik aufgezeigt werden.

Dazu wird zunächst als theoretische Einführung in die Thematik auf die Definition der Fachsprachendidaktik (Abschnitt 2), ihre bisherigen Entwicklungsetappen (Abschnitt 3) sowie auf die Unterrichtsformen (Abschnitt 4) eingegangen. Des Weiteren werden die Ergebnisse der durchgeführten Untersuchung von curricularen sowie institutionellen Entwicklungen im Bereich der Fachsprachendidaktik auf dem tertiären Bildungsniveau in Europa (Abschnitt 5) dargestellt. Sowohl im theoretischen Teil des Beitrags als auch in den präsentierten Ergebnissen der Untersuchung wird dem Status der Fachsprachendidaktik in Polen besondere Beachtung geschenkt. Den Beitrag runden verschiedene, sich aus der Analyse ergebende Schlussfolgerungen und die Darstellung der Entwicklungsperspektiven für die Fachsprachendidaktik an europäischen Hochschulen und Universitäten ab.

\section{Fachsprachendidaktik - definitorische Ambiguität}

Die Formulierung einer eindeutigen Definition für die Fachsprachendidaktik bereitet zahlreiche Probleme, von denen die nachstehenden unterschiedlichen Betrachtungsweisen auf das Phänomen zeugen. Fluck (1992) betont die Kommunikationsfähigkeit als Hauptziel und wesentliche Aufgabe der Fachsprachendidaktik, weshalb eine Definition die Prinzipien des Fachsprachen- und des Fachwissenserwerbs und deren Vermittlung einbeziehen müsse: „Der Fachsprachendidaktik (geht es) vor allem um die Frage, welche Ausschnitte fachlicher Kommunikation und welche sprachlichen Mittel für die Vermittlung fachlicher Inhalte und die Produktion und Rezeption von Fachtexten relevant sind“ (Fluck 1992: 16). Dagegen differenziert Grucza (2010) eine enge und eine breite Perspektive der Fachsprachendidaktik:

wir Ihnen, verehrte Frau Professor, für Ihre Unterstützung und anhaltende Ermutigung zur Aufnahme von Projektaktivitäten bedanken. 
Die Fachsprachendidaktik stellt einen besonderen Teilbereich der allgemeinen Glottodidaktik (Sprachendidaktik) dar, woraus sich ergibt, dass der Forschungsgegenstand der Fachsprachendidaktik als ein besonderer Teilbereich des Forschungsgegenstandes der Glottodidaktik im Allgemeinen zu betrachten ist, wobei hier zwischen einem allgemeinen Forschungsgegenstand sensu largo und einem allgemeinen Forschungsgegenstand sensu stricte unterschieden werden kann. Den Gegenstand der Fachsprachendidaktik sensu largo bilden Lehrer, der Lerner und der Kommunikationskanal zwischen ihnen. Den Gegenstand sensu stricte bilden der Lerner und seine Fachsprachenfähigkeiten/Fachsprachkompetenz (sein Fachidiolekt) (S. Grucza 2010: 42).

Die von S. Grucza präsentierte Unterscheidung fokussiert vor allem die Personen des Lehrers und die des Lerners als Hauptakteure im Prozess der Vermittlung und des Erwerbs von Fachsprachen.

Buhlmann und Fearns (2018: 235) weisen auf die typischen, distinktiven Merkmale der Fachsprachendidaktik hin:

- Behandlung berufs(feld)- und arbeitsbezogener Inhalte,

- Vermittlung von Kenntnissen über kommunikative Regeln am Arbeitsplatz,

- Behandlung der sprachlichen Fertigkeiten.

Die oben genannten Elemente spiegeln die Natur der Fachsprachendidaktik als praxisbedingten (arbeitsbezogene Inhalte), kommunikationsorientierten (Kommunikation am Arbeitsplatz) und immer bedeutender werdenden, interdisziplinären Bereich der angewandten Sprachwissenschaft wider. Die festzustellende Evolution der Didaktik der Fachsprachendidaktik verläuft von einer Prozessorientierung hin zu einer individualisierten Lernerorientierung, die durch eine starke Berufs- und Interdisziplinaritätsbezogenheit charakterisiert ist.

Charakteristisch für die Fachsprachendidaktik ist ein intensiver Einfluss von Bezugsdisziplinen (Abb. 1), die zur starken Bipolarität des Bereichs beitragen. Einerseits äußert sich dieser durch eine disziplintheoretische Abhängigkeit von didaktischen Entwicklungen, andererseits durch fachsprachliche Erkenntnisse (Adams 2010: 74). Die erwähnte Ambiguität des Bereichs stellt einen wichtigen Kritikgrund bezüglich einer fehlenden Einheit sowohl im Definieren der Fachsprachendidaktik als auch in der Bearbeitung ihrer theoretischen Fundierung dar.

\section{Entwicklung der Fachsprachendidaktik}

Die Fachsprachenforschung etablierte sich als Teildisziplin der Angewandten Linguistik als Konsequenz der textlinguistischen und pragmatischen Fundierung der Disziplin in den 60er-und 70er-Jahren des 20. Jahrhunderts (mehr dazu in Spillner 1998). Oldenburg (1992: 10) unterscheidet drei Phasen in der Entwicklung der Fachsprachenforschung. In der ersten Phase konzentrierten sich die 


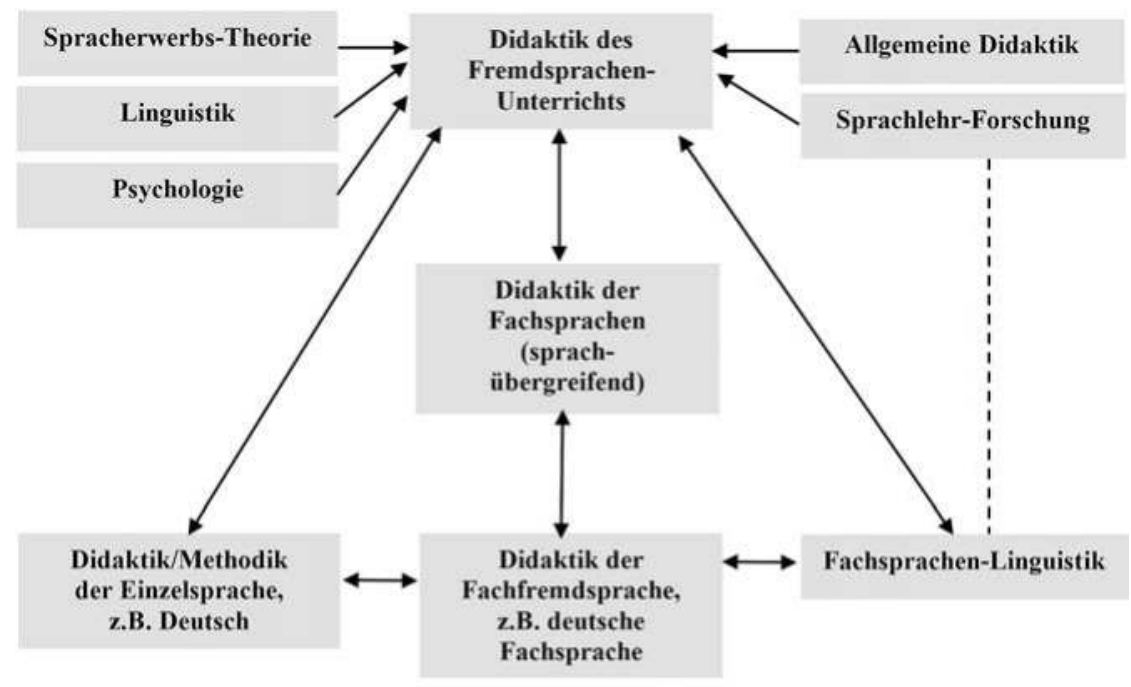

Ausgangssprachliche Einzelfach-Didaktik z.B. Physik
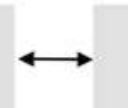

Methodik der EinzelfachSprachen, z.B. deutsche

Fachsprache Physik

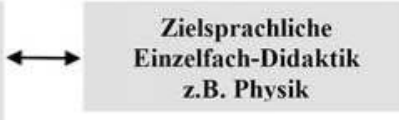

Zielsprachliche

z.B. Physik

Abb. 1: Systematisierung des fachsprachlichen Fremdsprachenunterrichts (nach Fluck 1992: 112)

quantitativen und qualitativen Studien auf die Beschreibung der Fachlexik als auffälligstes Merkmal der Fachsprachen (vgl. auch Hoffmann 1985: 112-115). Die zweite Phase zeichnete sich durch das zunehmende Interesse an den syntaktischen Strukturen der Fachsprachen aus. Dieses führte zur entscheidenden Ausdehnung des Forschungsbereichs der Fachsprachendidaktik. Die nachfolgende dritte Phase war durch das zunehmende Interesse an der Struktur und dem Inhalt von Fachtexten charakterisiert und stellte zugleich eine „pragmatischkommunikative Wende“ (Helbig 1986: 30) dar.

Mitte der 1980er-Jahre wurden zahlreiche Studien und Untersuchungen durchgeführt, die einen Überblick über den großen Bedarf an fachbezogenen Fremdsprachenkenntnissen in Industrie und Handel vermittelten (Oldenburg 1992: 14-15). Die angesprochenen Studien setzten sich zum Ziel, die in der Wirtschaft verwendeten Fremdsprachen, die jeweiligen Tätigkeitsfelder und die darin erforderlichen sprachlichen Fertigkeiten und Fähigkeiten zu ermitteln. Zusammen mit dem steigenden Bedarf an fremdfachsprachlichen Kenntnissen wuchs die Bedeutung ihrer entsprechenden Vermittlung, was zur Entstehung 
einer ,spezialisierten Unterrichtslehre' (Adams 2010: 75) beitrug. Es fehlte jedoch trotz zahlreicher fachsprachenmethodischer Ergebnisse an einer fachsprachendidaktischen Fundierung für die wichtigsten Felder der Fachsprachendidaktik (Fluck 2000: 3).

Gegenwärtig eröffnet die deutliche Interdisziplinarität der Fachsprachenforschung mit kommunikativ-kognitivem Fokus eine neue Perspektive auf die Didaktik und Methodik der Fachsprachenvermittlung. In Polen geht das Interesse an Fachsprachen und an deren intensiver Entwicklung auf den Anfang der 80erJahre des 20. Jahrhunderts zurück. Allerdings begann erst im Jahr 2000 ein landesweiter Prozess der Institutionalisierung der Fachsprachenforschung im tertiären Bereich und der Umsetzung der Ergebnisse in der Fachsprachendidaktik (S. Grucza 2010: 31-33, F. Grucza 2017: 239-240). Festzustellen ist vor allem eine Neugestaltung der Curricula und die Einführung von Fächern, die der Ausbildung qualifizierter Fachsprachenlehrkräfte dienen sollen. Auf dem Weg zur Etablierung der Fachsprachendidaktik auf tertiärem Niveau sollte betont werden, dass „Fachsprachen durch speziell ausgebildete Lehrer unterrichtet werden“ (S. Grucza 2010: 33) sollten. Darüber hinaus benötigt die Fachsprachendidaktik einen festen Platz in der universitären Bildungslandschaft (Zalipyatskikh 2017: 36-39, Prikoszovits 2017: 155).

\section{Formen der Vermittlung fachsprachlicher Inhalte}

Innerhalb der Fachsprachendidaktik haben sich unterschiedliche Unterrichtsformen etabliert, die den Erwerb und die Vermittlung von fachsprachlichen und berufsbezogenen Inhalten zum Ziel haben. Die Differenzierung dieser Formen führt häufig zu Missverständnissen. In Tabelle 1 wurden die drei Hauptunterrichtsformen des berufs- und fachbezogenen Fremdsprachenunterrichts und ihre Merkmale zusammengestellt.

Die besprochenen Unterrichtsformen kommen immer häufiger in der tertiären Bildung vor. Studienbegleitender Fremdsprachenunterricht wird in den nicht-philologischen Studienrichtungen angeboten, CLILiG an den technischen Universitäten. Fachsprachenunterricht bildet überwiegend an den philologischen Fakultäten ein Teil der Curricula. 
Tabelle 1: Unterschiede zwischen studienbegleitendem Fremdsprachenunterricht, Fachsprachenunterricht und CLILIG (Buhlmann \& Fearns 2018: 235)

\begin{tabular}{|l|l|l|}
\hline \multicolumn{1}{|c|}{$\begin{array}{c}\text { Studienbegleitender } \\
\text { Fremdsprachenunterricht }\end{array}$} & \multicolumn{1}{|c|}{ Fachsprachenunterricht } & \multicolumn{1}{|c|}{ CLILiG $^{3}$} \\
\hline $\begin{array}{l}\text { Entwicklung von Schlüssel- } \\
\text { qualifikationen und von } \\
\text { deren Gebrauch zur Bewäl- } \\
\text { tigung von Sprachaufgaben }\end{array}$ & $\begin{array}{l}\text { "elementbezogen, d. h., } \\
\text { kann die fachsprachliche } \\
\text { Grundlagenlexik gleichsam } \\
\text { punktuell und statisch ver- } \\
\text { mitteln, ohne die Problem- } \\
\text { orientierung des Fachun- } \\
\text { terrichts übernehmen zu } \\
\text { müssen“ Buhlmann \& Fea- } \\
\text { rns 2000:85) }\end{array}$ & $\begin{array}{l}\text { pädagogischer Ansatz, in } \\
\text { dem die Erst- (L1) und die } \\
\text { mittlung und zum Lernen } \\
\text { von Sachfächern dienen }\end{array}$ \\
\hline $\begin{array}{l}\text { Ziel: Erwerb berufsbezoge- } \\
\text { ner Fremdsprachenkennt- } \\
\text { nisse }\end{array}$ & $\begin{array}{l}\text { Ziel: Erwerb von Fertigkei- } \\
\text { ten, die es ermöglichen, } \\
\text { Fachtexte zu verstehen und } \\
\text { die Fachsprache adäquat zu } \\
\text { verwenden }\end{array}$ & $\begin{array}{l}\text { Ziel: Vermittlung von Sach- } \\
\text { fächern in der Fremdspra- } \\
\text { che }\end{array}$ \\
\hline
\end{tabular}

\section{Stand der Fachsprachendidaktik auf dem tertiären Bildungsniveau in Europa}

Im Europäischen Hochschulraum (EHEA ${ }^{4}$ ) wird immer mehr Wert auf die Qualität des Fremdsprachenunterrichts in Hochschulen und Universitäten gelegt. In einer Mitteilung vom 30.05.2017 über eine europäische Erneuerungsagenda für die Hochschulbildung hat die Europäische Kommission Folgendes festgestellt:

Es ist nicht einfach, gute Studienprogramme zu gestalten, aufzubauen und durchzuführen. Gute Lehrkräfte sind hierbei von entscheidender Bedeutung. $\mathrm{Zu}$ viele Hochschullehrkräfte sind nur wenig oder überhaupt nicht pädagogisch geschult, und systematische Investitionen in ihre kontinuierliche berufliche Entwicklung sind weiterhin die Ausnahme. Nationale und institutionelle Strategien, um guten Lehrkräften bessere Aufstiegsmöglichkeiten und Belohnungen zu bieten, sind inzwischen zwar weiter verbreitet, jedoch bei Weitem noch nicht die Norm (Europäische Kommission 2017).

Diese Feststellung bezieht sich auch auf die Fachsprachenlehrer. Das wachsende Interesse an Fachsprachen trägt zur Erhöhung des Angebots von Fachsprachenkursen an Universitäten und Hochschulen bei. Folglich beginnen Lehrer

3 Content and Language Integrated Learning in German (German language learning is combined with content from other subjects such as biology, physics, mathematics).

4 EHEA (European Higher Education Area), auf Deutsch: Der Europäische Hochschulraum ist ein durch die Bologna-Erklärung definierter Hochschulraum für Länder aus ganz Europa. 
häufig den Fachsprachenunterricht ohne spezielle fachsprachendidaktische Ausbildung.

Der Stand der Fachsprachendidaktik im europäischen Hochschulwesen war bisher ein weißer Fleck in der Fremdsprachenforschung. Diese Lücke wird jetzt teilweise durch die im Rahmen des Projekts TRAILs vorgenommenen Forschungen geschlossen. Nachfolgend werden die Hauptvoraussetzungen des Projekts geschildert und die Ergebnisse des ersten Forschungsziels - der Analyse der philologischen Studienprogramme an europäischen Hochschulen und Universitäten - präsentiert.

\subsection{Hauptvoraussetzungen des TRAILs-Projekts}

Das europaweite Projekt TRAILs (für: Erasmus+ „LSP Teacher Training Summer School ${ }^{\text {(5) }}$ ) zielt darauf ab, einen Beitrag zur Feststellung des Standes der Fachsprachendidaktik an europäischen Hochschulen und Universitäten zu leisten. ${ }^{6}$ Auf Grundlage der durchgeführten Bedarfsanalyse der Fachsprachenlehrer soll ein innovatives und in allen EHEA-Ländern umsetzbares Curriculum zur Fachsprachendidaktik erarbeitet werden.

Geplant wurden für das Projekt insgesamt fünf Forschungsschritte, die in den Jahren 2018 bis 2020 umgesetzt wurden und werden. Die erste Aufgabe, die die Analyse der Studienprogramme und Syllabi für künftige Fremd- und Fachsprachenlehrer voraussetzt, soll Ergebnisse liefern, ob Inhalte aus dem Bereich der Fachsprachendidaktik vermittelt werden und wenn ja, welche dies sind. Anschließend werden Fachsprachenlehrer aus ganz Europa zwecks der Ermittlung der Lehrerbedürfnisse befragt. Die im ersten und zweiten Schritt erhobenen Daten helfen bei der Identifizierung der Diskrepanzen zwischen dem Tatbestand und dem von den Fachsprachdidaktikern gewünschten Stand. Zudem tragen sie zur Entwicklung der Lernziele der Fachsprachendidaktik bei. Die zusammengestellten Lernziele bilden danach die Grundlage des Trainingsprogramms für die Sommerschule. Schließlich findet die Sommerschule für aktuelle und angehende Fachsprachendidaktiker im März 2021 in Zagreb (Kroatien) statt. Im Folgenden werden der methodologische Ansatz und die erzielten Ergebnisse des ersten Schrittes im Projektverlauf dargestellt.

5 Projektnummer 2018-1-FR01-KA203-048085.

6 An dem TRAILs-Projekt nehmen Forscher und Forscherinnen aus folgenden akademischen Institutionen teil: Universite de Bordeaux (Frankreich), Univerza V Ljubljani (Slowenien), Universita' Degli Studi Di Bergamo (Italien), Arcola Research Llp (Großbritannien), Universidad De Cadiz (Spanien), Sveuciliste U Zagrebu (Kroatien), Fakultet Strojarstva I Brodogradnje (Kroatien), Ade Hochschule Wilhelmshaven/Oldenburg/Elsfleth (Deutschland) und Adam-Mickiewicz-Universität (Polen). 


\subsection{Methodologische Grundlagen}

Mit dem ersten Schritt des TRAILs-Projekts wurde das Ziel verfolgt, den Stand der Lehrangebote zur Fachsprachendidaktik an Universitäten und Hochschulen des Europäischen Hochschulraumes zu untersuchen. Zu diesem Zweck wurden Studienprogramme überwiegend philologischer, humanistischer oder pädagogischer Fakultäten von 25 aus 48 EHEA-Ländern analysiert (Österreich, Belarus, Belgien, Bosnien und Herzegowina, Estland, Finnland, Frankreich, Großbritannien, Italien, Kroatien, Lettland, Litauen, Niederlande, Nordmazedonien, Deutschland, Polen, Portugal, Serbien, Slowakei, Slowenien, Spanien, Ukraine, Ungarn, Vatikan).

In der Anfangsphase erarbeiteten die Projektteilnehmer gemeinsam einen Fragebogen und übersetzten ihn in die Sprachen der teilnehmenden Länder. Der Fragebogen wurde in zwei Teile gegliedert. Mit dem ersten Teil wurden allgemeine Informationen zur Institution und zur Art des Lehrangebots ermittelt: Dazu zählten der Name der Universität/Hochschule und der Fakultät bzw. Abteilung, in welcher Studierenden Fachsprachendidaktik angeboten wird sowie Informationen zum Namen der Fremdsprachendidaktik-Einheit und der Form, in welcher die Fachsprache vermittelt wird (als vollständiger Studienkurs, als Modul oder als Fach innerhalb eines Studiengangs). Weitere Fragen griffen die erwarteten Lernergebnisse des Kurses auf, gaben Informationen zur durchschnittlichen Anzahl der teilnehmenden Studierenden und ermittelten, ob diese Einheit obligatorisch oder fakultativ ist.

Der zweite Teil des Fragebogens bezog sich auf den Aufbau und die Struktur der Fachsprachdidaktik-Einheiten. Damit wurden grundsätzlich Antworten auf folgende Fragen gesucht:

1. Im Rahmen welches Studienprogramms wird der Kurs angeboten (Bachelor, Master, Aufbaustudium, Promotionsstudium, andere)?

2. Können die Teilnehmer ein Zertifikat erwerben?

3. Wie vielen ECTS-Punkten entspricht das Programm?

4. Was sind die Zugangsvoraussetzungen für den Kurs (Qualifikationen, Voraussetzungen, Erfahrung)?

5. Welche Sprachen können die Teilnehmer nach Besuch des Kurses unterrichten?

6. In welcher Sprache wird der Kurs unterrichtet?

7. Welche Lehr- und Lernmethoden werden verwendet, um dieses Programm zu unterrichten?

8. Welche Lehr- und Lernmethoden werden in diesem Programm unterrichtet?

9. Welche Bewertungsmethoden werden zur Bewertung dieses Programms verwendet?

10. Welche Bewertungsmethoden werden in diesem Programm unterrichtet? 
11. Welche Informations- und Kommunikationstechnologien (IKT) werden verwendet, um dieses Programm zu unterrichten?

12. Welche Informations- und Kommunikationstechnologien (IKT) werden in diesem Programm unterrichtet?

13. Mit welchen Referenzmaterialien und Büchern wird dieses Programm unterrichtet?

14. Welches Referenzmaterial und welche Bücher empfiehlt das Programm den Teilnehmern für die Fachsprachendidaktik?

15. Welche Formen der praktischen Ausbildung bietet dieses Programm?

16. Wer ist für dieses Programm geeignet?

Die Fragebögen wurden aufgrund der öffentlich zugänglichen Daten, vor allem der Studienpläne, -programme sowie der Kurs-Syllabi von den Teilnehmern des TRAILs-Projekts selbst ausgefüllt. Falls sich einige Informationen den im Internet zugänglich gemachten Dokumenten nicht entnehmen ließen, wurde der Fragebogen an zuständige Personen der entsprechenden Hochschuleinrichtungen geschickt.

\subsection{Ergebnisse der Analyse - allgemeine Informationen}

Untersucht wurden insgesamt 1024 Hochschulinstitutionen aus 25 EHEA-Ländern. Die Umfrage umfasst 68 Hochschulinstitutionen aus 14 verschiedenen EHEA-Ländern, in welchen Studierenden Fachsprachendidaktik in unterschiedlicher Form angeboten wird. An einigen Hochschuleinrichtungen wurden mehrere Kurse mit Fachsprachendidaktik nachgewiesen, so dass sich die Gesamtzahl der Lehrangebote zur Fachsprachendidaktik insgesamt auf 88 beläuft. Im Folgenden werden nur die relevantesten und aufschlussreichsten Ergebnisse einer Online-Umfrage dargestellt, die die Partner an die zuständigen Personen der untersuchten Hochschuleinrichtungen geschickt haben bzw. alleine auf der Grundlage der im Internet auf den Internetseiten der Hochschuleinrichtungen zugänglich gemachten Daten ausgefüllt habe.

\subsubsection{Art der Lehrangebote zur Fachsprachendidaktik in Europa}

Die Mehrheit der angebotenen Lehreinheiten zur Fachsprachendidaktik sind kleinere Kurse, etwa Fächer oder Teile von Fächern. Oft werden Inhalte zur Fachsprachendidaktik im Rahmen der Fremdsprachendidaktik realisiert, dann aber in sehr begrenztem Umfang. Nur bei ca. 20 analysierten an den europäischen Universitäten und Hochschulen angebotenen Kursen handelt es sich entweder um vollständige Fachrichtungen oder um größere Module, die min- 
destens 10 ECTS-Punkte (also ungefähr 300 Stunden) garantieren. Allerdings können hier einzelne erhobene Daten fehlerhaft sein. Dies ist unter anderem auf terminologische Diskrepanzen in den Sprachen, in welchen die Umfrage durchgeführt wurde, zurückzuführen, aufgrund derer die Termini Kurs, Modul, Fach nicht immer richtig verstanden wurden. Dies wurde bei der Evaluation der Ergebnisse ersichtlich, nach denen einigen Einheiten sehr wenige ECTS-Punkte (wie etwa $0,1,5$ ) zuerkannt wurden, die aber an anderer Stelle als vollständige Kurse bezeichnet wurden. Daher gelten für uns bei der Beurteilung der Ergebnisse die garantierten ECTS-Punkte als maßgebend.

39 von 88 Kursen ( $44 \%$ ) sind für die Studierenden fakultativ und $24 \%$ obligatorisch. Im Falle von 28 Kursen (32\%) wurde diese Information nicht angegeben.

In der Regel wird die Fachsprachendidaktik auf Masterstudienniveau (59mal), in zwölf Fällen auf Bachelorstudienniveau und in einem Fall im Rahmen eines Promotionsstudiengangs realisiert. Fünf Mal wurden andere Leistungen wie Zertifikate genannt und in 13 Fällen wurde keine Antwort gegeben.

Ungefähr die Hälfte (52\%) der angebotenen Einheiten geben den Studierenden vollständige Qualifikationen zum Unterrichten der Fremdsprachen oder tragen teilweise zur Erreichung der Qualifikationen bei. $48 \%$ sind mit keinen Qualifikationen oder Zertifikaten verbunden.

Als Zugangsvoraussetzungen wurden meistens das entsprechende Sprachniveau, mindestens B2-C1, ein Bachelorabschluss und Grundkenntnisse der Fremdsprachendidaktik sowie abgeschlossene frühere Kurse genannt. Sonstige angegebene Zugangsvoraussetzungen wurden jeweils nur einmal genannt und sind damit für die Ermittlung des Standes der Fachsprachendidaktik von geringer Bedeutung.

\subsubsection{Art der Lehrangebote zur Fachsprachendidaktik in Polen}

In Polen wird Fachsprachendidaktik in unterschiedlicher Form an fünf Universitäten unterrichtet, und zwar an der:

1. Universität Warschau, Fakultät für Angewandte Linguistik, Institut für Angewandte Linguistik und Institut für Fachkommunikation und interkulturelle Studien,

2. Universität Rzeszów, Philologische Fakultät, Institut für Anglistik und Institut für Germanistik,

3. Nikolaus-Kopernikus-Universität Toruń, Philologische Fakultät, Lehrstuhl für Germanistik,

4. Maria-Skłodowska-Curie-Universität, Fakultät für Geisteswissenschaften, Institut für Germanistik und Institut für Angewandte Linguistik, 
5. Adam-Mickiewicz-Universität in Poznań, Neuphilologische Fakultät, Institut für Angewandte Linguistik, Fakultät für Anglistik, Institut für Englische Studien.

Insgesamt wurden elf verschiedene Formate ermittelt. In sieben Fällen wird Fachsprachendidaktik im Rahmen der Fremdsprachendidaktik realisiert. In vier Fällen wird der Fachsprachendidaktik ein getrenntes Fach gewidmet. Beachtenswert ist auch das Lehrangebot des Instituts für Fachkommunikation und interkulturelle Studien der Universität Warschau, in welchem Studierenden als zusätzliche Fachrichtung Fremd- und Fachsprachendidaktik zur Wahl steht.

Die Kurse zur Fachsprachendidaktik sind grundsätzlich obligatorisch, sobald sich ein Student für eine Fachrichtung oder ein Modul entscheidet, in dessen Rahmen Fachsprachendidaktik angeboten wird. Zehn von elf Kursen sind Teil eines Magisterstudiums, einer - an der Universität in Rzeszów - wird im Rahmen eines Bachelorstudiengangs durchgeführt. Nach Abschluss der Kurse erhalten allerdings nur diejenigen Studierenden eine Zulassung zum Unterrichten, die bereits ein Bachelorstudium in der Fachrichtung Didaktik abgeschlossen haben. Die zuerkannten ECTS-Punkte für die Kurse variieren zwischen 3 und 8, je nach dem mit den Kursen verbundenen Arbeitsaufwand (gemessen in Stunden).

\subsection{Ergebnisse der Analyse - Aufbau und Inhalt der Kurse}

Weitere Ergebnisse der Analyse umfassen die Sprachen, in welchen Fachsprachendidaktik geführt wird und welche die künftigen Arbeitssprachen der Kursteilnehmer sind. Gemäß den erzielten Umfrageergebnissen steht Englisch an der Spitze sowohl der Vermittlungssprachen der Fachsprachendidaktik als auch der künftigen Arbeitssprachen der Studierenden. In anderen Worten, $55 \%$ aller Fachsprachendidaktik-Programme, die Studierenden innerhalb des Europäischen Hochschulraumes angeboten werden, werden auf Englisch unterrichtet. Die Teilnehmer dürfen nach erfolgreichem Abschluss eines solchen Kurses Englisch unterrichten. Dem Englischen folgen das Deutsche und das Spanische mit einer relativ hohen Anzahl von Kursen. Mit etwas Abstand folgen die zwei Sprachen Russisch und Französisch. Weitere Sprachen sind grundsätzlich nur marginal repräsentiert.

Eine der anspruchsvollsten Aufgaben bei der Auswertung der Umfrage bestand in der Analyse der genannten Lernergebnisse. Da die Fachsprachendidaktik als Teil des Kurses der allgemeinen Fremdsprachendidaktik unterrichtet wird, waren die spezifischen Lernergebnisse ersterer oft mit allgemeinen Lernzielen und Lernergebnissen letzterer vermischt. Nach einer genaueren Untersuchung der Daten wurden die fachsprachendidaktischen Lernergebnisse in drei Gruppen geteilt: 


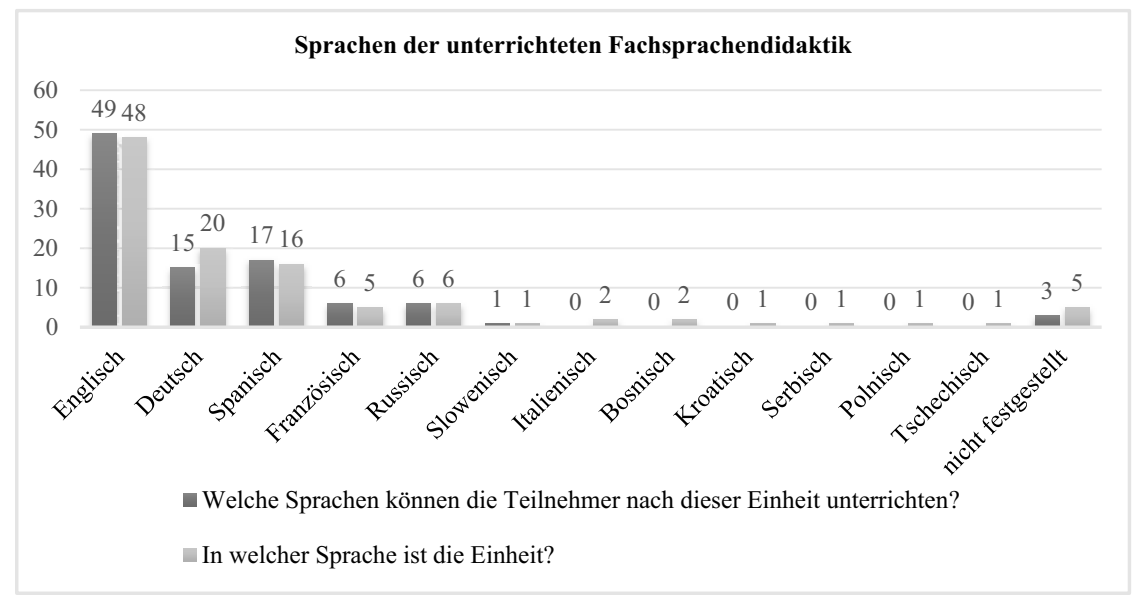

Abb. 2: Sprachen der unterrichteten Fachsprachenddidaktik

1. Wissen: Der Umfrage zufolge sollten nach dem Abschluss der Fachsprachendidaktik-Einheit folgende wissensbezogene Lernergebnisse erreicht werden:

- Identifizierung der konkreten Fachsprache und Kenntnis ihrer pragmatischen und kulturellen Aspekte,

- Kenntnis des aktuellen Forschungsstandes zur Fachsprachendidaktik und Nutzung des Wissens für die Unterrichtsprozesse,

- Kenntnis der Hauptterminologie und der erweiterten Terminologie aus dem Bereich der Methodik der Fachsprachen,

- Kenntnis der Methoden, Techniken und Instrumente der Fachsprachendidaktik,

- Kenntnis der Gemeinsamkeiten und Unterschiede zwischen Fremd- und Fachsprachendidaktik,

- Kenntnis der theoretischen Grundlagen der Fachsprachendidaktik, die sie von anderen verwandten Ansätzen unterscheiden, z. B. vom CLIL-Ansatz,

- Kenntnis der Textsorten, die in fachspezifischen Situationen Anwendung finden.

2. Didaktische Fähigkeiten: Von den Studierenden wird auch erwartet, dass sie nach dem Abschluss der Einheit fähig sein werden,

- den Fachsprachenunterricht kreativ, ziel- sowie bedürfnisorientiert zu planen,

- den Fachsprachenunterricht umzusetzen und zu bewerten,

- unterschiedliche Ansätze kritisch zu bewerten und diese an die Zielgruppe anzupassen, 
- Techniken für den Fachsprachenunterricht je nach Altersgruppe auszuwählen,

- Fachtexte kritisch zu bewerten und diese entsprechend dem geltenden Curriculum auszuwählen,

- Fachtexte mehrdimensional zu analysieren, um den Anforderungen der Fachsprachendidaktik Rechnung zu tragen.

3. Andere Kompetenzen: Von großer Bedeutung ist auch bei der Fachsprachendidaktik die Entwicklung sogenannter Soft Skills. Dazu gehören u. a.:

- Entwicklung der Lernerautonomie,

- Wissen über Gruppenmanagement,

- Notwendigkeit, sein Wissen während des gesamten Lebens zu vertiefen,

- Gebrauch von fortschrittlichen Informationstechnologien im Unterricht,

- Fähigkeit, die Analyse der Bedürfnisse der Zielgruppe durchzuführen.

Die Umfrageergebnisse haben auch eine Palette von Lern- und Lehrmethoden ergeben, die während des Fachsprachenunterrichts angewendet und unterrichtet werden. So wie im Falle der Zugangsvoraussetzung dominiert keine der genannten Methoden. Eine sinnvolle Klassifikation der angegebenen Methoden ist schwer möglich. Genannt wurden u. a. Frontalunterricht, Gruppenarbeit, Einzelarbeit, eklektischer Unterrichtsstil, Grammatik-Übersetzungsmethoden, strukturell-global-audiovisuelle Methoden, Analyse des Sprachaustauschs, Kritik am Lehren/Lernen, Vorlesungen, Textanalyse, Aussprachepraxis, Animationstechniken (pädagogische Differenzierung, Unterricht außerhalb der Mauern), kommunikativ-interkulturelle Fremdsprachendidaktik, korpusorientierter Fremdsprachenunterricht, Problemmethoden, grafische Aufzeichnungsmethoden und andere.

Eine ähnliche Vielfalt von Antworten lässt sich in Bezug auf die angewandten und unterrichteten Bewertungsmethoden nachweisen. Keine der an dieser Stelle gegebenen Antworten fiel deutlicher ins Gewicht als andere: schriftliche Aufgaben und Tests, Präsentationen und Projektarbeiten, Blogs, Aufsätze und Berichte, Portfolios, schriftliche Kolloquien, Bewertung von Unterrichtsmaterialien, Durchführung von Demonstrationskursen an den Schulen, Gestaltung von Lehrplänen, Unterrichtsplanung, Seminare, Beobachtungskurse, schriftliche oder mündliche Abschlussprüfungen, Interviews, Zusammenfassungen oder Rezensionen $\mathrm{zu}$ themenbezogenen gelesenen Artikeln, Analyse didaktischer Einheiten, Lehrplanprogramme, Entwicklung von Aktivitäten, die sich mit themenbezogenen Aspekten befassen, Gestaltung von Aufzeichnungen und Multimedia-Materialien.

Des Weiteren zeigen die Ergebnisse, dass bei der Vermittlung der Fachsprachendidaktik folgende IT-Programme Anwendung finden:

- MS Office/Libre Office/Open Office (75) 
- E-Mail (45)

- Sakai (39)

- Moodle (39)

- Ilia (39)

- Blackboard (39)

- Projektor (37)

- YouTube (34)

- Vimeo (34)

- Dailymotion (34)

- audiovisuelle Programme (33)

- Twitter (15)

- Snapchat (15)

- Linedin (15)

- Facebook (15)

- Skype (12)

- Hangouts (12)

- FaceTime (12)

- Smartphones (11)

- andere(11).

Beinahe jede Fachsprachendidaktik-Einheit setzt die Nutzung von Programmen zur Textverarbeitung, Tabellenkalkulation und Präsentation also die Büropakete Office voraus. Oft werden auch Lernplattformen wie Moodle, Ilias oder Blackboard sowie Videoportale wie YouTube verwendet. Seltener greifen die Lehrenden auf Social-Network-Tools wie Facebook, Linkedin, Snapchat oder Twitter zurück.

Die Antwort auf die letzte Frage bezüglich der im Kurs eingesetzten Fachliteratur brachte keine aufschlussreichen Ergebnisse. Die genutzte Literatur unterscheidet sich je nach Land und je nach unterrichteter Sprache.

\section{Zusammenfassung und Forschungsdesiderata}

Die zahlreichen Darstellungen zur Fachsprachendidaktik, die sich in der Vielfalt von methodisch-didaktischen Ansätzen und Methoden manifestieren, zeigen immer noch recht diffuse Vorstellung von diesem Phänomen unter Fachleuten und Fachsprachenlehrkräften. Die wiederum drückt sich in der fehlenden Einheit in Bezug auf theoretische Fundierung des Bereichs sowie Bearbeitung eines kohärenten Studienprogramms für angehende Fachsprachenlehrer aus.

Obwohl aus Zeitgründen nicht alle tertiären Bildungseinrichtungen aus dem Europäischen Hochschulraum untersucht werden konnten, gelten die aus 1024 Hochschuleinrichtungen erhobenen Daten zweifelsohne als repräsentativ und 
somit glaubwürdig. Die Ergebnisse weisen nach, dass Fachsprachendidaktik im europäischen Hochschulwesen eher marginal betrachtet wird. In nur $8 \%$ aller untersuchten akademischen Institutionen mit philologischen Fachrichtungen tragen die Studienprogramme der Fachsprachendidaktik Rechnung. Bei den von den TRAILs-Teilnehmern identifizierten Fachsprachendidaktik-Einheiten lassen sich (neben wenigen Gemeinsamkeiten wie die Anwendung bestimmter ITTools) grundsätzlich Unterschiede nachweisen. Sie betreffen einerseits formelle Aspekte wie die Form, in welcher die Fachsprachendidaktik unterrichtet wird, die Anzahl der Unterrichtsstunden, die Anzahl der zuerkannten ECTS-Punkte oder vorgesehene Qualifikationen, andererseits kommen die Differenzen auf der inhaltsbezogenen Ebene zum Ausdruck und beziehen sich auf die vorausgesetzten Lernziele und -ergebnisse, angewandten und unterrichteten Methoden, Techniken und Instrumente sowie das Bewertungssystem.

Der Notwendigkeit, zukünftige Fremdsprachenlehrer in Fachsprachendidaktik auszubilden, wird auch an den polnischen Universitäten und Hochschulen nach und nach Rechnung getragen. Das Auftreten von Elementen der Fachsprachdidaktik an sechs philologischen Fakultäten und im Rahmen der elf Studienrichtungen ist zwar eine gute Prognose für die Zukunft, erschöpft aber keineswegs den Bedarf an Ausbildung in diesem Bereich. Auch in Polen mangelt es an einer gewissen Standardisierung hinsichtlich der Form der fachsprachendidaktischen Unterrichtseinheit, Stundenzahl, Methoden oder Lernergebnisse im Bereich der Fachsprachdidaktik.

Die durchgeführte Untersuchung zeigt eindeutig, dass weitere Forschung in diesem Bereich wünschenswert ist. Die aktuelle Schwerpunktverschiebung bei der Fremdsprachendidaktik, von der Vermittlung der Allgemeinsprache hin zur Vermittlung der Fachsprachen, sollte unbedingt auch Änderungen in Studienprogrammen der philologischen Fachrichtungen, insbes. der philologischen Lehramt-Studiengänge, mit sich bringen. Künftige Fremdsprachenlehrer werden häufiger mit dem Unterrichten von Fachsprachen konfrontiert sein. Immer häufiger wird zu Recht dafür plädiert, Inhalte aus dem Bereich der Fachsprachendidaktik in die Studienprogramme zu integrieren und innerhalb des Europäischen Hochschulraumes zu standarisieren.

\section{Literaturverzeichnis}

Adams, Marina (Hrsg.) (2010). Historiographie von DaF als Fachsprachen-Disziplin in der $D D R$. Berlin: Frank \& Timme.

Buhlmann, Fearns (Hrsg.) (2000). Handbuch des Fachsprachenunterrichts. Berlin: Frank \& Timme. 
Buhlmann, Rosemare/Fearns, Annelise (Hrsg.) (2018). Handbuch des fach- und berufsbezogenen Deutschunterrichts DaF, DaZ, CLIL. Berlin: Frank \& Timme.

Baumann, Klaus-Dieter/Kalverkämper, Hartwig/Steinberg-Rahal, Kerstin (2000). Die Konzeption eines integrativen Fachsprachenunterrichts als aktuelle Herausforderung von Fachsprachenforschung und Fachsprachendidaktik. In: S. Cavagnoli/A. Schweigkofler (Hrsg.), Fachsprachen und Didaktik. Il linguaggi specialistic e la loro didattica. (S. 27-52). Bozen: Europäische Akademie.

Baumann, Klaus-Dieter (2000). Die Entwicklung eines integrativen Fachsprachenunterrichts - eine aktuelle Herausforderung der Angewandten Linguistik. In: K.-D Baumann/ H. Kalverkämper/K. Steinberg-Rahal (Hrsg.), Sprachen im Beruf. Stand-ProblemePerspektiven (S. 149-173). Tübingen: Narr.

Europäische Kommission (2017). Mitteilung der Kommission an das Europäische Parlament, den Rat, den Europäischen Wirtschafts- und Sozialausschuss und den Ausschuss der Regionen vom 30.05.2017 über eine europäische Erneuerungsagenda für die Hochschulbildung. Brüssel: Europäische Kommission.

Fluck, Hans-Rüdiger (Hrsg.) (1992). Didaktik der Fachsprachen: Aufgaben und Arbeitsfelder, Konzepte und Perspektiven im Sprachbereich Deutsch. Tübingen: Narr.

Fluck, Hans-Rüdiger (2000). Fachsprachen. Stuttgart: UTB.

Grucza, Sambor (2010). Sprache(n) - Fachsprache(n) - Fachsprachendidaktik. Studia Germanica Gedanensia, 22, 31-46.

Grucza, Franciszek (2017). Lingwistyka Stosowana. Historia - Zadania - Osiagnięcia. Dzieła zebrane. Tom 9. Warszawa: Wydawnictwo Naukowe Instytutu Komunikacji Specjalistycznej i Interkulturowej Uniwersytet Warszawski.

Helbig, Gerhard (Hrsg.) (1986). Entwicklung der Sprachwissenschaft seit 1970. Leipzig: Bibliographisches Institut.

Hoffmann, Lothar (Hrsg.) (1985). Kommunikationsmittel Fachsprache. Berlin: Frank \& Timme.

Oldenburg, Hermann (Hrsg.) (1992). Angewandte Fachtextlinguistik: „Conclusions“ und Zusammenfassungen. Tübingen: Narr.

Prikoszovits, Matthias (2017). Deutsch als Fremdsprache für den Beruf lernen -doch (ab) wann? Ein altersgruppen- und niveaustufenspezifischer Zugang zur Diskussion um die berufliche Ausrichtung von DaF-Curricula. Zeitschrift für Interkulturellen Fremdsprachenunterricht, 22 (2), 155-168. http://tujournals.ulb.tudarmstadt.de/index.php/ zif/ (Stand 15.06.2018).

Spillner, Bernd (1998). Mehrsprachigkeit, Kontrastive Linguistik, interkultureller Fachtextvergleich. Germanica. Jahrbuch für deutschlandkundliche Studien (Sofia), 5, 133142.

Zalipyatskikh, Natalya (2017). Didaktik der technischen Fachkommunikation: Methodologien, Konzepte, Evaluationen. Berlin: Frank \& Timme. 


\section{Teil 6: Übersetzen und Dolmetschen}


Open-Access-Publikation im Sinne der CC-Lizenz BY-NC-ND 4.0

(C) 2020, Vandenhoeck \& Ruprecht GmbH \& Co. KG, Göttingen ISBN Print: 9783847111863 - ISBN E-Lib: 9783737011860 


\title{
Zur Textur des Originalwerkes und der Übersetzung - über die lautlichen und graphematischen Herausforderungen der Übersetzung
}

\begin{abstract}
On the texture of the original work and the translation - about the phonetic and graphematic challenges of translation

The written and the phonetic form of texts represent a kind of surface which is called texture (Kozak 2009, Kubaszczyk 2016). Special texture elements are repetition and relief, which fulfill a poetic function in literary texts. The poetic function comes to the foreground through an appropriate selection and combination of the available language resources (Jakobson 1960/1971), which draws attention to the language itself. In this chapter, the most important figures of the repetition are discussed on the phonetic and graphematic level, jointly with examples of relief formation. Translation options for the figures discussed are also analysed. The goal is to sensitise translators to the texture elements. It is postulated to develop and increase the creative ability to combine language means in translation classes.
\end{abstract}

Keywords

texture, repetition, relief, poetic function, selection, combination, spelling, sound

\section{Einleitung}

Heutzutage werden literarische Texte vor allem lautlos gelesen. Das beeinflusst unsere Wahrnehmung sehr stark. Wir sehen sie, bevor wir sie hören - wenn wir sie überhaupt hören. Die Schrift hat das Lautliche verdrängt. Bevor jedoch die Schrift erfunden wurde und auch lange danach, hatte man die Literatur mündlich weitergegeben. Gedichte wurden vorgetragen, Epen vorgesungen. Aus unserer Kindheit kennen wir das vielleicht noch, als uns Märchen und andere Kindergeschichten erzählt oder vorgelesen wurden. Infolge technischen Fortschritts können wir die lautliche Seite der Literatur heute wieder dank den Hörbüchern genießen, und das ist gut so, denn wenn wir einen Text nur „mit den Augen u. dem Verstand erfassen" (was die lexikalische Beschreibung des Verbs lesen im Duden ist; vgl. Duden 2001), dann entgeht uns eventuell einiges. Ein Text kann dadurch an seinen 
Qualitäten verlieren, langweilig werden, vor allem, wenn bestimmte literarische Figuren und Stilmittel um des Lautes willen eingesetzt wurden. Das Lautliche wird glücklicherweise nie total verdrängt. Es ist zwar bei den mündlich vorgetragenen Texten besonders eminent, aber auch beim lautlosen Lesen irgendwie im Hintergrund immer noch präsent, wenn auch nicht so dominant.

Gleichzeitig stellen sowohl die Schrift als auch die lautliche Form der Texte eine Art Oberfläche dar, durch die wir hindurch müssen, um zum Sinn des Textes vorzudringen. Diese Oberfläche nenne ich nach Jolanta Kozak Textur ${ }^{1}$ (vgl. Kozak 2009). Es ist eine metaphorische Bezeichnung, denn unter Textur wird gewöhnlich eine Eigenschaft solcher Stoffe verstanden, die eine ausgedehnte Oberfläche haben und anfassbar sind wie Textilien, Holz oder Steine. Aber auch bei Gemälden sprechen wir von Textur.

Bei Textur in herkömmlicher Bedeutung spielen haptische, optische, ästhetische Qualitäten eine Rolle. Was die Textur in diesem Sinne mit der Textur des Textes verbindet, ist die Materialität des Zeichens. Bei einem Text geht es aber vor allem um die akustische und visuelle Oberfläche, wenn auch haptische Eigenschaften gleichfalls eine Rolle spielen können (das verwendete Papier oder eine andere Textunterlage, auf welcher ein Text niedergeschrieben oder gedruckt ist, ein Bildschirm, auf dem er ausgestrahlt wird, ein Stein, in den er eingemeißelt ist etc.).

Es gibt aber auch gemeinsame Merkmale der Gewebetextur und der Textur eines literarischen Textes, die ästhetische Funktionen erfüllen. Dazu kann man beispielsweise die Repetition rechnen. Bei der Textiltextur kann das die regelmäßige Wiederholung von geometrischen Mustern sein, in der Literatur die gezielte Wiederholung von Textsegmenten, die ein visuelles oder lautliches Muster ergeben.

Ein anderes Texturelement ist das Relief, welches in der bildenden Kunst ein Bildwerk bezeichnet, das sich plastisch vom Hintergrund abhebt, weil es aus einer Fläche erhaben herausgearbeitet oder in sie vertieft wurde (vgl. Duden 2001). Dieses Spiel zwischen Vordergrund und Hintergrund lässt sich in Texten auch beobachten, manche Mittel dienen der optischen oder lautlichen Hervorhebung, somit kann auch im sprachlichen Kunstwerk etwas im Relief dargestellt werden.

Die Textur wird manchmal von Übersetzern, die auf die Wiedergabe der denotativen Bedeutung fokussiert sind, missachtet, als eine Art „Verpackung“ für die zu übersetzenden Inhalte behandelt. Die von uns mehr oder weniger bewusst wahrgenommene Textoberfläche, die mal sehr glatt ist, mal aber auch rau, holprig, unausgefeilt und ungeschliffen sein kann (man sagt ja: „Die Erzählung liest sich glatt“, „Holprige Erzählweise“, „Der Text ist noch auszufeilen“), ist aber viel mehr als eine bloße Verpackung für die Bedeutung, und die eingesetzten

1 Kozak verwendet den polnischen Terminus faktura. 
Stilmittel sind mehr als nur ein Beiwerk, ein Ornament, das ausgelassen werden kann. Die Textur gestaltet den Sinn des Textes mit, daher ist sie auch sinntragend.

Diese Tatsache wird auf das Entschiedenste von Literaturtheoretikern in Bezug auf die poetische Funktion der Sprache hervorgehoben. So schreiben etwa Głowiński et al. (1972: 89), dass die poetische Funktion nicht darin bestehe, dass man irgendwelche Verzierungen, Ornamente, dekorative Elemente verwende. Vielmehr betonen die Autoren, dass die poetische Funktion vor allem ein semantisches Phänomen sei und dass die Äußerung, in der sie eine dominante Position einnimmt, etwas Anderes bedeute als eine Äußerung mit einer anderen dominanten Funktion. Bei der poetischen Funktion wird die Sprache selbst in den Vordergrund gerückt. Die poetische Funktion als „Zentrierung auf die Sprache um ihrer selbst willen“- so Jakobson (1971: 151) - „überträgt das Prinzip der Äquivalenz von der Achse der Selektion auf die Achse der Kombination.“ (ebd.: 153). Die entsprechende Selektion und Kombination sind auch die wichtigsten Verfahren bei der Übersetzung, wenn die poetische Funktion im Zieltext aufrechterhalten werden soll (vgl. Kubaszczyk 2019).

Die poetische Funktion der Sprache lässt sich natürlich nicht nur in der Literatur feststellen, dort kommt sie jedoch besonders häufig zum Tragen. Aber auch in der Werbung, in der Alltagsrede, vor allem bei geistreichen Aussagen sprachbewusster Sprecher und Sprecherinnen und in Wortspielen kommt sie vor.

Die Form des lautlichen Textaufbaus, der lautlichen Textorganisation und die Verschriftungsform eines Textes sind also von Bedeutung. Diese Bedeutung ist von den Übersetzern zu rekonstruieren. Und die Bedeutung, welche den Sprachmitteln zugeschrieben wird, hängt aufs Engste mit der Funktion zusammen, welche die jeweiligen Mittel in einer Äußerung erfüllen. Die Bestimmung dieser Funktion ist folglich erstrangig, denn je nach der dominanten Funktion werden andere Übersetzungsstrategien und Übersetzungsverfahren eingesetzt. Es ist daher wichtig, weil in der Übersetzung nicht nach dem Zeichenäquivalent, sondern nach dem Äquivalent der Zeichenfunktion gesucht wird (vgl. Kubaszczyk 2016: 8) und in einer der eigentlichen Übersetzung vorausgehenden Analyse wird nach der Erklärung und Begründung einer bestimmten Zeichenverwendung gefragt. Darauf hat bereits vor vielen Dekaden der polnische Übersetzungswissenschaftler Zenon Klemensiewicz (1955: 93) hingewiesen, indem er feststellte:

Die Aufgabe der Übersetzung besteht nicht darin, Elemente und Strukturen des Originalwerkes, Wörter, Sätze, Prosodeme, bildliche Redewendungen etc. zu kopieren oder zu rekonstruieren, um so weniger darin, sie zu verarbeiten, sondern sie liegt darin, deren Funktion zu erfassen und für die Elemente und Strukturen des Originalwerkes solche Elemente und Strukturen der eigenen Sprache einzuführen, die so weit möglich, Substitute und Äquivalente von derselben funktionalen Effizienz, Eignung und Effektivität 
wären, worin sich eben ihre Adäquatheit, Angemessenheit, Gleichanwendbarkeit und Gleichwertigkeit zeigt. ${ }^{2}$

Daher ist die richtige Bestimmung der Funktion der jeweiligen Zeichen die Bedingung sine qua non der gelungenen Übersetzung. Es kann natürlich sein, dass sich mehrere Funktionen überschneiden. Bei der Überlappung von Funktionen muss der Übersetzer Prioritäten setzen, oder wie es in der Übersetzungstheorie genannt wird, Invariante aufstellen, die aufgrund von mehrdimensionaler Analyse priorisiert werden.

Das Ziel des Aufsatzes ist, im Weiteren die wichtigsten Figuren der Wiederholung auf der lautlichen und graphematischen Ebene zu besprechen und Beispiele für Reliefbildung vorzuführen. In einem zweiten Schritt sollen Übersetzungsmöglichkeiten bzw. Übersetzungsprobleme aufgezeigt werden.

Die Probleme der Rekurrenz sind eines der Themen, mit denen sich die Stilistik befasst. Wie Izabela Prokop zu Recht betont, ist die Bedeutung der kontrastiven stilistischen Beschreibung für die Übersetzungswissenschaft kaum zu überschätzen. Die Problematik der stilistischen Übersetzbarkeit ergibt sich nach Prokop (2019: 213) daraus, dass Systeme der konnotativen Werte, die den Stil gestalten, sich in verschiedenen Sprachen nicht 1:1 entsprechen.

Möge der Jubilarin, die sich in ihrer Forschung unter vielen anderen Themen auch mit der kontrastiven Stilanalyse beschäftigte, mit diesen Ausführungen Ehre erwiesen werden.

\section{Repetition. Figuren der Wiederholung}

\subsection{Wiederholung von Wortmaterial}

Die Figuren der Wiederholung lassen sich sowohl auf der lautlichen als auch auf der graphematischen Textebene feststellen. Auf der graphematischen Ebene ist die Wiederholung in der sog. konkreten Poesie am signifikantesten, wo bestimmte Textsegmente, meistens einzelne Wörter, wiederholt werden. Als Beispiel kann das bekannte Gedicht schweigen von Eugen Gomringer angeführt werden, in dem ein einziges Wort als einziger Textbaustein vierzehnmal eingesetzt wird (vgl. Gomringer 1988):

2 [Übersetzung JK]. Polnischer Wortlaut: „Zadanie przekładu polega nie na kopiowaniu, nie na odtwarzaniu, tym mniej na przetwarzaniu elementów i struktur oryginału, wyrazów, zdań, prozodemów, zwrotów obrazowych itd., ale na uchwyceniu ich funkcji i wprowadzeniu za elementy i struktury oryginału takich elementów i struktur własnego języka, które by były w miarę możności substytutami i ekwiwalentami o takiej samej funkcjonalnej wydolności, zdatności i skuteczności, w czym właśnie okazuje się ich adekwatność, współmierność, współstosowalność, równowartościowość." 
schweigen schweigen schweigen schweigen schweigen schweigen schweigen schweigen schweigen schweigen schweigen schweigen schweigen schweigen

Mit der Repetition geht häufig ein anderes Verfahren einher. In diesem Gedicht wird symbolisch das Schweigen auch durch die zentrale Leerstelle dargestellt, die sich vom beschrifteten Hintergrund abhebt. Diese Leerstelle bildet ein Relief. Die Reliefbildung kann aber auch durch eine abweichende lexikalische Einheit erfolgen, wie im Gedicht ordnung (vgl.ebd.), die - in diesem Fall buchstäblich -, aus der Reihe tanzt":

ordnung ordnung
ordnung ordnung
ordnung ordnung
ordnung ordnung
ordnung ordnung
ordnung ordnung
ordnung unordn g
ordnung ordnung
ordnung ordnung
ordnung ordnung
ordnung ordnung
ordnung ordnung
ordnung ordnung

Diese Art der Wiederholung ist in der Übersetzung am leichtesten wiederzugeben, was die Übersetzungen in verschiedene Sprachen beweisen, was anhand der Übersetzung des Gedichts schweigen ins Polnische ${ }^{3}$ und Türkische ${ }^{4}$ zu sehen ist:

milczenie milczenie milczenie milczenie milczenie milczenie milczenie milczenie milczenie milczenie milczenie milczenie milczenie milczenie sessizlik sessizlik sessizlik sessizlik sessizlik sessizlik sessizlik sessizlik sessizlik sessizlik sessizlik sessizlik sessizlik sessizlik

Solche Ornamentsgedichte sind u. a. deswegen relativ einfach zu übersetzen, weil sie häufig eine völlige Desemantisierung des Wortes anstreben (vgl. Wysłouch 2001: 130).

3 Übersetzung JK.

4 https://www.lyrikline.org. [Übersetzung anonym]. 


\subsection{Homophone Übersetzung}

Der Verlust an Bedeutung bzw. an semantischem Inhalt lässt sich noch stärker im Falle mancher homophonen Wiederholungen beobachten. Hier lassen sich verschiedene Typen unterscheiden. Einer der Fälle ist die homophone oder angeblich homophone Übersetzung. Es geht dabei um eine lautliche Sequenz in einer Sprache, die in einer anderen Sprache wiederholt wird. Ein sehr interessantes Beispiel dafür findet man im spielerischen Text K.J. Jaworskis, der häufig irrtümlicherweise Julian Tuwim zugeschrieben wird, welcher ihn in der Sammlung „Pegaz dęba“ popularisierte ${ }^{5}$. In der polnischen Version verwendet der Dichter Laute, die den französischen Lauten ähnlich sind und er verschriftet sie dann so, dass sie das Französische vortäuschen. Die Schreibung des Textes ist irreführend, er ist nicht tatsächlich französisch, er hört sich nur französisch an und unterstützt wird diese spielerische Täuschung durch die Schreibung und Verwendung einiger französischer Worte. Eine solche lautliche Wiederholung, die in der Schriftform vorgibt, in einer anderen Sprache geschrieben zu sein, verfolgt das Ziel, zu amüsieren und den Text auffällig und interessant zu machen:

Oko trę, że mam ból Taki los komu żal? oko trę, pali sól O madame, kulą wal Ile trosk, ile burz, A ma krew kipi, wre, O madame, oto nóż $\mathrm{O}$, madame, oto mrę
O, cotrain, je m'emboulle, taquilosse, comme ou jalle?

$\mathrm{O}$, cotrain, palisoule

O madame, coulon valle!

Il est trosque, il est bouge,

à ma créve qui pis vrai

$\mathrm{O}$ madame, o tonouche

$\mathrm{O}$, madame, o tome rain!

Eine derartige lautliche Wiederholung kann aber auch gezielt in der tatsächlichen Übersetzung eingesetzt werden. Daher hat die homophone Übersetzung als eine besondere Art der lautlichen Wiederholung zu gelten. In diesem Falle wird der semantische Inhalt weitgehend zugunsten der lautlichen Nachahmung ignoriert. Diese Art der Übersetzung ahmt den Rhythmus und den Klang des Originals nach. Leser/Hörer werden verführt, werden „auf Abwege der Denotation“ ${ }^{\text {“6 }}$ gebracht. Mit anderen Worten soll der homophon übersetzte Text gleich oder sehr ähnlich wie das Original auf Kosten der Denotation (der denotativen Äquivalenz) klingen. Auf die homophone Übersetzung griff gelegentlich der polnische Übersetzer Stanisław Barańczak zurück, ein Beispiel führt Kozak an, wenn sie auf die Stelle aufmerksam macht, an der Barańczak „Vieni, vieni!“ (wörtlich: Komm,

5 Vgl. Kasperczak, Piotr. https://okruchy.pl, Tuwim 2008. Die Vornamen des Autors sind mir unbekannt.

6 Vgl. Kozak (2009: 133): „Tłumaczenie homofoniczne zwodzi tu czytelnika na manowce denotacji $[\ldots] .$. 
komm!) als „Wiemy, wiemy!“ (wörtlich: Wir wissen, wir wissen!) übertrug (vgl. Kozak 2009: 132f.) ${ }^{7}$.

Dieses Verfahren ist besonders gut geeignet, wenn vertonte Texte übersetzt werden sollen wie Lieder oder Libretti, vor allem wenn es sich um international bekannte Musikstücke handelt. Ein vortreffliches Beispiel liefert die polnische Übersetzung des Kehrreimes des weltweit berühmten Schlagers „Guantanamera“, der im Original lautet: „Guantanamera, guajira guantanamera, Guantanamera, guajira guantanamera“ (Martí 1929). Wojciech Młynarski übersetzte ihn wie folgt: „Bandanamera, napadła banda na mera, u jubilera, napadła banda na mera" (Młynarski 1999). Wie zu sehen ist, gibt es auch textintern eine lautliche Wiederholung: „Bandanamera“ als eine zwar sinnlose, aber enge lautliche Anknüpfung an das Original wird als eine Sinneinheit wiederholt (wörtlich: eine Bande hat den Maire [den Bürgermeister] überfallen).

Nach Kozak (2009: 133) ist die homophone Wiederholung meistens

ein närrisches Nachäffen des Originals. Sie hat jedoch diesen Vorteil und ist deswegen der semantischen Übersetzung überlegen, dass sie eine ikonische Erscheinung ist: sie zeigt uns die andere Sprache und lässt uns ihre graphische und lautliche Eigentümlichkeit schmecken und registriert dabei, was uns diese Sprache sagen würde, wenn wir nur ihre Textur adaptieren würden. ${ }^{8}$

Zugleich beweist jedoch die homophone Übersetzung, wie sie betont, dass die Textur des Textes aktiv an der Semiose beteiligt ist (ebd.: 137). Daher darf die Textur in der Übersetzung nicht missachtet werden.

\subsection{Homophonie als Scheinwiederholung}

Die Wiederholung auf der lautlichen Ebene kann mit der Wiederholung auf der graphematischen Ebene einhergehen, die graphematische Ebene kann aber auch die lautliche Wiederholung als Scheinwiederholung entlarven. Das können wir z. B. in folgendem Aphorismus von Stanisław Jerzy Lec beobachten, in dem der Schriftsteller zwei zwar homophone, aber nicht homographe adjektivische Komposita kontrastiert. Was sich als Wiederholung anhört, kann erst beim Lesen disambiguiert werden: „Nie wiem kto jest praworzadny, ja jestem prawożadny“ (Lec 2018: 20) [Hervorhebung JK]. Die Basis des Wortspiels sind hier die polni-

7 Es handelt sich hier um die Übersetzung einer Passage aus der Oper Don Giovanni Mozarts mit dem Libretto von Lorenz Da Ponte.

8 [Übersetzung JK]. Polnischer Wortlaut: „[...] powtórzenie homofoniczne jest na ogół błazeńskim przedrzeźnianiem tekstu oryginalnego. Ma jednak tę wielką zaletę, i swoistą przewagę nad tłumaczeniem semantycznym, że jest zjawiskiem ikonicznym: pokazuje nam drugi język, dając zasmakować jego graficznej i dźwiękowej specyfiki, a także rejestrując przy okazji, co mówiłby do nas ten język, gdybyśmy adoptowali samą jego fakturę.“ 
schen Allographen des gleichen Lautes [3]. Die abweichende Schreibung des Lautes bildet hier das Relief. Eine wortwörtliche Übertragung, die die poetische Funktion der Sprache ignorieren würde, wäre hier natürlich verfehlt. Wörtlich übersetzt müsste es heißen: Ich weiß nicht, wer rechtstreu ist, ich bin rechtsgierig. Ohne die humoristische Wiederholung verliert jedoch der geistreiche Aphorismus nicht nur jeglichen Reiz und wirkt trocken und fade, sondern man fragt sich auch, was diese Feststellung eigentlich soll. Es kommt also zu einer Störung auf der Ebene der Kohärenzstiftung. In solchen Fällen müssen andere Übersetzungsverfahren eingesetzt werden, die zugleich eine lautliche Wiederholung ermöglichen und einen ähnlichen Sinn stiften. Hier geht es folglich um die von Jakobson postulierte Übertragung des Prinzips der Äquivalenz von der Achse der Selektion auf die Achse der Kombination. Das Wortmaterial muss so selegiert werden, dass eine entsprechende Kombination erzielt wird. In dem besprochenen Fall wird eine lautliche Wiederholung mit der disambiguierenden Schreibung anvisiert. Hier bieten sich folglich Modulation oder Mutation als Übersetzungsverfahren an (vgl. Schreiber 1999: 152). Die Übertragung könnte beispielsweise lauten: Egal, wer Recht schaffen will, ich will rechtschaffen bleiben [Übersetzung JK]. In der Übersetzung wurde der denotative Inhalt geändert, damit ein „prägnant-geistreicher, in sich geschlossener Sinnspruch in Prosa, der eine Erkenntnis, Erfahrung, Lebensweisheit vermittelt“ (vgl. Duden 2001) kreiert werden kann.

\subsection{Wiederholung gleichlautender, aber nicht gleichgeschriebener Lautfolgen ohne Bedeutungsvariation}

Die homophone Rekurrenz kann rein ausdrucksseitig oder ausdrucksseitig und inhaltsseitig erfolgen. Mit der rein ausdrucksseitigen Wiederholung homophoner, aber nicht homographer Lautfolgen spielt der polnische Dichter Tadeusz Różewicz Różewicz (2002: 15) im Gedicht „Regression in die Ursuppe“ [Hervorhebungen $\mathrm{JK}]$ :

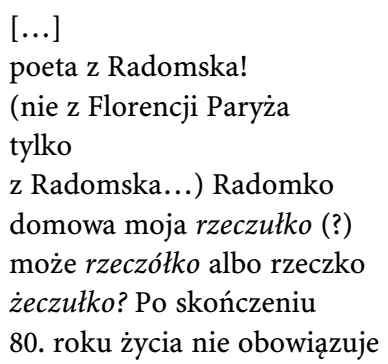


mnie ortografia

$[\ldots]$

Różewicz alterniert die Schreibung des Diminutivums des zweiten Grades vom Substantiv rzeka (Fluss). Er nutzt dabei die Tatsache aus, dass im Polnischen der Laut [3] entweder als $r z$ oder als $\dot{z}$ verschriftet wird. Auch der Laut [u] hat zwei Allographen $u$ und das sog. „geschlossene u“: ó. Die Schreibungsvarianten werden identisch ausgesprochen, aber nur eine Schreibweise des Diminutivums (die erste) ist kodifiziert und korrekt, die anderen Wörter sind fehlerhaft geschrieben. Mit der Rechtschreibung der beiden Laute haben nicht nur polnische Kinder Schwierigkeiten. Im Gedicht setzt sich der Dichter mit der altersbedingten Leistungsabnahme auseinander, die Regression ist auch in seiner Schreibverunsicherung zu sehen. Die abweichende Schreibung thematisiert also die Botschaft des Gedichts, die Vergesslichkeit, die mit dem Alter zunimmt und die Erkenntnis, dass sich alles „in eine merkwürdige Suppe des Todes“ verwandelt.

In der deutschen Übersetzung des Gedichtes kommt dem Übersetzer die Tatsache zu Hilfe, dass der Laut [s] im Deutschen auch unterschiedlich geschrieben werden kann (als $ß$ und Doppel-ss) und gerade die Schreibung des Substantivs Flu $\beta$ sich nach der Rechtschreibereform geändert hat, und heutzutage die Schreibung Fluss korrekt, Fluß aber inkorrekt ist, was gleichfalls zu einer Verunsicherung vor allem älterer Sprachnutzer führen kann. Zusätzlich kann auch der Laut [f] alternative Schreibweisen haben (wie in Phantasie/Fantasie ${ }^{9}$ ), was die Botschaft zusätzlich unterstützt und die Varianten ausbauen lässt:

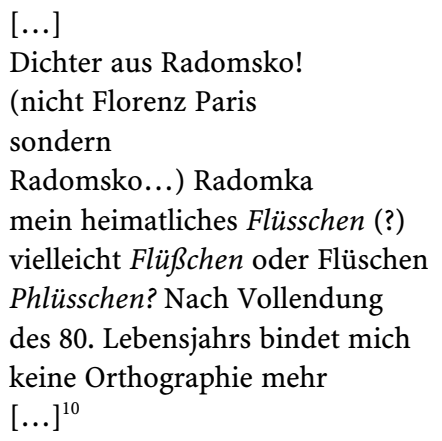

9 Zwar nur in Wörtern griechischen Ursprungs, der Rechtschreibunsichere kann das aber nicht (mehr) wissen.

10 Różewicz (2007). Übersetzt von Karl Dedecius. 


\subsection{Wiederholung gleichlautender, aber nicht gleichgeschriebener} Lautfolgen: Groß- und Kleinschreibung

Bei der Bedeutungsnuancierung kann im Falle der homophonen Wörter auch die Groß- und Kleinschreibung eine Rolle spielen. Im folgenden Aphorismus wird nic (nichts, das Nichts) wiederholt, bei der Repetition verwendet Stanisław Jerzy Lec aber die Großschreibung sowohl des Adjektivs als auch des Substantivs. Dadurch erzielt er nicht nur auf der semantischen, sondern auch auf der graphematischen Ebene eine Verstärkung (eine Hyperbel und Emphase): „Jest małe nic i jest Wielkie Nic“ (Lec 2018: 47) [Hervorhebung JK]. Die Differenzierung, welche mittels der Schreibung erfolgt, ist im Polnischen problemlos zu erzielen. Die Majuskel dient als ein Relief-Mittel und betont die Emphase. Da im Deutschen die Schreibung der Substantive normativ festgelegt ist, müssen bei der Übersetzung Wege gesucht werden, das semantische Spiel abzubilden. Der Aphorismus kann systemkonform und nichtsystemkonform übersetzt werden: Es gibt ein kleines Nichts und ein Großes Nichts./Es gibt ein kleines nichts und ein Großes Nichts [Übersetzung JK]. Im ersten Übersetzungsvorschlag wird ausgenutzt, dass im Deutschen Adjektive zur Verstärkung großgeschrieben werden können ${ }^{11}$. Im zweiten wird bewusst die nicht systemkonforme Schreibung im ersten Teil gebraucht, um die Differenz zwischen Groß und Klein noch stärker hervorzuheben. Das zeigt, dass der Übersetzer auch mit der Textur gezielt spielen kann.

\subsection{Lautliche Rekurrenz und Quantitätsprinzip}

Eine andere Funktion hat die alternierende Wiederholung im nächsten Beispiel, in dem die Autorin, Manuela Gretkowska, einen Vulgarismus verwendet, der das männliche Glied (Schwanz) bedeutet, aber auch als beleidigendes Schimpfwort auf den Menschen bezogen werden kann. Auch hier haben wir im Polnischen mit zwei Allographen des Lautes [h] zu tun, welcher mit dem Buchstaben $h$ oder mit ch geschrieben wird. Dieses Wort wird im Polnischen häufig fehlerhaft geschrieben. Gretkowska (2001: 125) greift das auf und lässt ihre Protagonistin die Bedeutung differenzieren, je nachdem wie lang der Schwanz ist. Die kürzere (inkorrekte) Form will sie für den kürzeren, beschnittenen vorbehalten. Man kann das als eine Realisierung des Ikonizitätsprinzips (diagrammatische Ikonizität: Quantitätsprinzip) betrachten. Das Prinzip besagt, dass je größer oder

11 Zum Beispiel ist es oft bei Elfriede Jelinek der Fall. Im Roman Die Klavierspielerin finden wir beispielsweise die Phrase Eigener Kopf: „[...] den Rest liefert der Eigene Kopf.“ (Jelinek 2005: 225-226). 
wichtiger das Denotat ist, desto mehr Sprachmaterial verwendet wird [Hervorhebung JK]:

Dobrze trafiłeś do szpitala, lecz się, „chuju“. Jestem za pisownią po polsku „huj“ dla krótszego, obrzezanego.

Die alternierende Wiederholung wurde in der Übersetzung nicht wiedergegeben. Die beiden Sätze wurden ausgelassen (vgl. Gretkowska 2004). Ist das als ein Scheitern der Übersetzerin Paulina Schutz zu werten? Hier könnte man wieder verschiedene Übersetzungsverfahren in Erwägung ziehen, z.B. die lexikalische Entlehnung könnte mit der synonymischen Wiederaufnahme kombiniert werden, was das Verständnis dieser Stelle bei dem deutschsprachigen Leser sichern würde:

Du bist wohl zu Recht ins Krankenhaus eingeliefert worden, du hast sie wohl nicht alle, du Schlappschwanz. Ich bin übrigens dafür, dass man im Polnischen das Wort „chuj“ für den längeren Schwanz und „huj“ für den kürzeren, beschnittenen verwendet. [Übersetzung JK]

\subsection{Paraphonische Wiederholung}

Paraphonie heißt anders Gleichklang und ist eine Art (ungewollte) Homophonie von Phrasen oder Sätzen. Unter Paraphonie wird eine Art Kakophonie („Missklang einer Folge von Tönen od. Lauten“ Duden 2001) verstanden, die zur Zweideutigkeit durch entsprechende Wortzusammenstellung führen kann. Im normalen Sprachgebrauch ist die Paraphonie zu meiden, da sie zu Missverständnissen führen kann. Die Paraphonie kann jedoch in einem Kalauer oder einem anderen Wortspiel gezielt eingesetzt werden, das auf der gleichen Lautung zweier Phrasen beruht („to pole/topole chcę kupić“12; „Widzą ciężarówki“/ „Widzą Cię żarówki“13; „Heisse Würstchen - Angenehm, Müller“14). Eine ausgezeichnete Illustration für die paraphonische Wiederholung bietet Jerzy Ficowskis Gedicht „Dziwna rymowanka“, in dem die Paraphonie zum Konstruktionsprinzip wird. Es sei nur der Anfang angeführt:

Pewien żarłok nie nażarty

raz wygłodniał nie na żarty.

I wywiesił szyld na płocie

12 Beispiel nach Sierotwiński (1986). Wörtlich: Ich möchte diesen Acker kaufen/Ich möchte Pappeln kaufen.

13 Dieses Beispiel wurde einem Cartoon von Paweł Jaroński entnommen, der die Paraphonie mit zwei verschiedenen Bildern veranschaulicht hat, vgl. Jaroński [online]. Wörtlich: Sie sehen LKWs./Glühbirnen sehen Dich.

14 Vgl. den Cartoon von Andre Sedlaczek, Heisse Würstchen. Sedlaczek [online]. 
że ochotę ma na płocie.

Tutaj na brak ryb narzeka,

bo daleko rybna rzeka. ${ }^{15}$

Sollte das Gedicht übertragen werden, müsste es neu geschaffen werden, indem in der Zielsprache gleichlautende Sequenzen gefunden werden, die das gleiche Prinzip realisieren. Der nachstehende sehr unvollkommene (und grammatikalisch nicht ganz korrekte) Übersetzungsvorschlag hat nur einen demonstrativen Charakter, in welche Richtung die Suche gehen könnte. Wollte jemand aber tatsächlich das Gedicht übersetzen, müsste er/sie von homophonen Ausdrücken oder Phrasen im Deutschen ausgehen und diese sinnvoll zu einer Geschichte zusammenstellen:

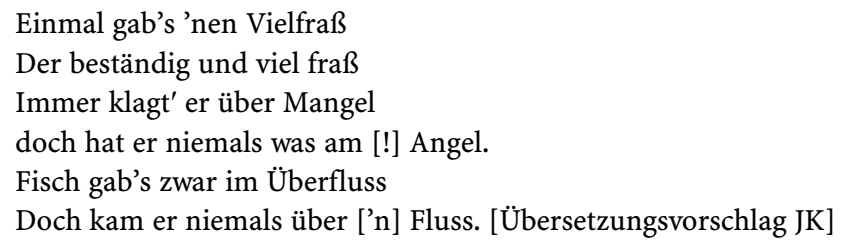

\subsection{Klassische Homonyme}

Der letzte Punkt, der noch zu erwähnen wäre, sind homonymische Wortspiele. In diesem Fall werden homonyme Wörter oder Ausdrücke zusammengestellt. Auf der lautlichen und graphematischen Ebene wird durch die Wiederholung des Gleichlautenden und Gleichgeschriebenen ein Aufmerksamkeitseffekt erzielt und durch den Bedeutungsunterschied wird die Hörerschaft/Leserschaft zur gedanklichen Arbeit angespornt oder einfach nur amüsiert. Ein Beispiel finden wir im Roman „Die Klavierspielerin“ von Elfriede Jelinek:

[...] und sie überspannte den Bogen. Sie ist eine überspannte Person [...]. ${ }^{16}$

[...] ona przeciagnęła strunę. Zresztą jest walnięta [...]. ${ }^{17}$

Diese von Ryszard Turczyn angefertigte Übersetzung kann hier nicht als zufriedenstellend gewertet werden, denn er hat nur die denotative Bedeutung beider Ausrücke wiedergegeben, ohne die poetische Funktion zu berücksichtigen. Um das zu erreichen, müsste sich der Übersetzer für eine andere Strategie entscheiden und auf Kosten der Denotation die Aussage so modifizieren bzw. modulieren, dass der Gleichklang auch im Zieltext erreicht wird oder zumindest

15 Ficowski, https://quotepark.com. [Hervorhebung JK].

16 Jelinek (2005: 272) [Hervorhebung JK].

17 Jelinek (2004: 335) [Hervorhebung JK]. 
die poetische Funktion mit anderen Mitteln kompensiert und hinübergerettet wird. Das Finden der passenden Lösung ist dadurch erschwert, dass die geleichlautenden Formen im Deutschen anderen Wortarten angehören. Will man die Wortarten beibehalten, muss man im Polnischen einen Formwandel wegen der Flexion in Kauf nehmen, was keine optimale Lösung ermöglicht (z. B.: [...] walnęła, (mocno) przeginając. Zresztą jest walnięta./[...] rąbnęła. Zresztą jest rąbnięta./[...] przegięła. Zresztą jest pogięta. Vorschläge JK). Erst wenn man die Aussage semantisch wandelt (das Verfahren der Mutation), kann eine homonyme Lösung zustande kommen, wodurch auch das Sprachspiel erhalten bleibt: „[...] dała czadu. Jakby była z Czadu.“ [Übersetzungsvorschlag JK].

Da solche sprachspielerischen Rekurrenzen für Jelineks Sprache typisch sind und ihr Sprachvermögen und ihren Esprit beweisen, sollten sie, wo es nur möglich ist, in der Übertragung ihrer Werke nachkreiert werden.

\section{Fazit}

Aus Platzgründen konnten nur die wichtigsten Erscheinungen besprochen werden, die die Textur eines literarischen Werkes beeinflussen. Diese poetologischen Textgestaltungsmittel sind an sich nicht unbekannt und einige von ihnen bereits im Rahmen der antiken Rhetorik behandelt. Übersetzer und Übersetzerinnen sind jedoch immer noch zu wenig dafür sensibilisiert, dass die poetische Funktion der Sprache in literarischen Werken meist vor anderen Funktionen Vorrang hat und dass im Fall einer gleichlautenden Repetition die Findung einer angemessenen Lösung nicht über eine einfache Selektion der Heteronyme, sondern auf dem Wege einer Kombination von gleichlautenden Sprachelementen der Zielsprache erfolgen muss, auch wenn dadurch der denotative Inhalt geändert wird. Daher ist es wichtig, dass im Verlauf des Übersetzungsunterrichts nicht nur Sachtexte, sondern auch anspruchsvolle literarische Kreationen übersetzt werden, welche den Studenten und Studentinnen Anlass dazu geben, mit der Sprache auf spielerische Art und Weise umzugehen. Dadurch kann ihre kreative Kombinationsfähigkeit entwickelt und gefördert werden und die angehenden Übersetzer werden darüber hinaus auch sprachlich sensibilisiert - für die Ausgangs- wie für die Zielsprache.

\section{Literaturverzeichnis}

Duden (2001). Deutsches Universalwörterbuch [CD-ROM]. Mannheim: Duden.

Ficowski, Jerzy (2020). Dziwna rymowanka. https://quotepark.com/pl/cytaty/358731-jerzyficowski-pewien-zarlok-nienazarty-raz-wyglodnial-nie-na-za/. 
Głowiński, Michał/Okopień-Sławińska, Aleksandra/Sławiński, Janusz (1972). Zarys teorii literatury. Warszawa: Państwowe Zakłady Wydawnictw Szkolnych.

Gomringer, Eugen (1988/2020). ordnung. http://www.gregorschroeder.de/konkrete-poe sie.html.

Gomringer, Eugen (1988/2020). schweigen. https://www.lyrikline.org/en/poems/schwei gen-10152.

Gretkowska, Manuela (2004). Polka (Übers. von Paulina Schutz). München: dtv.

Gretkowska, Manuela (2001). Polka. Warszawa: W.A.B.

Kasperczak, Piotr. okruchy. https://okruchy.pl/roztrzasania/oko-tre-ze-mam-bol-czylislow-kilka-o-satyrze-fonetycznej/.

Jakobson, Roman (1971). Linguistik und Poetik. In: J. Ihwe (Hrsg.), Literaturwissenschaft und Linguistik. Ergebnisse und Perspektiven (S. 142-178). Frankfurt a.M.: Athenäum.

Jaroński, Paweł (2020). Widzą Cię żarówki. https://memy.pl/mem/89856/Widza_cie_zaro wki.

Jelinek, Elfriede (2005). Die Klavierspielerin. Reinberg bei Hamburg: Rowohlt Taschenbuch Verlag.

Jelinek, Elfriede (2004). Pianistka (Übers. von Ryszard Turczyn). Warszawa: W.A.B.

Klemensiewicz, Zenon (1955). Przekład jako zagadnienie językoznawstwa. In: M. Rusinek (Hrsg.), O sztuce tłumaczenia (S. 85-97). Wrocław: Zakład Narodowy im. Ossolińskich.

Kozak, Jolanta (2009). Przekład literacki jako metafora. Między logos a lexis. Warszawa: PWN.

Kubaszczyk, Joanna (2019). „Ryby rzeczne są zazwyczaj niedorzeczne“. Czego nie potrafi maszyna, czyli o kompetencjach tłumacza i prawach przekładu w kontekście przekładu literackiego. Studia Litteraria Universitatis Iagellonicae Cracoviensis, 14(4), 219-232.

Kubaszczyk, Joanna (2016). Faktura oryginału i przekładu. Warszawa: PWN.

Lec, Stanisław Jerzy (2018). Myśli nieuczesane. Wszystkie. Warszawa: Noir sur Blanc.

Martí, José (1929/2020). Guantanamera. https://www.youtube.com/watch?v=a-cLbcVvg SE.

Młynarski, Wojciech (1999). Bandanamera. https://www.tekstowo.pl/piosenka,maryla_ro dowicz,banda_na_mera_guantanamera_html.

Prokop, Izabela (2019). Kilka uwag o kontrastywnej analizie stylu. Scripta Neophilologica Posnaniensia, 18, 207-219.

Różewicz, Tadeusz (2002). ,Regression' in die Ursuppe. In: ders.: Szara strefa (S. 15). Wrocław: Wydawnictwo Dolnośląskie.

Różewicz, Tadeusz (2002). ,Regression' in die Ursuppe. In: ders.: Nauka chodzenia = Gehen lernen (Übers. von Karl Dedecius, Bernhard Hartmann, Andrzej Słomianowski). Wrocław: Biuro Literackie.

Schreiber, Michael (1999). Übersetzungstypen und Übersetzungsverfahren. In: M. SnellHornby/H.G. Hönig/P. Kußmaul/P.A. Schmitt (Hrsg.), Handbuch Translation (S. 151154). Tübingen: Stauffenburg Verlag.

Sedlaczek, Andre (2020). Heisse Würstchen. https://de.toonpool.com/cartoons/Heisse\%20 W\%C3\%BCrstchen_73890.

Sierotwiński, Stanisław (1986). Słownik terminów literackich. Wrocław u. a.: Ossolineum. Tuwim, Julian (2008). Pegaz dęba czyli panopticum poetyckie. Warszawa: Wydawnictwo Iskry.

Wysłouch, Seweryna (2001). Literatura i semiotyka. Warszawa: PWN. 


\title{
Translation und Transkreation: Zu Differenzen der Begriffssystematik zwischen Theorie und Praxis ${ }^{1}$
}

\begin{abstract}
Translation and transcreation: On the theoretical and practical differences in terminological classification

This chapter deals with the problem of the demarcation between translation and other types of text transformation, which has acquired a new topicality in view of the growing diversification of tasks within translation practice and the corresponding changes in the job profile. Transcreation is described as a new phenomenon in professional translational marketing practice, still investigated only to a small extent in research to date, and put in relation to translation and creative translation, revealing considerable differences in the understanding of translation between theory and practice, which render communication and exchange between both more difficult.
\end{abstract}

\section{Keywords}

translation, transcreation, creative translation, translation of promotional texts, copywriting

\section{Einleitung}

Angesichts der wachsenden Diversifizierung der Aufgaben im Bereich der translatorischen Praxis und der damit einhergehenden Veränderungen des Berufsbildes von TranslatorInnen ${ }^{2}$ gewinnt erneut das Problem der Abgrenzung der Translation (hier Übersetzen) von anderen Arten der Texttransformation an Aktualität. ${ }^{3}$ Innerhalb des Fachübersetzens bilden Technical Writing (Erstellen

1 Dieser Artikel ist Frau Professor Izabela Prokop anlässlich ihres 70. Geburtstages gewidmet in Anerkennung ihrer Verdienste auf dem Gebiet der sprach- und translationswissenschaftlichen Forschung.

2 Der Beruf ist von Frauen dominiert (vgl. auch Schmitt 2002: 71). Um dem Ausdruck zu verleihen, wird hier die geschlechtergerechte Binnen-I-Form der Berufsbezeichnung verwendet. Davon bleiben Zitate sowie andere verwendete Personenbezeichnungen ausgeschlossen.

3 Die ursprünglich von der Translationswissenschaft vorgenommenen Abgrenzungsversuche (vgl. u. a. Schreiber 1993, 2006), sind gegenwärtig insbesondere für die Praxis von Interesse. 
von technischer Dokumentation) und Lokalisierung (Anpassung eines Produkts bzw. einer Dienstleistung an einen lokalen, sprachlich und kulturell fremden Markt mit dem Ziel der Vermarktung) bereits etablierte Teilaspekte. Einen der neusten Trends des translatorischen Sektors der Sprachindustrie, und genauer gesagt der translatorischen Marketingpraxis, bildet die Transkreation. ${ }^{4}$ Von der wachsenden Bedeutung der Transkreation als Aufgabe von TranslatorInnen bzw. TranskreativtexterInnen zeugen immer zahlreicher erscheinende sowohl praxisals auch forschungsbezogene Beiträge zum Thema (vgl. u. a. Gaballo 2012, Katan 2014, 2016, Gambier 2016, Benetello 2018, Spinzi \& Rizzo 2018). Katan (2016: 378) spricht sogar von einem „transcreational turn“, einer neuen Wende in der Translationswissenschaft, die für TranslatorInnen eine Chance bietet, unter den veränderten Berufs- und Marktbedingungen zu überleben.

Der vorliegende Beitrag diskutiert die Begriffe Translation und Transkreation aus der Sicht der translatorischen Forschung und der professionellen Berufspraxis im Hinblick auf begriffliche Differenzen und Kommunikationsprobleme. Besonderes Augenmerk gilt der Transkreation als einem neuem translatorischen Phänomen im Bereich des Marketing.

\section{Translation und Transkreation}

Zum Zweck eines Abgrenzungsversuches müssen beide Begriffe zunächst definiert werden, wobei hier die Translation stets auf das Übersetzen fokussiert ist. Die Definition von Translation aus der Sicht der Theorie fällt je nach gewähltem translationswissenschaftlichem Paradigma unterschiedlich aus. Die Ansätze des linguistischen Paradigmas fassen das Übersetzen als einen auf Invarianzforderungen beruhenden Rekodierungsprozess auf, wobei zwischen dem Ausgangsund Zieltext eine Äquivalenzrelation besteht. Diesem „eng“ betrachteten steht der „weitgefasste“ Translationsbegriff des handlungstheoretischen Paradigmas entgegen, u.a. der Skopostheorie, nach der die Translation die Funktion ihres Zweckes und der Ausgangstext lediglich ein Informationsangebot ist, der einer translatorischen Interpretation in einer konkreten Rezeptionssituation bedarf (vgl. Reiß \& Vermeer 1984). Da die Translation dabei als Kulturtransfer ${ }^{5}$ ver-

4 Das Konzept ist auch für das literarische Übersetzen relevant. Der Ausdruck transcriação wird u. a. von dem brasilianischen Dichter und Übersetzungstheoretiker Haroldo de Campos (1929-2003) in seiner „Anthropofagia“-Theorie (1992) verwendet. Damit wird die Idee des literarischen Übersetzens hervorgehoben, das eine radikale Operation der Transkreation durchführt, die mit der Freiheit von der Treue-Norm gekennzeichnet ist und weniger mit Übersetzen als mit Neuschöpfen zu tun hat (vgl. Borowski 2012). Zur Geschichte des Terminus Transkreation siehe Spinzi und Rezzo (2018: $4 \mathrm{ff}$.).

5 Zur Kulturbedingtheit der Translation siehe Prokop (1999). 
standen wird, gehen die Aufgaben der TranslatorInnen weit über die der Sprachmittlung hinaus.

Der Neologismus Transkreation ist eine zugleich elegant wie fachlich anmutende Kontamination der Wörter Translation und Kreation. Analysiert man die Internetauftritte der Dienstleister der Sprachindustrie, so stößt man auf verschiedene Auffassungen von Transkreation, nach denen das Konzept mal als Übersetzungsart, mal als keine Übersetzung, dann wieder als Übersetzungsmethode charakterisiert wird. Verwendet wird die Bezeichnung sowohl in Bezug auf den Prozess als auch auf das Produkt. Nach Schriver (2011), dem Gründer und CEO des auf globales Marketing spezialisierten Sprach- und Kulturkommunikationsdienstleisters GLOBO, ist Transkreation weder eine strenge Übersetzung noch die Erstellung einer von Grund auf neuen Botschaft. Bei der Transkreation wird eine Umsetzung vorgenommen, die der Botschaft der Marke treu bleibt und gleichzeitig die gewünschte Reaktion bei den Zieladressaten auslöst. Rike (2014: 8) betrachtet Transkreation als „Hot Topic in Translation Studies“ und weist darauf hin, dass bei der Transkreation, die hauptsächlich im Falle von Marketingtexten und Internetseiten praktiziert wird, sowohl verbale als auch nonverbale Aspekte eines Textes sprachlich und kulturell an die Zielgruppe angepasst werden. Der Unterschied zur Lokalisierung besteht nach Rike darin, dass die Lokalisierung hauptsächlich bei den Fachtextsorten Software und Gebrauchsanleitung angewendet wird und nicht mit der Idee der Kreativität in Verbindung steht, die der Transkreation eigen ist. Für Sattler-Hovdar (2016:21), Translatorin, Transkreativtexterin und Autorin eines praxisbezogenen Handbuchs, welches das Transkreationskonzept der Branche zu systematisieren versucht, ist für die Transkreation die Zugehörigkeit der Texte zu der Gruppe von Marketingtexten entscheidend. ${ }^{6}$ Diese Texte, zu denen die Autorin u. a. Unternehmensbroschüren, Produktkataloge, Pressemitteilungen, Kundenzeitschriften, Internetauftritte, Beiträge in den sozialen Netzwerken, Werbebriefe, Mitarbeiterzeitschriften zählt, sind für das Image und den Umsatz des Auftraggebers wichtig. Nach SattlerHovdar (2016: 25) besteht Transkreation aus zwei Phasen: der Übersetzung und der strategisch-kreativen Überarbeitung unter Berücksichtigung veränderter kultureller Bedingungen auf der Grundlage eines kreativen Briefings. ${ }^{7}$ Übersetzung wird dabei mit einer wörtlichen Übertragung gleichgesetzt, die von einer aus Adaption und Neutexten bestehenden Überarbeitung, einem kreativen (Nach-)Schöpfungsprozess, abgerundet wird. Folglich stellt Transkreation, die

6 Nach Sattler-Hovdar (2016: 28) handelt es sich bei 90 \% aller Fälle der Übersetzung von Marketingtexten um Transkreation und damit um den Aufwand des strategisch-kreativen Prozesses.

7 Das strategische Kreativbriefing ist eine Grundvoraussetzung für Transkreation. Es sollte das Unternehmen, das Produkt, die Marke, die Ziele beschreiben, die der Auftraggeber mit dem Text erreichen möchte (vgl. Sattler-Hovdar 2016: 32-36). 
die beiden Phasen umfassend als ,interkulturell adaptierende Übertragung imagesensibler Texte" (ebd. 43) bezeichnet werden kann, einen doppelten Aufwand dar, „zum einem die Arbeit des Übersetzers und zum zweiten zusätzlich noch die Arbeit eines Texters" (ebd. 25). Zu den Kompetenzen einer TranskreativtexterIn gehört daher „ein Höchstmaß an interkultureller Sensibilität“ (ebd. 20) und die Fähigkeit, griffig schreiben zu können (ebd. 31).

Stellt man die Definitionen von Translation und Transkreation gegenüber, so wird eine Überlappung der Begriffe deutlich. Aus der Perspektive der linguistischen Translationsansätze schließt Transkreation die Translation im engeren Sinne ein, kommt jedoch mit ihr allein nicht aus und erfordert eine zusätzliche Überarbeitung. Der Skopostheorie zufolge ist Transkreation jedoch mit der Translation identisch, für die eine zielkulturelle Adaption, stilistisch-kreative Überarbeitung wie auch Neukonzeption des Zieltextes selbstverständlich sind. Im Falle des Fachübersetzens, und darunter des Marketingtext-Übersetzens von Texten der fachexternen Kommunikation mit niedrigem Fachlichkeitsgrad, für das der funktionale Ansatz und damit die Anpassung des Translats an die zielsprachlichen und -kulturellen Normen gilt, kann Transkreation theoretisch mit der Translation im weiteren Sinne gleichgesetzt werden. Je nach Fassung des Translationsbegriffs schließt also die Transkreation die Translation ein oder sie gleicht ihr.

\section{Transkreation und kreatives Übersetzen}

Transkreation wird von der Translationsforschung oft mit kreativem Übersetzen des funktionalistischen Ansatzes verglichen bzw. gleichgestellt (vgl. u. a. Schmitt 2016: 267f.). Dies scheint jedoch aus folgenden Gründen problematisch. Zum einen basieren die beiden Konzepte, wie bereits festgestellt, auf unterschiedlichen Übersetzungsbegriffen - Transkreation auf dem engen, kreatives Übersetzen auf dem weiten Begriff, zum anderen liegen ihnen auch unterschiedliche Kreativitätsauffassungen (vgl. Schreiber 2017b) zugrunde.

Das Übersetzen oszillierte seit jeher je nach Textautorität zwischen Kunst und Handwerk. Da das Handwerk gegenüber der Kunst grundsätzlich nicht mit Kreativität assoziiert wird, galt das Fachübersetzen lange Zeit als keine schöpferische Tätigkeit. ${ }^{8}$ Kreatives Potenzial wurde lediglich dem künstlerischen

8 In der gängigen Meinung wird das Fachübersetzen weiterhin nicht als kreative Tätigkeit betrachtet (vgl. Risku 1998: 225). Dagegen weiß jede praktizierende ÜbersetzerIn, dass „Reproduzieren lediglich die Endphase einer mentalen Operationskette ist, in welcher Prozesse, wie Analysieren, Vergleichen, Analogisieren, Inferenzieren, Abwägen, Auswählen, Planen, Kombinieren etc. interaktiv in Verbindung treten“ (Wilss 1988: 8), die zu ihrer Durchführung durchaus Kreativität verlangen. 
Übersetzen zugesprochen ${ }^{9}$. Erst die funktionalistische Perspektive der Translationswissenschaft machte deutlich, dass auch zum Fachübersetzen Kreativität gehört $^{10}$, wobei je nach Fachtextsorte ein unterschiedliches Maß an Kreativität erforderlich ist. Den Gegenstand des Fachübersetzens bilden nicht nur informative und funktionskonstant zu übersetzende, sondern auch funktionsvariant $\mathrm{zu}$ übertragende und appellative (Werbe)Texte, die eine andere translatorische Behandlung als informative Texte verlangen. Aber auch hochgradig fachliche Texte technischen Inhalts, die auf den ersten Blick kein kreatives Potenzial aufweisen, bieten vielfältige Gelegenheiten zur translatorischen Kreativität, z. B. im Umgang mit Ausgangstext-Defekten oder Erscheinungen der Kulturspezifik (vgl. Schmitt 1999: 156-256). ${ }^{11}$ Grundsätzlich wird bei der Neuformulierung einer Botschaft in einer anderen Sprache stets ein gewisses Maß an Kreativität gefordert. Ein besonders hohes Maß an Kreativität wird bei pragmatisch bedingten Veränderungen impliziert, wenn, wie im Falle der Marketingtexte, Anpassungen an die Erwartungen und Bedürfnisse der zielkulturellen Rezipienten erforderlich sind. Übersetzen wird im Funktionalismus als Problemlösungsprozess angesehen, die translatorische Kreativität besteht schließlich auch in der Fähigkeit, geeignete Problemlösungsstrategien zu finden und anzuwenden.

In der Translationswissenschaft wurde die translatorische Kreativität unter verschiedenen Aspekten beleuchtet. ${ }^{12}$ Snell-Hornby und Vannerem (1986) richteten das Augenmerk von der Analyse des Ausgangstextes hin zu dem kreativen Prozess der Reproduktion, der zielsprachlichen Neugestaltung, und zeigten die kreative Rolle der ÜbersetzerInnen auf. Wilss (1996: 53) sah das Übersetzen als transformative Tätigkeit im Spannungsfeld zwischen Kreativität und Rekreativität an, da im Übersetzungsprozess der Zieltext neu formuliert

9 „When creativity is referenced or examined in translation studies, it is usually with regard to literary texts because, it is the nature of the literary text to invite creative engagement" (BoaseBeier 2007: 55).

10 Nach der Skopostheorie ist Kreativität erforderlich, um aus dem Informationsangebot des Ausgangstextes einen Zieltext zu kreieren, der in der Zielkultur die gewünschte Funktion erfüllt. So „ist [der Translator] nicht nur Mittler, sondern auch eigenständig kreativ tätig“ (Reiß \& Vermeer 1984: 7).

11 Die Kulturspezifik von Texten ist ein in der angewandten Linguistik häufig diskutiertes Thema. Siehe u.a. die didaktische Sicht auf das Problem bei Stork und Adamczak-Krysztofowicz (2016).

12 Pisarska (1989) erforscht die kreative Übersetzung von Metaphern in nichtliterarischen Texten, Kenny (2001) die lexikalische Kreativität mit korpuslinguistischen Methoden, LeeJahnke (2005) kreative Übersetzungsprozesse hinsichtlich ihres didaktischen Nutzens, Bălăcescu und Stefanink (2006) die Kreativität in der Rezeptionsphase des Ausgangstextes. Bayer-Hohenwarter (2011: 52) untersucht die translatorische Kreativität sowohl im Hinblick auf den Prozess als auch das Produkt und definiert sie als „Leistungsmerkmal translatorischen Handelns, das im Translationsprozess und/oder Produkt an Ausdrucksformen von Originalität, Flexibilität und Denkflüssigkeit erkennbar wird und im Idealfall in ein optimal angemessenes Translat mündet.“ 
wird, obwohl er sich stets auf den Ausgangstext bezieht. Neubert (1997) bezeichnete die Übersetzung als kreative Leistung und wies darauf hin, dass die Unterschiede zwischen der Ausgangs- und Zielsprache bei der Formulierung des Translats Änderungen erfordern. Nach Kußmaul (2000: 31), dem Autor der ersten Monographie über kreatives Übersetzen, sind es die obligatorischen Veränderungen des Ausgangstexts, aufgrund deren eine kreative Übersetzung entsteht. Diese stellt - in Einklang mit dem psychologischen Kreativitätsbegriff etwas (mehr oder weniger) Neues dar, was in einer Kultur von Experten mit Blick auf einen bestimmten Verwendungszweck als angemessen akzeptiert wird. Mit dem Kreativitätsbegriff von Kußmaul polemisiert Schreiber (2017b: 351f.), für den das Vorliegen einer Inkongruenz von Ausgangs- und Zielsprache als Grund für obligatorische Veränderungen kein hinreichendes Kriterium für eine kreative Übersetzung ist. Denn Probleme dieser Art werden mit Grammatikkenntnissen gelöst, ohne dass dabei etwas Neues entsteht. Entscheidend ist nach Schreiber nicht die Frage, ob eine obligatorische Veränderung stattfindet, sondern ob bereits eine etablierte Standardentsprechung vorliegt, die der Übersetzer/die Übersetzerin in einem Wörterbuch oder in einer existierenden Erstübersetzung nachschlagen kann. Ist dies der Fall, so haben Lexikographen bzw. frühere ÜbersetzerInnen bereits eine kreative Vorarbeit geleistet, auf die der Übersetzer/ die Übersetzerin aufbauen kann, ohne selbst kreativ werden zu müssen. Die translationswissenschaftliche Debatte über kreatives Übersetzen zeigt, dass das Phänomen definitorisch nicht leicht zu bestimmen ist.

Die Praktikerin Sattler-Hovdar (2016: 43-44) verbindet Kreativität mit der Übertragung von Marketingtexten, assoziiert jedoch Transkreation nicht mit kreativem Übersetzen, das sie auf das literarische Übersetzen bezieht. In der Transkreation von Marketingtexten spielt Kreativität zwar eine wesentliche, jedoch keine dominierende Rolle. Transkreation beruht vielmehr auf einem strategischen Vorgehen in Anlehnung an das kreative Briefing als auf purer Kreativität. ${ }^{13}$ Den starken Fokus auf Kreativität hin zur Bindung an die Strategie versucht Sattler-Hovdar mit dem Attribut strategisch-kreativ zu lenken, mit dem sie die Überarbeitungsphase der Transkreation bezeichnet. Daraus ist $\mathrm{zu}$ schließen, dass die erste Phase der Transkreation - das Übersetzen - unkreativ ist. Für Kußmaul ist dagegen Kreativität ein inhärentes Merkmal des Übersetzens, das er jedoch weiter als Sattler-Hovdar fasst. Durch die primäre Betonung des Strategischen bei der Transkreation wird das Augenmerk darauf gerichtet, was der Auftraggeber mit dem Text erreichen will und wie die Transkreativtex-

13 Aus diesem Grund bevorzugt Sattler-Hovdar (2016: 18-19) die Termini Adaption, Transadaption oder Übertexten. Sie verwendet jedoch „Transkreation“, weil sich der Terminus bereits in der Branche durchgesetzt hat, formuliert jedoch gegen ihn den Vorbehalt, er könnte den Kunden suggerieren, dass ihre Texte kreativ im Sinne von ungenau behandelt würden, wobei das Gegenteil - Genauigkeit als Grundvoraussetzung - wahr ist. 
terIn dieses Ziel am besten umsetzen kann. Daher ist es für die Kompetenz der TranskreativtexterInnen wichtiger, sich in den strategischen Aspekten der Markenpositionierung und Verbraucherpsychologie als in Kreativitätstechniken fortzubilden (ebd. 43), um „markenstrategisch und aus Konsumentensicht zu denken“ (ebd. 13). Kreativität kann eingesetzt werden, um die gewünschte Aufmerksamkeit der Zieltextadressaten zu erreichen, bleibt aber stets dem strategischen Ziel untergeordnet. Im Einzelnen wird Kreativität bei Recherchen, Ideenfindung, Anwendung von Kreativitätstechniken und Spiel mit Sprache (stilistischer Feinschliff, der die Texte geschmeidig, rhythmisch, dynamisch, taktvoll („Transkreation fürs Ohr“, ebd. 78-79) erscheinen lässt) stets mit dem Ziel eingesetzt, einen dem Briefing entsprechenden Zieltext zu schaffen. Anders als beim kreativen Übersetzen wird hier die Kreativität also im Vorfeld und nach Ablauf des Übersetzens eingesetzt. Während Neukonzeption (also das Texten) Kreativität erfordert, impliziert die Transkreationsleistung lediglich ein gewisses Maß an Kreativität, das aus unterschiedlichen sprachlich-kulturellen Besonderheiten der Ausgangs- und Zielsprache resultiert (ebd. 42). Kußmaul und Sattler-Hovdar sind sich darin einig, dass Kreativität durch sprachliche Inkongruenzen bedingt und ein graduelles Phänomen ist. Wichtiger als Kreativität ist außerdem für Sattler-Hovdar (2016: 43) die Disziplin und Bereitschaft zu umfassenden Recherchen sowie der Wille, „einen Text immer noch griffiger gestalten zu wollen“. Bei imagesensiblen Marketingtexten gehen auch die Gebote der Glaubwürdigkeit und der Relevanz vor Kreativität (ebd. 45-47), denn Texte sollten von der Zielgruppe als „nützlich, interessant, einzigartig und widerspruchsfrei“ (ebd. 48) empfunden werden. Zusammenfassend geht es in der Transkreation nach Sattler-Hovdar (2016: 70) darum, durch Verinnerlichung des Briefings, Brainstormings, Recherchen, Kreativitätstechniken in möglichst eingängiger Form und möglichst präzise die Intention des Auftraggebers zu vermitteln. Kreativität kommt dabei erst in der kreativen Schaffenspause zustande, nachdem die TranskreativtexterIn das Briefing verinnerlicht hat, in die Situation und Gedankenwelt des Empfängers eingetaucht ist, Recherchen durchgeführt, Unklarheiten geklärt, Fragen beantwortet, die Ideensammlung niedergeschrieben und eine Rohfassung des Zieltextes erarbeitet hat. Grundvoraussetzung für jegliche Form von Kreativität ist daher immer eine vollständige Vertrautheit mit der Thematik, den Spezifika des Auftrags und der intendierten Aussage, ob dies auf Erfahrung, Recherchen, Briefing oder bestenfalls auf einer Kombination aus allem beruht.

Stellt man die Konzepte der Transkreation und des kreativen Übersetzens zusammen $^{14}$, so ergeben sich zwischen ihnen doch Unterschiede, die Transkreation nicht mit kreativem Übersetzen gleichsetzen lassen.

14 Für Schreiber (2017b: 357) greifen die Kriterien für beide Konzepte zu kurz, da es sowohl 


\section{Schlussbetrachtung}

Die Begriffe Transkreation und Translation (Übersetzen und kreatives Übersetzen) decken sich teilweise bis vollständig je nach Paradigma und Verständnis der Translation. Die translationswissenschaftliche und die berufspraktische Perspektive gehen nicht nur von unterschiedlichen Übersetzungs-, sondern auch von abweichenden Kreativitätsbegriffen aus. Das kreative Übersetzen des Funktionalismus repräsentiert eine weite Sicht auf das Übersetzen, die durchaus mit dem begrifflichen Umfang der Transkreation verglichen werden kann. Die Transkreation grenzt den Übersetzungsbegriff dagegen auf den sprachlichen Transfer und die formale Wörtlichkeit ein. Während der theoretische Translationsbegriff inhaltlich stets ausgeweitet wurde, herrscht in der Praxis die gegenläufige Tendenz vor, den Translationsbegriff einzugrenzen und durch engere spezialisierte Begriffe abzulösen (vgl. Schreiber 2017a, 2017b). Dies scheint in vieler Hinsicht plausibel. Der Terminus Translation wurde Mitte der 1960er Jahre von der Translationslinguistik mit der Bedeutung des linguistischen Transfers geprägt. Seitdem sich die Translation jedoch vom Sprach- zum Kulturtransfer entwickelte, wird dieser Terminus der Komplexität des Begriffes nicht mehr gerecht. Im Laufe der fünf Jahrzehnte einer intensiven translatorischen Tätigkeit hat sich der Beruf verändert und ein Spektrum von Aufgaben und Dienstleistungen hervorgebracht, die immer weniger mit Translation im herkömmlichen Sinn zu tun haben. Anstatt Translation bzw. Übersetzen werden heute Software-, Spiele-, Multimedialokalisierung, Web-Globalisierung, Internationalisierung, Translationsmanagement, Transkreation u. a. betrieben. Translation, die in der Berufspraxis nach wie vor mit linguistischem Transfer assoziiert wird, scheint ausgedient $\mathrm{zu}$ haben. In der Theorie beansprucht zudem der weite Translationsbegriff der Skopostheorie, auf alle Textsorten und alle Arten der Texttransformationen anwendbar zu sein, was aus praktischer Sicht nicht immer klar ist. Der Translationsbegriff scheint auszuufern, wenn bspw. behauptet wird: „ein Translator bietet andere Information a uf andere Weise an" (Reiß \& Vermeer 1984: 123 H. i. O.) Dem stehen die reellen translatorischen Dienstleistungen der Praxis entgegen, die präzise benannt, voneinander abgegrenzt und preislich kalkuliert werden müssen. Zudem hat sich der Terminus „Übersetzung“

obligatorische Veränderungen gibt, die keine Kreativität erfordern (grammatische Transformationen) als auch Marketingtexte, die nicht kreativ übertragen werden (unübersetzte Werbeslogans). Der Forscher schlägt vor, statt dessen das Vorliegen einer Standardentsprechung, eines konventionalisierten Übersetzungsverfahrens oder einer wieder verwendbaren Erstübersetzung als Kriterium heranzuziehen, in welchen Fällen, der Übersetzer/die Übersetzerin nicht kreativ werden muss. Folglich ergibt sich die Notwendigkeit zur Kreativität immer dann, wenn eine Lücke vorliegt, die in der anzufertigenden Übersetzung zum ersten Mal zu schließen ist, indem eine neue Lösung geschaffen wird. 
in der Praxis desavouier ${ }^{15}$, weshalb die Tätigkeit durch neuere Ausdrücke aufgewertet werden soll. Diese neuen Bezeichnungen sollen auch dem Arbeitsaufwand, der sich hinter einer Aufgabe verbirgt, besser gerecht werden. z. B. im Falle der Transkreation deutlich machen, dass es sich dabei um mehr als nur sprachlichen Transfer handelt. Eine Ausweitung des Übersetzungsbegriffes, wie sie teilweise auch von der Forschung selbst kritisiert wird (vgl. u.a. Zybatow 2004), ist folglich für die Berufspraxis kontraproduktiv. Die Marketingpraxis distanziert sich vom Übersetzen, weshalb sie sich auch mit kreativem Übersetzen nicht identifizieren kann. Kreativität gehört aus der Sicht des Marketing zum Texten, und nicht zum Übersetzen. Während die Theorie auf Übersetzen beharrt, hat die Praxis den Terminus längst zugunsten neuerer Ausdrücke verworfen, die den Entwicklungstendenzen der Branche besser entsprechen. Trotz der terminologischen Abgrenzung ist jedoch auf Grund der Überlappung eine scharfe begriffliche Grenzziehung zwischen Transkreation und Translation nicht möglich. Vielmehr kann Transkreation daher als eine weitere Entwicklungsstufe der Translation betrachtet werden, die ihren Platz in einem Kontinuum der spezifischen translatorischen Handlungen - zwischen dem traditionsreichen literarischen und dem innovativen maschinellen Übersetzen - sucht. Die Translationswissenschaft sollte Transkreation auf jeden Fall mehr, als bisher erfolgt, in ihr Interessensspektrum einbeziehen, und sich mit dem neuen, noch recht unscharfen Begriff, auseinandersetzen, um einen theoretischen Rahmen auszuarbeiten. Dies ist u.a. aus dem Grunde lohnend, dass Marketingtexte im Wirkungsbereich der Humanübersetzung bleiben, während zahlreiche andere Fachtextsorten auf Grund des steigenden Automatisierungsgrades immer stärker in den Bereich der maschinellen Übersetzung fallen. Dafür muss aber die Theorie mehr Notiz von den Neuerungen der Praxis nehmen und die Praktiker müssen mit ihren Konzepten zu der Theorie durchdringen, damit bspw. Transkreation nicht bloß als neuer, attraktiv klingender Name für kreatives Übersetzen oder ein Modeschlagwort abgetan wird. Man muss Schreiber (2017b: 357) recht geben, wenn er sagt, dass wenn sich die gegenläufigen Tendenzen der Wissenschaft und Praxis in der Systematik der translatorischen Grundbegriffe fortsetzen, zwischen Translationsforschern und Praxisexperten gravierende Kommunikationsprobleme auftreten werden. Um dies zu verhindern, sollten neu entstandene Begriffe der Berufspraxis, wie Transkreation, von der Theorie systematisch bearbeitet werden. In der Angewandtheit der Translationswissen-

15 Für Sattler-Hovdar (2016: 86) sind „Übersetzungen in Misskredit geraten“, da sie viel zu wörtlich sind und im Falle von Marketingtexten daher mehr Schaden als Nutzen bringen können. Unter Übersetzung wird in der Branche eine „mehr oder weniger roboterhafte Reproduktion eines vorgegebenen Textes" verstanden. 
schaft als Teildisziplin der Angewandten Linguistik liegt die Chance, die Wissenschaft und Praxis besser zu integrieren.

\section{Literaturverzeichnis}

Bălăcescu, Ioana/Stefanink, Bernd (2002). Kognitivismus und übersetzerische Kreativität. Lebende Sprachen, 2, 50-61.

Bayer-Hohenwarter, Gerrit (2011). Kreativität in populärwissenschaftlichen und instruktiven Texten im Vergleich. Kein Raum für Kreativität beim Übersetzen von instruktiven Texten? trans-kom, 4 (1), 49-75. http.//www.trans-kom.eu/bd04nr01/trans-kom_04_ 01_03_Bayer-Hohenwarter_Kreativitaet.20110614.pdf (Stand 21.02.2020).

Benetello, Claudia (2018). When Translation is not enough: Transcreation as a conventiondefying practice. A practicioner's perspective. The Journal of Specialised Translation, $29,28-43$.

Boase-Beier, Jean (2007). Loosening the grip of the text. Theory as an aid to creativity. In: M. Perteghella/E. Loffredo (Hrsg.), Translation and creativity (S. 47-56). London: Bloomsbury Publishing.

Borowski, Gabriel (2012). Transkreacja: myśl przekładowa Haroldo de Camposa. Przekładaniec, 26, 87-107. DOI:10.4467/16891864PC.12.005.0838.

De Campos, Haroldo (1992). Metalinguagem e outras metas: ensaios de teoria e critica literária. Sao Paulo: Perspectiva.

Gaballo, Viviana (2012). Exploring the boundaries of transcreation in specialized translation. ESP Across Cultures, 9, 95-113.

Gambier, Ives (2016). Rapid and radical changes in translation and translation studies. International Journal of Communication, 10, 887-906.

Katan, David (2014). Uncertainty in the translation professions: time to transcreate? Cultus, 7, 10-19.

Katan, David (2016). Translation at the cross-roads: Time for the transcreational turn? Perspectives, 24 (3), 365-381.

Kenny, Dorothy (2001). Lexis and creativity in translation. A corpus-based study. Manchester: St. Jerome Pub.

Kußmaul, Paul (2000). Kreatives Übersetzen. Tübingen: Stauffenburg.

Lee-Jahnke, Hannelore (2005). Unterrichts- und Evaluierungsmethoden zur Förderung des kreativen Übersetzens. Lebende Sprachen, 50 (3), 125-132.

Pisarska, Alicja (1989). Creativity of translators: The translation of metaphorical expressions in non-literary texts. Poznań: Wydawnictwo Naukowe Uniwersytetu im. Adama Mickiewicza.

Prokop, Izabela (1999). Kulturbedingtheit des Translationsprozesses. In: A. Kątny/Ch. Schatte (Hrsg.), Das Deutsche von innen und von außen. Ulrich Engel zum 70. Geburtstag (S. 235-242). Wydawnictwo Naukowe Uniwersytetu im. Adama Mickiewicza.

Reiß, Katharina/Vermeer, Hans J. (1984). Grundlegung einer allgemeinen Translationstheorie. Tübingen: de Gruyter.

Rike, Sissel Marie (2014). Hot Topics in Translation Studies: Transcreation. Newsletter. European Society for Translation Studies, 44, 8-9. 
Risku, Hanna (1998). Translatorische Kompetenz. Kognitive Grundlagen des Übersetzens als Expertentätigkeit. Tübingen: Stauffenburg.

Sattler-Hovdar, Nina (2016). Translation - Transkreation. Vom Über-Setzen zum ÜberTexten. Berlin: Bundesverband der Dolmetscher und Übersetzer.

Schmitt, Peter A. (1999). Übersetzen und Technik. Berlin: Stauffenburg.

Schmitt, Peter A. (2002). Fachübersetzen - eine Widerlegung von Vorurteilen. In: J. Best/ S. Kalina (Hrsg.), Übersetzen und Dolmetschen. Eine Orientierungshilfe (S. 60-73). Tübingen/Basel: Francke Verlag.

Schmitt, Peter A. (2016). Handbuch Technisches Übersetzen. Berlin: Bundesverband der Dolmetscher und Übersetzer.

Schreiber, Michael (1993). Übersetzung und Bearbeitung. Zur Differenzierung und Abgrenzung des Übersetzungsbegriffs. Tübingen: Gunter Narr Verlag.

Schreiber, Michael (2006). A prototypical model of translation types. In: C. Heine, Carmen/ K. Schubert/H. Gerzymisch-Arbogast (Hrsg.), Text and translation. Theory and methodology of translation (S. 382-390). Tübingen: Gunter Narr Verlag.

Schreiber, Michael (2017a). Quo vadis, Übersetzungsbegriff? Tendenzen und Paradoxien. In: L. Heller (Hrsg.), Kultur und Übersetzung. Studien zu einem begrifflichen Verhältnis (S. 51-62). Bielefeld: transcript Verlag.

Schreiber, Michael (2017b). Kreativität in Translation und Translationswissenschaft. Zwei Fallbeispiele und ein Vorschlag. In: L. Cercel/M. Agnetta/M. T. Amido Lozano (Hrsg.), Kreativität und Hermeneutik in der Translation (S. 349-358). Tübingen: Narr Francke Attempto Verlag.

Schriver, Gene (2011). Linking language to the technology and communication process (GLOBO). https://www.gala-global.org/publications/linking-language-technology-andcommunication-process-0 (Stand 20.02.2020).

Snell-Hornby, Mary/Vannerem, Mia (1986). Die Szene hinter dem Text. „scenes-and-frames semantics“ in der Übersetzung. In: M. Snell-Hornby (Hrsg.), Übersetzungswissenschaft - Eine Neuorientierung (S. 184-205). Tübingen: Francke Verlag.

Spinzi, Cinzia/Rizzo, Alessandra (Hrsg.) (2018). Translation or transcreation? Discourses, texts and visuals. Cambridge: Cambridge Scholars Publishing.

Stork, Antje/Adamczak-Krysztofowicz, Sylwia (2016). „Kulturspezifik von Texten“. In: S. Ballweg (Hrsg.), Schreibberatung und Schreibförderung: Impulse aus Theorie, Empirie und Praxis (S. 83-95). Frankfurt a.M.: Peter Lang.

Wilss, Wolfram (1988). Kognition und Übersetzen. Zu Theorie und Praxis der menschlichen und der maschinellen Übersetzung. Tübingen: Niemeyer.

Wilss, Wolfram (1996). Übersetzungsunterricht. Eine Einführung. Tübingen: Gunter Narr Verlag.

Zybatow, Lew N. (2004). Was sagt die Wissenschaft zur Wissenschaft zur Translationswissenschaft. In: J. Albrecht/H. Gerzymisch-Arbogast/D. Rothfuß-astian (Hrsg.), Übersetzung - Translation - Traduction. Neue Forschungsfragen in der Diskussion. Festschrift für Werner Koller (S. 253-271). Tübingen: Gunter Narr Verlag. 
Open-Access-Publikation im Sinne der CC-Lizenz BY-NC-ND 4.0

(C) 2020, Vandenhoeck \& Ruprecht GmbH \& Co. KG, Göttingen ISBN Print: 9783847111863 - ISBN E-Lib: 9783737011860 


\author{
Alicja Sakaguchi \\ (Uniwersytet im. Adama Mickiewicza w Poznaniu)
}

\title{
Die Kunst des Verstehens und Übersetzens sakraler Texte. Ein kritischer Blick auf die Wiedergabe einiger Schlüsselinhalte auf der Grundlage der deutschen, polnischen und Esperanto-Bibelübersetzungen
}

\begin{abstract}
The art of understanding and translating sacral texts. A critical look at the rendering of some key content based on German, Polish and Esperanto Bible translations

This chapter discusses the art of understanding and translating sacral texts from a phenomenological point of view. First, it deals with the hermeneutic conditions and goals of the Bible interpretation. Subsequently, translations of selected key contents of the Bible in German, Polish and also in Esperanto are considered in a critical manner. The analysis of the selected key passages and key words suggests that the examined translations follow different intentions and principles of the respective translators and contract authorities of the Bible translations. In most cases, a strong ecclesiastical and discursive adaptation of the key contents of the text can be observed (individual expressions, utterances, presuppositions). On the other hand, contemporary text hermeneutics shows a blatant onesidedness in understanding the meaning of many biblical core contents. Namely, if culture, tradition, language, in the case of translations of the Bible, also by the theological world picture designed for a given institutional religion become the dominant features of religious hermeneutics, the personal qualities of biblical writers (the prophets) and their messages will be completely outshone.
\end{abstract}

\section{Keywords}

sacred language, translation of biblical key content, types of Bible translations, hermeneutic key, prophecy, prophetic inspiration, understanding, discursive bondage of thinking and understanding

\section{Die Kunst des Verstehens und ihre Bedeutung für die Übersetzungspraxis}

In den letzten dreißig Jahren hat sich die Übersetzungswissenschaft zunehmend mit den kognitiven Fähigkeiten und mentalen Prozessen von Übersetzern und Dolmetschern beschäftigt (z. B. Risku 1998, Presas 2004, Van Vaerenbergh 2004). Das Ziel der Untersuchungen in diesem Bereich ist ein tieferes Verständnis translatorischer Kompetenz und des translatorischen Handelns. Es geht dabei 
um die Fähigkeiten des Übersetzers und den Vorgang des Übersetzens. Die daraus gewonnenen Erkenntnisse sollen in der Übersetzer- und Dolmetscherausbildung angewendet werden. Es ist offensichtlich, dass die Psychologie, konkret: das kognitive Paradigma, für die Übersetzungstheorie und -praxis eine wesentliche Rolle spielt. Doch im Bereich der Übersetzungswissenschaft ebenso wie in der westlichen Linguistik sind außergewöhnliche kognitive Fähigkeiten und Vorgänge, wie das ganzheitliche Sehen oder die Autoreninspiration, ohne die das Verständnis und die Rezeption sakraler Texte kaum möglich sind, gänzlich aus dem Blick geraten.

Es sei hier angemerkt, dass das Thema dieses Beitrags von einem bislang vernachlässigten kognitivistischen Blickwinkel aus angegangen wird und das aus einer ganz persönlichen Motivation. Die Erläuterung des Hintergrunds meines Zugangs zu den sakralen Schriften findet sich in meinem Buch „Sprechakte der mystischen Erfahrung..." (Sakaguchi 2015) sowie in meiner interdisziplinären Untersuchung über religiöse Sprache (Sakaguchi 2011). Eine der Hauptthesen lautet, dass die Inspiration (lat. inspiratio ,Geisteinhauchung') heiliger und mystischer Schriften wie auch die Geistesgabe zur Deutung solcher Texte das Ergebnis der Prophetie beziehungsweise des mystischen Bewusstseins sind. ${ }^{1}$ Prophetie geht mit einer erweiterten oder vertieften Bewusstheit einher, die von den frühchristlichen Autoren erleuchtete Erkenntnis (Gnosis) oder unmittelbares Erfahrungswissen, das von Gott kommt, genannt wird. Schon der zweite Petrusbrief $1,19-21^{2}$, also die Bibel selbst, liefert uns eine Art Definition von Prophetie:

Dadurch ist das Wort der Propheten für uns noch sicherer geworden, und ihr tut gut daran, es zu beachten; denn es ist ein Licht, das an einem finsteren Ort scheint, bis der Tag anbricht und der Morgenstern aufgeht in eurem Herzen. Bedenkt dabei vor allem dies: Keine Weissagung der Schrift darf eigenmächtig ausgelegt werden; denn niemals wurde eine Weissagung ausgesprochen, weil ein Mensch es wollte, sondern vom Heiligen Geist getrieben haben Menschen im Auftrag Gottes geredet. (Hervorhebung durch die Autorin)

Die Schlüssel- oder Kerninhalte der biblischen Sprache gehen mit (universellen) spirituellen Formen der Propheten einher (ich nenne sie Sakralia). Diese seltene semantische Erscheinung (ja geistige Gabe) samt ihrem sprachlichen Ausdruck, ist eine Art geistiger Wortschatz der Mystiker und Propheten. Beispiele dafür sind Wörter und Ausdrücke wie: Wort Gottes, Haus meines Vaters, Gott erkennen, Siegel der Propheten, geistige Wiedergeburt, Auferstehung oder die herme-

1 Siehe dazu Kapitel VI. 6.3. ,Bewusstseinstypen: personales und transpersonales Bewusstsein in meinem Buch „Sprechakte der mystischen Erfahrung...“ (Sakaguchi 2015: 192-199). Vgl. ferner Heschel ([1936] 1962) sowie Albrecht ([1951] 2019).

2 Soweit nicht anders angegeben, beziehe ich mich durchgehend auf die „Neue Jerusalemer

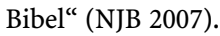


neutischen Begriffe, wie Bewusstseinsentleerung, Loslösung und Läuterung (purgatio) oder prophetische Eingebung. Sie gehen mit einer bestimmten Existenzweise eines Einzelnen einher und sind kulturübergreifend. Auch wenn solche Ausdrücke heute für viele Menschen altertümlich und verstaubt klingen, spielen sie für sakrale Texte eine Schlüsselrolle. Denn die entscheidenden Fragen, die die Menschen bewegen, sind über die Jahrtausende hin dieselben geblieben.

Der vorliegenden Betrachtung liegt ein phänomenologischer Ansatz zugrun$\mathrm{de}^{3}$. So wird hier versucht, die kognitive Basis zu erfassen, die für das jeweilige Denken, Verhalten und Handeln sowohl eines inspirierten biblischen Autors wie auch eines Übersetzers und Übersetzungsforschers verantwortlich ist. Diese Basis betrifft sowohl den weltlichen (profanen) wie auch den transzendenten (prophetisch-sakralen) Bereich mit seinen je eigenen Gesetzmäßigkeiten, Qualitäten, Kriterien und seinen je eigenen Sprachverwendungsweisen. Übersetzungsverfahren und translatorische Kompetenzen als solche, obwohl in diesem Zusammenhang auch sehr wichtig, stehen mithin nicht im Zentrum der vorliegenden Betrachtungen.

\section{Was ist Verstehen? Diskursive Gebundenheit von Denken und Verstehen und die folgenschwere Vernachlässigung des prophetischen Diskurses}

Im vorliegenden Beitrag wird das Verste h e n als Tätigkeit und Befähigung von Menschen verstanden, den Sinn und den Zusammenhang der sprachlichen Zeichen in Texten zu erfassen und adäquat weiter zu vermitteln. So wird das Verstehen als ein grundlegender Modus menschlicher Orientierung angesehen, ja als eine der wichtigsten geistigen (oder kognitiven) Fähigkeiten. Die Fähigkeit, den Sinn von etwas zu erfassen, etwas zu begreifen, etwas gut zu kennen, es richtig

3 Die phänomenologische Methode geht auf Edmund Husserl zurück. Sie wurde in der Linguistik bisher kaum eingesetzt. Die Phänomenologie geht davon aus, dass komplexe Sachverhalte aus einfacheren aufgebaut sind, welche wiederum aus noch einfacheren bestehen. Die Endpunkte dieser Begründungskette werden Phänomene genannt. Das Ziel der Phänomenologie ist eidetische Erkenntnis (gr. eidos , das in die Augen Fallende', also reine Erscheinung). So hat die Phänomenologie zum Ziel, Phänomene zu erhellen, die abseits unseres engeren Wahrnehmungsbereichs liegen. Die Verwendung der phänomenologischen Methode in der Linguistik verschafft prinzipiell die Möglichkeit, aufgrund des individuellen Sprachverhaltens (Idiolekt) die jeweilige existentielle Stufe des Menschen oder allgemein den Grad der inneren Reife zu bestimmen. Der Grad der inneren Reife wird hier als ein Aufrechthalten der Verbindung zur Realität begriffen. Weiterhin erlaubt die phänomenologische Methode, die Motive und Beweggründe zu erfassen, die den Handlungen und dem Sprachverhalten einzelner Menschen zugrunde liegen. Des Weiteren kann man mit Hilfe der phänomenologischen Methode der Bedeutung, dem Ziel oder der Botschaft verschiedenartiger Texte auf die Spur kommen. 
einzuordnen und damit sachgemäß umzugehen zu wissen, also das Verstehen schlechthin, kann man unter Umständen sogar als eine K u n s t betrachten. Ohne das Verstehen des Sinns und der Bedeutung einzelner Ausdrücke und Textaussagen ist jedenfalls ein bewusstes, verantwortungsvolles Übersetzen nicht möglich.

Während die Exegese die praktische Auslegung eines biblischen Textes ist, beleuchtet und klärt die Hermeneutik die Bedingungen und Ziele einer Auslegung. Als Symbolfigur gilt in der Hermeneutik der mythologische Götterbote Hermes. Die philosophische Disziplin Hermeneutik (gr. hermeneuein) beschäftigt sich danach mit dem Verstehen von Sinnzusammenhängen in menschlichen Lebensäußerungen aller Art: sowohl der weltlich-profanen wie auch der prophetisch-sakralen. Das ganzheitliche transzendente Verständnisvermögen eines Propheten oder Mystikers ist zugleich der hermeneutische Schlüssel zum Verständnis spiritueller Erfahrungen von Generationen, also im Grunde auch aller heiligen, inspirierten Schriften. Ich möchte hier nachdrücklich darauf hinweisen, dass eine derart spezifische Fähigkeit, für die wir einfach keine Worte haben, weder gesellschaftlich noch kulturell bedingt ist. Sie hängt weder von der konfessionellen Zugehörigkeit noch von der Ausbildung oder der Denkfähigkeit des Einzelnen noch von seiner Muttersprache ab. Das authentisch Religiöse liegt in derinneren Erfahrung und vertieften Erkenntnis des Einzelnen und nicht in äußeren Formen und Einflüssen, wie z. B. der Kultur oder Tradition, was von erstaunlich vielen immer wieder irrtümlich behauptet wird. Ein „Sprung" dieser Art, d.h. der Aufstieg oder die Verwirklichung, hat immer existentiellen, ja universellen Charakter, und die Sprache des Geisterfüllten ist mit dieser existentiellen inneren Wandlung aufs engste verknüpft. Soweit es sich also um die Übersetzung sakraler Texte handelt, ist zu beachten, dass der hermeneutische (oder exegetische) Schlüssel hierbei eine entscheidende Rolle spielt (siehe dazu Sakaguchi 2015 und Sakaguchi 2011).

Die landläufige Texthermeneutik geht jedoch seit Wilhelm Dilthey ${ }^{4}$ und Hans-Georg Gadamer (Boehm \& Gadamer [1976] 1979) davon aus, dass übliche Übersetzungen, wie alle sprachlichen Äußerungen auch, durch das kulturelle und ideologische Gedankengut, im Fall von Bibelübersetzungen auch durch das für eine gegebene (institutionelle) Religion entworfene theologische Weltbild, beeinflusst werden (so z. B. Berger \& Nord 2005: 144-145; Strecker \& Schnelle [1983] 1989: 130-131). Handelt es sich um herkömmliche, kirchlich-diskursiv verankerte Sichtweisen und Übersetzungsverfahren ${ }^{5}$ - sozusagen Christianisie-

4 Vgl. „Brockhaus Enzyklopädie“, Bd. 9 (1989: 709).

5 Siehe dazu Schreiber (1993: 286-288, Kap. 2.3.2.4., ,Ideologische Bearbeitung'). Das Kernproblem bezüglich des Zugangs zu heiligen Texten und unseres heutigen Umgangs mit ihnen innerhalb des religiös-institutionellen Rahmens wurde auch von Harald Schweizer in seinem Artikel ,Textlinguistik und Theologie“ (2000) angesprochen. Er schreibt: „Theologische/reli- 
rungen althergebrachter Schriften und Überlieferungen - ist diese Annahme durchaus berechtigt. Doch wenn man das gesamte menschliche Leben und die gesamten menschlichen Erfahrungen, Sicht- und Wahrnehmungsweisen (die weltlichen ebenso wie die prophetisch-sakralen) genauer ins Auge fasst, legt die landläufige moderne Texthermeneutik eine eklatante Einseitigkeit an den Tag. Viele moderne Forscher, darunter die Übersetzungswissenschaftler, sind nämlich der Überzeugung, dass biblische Texte Zeugnisse fremder Menschen aus einer anderen Kultur und Zeit seien. So bestünde der Hauptgrund der Verstehensschwierigkeit der Bibel in der „kulturellen Andersheit“ und resultiere folglich aus „mangelndem Kulturwissen“ heutiger Leser über die Welt, in der diese Texte entstanden sind ${ }^{6}$. Eine solch einseitige Einstellung soll in diesem Beitrag kritisch hinterfragt werden.

Es soll außerdem angemerkt werden, dass viele christliche Theologen dazu neigen, das Transzendente dem Konventionellen und Institutionellen unterzuordnen und damit zu verschmelzen. In diesem Sinne sollen „Produkte institutioneller Setzung“ (wie manche Dogmen, theologische Weltbilder, Bekenntnisformeln, Interpretationsweisen), die auf menschlicher Übereinkunft und Anerkennung beruhen, ihnen durch die Sprache verhelfen, eine „neue Wirklichkeit“ zu erzeugen. Wirklichkeit wird in einem solchen Verständnisrahmen all das, was geglaubt und was für wahr gehalten bzw. für wahr erklärt wird. In einem so begriffenen Rahmen, behauptet Gerhard Begrich (2005: 134), „wird mit der Sprache die Theologie und die Anthropologie verändert“. Doch das ist eine Illusion, ein gewaltiger Denkfehler. Denn der Realität ist es egal, was wir über sie denken und welche Gefühle wir dabei entwickeln, merkt z.B. Rolf Dobelli in

giöse Diskurse wären [...] strukturell darauf angelegt, Wissen und Erkenntnis zu verhindern bzw. das Nicht-Wissen zu verschleiern, wobei dem expliziten Wortsinn nach aber der gegenteilige Eindruck erweckt wird. So gesehen wäre die Nähe von TheologInnen zu sog. AgnostikerInnen groß, mit dem Unterschied, daß TheologInnen in soziologische Strukturen eingebettet sind (Kirchen - um nur für christliche Theologie zu reden), die die Unmöglichkeit, substantielles Wissen zu vermitteln, durch lebensbegleitende, stützende Rituale und [...] hierarchisch-autoritäre Strukturen auszugleichen versuchen." (Schweizer 2000: 795) Dieser kritische Kommentar legt nahe, dass uns die Prophetie samt dem umfassenden vertieften kognitiven Referenzrahmen (Bewusstseinstypen) für die Auslegung und das Verständnis religiöser Schriften fast völlig verloren gegangen ist, wobei aber die Absichten oder die Versuche, etwas zu verschweigen oder zu verhüllen, natürlich nicht der Bibel zur Last gelegt werden dürfen (was oft zu Unrecht erfolgt). Adolf Jülicher z. B. schreibt den Gleichnissen und damit der Botschaft Jesu einen verheimlichenden Zweck zu (vgl. Banschbach Eggen 2007: 9-40). Solche Versuche oder Maßnahmen sind allerdings lediglich auf menschliche Unzulänglichkeiten und gegebenenfalls auch auf die Situation im religiös-institutionellen Bereich zurückzuführen.

6 So z. B. Berger und Nord ([1999] 2005: 22), Nord (2004: 188), Berger und Nord (2005: 144), Nord (2011: 223-22); ähnliche Ansichten finden sich z.B. auch bei Strecker und Schnelle ([1983] 1989: 130-131). 
seinem der Kunst des klaren Denkens und Handelns gewidmeten Buch an (Dobelli 2017: 33-37).

Man kann also sehen, für wie außerordentlich ernst die Rolle der Sprache im religiösen Bereich genommen und für wie tief ihre suggestive und persuasive Kraft gehalten wird. Viele Menschen sind nämlich der Ansicht, dass - soweit es sich um transzendente Dinge handelt - die Wörter identisch mit dem seien, worauf sie eigentlich nur hinweisen. Sie wollen es nicht zur Kenntnis nehmen, dass der Sprache hier lediglich der Hinweischarakter zukommt; dass sie nur das Gewand ist, aber nicht die Sache selbst. Insgesamt lässt sich feststellen, dass der heutige religiöse Mensch die Erscheinungen der Religion nicht als Erleb n is, sondern als ein institutionell objektiviertes Ereignis kennt. Religiöse Erlebnisse wie die prophetische Erfahrung suchen ihre gegenständliche Beziehung erfahrungsgemäß aus ihrer Erlebnismaterie heraus (vgl. Reinach [1916/1917] 1989: 788; Albrecht [1951] 2019). Wenn aber Kultur, Philosophie, Geschichte oder gar Sprache zum dominierenden Merkmal religiöser Hermeneutik werden, werden die Charaktereigenschaften biblischer Autoren und ihre Botschaften gänzlich überstrahlt. Es dürfen also institutionelle Sachverhalte und Konventionen mit der Natur, mit der Wirklichkeit und mit der Wahrheit nicht verwechselt werden, wie es Adolf Reinach ([1913] 1989: 277) einst betonte. Aus diesem Grund dürfen Religiosität und Prophetie einerseits und Institution sowie Religion/Konfession andererseits nicht gleichgesetzt werden, denn sie sind grundverschieden.

Kurzum: Infolge zivilisatorischer Veränderungen im Abendland wurde die Prophetie als nicht mehr wahrgenommenes Phänomen an den Rand der Hauptschauplätze des wissenschaftlichen, kulturellen und gesellschaftlichen Lebens zurückgedrängt. Die prophetische Religiosität ist demzufolge im gegenwärtigen Zeitbewusstsein der Menschen des Westens nicht vorhanden. Bei genauer Betrachtung lässt sich aber andererseits erahnen, dass zumindest die Geisteswissenschaften ohne den Hintergrund der phänomenologischen Auslegung der Bibel - als eines Ausgangspunkts für das gesamte menschliche Leben und Handeln nicht wirklich denkbar sind. Diese wichtige Tatsache hebt Raif Georges Khoury (2013: 181), der Islamwissenschaftler syrischer Herkunft, hervor:

In der gemeinsamen biblisch geprägten Vergangenheit liegt zunächst die Möglichkeit, sich auf seine Wurzeln zu besinnen, die ein immenses Reservoir an gemeinsamer Spiritualität und Orientierung für Gegenwart und Zukunft bieten: Darin sind nicht nur jüdische und christliche Leitfiguren des Alten und Neuen Testaments zu finden, sondern auch die des Koran und darüber hinaus die der großen spirituell ausgerichteten Literatur mit ihrer typisch biblisch-semitischen Weisheit und ihren packenden Bildern und Ermahnungen, die einen gemeinsamen Weg öffnen.

Ähnlich Abraham Joshua Heschel, der jüdische Schriftgelehrte und Religionsphilosoph: „Kein anderes Buch liebt und achtet das Leben des Menschen so sehr“ 
wie die Bibel, kein anderes Werk reflektiert „die ewige Bewegung des Geistes“ wie sie und ist dabei „frei von jedem Hauch von Eigeninteresse“, sei es einer sozialen Schicht, eines Volkes oder irgendeiner Institution. Da es keinen anderen Spiegel auf der Welt gibt, der den Willen Gottes, sein Walten und seine Führung so untrüglich widerspiegelt, kann die Bibel durch kein anderes Werk ersetzt werden, bringt Heschel nachdrücklich in seinen Werken in Erinnerung (vgl. Heschel [1955] 2000: 184 und 189 sowie Heschel [1936] 1962).

\section{Die Bibel als inspirierte Schrift und die Folgerungen für die Übersetzung}

Wie bei allen Bibelübersetzungen stellt sich die Frage nach der Intention (oder der Handlungsabsicht) eines biblischen Autors. Die Frage nach der A u thentizität, nach der In spiration und sogar nach der Irrtu m sfreiheit des Ausgangstextes ist aber dabei ebenfalls vom Belang. Dass der Urtext der Bibel als historisch gewordene Urkunde und als inspirierte Schrift $\mathrm{zu}$ verstehen ist, wird heute sowohl von den christlichen Theologen und Exegeten als auch von den Vertretern überkonfessioneller Gruppen (wie z.B. den Vertretern der konkordanten Übersetzungsmethode) allgemein anerkannt. ${ }^{7}$ Doch der irdisch gesinnte, durch das positivistische Denken geprägte Mensch betrachtet die Bibel sehr oft als ein kulturell bedingtes fehlbares Menschenwort, als ein Buch wie jedes andere. Er erkennt nicht, dass die Bibel anders ist, dass sie vom Geist Gottes gehaucht und eingegeben ist (vgl. 2 Buch Samuel 23,2; 2 Timotheusbrief 3,16; 1 Korintherbrief 2,10-14). Diese Tatsache wird jedoch von den modernen westlichen Sprachwissenschaftlern und Übersetzern, darunter den Erforschern der religiösen Sprache, meist außer Acht gelassen. Hier zeigt sich, wie stark die eingefahrenen Wahrnehmungs- und Denkgewohnheiten verwurzelt sind.

Es soll nun versucht werden, möglichst knapp zu erläutern, was das Phänomen der religiösen Inspiration ist. Unter In s p ir a ti on wird im theologischen und damit auch im biblischen Kontext eine Eingebung religiöser (transzendenter) Inhalte verstanden (lat. inspiratio ,Beseelung', ,Einhauchen', aus in ,hinein' und spirare ,hauchen'; vgl. hebr. ruah, arab. ruh, lat. spiritus ,Atem', ,Seele', ,Geist'). Um also die Zeugnisse und Quellen der Propheten richtig zu erfassen und $\mathrm{zu}$ übersetzen, muss man sich unbedingt Folgendes vor Augen halten: Die Äußerungen und Texte solcher Verfasser sind von einem jeweilig be s o nders ge -

7 So z. B. der „Katechismus der Katholischen Kirche - Kompendium“, Nummer 101-102: „Die Heilige Schrift wird [...] als inspiriert bezeichnet und lehrt ohne Irrtum jene Wahrheiten, die zu unserem Heil notwendig sind. Der Heilige Geist inspirierte nämlich die menschlichen Verfasser, die das aufgezeichnet haben, was er uns lehren wollte.“ 
arteten persönlichen Erfahrungsgut mitgetragen und mitbestimmt. Die Äußerungen jener Autoren, die das sehen, was andere gerade nicht sehen und verstehen können, haben zum Ziel, auf die anderen einzuwirken - ihre Adressaten wachzurütteln, zu ermahnen, zu rügen, sie zur Konfrontation und zum Nachdenken anzuregen, aber sie auch zu beruhigen. Die Menschen sollen aber vor allem von den meisten menschengemachten Übeln, wie Konflikte, Kriege, Terrorismus, bewahrt werden. So ist die Rolle der Propheten auszureißen und niederzureißen, zu vernichten und einzureißen, aufzubauen und einzupflanzen, worauf das Buch Jeremia $(1,10)$ ausdrücklich hinweist, wobei die prophetische Botschaft zugleich bloßstellend, unheildrohend und heilstiftend ist.

Die Propheten Israels waren Männer und Frauen. Im Hinblick auf ihre Tätigkeit werden die Propheten jedoch nicht ausgebildet. Sie sind auch keine religiösen Autodidakten, die durch eigenes Suchen und Finden und durch eigene Anstrengung ihre speziellen Erkenntnisse und ihr vertieftes Verständnis erlangen (vgl. Heschel [1936] 1962: 2). So ist das, was sie bekunden, Wissen „aus erster Hand“, nicht aus Büchern oder von anderen Vermitteltes. Die Propheten werden auch von keinem Amt eingesetzt (vgl. ebd.: 23; Friedmann 2007: 45). Nicht einmal aus sich selbst heraus entscheiden sie sich für ihre Berufung. Die folgenden Aussagen biblischer Propheten weisen auf diesen spezifischen Sachverhalt hin: Der Herr ließ mich schauen (Buch Jeremia 24,1); Das Wort des Herrn erging an mich (Buch Ezechiel 12,21; 23,1; 33,1); Seine Hand (...) packte (mich) und er (hielt) mich davon ab (...), auf dem Weg dieses Volkes zu gehen (Buch Jesaja 8,11). Ähnlich der Koran: Ich habe ihnen nur das ausgerichtet, was Du [d.h. Gott - A.S.] mir befohlen [aufgetragen - A.S.] hast (Koran 5: 117). Die Propheten werden außerdem nirgendwo in der Bibel als fehlerfrei und vollkommen dargestellt, sondern ganz und gar als gebrechliche Menschen. Die Propheten sehen genau den Unterschied zwischen Willkürakten, Scheinwahrheiten, Gaukelbildern und Fiktion einerseits und der objektiven Realität andererseits. Diese Tatsache möchte ich hier hervorheben und zum Ausdruck bringen, dass nicht ästhetischpoetische, logische oder überhaupt inhaltsimmanente Qualitäten, sondern die transzendente Herkunft den Reden der Propheten Ansehen und Wert verleiht.

Die Gaben der höchsten spirituellen Erfahrung werden im Griechischen gnosis tou Theou (Buch Weisheit 1,13) und epignosis (Hebräerbrief 10,26; 2 Petrusbrief 1,2; 2,20), im Sanskrit paramâ vidyâ ,höchstes Wissen', im Japanischen jōchi ,höheres Wissen' und im Zen-Buddhismus satori wörtlich ,Verstehen' genannt. Ein solches Erfahrungsgut speist sich nicht nur aus dem unmittelbaren mystischen oder prophetischen Erleben, sondern auch aus den Inhalten der geistigen Schau eines Propheten ${ }^{8}$ und aus dem so genannten „eingegossenen Wissen“ (gr.

8 Die Natur einer solchen geistigen Schau (hebr. hazon) gründet nicht auf der landläufigen 
propheteia ,prophetische Inspiration', hebr. nevua) ${ }^{9}$. Prophetie ist also eine Geistesgabe. Man wird vom mystischen Erleuchtungserlebnis meist unerwartet in Besitz genommen und völlig ergriffen. Theistische Propheten (wie Mose, Jesaja oder Mohammed) sehen es als die höchste Frucht des Wirkens einer höheren geistigen Macht an. Es fällt einem Einzelnen sozusagen als Geschenk zu und kann durch den Willen nicht erzwungen werden. Deshalb kann man Prophetie in einem Lernprozess nicht erwerben.

Das bereits oben angesprochene inspirierte Wissen ist also mit spirituellen Formen der Propheten (oder der Erleuchteten) aufs engste verbunden. Ich nenne solche besonderen Erscheinungen „Sakralia“. Sakralia spiegeln archetypische religiöse Erfahrungen, Vorgänge und Heilserlebnisse wider, sind aber für Außenstehende und in praktischer Spiritualität nicht Geübte normalerweise unverständlich und unklar. Der Apostel Paulus spricht über diese ganz andere Sphäre der Erfahrung so: Und mein Wort und meine Predigt geschahen nicht mit überredenden Worten menschlicher Weisheit, sondern in Erweisung des Geistes und der Kraft, damit euer Glaube nicht stehe auf Menschenweisheit, sondern auf Gottes Kraft (1 Korintherbrief 2,4-5, nach der LUT 1984). Angesichts dessen ist es wichtig, sich darüber im Klaren zu sein, dass der Kern der religiösen Sprache in den Akten mystischer Erlebnisse verwurzelt ist. Dieses Gut ist in Form des sehr spezifischen lexikalischen Inventars und sehr spezifischer Wendungen - eben der Sakralia fassbar (näheres dazu bei Sakaguchi 2011: 262-274 und Sakaguchi 2013).

\section{Typen der Bibelübersetzungen}

Werfen wir nach diesen allgemeinen Bemerkungen einen Blick auf die Bibel. Es ist hinlänglich bekannt, dass kein anderes Buch so oft und so unterschiedlich übersetzt wurde wie sie. Es ist heute selbstverständlich, dass eine Bibelübersetzung aus den biblischen Originalsprachen angefertigt wird, also für das Alte Testament aus dem Hebräischen und teilweise Aramäischen, für die Spätschriften des Alten Testaments (Deuterokanonische Schriften/Apokryphen) und das Neue Testament aus dem Griechischen.

Traditionelle Übersetzungen der Bibel kommen auf verschiedene Weise zustande. Klaus Berger und Christiane Nord nennen in ihrem Buch „Translationskompetenz und Bibelübersetzung“ (2005: 143) die folgenden drei Typen:

intellektuellen Wissensordnung oder einer philosophischen Idee oder auch Spekulation, sondern auf der höheren Erkenntnisordnung samt den Geboten des rechtschaffenen Lebens.

9 Näheres dazu findet sich im Kapitel IV. 6. ,Weltliche (profane) und transzendente (prophetisch-sakrale) Akte aus integrativer Sicht' meines Buchs „Sprechakte der mystischen Erfahrung [...]“" (Sakaguchi 2015). 
a) Übersetzungen, bei denen kein individueller Übersetzer angegeben ist, wie z. B. die King James Authorized Version (KJV) oder die New International Version (NIV 1984) (=,,anonyme Übersetzungen“);

b) Übersetzungen, die im Wesentlichen von einem Autor oder einer Autorin verantwortet werden, wie z. B. die Übersetzung Martin Luthers (LUT [... revidierte Fassung 1984]) oder die Übersetzung des katholischen Theologen Fridolin Stier (1989) (=„Autorenübersetzungen“); und

c) Übersetzungen, die von Übersetzergruppen aus zwei oder mehr, manchmal von Gremien von Theo-logen, Theologinnen (seltener!), oft auch unter Mitarbeit von Vertretern anderer Disziplinen (darunter jedoch nicht die Übersetzungswissenschaft) verfasst sind, z. B. die Gute Nachricht Bibel (GN, [...] in der Ausgabe 1997 verwendet) (=„Gremienübersetzungen“).

Berger und Nord vermerken an der gleichen Stelle: „Sowohl die Übersetzungen der ersten [hier: a)] als auch der dritten Kategorie [hier: c)] erwecken den Anschein von Anonymität und damit für uneingeweihte Leserinnen und Leser sicher auch von Objektivität. Der Übersetzungsprozess durchläuft zahlreiche Revisionsphasen (mit und ohne Rückbindung an die Ausgangstexte) und ist unter der Mitwirkung von Vertretern verschiedener Konfessionen [also auch verschiedener diskursiver Ansätze - A.S.] durch Kompromisse gekennzeichnet.“ (Berger \& Nord 2005: 143) Es handelt sich dabei meist um glaubenskonforme Anpassung an eine andere Religion (konfessionell geprägte Terminologie und die damit einhergehende Suggestivität, Zusätze, Kommentare, ideologische Umfunktionierung). Aus der westlichen Übersetzungsgeschichte sind hier insbes. ,Christianisierungen` bekannt (vgl. Schreiber 1993: 287). So wurde das Alte Testament nur christozentrisch ${ }^{10}$, als ein auf das Neue Testament verweisendes Buch betrachtet und interpretiert. Die Übersetzungen einzelner Übersetzer andererseits (hier: Kategorie b) spiegeln im Allgemeinen deren fachliche und biographische Besonderheiten wider (vgl. Nord 2004: 186). So war z. B. das Ziel des evangelischen Theologen Ulrich Wilckens (1970) vor allem einen Kommentar zu schreiben. Es nehmen dabei Übersetzung und Kommentar (auf dem unteren Teil jeder Seite) jeweils ungefähr gleichen Raum ein. Bei den Gremienübersetzungen (Kategorie a und c) werden indes die Ziele in der Regel sehr allgemein angegeben. Die Übersetzung der „Gute Nachricht Bibel“ (GN) durch die Deutsche Bibelgesellschaft ist beispielsweise in interkonfessioneller Zusammenarbeit der evangelischen und der katholischen Kirche sowie der Freikirchen entstanden. Sie stellt den ersten Typ deutscher Bibelübersetzung dar nach der so genannten „kommunikativen Übersetzungsmethode“ mit drei Hauptverfahren:

10 Unter Christozentrik versteht man in der christlichen Theologie die Auffassung, dass Jesus Christus die zentrale Stellung in der Schöpfungs- und Heilsgeschichte einnimmt, die alles andere bedingt und ordnet (vgl. Vorgrimmler 2000: 118). 
Umstrukturierung, Explikation und Kontextorientierung. Deren Ziel ist es, „vor allem den Sinn des Textes möglichst klar und verständlich zum Ausdruck zu bringen “. Nach der Auffassung ihrer Übersetzer verbinde die GN in der aktuellen Fassung von 1997 „Leichtverständlichkeit mit großer Genauigkeit und voller Ausschöpfung des Textsinns“. Doch der „kommunikative“ und mithin pragmatische (einseitig handlungsorientierte, auf die beabsichtigte Wirkung zielende) Ansatz dieser Übersetzungsmethode entspricht genau dem „postmodernen“ Zeitgeist, der unter den Christen zu einer erschreckenden Verflachung der biblischen Lehre und Verkündigung geführt hat (vgl. die kritischen Bemerkungen dazu bei Ebertshäuser 2006 und Lapide 2004).

Es soll an dieser Stelle noch eine weitere eher seltene, wenn nicht sogar die bislang einzige Übersetzung biblischer Texte erwähnt werden, die durch ein Team verantwortet wurde, dem ein evangelischer Neutestamentler und eine Übersetzerin und Übersetzungswissenschaftlerin angehört. Es handelt sich um die Übersetzung des Neuen Testaments und frühchristlicher Schriften durch die bereits oben zitierten Autoren Klaus Berger und Christiane Nord ([1999] 2005). Nord folgt den neueren Einsichten der modernen „funktionalen“ Übersetzungstheorie, die zum Ziel hat, die Fremdheit der Texte bestimmten Adressaten verstehbar zu machen. Durch die Verbindung von Theorie und Praxis dieser Übersetzung soll der Grundsatz „je wörtlicher, desto sinngetreuer" widerlegt werden. Es sollen aber darin andererseits auch etwaige Inkongruenzen zwischen den prophetischen Sinngehalten und dem offiziellen theologischen Weltbild, also „Häresien“ (Irrlehren), vermieden und glaubenskonform (für die christliche Glaubenslehre) dargestellt werden.

Die sich als inspiriert verstehenden Schriften (vor allem die prophetischen und die Offenbarungstexte der Bibel selbst) können jedenfalls nicht im Handumdrehen verstanden werden. Die Bedeutung ihrer Aussagen bleibt meistens dem Blick von außen verborgen. Deshalb bedürfen sie einer sorgfältigen Klärung nicht nur des Sinns ihrer Aussagen (Lexik - Grammatik - Rhetorik - Sprechakt Text), sondern auch der sog. „textexternen Faktoren“, wie die spirituelle Verfassung des Autors, seine Botschaft bzw. Intention. Die Erwartungen der Zielleser spielen hierin eine geringere Rolle.

\section{Ein Blick auf die Vorgehensweisen und Prinzipien bei der Übersetzung biblischer (sakraler) Texte}

Das Spektrum der Bibelübersetzungen erstreckt sich im Grunde zwischen zwei Extremen: Die Übersetzung kann versuchen, den Wortlaut der Ausgangssprache in der Zielsprache möglichst genau nachzubilden oder die Übersetzung kann 
versuchen, den Sinn des originalen Textes „mit eigenen Worten“ frei und unmittelbar verständlich wiederzugeben. Jede neue Bibelübersetzung hat ihren eigenen Charakter mit jeweils eigenen Stärken und Schwächen. Eine Übersetzung, die das einzig richtige Verständnis des Inhalts für jeden möglichen Empfänger „unfehlbar“ garantiert, gibt es jedenfalls nicht ${ }^{11}$.

Die Übersetzungen der Bibel, z.T. auch Übersetzungen anderer sakraler Schriften, folgen verschiedenen Absichten und Prinzipien, die bekanntermaßen nicht immer vereinbar sind. Deshalb ist eine Klassifizierung der einschlägigen Bibelübersetzungen nur näherungsweise möglich. Im Folgenden sollen einige wichtigere Übersetzungstypen samt ihren je eigenen Vorgehensweisen und Prinzipen kurz dargestellt werden. Die vorliegende Einordnung richtet sich in etwa nach der Häufigkeit ihres Auftretens.

Übersetzungstyp: Übersetzungen im Sinne sprachlich-diskursiver (hier: institutionell-
konfessioneller) Anpassung
Merkmale: Konfessionell, darunter auch dogmatisch motivierte Übersetzungen, neigen
mehr oder weniger bewusst dazu, solche Ausdrücke und Formen zu bevorzugen, die mit
den bestehenden theologischen Weltsichten, angelernten dogmatischen Sätzen und
Sichtweisen übereinstimmen. Deshalb sind die Übersetzungen dieses Typs einer Reihe
von Glaubenspräsuppositionen verpflichtet und sollen mit dem tradierten Sprachge-
brauch, wie z. B. dem römisch-katholischen, übereinstimmen. In diesem Sinne werden
Fragmente und längere Textabschnitte mit eigenen glaubenskonformen Erläuterungen
und Kommentaren versehen. Es werden dabei aber auch manche biblischen Kernbegriffe
umgedeutet, wie z.B. Vollständigsein, Ganzsein (heb. tamim) oder heilig (heb. qādosch,
es bedeutet ,zu unterscheiden` oder einfach ,anders` zu sein). Mit hebr. qādosch, gr.
hagios und lat. sanctus soll ein eigentümliches Moment seelischer Regungen und
Erlebnisse mit prophetischem und Geheimnischarakter zum Ausdruck gebracht werden,
der das Alltägliche überschreitet. In allen drei Sprachen (Hebräisch, Griechisch und
Lateinisch) weisen diese Ausdrücke auf die höchste Stufe der Entwicklung hin und
werden in übertragener, keineswegs aber ihrer ursprünglichen Bedeutung nach mit heilig
übersetzt (vgl. Otto ([1917] 2004: 6-7). Unter „heilig“ wird nämlich heutzutage das
absolute sittliche Prädikat verstanden. „So kann es scheinen“, vermerkt Gerda Walther
([1955] 1976: 186), „als sei der vollkommen ethische Mensch auch ein vollkommener
Heiliger, als entspräche der vollkommen ethische Mensch ohne weiteres dem vollendeten
Heiligen und umgekehrt. Eine derartige Auffassung ist aber durchaus irrig.“ Damit aber
die Bedeutung des Wortes heilig von der im westlichen Kulturkreis eingewurzelten

11 Vgl. „Kirchen-Lexikon oder Encyklopädie der katholischen Theologie...“ (1855: 932-952); vgl. auch https://www.bibelwerk.de/Bibel.12790.html/Bibelübersetzungen+im+Vergleich.85 153.html.

12 Um all das, was als „Wunder“ hinlänglich bezeichnet wird, zu begreifen, muss sich unser Geist weiten und sich den sinnbildlichen, allegorischen oder metaphorischen Bedeutungen von Wörtern und Ausdrücken öffnen. Bei Wundern in Bezug z. B. auf Jesu geht es um eine geistige mystische, also geheimnisvolle, Wirklichkeit, die weit über das hinausgeht, was die heutigen (Natur-)Wissenschaftler erfassen und erklären können. Die Natur und die Wirklichkeit biblisch geschilderter mystischer Vorgänge, also der Ereignisse, der Erfahrungen und der Einblicke, über die die Bibel (unter Zuhilfenahme von Wunder, Engel, Gebet, Auferstehung oder Himmelfahrt) berichtet, ist jedenfalls sinnlich-physikalisch nicht greifbar. 
sittlich-moralischen Konnotation unterschieden werden könne, hat Rudolf Otto ([1917] 2004: 7) hierfür ein denominales Adjektiv numinos (vom lat. numen ,Gottheit ursprünglich ,Wink', faktisch ,göttliche Kraft/Macht', ,Walten und Wirken der Gottheit selbst') gebildet, das eine ganz andere Qualität aufweist als heilig in der gängigen, populären Verwendungsweise. Es geht hier nämlich um den Ausdruck dessen, was ganz anders, außergewöhnlich, unbekannt, nicht landläufig, also transzendent ist.

Andere Begriffe wiederum werden in derartigen Übersetzungen zugegeben, wie z.B. Prophezeiungen oder Wunder ${ }^{12}$. Universelle Schlüsselinhalte (bei mir: Sakralia), wie Logos oder Wahrheit, werden durch doktrinär motivierte Ausdrücke, wie der wahre Glaube ersetzt. Manche religiösen Ausdrücke, wie z. B. Himmelfahrt, werden im Sinne besserer Verständlichkeit für die durchschnittlichen Gläubigen vergegenständlicht, d.h. $\mathrm{zu}$ einem sinnlich-materiellen Gegenstand gemacht. In der biblischen allegorischen Handlung, wie z.B. dem Aufbrechen zum Treffen mit dem Bräutigam (das Hohelied), kommt zum Ausdruck, dass religiöse Allegorie sich auf die transzendente Wirklichkeit und Heilsgeschichte bezieht und diese vergegenwärtigt. Doch diese Allegorie wurde später durch die christlichen Theologen umgedeutet: das Gegensatzpaar Braut und Bräutigam wird nämlich als allegorische Darstellung der christlichen Kirche und Jesus Christus gedeutet.

Da das menschliche Gehirn unverhältnismäßig schwach auf immaterielle, komplexe und deutungsbedürftige Dinge und Phänomene reagiert, werden manche biblische Aussagen und Kernbegriffe immer wieder vereinfacht oder umgedeutet. Im Sinne der evangelischlutherischen Tradition nun werden alttestamentliche Namen im NT z. T. in griechischer Form (Jesse, hebr. Isaias, Gott hilft', ,Gott heilt') angegeben. Der Name Gottes selbst (hebr. Adonai ,mein Herr') wird mit Großbuchstaben HERR ausgeschrieben, um hervorzuheben, dass es sich hier um nichts Anderes (etwa um einen weltlichen Herrscher) als nur den Namen Gottes handelt (neuere Ausgaben Herr).

Beispiele: NJB (Einheitsübersetzung, römisch-katholisch) (2007); „Elberfelder Bibel“ (evangelisch) (2010); „Biblia Tysiąclecia“ (1990); „Biblio“ (2006).

Übersetzungstyp: Übersetzungen im Sinne der Anpassung an die Gegenwartssprache („kommunikative Übersetzungsmethode“)

Merkmale: Übersetzungen in die Gegenwartssprache sind darum bemüht, die Aussagen des Urtextes so wiederzugeben, dass sie für einen Gläubigen der Gegenwart leichter verständlich werden. Hier lässt sich oft der Einfluss des Übersetzers auf den Urtext feststellen. Im Sinne eines solchen Vorgehens wird manchmal stark von der sprachlichen Struktur des Urtextes abgewichen, sodass sich hierin letztendlich die Gefahr birgt, den von den inspirierten Autoren intendierten Sinn zu entstellen oder zu verfehlen. So ersetzen manche modernen Bibelübersetzer sogar den Kernbegriff der sakralen Sprache, das Wort Gottes selbst, durch Botschaft Gottes.

Beispiele: GN (1997); „Hoffnung für alle“ (2002).

Übersetzungstyp: Strukturtreue Übersetzungen

Merkmale: Strukturtreue Übersetzungen versuchen, die sprachliche und inhaltliche Struktur des Urtextes so genau wie nur möglich in die Zielsprache zu übertragen. Von

13 „Die Schrift“ ist die deutsche Übersetzung des Tanachs, der hebräischen Bibel, durch die jüdischen Philosophen Martin Buber und Franz Rosenzweig. Sie entstand von 1926 bis 1938, bis 1929 in gemeinsamer Arbeit von Buber und Rosenzweig. Nach dem Tod Rosenzweigs wurde sie von Buber allein weitergeführt und vollendet. Von 1954 bis 1962 überarbeitete Buber den Text noch einmal. Die Übersetzung entstand in „eigenem“ Auftrag, das heißt, es gab keinen institutionellen Auftraggeber, wie beispielsweise eine Synagogengemeinde oder eine Kirche. Die Übersetzer waren dadurch frei, ihre eigenen Auffassungen bezüglich des 
besonderer sprachlicher und religionsgeschichtlicher Bedeutung ist die als extrem strukturtreu geltende Übersetzung Martin Bubers (begonnen 1925 zusammen mit Franz Rosenzweig, abgeschlossen 1961). Diese Übersetzung hat zum Ziel, die aus dem Geist des Judentums herrührenden Besonderheiten der hebräischen Syntax und die biblischen Sinnbilder in der deutschen Sprache wiederzugeben (vgl. Lapide 2004; „Brockhaus Enzyklopädie“, Bd. 3, 1987: 270). Deshalb ist diese Übersetzung um die wörtliche Übersetzung in engster Nähe zum hebräischen Urtext bemüht. Sie verzichtet auf glättende Füllwörter. Für jedes hebräische Wort wird unabhängig vom jeweiligen Sinnzusammenhang eine gleichbleibende deutsche Entsprechung verwendet. Darüber hinaus werden wurzelverwandte hebräische Wortgruppen durch wurzelverwandte deutsche Wortgruppen wiedergegeben (z. B. chäsäd - Huld; chasad - hold sein; chasidim - die Holden). Zu den weiteren Merkmalen dieser Übersetzungsmethode gehört die genaue Wiedergabe der hebräischen Namensformen. So werden in Bezug auf Gott Personalpronomen in der Großschreibung (ER, DU) verwendet. Die Zielgruppe bilden Leser, die in der deutschen Übersetzung dem hebräischen Original möglichst nahekommen wollen und bereit sind, ein hohes Maß an Einfühlung aufzubringen. Beispiel: „Die Schrift“, übers. von Buber \& Rosenzweig (1992). ${ }^{13}$

Übersetzungstyp: Sinn- oder inhaltstreue und zugleich wirkungstreue Übersetzungen Merkmale: Die Mischformen stellen Kompromisse zwischen den oben genannten Typen dar, wobei entweder das kommunikative oder das strukturtreue Prinzip bevorzugt wird und das jeweils entgegengesetzte Prinzip zum Ausgleich der Nachteile herangezogen wird. Das Ziel solcher Übersetzungen ist eine „sinn- oder inhaltstreue Wiedergabe des Urtextes“ (vgl. http://www.geistigenahrung.org/ftopic566410.html). Ein Übersetzungsverfahren, bei dem die Sinnwiedergabe im Vordergrund steht, zeichnet sich durch eine weitgehende Befreiung von den Formzwängen aus. Sinntreue Übersetzungen versuchen also, die Kerninhalte der Bibel, die bei anderen Übersetzungen weniger oder gar nicht beachtet werden, so treu wie möglich wiederzugeben. Manche solcher Übersetzungen sind auch darum bemüht, die Wirkungstreue der Aussagen beizubehalten. So bieten sie eine sinnvolle Ergänzung zu anderen Übersetzungstypen an, in denen eben diese Anforderung missachtet wird. Manche selteneren inhaltstreuen Übersetzungen, obwohl insgesamt auch nicht ganz fehlerfrei (wie z.B. LUT 1984), gehen die Schrift aus einer anderen, nämlich der mystisch-prophetischer Perspektive an.

Beispiele: LUT $(1912,1984)$.

Übersetzungstyp: Konkordante Übersetzungsmethode

Merkmale: Eine Bibelkonkordanz, die alle oder alle wichtigen in der Bibel vorkommenden Wörter und eventuell eine Definition solcher Wörter sowie die Belegstellen oder

Übersetzungsprozesses umzusetzen. Sie korrigierten ihre Arbeiten gegenseitig, sie ließen ihre Übersetzungsmanuskripte von Dritten vorlesen und verglichen ihre Fassungen mit gängigen deutschen, englischen, französischen und italienischen Übersetzungen. „Die Schrift“ ist weitestgehend eine konkordante Übersetzung. Sie strebt an, sowohl den sprachlichen Charakter wie auch den Inhalt des Urtextes so getreu wie möglich wiederzugeben (Vgl. https://de. wikipedia.org/wiki/Die_Schrift).

14 Mit Okkurrenz (auch Vorkommen) bezeichnet man in der Sprachwissenschaft die Häufigkeit, mit der ein bestimmtes sprachliches Element (ein Wort oder eine Struktur) in einem komplexeren sprachlichen Gefüge zu finden ist.

15 Vgl. https://de.wikipedia.org/wiki/Konkordante_Bibelübersetzung.

16 Leopold Zunz, ein deutscher Wissenschaftler jüdischer Herkunft und Vorkämpfer der Emanzipation der Juden in Deutschland, leitete eine Gruppe jüdischer Wissenschaftler, die von 1839 an eine Übersetzung der Heiligen Schrift unter dem Titel „Die vier und zwanzig 


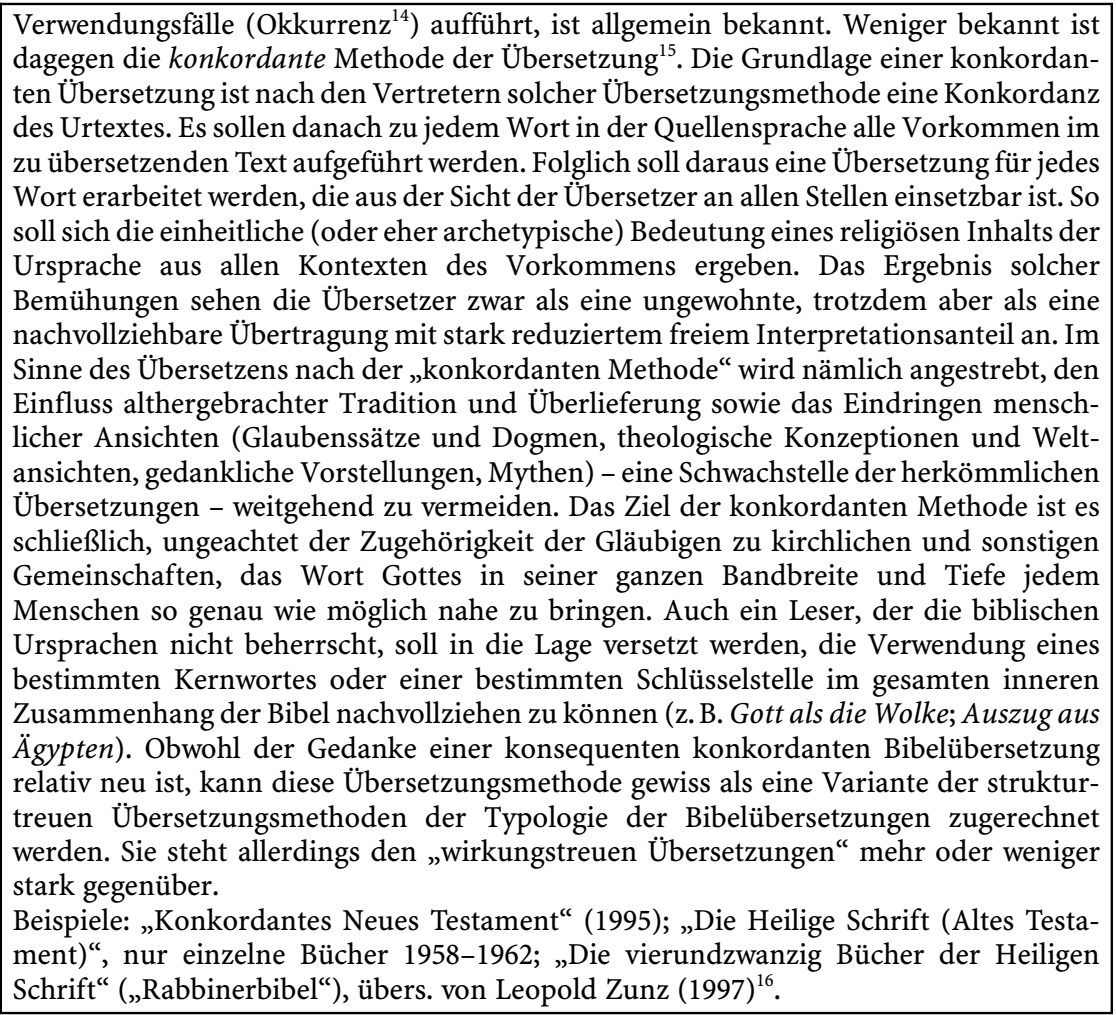

Der Übersetzer legt normalerweise aufgrund der eigenen Einschätzung fest, ob ein Ausdruck der Originalsprache in der unveränderten Form übernommen wird oder eine der - mehr oder weniger - gängigen Methoden des Übersetzens angewandt werden soll. Insoweit soll eine Bewertung der Güte einer Bibelübersetzung natürlich die Ziele ihrer jeweiligen Übersetzer berücksichtigen. Sie hängt allerdings weniger von den Erwartungen, Wünschen und Verstehensfähigkeiten durchschnittlicher Gläubiger $a b$, was heute fälschlicherweise oft angenommen wird (einseitig handlungsorientierte, pragmatische Argumentationsweise). Übersetzer, die nur die Bedürfnisse und Erwartungen des Zielpublikums oder gar einer Institution (z.B. kirchlichen) im Blick haben, verlieren bestimmt den Ausgangstext aus den Augen. Strukturtreue Übersetzungen lassen sich hinsichtlich ihrer Strukturtreue wohl leichter bewerten, da die Einhaltung der Vorgaben überprüfbar ist. Bei den wirkungstreuen Übersetzungen dagegen kann oft nicht „objektiv“ festgestellt werden, ob die Übersetzung im Detail tatsächlich

Bücher der Heiligen Schrift. Nach dem masoretischen Texte" herausgaben. Sie wurde später die „Rabbinerbibel“ genannt. 
dieselbe Wirkung bei den Lesern und Hörern hervorrufen mag wie die Originalfassung. Bibelübersetzungen oder zumindest Übersetzungen ihrer Schlüsselinhalte lassen sich jedoch in vielen Fällen keinen methodisch fest abgegrenzten Kategorien zuordnen.

Wie dem auch sei, feststeht, dass ohne den hermeneutischen $S c h l u ̈ s s e l$ sich nicht genau feststellen lässt, ob der jeweilige Übersetzungsvorschlag begründet, zufällig oder gar willkürlich ist. Kurzum: Die inhaltstreuen und die wirkungstreuen Übersetzungen dürfen sehr spezifische innere Prädispositionen der jeweiligen Bibelautoren (wie innere Gnosis oder inneres Sehen) keinesfalls außer Acht lassen.

\section{Ausgewählte Beispiele der biblischen Schlüsselinhalte anhand der Bibelübersetzungen in deutscher und polnischer Sprache sowie in Esperanto}

Im Folgenden soll anhand ausgewählter Beispiele veranschaulicht werden, wie die modernen Übertragungen die Schlüsselinhalte der Bibel wiedergeben. Es soll dabei der Blick aber auch auf die textrelevanten grammatischen Verfahren (wie z.B. Satzmodi) geworfen werden. Hier stellen sich Fragen wie: Sind die Übertragungen wirklich dem Urtext getreu? Geben sie ein besseres Verständnis dessen, was die biblischen Autoren übermitteln wollten oder verdunkeln sie eher ihre Botschaften und mithin das geistige Verständnis der Schrift? Wir können uns der Beantwortung dieser Fragen nähern, indem wir aufmerksam die Schlüsselbegriffe (oder Kerninhalte) selbst anhand ausgewählter Abschnitte verschiedener Übertragungen miteinander vergleichen. Liest man die modernen Übersetzungen jedoch für sich allein, fallen viele Veränderungen des Sinnes gar nicht ohne weiteres auf. Doch die Sicht von innen, sozusagen mit dem „geistigen Auge“, bringt ernste und schwerwiegende Abweichungen von den inspirierten Texten ans Licht.

a) Wie bereits oben kritisch angemerkt, neigen die modernen Bibelübersetzer verstärkt dazu, den Kernbegriff der sakralen Sprache, das Wort Gottes (gr. logon tou theou, lat. verbum dei) selbst, durch Botschaft Gottes zu ersetzen. Hier ein Beispiel aus dem zweiten Korintherbrief 2,17. In GN (1997) lesen wir: Viele verbreiten die Botschaft Gottes, wie man ein Geschäft betreibt. Ich dagegen verkünde sie völlig uneigennützig. Ich rede als einer, der Christus dient. Was ich sage, kommt von Gott, dem ich mich verantwortlich weiß. (2 Korintherbrief 2,17) Im Unterschied dazu steht in der LUT (1984): Wir sind ja nicht wie die vielen, die mit dem Wort Gottes Geschäfte machen; sondern wie man aus Lauterkeit und aus Gott reden muss, so reden wir vor Gott in 
Christus. Im Zuge der modernen Übersetzungen wird vielerorts der sakrale Begriff Wort Gottes beziehungsweise Wort des Herrn aufgelöst und durch andere Bezeichnungen ersetzt. Ein solches Vorgehen bezeugt damit offensichtlich das mangelnde Verständnis prophetisch-sakraler Redeweisen (oder genauer: prophetischer Diskurse) seitens der Übersetzer: Die dem griechischen Original $\lambda$ oyos sowie seiner lateinischen Wiedergabe verbum näherstehende Grundvokabel WORT (die hebräische Wortwurzel DBR, vokalisiert DaBhaR, hebräisch דבר, davar) etwa im Sinne von ,Gesetz Gottes‘, durch Jahrhunderte langen Gebrauch als Übersetzungsäquivalent legitimiert, wird durch den farblosen Übersetzungsneologismus Botschaft ersetzt. Die dem Ausdruck WORT aufgrund seines fundamentalen Charakters und seiner „Patina“ potentiell anhaftende Konnotation zeitewiger Wahrheit und Sakralität wird dadurch aufgegeben. Vergleichen wir z.B. den Prolog aus dem Johannesevangelium 1,1: Im Anfang war das Wort [oder Logos - A.S.], und das Wort war bei Gott, und das Wort war Gott. (Evangelium nach Johannes 1,1) Diese (prophetische) Verkündigung bezieht sich auf Urphänomene (apriorische Tatsachen), die an und für sich eine ausnahmslose Geltung besitzen. Sie sind unabhängig von den bestehenden Konventionen, von den herrschenden Weltbildern und Weltsichten, von den Geisteshaltungen, Traditionen, von der Organisation etc. Biblische Verkündigungen wollen uns etwas sehen lassen, dessen wir vorher nicht gewahr waren. Doch die Stelle in Johannes $1,1 \mathrm{ff}$. ist wohl eine der am meisten missverstandenen und von Vertretern der Dreifaltigkeitslehre benutzten „Beweisstellen“ aus der Bibel für ihre Behauptung, dass Jesus Gott sei. ${ }^{17}$ Das Wort logos wurde hier als (das fleischgewordene) Wort übersetzt. In Johannes 1,14 lesen wir: Und das Wort ist Fleisch geworden (gr. kai ho logos sarx egeneto). Einfleischung, Fleischwerdung (lat. incarnatio; von incarnare, Fleisch werden') ist ein theologischer Fachausdruck; er geht auf die lateinische Übersetzung des Johannesevangeliums 1,14 Et Verbum caro factum est zurück. In diesem Evangelium wird Jesus als das fleischgewordene Wort (oder Logos) dargestellt. Es geht hier um das Phainomenon der Fleischwerdung des göttlichen Archetyps.

Dem Wort logos werden in dem „Langenscheidts Großwörterbuch Altgriechisch-Deutsch“ von Menge und Güthling (26. Aufl. 1987) folgende Bedeutungen zugeschrieben: ,Rede', ,Aussage', ,Äußerung', ,Wort', ,Kunde', ,Gedanke', ,Überlegung', ,Bedeutung' u.a. Im Laufe der Geschichte deuteten griechische Philosophen den Ausdruck logos auch als „das göttliche

17 Die Bibel indes lehrt, Gott ist kein Mensch (Gott ist nicht ein Mensch, Buch Numeri 23,19; Denn Ich bin Gott und nicht ein Mensch, Buch Hosea 11,9; Gott ist Geist, Johannesbrief 4,24; 2 Korintherbrief 3,17). Gott ist kein Mensch, aber Jesus, der Sohn Gottes, der Prophet, war ein Mensch, daher kann Jesus nicht als Gott betrachtet werden. 
Grundprinzip“, als „Weltseele“, bzw. brachten das Wort logos mit einem „Denken Gottes“, ja dem Wort Gottes selbst, in Zusammenhang. Doch im Laufe der Jahrhunderte vollzog sich eine schrittweise Sinnverengung von Davar-Logos-Verbum-Wort (vgl. Lapide 2004, S. 22-23). In der japanischen Übersetzung dieser Stelle beispielsweise wurde logos nicht mit Wort, sondern mit Wort Gottes (mikotoba) übersetzt. Dieses Beispiel soll verdeutlichen, dass diese Verkündigung zu Anfang des Johannesevangeliums nicht losgelöst vom Rest dieses geistigen Evangeliums gelesen und recht verstanden werden kann. Es wird auch deutlich, wie schnell man diese Verse aufgrund einer vorgefassten und mithin trinitarisch beeinflussten Denkweise so verstehen kann, dass sich immer wieder Widersprüche ergeben und das Ganze seine eindeutige Bedeutung verliert.

b) Der Vorgang der eigenen in neren Wandlung, des Wiedergebor en We rd en s (gr. morphe ,Form', -morphia,-gestaltigkeit'; hebr. schub, in der Septuaginta als metanoia übersetzt - ,innere Wandlung', Wiedergeburt durch das geheimnisvolle Wirken des Geistes', vgl. Johannesbrief 3,1-13) ist das zentrale Anliegen des Neuen Testaments und der spirituellen apokryphen Evangelien, wie z.B. des Thomas- oder Philippusevangeliums. In dieser biblischen Weisung mag der Stammvater Abraham als unser Vorbild dienen, welchem Gott empfahl: Wandle vor mir und sei ganz! [im Sinne eines umfassenden Ganzheitsdenkens, erweiterten Bewusstseins] (Buch Genesis 17,1, nach der Übers. von Lapide 2004: 203). Durch die seelisch-geistige Wiedergeburt kann der Mensch zu seinem wahren Ursprung, zu seiner spirituellen Urexistenz, zum Ur-Einen zurückkehren. Wenn also Gott Abraham empfahl: Gehe vor mir und sei ganz!, dann ist das hebräische Adjektiv tamim, mit Sicherheit anders zu lesen ${ }^{18}$. Es geht bei dem hebräischen Adjektiv tamim [taw-meem] im Kontext von Genesis 17,1 nicht um die Verhaltens- oder Handlungsweise des Menschen, sondern um dessen Existenzweise, um die Frage, wer er ist. ,Vollständigsein', ,Ganzsein', ,integriert', ,frei von Mängeln“ ist hier mit Sicherheit gemeint. In den meisten westlichen Bibelübersetzungen wird dieses Attribut aber fälschlich wiedergegeben als ,rechtschaffen', ,makellos', ,fromm'. Ein solches Vorgehen läuft auf ein einseitiges Verständnis des Menschen hinaus. Hier zeigt sich die Tendenz, das ReligiösProphetische (als eine besondere Geistesgabe) innerhalb der Grenzen gewöhnlicher Erfahrung einzufangen. Dieses (Interpretations- und mithin Übersetzungs-) Übel betrifft vor allem das westliche Christentum. Es kann dadurch erklärt werden, dass die authentisch religiöse, existentielle Situation im Sinne eines Wegs zur Erfahrung Gottes von den institutionellen Religionen aus der Sphäre des Überbewussten in die Sphäre der Moral des indivi-

18 Vgl. https://www.theology.de/downloads/hebr500.pdf. 
duellen Handelns und des Handelns religiöser Gemeinschaften verschoben wurde. Infolgedessen ist das Moment der religiösen Offenbarung (mit ihrem Aspekt der Immanenz und Transzendenz) in den Bereich der Ethik und der reinen Begriffsbildung gerückt. Weitere Beispiele solcher nicht adäquaten, wohl durch die kircheninstitutionellen Diskurse beeinflussten Übersetzungen dieser Stelle, finden sich in NJB (2007): Geh deinen Weg vor mir und sei rechtschaffen und der polnischen „Biblia Tysiąclecia“ (1990): Służ Mi i badź nieskazitelny. Außerdem: „King James 2000 Bible“ KJV: Walk before me, and be you perfect oder Esperanto: Iradu antaŭ mi kaj estu senpeka („Biblio“ $\left.2006^{19}\right)$. Auch die Übersetzung von Luther (1984) scheint nicht einwandfrei zu sein: Wandle vor mir und sei fromm.

c) Die folgende Stelle aus dem Alten Testament veranschaulicht, dass selbst bei einem Priester oder falschen Lehrer Jesaja, der alttestamentliche Prophet, nur ablehnenden Spott erntet. So fragt Jesaja bitter spottend: ,Wen', sagen sie, , will der denn Erkenntnis lehren? Wem will er Offenbarung zu verstehen geben'? (Buch Jesaja 28,9, nach der LUT 1984) Im Unterschied dazu wird diese Stelle in der NJB so übersetzt: Wen will der Mann denn Erkenntnis lehren, wem das Gehörte erklären? (Buch Jesaja 28,9, NJB 2007) Ähnlich die Übersetzung von Zamenhof ins Esperanto: Al kiu li instruas scion, kaj al kiu li aŭdigas predikon? - Wen lehrt er Erkenntnis, und wem bringt er die Predigt bei? („Biblio“ 2006). In der NJB wurde der sakrale Kernbegriff Offenbarung durch einen profanen Ausdruck das Gehörte ersetzt und in der Esperanto-Übersetzung durch prediko (,Predigt') statt revelacio (,Offenbarung'). Außerdem weist die Äußerung Jesajas in der indirekten Rede mit der Personenverschiebung (1. > 3.) (sie sagen) formal darauf hin, dass er einen Übelstand schildert, der nicht ihn (den Propheten) selbst, sondern andere, nämlich damalige Priester und Irrlehrer, betrifft. In der NJB (2007) wurde jedoch jene Funktion „aus der Sicht des Propheten“ nahezu vollständig aufgehoben, so dass die ironische Redeabsicht des Verfassers dieser Zeilen, also Jesajas, nicht mehr eindeutig zu erkennen ist. Pragmatisch gesehen mag der Ausdruck (der

19 In den Plansprachen wie Esperanto gibt es mittlerweile drei Bibelübersetzungen. Ludwig L. Zamenhof, der Begründer des Esperanto, hat bereits die ganze hebräische Bibel (Tanach) ins Esperanto übersetzt. Er habe die Übersetzungsarbeit im März 1915 abgeschlossen. Doch wegen der Unmöglichkeit, das Übersetzungsmanuskript während des Ersten Weltkriegs nach Großbritannien zu Publikationszwecken per Post zu senden, ist das Werk erst zwei Jahre nach seinem Tode (1917) dort eingetroffen. Zamenhofs Übersetzung wurde in den Jahren 19191926 vom Londoner Biblia Komitato (Biblisches Komitee) gelesen, korrigiert und sprachlich (aus römisch-katholischer und protestantischer Sicht) angeglichen. Das Neue Testament, das vom Britischen Komitee (beste-hend aus Londoner Priestern) bereits übersetzt und im Jahre 1912 erschienen war, wurde folglich zusammen mit der Übersetzung des Alten Testaments von Zamenhof als die gesamte Bibel im Jahre 1926 veröffentlicht (vgl. „Enciklopedio de Esperanto“ 1986: 49). 2006 ist eine neue Auflage der Esperanto-Bibel in Prag erschienen. 
Mann) der rhetorischen Figur der Antonomasie im Sinne „der Autor dieser Zeilen“ entsprechen, um das Personalpronomen „ich“ zu ersetzen. Doch diese Form kann in dem gegebenen Kontext bestimmt nicht als eine adäquate Lösung betrachtet werden.

d) Fehldeutungen der Schlüsselinhalte der Bibel sollen nun an einem weiteren Beispiel veranschaulicht werden. Betrachten wir die Weisung aus dem Evangelium nach Lukas 17,21. In der älteren Fassung der LUT aus dem Jahr 1912 lesen wir: Das Reich Gottes ist inwendig in euch. Im Thomasevangelium wird das darin enthaltene Konzept des Spiegelbilds und das Nebeneinander von Immanenz und Transzendenz noch genauer formuliert: Inwendig in euch ist das Königreich und außerhalb von euch (Thomasevangelium, Log. 3). Im griechischen Text tritt entos humon, in euch“ im Sinne von „in eurem Inneren" auf (gr. Basileia tou Theou entos humon estin; Engl. The Kingdom of the God is inside of you). Diese Äußerungen weisen auf die Natur und auf das innere Potential des Menschen hin, Gott als verstehbare Wirklichkeit $\mathrm{zu}$ fassen und zu begreifen und aus der existentiellen Starre (oder dem existentiellen Schlaf) erweckt zu werden (Ebenbildlichkeit Gottes, Buch Genesis 1,26-27; Buch Weisheit 2,23; gr. eikon tou Theou). Doch die NJB (2007) übersetzt diese Verkündigung höchst eigenwillig: Das Reich Gottes ist (schon) mitten unter euch (Evangelium nach Lukas 17,21), genauso wie die „Neue Genfer Übersetzung“ (2011): Das Reich Gottes ist mitten unter euch. Ähnlich die Esperanto-Übersetzung: La regno de Dio estas inter vi - Das Reich Gottes ist mitten unter euch. Mit der Präpositionalphrase mitten unter euch/inter vi wird suggeriert, dass das Reich Gottes ein weltliches Reich sei, was einfach eine Missdeutung der biblischen Botschaft ist. Diese psychologistisch verfälschte Deutungsweise, die den für die Heilsgeschichte so entscheidenden dynamischen Moment der seelisch-geistigen Erfahrung und der Einfleischung auf die Ebene des ausschließlich Weltlichen, „Objektiven“ zu setzen versucht, hat bedauerlicherweise auch in die neuere Fassung der LUT aus dem Jahr 1984 Eingang gefunden. Dort lesen wir nämlich: Das Reich Gottes ist mitten unter euch (Evangelium nach Lukas 17,21).

e) Wer die unmittelbare Nähe Gottes erfährt, dem eröffnet sich die Möglichkeit, seinen Namen zu kennen, indem er seine Gegenwart erfasst und sich von seinen Energien tragen lässt. Im Buch Psalmen 91,14 lesen wir: Weil er an mir hängt, will ich ihn retten; ich will ihn schützen, denn er kennt meinen Namen. (NJB 2007). Im Unterschied dazu wird in der polnischen „Biblia Tysiąclecia“ (1990) das hebräische kī jāda '̌́emī (bo poznał Imię moje - denn er hat meinen Namen erkannt) völlig falsch übersetzt: Ja go wybawię, bo przylgnał do Mnie; osłonię go, bo uznał moje imię - ... denn er hat meinen Namen anerkannt. Die Ursache für diese Änderung des Sinns dieser Verkündigung kann man in der landläufigen dogmatischen Denkweise suchen, nämlich die gläubige 
Anerkennung Jesu als Erlöser in der für das Christentum so markanten Glaubensweise pistis, die wesentlich auf der Deklaration und Anerkennung der vorgegebenen Glaubensinhalte beruht. Eine derartige Glaubensweise spiegelt das Zitat aus dem „Katechismus der katholischen Kirche - Kompendium“, Nummer 50-53 wieder: „In seiner Güte und Weisheit offenbart sich Gott dem Menschen. In Taten und Worten offenbart er sich selbst und seinen gnädigen Ratschluss, den er in Christus für das Menschengeschlecht von Ewigkeit her gefasst hat. Dieser Ratschluss besteht darin, durch die Gnade des Heiligen Geistes alle Menschen als seine Kinder in seinem einzigen Sohn anzunehmen und am göttlichen Leben teilhaben zu lassen." (Hervorhebung durch die Autorin).

Ganz ähnlich: Ach, töricht ist mein Volk; mich kennen sie nicht. Sie sind unverständige Kinder, ja, sie sind ohne Einsicht. Sie wissen, wie man Böses tut, aber Gutes zu tun wissen sie nicht. (Buch Jeremia 4,22, NJB 2007) Im Unterschied dazu die polnische Übersetzung in „Biblia Tysiąclecia“ (1990): Tak, niemądry jest mój naród, nie uznają Mnie. Sa dziećmi bez rozwagi, nie maja wcale rozsadku. Madrzy sa bowiem w popetnianiu nieprawości, lecz dobrze czynić nie umieja - Ja, töricht ist mein Volk, sie erkennen mich nicht an. Sie sind unverständige Kinder... (Buch Jeremia 4,22, „Biblia Tysiąclecia“ 1990). Ähnlich die Esperanto-Übersetzung: Ĉar Mia popolo estas freneza, Min ili ne rekonas; ili estas malsấaj infanoj, ili ne komprenas; saĝaj ili estas por fari malbonon, sed fari bonon ili ne povoscias - Weil Mein Volk töricht (ganz verblendet) ist, anerkennen sie Mich nicht; sie sind unverständige Kinder... („Biblio“ 2006). Die dogmatische Hintergrundannahme, nämlich die gläubige Anerkennung Jesu als Erlöser in der für die christlichen Kirchen so typischen Glaubensweise pistis, liegt auch dieser Übersetzung der prophetischen Feststellung Jeremias zugrunde. Die Gefahr für die Missdeutung solcher Stellen rührt nicht zuletzt auch aus der Tatsache her, dass im Christentum normalerweise zwei unterschiedliche Weisen der Beziehung zu Gott nicht auseinandergehalten werden: (1) das ehrfürchtige, hingebende Vertrauen auf Gott und das Gefühl, von ihm durchdrungen zu sein (hebr. emuna), und (2) die Deklaration dogmatischer Sätze über ihn - Glaubenslehre (gr. pistis). ${ }^{20}$

f) Das folgende Beispiel veranschaulicht den Ersatz des für die prophetischsakrale Rede so charakteristischen Optativs (und auch des Imperativs) durch eine indikativische Form im Präsens. In der LUT (1984) lesen wir: Wenn wir im Geist leben, so lasst uns auch im Geist wandeln! (Galaterbrief 5,25). In der Übersetzung der NJB (2007) jedoch wird der Optativ merklich abgeschwächt:

20 Weiteres zu diesen zwei menschlichen Erfahrungsweisen findet sich in meinem Buch (Sakaguchi 2015, Anhang, Kap. 1 Vertrauen-emuna, Wissen-gnosis und Glaube-pistis). 
Wenn wir aus dem Geist leben, dann wollen wir auch dem Geist folgen. Die indikativische Form im Hauptsatz dann wollen wir auch dem Geist folgen bezieht das eigentliche seelische Anliegen, d.h. den Wunsch oder Weg nach einer inneren Wandlung, auf die Ebene etwa eines Entschlusses oder eines Berichts und nicht auf eine Forderung, auf ein Verlangen oder sogar auf ein Gebet. Dabei wird nicht nur die ausdrückliche Wunschform abgeschwächt, sondern auch der religiöse Schlüsselbegriff im Geist wandeln durch einen mehrdeutigen, weltlichen Ausdruck dem Geist folgen ersetzt.

g) Auch das folgende Übersetzungsbeispiel aus dem alttestamentlichen Buch Micha illustriert diskursive Nichtadäquatheit. Um die Verirrten auf den rechten Weg zu leiten und die betörten Gewissen aufzurütteln, greifen die Propheten auch die Irrlehrer (oder die falschen Propheten) mit den Mitteln der Ironie und des Spotts hart an, all jene, ... die da predigen, es werde gut gehen, wenn man ihnen zu fressen gibt; wer ihnen aber nichts ins Maul gibt, dem predigen sie, es werde ein Krieg kommen (Buch Micha 3,5, nach der LUT 1984). Zum Vergleich die Esperanto-Übersetzung: ...profetoj, kiuj erarigas mian popolon, kiuj mordas per siaj dentoj kaj predikas pacon, kaj kontraŭ tiu, kiu ne donas ion en ilian buŝn, ili predikas militon - ...Propheten, die mein Volk verführen, diejenigen, die etwas zu beißen haben, predigen den Frieden, denjenigen aber, der ihnen nichts in den Mund steckt, predigen sie den Krieg („Biblio“ 2006). Der alttestamentliche Prophet Micha brandmarkt und verspottet aufs schärfste „käufliche Priesterschaft und Geistlichkeit mit dienstbeflissenem Eifer“, die gerne bereit ist, „moralischer Verkommenheit gegenüber ein Auge zuzudrücken, alles gut und schön zu finden und die Bessergesinnten irrezuführen“ (vgl. Steif 1906: 580). Zum Vergleich die NJB: Haben sie etwas zu beißen, dann rufen sie: Friede! Wer ihnen aber nichts in den Mund steckt, dem sagen sie den Heiligen Krieg an (Buch Micha 3,5, NJB 2007). Der Versuch, weltliche Ereignisse wie einen Krieg auf die Stufe des scheinbar Religiösen zu erheben (heiliger Krieg), kann unumgänglich zu Ausschweifungen und Irrtum führen. Denn der Ausdruck heiliger Krieg gehört vor allem zum lexikalischen Inventar kirchlich-institutioneller und national-ideologischer Diskurse. Als „heiligen Krieg“ bezeichnet man üblicherweise eine kollektive organisierte Gewaltanwendung, die wegen einer „religiösen“ Idee, einer vermeintlich religiösen Verpflichtung oder zur Verteidigung „heiliger" Bereiche geführt wird. Derartige Motive treten gelegentlich in Gesellschaftsordnungen und diskursiven Gemeinschaften auf, in denen weltliche und religiöse Sphäre miteinander verschmolzen und semantisch eingeebnet ist. Der Begriff ,heiliger Krieg' wurde im Christentum seit dem Hochmittelalter für die Kreuzzüge und die ihnen eigenen (kirchlichen) Diskurse benutzt. Da dieser Begriff im Tanach (der biblischen Textsammlung mit normativem Charakter für das Judentum) nur einmal vor- 
kommt (Buch Joel 4,9), ist der ideologisch geprägte Begriff und Ausdruck heiliger Krieg als Ersatz für Krieg (im Sinne organisierter militärischer Auseinandersetzung zur gewaltsamen Durchsetzung meist politischer Ziele) mithin als völlig ungeeignet anzusehen.

h) Das folgende Beispiel eines biblischen Schlüsselinhalts stammt aus Buch Exodus 3,14. In der LUT (1984) lesen wir: Gott sprach zu Mose: Ich werde sein, der ich sein werde. [Ich bin, der ich bin] Und sprach: So sollst du zu den Israeliten sagen: ,Ich werde sein' [Ich bin], der hat mich zu euch gesandt. „Hoffnung für alle“ 2002 übersetzt diese Stelle jedoch so: Gott antwortete: ,Ich bin euer Gott, der für euch da ist'. Darum sag den Israeliten: ,Ich bin für euch $d a^{\prime}$ hat mich zu euch gesandt. In GN (1997) finden wir eine ebenso fragwürdige, vom Original abweichende, Übersetzung dieser Stelle: Gott antwortete: ,Ich bin da', und er fügte hinzu: ,Sag zum Volk Israel: ,Der Ich-bin-da hat mich zu euch geschickt'.' In diesem Abschnitt handelt es sich um die öffentliche (ekklesiale) Seite der Offenbarung Gottes auf dem Sinai unter der Beteiligung von Mose. Ich bin, der ich bin ist dabei als die Selbstvorstellungsformel Gottes (Bund Gottes mit dem Volk) zu verstehen, die sich in keine starren Begriffe fassen lässt. Sprechakttheoretisch handelt es sich hier um die biblische Verkündigung, die das Zentrum aller theistischen heiligen Schriften ausmacht. Gott offenbart sich Mose als der ewig Seiende, als eine Realität, die schon immer da war. Der sakrale Ausdruck und mithin die Chiffre Ich bin, der ich bin ist ein Beispiel einer (sozusagen) ewigen „Sprache“ Gottes. Die Verkündigung (und damit auch die Selbstoffenbarung Gottes) wird von den Übersetzern der „Hoffnung für alle“ (2002) in einer unzulässigen Weise entstellt und auf die profane, menschenzentrierte, kollektive Verständnisebene herabgesetzt, und zwar: Gott ist für mich da. Diese Übersetzung bezeugt eine modernexegetisch gefärbte Verzerrung der biblischen Aussage. Die Entstellung bezeugt letztendlich den einseitigen weltlichen Geist der Postmoderne in den modernen Übertragungen der Bibel. Das Sakrale wird nämlich dort an die menschlichen Bedürfnisse und Wünsche angepasst. Die Formulierung in der GN (1997) wiederum grenzt in ihrem mangelnden Verständnis des biblischen Schlüsselereignisses (Prophetie) und ihrer Botschaften und vor allem in ihrer Unbekümmertheit geradezu an Gotteslästerung: „Der Ich-bin- da“ hat mich zu euch geschickt.

Immerhin ist Jahwe der am häufigsten genannte biblische Eigenname. JHWE (außerhalb des Judentums ausgeschrieben als Jahwe oder Jehova) ist eigentlich kein Wort und auch nicht die Stellvertretung eines Namens, sondern eher eine Chiffre für etwas, was menschlichem Verstand und menschlicher Vorstellung entrückt ist. So nennt sich Jahwe, wie dies nach Exodus 3,14 oft übersetzt wird: Ich bin, der Ich sein werde, nach der Septuaginta Ich bin der Seiende oder nach Buber Ich werde da sein, als Der Ich da sein werde. Um der 
textwidrigen Maskulinisierung Gottes auszuweichen, schlägt Pinchas Lapide (2004: 201) den Ausdruck Ich werde sein, wer Ich sein werde vor und nicht, wie in den meisten deutschen Bibelübersetzungen fälschlich wiedergegeben Ich werde sein, der Ich sein werde. Die so oft in der Umgangssprache gebrauchten Ausdrücke Herrgott und poln. Pan Bóg sind insoweit inadäquat.

\section{Schlussfolgerungen und Ausblick}

Die oben exemplarisch dargestellten Beispiele der Schlüsselstellen der Bibel sollen einen ersten Eindruck vermitteln, dass die Sprache überhaupt ein guter Indikator für diskursive Eingebundenheit ist. Im vorliegenden Fall geht es vor allem um christliche kirchlich-diskursiv verankerte Sichtweisen und dementsprechend festgefahrene Übersetzungsverfahren. Dieses Übel tritt in Form von dogmatisch oder ideologisch motivierten Verformungen mancher Schlüsselinhalte der Bibel zu Tage. Hinzu kommt die Tatsache, dass heute der Begriff ,Präsupposition' lediglich in den Kontext des impliziten, soziokulturell konventionalisierten Hintergrundwissens gestellt wird. Dafür ist mit Sicherheit die gängige Überzeugung verantwortlich, nach der die Bedeutungen der Ausdruck kulturbedingter Erfahrungen seien. So halten viele heutige Forscher nur soziokulturelle Komponenten oder Voraussetzungen des Äußerns und Verstehens von Sätzen für rekonstruierbar (Hintergrundwissen oder, genauer gesagt, Hintergrundannahmen). Das bedeutet, dass nur das, was sich äußerlich manifestiert, von ihnen als Präsupposition zur Kenntnis genommen wird. Psychische Faktoren dagegen, darunter zwei grundlegende Bewusstseinstypen: ein personales und ein transpersonales Bewusstsein, lassen zeitgenössische Sprachforscher in der Regel außer Acht. Dies kann dadurch erklärt werden, dass es einem gegenwärtigen westlichen Menschen besonders schwerfällt, andersartige Hintergrundannahmen als nur soziokulturelle Annahmen oder historisches Alltagswissen eines durchschnittlichen Menschen in seine wissenschaftlichen Erörterungen einzubeziehen.

Es wurde bereits oben darauf hingewiesen, dass die Frage der richtigen Übersetzung von Schlüsselinhalten der Bibel und anderer heiliger Schriften eine höchst geistige Frage ist, die in erster Linie nach inneren, geistigen (und nicht äußeren!) Faktoren entschieden werden kann. In den heiligen inspirierten Texten muss man nämlich nach geistigen und nicht profan-literarischen Bedeutungen suchen. Die Übersetzung inspirierter sakraler Texte unterscheidet sich nämlich von der literarischen Übersetzung oder der (weltlich-profanen) Fachtextübersetzung entscheidend. Diese Tatsache hat natürlich Folgerungen für einen bewussten Übersetzer der Bibel. Denn er muss sich dessen bewusst sein, dass er archetypische (und nicht nur kulturbedingte!) Inhalte in Form spezifischer 
Ausdrücke, Äußerungen und Sätze in eine andere Sprache übertragen muss, die seine landläufigen übersetzerischen Fähigkeiten normalerweise weit übersteigen. Als besonders wichtig ist dabei zu beachten, dass der Übersetzer seine eigenen Weltsichten und Überzeugungen, seine Einstellungen, seine kulturell bedingten Vorstellungen, seine eingefahrenen Denkgewohnheiten oder gar platt-moralisierende Interpretationen biblischer Schlüsselstellen in seine Übersetzung nicht einfließen lassen darf. Die Tendenz dagegen, biblische Inhalte so zu interpretieren, dass sie mit bestehenden theologischen Weltsichten, angelernten dogmatischen Sätzen ${ }^{21}$ und dogmatischen Verkündigungen ${ }^{22}$, kurz: offiziellen Lehren, kompatibel sind, führt unweigerlich dazu, dass fehlbare, kulturell bedingte Menschenworte als Wort Gottes ausgegeben werden. Die in den einschlägigen Publikationen oft erwähnten Kriterien, wie „Loyalität“, „Professionalität“, oder sogar theologische Bildung und Glaube des Übersetzers, sind hier bei weitem unzureichend. Aus diesem Grunde ist die Übersetzung inspirierter sakraler Schriften, wie der Bibel, aufgrund der sehr spezifischen Anforderungen und Merkmale als eine eigene Kategorie des Übersetzens oder gar als ein eigener Übersetzungstyp anzusehen. Ein solcher Übersetzungstyp darf grundsätzlich keinem Gruppendruck unterworfen werden.

Die stillschweigende Grundvoraussetzung der heute meist eingesetzten Übersetzungsmethode der Bibel erweist sich allerdings als problematisch. Denn das ewige Wort Gottes wird darin als zeitlich und kulturell gebunden und als relativ und beliebig angesehen. Es wird letztlich auch geleugnet, dass das Wort Gottes zu allen Zeiten und für alle Menschen, gleich welcher Kultur und Konfession, Gültigkeit und Aussagekraft hat. Die hier exemplarisch angeführten Schlüsselstellen der Bibel dürften nahelegen, dass sie alle bis auf die Übersetzung Luthers sehr weit vom Original abweichen.

Es lässt sich ebenfalls beobachten, dass manche Gruppen von Menschen die Achtung gebietende Art und Weise, in der das Wort Gottes vermittelt wird, ablehnen und stattdessen eine banalere, ihrem jeweiligen geistigen Niveau entsprechende vorziehen. Demgegenüber muss eine zuverlässige Übersetzung

21 Solche dogmatischen Sätze betreffen innerhalb der katholischen Kirche z. B. die Inkarnation des Logos (Fleischwerdung Jesu Christi), die Auferstehung, die Himmelfahrt oder die Himmelfahrt Mariens (Mariä Aufnahme in den Himmel).

$22 \mathrm{Zu}$ den Dogmen gehört z. B. die Verkündigung an Mariä (NT: Lukasevangelium 1,26-38), die innerhalb der christlichen Religion die exegetische Grundlage für die Lehre der Mariologie, besonders jene der unbefleckten Empfängnis Mariens, bildet. Bestimmte, später auch auf Jesus, aber auch auf andere religiös bedeutsame Gestalten (Religionsstifter) angewandte Urmythen (z. B. von der jungfräulichen Geburt oder der Auferstehung nach dem Tod) entstammen allerdings ursprünglich nicht dem Christentum und nicht der Bibel, sondern sind um Jahrtausende älter und tauchen in verschiedener Form in vielen Religionen und Kulturen unabhängig voneinander auf. Der Tod und die Wiederauferstehung sind nämlich das Kernthema uralter Mythen. 
darum bemüht sein, die prophetischen Aussagen, darunter die Sakralia, möglichst getreu wiederzugeben. Die Forderung an eine gelungene Übersetzung müsse also sein, die Worte des Originals so genau und getreu wie möglich wiederzugegeben, d.h., jeder sakrale Kernbegriff soll angemessen, ohne semantische Abweichungen übersetzt werden, ohne Sinnverfälschung und ohne eigenwillige Hinzufügungen oder Weglassungen. Die nächste Forderung indes erweist sich in der Praxis als viel schwerer umsetzbar. Sie lautet: Eine gelungene wortgetreue Übersetzung sollte möglichst verständlich sein und dabei eine der Bibel angemessene (sakrale) Diskursart wahren. Wie auch immer, das Wort Gottes kann nur dann seine erbauende Kraft entfalten, wenn es von seinem Leser verstanden wird. Karl-Heinz Vanheiden rät diesbezüglich: „Ein Leser, der die biblischen Grundsprachen nicht beherrscht, verwendet am besten eine wortgenaue und eine sinngenaue Übersetzung nebeneinander. Und wenn er beim Lesen eines Verses feststellt, dass in beiden Übersetzungen derselbe Sinn deutlich wird, kann er

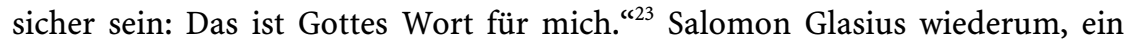
deutscher lutherischer Theologe, gibt den Lesern u.a. folgenden Ratschlag: „Möge man nach jedem gelesenen Kapitel mit Andacht erwägen, welche Kernsprüche darin vorkommen, welche Lehre, Trost oder Ermahnung man darin gefunden hätte [...].“ (Zit. nach Köster 1995: 175) Das verstehende Lesen heiliger Texte ist immer auf das Verhalten und Handeln ausgerichtet (illokutive Funktion). Doch nichts und niemand kann den Menschen, weder den Übersetzer der Bibel selbst noch seinen Leser, in seinem Ringen um das rechte Verständnis der Schrift ersetzen.

Die Bibel will den Menschen vergegenwärtigen, dass das Wort Gottes auf festem, unerschütterlichem Fundament gründet und dass göttliche Weisheit unvergleichlich viel höher steht als menschliche Gedanken, Wünsche, Ideen und Einfälle. Indem eine Reihe moderner Bibelübersetzungen dazu beiträgt, vor allem die prophetischen Verkündigungen und Weisungen zu banalisieren, bahnt sie so den Weg für irrige, oft realitätsferne Vorstellungen. Die schwammigen, zum Teil weit voneinander abweichenden und sinnentstellenden Textwiedergaben können deshalb für den Menschen nicht mehr ein Mittel sein, gegenüber von Missständen und Gefahren in der Gesellschaft bewusst zu sein, wachsam zu denken und kritisch zu handeln. Die prophetische Erfahrung und die Offenbarung werden in den meisten modernen Übertragungen der Bibel an verschiedenen Stellen durch irdische Begriffe ersetzt, wie z. B. grenzenlose Liebe, Güte oder gar Buße (z. B. NJB 2007). Und noch etwas anderes: Wenn eine Übersetzung die Kernbegriffe der Bibel auflösen will (etwa durch ausgiebigen Gebrauch von umgangssprachlichen Formen), geht sehr viel von dem Sinn des Urtextes verloren.

23 Karl-Heinz Vanheiden (https://www.derbibelvertrauen.de/der-bibel-vertrauen/bibelueber setzungen/27-diegrundlagen/15-die-beiden-uebersetzungstypen.html). 
Eine angemessene, bewusste, direkte Übersetzung aus den Originalsprachen muss also die Aussageabsichten der biblischen Autoren unbedingt berücksichtigen. Anderenfalls werden die biblischen Aussagen und Botschaften auf eine unzulässige Weise verformt und entstellt und folglich auf die profane, menschenzentrierte Verständnisebene herabgesetzt. Wollen wir heute den Quellen und dem Wahrheitsgehalt biblischer Aussagen phänomenologisch nachspüren, muss der umfassende kognitive Referenzrahmen, d.h. das prophetische Bewusstsein und das durch tiefe spirituelle Erfahrung bereicherte und bestätigte Wahrnehmen, Wissen und Verstehen (Inspiration), unbedingt beachtet werden.

Obwohl bei den inhalts- und wirkungstreuen Übersetzungen sowie bei der konkordanten Methode Beliebigkeit weitgehend ausgeschlossen werden kann, da ein archetypischer, einheitlicher Wortsinn gefunden werden muss, der im Prinzip in jedem prophetisch-sakralen Kontext einsetzbar ist, sind auch bei ihnen einige Alternativen möglich. Das bedeutet, dass die subjektive Wahl des Übersetzers auch hier nicht ganz ausgeschlossen werden kann. Doch im Fall der Übersetzung heiliger Schriften ist der Einfluss des Übersetzers stark eingeschränkt, so im Gegensatz zu der Übersetzung weltlich-profaner literarischer Texte.

Abschließend sei festgestellt, dass alle Texte, hier vor allem die prophetischen und die Offenbarungstexte der Bibel, sorgfältiger Klärung ihres Sinns bedürfen, wenn sie allgemein schwer zu verstehen sind. Und es ist schließlich die Aufgabe der Hermeneutik, die wahre Intention der inspirierten Autoren zu ermitteln immer mit gebührender Rücksicht auf die Tatsache, dass den biblischen Autoren aufgetragen ist, ihre Botschaft so exakt wie möglich auszudrücken und zu verkünden, und dabei weder etwas wegzulassen noch etwas hinzufügen (vgl. Buch Jeremia 26,2; Evangelium nach Matthäus 4,4). Dieser demütigen Grundhaltung, möglichst nah am Wort Gottes zu sein (Buch Jeremia 23,36), fernab mancher historisierenden, dogmatisierenden, weltanschaulichen Auslegung oder gar blinden religiösen Eifers, sollte jeder bewusste und verantwortliche Übersetzer der sich als inspiriert verstehenden Schriften Rechnung tragen.

\section{Literaturverzeichnis}

Albrecht, Carl ([1951] 2019). Psychologie des mystischen Bewusstseins. Neu eingeführt und durchges. von Franz K. Wöhrer. München: Aphaia Wissenschaft.

Banschbach Eggen, Renate (2007). Gleichnis, Allegorie, Metapher. Zur Theorie und Praxis der Gleichnisauslegung. Tübingen: Narr Francke Attempto.

Begrich, Gerhard (2005). Der blonde David. Ein Beitrag zur Interpretation, Kultur und Übersetzung der (hebräischen) Bibel. In: H. Salevsky (Hrsg.), Kultur, Interpretation, Translation (S. 131-142). Frankfurt a.M., Berlin: Peter Lang. 
Berger, Klaus/Nord, Christiane (2005). Das Neue Testament - neu übersetzt. Funktionsgerechtheit und Loyalität bei der Übersetzung neutestamentlicher und frühchristlicher Texte. In: H. Salevsky (Hrsg.), Kultur, Interpretation, Translation (S. 143-153). Frankfurt a.M., Berlin: Peter Lang.

Betz, Otto/Schram, Tim (2006). Brockhaus Enzyklopädie in 24 Bänden. Düsseldorf: Brockhaus.

Dobelli, Rolf (2017). Die Kunst des guten Lebens. München: Pieper.

Ebertshäuser, Rudolf (2006). Gottes Wort oder Menschenwort? Moderne Bibelübersetzungen unter der Lupe. Augustdorf: Betanien Verlag.

Enciklopedio de Esperanto: I. volumo. (1986). L. Kökeny, V. Bleier, la lingvo-fakon: K. Kalocsay (Hrsg.. ) iniciatinto-ĉefredaktoro: I. Sirjaev. Represo de la unua eld. Budapest: Hungara Esperanto-Asocio.

Friedmann, Richard Elliot (2007). Wer schrieb die Bibel? So entstand das Alte Testament (Original: Who wrote the Bible?). Köln: Anaconda.

Gadamer, Hans-Georg/Boehm, Gottfried (Hrsg.) ([1976] 1979). Seminar philosophische Hermeneutik. Frankfurt a.M.: Suhrkamp.

Heschel, Abraham Joshua ([1936] 1962). Die Prophetie. Kraków: Polska Akademia Umiejętności.

Heschel, Abraham Joshua ([1955] 2000). Gott sucht den Menschen. Eine Philosophie des Judentums. 5. Aufl. Berlin: Jüdische Verlagsanstalt. (Original: God in Search of Man..., New York 1955).

Khoury, Raif Georges (2013). Die Magier des Wortes und die Bedeutung ihrer Werke für ein konfliktfreies, religiöses und politisches Zusammenleben zwischen Juden, Christen und Muslimen im Vorderen Orient. In: M. Enders/R. Kühn (Hrsg.), Kritik gegenwärtiger Kultur. Phänomenologische und christliche Perspektiven (S. 178-204). Freiburg, München: Karl Alber.

Kirchen-Lexikon oder Encyklopädie der katholischen Theologie und ihrer Hilfswissenschaften: 1 Bd. (1855). Aaron - Bibelübersetzungen. Freiburg: Herder.

Köster, Uwe (1995). Studien zu den katholischen deutschen Bibelübersetzungen im 16., 17. und 18. Jahrhundert. Münster: Aschendorffische Verlagsbuchhandlung.

Lapide, Pinchas (2004). Ist die Bibel richtig übersetzt? Band 1 und 2. Gütersloh: Gütersloher Verlagshaus.

Menge, Hermann/Güthling, Otto (1987). Langenscheidts Großwörterbuch AltgriechischDeutsch. Berlin, München: Langenscheidt.

Nord, Christiane (2004). Translationskompetenz und Bibelübersetzung. In: E. Fleischmann/P.A. Schmitt/G. Wotjak (Hrsg.), Translationskompetenz (S. 183-198). Tübingen: Stauffenburg.

Nord, Christiane (2011). Funktionsgerechtigkeit und Loyalität. Die Übersetzung literarischer und religiöser Texte aus funktionaler Sicht. Berlin: Frank \& Timme.

Otto, Rudolf ([1917] 2004). Das Heilige. Über das Irrationale in der Idee des Göttlichen und sein Verhältnis zum Rationalen. München: Beck.

Presas, Marisa (2004). Translatorische Kompetenz als Expertenwissen: eine Annäherung aus kognitiv-psychologischer Sicht. E. Fleischmann/P.A. Schmitt/G. Wotjak (Hrsg.), Translationskompetenz (S. 199-207). Tübingen: Stauffenburg. 
Reinach, Adolf ([1913] 1989). Die apriorischen Grundlagen des bürgerlichen Rechtes. In: K. Schuhmann/B. Smith (Hrsg.), Sämtliche Werke. 2 Bände (S. 141-278). München u.a.: Philosophia Verlag.

Reinach, Adolf ([1916/1917] 1989). Aufzeichnungen [religiöse] (1916/1917). In: K. Schuhmann/B. Smith (Hrsg.), Sämtliche Werke. 2 Bände (S. 589-611). München u. a.: Philosophia Verlag.

Risku, Hanna (1998). Translatorische Kompetenz. Kognitive Grundlagen des Übersetzens als Expertentätigkeit. Tübingen: Stauffenburg.

Sakaguchi, Alicja (2011). Język, mistyka, proroctwo. Od doświadczenia do wysłowienia. Poznań: Wydawnictwo Naukowe Uniwersytetu im. Adama Mickiewicza.

Sakaguchi, Alicja (2013). Warum werden heute Sakralia nicht mehr verstanden? Einige Bemerkungen zur religiösen (Fach-)Sprache. In: F. Grucza, (Hrsg.): Akten des XII. Internationalen Germanistenkongresses Warschau 2010: Vielheit und Einheit der Germanistik weltweit (S. 111-118). Frankfurt a.M., Berlin etc.: Lang.

Sakaguchi, Alicja (2015): Sprechakte der mystischen Erfahrung. Eine komparative Studie zum sprachlichen Ausdruck von Offenbarung und Prophetie. Freiburg, München: Karl Alber.

Schreiber, Michael (1993). Übersetzung und Bearbeitung. Zur Differenzierung und Abgrenzung des Übersetzungsbegriffs. Tübingen: Gunter Narr.

Schweizer, Harald (2000). Textlinguistik und Theologie. In: K. Brinker/G. Antos/W. Heinemann/S.F. Sager (Hrsg.), Ein internationales Handbuch zeitgenössischer Forschung. 1. Halbband: Textlinguistik (S. 790-797). Berlin, New York: de Gruyter.

Steif, Max (1906). Die Ironie in der Bibel. Ost und West: illustrierte Monatsschrift für das gesamte Judentum, 8.-9. August, 575-582.

Strecker, Georg/Schnelle, Udo ([1983] 1989). Einführung in die neutestamentliche Exegese. 3., durchgesehene und ergänzte Auflage. Göttingen: Vandenhoeck \& Ruprecht.

Vanheiden, Karl-Heinz (2019). Zwei Übersetzungstypen. https://www.derbibelvertrauen. de/der-bibel-vertrauen/bibeluebersetzungen/27-die-grundlagen/15-die-beiden-ueber setzungstypen.html.

Van Vaerenbergh, Leona (2004). Kognitive Prozesse und die Kompetenz(en) des Übersetzers. In: E. Fleischmann/P.A. Schmitt/G. Wotjak (Hrsg.), Translationskompetenz (S. 255-267). Tübingen: Stauffenburg.

Vorgrimmler, Herbert (2000). Neues Theologisches Wörterbuch. Freiburg: Herder.

Walther, Gerda ([1955] 1976). Phänomenologie der Mystik. 3. Aufl. Olten, Freiburg im Breisgau: Walter. (1. Aufl. 1955).

Internetquellen

https://www.bibelwerk.de/Bibel.12790.html/Bibelübersetzungen+im+Vergleich.85153.html. http://www.geistigenahrung.org/ftopic5664-10.html.

Katechismus der Katholischen Kirche - Kompendium. https://kathleben.de/wp-content/up loads/2016/06/Katechismus-der-Katholischen-Kirche-Kompendium.pdf.

https://de.wikipedia.org/wiki/Die_Schrift.

https://www.theology.de/downloads/hebr500.pdf.

https://de.wikipedia.org/wiki/Konkordante_Bibelübersetzung. 


\section{Zitierte Bibelübersetzungen}

Biblio (2006). La Malnova Testamento tradukita de Ludoviko Lazaro Zamenhof kun la Duakanonaj Libroj tradukitaj de Gerrit Berveling. La Nova Testamento tradukita de la Brita Komitato. Dobřichovice (Praha): Kava-Pech.

Elberfelder Bibel mit Erklärungen (2010). Haan: Brockhaus.

GN (1997). Gute Nachricht Bibel. Altes und Neues Testament. Stuttgart: Deutsche Bibelgesellschaft 1997.

Heilige Schrift (Altes Testament), die: eine konkordante Wiedergabe; in der Weise entworfen, daß der deutsche Leser in engste Verbindung mit Gottes ursprünglichem Wort gebracht wird... (1958-1962). 2. Bände: Genesis und Jesaja. Adliswil: Konkordanter Verl. Hoffnung für alle. Die Bibel (2002). Basel, Gießen: Brunnen Verlag.

KJV (o. J.): The Holy Bible containing the Old and New Testament, trans. out of the original tongues and with the former translations diligently compared and revised by $\mathrm{HiSM}$ jesty's special command. Cambridge: Cambridge University Press.

Konkordantes Neues Testament: mit Stichwortkonkordanz (1996). Pforzheim: Konkordantes Verlag. (6. Aufl.)

LUT (1912). Die Bibel oder die ganze Heilige Schrift des Alten und des Neuen Testaments; nach der deutschen Übersetzung D. Martin Luthers; nach dem 1912 vom Deutschen Evangelischen Kirchenausschuß genehmigten Text. Stuttgart: Privilegiertes Württemberg. Bibelamt.

LUT (1984). Die Bibel nach der Übersetzung Martin Luthers. Bibeltext in der revidierten Fassung von 1984. Durchges. Ausgabe in neuer Rechtschreibung 2006. Stuttgart: Deutsche Bibelgesellschaft 2006.

Neue Genfer Übersetzung NGÜ NT + PS (2011). Romanel-sur-Lausanne: Genfer Bibelgesellschaft.

NJB (2007). Neue Jerusalemer Bibel: Einheitsübersetzung. Neu bearbeitete und erweiterte Ausgabe. Freiburg, Basel, Wien: Herder.

Neue Testament und frühchristliche Schriften, das. Vollständige Sammlung aller ältesten Schriften des Urchristentums (2005). Übers. von Klaus Berger und Christiane Nord. Frankfurt a.M., Leipzig: Insel Verlag.

NIV (1984). New international version of the New Testament. Colorado Springs, Mexico City, Herrljunga, Nairobi, Hyderabad, Bangkok: International Bible Society.

Biblia Tysiaclecia (1990). Pismo Święte Starego i Nowego Testamentu. W przekładzie z języków oryginalnych. Wydanie trzecie poprawione. Poznań, Warszawa: Pallottinum.

Schrift, die (1992): Aus dem Hebräischen verdeutscht von Martin Buber gemeinsam mit Franz Rosenzweig, 4 Bände (Taschenbuch), Deutsche Bibelgesellschaft.

Stier (1989). Das Neue Testament. Stier, Fridolin (Übers.); Beck, Eleonore/Miller, Gabrielle/ Sitarz, Eugen (Hgg.). München, Kösel/Düsseldorf: Patmos.

Vierundzwanzig Bücher der Heiligen Schrift, die (1997). Nach dem masoretischen Text übers. von Leopold Zunz, Tel-Aviv: Sinai Verlag in Zusammenarbeit mit dem DORONIA Verlag Stuttgart [Eine hebräisch-deutsche Ausgabe, später „Rabbinerbibel“].

Wilckens, Ulrich (Übers.) (1970). Das Neue Testament. Hamburg: Furche. 


\title{
Postulate hinsichtlich der Entwicklung der Fähigkeit zur Selbstbewertung eigener Dolmetschleistungen - Vorschlag einer Feldübung für Studierende der Angewandten Linguistik mit dem Schwerpunkt Übersetzen/Dolmetschen
}

\begin{abstract}
Postulates regarding the development of the ability to self-assess one's own interpreting proposal for a field exercise for students of Applied Linguistics specializing in translation/interpreting

This chapter presents some postulates concerning the development of the ability to selfassess one's own interpretations in students of applied linguistics specializing in translating/interpreting. They are formulated on the basis of the analysis of the results of a field exercise in guide interpreting designed for students. First, the concept of the field exercise and its theoretical basis are described. Next, the self-assessment form and the phases of the field exercise are presented. Finally, the discussion moves to the findings of quantitative and qualitative analyses of the students' self-assessments. The results show that the students are not able to perform an accurate, realistic and objective self-evaluation because of their shortages in foreign language skills. They can neither develop proper interpreting skills nor the ability to self-assess. Because of their poor awareness of these facts they show excessive optimism considering evaluation of their interpreting. Field exercises like the one presented in this chapter could be a suitable remedy, as they can make such students' language and interpreting deficits clearer to them.
\end{abstract}

Keywords

guide interpreting, interpreting quality, interpreting exercises, field exercise, self-assessment of interpreting

\section{Situierung und theoretische Grundlagen des Konzepts der Feldübung}

In diesem Beitrag wird ein Konzept für die Entwicklung der Fähigkeit zur Selbstbewertung dargestellt, das durch die Autorin ursprünglich für Studierende des zweisemestrigen postgradualen Wochenendstudienganges Studia Podyplomowe Kształcenia Tłumaczy Języka Niemieckiego (dt. Aufbaustudium zur 
Ausbildung von Übersetzern und Dolmetschern der Deutschen Sprache $)^{1}$ entwickelt und bei Dolmetschübungen eingesetzt wurde. Die neunjährige Erfahrung im Dolmetschunterricht für Aufbau- und Magisterstudierende zeigt, dass das Konzept nach wie vor aktuell ist und, da die beiden Gruppen ähnliche Defizite aufweisen, auch in Dolmetschübungen für Magisterstudierende des neu gebildeten selbstständigen Studienfaches Angewandte Linguistik Platz finden sollte.

Bei der Konzipierung der Lehrinhalte und -formen der im Lehrplan des Aufbaustudiums angebotenen Kurse stellten die berufliche Praxis und die Prüfungsanforderungen den Schwerpunkt dar, aber der notwendige Bezug auf die Theorie der Translationswissenschaft war ebenfalls gegeben. Die Autorin konzentrierte sich in den durch sie geleiteten Übungen Konsekutivdolmetschen I (im ersten Studiensemester, 20 Stunden) und Konsekutivdolmetschen II (im zweiten Studiensemester, 20 Stunden) auf die Entwicklung der Dolmetschkompetenz und Übung des Konsekutivdolmetschens in verschiedenen Situationen, die im beruflichen Alltag in Polen vorkommen können, denn in Anbetracht der Tatsache, dass die meisten Aufbaustudierenden polnische Muttersprachlerinnen und Muttersprachler, größtenteils ausgebildete Germanistinnen und Germanisten, darunter berufstätige Deutschlehrerinnen und -lehrer, waren, wurde angenommen, dass sie über die notwendige Sprach- und dementsprechend Kulturkompetenz verfügten und dass man sich - in Anlehnung an das Kompetenzmodell für Dolmetschen von Pöchhacker (2001) - dementsprechend auf die Entwicklung anderer Bereiche der translatorischen Kompetenz als die fremd- und muttersprachliche Kompetenz konzentrieren konnte, weil bei den Studierenden die Voraussetzungen für die Entwicklung der Dolmetschkompetenz gegeben waren. ${ }^{2}$

1 Das Aufbaustudium wurde am Institut für Angewandte Linguistik der Adam-MickiewiczUniversität Posen in den Jahren 2006-2015 angeboten. Der Studiengang war eine Antwort auf den Marktbedarf, erweckt durch das Gesetz über den Beruf beeidigter Übersetzer und Dolmetscher vom 25. November 2004 in der damals geltenden Fassung (pln. GBl. Dziennik Ustaw Nr. 273, Pos. 2702) und zielte daher - bei künftigen Examenskandidatinnen und -kandidaten auf die Vorbereitung der Aufbaustudentinnen auf das ministeriale Examen und - sowohl bei künftigen Examenskandidatinnen und -kandidaten als auch bei sonstigen Studierenden - auf die Entwicklung der translatorischen Kompetenz sowohl im Bereich des Übersetzens als auch des Dolmetschens ab.

2 Das Kompetenzmodell für Dolmetschen von Pöchhacker (2001: 21-22) basiert auf drei Grundkomponenten: Sprach- („Sprachen können“), Kultur- („Kulturen kennen“) und Dolmetschkompetenz („Dolmetschen können“). Die Kompetenzentwicklung verläuft nach diesem Modell graduell von „unten“ nach „oben“. Zuerst entwickelt sich somit die Sprachkompetenz, die selbstverständlich die fremd- und muttersprachliche Kompetenz umfasst, und erst danach - auf der Grundlage der Sprachkompetenz - die Kulturkompetenz. Auf der Sprach- und Kulturkompetenz baut dann die Dolmetschkompetenz auf, welche die Transferund Verhaltenskompetenz umfasst, d. h. die Kompetenz, sich als Dolmetscher situationsadäquat und den berufsethischen Normen entsprechend während, vor und nach dem Dolmetscheinsatz verhalten zu können. Die Dolmetschkompetenz bildet sich analog zur Sprach- 
Lipiński (2000: 169) meint mit Recht, dass keine Einrichtung zur Ausbildung von Dolmetschern (und Übersetzern) im Stande ist, ihre Absolventinnen auf sämtliche beim Dolmetschen (und Übersetzen) auftauchenden Probleme vorzubereiten. Angesichts dieser nicht zu verneinenden Tatsache müssen Ausbildungseinrichtungen ihren Absolventen zumindest ein geeignetes Instrumentarium mit auf den beruflichen Weg geben, indem sie die Studierenden dabei unterstützen, Kompetenzen zu entwickeln, die sie sich in ihrem beruflichen Leben bei der selbstständigen Weiterentwicklung ihrer Dolmetschkompetenz zunutze machen können. Hierzu gehört die Fähigkeit zur Selbstbewertung eigener Dolmetschleistungen, die im Studium entwickelt werden sollte.

Um dieser Forderung gerecht zu werden, wurden die Übungsformen in den Lehrveranstaltungen Konsekutivdolmetschen I und Konsekutivdolmetschen II möglichst breit gefächert. Geübt wurde bilaterales (Gespräche, Diskussionen) und unilaterales Dolmetschen (Power Point-Präsentationen, Ansprachen: mit und ohne Notizennahme - je nach Sequenzlänge), mit und ohne Vorbereitung, um so die Bedeutung der Vorbereitung zu zeigen. Um den Studierenden ihre Stärken und Defizite vor Augen zu führen, wurden sie bei einer Übung zum Vortragdolmetschen mit einer Kamera aufgenommen und die Auftritte wurden im Anschluss im Forum bewertet. Des Weiteren wurde in jedem Semester eine Feldübung zum Betreuungsdolmetschen durchgeführt, die auf die Entwicklung der Fähigkeit zur Selbstbewertung abzielte.

Obwohl es den Alltag vieler Dolmetscher prägt, wird das Betreuungsdolmetschen selten in der einschlägigen Literatur als eine (separate) Dolmetschsorte erwähnt, weil sie „gemeinhin gar nicht als Spezifikum“ angesehen wird (Matyssek 1989: 7-8). Als Betreuungsdolmetschen bezeichnet Matyssek Dolmetscheinsätze bei der sprachlichen Kommunikation von Frage- und Antwortsituationen oder bei sprachlicher Umsetzung von Ausführungen $\mathrm{zu}$ einem relativ begrenzten und deswegen sachlich und sprachlich leicht zu erarbeitenden Sachgebiet wie etwa bei (Stadt-)Führungen und Besichtigungen sowie bei Informationsständen auf Messen. $^{3}$

und Kulturkompetenz graduell von „unten“ nach „oben“ aus, d. h. zuerst entwickelt sich die Transferkompetenz und erst dann die Verhaltenskompetenz.

3 Matyssek (1989: 7-8) führt das Betreuungsdolmetschen am Rande seiner Ausführungen zum Verhandlungsdolmetschen auf und ist der Ansicht, dass es von der Dolmetschkompetenz („vom dolmetscherlichen Können“) her ebenso (relativ) einfach wie das Verhandlungsdolmetschen sei, weil die Translationsaufgabe hierbei darauf beruht, kürzere Textsequenzen unilateral, z. B. Ausführungen eines Fremdenführers, bzw. bilateral, z. B. ein Gespräch zwischen anderssprachigen Partnern, zu verdolmetschen. Anders als das unilaterale Konsekutivdolmetschen längerer Textsequenzen mit seinen Anforderungen an ein gut trainiertes Gedächtnis und eine gut beherrschte Notizentechnik bzw. das Simultandolmetschen mit seinem Gleichzeitigkeitsvollzug erfordere das Betreuungsdolmetschen damit keine besonderen ,dolmetschspezifischen Kenntnisse und Fähigkeiten'. Gefragt seien hierbei lediglich 
Die Matysseksche Fähigkeit „als Dolmetscher rollengerecht zu handeln“ ist mit der Pöchhackerschen Verhaltenskompetenz gleichzusetzen und diesem Kompetenzbereich ist die Fähigkeit zur korrekten, realistischen und (möglichst) objektiven Selbstbewertung der Qualität eigener Dolmetschleistungen zuzuordnen, denn diese Fähigkeit ermöglicht es (künftigen) Dolmetschern, bei der Nacharbeitung eines Dolmetscheinsatzes (möglichst) sachlich, d. h. auf der Grundlage von festgelegten Kriterien, beurteilen zu können, unter welchen Aspekten ihre Leistung qualitativ gut war, was nicht so gut gelungen ist und woran deswegen noch gearbeitet werden muss, um so aus dem Dolmetscheinsatz zu lernen und dadurch die Dolmetschkompetenz weiterzuentwickeln. Beobachtungen der Autorin im Unterricht und in der Praxis zeigten, dass es Dolmetschanfängern in der Regel an dieser Fähigkeit mangelt, und stellten den direkten Anstoß für die Konzipierung des in diesem Beitrag präsentierten Konzepts für die Entwicklung der Fähigkeit zur Selbstbewertung eigener Dolmetschleistungen durch Studierende dar.

Das Hauptziel des Konzepts war, dass die Aufbaustudierenden im Rahmen einer Feldübung bei einer Fremdenführung unter möglichst authentischen Bedingungen alle Phasen eines Betreuungsdolmetscheinsatzes durchlaufen und auf dieser Grundlage das rollengerechte Handeln als Dolmetscherinnen unter besonderer Berücksichtigung der Fertigkeit zur selbstständigen Bewertung der Qualität eigener Dolmetschleistungen erlernen. Damit sie Schlüsse aus der Selbstbewertung in die Praxis umsetzen und bei der Entwicklung der Dolmetschkompetenz Fortschritte sehen konnten, wurde die Feldübung zweimal durchgeführt. ${ }^{4}$

\section{Der Selbstbewertungsbogen}

Zur Bewertung der Qualität von Dolmetschleistungen als einem der wichtigsten Aspekte des Dolmetschens äußern sich nicht nur Dolmetschwissenschaftler, sondern vor allem auch Dolmetschdidaktiker, die oft zugleich auch praktizierende Dolmetscher sind. Kurz $\left(1999^{2}\right)$ stellt beispielsweise verschiedene Per-

kommunikative Fähigkeiten, gute Sprach- und Sachkenntnisse auf dem jeweiligen Gebiet sowie die Fähigkeit, in der jeweiligen Dolmetschsituation als Dolmetscher rollengerecht zu handeln. Die Arbeit des Dolmetschers wird beim Betreuungsdolmetschen auch durch weitere Faktoren erleichtert: wenig Zeitdruck, direkter Kontakt zum AS-Textproduzenten und dem/ den ZS-Textrezipienten, unmittelbare Rückkoppelung mit dem AS-Textproduzenten und dem/den ZS-Textrezipienten, um eventuelle Probleme klären zu können.

4 Die Feldübung wurde im zweiten Studiensemester mit dem Unterschied wiederholt, dass die Stadt Posen besichtigt wurde und die Studierenden bei der inhaltlich-fachsprachlichen Vorbereitung auf den paraauthentischen Dolmetscheinsatz selbstständiger arbeiten mussten. 
spektiven bei der Bewertung von Dolmetschleistungen dar. Kalina (2001) geht auf die Notwendigkeit der Aufklärung über die Problematik der Sicherung guter Qualität von professionellen Dolmetschleistungen ein und (2002) auf die Notwendigkeit der Formulierung objektiver Kriterien zur Beurteilung der Qualität von Dolmetschleistungen und die Defizite von Rezipientenbefragungen als Instrumente zur Qualitätsbeurteilung. Riccardi (2001) präsentiert eine Liste mit Mikrokriterien für die Bewertung von Dolmetschleistungen während der Ausbildung im Unterricht und bei Prüfungen. Mack (2002) bemerkt in Anlehnung an Reiß (1989: 72), dass Qualitätsbeurteilung eine nicht rein intuitive und subjektive, sondern argumentative und intersubjektiv nachvollziehbare Feststellung, Beschreibung und Bewertung von Dolmetschleistungen bedeuten soll und formuliert Leitlinien für eine wissenschaftliche Dolmetschkritik.

Drei Grundaspekte für die Bewertung studentischer Dolmetschleistungen sind nach Kutz (1997 zit. bei Kurz 1999: 393): Gesamteindruck und Verhalten des Dolmetschers, Wiedergabe des Inhalts des AS-Textes und sprachliche Realisierung der Verdolmetschung. Unter Berücksichtigung dieser drei Aspekte sowie in Anlehnung an Riccardi (2001: 274-275), Chyży (2010: 371-372) und eigene Erfahrungen als Didaktikerin und praktizierende Dolmetscherin wurde ein Selbstbewertungsbogen erstellt, der durch Studierende bei der selbstständigen Evaluierung ihrer Dolmetschleistungen bei der Feldübung auszufüllen war. Um eventuelle Missverständnisse zu vermeiden, wurde der Selbstbewertungsbogen in polnischer Sprache abgefasst. Er beinhaltete mehrere Punkte und eine Anweisung:

Inhaltlich-sprachliche Bewertung

\begin{tabular}{|l|l|l|l|}
\hline 1 & 2 & 3 & 4 \\
\hline$(+)$ bzw. $(-)$ & Aufnahme & Kommentar zu Rubrik 2 & Korrekturvorschlag \\
\hline & & & \\
\hline
\end{tabular}

\section{Beschreibende Bewertung}

Inhaltliche Korrektheit:

Sprachliche Korrektheit:

Haltung:

Gesamteindruck:

Neue Termini, die während der Feldübung gelernt wurden bzw. „gefehlt“ haben: 
Erklärungen zur inhaltlich-sprachlichen Bewertung

\section{Rubrik 1}

Hierin tragen Sie bitte folgende Bezeichnungen ein:

(+) etwas, was wir für eine besonders gute Lösung halten

(-) Fehler (grammatisch, lexikalisch, kommunikativ [falsch wiedergegebener Inhalt])

\section{Rubrik 2}

Hierin tragen Sie bitte die jeweiligen Sätze bzw. Phrasen ein (so viel, wie viel notwendig ist, um den Kontext zu verstehen), z. B.: ... * die neueste archäologische Entdeckungen ...

\section{Rubrik 3}

Hier erklären Sie bitte, worin der Vorteil der gegebenen Lösung bzw. der Fehler daran liegt, warum Sie eben so verdolmetscht haben, und warum Sie denken, dass es (+) bzw. (-) ist, z. B.: grammatischer Fehler, falsche Deklinationsendung „ $\mathrm{e}^{\text {“ }}$ statt „en“ im Ausdruck „,neueste archäologische“, nach dem bestimmten Artikel wird bei Adjektiven im Plural immer die Deklinationsendung „en“ gebraucht.

\section{Rubrik 4}

Hierin tragen Sie bitte Ihren richtigen Vorschlag/die Korrektur ein:

... die neuesten archäologischen Entdeckungen ...

\section{Erklärungen zur beschreibenden Bewertung}

Inhaltliche Korrektheit (Habe ich dem Empfänger das, was die Fremdenführerin gesagt hat, korrekt, ohne Sinnentstellungen und unnötige Kürzungen vermittelt? Mit anderen Worten: Würde der deutsche Empfänger aus der Verdolmetschung dieselben Informationen erhalten wie der polnischen Empfänger aus den Originalausführungen der Fremdenführerin?)

\section{Sprachliche Korrektheit (Lexik und Grammatik)}

Haltung („rhetorischer Eindruck“: Lautstärke, selbstbewusstes Auftreten, Flüssigkeit, Füllpausen, Aussprache und Wortbetonung) 
Gesamteindruck (allgemein: A. Aus welchen Gründen halte ich die Verdolmetschung für gelungen? B. Was hat mir keine Schwierigkeiten bereitet? C. Was hat mir Schwierigkeiten bereitet? D. Woran sollte ich arbeiten?)

Bitte scheuen Sie nicht, Vorteile einzutragen: Es geht darum, dass Sie lernen, sich selbst objektiv zu betrachten und Ihre Stärken zu bemerken!

Durch die so gesetzten Schwerpunkte bei der Selbstevaluierung wollte man der Forderung gerecht werden, dass die Bewertung von Dolmetschleistungen keinesfalls eine reine Beurteilung sprachlicher Korrektheit bedeuten sollte, „weil die Adäquatheitsbedingungen nicht nur von sprachlichen Gegebenheiten (im Sinne des Verhältnisses zwischen Quell- und Zielsprache) abhängen, sondern im weitesten Sinne von den relevanten Faktoren der Kommunikationssituation bestimmt werden“ (Verbmobil 1991: 15 zit. bei Kautz 2000: 413).

\section{Die Phasen der Feldübung}

Das Konzept definierte den folgenden Ablauf der Übung zum Betreuungsdolmetschen, der den Ablauf eines realen Dolmetscheinsatzes nachzuahmen hatte:

\subsection{Vorbereitung auf den Dolmetscheinsatz}

Im Rahmen der Vorbereitung als Vorphase des eigentlichen Dolmetscheinsatzes muss sich der Dolmetscher auf allen, aber vor allem auf den thematischen und sprachlichen Ebenen vorbereiten: sich mit dem jeweiligen Fachgebiet inhaltlich vertraut machen, darüber die neuesten Informationen einholen (Kutz 2002: 186) und so seine Wissensdefizite schließen (Kautz 2000: 293), z. B. durch Internetrecherche, Bearbeitung von Paralleltexten und Erstellung themenorientierter Glossare.

\section{A. Aufgabenformulierung und Erklärung des Übungskonzepts}

$\mathrm{Zu}$ Beginn des ersten Studiensemesters (Oktober) wurde den Studierenden mitgeteilt, dass sie zu Semesterende (Januar) im Rahmen einer Feldübung bei einer Fremdenführung auf der Dominsel und im Posener Dom die Ausführungen einer Fremdenführerin ins Deutsche übertragen, dabei mit einem Diktiergerät aufgezeichnet und später nach Anweisungen in einem Selbstbewertungsbogen eine Selbstbewertung der eigenen Dolmetschleistung vornehmen werden. Bei der Fremdenführung wird eine deutsche Muttersprachlerin anwesend sein, die Polnisch zumindest versteht. Die Fremdenführerin, die eine polnische 
Muttersprachlerin ist, spricht Deutsch. ${ }^{5}$ Zwecks terminologischer und inhaltlicher Vorbereitung auf die Feldübung wurde den Studierenden per E-Mail geschickt: eine Liste mit Fachwortschatz in polnischer Sprache (120 Fachwörter bzw. Fachphraseologismen; die Studierenden sollten deutsche Äquivalente finden), Links zu Internet-Seiten und einige PDF-Materialien, die bei der inhaltlichen Vorbereitung nützlich sein konnten. Das Datum der Feldübung wurde sogleich angegeben und angesagt wurde auch, dass die Wortschatzliste vor der Feldübung im Unterricht gemeinsam besprochen und dann in der letzten Lehrveranstaltung vor der Feldübung eine Klausurarbeit zu diesem Wortschatz geschrieben werde. Zwischen der Besprechung des Vokabulars im Unterricht und der Klausurarbeit wurden zwei Wochen eingeplant, damit die Aufbaustudierenden den Wortschatz auswendig lernen konnten. Die Fertigkeit, die in dieser Etappe eingeübt wurde, war die Fähigkeit zur gezielten Vorbereitung auf einen Dolmetscheinsatz, auch wenn die Vorbereitung teilweise gesteuert wurde. Die teilweise Steuerung war insofern begründet, dass fast alle Aufbaustudierenden noch nie einen realen (kommerziellen) Dolmetschauftrag ausgeführt hatten.

\section{B. Selbstarbeit}

Die Studierenden hatten zwei Monate Zeit, um sich terminologisch und inhaltlich vorzubereiten. Der Verlauf dieser Phase wurde den Aufbaustudierenden zur selbständigen Arbeit überlassen. Im regelmäßigen Unterricht wurde in dieser Zeit der im Lehrplan vorgesehene Stoff durchgenommen. Die Lehrinhalte und -formen und die Übungen dienten selbstverständlich neben der Entwicklung der Dolmetschkompetenz auch der Vorbereitung auf die Feldübung, die für die Studierenden als Krönung des Kurses im ersten Studiensemester eine Art Bewährungsprobe darstellen sollte.

5 Dadurch dass die Fremdenführerin Deutsch sprach, konnte sie eventuelle Probleme der Studierenden mit der Verdolmetschung, besonders in Bezug auf die Sinnwiedergabe (Sinnentstellungen, Auslassungen und die Fachlexik) bemerken und dementsprechend reagieren: Ihre Ausführungen entweder wiederholen, etwas zusätzlich erklären bzw. die AS-Textsequenzen kürzen, wobei sie von der Autorin gebeten wurde, nur in Extremfällen einzugreifen, wenn ein völliger Abbruch der Kommunikation drohte, sonst sollte sie sich so verhalten, als ob sie keine Probleme bemerkt hätte, damit die Studierenden im Rahmen des Monitoring der eigenen Dolmetschleistung die Gelegenheit zur Anwendungen von Notlösungen (Selbstkorrekturen, Klärungsfragen, Bitten um Wiederholung bzw. Kürzung der Ausführungen) hätten. 


\section{Besprechung des Wortschatzes im Forum}

Bei der Besprechung des Vokabulars im Unterricht ging es nicht um die bloße Angabe der in Frage kommenden Lösungen durch die Dozentin (Autorin), sondern es wurde gemeinsam besprochen, was die Begriffe bedeuten und welche Äquivalente daher korrekt sind. Als Bezugsquellen dienten dabei monolinguale Wörterbücher der deutschen Sprache, Bildwörterbücher, Lexika und Internetseiten. Nach zwei Wochen wurde die angesagte Klausur zu dem Wortschatz geschrieben. Des Weiteren wurde im Unterricht der Selbstbewertungsbogen nebst Anweisungen zu dessen Ausfüllen erörtert. Um technische Probleme mit dem Aufzeichnen der Verdolmetschungen zu vermeiden, wurde auch gezeigt, wie das Diktiergerät zu bedienen war.

\subsection{Feldübung: Fremdenführung}

Die Feldübung stellte den eigentlichen Dolmetscheinsatz dar und gab den Studierenden eine Gelegenheit ihre Transfer- und Verhaltenskompetenz unter fast authentischen Bedingungen zu üben. Bei der Feldübung wurden Fähigkeiten zum Vollzug dolmetschspezifischer Handlungen wie Rezeption, Transposition, Reproduktion, Monitoring der eigenen Dolmetschleistung und Memorisierung geübt. Während der Feldübung machte sich sowohl die Dozentin als auch die Muttersprachlerin Notizen zu den Verdolmetschungen der Aufbaustudierenden, wobei sich die erstgenannte auf die Haltung, gestisch-rhetorische Präsentation und inhaltliche Vollständigkeit und die zweitgenannte auf die kommunikative, inhaltliche und sprachliche Ebene konzentrierte. Um die Fremdenführung möglichst authentisch $\mathrm{zu}$ gestalten und den Aufbaustudierenden auch die Möglichkeit zu geben, mit der Verdolmetschung unerwarteter Rezipientenfragen konfrontiert zu werden, haben die Dozentin und die Muttersprachlerin ab und zu in deutscher Sprache Fragen zu den Aufführungen der Fremdenführerin gestellt, die es ins Polnische und dann die Antworten ins Deutsche zu verdolmetschen galt.

\subsection{Nachbereitung und Selbstbewertung}

Die Nachbereitung, die hier eine teilweise gesteuerte Analyse der eigenen Dolmetschleistung durch die Studierenden in Hinsicht auf kommunikative, inhaltliche und sprachliche Korrektheit sowie auf nichtverbale Phänomene wie Haltung, Gestik und sprecherische Qualitäten bedeutete, wurde in zwei Etappen 
vorgenommen, teilweise im Forum und teilweise individuell durch die Erstellung von Textnotationen und das Ausfüllen der Selbstbewertungsbögen.

\section{A. Besprechung im Unterricht direkt nach der Feldübung}

Direkt nach der Feldübung wurden am nächsten Tag im Unterricht als eine Art Voranalyse die kommunikative und inhaltliche Korrektheit sowie die nichtverbalen Phänomene besprochen. Die Voranalyse wurde in Anlehnung an die Notizen und Eindrücke der an der Feldübung beteiligten deutschen Muttersprachlerin und der Dozentin sowie die Mini-Selbstbewertungsbögen durchgeführt. Die Mini-Selbstbewertungsbögen beinhalteten lediglich zwei Rubriken: „als besonders gelungen an meinem Dolmetscheinsatz empfinde ich:..." und „ich muss noch arbeiten an:...“. Die Studierenden benannten vor allem Probleme mit der Stressbewältigung, Memorisierung und Lexik. Die Dozentin und Muttersprachlerin gingen auch auf grobe bzw. sich oft wiederholende sprachliche Fehler, darunter die Wortbetonung, ein.

\section{B. Selbstevaluierung der Dolmetschleistungen durch die Studierenden}

Im Rahmen der eigentlichen Selbstbewertung ihrer Dolmetschleistungen mussten die Studierenden die per E-Mail zugeschickten Aufzeichnungen ihrer Verdolmetschungen und der entsprechenden Ausführungen der Fremdenführerin niederschreiben, wobei sie nicht nach wissenschaftlichen Regeln zur Anfertigung von Transkriptionen vorgehen mussten, da solch eine Vorgehensweise nicht von professionellen Dolmetschern erwartet wird, im Anschluss ihre Dolmetschleistung nach den im Selbstbewertungsbogen vorgegebenen Kriterien bewerten und die Textnotation unter Berücksichtigung der Tatsache, dass es sich hierbei um den lediglich niedergeschriebenen gesprochenen Text einer Verdolmetschung handelt, sprachlich korrigieren und beides der Autorin zwecks Beurteilung ihrer Fähigkeit zur Selbstbewertung mailen. Es wurde betont, dass nicht die sprachliche Korrektheit der Verdolmetschung, sondern die Fähigkeit zur Selbstbewertung und -korrektur beurteilt wird.

\subsection{Verifizierung der Selbstbewertungsbögen durch die Autorin}

Schwerpunkt bei der Verifizierung der Selbstbewertungsbögen war die Bewertung der Fähigkeit zur Selbstkorrektur in Bezug auf die Sinnwiedergabe, wodurch zwangsläufig Schlüsse auf die Dolmetschkompetenz der Studierenden gezogen werden konnten. Unter Mitwirkung von deutschen Muttersprachlern wurde aber auch die Fähigkeit zur sprachlichen Selbstkorrektur verifiziert, was wiederum die 
Beurteilung der Sprachkompetenz ermöglichte. Nach der Verifizierung erhielten die Studierenden ein Feedback: eine beschreibende Beurteilung und den Selbstbewertungsbogen mit detaillierten Kommentaren sowie inhaltlichen und sprachlichen Korrekturen der Autorin. Damit wollte man den Studierenden vor Augen führen, was sie richtig erkannt und welche Fehler sie übersehen haben.

\section{Die Auswertung der Fähigkeit der Studierenden zur Selbstbewertung}

Die binnen neun Jahren bei den für jeden Studiengang zweimal durchgeführten Feldübungen gesammelten Aufzeichnungen nebst Selbstbewertungsbögen ergeben ein sehr umfangreiches empirisches Material, das es gilt, einer genauen wissenschaftlichen Analyse zu unterziehen, um methodisch untermauerte Schlüsse ziehen zu können. Es wird durch die Autorin im Rahmen eigener Forschungsarbeit schrittweise aufbereitet. Auf der Grundlage der Verifizierung von Selbstbewertungsbögen zwecks des Feedbacks für die Studierenden ließen sich jedoch Gemeinsamkeiten feststellen und vorläufige Schlüsse ziehen, die bereits bei einigen stichprobenweise durchgeführten qualitativen und quantitativen Voruntersuchungen als Thesen dienten. Die Untersuchungen wurden im Rahmen von unter Betreuung der Autorin geschriebenen Bachelorarbeiten durchgeführt. Hinsichtlich der inhaltlichen Korrektheit der Inhaltswiedergabe (und somit hinsichtlich der Dolmetschkompetenz) wurden in den Voruntersuchungen Expandierungen und Omissionen analysiert. Hinsichtlich der sprachlichen Korrektheit wurden sprachliche Fehler aller Art, außer Fehler bei der Aussprache, untersucht, d. h. grammatische, lexikalische und Interferenzfehler zwischen dem Polnischen und Deutschen sowie der Gebrauch des Fachwortschatzes.

Wąsik (2013) hat in einer quantitativen Inhaltsanalyse der inhaltlich-sprachlichen Selbstbewertungen in einer Stichprobe von 16 Selbstbewertungsbögen und entsprechenden Textnotationen durch Vergleich aller begangenen und der durch die Studierenden identifizierten Fehler untersucht, welche Fehlerkategorien nicht erkannt wurden und wie oft diese nicht erkannt wurden. Insgesamt wurden durch die Studierenden nur knapp über ein Viertel aller begangenen Fehler (167 von 580 Fällen) erkannt. ${ }^{6}$ Die häufigste Kategorie unter den begangenen Fehlern waren grammatische Fehler (290 Fälle), von denen fast drei Viertel (209 Fälle $72 \%)$ nicht erkannt wurden. Die zweithäufigste Kategorie stellten inhaltliche

\footnotetext{
6 Ursache für diese enorme Diskrepanz kann neben der mangelhaften Sprachkompetenz auch eine zu geringe Sorgfalt beim Ausfüllen der Selbstbewertungsbögen sein, worauf manche Selbstbewertungen hindeuteten: lakonisch, ohne eingehende Kommentare, mit nicht allen erkannten und korrigierten Fehlern in den angeführten Textabschnitten.
} 
Fehler (154 Fälle) dar, von denen über drei Viertel (118 Fälle - 77 \%) nicht erkannt wurden. Diesen folgten lexikalische Fehler mit 93 Fällen, von denen fast zwei Drittel (59 Fälle - 63 \%) von den Studierenden nicht identifiziert wurden. Unter den grammatischen Fehlern war der falsche Artikelgebrauch die häufigste nicht erkannte Subkategorie (nur neun erkannte von 73 Fällen), wobei dies ein Phänomen in der deutschen Sprache ist, das Polen große Schwierigkeiten bereitet, da es in der polnischen Sprache nicht vorkommt. An zweiter Stelle befanden sich falsche Konstruktionen (nur zehn erkannte von 51 Fällen), meistens Konstruktionen, die wortwörtlich aus der polnischen Sprache übernommen wurden. Bei inhaltlichen Fehlern stellten Omissionen die häufigste Subkategorie (104 Fälle) dar, von denen nur ein Fünftel (21 Fälle) identifiziert wurde. ${ }^{7}$

Vor dem Hintergrund der Ergebnisse dieser Untersuchung sind die Ergebnisse der qualitativen Inhaltsanalyse der beschreibenden Selbstbewertungen der inhaltlichen und sprachlichen Korrektheit sowie des Gesamteindrucks in derselben Stichprobe von 16 Selbstbewertungsbögen und entsprechenden Textnotationen erstaunlich (vgl. Wąsik 2013). Von der Hälfte der 16 Studierenden wurde die inhaltliche Korrektheit ihrer Dolmetschleistungen als eher positiv und von drei Personen als positiv bewertet. Auch die sprachliche Korrektheit wurde von der Hälfte der Studierenden als positiv bewertet und von einer Person als positiv. Bewertungen von drei Personen fielen neutral aus.

Das Kriterium bei der Selbstbewertung, das eine Art abschließende Beurteilung darstellte und sich verhältnismäßig im größten Grade auf subjektive Eindrücke bezog, war der Gesamteindruck. Zu erwarten war, dass die Bewertung der vorher im Selbstbewertungsbogen aufgelisteten Kriterien, vor allem die inhaltlich-sprachliche Bewertung im tabellarischen Teil, einen wesentlichen Einfluss auf die Betrachtung dieser Kategorie hatte. Die studentischen Selbstbeurteilungen in Bezug auf den Gesamteindruck konnten analog zu den Selbstbewertungen der inhaltlichen und sprachlichen Korrektheit ausgewertet werden: Bei sieben Personen wurden sie der Kategorie „eher positiv“ und bei einer „positiv“ zugeordnet. Vermutlich haben die Studierenden somit bei der Beurteilung des Gesamteindrucks keinen Bezug auf ihre eigenen Bewertungen der inhaltlichsprachlichen Korrektheit im tabellarischen Teil der Selbstbewertungsbögen genommen, welche, auch wenn sie im Hinblick auf die erkannten Fehler nicht vollständig waren, eindeutig auf die Defizite der Verdolmetschungen hinwiesen, und/oder die aufgezeichneten bzw. niedergeschriebenen Verdolmetschungen mit den Ausführungen der Fremdenführerin überhaupt nicht bzw. nicht sorgfältig genug verglichen. Vorausgesetzt jedoch, dass sie die Aufgabe ernst genommen haben, deutet beides auf eine mangelhafte Fähigkeit zur Selbstbewer-

7 Omissionen sind natürlich nicht immer als Fehler zu betrachten, denn sie können auch eine Dolmetschstrategie darstellen. 
tung hin, denn sie haben das Urteil über den Gesamteindruck ohne Bezug auf objektive Tatsachen gefällt.

Fazit: Der Vergleich von Ergebnissen der quantitativen und qualitativen Untersuchung, genauso wie die Ergebnisse weiterer Voruntersuchungen, bestätigen die durch Autorin bei der Verifizierung der studentischen Selbstbewertungsbögen gemachten Beobachtungen, dass die meisten Aufbaustudierenden, obwohl sie größtenteils ausgebildete Deutschlehrerinnen und -lehrer waren, über die notwendige Sprachkompetenz, auf der die Dolmetschkompetenz mit Erfolg aufbauen könnte, nicht verfügten. Aufgrund der mangelhaften fremdsprachlichen Kenntnisse verfügten sie auch nicht über die Fähigkeit zur realistischen, objektiven Selbstbewertung eigener Dolmetschleistungen und zeigten sich zu optimistisch: Da sie sich ihrer fremdsprachlichen Defizite nicht bewusst waren, waren sie auch nicht im Stande die Defizite ihrer Dolmetschkompetenz zu bemerken.

\section{Postulate hinsichtlich der Entwicklung der Fähigkeit zur Selbstbewertung eigener Dolmetschleistungen durch Studierende}

Aus dem Vorstehenden ergeben sich folgende Postulate hinsichtlich der Entwicklung der studentischen Fähigkeit zur Selbstbewertung: In Dolmetschübungen, z. B. auch im Rahmen von Projektarbeit in kleineren Gruppen, sollten regelmäßig Feldübungen durchgeführt werden, bei denen Studierende aufgezeichnet werden und ihre Verdolmetschungen unter Einsatz schriftlicher Selbstbewertungsbögen bewerten müssen, da diese Übungsform es ermöglicht, unter fast authentischen Bedingungen alle Elemente der Dolmetschkompetenz, darunter die Fähigkeit zur Selbstbewertung, und alle Phasen eines Dolmetscheinsatzes zu üben. Die Feldübungen sollten immer eine Selbstbewertung in schriftlicher Form, verifiziert durch Dozentinnen, mit einbeziehen, weil damit Studierenden am besten objektiv vor Augen geführt werden kann, welche Defizite ihre Sprach- und Dolmetschkompetenz aufweist. Sowohl während des gesamten Bachelor- als auch Magisterstudiums Angewandte Linguistik mit Schwerpunkt Übersetzen/Dolmetschen sollten Studierenden Übungen zur intensiven dolmetschorientierten Erweiterung der fremdsprachlichen Kompetenz angeboten werden. Dolmetschübungen sollten bereits im Lehrplan des Bachelorstudienganges Platz finden, weil es im Magisterstudiengang oft zu spät für die Entwicklung der Dolmetschkompetenz und der Fähigkeit zur Selbstbewertung ist, da die fremdsprachliche Kompetenz wegen automatisierter Fehler, deren sich Studierende nicht bewusst sind, dermaßen defizitär ist, dass darauf die Dolmetschkompetenz nicht mehr aufgebaut werden kann. 


\section{Literaturverzeichnis}

Chyży, Radosław (2010). Zum Begriff der Qualität der Verdolmetschung. In: A. Małgorzewicz (Hrsg.), Translation: Theorie - Praxis - Didaktik (S. 269-375). Wrocław, Dresden: Oficyna Wydawnicza ATUT - Wrocławskie Wydawnictwo Oświatowe/Neiße Verlag.

Kalina, Sylvia (2001). Zur Professionalisierung beim Dolmetschen - Vorschläge für Forschung und Lehre. In: A. F. Kelletat (Hrsg.), Dolmetschen. Beiträge aus Forschung, Lehre und Praxis (S. 51-64). Frankfurt a.M.: Peter Lang.

Kalina, Sylvia (2002). Fragestellungen der Dolmetschwissenschaft. In: J. Best/S. Kalina (Hrsg.), Übersetzen und Dolmetschen: eine Orientierungshilfe (S. 30-43). Tübingen, Basel: Francke.

Kautz, Ulrich (2000). Handbuch Didaktik des Übersetzens und Dolmetschens. München: Iudicium. Goethe-Institut.

Kurz, Ingrid (1999). Dolmetschleistungen. In: M. Snell-Hornby/H.G. Hönig/P. Kussmaul/ P.A. Schmitt (Hrsg.), Handbuch Translation (S. 391-394). Tübingen: Stauffenburg Verlag. Kutz, Wladimir (2002). Dolmetschkompetenz und ihre Vermittlung. In: J. Best/S. Kalina (Hrsg.), Übersetzen und Dolmetschen: eine Orientierungshilfe (S. 184-195). Tübingen, Basel: Francke.

Lipiński, Krzysztof (2000). Vademecum tłumacza. Kraków: Idea.

Mack, Gabriele (2002). Die Beurteilung professioneller Dolmetschleistungen. In: J. Best/ S. Kalina (Hrsg.), Übersetzen und Dolmetschen: eine Orientierungshilfe (S. 110-119). Tübingen/Basel: Francke.

Matyssek, Heinz (1989). Handbuch der Notizentechnik für Dolmetscher. Ein Weg zur sprachunabhängigen Notation. Teil I. Heidelberg: Julius Groos Verlag.

Pöchhacker, Franz (2001). Dolmetschen und translatorische Kompetenz. In: A. F. Kelletat (Hrsg.), Dolmetschen. Beiträge aus Forschung, Lehre und Praxis (S. 19-36). Frankfurt a.M.: Peter Lang.

Reiß, Katharina (1989). Übersetzungstheorie und Praxis der Übersetzungskritik. In: F. G. Königs (Hrsg.), Übersetzungswissenschaft und Fremdsprachenunterricht. Neue Beiträge zu einem alten Thema (S. 71-93). München: Goethe-Institut.

Riccardi, Alessandra (2001). Die Bewertung von Dolmetschleistungen während der Ausbildung und in der Berufspraxis. In: A. F. Kelletat (Hrsg.), Dolmetschen. Beiträge aus Forschung, Lehre und Praxis (S. 267-277). Frankfurt a.M.: Peter Lang.

Wąsik, Ewelina (2013). Die Dolmetscherfähigkeit zur Selbstbewertung anhand einer Analyse studentischer Selbstbewertungsbögen; (eine unveröffentlichte Bachelorarbeit) am AMU Poznań.

Verbmobil Konsortium (Hrsg.) (1991). Verbmobil - mobiles Dolmetschgerät - Studie. München. 


\title{
Culture in translation: A comparative analysis of English originals and Polish dubbed versions of computer-animated film productions
}

\begin{abstract}
The aim of the research presented in this chapter was to indicate the multiplicity of culturerelated references in audio-visual English-Polish translation on the example of a longer form, which is a computer animated film containing humorous elements. The film selected for the analysis is the Polish dubbed version of the American blockbuster from 2004 Shrek 2. The culture-related fragments detected and analysed have been divided into four categories and marked out as: 1) linguistic references (quotations or paraphrases from popular Polish books, films and songs); 2) cultural references (expressions or proper names known and characteristic exclusively of Polish culture);3) everyday language (popular, colloquial and slang sayings, idiomatic expressions, proverbs, language styled to be archaic and elements of regional dialects); 4) personal references to well known Poles, celebrities and fictional characters. As a result of the analysis undertaken, 90 culture-related film script fragments were detected. Moreover, having been confronted with the original version, in many cases they seem to be much funnier than the original phrases.
\end{abstract}

Keywords

audio-visual translation, dubbing, computer animated films, humour, culture

\section{Introduction}

Audio-visual translation (AVT) dates back to the very beginning of cinema and cinematography. It has evolved over the decades and become specialised within narrow varieties, the most important of which are dubbing, subtitles and voiceover (Pisarska \& Tomaszkiewicz 1996; Tomaszkiewicz 2006, Chaume 2012). At first, at the beginning of cinematography, it was limited to subtitles, for obvious reasons - as all films were silent, so even original productions were subtitled in their native versions. This was conditioned by the technological barrier making it impossible for sound to be conveyed in the film. Soon, in the era of silent movies, naturally, subtitles were translated into other languages as the film distribution spread around the world. At that time, subtitles were present in a form of or- 
namented 'title cards' containing written indications of the plot and key dialogue lines. Silent films also used 'intertitles' to narrate the story points or sometimes even to comment on the action for the audience. The interest of this chapter is focused on dubbing, and narrowed down to the relatively new genre of computeranimated films, which evolved from cartoons and was initiated by the Toy story production from 1995. However, it was definitely the Shrek saga ${ }^{1}$ which constituted a breakthrough in the area of dubbing and contributed to the further development of the genre to its present state. And although all its sequels turned out to be commercial successes, only the Shrek 2 production was similarly appreciated by the critics, compared to its predecessor. That is why this particular film has been selected for the analysis of the translation of its dubbed version into Polish. The aim of the article is to present the multiplicity of culture-related elements detected in the translation of the film and confront them with the strategies of translating humour (including wordplay) and cultural elements defined in the literature on the subject. It is interesting which of them have been selected by the Polish translator to obtain the dubbed version, which turned out to be even more humorous to the Polish audience than the original one.

\section{Strategies in audio-visual translation}

Dubbing is one of the audio-visual translation techniques, definitely the most prevalent nowadays, consisting in replacing the dialogues in the source language by their versions in the target language. It is not popular in Eastern and Central Europe, including Poland, where subtitles still dominate in films shown in cinemas and voiceover is the most common in television productions. Currently, the films which are dubbed in Polish versions are mainly animated productions for children, and films which can be watched by whole families (Adamowicz-Grzyb 2013). The techniques of dubbing and subtitling are also used in the process of translation of computer games (Sajna 2013). However, problems in translation appear already at the stage of translating titles of productions (Surdyk \& Urban 2016).

According to Dore (2008: 58), the process of dubbing consists of several stages. At first, the translator delivers a 'raw translation', and later on the dubbing team alters it to match the lip movement of the actors, called 'lip-sync', and synchronises it with paratextual elements, such as the actors' intonation, gestures

1 A series of films which started with the release of Shrek (2001), continued with Shrek 2 (2004), Shrek the Third (2007), Shrek Forever After (2010), all of them constituting the basic saga; and a few minor productions - holiday-themed short productions: Shrek the Halls (2007) and Scared Shrekless (2010), as well as a spin-off prequel film entitled Puss in Boots (2011). All of the films were translated into the Polish dubbed version by Bartosz Wierzbięta. 
and posture. Clearly, it is an extremely involving, demanding and complicated process (and at the same time costly), which is confirmed by other researchers (e.g. Pisarska \& Tomaszkiewicz 1996; Sikora 2013), as it is not only pure text translation but, in fact, audio and visual translation. The pace of dialogues in the original version must be perfectly synchronised with the dubbed version. To achieve this, many details like even the length and quality of syllables have to be taken into consideration (Adamowicz-Grzyb 2013).

The process of translating dubbing, especially in comedy computer-animated productions, most often includes translation of humour (Bremmer \& Roodenburg, 1997, Rębkowska 2016) with the use of different strategies. The most popular humorous element present in comedies is the wordplay, also known as punning. There are various strategies for translating wordplay.

Dirk Delabastita (1989: 128) proposes quite a precise model which deals specifically with the translation of puns, and presents a typology consisting of eight strategies of translating wordplay:

1. Pun $\rightarrow$ pun: The source-text pun is translated into the target language with mirror adjustments, which may be more or less different from the original wordplay in terms of formal and semantic structure, or textual function.

2. Pun $\rightarrow$ non-pun: The pun is treated as a non-punning phrase, which may save both components of the wordplay despite a non-punning conjunction, or choose one of the components at the sacrifice of suppressing the other; of course it may also happen that both pun components are translated 'beyond recognition'.

3. Pun $\rightarrow$ related rhetorical device: The pun is replaced by some related rhetorical device e.g. repletion, rhyme, irony, paradox, alliteration, referential vagueness, which aims to recover a part of the original humorous effect.

4. Pun $\rightarrow$ zero: A part of the text containing the pun is omitted altogether.

5. Pun ST = pun TT: The translator reproduces the original pun and possibly its immediate environment in its original formulation.

6. Non-pun $\rightarrow$ pun: The translator adds a pun in textual positions where the original text has no wordplay in order to compensate for a pun lost elsewhere, or for any other reason.

7. Zero $\rightarrow$ pun: An entirely new textual material is added containing a wordplay that has no apparent reason to appear in the source text, except for its compensatory function.

There is also an eighth technique called 'editorial', which stands for including explanatory notes or footnotes, comments in the translator's foreword or presentation of different allegedly complementary solutions to one and the same source-text problem, etc. However, Dore (2008: 130) notices that this technique 
"cannot be applied to audiovisual text since endnotes or footnotes cannot be used in spoken texts".

\section{Culture-based humour}

Many scholars (e.g. Díaz \& Remael 2007; Lew 2000; Zabalbeascoa 1996) are convinced that audiovisual humour is perfectly translatable. However, some (Zabalbeascoa 1996: 2) warn against such a false assumption, saying that the problems of translating audiovisual texts are purely linguistic and "can be reduced to questions of grammar, lexis or semantic structure", adding that it is also important "to be aware of the number of factors involved in determining the outcome of the audiovisual translation activity" (ibid.). Culture-based humour requires specific and adequate treatment and a proper selection of translation strategies.

Pedersen (2005: 3-4) enumerates seven strategies regarding culture-based humour which are at the translator's disposal:

1. Finding an official equivalent: this technique involves the use of a pre-prepared target language version of cultural elements.

2. Retention: by applying this technique, a translator does not modify the source text or does not provide any guidance to the target audience. Retention is frequently used to render proper names.

3. Specification: this strategy enables the translator to leave the cultural reference so that it remains untranslated. However, the translator provides some information for the target audience.

4. Direct translation: this strategy is divided into two subcategories - calque and shift. It is used to render the source text without making any alterations. The strategy can be used, for instance, to render names of institutions and companies.

5. Generalisation: the culture-related element is generalised in the translation although in the source language it refers to something more specific.

6. Substitution: a joke can be either paraphrased or replaced by another joke which is more suitable for the target viewers.

7. Omission: a culture-based joke is omitted by the translator and, therefore, it does not appear at all. However, the use of omission may indicate less care taken by the translator.

It should be remembered that, above all, the aim of the translator is to transmit the sense of a text which will be understandable in the target culture (Tomaszkiewicz 2006). 


\section{Translation analysis}

The total running time of the film Shrek 2 is 1:32:23 (92 minutes and 23 seconds), while the real time of the film (excluding initial and final credits) is 1:22:23 (82 minutes and 23 seconds). Having analysed the whole film multiple times, a total of 90 potentially culture-related translations have been detected. The quotations, numbered from 1 to 90, with their direct translations (from Polish back into English) and explanations together with the source texts are presented in Table 1 below. They have been divided into four categories marked with different colours: 1. linguistic references labelled by the author as 'cultural/intermedial intertextuality' to which quotations, paraphrases from popular Polish books, films and songs have been classified (dark grey colour - 13 fragments);

2. cultural references - among which there are expressions, proper names known and characteristic exclusively for the Polish culture (grey colour - 31 fragments);

3. everyday language - covering popular, colloquial and slang sayings, idiomatic expressions, proverbs, and elements of regional dialects (white colour -40 fragments);

4. personal references - concerning very well known people in Poland, celebrities and fictional characters (black colour - 6 fragments).

All the selected fragments have been analysed by means of Delabastita's (1989) strategies for translating wordplay and Pedersen's (2005) strategies for translating culture-based humour, both considered as the most appropriate in this case in order to establish the dominant strategies applied in the Polish dubbed version of the film.

Table 1: Cultural references in Shrek 2

\begin{tabular}{|c|c|c|c|c|}
\hline No & Time & Polish dubbed version & $\begin{array}{l}\text { Literal meaning/Ex- } \\
\text { planation }\end{array}$ & Original English text \\
\hline 1. & & Książę z bajki. & $\begin{array}{l}\text { Prince from a fairytale. (A } \\
\text { common saying in Polish.) }\end{array}$ & Prince Charming. \\
\hline 2. & $1: 40$ & $\begin{array}{l}\text { Niepomny przepisów } \\
\text { BHP... }\end{array}$ & $\begin{array}{l}\text { Disregarding the Health and } \\
\text { Safety Regulations... } \\
\text { (Comical use of a formal } \\
\text { term.) }\end{array}$ & risking life and limb \\
\hline 3. & $2: 25$ & hajtnęła się była & $\begin{array}{l}\text { She had got hitched (slang, } \\
\text { styled to be archaic - with } \\
\text { use of Past Perfect Tense } \\
\text { perceived as old-fashioned } \\
\text { in Polish and rarely used). }\end{array}$ & $\begin{array}{l}\text { She's on her } \\
\text { honeymoon. }\end{array}$ \\
\hline 4. & $2: 27$ & Zali z kim? & $\begin{array}{l}\text { Whoe'er with? (styled to be } \\
\text { archaic) }\end{array}$ & $\begin{array}{l}\text { Honeymoon? With } \\
\text { whom? }\end{array}$ \\
\hline
\end{tabular}


Table 1 (Continued)

\begin{tabular}{|c|c|c|c|}
\hline No Time & Polish dubbed version & $\begin{array}{l}\text { Literal meaning/Ex- } \\
\text { planation }\end{array}$ & Original English text \\
\hline $4: 55$ & $\begin{array}{l}\text { Jednak nie ma to jak w } \\
\text { domu }\end{array}$ & $\begin{array}{l}\text { A popular Polish saying. } \\
\text { "Yet, there's no place like } \\
\text { home". }\end{array}$ & It's so good to be home! \\
\hline $5: 25$ & $\begin{array}{l}\text { Zajmowałem się } \\
\text { waszym M4 }\end{array}$ & $\begin{array}{l}\text { I took care of your three- } \\
\text { bedroomed apartment. } \\
\text { "M4" used comically, } \\
\text { referring to a term used back } \\
\text { in the communist time, } \\
\text { denoting the size of an } \\
\text { apartment.) }\end{array}$ & $\begin{array}{l}\text { Taking care of your love } \\
\text { nest for you. }\end{array}$ \\
\hline $5: 38$ & Pies na glony & $\begin{array}{l}\text { He's a seaweed-chaser (a } \\
\text { reference to an aquarium } \\
\text { pet, purposefully made to } \\
\text { refer to the term "skirt- } \\
\text { chaser") }\end{array}$ & $\begin{array}{l}\text { That Shrek is a rascally } \\
\text { devil. }\end{array}$ \\
\hline $6: 15$ & $\begin{array}{l}\text { Nie musisz mi, jak } \\
\text { łopatą. }\end{array}$ & $\begin{array}{l}\text { You don't need to explain as } \\
\text { if I were dumb (a Polish } \\
\text { expression "explain with a } \\
\text { shovel") }\end{array}$ & $\begin{array}{l}\text { You don't have to worry } \\
\text { about a thing. }\end{array}$ \\
\hline $7: 05$ & Wystarczy Rysiu. & $\begin{array}{l}\text { Enough, Dick. (The Polish } \\
\text { equivalent of the diminutive } \\
\text { of the name has the same } \\
\text { comical connotations and is } \\
\text { similar to the English } \\
\text { original in form.) }\end{array}$ & Enough, Reggie. \\
\hline 10. $7: 17$ & Zasiedmiogórogrodu & $\begin{array}{l}\text { the } \\
\text { Beyondsevenmountainburg } \\
\text { (A reference to Polish } \\
\text { fairytale tradition of starting } \\
\text { the story with "beyond seven } \\
\text { mountains, beyond seven } \\
\text { forests...") }\end{array}$ & $\begin{array}{l}\text { the Kingdom of Far, Far } \\
\text { Away }\end{array}$ \\
\hline 11. $8: 07$ & Orkiestra nadęta & $\begin{array}{l}\text { A pompous band (both for } \\
\text { being pompous as well as } \\
\text { consisting of wind } \\
\text { instruments) }\end{array}$ & $\begin{array}{l}\text { Sergeant Pompous and } \\
\text { the Fancy Pants Club } \\
\text { Band }\end{array}$ \\
\hline 12. $8: 32$ & $\begin{array}{l}\text { Patrz, nie wiedziałem, } \\
\text { że to transakcja } \\
\text { wiązana! }\end{array}$ & $\begin{array}{l}\text { Look, I didn't know that it's } \\
\text { a tie-in transaction! (A } \\
\text { business term.) }\end{array}$ & $\begin{array}{l}\text { Well, there's some fine } \\
\text { print for you! }\end{array}$ \\
\hline 13. $8: 40$ & ...a zupa była za słona & $\begin{array}{l}\text {... and the soup was too salty } \\
\text { (A term used to refer to } \\
\text { domestic violence.) }\end{array}$ & And that's final! \\
\hline 14. $9: 08$ & $\begin{array}{l}\text { "Na ciągniku rolnik } \\
\text { worek ma..." }\end{array}$ & $\begin{array}{l}\text { "The farmer has a sack on } \\
\text { his tractor" (Donkey singing } \\
\text { a song from communist } \\
\text { Poland.) }\end{array}$ & $\begin{array}{l}\text { Rawhide! Move'em on! } \\
\text { Head'em up! }\end{array}$ \\
\hline 15. $11: 31$ & Alu-felgi & Alu rim. & Hey, good-looking! \\
\hline
\end{tabular}


Table 1 (Continued)

No Time Polish dubbed version Literal meaning/Explanation

16. 12:10 Bryczka niczego sobie Not a bad ride (referring to a Nice day for a parade, car)

huh?

17. 13:53 Zielony pasztet

A green pâté (The word

Who on earth are they? "pâté" also stands for

"nuisance" or "ugly" in

Polish.)

18. $14: 10$ Spadajmy!

Let's scram! (Literally: “Let's Now let's go! fall down!")

19. 14:25 Damy w długą i cześć. Literally: We will give in to a We can make a run for it long one and bye. (A

popular idiomatic

expression in Polish

meaning to run away

quickly)

20. $14: 50$ Więc, ten...

Literally: So, this... (A Well, um...

popular interlude in Polish)

21. $15: 50$ mordy kochane

dear muzzles (A sarcastic, What's happening,

political reference - a way of everybody?

addressing each other by the

"elites".)

22. 16:01 nie róbmy draki, od tego są rumaki

A rhyme standing for: “Let's That's me: The noble not make a fuss, steeds do steed. that"

23. 16:13 Zupa pycha, psze pani Yummy soup, ma'm.

Great soup, Mrs. Q.

24. 17:13 ten typ tak ma this type is this way (from a For his type, yes. popular song by a Polish singer Ryszard Rynkowski [2003])

25. 17:33 Dla mnie bomba, siostro!

I think it's fantabulous,

Don't mind if I do, sister. Literally: "To me it's a Lillian.

bomb, sister!” (A colloquial way of expressing appreciation.)

26. 21:21 Idź się wypchaj do tapicera!

Go get stuffed by an

upholsterer!

We didn't like you,

27. 22:04 I tak już miałam się zbierać.

28. 22:18 Jedziemy, panie Zdzisiu

I was about to go anyway. I need to go, anyway.

Let's go, Mr Zdzisiu. (A diminutive from an oldfashioned male name.)

Let's go, Kyle.

And then I grabbed you.

Our first kiss.

29. 24:26 I wtedy cię capnąłem.

30 24:33 Życie to nie telenowela, mój drogi.

$$
\text { dear. }
$$

Oh, stop being such a

31. $24: 52$ rozchodzę to na drama king. balkonie I'll walk it off on the balcony. I'll just stretch it out (A colloquial saying used to here for a while. ignore some minor physical discomforts.) 
Table 1 (Continued)

\begin{tabular}{|c|c|c|c|}
\hline No Time & Polish dubbed version & $\begin{array}{l}\text { Literal meaning/Ex- } \\
\text { planation }\end{array}$ & Original English text \\
\hline 32. $26: 06$ & $\begin{array}{l}\text { kuchnia na wynos } \\
\text { sióstr Magdalenek }\end{array}$ & $\begin{array}{l}\text { take-away kitchen of } \\
\text { Magdalene sisters }\end{array}$ & Friar's Fat Boy! \\
\hline 33. $26: 16$ & zestaw z niespodzianką & $\begin{array}{l}\text { a set with a surprise (Popular } \\
\text { at restaurants offering fast } \\
\text { food.) }\end{array}$ & the Medieval Meal. \\
\hline 34. $28: 32$ & $\begin{array}{l}\text { Kolego, patrz mnie na } \\
\text { usta. Jest tylko jeden } \\
\text { szpeniol, co weźmie tę } \\
\text { chryję na warsztat }\end{array}$ & $\begin{array}{l}\text { Buddy, read my lips. There is } \\
\text { one bloke who might take } \\
\text { this mess on. (very } \\
\text { colloquial and regional) }\end{array}$ & $\begin{array}{l}\text { Hey, buddy, let me clue } \\
\text { you in. There's only one } \\
\text { fellow who can handle a } \\
\text { job like that }\end{array}$ \\
\hline 35. $28: 39$ & $\begin{array}{l}\text { Radia to on słucha } \\
\text { tylko w pogodę }\end{array}$ & $\begin{array}{l}\text { He only listens to the radio } \\
\text { when there's weather. (A } \\
\text { reference to the "Weather } \\
\text { Radio" ["Radio Pogoda"] } \\
\text { from Poland at which two } \\
\text { male journalists, who } \\
\text { dubbed the ugly sisters, used } \\
\text { to work - Wojciech Mann } \\
\text { and Krzysztof Materna.) }\end{array}$ & $\begin{array}{l}\text { He don't like to be } \\
\text { disturbed. }\end{array}$ \\
\hline 36. $30: 58$ & Śpiąca królewna & $\begin{array}{l}\text { Sleeping Princess. (A Polish } \\
\text { equivalent of this character) }\end{array}$ & Sleeping Beauty \\
\hline 37. $31: 25$ & Księżna Fiona z Bajki & $\begin{array}{l}\text { Princess Fiona from a } \\
\text { fairytale. (Analogically to } \\
\text { the expression "Prince from } \\
\text { a fairytale".) }\end{array}$ & Mrs. Fiona Charming. \\
\hline 38. $32: 56$ & $\begin{array}{l}\text { A tu masz krzak, co go } \\
\text { sadzili harcerze }\end{array}$ & $\begin{array}{l}\text { And here's the bush which } \\
\text { the scouts planted }\end{array}$ & $\begin{array}{l}\text { The bush shaped like } \\
\text { Shirley Bassey! }\end{array}$ \\
\hline 39. $33: 22$ & Teściu przytul mnie! & Father-in-law, hug me! & $\begin{array}{l}\text { Now let's go bond with } \\
\text { Daddy. }\end{array}$ \\
\hline 40. $34: 28$ & jam Puszek Okruszek & $\begin{array}{l}\text { I am Puss the Crumb (the } \\
\text { name for "Puss the Crumb" } \\
\text { rhyming in Polish comes } \\
\text { from a famous song } \\
\text { performed by Natalia } \\
\text { Kukulska [1986]) }\end{array}$ & $\begin{array}{l}\text { Now, ye ogre, pray for } \\
\text { mercy from... Puss... in } \\
\text { Boots! }\end{array}$ \\
\hline 41. $34: 34$ & umarł w butach & died with his boots on & Puss... in Boots! \\
\hline 42. $35: 36$ & na dzień dobry & $\begin{array}{l}\text { for starters (literally: for } \\
\text { good morning) - a popular } \\
\text { saying }\end{array}$ & $\begin{array}{l}\text { Almost everybody that } \\
\text { meets you wants to kill } \\
\text { you. }\end{array}$ \\
\hline 43. $37: 18$ & na dobre i na złe & $\begin{array}{l}\text { For good and bad. - A } \\
\text { reference to a popular Polish } \\
\text { tv soap opera of the same } \\
\text { title. }\end{array}$ & $\begin{array}{l}\text { Ain't no stoppin' us } \\
\text { now! }\end{array}$ \\
\hline 44. $37: 25$ & Mamy zniżki na basen & $\begin{array}{l}\text { we have discounts for the } \\
\text { pool }\end{array}$ & We've got jackets. \\
\hline 45. $38: 06$ & Nie dąsaj się! & Don't sulk! & Lighten up! \\
\hline
\end{tabular}


Table 1 (Continued)

\begin{tabular}{|c|c|c|c|}
\hline No Time & Polish dubbed version & $\begin{array}{l}\text { Literal meaning/Ex- } \\
\text { planation }\end{array}$ & Original English text \\
\hline 46. $39: 12$ & $\begin{array}{l}\text { Tuszę, że podroby dla } \\
\text { chartów, Wasza } \\
\text { Wysokość. }\end{array}$ & $\begin{array}{l}\text { I take that this is offal for the } \\
\text { dogs, Your Majesty. (styled } \\
\text { to be archaic) }\end{array}$ & $\begin{array}{l}\text { That would be the dog's } \\
\text { breakfast, Your Majesty. }\end{array}$ \\
\hline $47 \quad 40: 26$ & $\begin{array}{l}\text { Wygląda na obiekt } \\
\text { chroniony. }\end{array}$ & $\begin{array}{l}\text { It looks like a secure area. } \\
\text { (Comical use of a formal } \\
\text { term.) }\end{array}$ & $\begin{array}{l}\text { That's the old Keebler's } \\
\text { place. (referring to a } \\
\text { famous American } \\
\text { cookie and cracker } \\
\text { manufacturer - The } \\
\text { Keebler hardly or not } \\
\text { known in Poland) }\end{array}$ \\
\hline 48. $40: 45$ & Ale jajca! & $\begin{array}{l}\text { What a blast! (Literally: } \\
\text { "What kind of eggs!") }\end{array}$ & He makes me laugh. \\
\hline 49. $40: 58$ & Torcik Wedlowski & $\begin{array}{l}\text { the Wedel cake (a famous } \\
\text { Polish cake) }\end{array}$ & $\begin{array}{l}\text { Coffee and a Monte } \\
\text { Cristo. (a popular cake } \\
\text { in the U.S.) }\end{array}$ \\
\hline 50. $41: 07$ & Jesteśmy z Sanepidu. & $\begin{array}{l}\text { We're with the sanitary } \\
\text { services. }\end{array}$ & We're from the union. \\
\hline 51. $41: 23$ & $\begin{array}{l}\text { W męskiej skończył się } \\
\text { papier. }\end{array}$ & $\begin{array}{l}\text { the toilet paper has run out } \\
\text { in the men's room. }\end{array}$ & $\begin{array}{l}\text { We don't even have } \\
\text { dental. }\end{array}$ \\
\hline 52. $41: 33$ & Rozumiemy się? & $\begin{array}{l}\text { Do we understand each } \\
\text { other? (A reference to a } \\
\text { citation from a classic Polish } \\
\text { tv comedy series } \\
\text { "Alterntaywy 4" [1983].) }\end{array}$ & $\begin{array}{l}\text { Know what I'm saying? } \\
\text { Huh? }\end{array}$ \\
\hline 53. $43: 10$ & Sierotka Marysia & $\begin{array}{l}\text { Mary the Orphan. (Refers to } \\
\text { a popular Polish fairytale.) }\end{array}$ & The Golden Bird \\
\hline 54. $43: 57$ & hala jak talala, co stary? & the hall is grand, right pal? & TGIF, eh, buddy? \\
\hline 55. $44: 00$ & $\begin{array}{l}\text { Byle do fajrantu i na } \\
\text { chatę, co nie? }\end{array}$ & $\begin{array}{l}\text { To the end of the shift and } \\
\text { off home, right? [A popular } \\
\text { saying. This fragment refers } \\
\text { actually to the original } \\
\text { phrase in } 54 \text {. above and is to } \\
\text { some extent its equivalent in } \\
\text { Polish. However, for some } \\
\text { reasons, the translator } \\
\text { decided to shift it to this } \\
\text { place.] }\end{array}$ & $\begin{array}{l}\text { Working hard or hardly } \\
\text { working, eh, Mac? }\end{array}$ \\
\hline 56. $44: 34$ & $\begin{array}{l}\text { jak się trzeba będzie } \\
\text { komuś podlizać }\end{array}$ & $\begin{array}{l}\text { if we need to suck up to } \\
\text { somebody }\end{array}$ & $\begin{array}{l}\text { If we need an expert on } \\
\text { licking ourselves. }\end{array}$ \\
\hline 57. $44: 38$ & Ten pomysł jest do bani & $\begin{array}{l}\text { This idea sucks. (A } \\
\text { colloquial saying.) }\end{array}$ & This is a bad idea. \\
\hline 58. $44: 40$ & Filuj na ochronę! & Look out for the guards! & Go keep watch! \\
\hline 59. $44: 48$ & $\begin{array}{l}\text { Jak mawiał kierownik } \\
\text { schroniska - pierwsze } \\
\text { koty za płoty. }\end{array}$ & $\begin{array}{l}\text { As the shelter director used } \\
\text { to say - the first pancake is } \\
\text { always spoiled (literally: "the } \\
\text { first cats out the fences") }\end{array}$ & $\begin{array}{l}\text { I was the great cat } \\
\text { burglar of Santiago de } \\
\text { Compostela. }\end{array}$ \\
\hline
\end{tabular}


Table 1 (Continued)

\begin{tabular}{|c|c|c|c|}
\hline No Time & Polish dubbed version & $\begin{array}{l}\text { Literal meaning/Ex- } \\
\text { planation }\end{array}$ & Original English text \\
\hline 60. $45: 02$ & wleźć na meblościankę & $\begin{array}{l}\text { get on the furniture (The } \\
\text { term "meblościanka" - } \\
\text { literally "furniture-wall" - } \\
\text { refers to a typical piece of } \\
\text { furniture characteristic } \\
\text { especially for the } \\
\text { communist time in Poland, } \\
\text { consisting of a set of } \\
\text { bookshelves and a } \\
\text { wardrobe.) }\end{array}$ & I'll be giggling to myself. \\
\hline 61. $45: 13$ & Walerianka. & $\begin{array}{l}\text { Valerian drops. (A reference } \\
\text { to a popular medicine made } \\
\text { of herbs pacifying nervous } \\
\text { diseases.) }\end{array}$ & Hex Lax? \\
\hline 62. $45: 21$ & Żyj długo i szczęśliwie! & Live long and hapilly! & Happily Ever After. \\
\hline 63. $45: 27$ & Jak ten młody bóg & Literally: like a young god & Beauty Divine. \\
\hline 64. $47: 46$ & Nie spoć się skarbie! & $\begin{array}{l}\text { Don't get sweaty, } \\
\text { sweetheart! (A reference to a } \\
\text { citation from a classic Polish } \\
\text { comedy film Kingsajz } \\
\text { [1987].) }\end{array}$ & Oh, put it away, Junior! \\
\hline 65. $48: 28$ & $\begin{array}{l}\text { Środek ścisłego } \\
\text { zarachowania }\end{array}$ & Classified stock. & Jug o’voodoo. \\
\hline 66. $48: 39$ & $\begin{array}{l}\text { W tym mogą być } \\
\text { promile! }\end{array}$ & $\begin{array}{l}\text { This could have per mils } \\
\text { [usually of alcohol] in it! (A } \\
\text { reference to a classic Polish } \\
\text { film Seksmisja [Eng.: } \\
\text { Sexmission [1984].) }\end{array}$ & You'll have a reaction. \\
\hline 67. $51: 21$ & $\begin{array}{l}\text { W czasie deszczu osły } \\
\text { się nudzą, panie proszą } \\
\text { panów (piosenka } \\
\text { śpiewana przez osła) }\end{array}$ & $\begin{array}{l}\text { When it's raining, donkeys } \\
\text { are bored. (A reference to a } \\
\text { classic Polish children's } \\
\text { song "W czasie deszczu } \\
\text { dzieci się nudzą" [1962] } \\
\text { performed by Barbara } \\
\text { Krafftówna - Eng.: "When } \\
\text { it's raining children are } \\
\text { bored".) }\end{array}$ & $\begin{array}{l}\text { The sun'll come out... } \\
\text { Tomorrow. }\end{array}$ \\
\hline 68. $53: 44$ & Jesteś ze Stanów? & Are you from the States? & Are you from Europe? \\
\hline 69. $53: 59$ & Odgłos paszczy. & $\begin{array}{l}\text { A mouth sound (A reference } \\
\text { to a citation from a classic } \\
\text { Polish comedy film Rejs } \\
\text { [1970], Eng.: The Cruise.) }\end{array}$ & I can whinny. \\
\hline 70. $54: 20$ & $\begin{array}{l}\text { Przeczytaj ulotkę lub } \\
\text { skonsultuj się z } \\
\text { lekarzem lub } \\
\text { farmaceutą }\end{array}$ & $\begin{array}{l}\text { Read the leaflet or consult } \\
\text { your doctor or a pharmacist } \\
\text { (A typical drug label term.) }\end{array}$ & $\begin{array}{l}\text { Not intended for heart } \\
\text { patients or those with... } \\
\text { nervous disorders. }\end{array}$ \\
\hline
\end{tabular}


Table 1 (Continued)

\begin{tabular}{|c|c|c|c|}
\hline No Time & Polish dubbed version & $\begin{array}{l}\text { Literal meaning/Ex- } \\
\text { planation }\end{array}$ & Original English text \\
\hline 71. $56: 10$ & $\begin{array}{l}\text { Dziękuję } \\
\text { waszmościowie. } \\
\text { Pewnego dnia się } \\
\text { odwdzięczę. }\end{array}$ & $\begin{array}{l}\text { "Thank you, masters [styled } \\
\text { to be archaic]. One day, I will } \\
\text { repay you." A sentence } \\
\text { almost identical to the one } \\
\text { said by Bohdan Chmielnicki } \\
\text { to Jan Skrzetuski in a film } \\
\text { adaptation (by Jerzy } \\
\text { Hoffman) of a classic novel } \\
\text { With fire and sword (Pl: } \\
\text { Ogniem i mieczem) by } \\
\text { Henryk Sienkiewicz. }\end{array}$ & $\begin{array}{l}\text { Thank you, gentlemen! } \\
\text { Someday, I will repay } \\
\text { you. }\end{array}$ \\
\hline 72. $57: 43$ & Cześć, mogę cię zjeść? & $\begin{array}{l}\mathrm{Hi} \text {, may I eat you? (Rhyming } \\
\text { in Polish.) }\end{array}$ & Hello, handsome. \\
\hline 73. $58: 08$ & $\begin{array}{l}\text { Dla ciebie maleńka } \\
\text { choćby i "Pedigree" }\end{array}$ & $\begin{array}{l}\text { For you, baby, I could be } \\
\text { even "Pedigree". (A } \\
\text { reference to a popular pet } \\
\text { food.) }\end{array}$ & $\begin{array}{l}\text { - Shrek? - For you, } \\
\text { baby... I could be. }\end{array}$ \\
\hline 74. $60: 53$ & $\begin{array}{l}\text { Zostaw całą flaszkę, } \\
\text { Wojtuś. }\end{array}$ & $\begin{array}{l}\text { Leave the whole bottle, } \\
\text { Wojtus. (A diminutive from } \\
\text { the actual name of the } \\
\text { person who dubbed the } \\
\text { character - Wojciech Mann, } \\
\text { a famous Polish journalist } \\
\text { and a TV and radio } \\
\text { presenter.) }\end{array}$ & $\begin{array}{l}\text { Just leave the bottle, } \\
\text { Doris. }\end{array}$ \\
\hline 75. $60: 55$ & $\begin{array}{l}\text { Co, zrobili was } \mathrm{w} \\
\text { konia? }\end{array}$ & $\begin{array}{l}\text { You've been hoaxed, huh? } \\
\text { Literally: They've made a } \\
\text { horse out of you [The } \\
\text { Donkey's new incarnation } \\
\text { after taking a magical potion } \\
\text { is a horse]. }\end{array}$ & Hey. Why the long face? \\
\hline 76. $61: 08$ & $\begin{array}{l}\text { nienawidzę } \\
\text { poniedziałków }\end{array}$ & $\begin{array}{l}\text { A possible reference to a title } \\
\text { of a classic Polish comedy } \\
\text { "Nie lubie poniedziałku" } \\
\text { (Eng.: "I don't like } \\
\text { Monday"[1971].) }\end{array}$ & \\
\hline 77. $63: 27$ & $\begin{array}{l}\text { Czy jest suchy chleb dla } \\
\text { konia? }\end{array}$ & $\begin{array}{l}\text { Is there some dry bread for } \\
\text { the horse? (A reference to a } \\
\text { citation by the actor Jarema } \\
\text { Stpowski from a classic } \\
\text { Polish TV series "Wojna } \\
\text { domowa" [1965-1966], Eng.: } \\
\text { A house war. The term in } \\
\text { Polish is ambiguous as it } \\
\text { also means "A civil war".) }\end{array}$ & $\begin{array}{l}\text { Um... Mary! A talking } \\
\text { horse! }\end{array}$ \\
\hline 78. $66: 50$ & odbiła szajba & $\begin{array}{l}\text { A Polish colloquial saying } \\
\text { meaning "to go nuts". }\end{array}$ & $\begin{array}{l}\text { He's completely lost his } \\
\text { mind! }\end{array}$ \\
\hline
\end{tabular}


Table 1 (Continued)

\begin{tabular}{|c|c|c|c|}
\hline No Time & Polish dubbed version & $\begin{array}{l}\text { Literal meaning/Ex- } \\
\text { planation }\end{array}$ & Original English text \\
\hline 79. $67: 11$ & $\begin{array}{l}\text { To moja, sypana. Po } \\
\text { torebkach mam zgagę. }\end{array}$ & $\begin{array}{l}\text { This is my tea, leaf. I get } \\
\text { heartburn after teabags. }\end{array}$ & $\begin{array}{l}\text { That's mine. Decaf. } \\
\text { Otherwise I'm up all } \\
\text { night. }\end{array}$ \\
\hline 80. $69: 36$ & $\begin{array}{l}\text { Masz jeszcze kontakt ze } \\
\text { Żwirkiem, bo } \\
\text { Muchomorek mi się } \\
\text { skarży? }\end{array}$ & $\begin{array}{l}\text { Are you still in touch with } \\
\text { Żwirek, because } \\
\text { Muchomorek is } \\
\text { complaining to me? (Żwirek } \\
\text { and Muchomorek are } \\
\text { famous characters from a } \\
\text { Czechoslovakian classic TV } \\
\text { cartoon Pohádky z mechu a } \\
\text { kapradí [Eng.: The Tales of } \\
\text { Moss and Fern, 1968] } \\
\text { popular in Poland.) }\end{array}$ & $\begin{array}{l}\text { Do you still know the } \\
\text { Muffin Man? Because } \\
\text { we're gonna need flour. } \\
\text { (A popular cartoon } \\
\text { character) }\end{array}$ \\
\hline 81. $69: 50$ & $\begin{array}{l}\text { Pozdrowienia od } \\
\text { Muchomorka, } \\
\text { młynarzu! }\end{array}$ & $\begin{array}{l}\text { Regards from Muchomorek, } \\
\text { miller. (A reference to the } \\
\text { above Czechoslovakian } \\
\text { cartoon [1968] popular in } \\
\text { Poland.) }\end{array}$ & $\begin{array}{l}\text { Fire up the ovens, } \\
\text { Muffin Man! }\end{array}$ \\
\hline 82. $71: 47$ & $\begin{array}{l}\text { Czemu wcięło chłopów } \\
\text { stąd... }\end{array}$ & $\begin{array}{l}\text { A reference to a popular pop } \\
\text { song performed by Danuta } \\
\text { Rinn (“Gdzie ci mężczyźni?" } \\
\text { [1974] - "Where have all } \\
\text { those men gone?") }\end{array}$ & $\begin{array}{l}\text { Where have all the good } \\
\text { men gone. }\end{array}$ \\
\hline 83. $72: 15$ & Czadu! & $\begin{array}{l}\text { A popular saying } \\
\text { encouraging to act } \\
\text { spontaneously and wildly. }\end{array}$ & Hit it! \\
\hline 84. $74: 32$ & Pij mleko! & $\begin{array}{l}\text { Drink milk. (a reference to a } \\
\text { Polish social campaign) }\end{array}$ & Be good. \\
\hline 85. $75: 44$ & podrzućcie jej świnię & $\begin{array}{l}\text { play a trick on her (literally: } \\
\text { "throw a pig to her") }\end{array}$ & Pigs und blanket! \\
\hline 86. $76: 26$ & $\begin{array}{l}\text { Spółka z o.o. czyli kot i } \\
\text { osioł! }\end{array}$ & $\begin{array}{l}\text { Donkey Ltd., alias, puss and } \\
\text { donkey }\end{array}$ & $\begin{array}{l}\text { - Pray for mercy, from } \\
\text { Puss... - And Donkey! }\end{array}$ \\
\hline 87. $77: 53$ & beknął & $\begin{array}{l}\text { He burped. (Ambiguous - in } \\
\text { Polish the expression also } \\
\text { refers to an idiom meaning } \\
\text { in English "to kick the } \\
\text { bucket".) }\end{array}$ & He croaked. \\
\hline 88. $78: 23$ & $\begin{array}{l}\text { Czy ujdzie mi płazem } \\
{[\ldots] ?}\end{array}$ & $\begin{array}{l}\text { Will I get away with it } \\
\text { (literally: "Will I get the } \\
\text { amphibious side of it" - } \\
\text { "amphibious side" in Polish } \\
\text { is identical to "the flat side of } \\
\text { a sword") }\end{array}$ & $\begin{array}{l}\text { Will you accept an old } \\
\text { frog's apologies... }\end{array}$ \\
\hline
\end{tabular}


Table 1 (Continued)

\begin{tabular}{|c|c|c|c|}
\hline No Time & Polish dubbed version & $\begin{array}{l}\text { Literal meaning/Ex- } \\
\text { planation }\end{array}$ & Original English text \\
\hline 89. $79: 29$ & $\begin{array}{l}\text { Boże w niebiesiech, nie } \\
\text { będę tu płakać, } \\
\text { przecież nie będę tu } \\
\text { płakać. }\end{array}$ & $\begin{array}{l}\text { Dear God in heaven, I will } \\
\text { not cry, I say I will not cry } \\
\text { here. (A reference to a } \\
\text { citation from a classic Polish } \\
\text { novel Potop by Henryk } \\
\text { Sienkiewicz [Eng.: The } \\
\text { Deluge].) }\end{array}$ & $\begin{array}{l}\text { Whatever happens, I } \\
\text { must not cry! You } \\
\text { cannot make me cry! }\end{array}$ \\
\hline 90. $84: 18$ & $\begin{array}{l}\text { Amigo, idę z kociakami } \\
\text { na Whiskas. }\end{array}$ & $\begin{array}{l}\text { Amigo, I'm going with the } \\
\text { kitties for Whiskas. } \\
\text { (Probably the most popular } \\
\text { cat food in Poland.) }\end{array}$ & $\begin{array}{l}\text { Amigo, we are off to the } \\
\text { Kit-Kat Club. } \\
\text { (The Kit-Cat Club } \\
\text { [sometimes Kit-Kat } \\
\text { Club] was an early 18th- } \\
\text { century English club in } \\
\text { London with strong } \\
\text { political and literary as- } \\
\text { sociations. Also KitKat- } \\
\text { Club - a nightclub in } \\
\text { Berlin.) }\end{array}$ \\
\hline
\end{tabular}

\section{Conclusions and further research perspectives}

It seems that the dominant strategy used in the process of translation of the film analysed above in the area of wordplay was Delabastita's (1989) seventh type ("Zero $\rightarrow$ pun: Entirely new textual material is added containing a wordplay that has no apparent reason in the source text except as a compensatory device"). So, it means that the translator enriched some fragments and transformed them into puns although the original text did not contain any. The following fragments have been classified into this category: $7,11,13,21,22,26,41,72,75,85$. Only in the case of fragment 55, the translator applied the strategy of the sixth type "Nonpun $\rightarrow$ pun: The translator adds a pun in textual positions where the original text has no wordplay in order to compensate for a pun lost elsewhere, or for any other reason", which means that the translator compensated a missing translation of a pun in one place by putting it in another place.

When it comes to the translation of culture-related content, the dominant strategy, according to Pedersen's classification (2005), was 'substitution'. However, occasionally 'finding an official equivalent' and 'generalisation' appear as well. Below there are presented the total numbers of fragments identified in each of the categories:

- 'Finding an official equivalent' fragments: 1, 10, 20, 24, 27, 36, 37, 62, 63, 76.

- 'Generalisation' fragments: 44, 47, 90. 
- 'Substitution' fragments: 2-9, 11-19, 21-23, 25-26, 28-35, 38-43, 45-46, 48-61, 64-75, 77-89.

The crucial thing is that a large number of all the 90 fragments selected for the analysis from the film are indeed culture-related only in the Polish dubbed version while in the original version they were not meant to be such. Therefore, in the case of many translated fragments classified as substitution, the original ones did not contain any jocular elements. Moreover, the puns detected in them are present also only in the Polish dubbing, so the translator applied Delabastita's (1989) seventh type of strategy (Zero $\rightarrow$ pun). Some of the translations (like, for example, number 76) may only seem to be culture-related and might have been misinterpreted in the analysis, but it cannot be excluded that they were still not accidental in the associations they raised, and that the translator selected deliberately these particular connotations. Perhaps, the only person that could answer all these questions is the translator himself.

The outcome of the research presented above raises further questions which may lead to a continuation of the study into the strategies used in the translation of computer animated films concerning culture-related elements and wordplay. The author does not exclude analysing the other episodes of the Shrek saga to broaden the corpus of data and ultimately construct a didactic game serving mastering the skills of intercultural competence (Andrzejewska-Kwiatkowska \& Stöckmann 2009; Barłóg 2010; Stöckmann \& Zator 2010) in translation studies among students of neophilological majors.

\section{References}

Adamowicz-Grzyb, Grażyna (2013). Tłumaczenia filmowe w praktyce. Warszawa: Fortima - Tłumaczenia - Edukacja - Media.

Andrzejewska-Kwiatkowska, Joanna/Stöckmann, Britta (2009). Czy jesteś kompetentny interkulturowo? Projekt gry sytuacyjnej. Homo Ludens, 1(1), 25-32.

Barłóg, Cecylia (2010). Konceptualizacja gier w kontekście treningu interkulturowego. Homo Ludens. 1(2), 9-18.

Bremmer, Jan/Roodenburg, Herman (1997). A cultural history of humour from antiquity to the present day. Cambridge and Oxford: Polity Press and Blackwell Publishing.

Chaume, Frederic (2012). Audiovisual translation: Dubbing. Manchester: St. Jerome.

Delabastita, Dirk (1989). Translation and mass-communication: Film and TV translation as evidence of cultural dynamics. Babel, 35(4), 193-218.

Dore, Margherita (2008). The audiovisual translation of humour: Dubbing the first series of the TV comedy programme Friends into Italian. $\mathrm{PhD}$ Thesis defended at The Lancaster University. https://www.academia.edu/16946977/MD_PHD_THESIS_The_Audiovisu 
al_Translation_of_Humour_Dubbing_the_First_Series_of_the_TV_Comedy_Pro gramme_Friends_into_Italian [access: 12.02.2019].

Lew, Robert (2000). Dowcip językowy w świetle najnowszych językoznawczych teorii humoru. In: Stanisław Gajda/Dorota Brzozowska (eds.), Świat humoru (pp. 127-135). Opole: Uniwersytet Opolski.

Pedersen, Jan (2005). How is culture rendered in subtitles. In: Heidrun Gerzymisch-Arbogast/Sandra Nauert (eds.), Conference proceedings Challenges of multidimensional translation. Advanced translation research center (ATRC) (pp. 113-130). Saarbrücken: Saarland University. http://www.translationconcepts.org/pdf/MuTra_2005_Proceed ings.pdf [access date: 23.05.2019].

Pisarska, Alicja/Tomaszkiewicz, Teresa (1996). Wspótczesne tendencje przekładoznawcze: Podręcznik dla studentów neofilologii. Poznań: Wydawnictwo Naukowe Uniwersytetu im. Adama Mickiewicza.

Rębkowska, Agata (2016). Humor w badaniach nad przekładem. Romanica Wratislaviensia LXIII, 157-174.

Sajna, Mateusz (2013). Translation of video games and films - a comparative analysis of selected technical problems. Homo Ludens, 1(5)/2013, 219-232.

Sikora, Iwona (2013). Dubbing filmów animowanych. Nysa: Oficyna Wydawnicza PWSZ. Stöckmann, Britta/Zator, Joanna (2010). Jak widzę siebie? Jak widzą mnie inni? Eliminowanie uprzedzeń i stereotypów narodowych za pomocą gier stosowanych podczas nauczania języka obcego. Homo Ludens, 1(2), 175-190.

Surdyk, Augustyn/Urban, Anna (2016). Mistranslations of film titles: Between fidelity and advertising. Glottodidactica. An International Journal of Applied Linguistics, 43 (1). 153-169.

Tomaszkiewicz, Teresa (2006). Przekład audiowizualny. Warszawa: PWN.

Zabalbeascoa, Patrick (1996). Translating jokes for dubbed television situation comedies. The Translator, 2(2), 235-257. 
Open-Access-Publikation im Sinne der CC-Lizenz BY-NC-ND 4.0

(C) 2020, Vandenhoeck \& Ruprecht GmbH \& Co. KG, Göttingen ISBN Print: 9783847111863 - ISBN E-Lib: 9783737011860 


\section{Teil 7: Linguistic landscapes}


Open-Access-Publikation im Sinne der CC-Lizenz BY-NC-ND 4.0

(C) 2020, Vandenhoeck \& Ruprecht GmbH \& Co. KG, Göttingen ISBN Print: 9783847111863 - ISBN E-Lib: 9783737011860 


\title{
Neue Herausforderungen für Angewandte Linguistik - Sprachlandschaften? Visuelle Mehrsprachigkeit in den Linguistic Landscapes der deutsch-polnischen Grenzregion
}

\begin{abstract}
New challenges for applied linguistics - Linguistic landscapes? Visual multilingualism in the linguistic landscapes of the German-Polish borderlines

This chapter focuses on a sub-field of Applied linguistics which has been intensively discussed since the 2000s - namely Linguistic landscapes. The phenomenon of the visual manifestation of language(s) can be viewed from various aspects, i. e. foreign language teaching, sociolinguistics, conversation analysis. All these aspects belong to the fixed repertoire of the research fields of Applied linguistics. In the present text, the sociolinguistic perspective is taken above all in order to grasp the language in the public border area. The central question here is in which contexts the foreign language (predominantly German) signs occur, i.e. to which types of discourse the recorded language occurrence belongs. The analysis shows that the linguistic landscapes of the Polish border cities do not differ at all in terms of the types of discourse. In none of the cities, a manifestation of transgressive signs in German could be recorded. In both Słubice and Świnoujście, linguistic signs appear in infrastructural and commercial discourse. In the commercial discourse, similarities were found, especially with regard to the occurrence of multilingual signs in the field of health care services.
\end{abstract}

Keywords

German-Polish borderland, linguistic landscapes, visual multilingualism

\section{Einleitung}

Städte sind ein Ort diverser sozioökonomischer, politischer und nicht zuletzt linguistischer Prozesse. Städte beinhalten Sprache, sowohl die geschriebene als auch die gesprochene. Die Sprachlandschaften als Phänomen betreffen schriftfixierte Sprache, also Texte. Texte können wiederum separat vorkommen, sie ergeben aber in ihrer Gesamtheit in einer Stadt konkrete Kontexte, die als Diskurstypen betrachtet werden können.

Linguistisch interessant sind sicherlich Großstädte, wo Veränderungen im Sprechhandeln sowie in der Sprachlandschaft multifaktoriell abhängig sind und 
intensiver als in den Kleinstädten oder auf dem Lande verlaufen. In diesem Beitrag wird das Sprachvorkommen in der deutsch-polnischen Grenzregion fokussiert. Ähnlich wie in der Großstadt treffen an der Grenze aufeinander Menschen mit ihren diversen sprachlichen und kulturellen Prägungen. Jańczak (vgl. 2018b: 191) hebt in ihren Analysen des deutsch-polnischen Grenzraumes hervor, dass in der Grenzregion vermehrt zu Sprachkontakt kommt. Dies resultiert auch in visueller Mehrsprachigkeit.

Ziel meiner Untersuchung ist die Ähnlichkeiten und Unterschiede zwischen dem erfassten Sprachvorkommen (schriftbasierte Kommunikation) in verschiedenen polnischen Grenzstädten zu analysieren und dieses den möglichen Diskurstypen zuzuordnen. Bevor in diesem Beitrag näher auf die Forschungsfragen eingegangen wird, soll das Phänomen der Sprachlandschaft (Linguistic Landscape - LL) als Forschungsobjekt der Angewandten Linguistik verortet werden. Darauf folgt eine kurze Vorstellung der Städte, deren LL analysiert werden soll, um zu dem Forschungsdesign sowie den Ergebnissen der Untersuchung zu gelangen. Diese werden kommentiert und mit einem Ausblick versehen.

\section{Linguistic Landscapes als neue Tendenz in der Angewandten Linguistik?}

Sprachlandschaften, vor allem bekannt als Linguistic Landscapes (LL), sind als Phänomen nicht gänzlich eine neue Erscheinung in der Linguistik, auch nicht in der Angewandten Linguistik. Bevor Ansätze und Tendenzen um die Linguistic Landscapes herum skizziert werden, beschäftige ich mich kurz mit der Angewandten Linguistik, um im Rahmen dieser die Untersuchungen zu den Sprachlandschaften zu verorten.

In diesem Abschnitt konzentriere ich mich einerseits auf die Angewandte Linguistik im Allgemeinen und andererseits fokussiere ich bewusst die in Polen etablierten Schwerpunkte dieser Disziplin. Die Angewandte Linguistik ist in den 1960er Jahren verwurzelt. Harden (2006: 24) hat aber nicht Unrecht, wenn er eine viel längere zeitliche Perspektive zeichnet: „Angewandte Linguistik ist zumindest so alt wie die ersten Versuche, Sprache schriftlich oder in irgendeiner anderen Form zu fixieren, vielleicht auch schon deutlich älter“. Dieser Aspekt ist auch sichtbar, wenn die Forschungsfelder der Angewandten Linguistik (z.B. Diskursanalyse, Rhetorik, Soziolinguistik, Übersetzungswissenschaften, Sprachpolitik, Zweitsprachenerwerb) in Erscheinung treten. Damit wird klar, dass es sich um viel mehr als um second language acquisition research - wie die Disziplin im angloamerikanischen Raum verstanden wird - handelt. Angewandte Linguistik erscheint hier als Mittler und Bindeglied zwischen der traditionellen Sprach- 
wissenschaft mit Sprachtheorien und praktischer Erforschung alltäglicher Sprachphänomene. Sie kann als Antwort der 1960er Jahre auf die „weit verbreitete Frustration hinsichtlich der sich immer klarer abzeichnenden Praxisferne linguistischer Forschung " (Harden 2006: 24) verstanden werden. Die Praxis und Lösung von sprachbezogenen Problemen rückte immer mehr in den Mittelpunkt. Die Ausweitung des wissenschaftlichen Fokus „vom Problemverstehen auf Problemlösen" (vgl. Knapp 2006: XXI) war prägend für die Angewandte Linguistik, die sich „mit der Beschreibung, Erklärung und Lösung von lebensund gesellschaftspraktischen Problemen in den Bereichen von Sprache und Kommunikation befasst" (Knapp 2006: XXII). Szerszeń (vgl. 2010: 262) oder Grucza (vgl. 2017: 7) als Vertretende ${ }^{1}$ polnischer Angewandten Linguistik erklären, dass im Fokus der Forschungsinteressen dieser Disziplin immer der Mensch mit seinen Äußerungen in mündlicher und/oder schriftlicher Form steht. Die Angewandte Linguistik in Polen beschäftigt sich der Fachliteratur (vgl. Szerszeń 2010: 262) zufolge mit den Spezifika der Kommunikation, die ein konkreter Mensch oder Gruppe von Menschen betreibt. In der Anfangsphase widmete sie sich neben den unterschiedlichen Fragen zum Polnischen vor allem der Fremdsprachenmethodik und -didaktik. Mit der Zeit kamen auch Forschungsfragen übersetzungswissenschaftlichen Charakters dazu. Wichtig sind ebenso polnische Erkenntnisse von Angewandter Linguistik, von denen Grucza (vgl. 2017: 209ff.) berichtet und diese zusammenfassend als Beiträge zur kontrastiver Linguistik, angewandter Phonetik, Lexikographie, Fachsprachenerforschung oder Logopädie zu nennen sind.

Die Forschungsinteressen der polnischen Angewandten Linguistik unterscheiden sich nicht wirklich von den deutschsprachigen Schwerpunkten der Disziplin. Dazu gehören ebenso u. a. Werbekommunikation, technische Kommunikation, Hypertext und Texttechnologie, interkulturelle Kommunikation, klinische und forensische Linguistik, Mehrsprachigkeit, Fremdsprachenunterricht, Fachsprachenkommunikation, Herkunftssprachenunterricht und nicht zuletzt Linguistic Landscapes. Harden (2006: 26) zählt beispielsweise die folgenden Forschungsfelder der Angewandten Linguistik auf:

- Sprache und Gesellschaft,

- Sprache und Individuum,

- Sprache und Sprache,

1 An dieser Stelle möchte ich die wissenschaftlichen Verdienste von Frau Prof. Dr. habil. Izabela Prokop um die Entwicklung der Angewandten Linguistik an der Universität Poznań und in Polen besonders würdigen. Ihre Arbeiten, die u. a. gesprochene Sprache (vgl. 1995, 2010), Fachkommunikation (vgl. 1989) sowie Stereotypenforschung (vgl. Prokop 1995) betreffen, waren für Generationen polnischer Linguisten und Linguistinnen prägend. Anlässlich des Geburtstags von Frau Prof. Prokop möchte ich der Jubilarin herzlichst gratulieren und mich für die wissenschaftlich erquickenden Gespräche bedanken. 
- Sprachvermittlung und Spracherwerb sowie

- Sprachverwendung und Sprachgebrauch ${ }^{2}$.

Beim näheren Betrachten der Phänomene, die unter diese Kategorien fallen, wird es klar, dass Linguistic Landscapes (Sprachlandschaften) nicht nur über den individuellen Gebrauch einer Sprache informieren, sondern vor allem die gesellschaftliche Wirkung und Wahrnehmung von Sprachgebrauch reflektieren. Darüber hinaus können sie aus der fremdsprachendidaktischen Perspektive betrachtet werden sowie als Teil einer Diskursforschung fungieren. Sie stehen also Fest im Spannungsfeld der Forschungsinteressen Angewandter Linguistik, was auch Ben-Rafael et al. (vgl. 2010) vermerken. Linguistic Landscapes stellen des Weiteren eine zeitgemäße Erweiterung korpusbasierter Untersuchungen zu Sprachpolitik, visueller Mehrsprachigkeit oder Spracheinstellungen dar, die ebenso im Rahmen Angewandter Linguistik erforscht werden.

Nach der Verortung der Linguistic Landscapes im Rahmen der Angewandten Linguistik sollten im nächsten Schritt die Sprachlandschaften selbst und ihre Analyseverfahren näher charakterisiert werden. An dieser Stelle ist der Beitrag von Landry und Bourhis (1997) zu würdigen, in dem das Phänomen neu umrahmt wird. Die Forscher haben das einzelne Schriftvorkommen (in Form von Texten oder Textbausteinen) global als Landschaft gefasst und zum eigenständigen Forschungsthema erhoben. Landy und Bourhis (1997: 25) charakterisieren Linguistic Landscapes als:

The language of public road signs, advertising billboards, street names, place names, comercial shop signs, and public signs on goverment buildings combines to form the linguistic lndscapes of a given territory, region, or urban agglomeration."

Untersucht wird also das Schriftvorkommen (die Sprache), häufig in Form visueller Mehrsprachigkeit, die „sich auf Hinweis-, Informations- und Geschäftsschildern, Plakaten, Aufklebern sowie Graffitis [zeigt] (Mühlan-Meyer 2018: 260).

Jede Sprachlandschaft kann eine oder mehrere Funktionen haben. In der Fachliteratur (vgl. Landy \& Bourhis 1997: 25) werden zwei Hauptfunktionen aufgezählt: informative (Informationen zu Nutzern und Bewohnern einer Ge-

2 Die präsentierte Liste soll nicht als abschließende Aufzählung von Forschungsfeldern der Angewandten Linguistik verstanden werden. Nach de Cillia (vgl. 2010: 10) fungiert Angewandte Linguistik als Dachbegriff für interdisziplinäre Zugänge in der Sprachwissenschaft wie Psycholinguistik, Soziolinguistik, Ethnolinguistik, Korpuslinguistik oder Forensische Linguistik. Die von de Cillia (vgl. 2010: 10) darüber hinaus aufgelisteten Teilbereiche wie Sprachvermittlung, mündliche und schriftliche Kommunikation, mediale, öffentliche und mehrsprachige Kommunikation, Diagnostizieren und Therapieren gehören zum Forschungsfeld der Angewandten Linguistik. 
gend) und symbolische (An- oder Abwesenheit einer Sprechergruppe) ${ }^{3}$. Diese Funktionen greifen vor allem die sprachliche Superdiversity der Großstädte auf, weil sie Aussagen zu der (vorhandenen oder nicht vorhandenen) Manifestation von Minderheitensprachen oder zu historischen Prozessen treffen, die sich in der öffentlich sichtbaren Sprache widerspiegeln.

Die schriftfixierten Äußerungen im öffentlichen Raum (Linguistic Landscapes) sind heutzutage ebenso und vor allem unter den Aspekten des Konsumtourismus oder der Arbeitsmigration zu betrachten und können auch so im Zusammenhang mit diesen Erscheinungen interpretiert werden. So lässt sich beispielsweise die Beschilderung der Restaurants, Bars und Kneipen an der Ostsee in den polnischen Kurorten kontextgebunden erklären: Linguistic Landscapes haben hier eine persuasive Funktion, was anhand von mehrsprachigen Aufstellspeisekarten (vgl. Lisek 2018: 223) festgehalten wurde.

Mittelbar abhängig von der Funktion eines Zeichens im Linguistic Landscape wird das Sprachvorkommen in inoffizielles oder privates (Bottom-Up-Perspektive) sowie offizielles oder staatliches (Top-Down-Perspektive) gegliedert. Zu der ersten Kategorie gehören z.B. Werbeschilder, Veranstaltungsplakate, aber auch wiederrechtlich platzierte Graffiti oder Aufkleber. Die zweite Kategorie repräsentieren Straßenschilder, Gedenktafeln oder Hinweisschilder.

Aus der Menge von unterschiedlichen Funktionen und Arten der Zeichen im schriftlich fixierten Sprachvorkommen lassen sich verschiedene Kontexte ableiten. Diese ergeben wiederum diverse Diskurstypen, die zugrunde der zentralen Forschungsfrage dieses Beitrages liegen.

\section{Städte an der Grenze}

Bevor näher auf das Forschungsdesign und die dazu gehörigen Forschungsfragen eingegangen wird, soll ein kurzer Blick auf die ausgewählten Städte - Świnoujscie und Słubice erfolgen. Diese Charakteristik soll zu einem den Lesenden die (ihnen vielleicht unbekannten) Städte näher bringen, zu anderem auf die „grenzüberschreitende Hybridität“ solcher Grenzstädte (Jańczak 2018b: 191) aufmerksam machen.

3 Darüber hinaus soll bedacht werden, dass es sich hier um Sprache in Schriftform handelt. Deswegen greife ich bewusst die Schriftfunktionen von Auer (vgl. 2010: 272) explizite auf: Bennen und Charakterisieren, Zugehörigkeit markieren, Gebrauchsweisen vorschlagen oder verbieten, Wege weisen, Ermahnen und Gedenken. Die hier aufgelisteten Funktionen sind auch Funktionen der Sprachlandschaften, auch wenn die unterschiedlichen Autoren diese nicht direkt als solche deklarieren. Die Funktionen werden noch einleuchtender, wenn sie beispielsweise mit Diskurstypen im Sinne von Scollon und Scollon (2003) zusammengestellt werden. 
Gemeinsam ist den Städten die Tatsache, dass diese an der Grenze zu Bundesrepublik Deutschland liegen und sie bis zur $1945 \mathrm{zu}$ Deutschland gehörten. Dieser historische Fakt prägt sicherlich auch das gesellschaftliche Miteinander in der heutigen Grenzregion und hat auch mittelbar Einfluss auf die Manifestation der Mehrsprachigkeit in den untersuchten Städten. Bezüglich der Einwohnerzahl $^{4}$ unterscheiden sich die Städte. Świnoujscie hat fast 41.000 Einwohner, Słubice dagegen ca. 17.000 Einwohner. Die deutlich niedrigere Einwohnerzahl resultiert aus der Tatsache, dass Słubice vor dem Zweiten Weltkrieg ein Stadtteil von Frankfurt (Oder) waren. Świnoujście ist zu damaligen Zeiten ein bekannter Kurort gewesen, was auch heute unter den Touristen beliebt ist. Die Einwohnerzahlasymmetrie zieht sich über die Grenze hinweg. Die Stadt Słubice grenzt an die einwohnerstärkere Universitätsstadt Frankfurt (Oder), dagegen Ahlbeck, also die Grenzstadt zu Świnoujście, gehört einer Gemeinde mit ca. 9000 Menschen an.

Beide polnischen Städte werden von Tagestouristen besucht, d.h. von Personen, die für einen Tag oder nur für ein paar Stunden die Grenze passieren, um einzukaufen, zu essen oder Dienstleistungen in Anspruch (Friseur, Zahnarzt) zu nehmen. Świnoujście wird nicht nur von Tagestouristen besucht, was aus seiner Lage und kulturellen und gastronomischen Angeboten resultiert. Słubice können nicht von der Nähe zur Ostsee profitieren, obwohl sie im Odertal liegen. Andererseits ist zu überlegen, ob Besucher aus einer größeren Stadt wie Frankfurt (Oder) nicht Auswirkung auf das Dienstleistungsangebot in Słubice und somit Einfluss auf die Manifestation der Mehrsprachigkeit haben.

\section{Forschungsdesign und Datenmaterial}

Aufgrund der Grenznähe wird davon ausgegangen, dass die Sprachlandschaft der ausgewählten Städte - Świnoujscie und Słubice - von der Manifestation deutschsprachiger visueller Mehrsprachigkeit geprägt ist. Diese Annahme resultiert aus vorliegenden Analysen zu der deutsch-polnischen Sprachlandschaft (vgl. Lisek 2017, Lisek 2018, Lisek 2019) sowie Erkenntnissen zum Sprechhandeln in der Grenzregion (vgl. Jańczak 2016, Jańczak 2018a, Jańczak 2018b). Neuartig an der Untersuchung ist die Zusammenstellung der Linguistic Landscapes zweier polnischer Grenzstädte, die sich durch touristische Angebote, Einwohnerzahl und Stadtprofilierung ${ }^{5}$ unterscheiden.

4 Die Einwohnerzahlen wurden zum Stichtag 31.12.2017 dem polnischen Statistikportal https:// www.polskawliczbach.pl/ (31.03.2020) entnommen.

5 Unter der Stadtprofilierung ist die eindeutige Festlegung von Świnoujście auf die Touristik gemeint. Dies ist nicht in diesem Ausmaß für Słubice möglich, zumal Świnoujście eine Meeresgrenze zu Schweden hat. Słubice hat wiederum ein grenzüberschreitendes Forschungsin- 
Im Mittelpunkt der Analyse steht die Frage, in welchen Kontexten die fremdsprachigen (vorwiegend deutschen) Zeichen auftreten, d.h. zu welchen Diskurstypen das erfasste Sprachvorkommen gehört. Um die Frage zu beantworten, werden Bilddaten ${ }^{6}$ ausgewählter Straßen von Świnoujscie und Słubice herangezogen. Beide Untersuchungsabschnitte (jeweils eine Straße) befinden sich in der Nähe eines Grenzübergangs und sind zentral in der jeweiligen Stadt gelegen. Die fotografische Dokumentation des Sprachvorkommens in den Straßen wird sowohl quantitativ (Beschreibung des Sprachmaterials) als auch qualitativ (interpretative Zuordnung der visuellen Mehrsprachigkeit den Diskurstypen) genutzt. Die Analyse wurde anhand von 66 Untersuchungseinheiten aus Słubice und von 58 Untersuchungseinheiten aus Świnoujście durchgeführt.

Die Beschreibung des Sprachvorkommens kann aus vielen Perspektiven erfolgen. Die textsortenlinguistische Richtung ist bei einem allgemeinen Überblick über die Sprachlandschaft einer Stadt wenig produktiv, auch wenn bei dieser Analyse des Linguistic Landscapes der situative Kontext oder Textfunktion eine Rolle spielen. Auch aus der sprachpolitischen Perspektive lassen sich Sprachlandschaften gut betrachten, d.h. inwieweit die Sprachgesetze eines Staates einen Einfluss auf die visuelle Mehrsprachigkeit haben. Die Kommunikationswissenschaft würde das Sprachvorkommen als Meso-Kommunikation (vgl. Domke 2014) auf ihre Ortsgebundenheit oder besondere medial-materiale Geprägtheit fokussieren. Aus den obigen Überlegungen ergeben sich folgende quantitative Untersuchungskategorien:

a) Anzahl der Sprachen,

b) Nachhaltigkeit der Zeichen in der Sprachlandschaft,

c) Situierung der Fremdsprache in Bezug zum Polnischen,

d) Charakter der visuellen Manifestation (institutionell vs. privat) von Zeichen.

Die quantitativen Merkmale des analysierten Linguistic Landscapes einer Stadt werden im weiteren Schritt qualitativ nach den Kontexten, in denen sie vorkommen, klassifiziert. Die Kontexte werden nach Diskurstypen geordnet. Nach Scollon und Scollon (2003: 167) werden infrastrukturelle (Wegweiser, Straßenschilder), regulatorische (Verkehrs- und Verbotsschilder), kommerzielle (Informationen zu Angeboten, Werbung, Eigennamen der Geschäfte) sowie transgressive (unautorisiert angebrachte Aufkleber, Graffiti) Diskurstypen unter-

stitut sowie eine Außenstelle der Adam-Mickiewicz-Universität Poznań (Collegium Polonicum) und ist eine Grenzstadt im Binnenland.

6 Die Rohdaten entstammten separaten Analysen zum Sprachvorkommen und Sprachpolitik einzelner Städte (vgl. Lisek 2017, Lisek 2019) und werden unter kritischer Berücksichtigung der Vergleichbarkeitsaspekte für diese Untersuchung aggregiert. Die Vergleichbarkeit wird u.a. durch die Länge des Abschnitts (400 m) gewährleistet. 
schieden. Diese Klassifikation schient umfangreich genug zu sein, um die unterschiedlichen Kontexte der visuellen Mehrsprachigkeit zu erfassen.

\section{Ergebnisse und Ausblick}

Im ersten Schritt werden die Sprachlandschaften einzelner Städte fokussiert, um dann im nächsten Schritt mögliche Unterschiede oder Ähnlichkeiten zu charakterisieren und zu interpretieren.

\subsection{Sprachlandschaften in Słubice}

$60 \%$ der Zeichen haben einen eindeutig einsprachigen Charakter. Die restlichen $40 \%$ von den Zeichen zeigen visuelle Mehrsprachigkeit, die sowohl auf zweisprachigen als auch dreisprachigen Schildern erfasst wurde. Diese Tatsache ist nicht ganz überraschend, auch wenn angenommen wird, dass die Oderbrücke zu einer größeren Stadt führt - Frankfurt (Oder) - und somit ein größerer Menschenverkehr aus dem Nachbarland zu erwarten wäre. Die $40 \%$ der visuellen Mehrsprachigkeit zeigen zwar, dass Słubice gute Einkaufsmöglichkeit für ausländische Kunden bietet, jedoch die touristische Komponente fehlt und somit das Angebot entsprechend geringer ausfällt. Dies lässt sich am Linguistic Landscape der Stadt festmachen.

Mehrsprachige Schilder, die die deutsche Sprache beinhalten, machen $70 \%$ dieser aus. Der restliche Anteil entfällt auf englischsprachige Zeichen. Meine Analyse fokussiert die deutschsprachige Manifestation der visuellen Mehrsprachigkeit. Die Mehrheit der Schilder ist Bottom-Up (privat), vereinzelte Schilder sind Top-Down (institutionell). Zu den letzteren gehören Wegweiser zu Post und Polizei sowie eine Information zu der Stadt. Alle mehrsprachigen Schilder sind ein fester Beststandteil der Infrastruktur, d.h. sie prägen auf nachhaltige Art und Weise die Sprachlandschaft von Słubice. Das Sprachmaterial auf Deutsch ist zu $35 \%$ auf der gleichen Höhe wie Polnisch, zu $59 \%$ unter Polnisch und nicht zuletzt zu $6 \%$ über Polnisch positioniert. Dies sollte nicht überraschen, da es sich um eine polnische Stadt handelt und die Empfänger der Zeichen primär polnischsprachig sind. Darüber hinaus spielt hier die Sprachpolitik des Staates eine wesentliche Rolle. Diese bevorzugt eindeutig die Amtssprache, also Polnisch.

Die untersuchte visuelle Mehrsprachigkeit, die vor allem die deutsche Sprache fokussiert, lässt sich unterschiedlichen Diskurstypen zuordnen. Interessant ist zu beobachten, dass keiner der Schilder den transgressiven Diskurstypen zugeschrieben werden kann. Dies ist sicherlich darauf zurückzuführen, dass transgressive Zeichen eines unerlaubten Charakters sind und solche oft aus Protest- 
bewegungen resultieren oder für etwas werben. Da Słubice nicht eine Großstadt ist, ergibt auch keinen Sinn, in einer Sprache für etwas zu werben oder gegen etwas zu protestieren, wofür es keine oder wenige Empfänger gibt.

Die Mehrheit der Zeichen (71 \%) gehört dem kommerziellen Diskurs, was wiederum auf die Touristik zurückzuführen ist. Die restlichen Zeichen (29\%) gehören dem infrastrukturellen Diskurs, in dem für die Grenznähe interkulturell sensibilisiert wird. Die Manifestation der visuellen Mehrsprachigkeit betrifft vor allem Dienstleistungen aus den Branchen wie Bau, Medizin, Innenausstattung. Dies sind auch Bereiche, die Stammkundschaft aus Deutschland - insbesondere aus Frankfurt (Oder) und den benachbarten Ortschaften in Brandenburg haben müssen.

\subsection{Sprachlandschaften in Świnoujście}

In Świnoujście haben $45 \%$ untersuchter Zeichen einen eindeutig einsprachigen Charakter. Die weiteren $55 \%$ der Zeichen haben einen mehrsprachigen Charakter. Die Schilder sind zwei-, drei-, vier- oder sogar sechssprachig. Hier zeichnet sich die touristische Stärke einer Stadt ab, die sich in der visuellen Mehrsprachigkeit wiederspiegelt.

Unter den mehrsprachigen Schildern ist die deutsche Sprache auf $56 \%$ vorzufinden. Weitere Zeichen repräsentieren überwiegend das Englische, es wurden ebenso Schwedisch, Italienisch und Norwegisch ausgemacht. Ähnlich wie bei den Zeichen in Słubice konzentriere ich mich in meiner Analyse auf die Zeichen mit deutscher Manifestation in visueller Mehrsprachigkeit. Die Mehrheit der Schilder hat einen privaten Charakter (Bottom-Up). Auch Top-Down-Schilder sind vertreten. $\mathrm{Zu}$ den letzten gehören Gedenktafeln sowie eine Information $\mathrm{zu}$ Radwegen, dem Fährverkehr sowie zu ehemaligen Straßennamen. Bis auf drei Einheiten sind alle Schilder fest eingebaut und gehören nachhaltig zum Linguistic Landscape der Stadt. Die deutschsprachigen Zeichen befinden sich zu $12 \%$ über den polnischen Zeichen, zu $5 \%$ sind sie gleich mit Polnischem positioniert und zu $83 \%$ unter dem Polnischen gesetzt. Somit ist eindeutig die Sprachpolitik des Staates zu erkennen, die eine Amtssprache bevorzugt. Die Angebote der Tourismusbranche sind auf Schildern in Schriftform zu beobachten, diese richten sich anscheinend in erster Linie an die polnischsprachigen Touristen.

Die visuelle Mehrsprachigkeit, die insbesondere die deutsche Sprache fokussiert, gehört zu unterschiedlichen Diskurstypen. Ähnlich wie in Słubice ließen sich keine transgressiven Diskurse festmachen. Es wurde ein Schild ausgemacht, das ein $1 \%$ des regulatorischen Diskurstyps darstellt. Weitere Kontexte repräsentieren zu $49 \%$ infrastrukturelle und $50 \%$ kommerzielle Diskurse. Die mehrsprachige visuelle Manifestation der letzten Kategorie betrifft vor allem Handel und Dienst- 
leistungen in Bereichen der Gesundheitsversorgung. Der starke Anteil der mehrsprachigen Zeichen am infrastrukturellen Diskurstyp deutet auf zahlreiche fremdsprachige Menschen hin, die in der Stadt verkehren und somit auch Informationen in einer anderen Sprache als Polnisch erforderlich sind.

\subsection{Zusammenfassende Bemerkungen und Forschungsdesiderata}

Zwischen den analysierten Linguistic Landscapes bestehen sowohl Unterschiede als auch Gemeinsamkeiten. Einen klaren Unterschied macht der Anteil der mehrsprachigen Zeichen aus, dieser ist in Świnoujście um 15 \% höher als in Słubice. Einen weiteren Unterschied stellt der Anteil der deutschen Sprache in der Manifestation der visuellen Mehrsprachigkeit in den Sprachlandschaften der Städte dar. Dieser ist wiederum in Słubice um 14 \% höher als in Świnoujście und beträgt 70 \%.

Des Weiteren bestehen mehrere Ähnlichkeiten zwischen den Sprachlandschaften. Auf Schildern in beiden Städten ist die deutsche Sprache nicht auf gleicher Höhe mit Polnisch positioniert. Zu einem resultiert es aus der staatlichen Sprachpolitik, die die polnische Sprache als Amtssprache bevorzugt. Zu anderem ist es eine Folge der Ausrichtung der im privaten Auftrag erstellten Schilder, Tafeln und Aushänge, die sich an polnischsprachige Empfänger richten.

In Bezug auf die Diskurstypen unterscheiden sich die Sprachlandschaften der Städte nahezu gar nicht. In keiner der Städte konnte eine deutschsprachige Manifestation von transgressiven Zeichen erfasst werden. Es wird davon ausgegangen, dass die unautorisierten Aufkleber oder Graffiti aufgrund ihrer persuasiven oder emotiven Funktion und somit einer primären Ausrichtung an das polnischsprachige Publikum keinen Bestand haben können. Sowohl in Słubice als auch in Świnoujście treten die Zeichen im infrastrukturellen und kommerziellen Diskurs auf. Zwar ist der Anteil der Zeichen im infrastrukturellen Diskurstyp im Linguistic Landscape in Świnoujście um 21 \% höher, aber dies ist auf die Stadtgröße sowie den Touristenverkehr zurückzuführen. Somit ist tatsächlich kein Unterschied feststellbar. Auch im kommerziellen Diskurs wurden Ähnlichkeiten festgestellt, insbesondere im Hinblick auf den Bereich der Dienstleistungen in der Gesundheitsversorgung.

Somit kristallisiert sich eine Tendenz heraus, die an die Größe sowie den Charakter der Stadt zurückzuführen ist. Die Diskurstypen unterscheiden sich nicht wesentlich voneinander. Der Unterschied liegt in der ausgemachten Anzahl der deutschsprachigen Zeichen sowie in prozentueller Verteilung der Zeichen in den jeweiligen Diskurstypen. Der Grenzraum bleibt nach wie vor ein spannender Ort mit Raum für unterschiedliche Texte und Diskurstypen, die im Linguistic Landscape vorkommen und für den sie geschaffen werden, da sich die Bedürfnisse der Einwohner, Geschäftsleute und Touristen ändern können. Interessante 
Ergebnisse zu Sprachlandschaften wären bei vergleichenden deutsch-polnischen Studien zu erwarten.

Darüber hinaus ist für Folgeuntersuchungen zu überlegen, ob die deutsche Sprache in den Grenzstädten aufgrund der Nähe zur Sprachgemeinschaft als lingua culturalis (vgl. Raasch 2008: 14) zu sehen ist oder, ob Deutsch viel mehr lingua oeconomica der Grenzregion sei? Darauf würde der hier erfasste kommerzielle Diskurstyp mehrsprachiger Zeichen im Linguistic Landscape hindeuten. Dies wäre auch ein mögliches Forschungsdesiderat, das aus der hier präsentierten Analyse hervorgeht. Eine weitere Erforschung von Linguistic Landscapes kombiniert mit gesprächslinguistischen Ansätzen Angewandter Linguistik oder einem weiteren Forschungsfeld wie interkulturelle Kommunikation würden sicherlich einen Beitrag zur Entwicklung der Disziplin beitragen.

\section{Literaturverzeichnis}

Auer, Peter (2010). Sprachliche Landschaften. Die Strukturierung des öffentlichen Raums durch die geschriebene Sprache. In: A. Deppermann/A. Linke (Hrsg.), Sprache intermedial - Stimme und Schrift, Bild und Ton (S. 271-298). Berlin: De Gruyter.

Ben-Rafael, Eliezer/Shohamy, Elana/Barni, Monica (2010). Linguistic landscape in the City. Bristol [u.a.]: Multilingual Matters.

Cillia de, Rudolf (2010). Angewandte Linguistik/angewandte Sprachwissenschaft. In: H. Barkowski/H-J. Krumm (Hrsg.), Fachlexikon Deutsch als Fremd- und Zweitsprache (S. 10). Tübingen, Basel: Francke UTB.

Domke, Christine (2014). Die Betextung des öffentlichen Raumes: eine Studie zur Spezifik von Meso-Kommunikation am Beispiel von Bahnhöfen, Innenstädten und Flughäfen. Heidelberg: Universitätsverlag Winter $\mathrm{GmbH}$.

Grucza, Franciszek (2017). Lingwistyka stosowana. Historia - Zadania - Osiagnięcia. Dzieła zebrane. Tom 9. Warszawa: Wydawnictwo Naukowe Instytutu Komunikacji Specjalistycznej i Interkulturowej Uniwersytet Warszawski.

Harden, Theo (2006). Angewandte Linguistik und Fremdsprachendidaktik. Tübingen: Narr. Jańczak, Barbara Alicja (2018a). „Jesteśmy tu wszyscy w Polsce i mówimy po polsku“ Eigen- und Fremdwahrnehmung anhand der Kommunikationsstrategien von Bewohnern polnischer Grenzstädte. In: I. Wowro/M. Jakosz/R. Kozieł (Hrsg.), Sprachliche Dimensionen der Fremdheit und Andersartigkeit (S. 237-254). Frankfurt a.M.: Peter Lang GmbH Verlag.

Jańczak, Barbara Alicja (2018b). „Linguistische Grenzschaften“: Kommunikationsstrategien im deutsch-polnischen Grenzland am Beispiel von Bewohnern der polnischen Städte Zgorzelec und Łęknica. In: B. Hufeisen/D. Knorr/P. Rosenberg/C. Schroeder/A. Sopata/T. Wicherkiewicz (Hrsg.), Sprachbildung und Sprachkontakt im deutsch-polnischen Kontext (S. 189-217). Frankfurt a.M.: Peter Lang GmbH Verlag.

Jańczak, Barbara Alicja (2016). Deutsch-polnische Grenzschaft: Sprachgebrauch im transnationalen Raum der Grenzmärkte im deutsch-polnischen Grenzland. In: A. Tölle/ 
R. Wehrhahn (Hrsg.), Translokalität und lokale Raumproduktionen in transnationaler Perspektive (S. 119-131). Berlin: Logos Verlag.

Knapp, Karlfried (2006). Vorwort. In: K. Knapp u. a. (Hrsg.), Angewandte Linguistik. Ein Lehrbuch (S. XIX-XXV). Tübingen, Basel: Francke UTB.

Landry, Rodrigue/Bourhis, Richard Y. (1997). Linguistic landscape and ethnolinguistic vitality: An empirical study. Journal of Language and Social Psychology, 16(1), 23-49.

Lisek, Grzegorz (2017). Sprachpolitik und ihr Einfluss auf polnische linguistic Landscapes in der deutsch-polnischen Grenzregion. In: A. Weigl/N. Nübler/K. Naumann/M. VölkelBill/S. Grahl/T. Lis (Hrsg.), Junge Slavistik im Dialog VI. Beiträge zur XI. Internationalen Slavistischen Konferenz (S. 169-180). Hamburg: Verlag Dr. Kovač.

Lisek, Grzegorz (2018). Überlegungen zu Aufstellspeisekarten im grenznahen Linguistic Landscape - metrolinguistische Perspektive. In: V. Janíková/A. Brychová/J. Veličková/ R. Wagner (Hrsg.), Sprachen verbinden. Beiträge der 24. Linguistik- und Literaturtage, Brno/Tschechien 2016 (S. 217-228). Hamburg: Verlag Dr. Kovač.

Lisek, Grzegorz (2019). Polnisch und Deutsch im Kontakt. Zur Problematik der Linguistic Landscapes am Beispiel von Słubice. In: E. Kegyes/R. Kriston/M. Schönenberger (Hrsg.), Sprachen, Literaturen und Kulturen im Kontakt. Beiträge der 25. Linguistikund Literaturtage, Miskolc/Ungarn, 2017 (S. 281-292). Hamburg: Verlag Dr. Kovač.

Mühlan-Meyer, Tirza (2018). Mehrsprachigkeit in der Linguistic Landscape der Metropole Ruhr mit besonderer Berücksichtigung des Polnischen. In: B. Hufeisen/D. Knorr/P. Rosenberg/C. Schroeder/A. Sopata/T. Wicherkiewicz (Hrsg.), Sprachbildung und Sprachkontakt im deutsch-polnischen Kontext (S. 259-295). Frankfurt a.M.: Peter Lang.

Prokop, Izabela (1989). Kommunikation im Büro - erste Analyseergebnisse. In: M. Pätzold/P. Lindemann (Hrsg.), Kommunikationstagung 1989. Internationale Arbeitstagung in Wulkow 18.-20. April 1989 (S. 116-123). Berlin: Akademie der Wissenschaften der DDR, Zentralinstitut für Sprachwissenschaft.

Prokop, Izabela (1995a). Fremdbilder, Stereotype, Vorurteile. In: M. Czyżewski/E. Gülich/ H. Hausendorf/M. Kastner (Hrsg.), Nationale Selbst- und Fremdbilder im Gespräch. Kommunikative Prozesse nach der Wiedervereinigung Deutschlands und dem Systemwandel in Ostmitteleuropa (S. 180-202). Opladen: Westdeutscher Verlag.

Prokop, Izabela (1995b). Erotetische Sprechakte im Deutschen und im Polnischen anhand natürlicher Gespräche. Poznań: Wydawnictwo Naukowe Uniwersytetu im. Adama Mickiewicza.

Prokop, Izabela (2010). Aspekty analizy pragmalingwistycznej (Aspekte der pragmalinguistischen Analyse). Poznań: Wydawnictwo Naukowe Uniwersytetu im. Adama Mickiewicza.

Raasch, Albert (2008). Grenzkompetenz - von der Definition zur Evaluation zur Anwendung. In: M. Bien-Lietz/T. Vogel (Hrsg.), Frühstart in die Nachbarsprache. Handbuch für den Spracherwerb in der deutsch-polnischen Grenzregion (S. 9-15). Frankfurt O.: Europa-Universität Viadrina.

Scollon, Ronald/Scollon, Suzanne (2003). Discourses in place: Language in the material world. London, New York: Routledge Routledge, Taylor \& Francis Group.

Szerszeń, Paweł (2010). Polska szkoła lingwistyki stosowanej: Kilka uwag na kanwie monografii F. Gruczy pt. „Lingwistyka stosowana. Zarys jej historii, zadań i dokonań“. Lingwistyka Stosowana/Applied Linguistics/Angewandte Linguistik, 2, 261-269. 


\title{
Danuta Wiśniewska \\ (Uniwersytet im. Adama Mickiewicza w Poznaniu)
}

\section{Linguistic landscape, murals and language learning}

\begin{abstract}
This chapter argues that the linguistic landscape provides verbal and visual input for language learning. Language learners may benefit from activities based on the art present in open public spaces, specifically on the analysis and interpretation of murals. It suggests that the use of murals in classroom and out-of-classroom activities provides numerous opportunities for the development of all language skills at almost any level of language competence, and makes it possible to combine learning focused on both form and meaning. In addition, language learning may enhance thinking and talking about murals within their socio-cultural context by providing linguistic means for analysis and interpretation.
\end{abstract}

Keywords

Linguistic landscape, language learning, multimodality, murals

\section{Introduction}

Today (foreign) language education is not limited solely to teaching/learning the lexis and grammar structures, and the skills necessary to use the language efficiently, including the basic ones of reading, writing, listening and speaking. The language is researched, learned and taught as a means of communication inextricably related to "social relations, cultural models, power and politics, perspectives on experience, values and attitudes, as well as things and places in the world" (Gee 2008: 1) and as a means to "think about and act on the world, on the other" (Gee 2001: 714). Thus, language has always empowered its speakers to function in society. Recently, however, verbal language has been considered as just one of the cultural and social semiotic resources, in other terms - modes for meaning making (Kress \& van Leeuwen 2001; Jewitt \& Kress 2003). Shohamy (2015: 168) wondered:

Does an expanded meaning of 'language' need to be limited only to words? Is a movement, a dance, food, bodies not languages that provide and send meanings to others? The term 
'language' needs to be expanded to all these other devices which take place in the 'practiced', 'conceived' and 'lived' spaces.

Kress (2015) argues that teaching a language should not mean teaching only one of the meaning-making modes. Instead, language education which is focused on meaning-making and communication should exploit the modes in combination, thus enriching the purely linguistic approach. That is not to say that in the teaching of languages so far other modes than linguistic one have not been attended to; it means that greater attention to the socio-cultural as well as technological aspects of language teaching requires more attention and wider use of suitable combinations of modes.

Foreign language learning and teaching is closely related to and conditioned by the world around us, especially as foreign language education is a response to the needs of societies which learn and use languages other than their mother tongues for work, education, travel, leisure, politics, or economy. Even in countries which are basically monolingual (however, not many states are purely monolingual, after all there are minority or regional languages present in almost every country) foreign languages, along with the official language of the state, appear in the public space on a variety of occasions, in brand names, advertisements, names of services and shops, on vehicles, in train stations, museums, restaurants, offices, hospitals, street-art. The presence of foreign tongues in everyday encounters in public spaces, in forms often combined with images, easily accessible to potential readers/viewers, named linguistic landscape (Landry \& Bourhis 1997:25) makes it a potentially valuable source for language learning and teaching (Cenoz \& Gorter 2008).

This chapter will seek to analyse one of the more frequent and visible components of the linguistic landscape in relation to foreign language learning, namely murals. It will look at bidirectional relations between language learning and murals. First, the focus will be on the ways language education is suitable for preparing for the reading of mural texts and, secondly, on how the activities related to the reading of mural texts may support language learning/teaching.

\section{Linguistic landscape}

The linguistic landscape, originally conceived of as "the language of public road signs, advertising billboards, street names, place names, commercial shop signs and public signs on governmental buildings" (Landry \& Bourhis 1997: 25), and later also as the language of graffiti (Pennycook 2009, 2010), the language of tourist post cards (Jaworski 2010), tourist signs (Kallen 2009), memory and monuments (Abousnnouga \& Machin 2009), street art (Guilat 2010) and of cy- 
berspace (Ivkovic \& Lotherington 2009; Troyer 2012) has been the object of the sociolinguistic and semiotic studies for over forty years. Throughout this time, the theoretical perspectives have been changing and the scope and objects of research have widened immensely. The main focus of linguistic landscape research is on the use of language in its written form in the public space (Gorter 2006) and the main aim of the research is "to add another view to our knowledge about societal multilingualism by focusing on language choices, hierarchies of languages, contact-phenomena, regulations, and aspects of literacy" (Gorter 2013: 191). The sociolinguistic and semiotic studies of the linguistic landscape have been subsequently enriched with the focus on multimodality (Zabrodskaja \& Milani 2014; Pütz \& Mundt 2018,) and geosemiotics (Scolon \& Scollon 2003).

Studies on the linguistic landscape are naturally conducted mainly in multilingual societies (such as Canada, Belgium, Finland, Luxembourg, Spain), in which the co-existence of languages makes the linguistic landscape more varied and visible, significantly influences the appearance of cities, reveals the inhabitants' identities and at times leads to social and/or political conflicts. Fewer studies have been conducted in monolingual contexts, however they are not excluded from this type of research, although their research aims will in many cases be different (Coluzzi 2012; Zabrodskaja 2014; Wiśniewska 2019). In reality, although states are normatively monolingual, other languages appear in the public space as well. The presence of English is almost obvious, due to its role in the sphere of economy, politics, culture, technology, tourism, and to global trends of using English for the feeling of belonging to a modern world marked by the omnipresence of English. Other languages and other scripts have also been commodified, mainly for advertising purposes (French, Italian, Chinese) or for making goods and services available to immigrants and tourists. Besides, in officially monolingual countries, dialects, regional varieties or minority languages are at play, influencing to a certain degree the linguistic landscape in, for example, place names, information, announcements written in two languages.

Jaworski and Thurlow (2011:2) rightly observe that written discourse is not the only discourse present in the public space and that it "interacts with other discursive modalities: visual images, nonverbal communication, architecture and the built environment". The ubiquitous presence of multimodal texts in urban spaces has led some writers to coin the metaphor of the city as text, followed by the further concept of "reading the city" and assuming the presence of a reader or readers (Barthes 1988: 418). The metaphor, even if not explicit, has been present in literary works (e.g. Hessel 1929; Calvino 1972; Benjamin 1997, Krakauer 2001) as well as in semiotic considerations (e.g. Żyłko 2007; Chmielewska 2010, Koschany 2013). In the light of the concept of the city as text, we are all engaged in knowing the city, understanding and interpreting it, or in other words, in reading the city. In this context, the statement "The city gives food for thought" (Ko- 
schany 2014: 74) is well justified, and may be complemented by the statement that the city might be food for language education as well.

\section{Linguistic landscape and language pedagogy}

The socio-linguistic and semiotic studies of the linguistic landscape are numerous, while the studies seeking to develop a common ground for the linguistic landscape and language education are much more recent and rather scant, although today we may observe an increase in the interest in the search for possibilities of linking "reading the city" with (foreign) language pedagogy. The need to prepare learners to understand the linguistic landscape and to make use of it for their benefits has been expressed by Clemente et al. (2012: 271):

Being able to read, understand and critically question the different landscapes that surround us and their changes through time means, imperatively, that we have to conceive literacy in a particularly complex sense. Clearly, to practice and accomplish this perspective involves an interdisciplinary and truly holistic educational approach early on in official schooling.

It is, then, not enough to develop verbal literacy, that is the ability to read and write, rather, education needs to aim at the development of multimodal literacy, as Alvermann (2002: viii) indicates; performative, visual, oral, semiotic, which are all needed to construct and reconstruct texts. What has also been underlined is the key role of formal education. Although the significance of the informal environments has not been neglected, it is the role of formal schooling to help learners become aware of the language and image diversity in the world that surrounds them (Sayer 2010; Bever 2012). The advantage of using the linguistic landscape in language education is its authenticity, even if foreign language texts appear in a monolingual context. However, such texts have not been produced for educational purposes, but for some "real" informational or symbolic aims (Hewitt-Bradshow 2014). Cenoz and Gorter (2008) have made several points to advocate that the linguistic landscape has a potential for language learning, and the development of verbal and non-verbal literacies as a source of language input, and of diversified texts which fulfil multiple functions, hence being suitable for the study of pragmatic functions of verbal and non-verbal texts.

Linguistic landscape is not only a source of language input. It also creates opportunities for learners and teachers to engage in small-scale research into the languages in the surrounding environment. Collecting data from a research area and personal engagement in the research process raises the participants' awareness of multilingualism and the role of individual languages (Hancock 2012). Cenoz and Gorter (2008) emphasise that informal language learning in the 
case of linguistic landscape is possible owing to frequent or intensive exposure to languages. If such informal learning were supported by learners' research into the linguistic landscape, it could result in raising language awareness, in better understanding of their attitudes towards languages and development of autonomy as language learners.

The idea of including the linguistic landscape in foreign language teaching practice has become attractive both for practitioners and scholars. Research literature reveals that generally the studies outside and inside school activities are aimed at raising learners' awareness of the diversity of cultures, languages, texts and modes (Scarvaglieri 2007; Dagenais et al. 2009; Bever 2012; Hewitt-Bradshow 2014; Aladjem \& Jou 2016). Some research shows that the work on linguistic landscape can also be done with children (Dagenais et al 2009; Chern \& Dooley 2014; Roos \& Nicholas 2019), as well as with students (Malinowski 2010; Chesnut et al. 2013; Hewitt-Bradshaw 2014), international students (Bagna et al. 2018) and pre-service teachers (Kim 2017).

\section{Linguistic landscape and murals}

Mor-Sommerfeld and Johnson (2012: 155) conceive of spaces of the linguistic landscape as multiple and multifaceted, conspicuous or concealed, artistic or linguistic, written or painted, static or dynamic, human and social. In such a broadly defined linguistic landscape, there is a place for visual or visual-verbal art. This is not art hidden in galleries but art of the public space, accessible to everybody to look at and read - murals. This form of art has been known since ancient times as a means of communication within societies before writing had been invented, or was used by elite groups only. At times, it was a tool of propaganda, at others a resource for current information (Łabądź 2019).

Contemporary murals are a form of usually large-format public art performed on walls in urban spaces which recently have begun to function as a "[S]ymbol of modernity and trendiness, enriching the material dimension of the city and gentrifying" (Mokras-Grabowska 2014) and as a symbol of places (Skinner \& Jolliffe 2017: 6). Currently, muralism to some extent has lost its contesting character, and instead, in contrast to graffiti, is largely institutionalized, and the works are frequently designed and produced in cooperation with organizations and local societies. However, the beginnings of modern mural painting are closely related to politics. The outbreak of the Mexican revolution in 1910, against an authoritarian government, British and American capitalism and the Church, was supported by the artists of the mural movement who, in their monumental wall paintings, aimed to express the life and struggles of Mexican peasants and workers during the revolution which lasted almost 10 years (Greele 2012). After the revolution, the new 
government invited the muralists to paint pictures in the public space which could visually convey the new ideas about the new political and social order, identity, history and culture to a basically illiterate society (Łabądź 2019). In fact, the work of the three great Mexican painters, Diego Rivera, José Clemente Orozco, David Alfaro Siqueiros, was deeply engaged in the political and cultural problems of the country and played the role of mediator between state and nation in the turbulent post-revolutionary period (Greele 2012).

Northern Ireland is another country where murals enjoyed, and still enjoy, great popularity for expressing political and social views. The murals here were first painted at the beginning of the twentieth century by the Loyalists, who used the paintings to declare their loyalty to the British crown. The Republicans engaged in mural painting only in the early 1970s, to express their political views, but also covering wider issues such as prison conditions, media censorship, police brutality, the Irish Republican Army, the Irish language and women's rights (Lyell 2001). Both sides of the conflict in Ireland provoked visually with their political views, aggression, and manifestation of their strength.

American and European muralism developed in two directions, towards the decorating of buildings and advertising goods, or for expressing societal and political problems. The aim was to make art and its message accessible to a society which could not afford to visit art galleries.

In the $20^{\text {th }}$ century, mural art has been developing on almost every continent, partly losing its politically and socially engaged voice on one hand, on the other fulfilling a number of other functions. Skinner and Jollifee (2017: $3 \mathrm{ff}$ ) grouped these functions into economic, political, commemorative, commercial and artistic. Murals which belong to the economic group are those painted for decorative purposes, development and urban revitalization. Most often they are found in towns and on mural tourist trails. Murals that have been motivated politically express political views, positions and resistance. They are found mainly in conflict areas during political campaigns. Murals which commemorate people and events are also popular, being painted in places significant for the local society. Commercial murals deal with the promotion and advertising of goods and sales, and are placed on commercial buildings or in other places where the messages can be easily seen by potential consumers. Finally, there are murals with a more individual, artistic expression, including poetry and artistic typography (Skinner \& Jollifee 2017: 10; Łabądź 2019: 114). Murals are also seen as a way to consolidate local identity and develop community (Gralińska-Toborek 2019), and as a tourist attraction (Jażdżewska 2017). An important feature of muralism is that the works of art are painted by members of the community for the community (Kang \& Gammel 2011: 267), though recently the authors are often specially invited painters from all over the world. Finally, their educational 
value cannot be overlooked. For example, Irish murals have aimed at teaching Irish history, culture, aspirations and dreams.

Mural art aims to communicate ideas and messages to viewers, to enter into a dialogue with others and inspire others to think. Placed in public space, they are available to every user of this space. Placed in galleries or private collections, they partly lose their communicative power, the idea of dialogue, and their influence on the society is largely diminished.

Murals make meanings using at least two modes, visual and textual. They communicate meaning through image and text, wherein the relation between these two is unbalanced. Usually the image is more important than the text, though at times the text becomes an image itself, as in the case of typographic murals (Gralińska-Toborek 2019). Whatever the means are, murals cannot be "read" without consideration of the socio-cultural context in which they have been painted.

In the following sections, the focus will be on the bidirectional relationship between murals as visual or verbal/visual elements of the linguistic landscape, and (foreign) language learning and teaching. I will attempt to show that linguistics and language teaching might be helpful in 'reading' murals, while murals might be an interesting and motivating resource for language learning and teaching.

\section{Language learning for 'reading' murals}

Van Leeuwen (2004: 18) has suggested that many linguistic concepts and methods may be directly applicable to the study of visual communication, which should result in better understanding, especially of the fact that we need a language which makes the dialogue with the visual and about the visual easier. Avgerinou and Ericson (1997: 286), in the context of visual literacy development, argue that in order to decode the meaning hidden behind a visual or visual-verbal artifact, learners need means to describe the graphic elements composing the image, to analyse the arrangement of these elements, interpret the messages and to express their appreciation. Similarly Pauwels (2008: 80) sees the need for the relevant language as crucial for a meaningful encounter and understanding of a piece of art, and for the development of skills which will help to communicate messages transferred visually, especially that, as Pauwels (2008: 80) continues, "we have a very limited set of words at our disposal to talk intelligibly about visual artifacts and experiences", which makes people refrain from talking. Therefore, on the one hand, there is a need for the development of visual literacy, and so the ability to consciously deal with images, develop an awareness of their presence, identify their genre (photograph, map, graffiti, mural), place visual representations in a social, political or cultural context, and to identify any personal ex- 
pression in the image (Pauwels 2008). On the other hand, there is a need for verbal resources to achieve the above aims.

One of the best known methods for analysing art is that developed by Erwin Panofsky (1939/1972). If this method was used in the analysis of a mural, we would need adequate lexical and syntactic resources to do so. The first, preiconographic stage of the analysis entails the identification of forms (lines, colours, shapes, details, poses, texture), relations (proximity, similarity) and objects (human beings, animals, plants, etc.). The richer the learners' lexicon and grammar structures, the more complete the description at the denotative level. From this level, the analysis proceeds to the iconographic stage, "in the narrower sense of the word", as Panofsky puts it (1939/1972: 6), which assumes knowledge of literary sources and involves the identification of symbolic images, stories and allegories, the sphere of "conventional subject matter". During the third stage of "iconography in a deeper sense", the interest lies in synthesis, and in situating the hitherto analyses within a broader cultural, social and political context to enhance the interpretation of the images. These two steps need both experience and knowledge, and more advanced language competence is required to talk about the image. The language competence refers both to $\mathrm{L} 1$ as well as to $\mathrm{L} 2 / \mathrm{L} 3$, where especially thorough linguistic preparation is essential. The analysis of an art work in a foreign/second language has some additional values. Vygotsky (1960: 110) argued that, for a child, being able to express one's thought in more than one language will enable him to see his/her language as one of many, and will lead him/her to become aware of his linguistic operations.

The second argument that (foreign) language learning may support a meaningful dialogue about art comes from a comparison of the functions of language and art. Is there an analogy between verbal language and the language of art? Is art a language in itself? There is no unanimous answer to these problematic questions. According to Tatarkiewicz (1979: 20), from the point of view of the functions the two systems realise, art and language are considered autonomous semiotic systems which become closer only in their expressive function. Barasch (1997) provides a thorough overview of these everlasting considerations, with the conclusion that the two systems at least share a communicative function. Treichl (2017: 19) holds a similar view, that art and language are two distinct semiotic systems, but tightly connected, since each system has some features of the other and shares some aims and functions, such as communication, creation of personal and social identity, transmission of emotions or ideology.

We may attempt to explore whether the language functions developed by Jakobson (1985) can be useful in talking about art. According to Jakobson (1985), verbal language performs the following functions which can be applied for the analysis of a word, a text or an image: emotive, poetic, referential, phatic, conative and metalingual. It would be interesting to see whether the functions of language 
could be applied to mural painting, and how language learning may help in describing, analysing and interpreting murals, taking as a point of departure, the functions of language and art. The emotive (or expressive) function is related to the attitudes and emotions of the addresser, the person who "sends" the message - in our case, the painter of the mural. This function will require from learners more advanced and refined language with which they may talk about the painter's attitudes towards the topic of the mural, or to try to identify the painter's emotional states concerning the topic. The poetic function focuses on the message itself, not on its content but on the linguistic features of the message, "[J]ust as the analysis of painting [...] deals with pictorial structure, 'poetics' deals with problems of verbal structure." (Jakobson 1985: 147). If we reverse this comparison, we could apply it to mural painting and relate poetic function to pictorial structures. In discussing this function of a painting, the language learners will need to use their lexical and syntactical resources to talk about the organization of the mural, its form and style, how the final effect has been achieved and what effect it has on the learner. The conative function, its orientation or appeal towards someone, in a purely visual mural may be realised indirectly, through image, in verbal-visual murals also by the use of words, mostly vocative or imperative. The learners then will talk about their attitudes towards the mural, their reactions, the influence the mural has had on them, their understanding of it and, hence, they will use very personal, emotionally laden language. The knowledge of how to communicate emotions and the skills to do it will be very helpful. The referential function is oriented towards the context, external conditions, historical, social, cultural events and economic factors. A good range of vocabulary and grammar structures will be helpful not only in talking about murals, but in searching for information and for reading purposes.

Why should we bother about being able to talk about art? The answer seems to be very simple: because people do talk about art, because art is taught at schools and universities, is discussed in committees, is written about (O'Toole 1994: 4). Moreover, as research shows, while the viewer is speaking about the painting, his attention to the painting and its details is more focused (Klein et al. 2014). Why should we bother about being able to talk about murals? Mural art is found in the majority of cities across the globe. It is found in the immediate surroundings of the communities where we live. Apart from decorative purposes, it appeals, provokes, conveys political and social messages, commemorates, finally, it is in fashion.

In this section, I have argued that the ability to read and interpret murals can be enhanced by adequate language practice. Searching for the best ways of expression in a foreign language might make the learner become more attentive to the content and artistic or social value of an artwork, might help to express meanings more precisely and might lead to comparisons between languages, as well as comparisons between different modes of expression, verbal and visual. In 
the next section, I would like to look at murals from a different angle, that is, their applicability in learning/teaching foreign languages.

\section{Murals and language learning/teaching}

In this section, the problem will be reversed, and the question is: why use murals in teaching foreign languages? An exhaustive list of arguments for applying art in general in the foreign language classroom has been presented by Ortuño (1994: 501). The arguments fall into several categories, referring to:

1) motivation, lowering anxiety, creating a non-threatening environment, enhancement of engagement and learner-centered teaching,

2) exposure to social, historical, geographical, and religious context for the acquisition of language skills,

3) development of analytical thinking skills,

4) active exploration of the language,

5) capability of talking about art and exploration of art-related concepts.

The above arguments apply also to the use of murals, as one of many kinds of visual art. Further arguments, concerning murals, are the following:

6) murals are easily available in the public space or in virtual public space, where one can find murals not only from his/her own city or country but from all over the world and from different periods in history,

7) provide both verbal and visual input for interpretation,

8) if murals do not serve merely decorative purposes, they deal with important up-to-date political, social, cultural, existential issues, often being provocative, thus providing grounds for the development of content-related lexical and grammar structures,

9) they are connected to other texts - blogs, articles, films, thus providing grounds for the development of not only speaking skills, but also listening and reading,

10) they provide opportunities for writing practice.

In what follows, I will discuss some of the areas in which work with murals can enhance learning a foreign language.

The study by Bruder and Ucok (2000), in which they analysed viewers' utterances about paintings in the gallery disclosed some regularities in the use of language structures, grouped by the authors into three broad categories: evaluation of a piece of art, attraction and storytelling. The examples of recorded fragments of discussions among art gallery visitors indicate what language structures can be practiced during art-based lessons. The category of evaluation 
has been further divided into four sub-categories: preferences, judgments, and disclaimers. While talking about preferences, i.e., the most frequent topic, the viewers used statements such as "I don't like it," "I really like this painting a lot and also the color," "I love the lighting in it". Judgments included evaluations of paintings. Resolved judgments reflected decisive conclusions which were expressed in the following words: "For me it's ugly ... they look like monsters ... the persons look like cannibals ... it's ugly for me. I don't like the colors"; "You know, it kind of surprises me because the artist is obviously a good artist. The artist would have thought of that... The artist is too good an artist to have made such a mistake". In contrast, reserved judgments expressed uncertainty, considerations, reflection, as in the following examples: "I haven't got to a clarity of a decision on it."; "If I look at it long enough, I might be able to see more". Disclaimers were used to distance oneself from judgment: "I like it, I don't know"; "I don't know why, but I don't like it"; "Seems like he could do it better. I mean it's just a few lines, I don't know". The second category of statements which appeared in the viewers' talk were those which referred to attraction generally: "I find it very moving"; "It's really eye catching"; "It just draws me into it"; "It really hits you", and to particular elements of the painting which attracted the attention, such as colour: "I was struck initially by the vibrancy of the colors"; subjectmatter: "I guess I like more realistic paintings, and this one is realistic; you can see all the features of divisions"; puzzlement: "[I'm] trying to understand what the artist was doing by the, first of all, the separation of the right side and the left side", "I am trying to figure out what the artist is trying to do, but I am confused; and the paining technique: 'I like the way she uses paint. It is very gestural, very ... which adds more to the feeling of violence"' . The final category identified by Bruder and Ucock is storytelling which includes enigmatic, imaginative and reflective stories for which narrative skills are significant.

The research reveals what language people need to talk about art but, at the same time, shows that talking about art gives an opportunity to develop foreign language lexis and structures. The conclusion formulated by the authors emphasises indirectly why it is profitable from the wider perspective to combine art and language learning:

We have seen that art viewership is a social process. Through interactively sharing their own experiences, competencies, and judgments, viewers express and construct aspects of themselves and others. This is certainly no less true in aesthetic experience than elsewhere. Viewers verbalize (emphasis mine) their experience of artwork in order to disclose its meaning to themselves and others. In so doing, they afford themselves meaning-and, perhaps, a sense of the meaningful-that is not otherwise available. Art talk may, therefore,

1 All quoted examples have been selected from the research results reported by Bruder and Ucock (2000: 341-347). 
be understood as a social institution by which we viewers explore and engage in that most fundamental of all artifices: ourselves (Bruder \& Ucock 2000: 357).

Malinowski (2015), inspired by the work of Henri Lefebvre (1974/1991), views language learning in the linguistic landscape, of which murals are a part, as situated within three spaces, perceived, conceived and lived. In each of the spaces, a different type of language learning takes place, which roughly parallel the stages of reading art proposed by Panofsky (1939/1972). The perceived space involves reading, listening, observation and documentation. In this space, the identification of murals in the cities, or on the Internet can be situated and followed by a description of visual and/or verbal elements. This stage will be an occasion for practicing the relevant vocabulary for describing the physical features of objects. Activities in the conceived space, the space of knowledge, require much more effort and skills but, at the same time, create opportunities for the use of language for authentic purposes, such as searching for information about the artists and their works, studying historical or sociological texts, current journals or blogs on mural art. Listening comprehension may be practiced while listening, for example, to interviews with the mural artists or viewers. More advanced activities will involve discussions of the genre of the mural, comparisons with other types of painting or street art in general, or comparison between works of the same artist or works of different artists. The study of the places where murals are situated is to be taken into consideration as well, since it is the environment, both built and natural, which provides the context for the interpretation of a particular element of the linguistic landscape (Scollon \& Scollon 2003: 9). The work on the relationships between the two different systems, a mural and its environmental context, requires integrating language skills. Finally, the lived space, holistic and experiential, is a space of social relations (Lefebvre 1991) in which learners participate and in which meanings and experiences are discussed. Shohamy et al. (2010: 237) regard Lefebvre's concept of 'lived space' as a space where attitudes and perceptions become central to convey how linguistic landscape is experienced; in our case, how a mural is experienced by its viewers. Within this space, the ways of expressing emotional, moral and intellectual preferences and attitudes in dialogue with others (conversations, interviews, plays, dramas) or with oneself (diaries, poetry writing, storytelling) can be practiced.

\section{Conclusion}

In this chapter, I argued that murals, modern and historical, can play a role in mastering a foreign language, similarly to other forms of art, by providing 'food for thought' and varied opportunities of language learning and use. Also, I at- 
tempted to show that learning a foreign language can help 'reading' murals, by providing appropriate language resources and creating conditions for more focused attention on mural details. Albers and Sanders (2010: 4) observed that: "[M]ultimodal literacies instruction is pedagogy with a fundamental philosophical orientation that holds that children (and adults) learn best when engaged in complex, socially constructed, personally relevant, creative composition and interpretation of texts that incorporate a variety of meaningful communicative modes or symbol systems". A well considered use of murals is a good example of such instruction.

Murals can be exploited almost on any level of language competence and at any age. For instance, younger learners may enjoy work with the mural "Opowieść Śródecka" (Eng.: Śródka story, Poznań). This might begin with a description (shapes, colours, objects, people, actions), proceed to a discussion on likes and dislikes concerning the mural, a search for information about the district and telling its story, a simple conversation with peers about their attitudes towards the mural. Another example is the "Welcome Wall" mural by Keir Johnson and Ernel Martinez, painted at the intersection of Broadway and Fillmore in Buffalo, which spells out the word "welcome" in 13 languages (access on the Internet). It offers a great opportunity to talk about shapes and colours, but also to build language awareness by identifying languages in the mural, matching it with information about countries. The series of murals painted on Coit Tower in San Francisco, California, by the Spanish mural painter José Moya del Piño (access on the Internet) provides rich visual material for practicing verbs and tenses since each mural presents how people lived, worked and played in the 1930s in America. They are a springboard to learn and discuss the history, to learn about the town, to visit the Coit Tower museum website, to listen to videos dedicated to this place. All this provides rich material to practice all the language skills in authentic interaction with the artifacts, and with others. More advanced language learners, young adults and adults, will profit from working with the more abstract, poetic and profound murals by the South African mural artist Faith47 (access on the Internet), which deal with social and existential issues. This type of mural, besides the development of language skills, will create opportunities for self-expression in a variety of forms, for example, drama, talking about art, exchange of ideas, presentation of one's own attitudes towards the topic of a mural.

The list of examples of murals and proposals for activities is almost endless, especially that murals and other accompanying materials can be easily found either in the immediate environment or in the virtual environment. The use of murals in teaching language allows for in-class and out-of-class activities. It will provide grounds for combining language learning, content learning and development of social, political, and art awareness. Language learning, on the other 
hand, facilitates the development of skills which enhance talking about murals and art in general.

\section{References}

Abousnnouga, Gill/Machin, David (2010). War monuments and the changing discourses of nation and soldiery. In: Adam Jaworski/ Crispin Thurlow (eds.), Semiotic landscapes. Language, image, space (pp. 219-240). London, New York: Continuum.

Aladjem, Ruthi/Jou, Bibiana (2016). The linguistic landscape as a learning space for contextual language learning. Journal of Learning Spaces, 5(2), 66-70.

Alvermann, Donna E. (2002). Preface. In: Donna Alvermann (ed.), Adolescents and literacies in a digital world (pp. i-ix). New York: Peter Lang.

Avgerinou, Maria D./Erickson, John (1997). A review of the concept of visual literacy. British Journal of Educational Technology, 28(4), 280-291.

Bagna, Carla/ Gallina, Francesca/Machetti, Sabrina (2018). L'approccio del Linguistic Landscape applicato alla didattica dell'italiano L2 per studenti internazionali. In: Carmel M. Coonan/Ada Bier/Ellena Ballarin (eds.), La didattica delle lingue nel nuovo millennio. Le sfide dell'internazionalizzazione. (pp. 219-232). Venezia: Edizioni Ca'Foscari.

Barash, Moshe (1997). The language of art. Studies in interpretation. New York, London: New York University Press.

Barthes, Roland (1988). Semiotic challange. Oxford: Blackwell Publishers.

Benjamin, Walter (1997). Ulica jednokierunkowa. Warszawa: Wydawnictwo Alatheia.

Bever, Olga (2012). Linguistic landscapes and environmental print as a resource for language and literacy development in multilingual contexts. In: Sanz Montserrat/ José Manuel Igoa (eds.), Applying language science to language pedagogy. Contributions of linguistics and psycholinguistics to second language teaching (pp. 321-341). Newcastle upon Tyne: Cambridge Scholars.

Bruder, Kurt A./Ucok Ozum (2000). Interactive art. Interpretation: How viewers make sense of paintings in conversation. Symbolic Interaction, 23(4), 337-358.

Calvino, Italo (1972). Le città invisibili. Torino: Einaudi.

Cenoz, Jasone/Gorter, Durk (2008). The linguistic landscape as an additional source of input in second language acquisition. IRAL, 46, 267-287.

Chern, Chiou-Ian/Dooley, Karen (2014). Learning English by walking down the street. ELT Journal, 68(2), 113-123.

Chestnut, Michael/Lee, Vivian/Schulte Jenna (2013). The language lessons around us: Undergraduate language pedagogy and linguistic landscape research. English Teaching. Practice and Critique, 12(2), 102-120.

Chmielewska, Ella (2010). Semiosis takes place or radical uses of quaint theories. In: Adam Jaworski/Crispin Thurlow (eds.), Semiotic landscapes. Language, image, space (pp.274291). London, New York: Continuum.

Clemente, Mariana/Andrade, Ana Isabel/Martins, Filomena (2012). Learning to read the world, learning to look at the linguistic landscape: A study in the first years of formal education1. In: Christine Hélot/Monica Barni/Rudi Janssens/Carla Bagna (eds.), Lin- 
guistic landscapes, multilingualism and social Change (pp. 267-285). Frankfurt a.M. et al.: Peter Lang.

Coluzzi, Paolo (2012). Multilingual societies vs. monolingual states: The linguistic landscape in Italy and Brunei Darussalam. In: Durk Gorter/Heiko F. Marten/Luk Van Mensel (eds.), Minority languages in the linguistic landscape (pp. 225-242). London: Palgrave Macmillan.

Dagenais, Diane/Moore, Danièle/Sabatier, Cécile/Lamarre, Patricia/Armand, Françoise (2009). Linguistic landscape and language awareness. In: Elana Shohamy/Durk Gorter (eds.), Linguistic landscape. Expanding the scenery (pp. 253-269). New York, London: Routledge.

Gee, James Paul (2001). Reading as situated language: A sociocognitive perspective. Journal of Adolescent \& Adult Literacy, 44(8), 714-725.

Gee, James, Paul (2008). Social linguistics and literacies. New York: Routledge.

Gorter, Durk (2013). Linguistic landscapes in a multilingual world. Annual Review of Applied Linguistics, 33, 190-212.

Gralińska-Toborek, Agnieszka (2019). Graffiti i street art. Słowo, obraz, działanie. Łódź: Wydawnictwo Uniwersytetu Łódzkiego.

Greeley, Robin A. (2012). Muralism and the state in post-revolution Mexico, 1920-1970. In: Alejandro Anreus/Leonard Folgarait/Robin A. Greeley (eds.), Mexican muralism. A critical history (pp. 13-36). Berkeley et al.: University of California Press.

Guilat, Yael (2010). 'The Holy Ark in the Street': Sacred and secular painting of utility boxes in the public domain in a small Israeli town. In: Elana Shohamy/Eliezer Ben-Rafael/ Monica Barni (eds.), Linguistic landscape in the city (pp. 37-54). Bristol et al.: Multilingual Matters.

Hancock, Andy (2012). Capturing the linguistic landscape of Edinburgh: A pedagogical tool to investigate student teachers' understandings of cultural and linguistic diversity. In: Christine Hélot/Monica Barni/Rudi Janssens/Carla Bagna (eds.), Linguistic landscapes, multilingualism and social change (pp. 249-266). Frankfurt a.M.: Peter Lang.

Hessel, Franz (1979). Spazieren in Berlin. Beobachtungen in Jahr 1929. Berlin: Buchverlag der Morgen.

Hewitt-Bradshaw, Iris (2014). Linguistic landscape as a language learning and literacy resource in Caribbean Creole contexts. Caribbean Curriculum, 22, 157-173.

Jakobson, Roman (1985). Closing statement. Linguistics and Poetics In: Robert E. Innis (ed.), Semiotics. An introductory anthology (pp. 350-377). Bloomington: Indiana University Press.

Jaworski, Adam (2010). Linguistic landscapes on postcards: Tourist mediation and the sociolinguistic communities of contact. Sociolinguistic Studies, 4(3), 469-594.

Jaworski, Adam/Thurlow, Crispin (2011). Introducing semiotic landscapes. In: Adam Jaworski/C. Thurlow (eds.), Semiotic landscapes. Language, image, space (pp.1-40). London, New York: Continuum.

Jewitt, Carey/Kress, Gunther (2003). Multimodality, literacy and school English. In: Dominic Wyse/Richard Andrews/James Hoffman (eds.), The Routledge international handbook of English language and literacy teaching (pp. 342-353). New York: Routledge. 
Kallen, Jeffrey (2009). Tourism and representation in the Irish linguistic landscape. In: Elana Shohamy/Durk Gorter (eds.), Linguistic landscape. Expanding the scenery (pp. 270-283). New York, London: Routledge.

Kang Song, Young Imm/Gammel, Jo Ann (2011). Ecological mural as community reconnection. The International Journal of Art \& Design Education, 30(2), 266-278.

Kim, Sungwoo (2017). Linguistic landscape in pre-service teacher education: Potentials and considerations. Multimedia-assisted Language Learning, 20(4), 66-98.

Klein, Christoph/Betz, Juliane/Hirschbuehl, Martin/Fuchs, Caroline/Schmiedtová, Barbara/Engelbrecht, Martina/Mueller-Paul, Julia/Rosenberg, Raphael (2014). Describing art - An interdisciplinary approach to the effects of speaking on Gaze Movements during the beholding of paintings. PLoS One, 9(12). https://www.ncbi.nlm.nih.gov/pmc/ articles/PMC4262203/.

Koschany, Rafał (2013). Semiotyka miasta: Od lektury "tekstu” do interpretacji jako praktyki miejskiej. Studia Kulturoznawcze, 1(3), 109-124.

Koschany, Rafał (2014). Zamieszkiwanie jako interpretacja: Uwagi na temat hermeneutyki miasta. Studia Kulturoznawcze, 1(5), 65-78.

Krakauer, Siegfried (2001). Ulice. Literatura na Świecie, 8, 9.

Kress, Gunther (2015). Semiotic work: Applied linguistics and social semiotic account of Multimodality. AILA Review, 28, 49-71.

Kress, Gunther/Van Leeuwen,Theo (2001). Multimodal discourse: The modes and media of contemporary communication. London: Arnold.

Landry, Rodrigue/Bourhis, Richard Y. (1997). Linguistic landscape and ethnolinguistic vitality. An empirical study. Journal of Language and Social Psychology, 16, 23-49.

Lefebvre, Henri (1991). The production of space: Oxford: Blackwell.

Lyell, Davies (2001). Artworks: Republican murals, identity, and communication in Northern Ireland. Public Culture, 13(1), 155-158.

Łabądź, Justyna W. (2019). Street art, sztuka ulicy. Warszawa: Wydawnictwo SBM.

Malinowski, David (2010). Showing in the Korean linguistic cityscape In: Elana Shohamy/ Eliezer Ben-Rafael/Monica Barni (eds.), Linguistic landscape in the city (pp. 199-213). Bristol.: Multilingual Matters.

Malinowski, David (2015). Opening spaces in learning in the linguistic landscape. Linguistic Landscape, 1/2, 95-113.

Mor-Sommerfeld, Aura/Johnston, John (2012). Linguistic landscape - the seeing and writing of art. In: Christine Hélot/Monica Barni /Rudi Janssens /Carla Bagna (eds.), Linguistic landscape, multilingualism and social change. (pp. 155-168). Frankfurt a.M.: Peter Lang.

Ortuño, Marian Mikaylo (1994). Teaching language skills and cultural awareness with Spanish paintings. Hispania, 77(3), 500-511.

O'Toole, Michael (1994). The language of displayed art. Rutherford et al.: Fairleight Dickinson University Press.

Panofsky, Erwin (1939/1972). Studies in iconology. Oxford: Westview Press.

Pawuels, Luc (2008). Visual literacy and visual culture: Reflections on developing more varied and explicit visual competencies. The Open Communication Journal, 2, 79-85.

Pennycook, Alastair (2009). Linguistic landscapes and the transgressive semiotics of graffiti. In: Elana Shohamy/Durk Gorter (eds.), Linguistic landscape. Expanding the scenery (pp. 302-312). New York, London: Routledge. 
Pennycook, Alastair (2010). Spatial narrations: Graffscapes and city souls. In: Adam Jaworski/Crispin Thurlow (eds.), Semiotic landscapes. Language, image, space (pp.137150). London, New York: Continuum.

Pütz, Martin/Mundt, Neele (eds.) (2018). Expanding the linguistic landscape: Linguistic diversity, multimodality and the use of space as a semiotic resource. Bristol: Multilingual Matters.

Roos, Jana /Nicholas, Howard (2019). Using young learners' environments for EFL learning. Ways of working with linguistic landscapes. AILA Review, 32 (1), 91-111.

Sayer, Peter (2009). Using the linguistic landscape as a pedagogical resource. ELT Journal, 64 (2), 143-154.

Scarvaglieri, Claudio (2017). Educational landscaping: A method for raising awareness about language and communication. Language Awareness, 26 (4), 325-342.

Scinner, Jonathan/Jollifee, Lee (2017). "Wall-to-wall coverage": An introduction to murals tourism. In: Jonathan Skinner/Lee Jollifee (eds.), Murals and tourism. Heritage, politics and identity (pp. 3-24). London, New York: Routledge.

Scollon, Ron/Scollon, Suzie Wong (2003). Discourses in place: Language in the material world. London, New York: Routledge.

Shohamy, Elana (2015). LL research as expanding language and language policy. Linguistic Landscape. An International Journal, 1(1/2), 152-171.

Tatarkiewicz, Władysław (1970). Sztuka i język. Dwa wieloznaczne wyrazy. Studia Semiotyczne, 1(1), 11-22.

Treichl, Christiane (2017). Explorations in (post) modern thought and visual culture. Kassel: Kassel University Press.

Trumper-Hecht, Nira (2010). Linguistic landscape in Mixed Cities in Israel from the perspective of "Walkers": The case of Arabic. In: Elana Shohamy/Eliezer Ben-Rafael/ Monica Barni (eds.), Linguistic landscape in the city. (pp. 235-251). Bristol et al.: Multilingual Matters.

Van Leeuween, Theo (2004). Ten reasons why linguists should pay attention to visual communication. In: Philip LeVine/Ron Scollon (eds.), Discourse and technology. Multimodal discourse analysis (pp. 7-19). Washington D.C.: Georgetown University Press.

Vygotsky, Lew S. (1962). Thought and language. Boston: MIT Press.

Waliński, Jacek (2014). Implementing linguistic landscape investigations with m-learning for intercultural competence development. International Journal of Mobile and Blended Learning, 4(1), 15-25.

Wiśniewska, Danuta (2018). Reading signs in the linguistic landscape. In: Camilla Badstübner-Kizik/Verra Janikowa (eds.), Linguistic Landscape und Fremdsprachendidaktik (pp.115-136). Berlin: Peter Lang.

Zabrodskaja, Anastassia (2014). Tallin: Monolingual from above and multilingual from below. International Journal of the Sociology of Language, 228, 105-130.

Zabrodskaja, Anastassia/Milani, Tomasso M. (2014). Signs in context: Multilingual and multimodal texts in semiotic space. International Journal of the Sociology of Language, $228,1-6$.

Żyłko, Bogusław (2007). Miasto jako przedmiot semiotyki kultury. Uwagi metodologiczne. Estetyka i Krytyka, 12 (1), 65-72. 
Open-Access-Publikation im Sinne der CC-Lizenz BY-NC-ND 4.0

(C) 2020, Vandenhoeck \& Ruprecht GmbH \& Co. KG, Göttingen ISBN Print: 9783847111863 - ISBN E-Lib: 9783737011860 


\section{Autorenverzeichnis}

Sylwia Adamczak-Krysztofowicz, Prof. Dr., Uniwersytet im. Adama Mickiewicza w Poznaniu, Institut für Angewandte Linguistik, Lehrstuhl für Fremdsprachendidaktik und interkulturell orientierte Studien, Arbeitsschwerpunkte: Fremdsprachendidaktik, interkulturelles Lernen, Begegnungspädagogik, integrative Hörverstehensentwicklung, neue Medien und Hassrede; adamczak@amu.edu.pl.

Magdalena Aleksandrzak, Prof. Dr., Uniwersytet im. Adama Mickiewicza w Poznaniu, Institut für Angewandte Linguistik, Lehrstuhl für Fremdsprachendidaktik und interkulturell orientierte Studien, Arbeitsschwerpunkte: Entwicklung und Evaluierung der Fertigkeit Sprechen auf fortgeschrittenem Niveau, fremdsprachliche Unterrichtskommunikation, Interaktion in der fremdsprachlichen Bildung sowie autonomes Fremdsprachenlernen und -lehren; madaalek@amu.edu.pl.

Klaus-Dieter Baumann, Prof. Dr., Universität Leipzig, Institut für Angewandte Linguistik und Translatologie, Arbeitsschwerpunkte: Fachkommunikationsforschung (u.a. in den Bereichen Medizin, Jura, Psychologie, Soziologie), Emotionsforschung, Übersetzungswissenschaft, Kognitionsforschung und Kulturwissenschaft; klaudiebau@aol.com.

Małgorzata Bielicka, Prof. Dr., Uniwersytet im. Adama Mickiewicza w Poznaniu, Institut für Angewandte Linguistik, Lehrstuhl für Mehrsprachigkeitsforschung, Arbeitsschwerpunkte: Autonomie und Motivation im Fremdsprachenlernen, Fremdsprachendidaktik, früher Fremdsprachenunterricht, bilinguales Lernen und Lehren; malgorzata.bielicka@amu.edu.pl.

Katarzyna Bieniecka-Drzymała, Mag., Uniwersytet im. Adama Mickiewicza w Poznaniu, Institut für Angewandte Linguistik, Lehrstuhl für Fremdsprachendidaktik und interkulturell orientierte Studien, Arbeitsschwerpunkte: Fremdsprachendidaktik, Lernerautonomie, Sprachlern-Coaching, Fachsprachentranslation und Translationsdidaktik; K.Drzymała@amu.edu.pl. 
Hanka Błaszkowska, Dr., Uniwersytet im. Adama Mickiewicza w Poznaniu, Institut für Angewandte Linguistik, Lehrstuhl für Linguistische Studien zur Translation, Arbeitsschwerpunkte: Kontrastive Linguistik, Theorie, Praxis und Didaktik des Übersetzens und Dolmetschens für das Sprachenpaar Deutsch Polnisch; Hanka.Blaszkowska@amu.edu.pl.

Elżbieta Dziurewicz, Dr., Uniwersytet im. Adama Mickiewicza w Poznaniu, Institut für Angewandte Linguistik, Lehrstuhl für Kultur- und Mediendidaktik, Arbeitsschwerpunkte: Phraseologie des Deutschen, Fremdsprachendidaktik, Lexikographie; edziu@amu.edu.pl.

Martyna Gabrych, Uniwersytet im. Adama Mickiewicza w Poznaniu, margab2 @st.amu.edu.pl.

Magdalena Jurewicz, Dr., Uniwersytet im. Adama Mickiewicza w Poznaniu, Institut für Angewandte Linguistik, Lehrstuhl für Linguistische Studien zur Translation, Arbeitsschwerpunkte: Pragmalinguistik, Konsekutivdolmetschen, Didaktik und Methodik des Dolmetschens; magdalen@amu.edu.pl.

Joanna Kic-Drgas, Dr., Uniwersytet im. Adama Mickiewicza w Poznaniu, Institut für Angewandte Linguistik, Lehrstuhl für Mehrsprachigkeitsforschung, Arbeitsschwerpunkte: Fremdsprachendidaktik und -methodik, Fachsprachendidaktik und -methodik, Fremdsprachengeragogik, Fachsprachen (Wirtschaftssprache, technische Sprache); j.drgas@amu.edu.pl.

Lucyna Krenz-Brzozowska, Dr., Uniwersytet im. Adama Mickiewicza w Poznaniu, Institut für Angewandte Linguistik, Lehrstuhl für Linguistische Studien zur Translation, Arbeitsschwerpunkte: Allgemeine Übersetzungswissenschaft (Methodologie der Übersetzungswissenschaft), Dolmetschwissenschaft (Liaison-, Konsekutiv- und Simultandolmetschen, Notation, Dolmetschqualität), Fachübersetzen und Übersetzungsqualität; Lucyna.Brzozowska@amu.edu.pl.

Barbara Kryk-Kastovsky, Prof. Dr., Universität Wien, Institut für Anglistik und Amerikanistik, Arbeitsschwerpunkte: Pragmatik, Diskursanalyse, Textlinguistik, Sprachphilosophie, Semiotik, interkulturelle Kommunikation; barbara.krykkastovsky@univie.ac.at.

Joanna Kubaszczyk, Prof. Dr., Uniwersytet im. Adama Mickiewicza w Poznaniu, Institut für Angewandte Linguistik, Lehrstuhl für Kontrastive Studien, Arbeitsschwerpunkte: Übersetzungsforschung, Didaktik des Dolmetschens, kontrastive Wortbildung, kognitive Linguistik, Semantik; gluck@amu.edu.pl. 
Paweł Kubiak, Dr., Uniwersytet im. Adama Mickiewicza w Poznaniu, Institut für Angewandte Linguistik, Lehrstuhl für Linguistische Studien zur Translation, Arbeitsschwerpunkte: Übersetzungswissenschaft, Kulturwissenschaft, Politolinguistik, Metalexikographie; pawel.kubiak@amu.edu.pl.

Grzegorz Lisek, Dr., Universität Greifswald, Institut für Slawistik, Lehrstuhl für Slawische Sprachwissenschaft, Arbeitsschwerpunkte: Sprachpolitik (besonders in Polen und Deutschland), Linguistic Landscape Forschung, E-Learning, Fachsprachen; grzegorz.lisek@uni-greifswald.de.

Krystyna Mihułka, Prof. Dr., Uniwersytet Rzeszowski, Institut für Germanistik, Lehrstuhl für Glottodidaktik und Interkulturelle Kommunikation, Arbeitsschwerpunkte: Glottodidaktik, interkulturelles Lernen, interkulturelle Kommunikation, deutsch-polnische Beziehungen nach dem Jahr 1989 - gesellschaftliche Perspektive, Stereotype und Vorurteile (vor allem in den DACHL-Ländern und in Polen); kmihulka@gmail.com.

Norbert Nübler, Prof. Dr., Christian-Albrechts-Universität zu Kiel, Institut für Slavistik, Lehrstuhl für Slavistische Sprachwissenschaft, Arbeitsschwerpunkte: Geschichte der slavischen Sprachen und Toponomastik, Syntax unter dependenzgrammatischen Gesichtspunkten, Fachsprache des Rechts, Aspektologie des Russichen, Polnischen und Tschechischen; nuebler@slav.uni-kiel.de.

Anna Pieczyńska-Sulik, Dr., Uniwersytet im. Adama Mickiewicza w Poznaniu, Institut für Angewandte Linguistik, Lehrstuhl für Kontrastive Studien, Arbeitsschwerpunkte: Kontrastive Sprachwissenschaft (Deutsch - Polnisch), angewandte Semiotik, Translatorik; sul@amu.edu.pl.

Aleksandra Putowska, Mag., Uniwersytet im. Adama Mickiewicza w Poznaniu, Institut für Angewandte Linguistik, Lehrstuhl für Mehrsprachigkeitsforschung, Arbeitsschwerpunkte: Mehrsprachigkeit, Psycholinguistik, Drittspracherwerb, Glottodidaktik; aleksandra.putowska@amu.edu.pl.

Paweł Rybszleger, Dr., Uniwersytet im. Adama Mickiewicza w Poznaniu, Institut für Angewandte Linguistik, Lehrstuhl für Linguistische Studien zur Translation, Arbeitsschwerpunkte: Textlinguistik, Pragmalinguistik, interkulturelle Kommunikation, Medienlinguistik (Sprache des Internets, soziale Medien, Bürgerjournalismus); pawerybs@amu.edu.pl. 
Alicja Sakaguchi, Prof. Dr., Uniwersytet im. Adama Mickiewicza w Poznaniu, Institut für Angewandte Linguistik, Lehrstuhl für Linguistische Studien zur Translation, Arbeitsschwerpunkte: Allgemeine und angewandte Sprachwissenschaft, Esperantologie und Interlinguistik, Mystik und mystische Theologie, religiöse Sprache, Phänomenologie; a.sakaguchi@gmx.de.

Teresa Siek-Piskozub, Prof. Dr., Uniwersytet im. Adama Mickiewicza w Poznaniu, Fakultät für Anglistik, Lehrstuhl für Englische Angewandte Sprachwissenschaft und Didaktik des Englischen, Arbeitsschwerpunkte: Theorien zum Fremdsprachenlernen und -lehren, Untersuchungen zu den Prozessen des Kompetenzerwerbs in der Nicht-Muttersprache, psycholinguistische Theorien, interkulturelle Kommunikationskompetenz; piskozub@amu.edu.pl.

Justyna Sobańska, Uniwersytet im. Adama Mickiewicza w Poznaniu, js94260 @st.amu.edu.pl.

Aldona Sopata, Prof. Dr., Uniwersytet im. Adama Mickiewicza w Poznaniu, Institut für Angewandte Linguistik, Lehrstuhl für Mehrsprachigkeitsforschung, Arbeitsschwerpunkte: Psycholinguistik, Zweitspracherwerb im natürlichen und schulischen Kontext, Erstspracherwerb, Frühfremdsprachenunterricht, frühe kindliche Zweisprachigkeit, Entwicklung der Mehrsprachigkeit, Glottodidaktik; sopata@amu.edu.pl.

Augustyn Surdyk, Dr., Uniwersytet im. Adama Mickiewicza w Poznaniu, Institut für Angewandte Linguistik, Lehrstuhl für Interkulturelle Kommunikation und Ludologische Untersuchungen, Arbeitsschwerpunkte: Sprachwissenschaft, Angewandte Linguistik, Translatorik, Glottodidaktik (Englisch als Fremdsprache), Lernerautonomie, kontrastive Grammatik des Englischen und Polnischen, Phonetik des Englischen, Sprachphilosophie, Psycholinguistik, Soziolinguistik, interkulturelle Kommunikation, Ludologie, kommunikative und sprachliche Spiele u.a. im Fremdsprachenlehren, E-learning und in der Pädagogik, neue Technologien; SurdykMG@amu.edu.pl.

Anna Szczepaniak-Kozak, Prof. Dr., Uniwersytet im. Adama Mickiewicza w Poznaniu, Institut für Angewandte Linguistik, Lehrstuhl für Fremdsprachendidaktik und interkulturell orientierte Studien, Arbeitsschwerpunkte: Intersprache und interkulturelle Pragmatik, Didaktik und Methodik des Englischen als Fremdsprache, Fremdsprachenandragogik, interkulturelle Kommunikation und verbundene Kompetenzen, Forschungsmethodologie in der angewandten Linguistik, Integration von Migranten; annkozak@amu.edu.pl. 
Aleksandra Wach, Prof. Dr., Uniwersytet im. Adama Mickiewicza w Poznaniu, Fakultät für Anglistik, Lehrstuhl für Englische Angewandte Sprachwissenschaft und Didaktik des Englischen, Arbeitsschwerpunkte: Grammatik der Zweitsprache lernen und lehren, Autonomie der Lerner und Lehrer der Zweitsprache; Rolle der Erstsprache beim Lernen und Lehren der Zweitsprache; waleks@wa.amu. edu.pl.

Danuta Wiśniewska, Prof. Dr., Uniwersytet im. Adama Mickiewicza w Poznaniu, Institut für Angewandte Linguistik, Lehrstuhl für Didaktik des Englischen, Arbeitsschwerpunkte: Soziolinguistik (besonders Linguistic Landscape Studies), Forschungsmethodologie in der Angewandten Linguistik, Lehren und Lernen des Englischen als Fremdsprache, Bildung und Entwicklung der Fremdsprachenlehrer, Analyse der Forschungsgattungen; wis@amu.edu.pl.

Stephan Wolting, Prof. Dr., Uniwersytet im. Adama Mickiewicza w Poznaniu, Institut für Angewandte Linguistik, Lehrstuhl für Interkulturelle Kommunikation und Ludologische Untersuchungen, Arbeitsschwerpunkte: Interkulturelle Hermeneutik, Thanatologie in kultureller Perspektive, kreatives und biographisches, inter- bzw. transkulturelles Schreiben; wolting@amu.edu.pl.

Joanna Woźniak, Dr., Uniwersytet im. Adama Mickiewicza w Poznaniu, Institut für Angewandte Linguistik, Lehrstuhl für Kontrastive Studien, Arbeitsschwerpunkte: Kontrastive Phraseologie des Deutschen und Polnischen, Fachsprachen, Translatorik, Textlinguistik; woa@amu.edu.pl. 
Open-Access-Publikation im Sinne der CC-Lizenz BY-NC-ND 4.0

(C) 2020, Vandenhoeck \& Ruprecht GmbH \& Co. KG, Göttingen ISBN Print: 9783847111863 - ISBN E-Lib: 9783737011860 